\title{
DISCOVERY AND OPTIMISATION OF BACTERIAL NITROREDUCTASES FOR USE IN ANTI-CANCER GENE THERAPY
}

\author{
By
}

Gareth Adrian Prosser

A thesis

Submitted to the Victoria University of Wellington in fulfilment of the requirements for the degree of Doctor of Philosophy

In Biotechnology

Victoria University of Wellington 



\section{Abstract}

Nitroaromatic prodrugs are biologically inert compounds that are attractive candidates for anti-cancer therapy by virtue of their ability to be converted to potent DNA alkylating agents by nitroreductase (NTR) enzymes. In gene-directed enzyme-prodrug therapy (GDEPT), NTR-encoding therapeutic transgenes are delivered specifically to tumour cells, whereupon their expression confers host cell sensitivity to subsequent systemic administration of a nitroaromatic prodrug. The most well studied NTRGDEPT system involves reduction of the aziridinyl dinitrobenzamide prodrug CB1954 by the Escherichia coli NTR NfsB. However, low affinity of this enzyme for CB1954 has so far limited the clinical efficacy of this GDEPT combination. The research described in this thesis has primarily sought to address this limitation through identification and optimisation of novel NTR enzymes with improved nitroaromatic prodrug reductase activity.

Efficient assessment of NTR activity from large libraries of candidate enzymes requires a rapid and reliable screening system. An E. coli-based assay was developed to permit indirect assessment of relative rates of prodrug reduction by over-expressed NTRs via measurement of SOS response induction resulting from reduced prodrug-induced DNA damage. Using this assay in concert with other in vitro and in vivo tests, more than 50 native bacterial NTRs of diverse sequence and origin were assessed for their ability to reduce a panel of clinically attractive nitroaromatic prodrugs. Significantly, a number of NTRs were identified, particularly in the family of enzymes homologous to the native E. coli NTR NfsA, which displayed substantially improved activity over NfsB with CB1954 and other nitroaromatic prodrugs as substrates. This work also examined the roles of $E$. coli DNA damage repair pathways in processing of adducts induced by various nitroaromatic prodrugs. Of particular interest, nucleotide excision repair was found to be important in the processing of DNA lesions caused by 4-, but not 2-nitro group reduction products of CB1954, which suggests that there are some parallels in the mechanisms of CB1954 adduct repair in E. coli and mammalian cells. Finally, a lead NTR candidate, YcnD from Bacillus subtilis, was selected for further activity improvement through site-directed mutagenesis of active site residues. Using SOS screening, a double-site mutant was identified with 2.5 -fold improved activity over the wildtype enzyme in metabolism of the novel dinitrobenzamide mustard prodrug PR104A.

In conclusion, novel NTRs with substantially improved nitroaromatic prodrug reducing activity over previously documented enzymes were identified and characterised. These results hold significance not only for the field of NTR-GDEPT, but also for other biotechnological applications in which NTRs are becoming increasingly significant, including developmental studies, antibiotic discovery and bioremediation. Furthermore, the in vitro assays developed in this study have potential utility in the discovery and evolution of other GDEPT-relevant enzymes whose prodrug metabolism is associated with genotoxicity. 


\section{Acknowledgements}

The realisation and transformation of four years of back-breaking labour into the 200 odd pages of scientific ramble that lie before you today would not have been achievable without the aid, advice, support and general comradery of everyone around me. I am particularly indebted to Victoria University of Wellington for providing an atmosphere of learning and support that fostered my scientific appetite, and the Tertiary Education Commision for providing me with financial freedom for the first three years. Also, many thanks to the crew at the Auckland Cancer Society Research Centre, in particular Adam Patterson, Jeff Smail, Bill Wilson, Chris Guise, Sophie Syddall and Alex Mowday, for providing many of the tools, reagents and ideas that made my research possible.

To Dave, my supervisor and mentor. Without your tutorage and encouragement I would have remained a simple ignoramus, lacking insight into the stunningly fascinating world of nitroreductases and nitroaromatic prodrugs. I owe all of my scientific achievements and knowledge to you. For your own benefit, please help me get a job at Oxford.

To my fellow lab and office mates, past and present, I offer my most sincere apologies for all you have had to endure throughout our coupled working existence. In particular, my heart-felt appreciation goes out to Sha, for keeping me insane in the face of reason, to Jeremy for teaching me that organisation skills are not essential for becoming a great scientist, to Elsie for letting me do all her work for her; to Nate for introducing me to the blob fish, and to Janine for initially showing me how wonderful a life with E. coli can be. Big thanks also to all the administrative, technical and support staff in the VUW Biology department for all their fantastic work behind the scenes keeping my research afloat and possible. Craig especially, you have been a blessing to our lab. The shaking incubator wouldn't be in its current state of constant malfunction without you.

To my less laboratory-oriented friends and acquaintances, thank you for all the support, friendship and feigned interest in my work you have offered me through my years of academic isolation. Special mentions go to Bran, for giving me all his free concert tickets, to Andy, for loving Jim Carrey just that little bit more than me, and to Duncan, for constantly reminding me just how dirty my trousers actually are. Also, thanks to the folks at St. Mary's and North City Oasis in Whitby for all your kind support and prayers, and for letting me be a part of such a wonderful and caring community.

Alex, the Bright Star in the midst of my chaotic existence. Thank you for being you, for always being there and for understanding the pains and tribulations of being a grad student. I only hope I can be of equal assistance during your impending deom $\mathrm{PhD}$ studies.

Finally, to my parents: Be proud of me. I may actually start earning some money now. 


\section{Table of Contents}

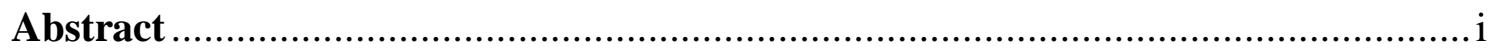

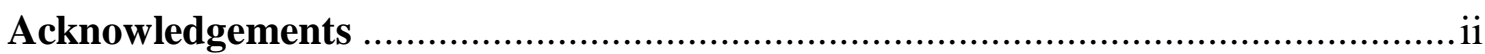

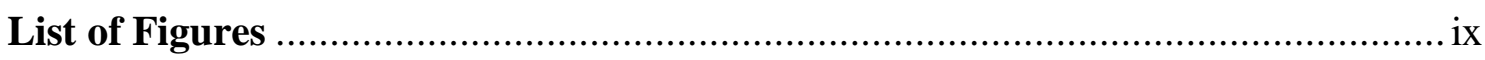

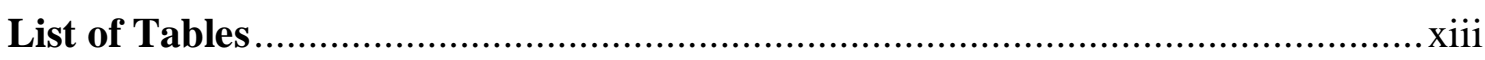

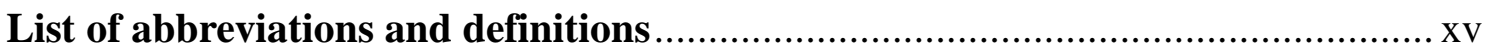

Chapter 1: Introduction ........................................................................... 1

1.1. Chemotherapy for cancer ...................................................................... 1

1.2. Targeting tumour hypoxia …………………………………………... 2

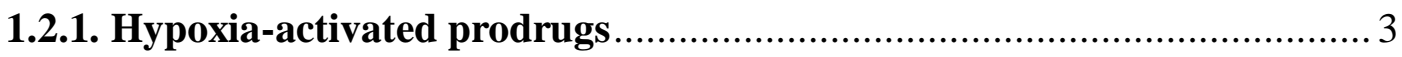

1.2.1.1. $N$-oxide and quinone hypoxia-activated prodrugs............................ 4

1.2.1.2. Nitroaromatic hypoxia-activated prodrugs ……………………..... 5

1.2.1.2.1. Dinitrobenzamide mustards (DNBMs) ...................................... 7

1.2.2. Combining hypoxia-activated prodrugs with other cancer therapies ... 10

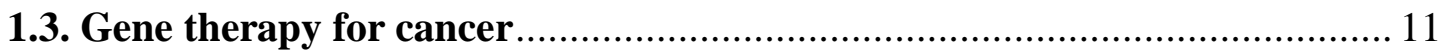

1.4. Gene-Directed Enzyme-Prodrug Therapy (GDEPT) …………………….... 12

1.4.1. Herpes simplex virus thymidine kinase - ganciclovir .............................. 13

1.4.2. Cytosine deaminase - 5-fluorocytosine ................................................. 14

1.4.3. Cytochrome P450 - cyclophosphamide. …………………………......... 16

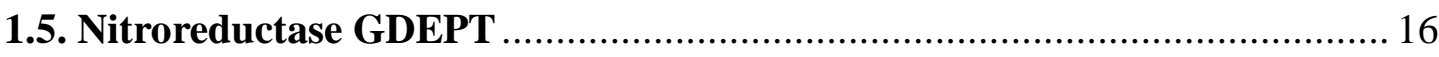

1.5.1. CB1954 and the Walker 256 Carcinoma ………………………......... 17

1.5.2. CB1954 and Escherichia coli NfsB ...................................................... 19

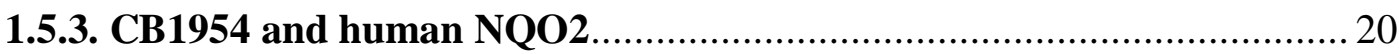

1.5.4. $E$. coli $\mathrm{NfsB}$ and alternative nitroaromatic prodrugs ............................ 21

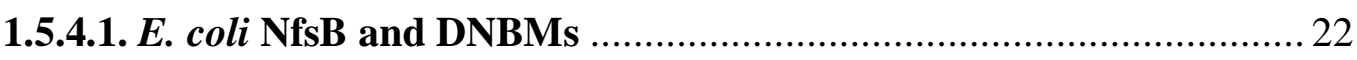

1.6. Alternative enzymes for cancer gene therapy ………………………….... 23

1.6.1. The bacterial nitroreductases: phylogenetic and biochemical properties

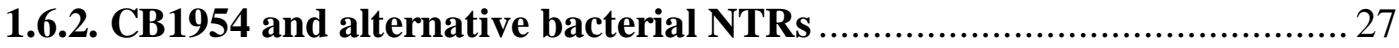

1.6.3. Enzyme engineering to improve activation of anti-cancer prodrugs..... 29

1.7. Enzyme activity screening technologies...................................................... 30

1.7.1. Screening for nitroaromatic prodrug reduction.................................... 31

1.7.1.1. Use of the $E$. coli SOS response as a screening tool ......................... 32

1.8. Research objectives ..................................................................................... 34 


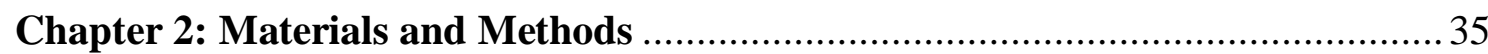

2.1. Chemicals, enzymes, reagents, media ..................................................... 35

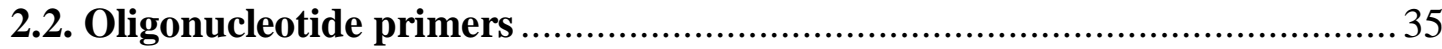

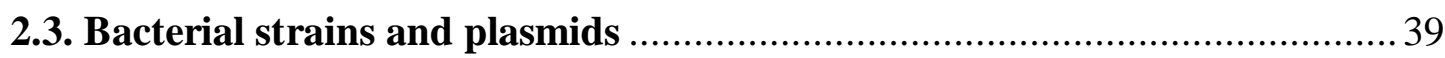

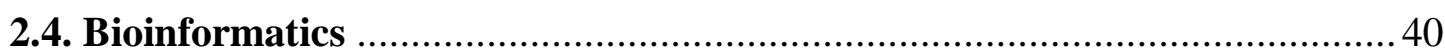

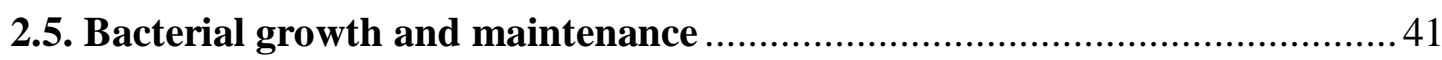

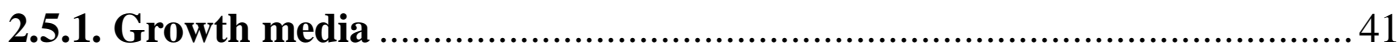

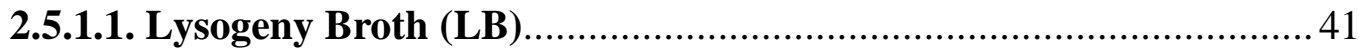

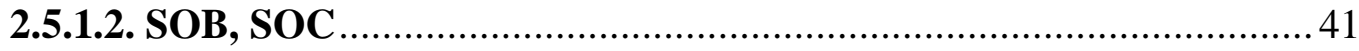

2.5.1.3. ZYP5052 (autoinduction medium) ............................................... 41

2.5.1.4. M63 minimal medium ............................................................ 42

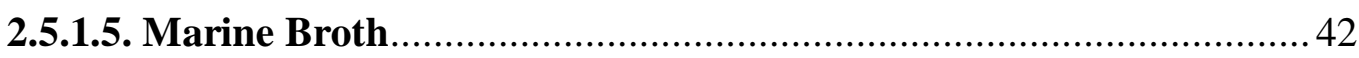

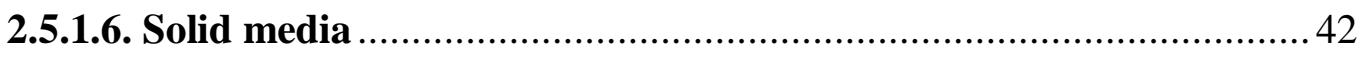

2.5.2. Antibiotics and other media supplements ....................................... 43

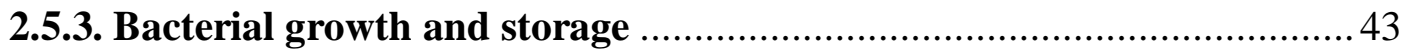

2.6. Cloning methods, routine molecular biology …..................................... 43

2.6.1. Preparation of genomic DNA (gDNA) ................................................ 43

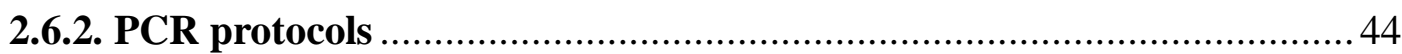

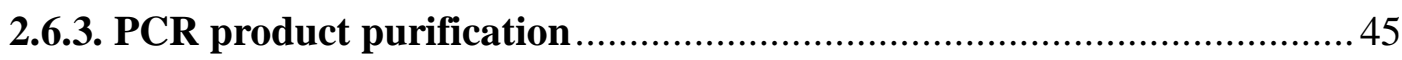

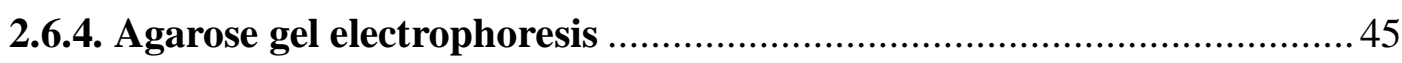

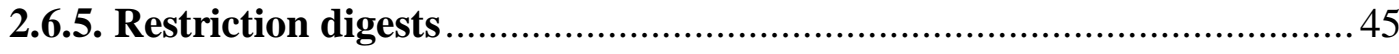

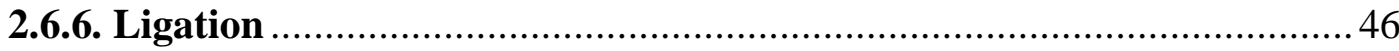

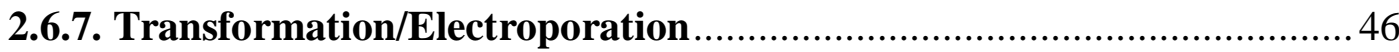

2.6.7.1. Generation of chemically competent cells .................................... 46

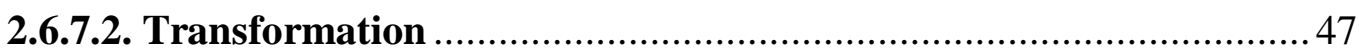

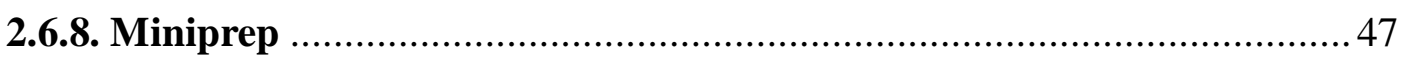

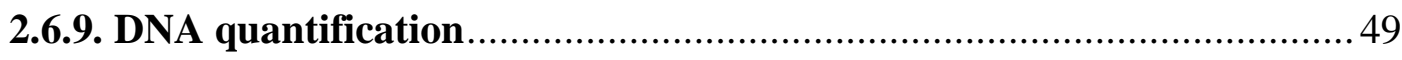

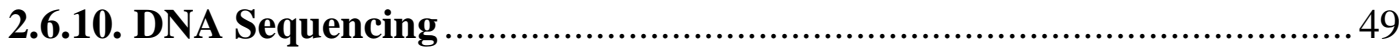

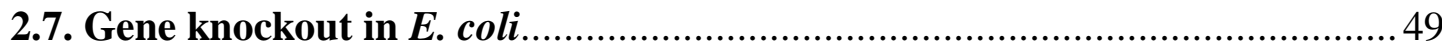

2.7.1. Gene knockout PCR amplification.................................................... 49

2.7.2. Preparation of electrocompetent cells ............................................... 50

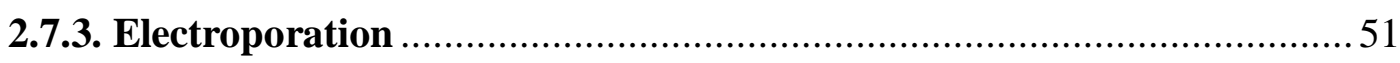

2.7.4. Curing of kanamycin resistance cassette .......................................... 51

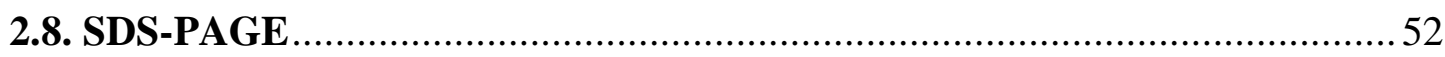

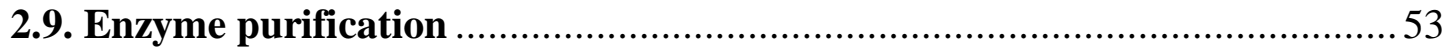


2.9.1. Enzyme expression in LB media ...................................................... 53

2.9.2. Enzyme expression in ZYP-5052 media............................................ 54

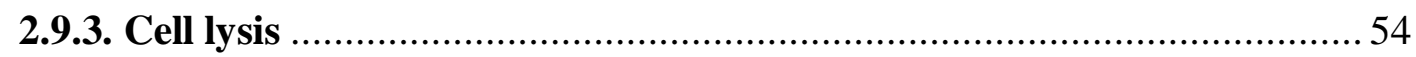

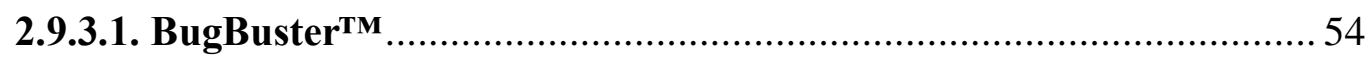

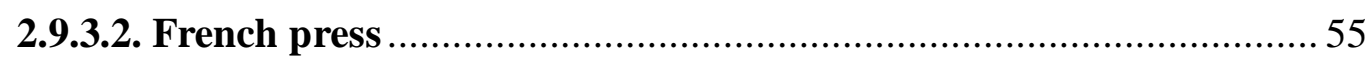

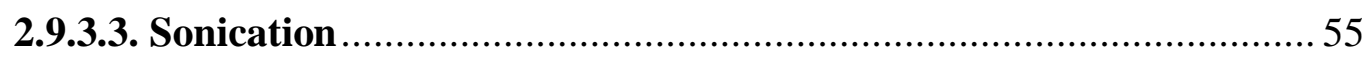

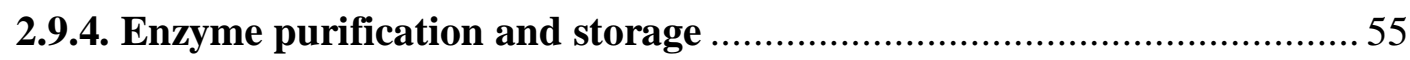

2.9.5. Removal of His6 tags from purified enzymes ................................... 56

2.9.6. Determination of enzyme concentration .............................................. 56

2.10. Determination of substrate extinction coefficients .................................. 56

2.11. In vitro kinetics of purified enzymes ......................................................... 56

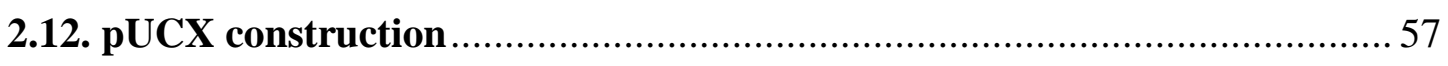

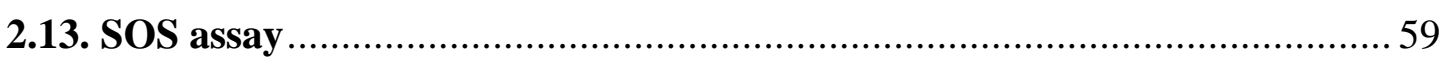

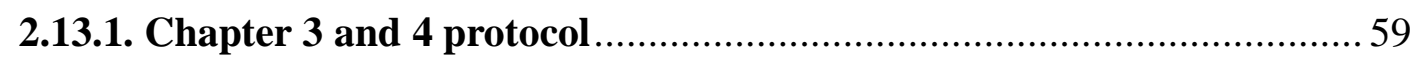

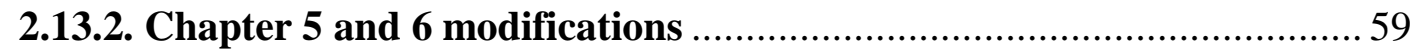

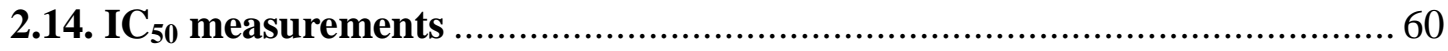

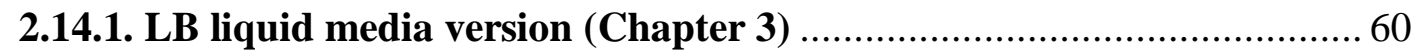

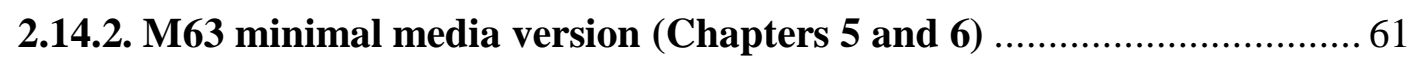

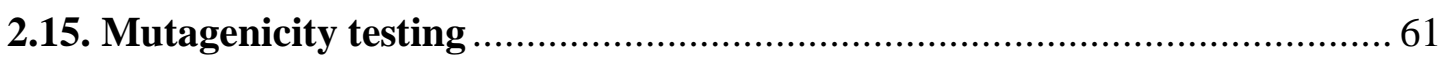

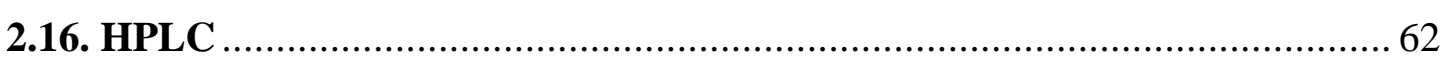

2.16.1. Identification of CB1954 nitroreduction products ...........................62

2.16.2. Identification of PR-104A nitroreduction products ......................... 62

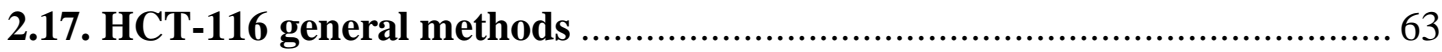

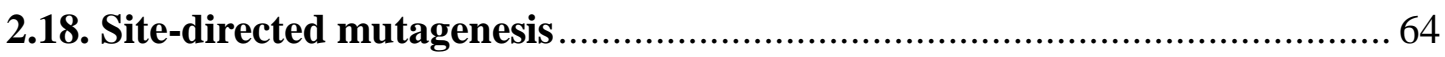

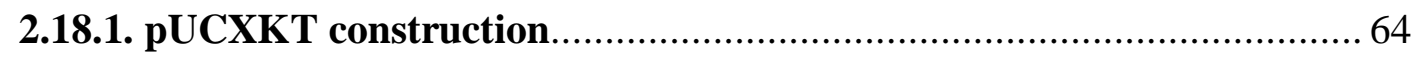

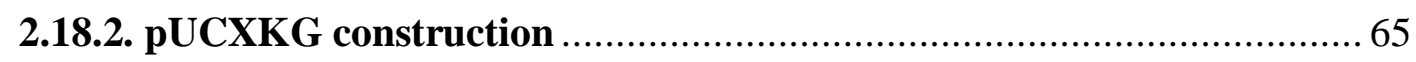

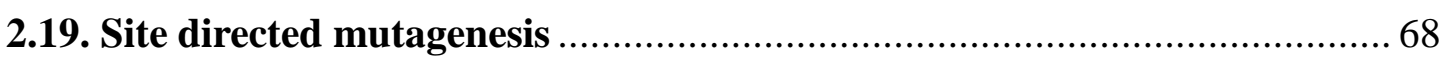

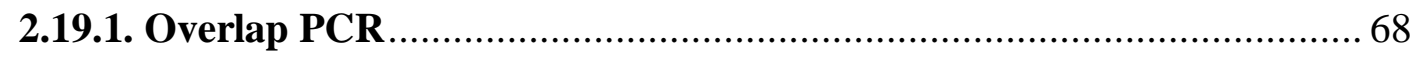

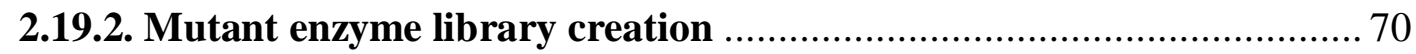

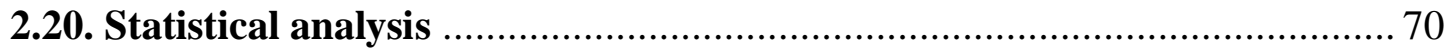

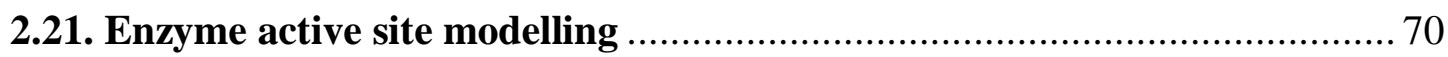

Chapter 3: Screening putative nitroreductases of $E$. coli for CB1954-reducing activity: validation of the SOS assay as a prodrug reduction screen .................... 71

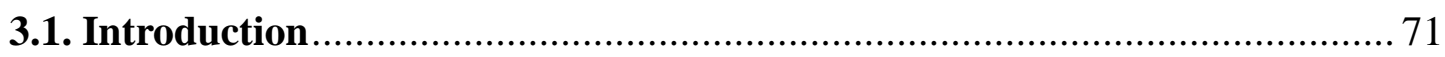

3.2. Initial testing and optimisation of SOS assay ...................................... 71 
3.2.1. Choice and optimisation of reporter strain...................................... 71

3.2.2. NTR over-expression and SOS assay optimisation............................ 73

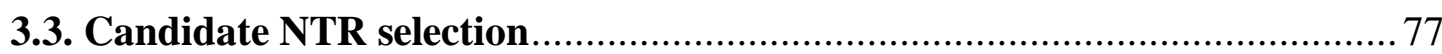

3.4. Screening candidate NTRs for CB1954-reductase activity ........................ 78

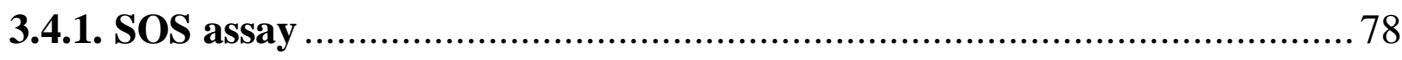

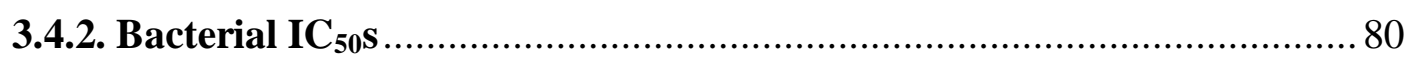

3.4.3. In vitro kinetics of purified NTR candidates with CB1954 .................. 81

3.4.4. In vivo cytotoxicity in HCT-116 human colon carcinoma cell line ......... 84

3.4.5. Identification of reduced CB1954 metabolites generated by the different

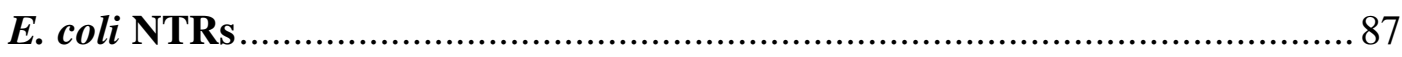

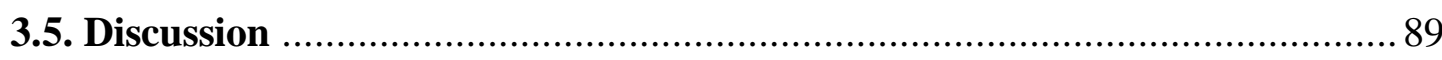

Chapter 4: Contribution of various DNA repair pathways to CB1954-adduct processing, and their effects on the SOS assay ............................................. 95

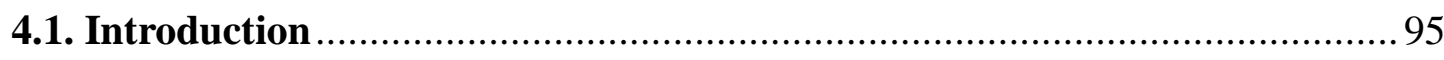

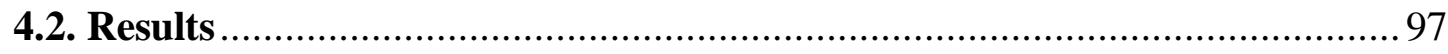

4.2.1. Development of an improved Miller Unit calculation ..........................97

4.2.2. Identification of novel NTRs for reduction of either the 2- or 4- $\mathrm{NO}_{2}$ of

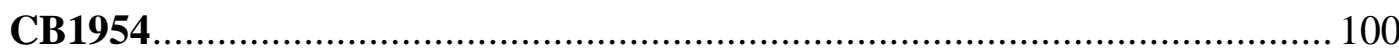

4.2.3. Generation and phenotypic characterisation of $\Delta a d a \Delta o g t, \Delta m u t S$ and $\Delta u v r B$ gene knockout strains of $E$. coli SOS-R1 .................................... 101

4.2.4. CB1954 SOS induction in DNA repair deficient and proficient strains of

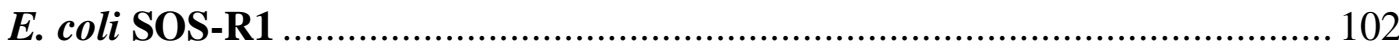

4.2.5. Role of NER in processing adducts induced by alternative nitroaromatic

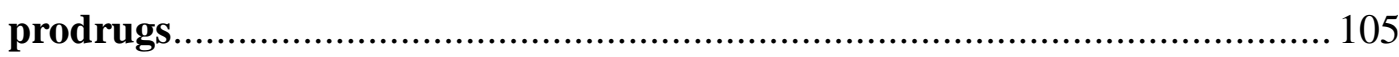

4.2.6. Mutagenicity of CB1954 reduction products .................................... 107

4.2.7. Mutational spectra (nucleotide substitutions, sequence specificity) of

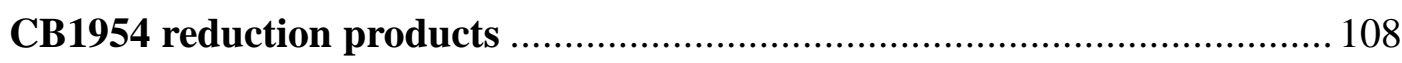

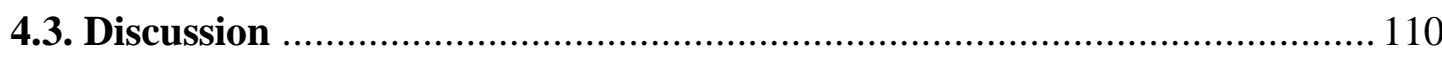

Chapter 5: Screening a phylogenetically diverse library of bacterial nitroreductases for nitroaromatic prodrug reduction.................................. 115

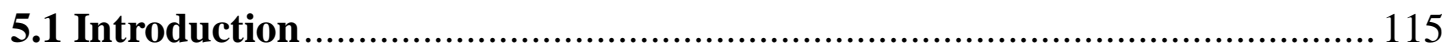

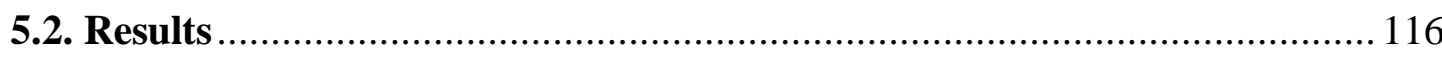

5.2.1. Further optimisation of the SOS assay ......................................... 116

5.2.2. Generation and expression of a core library of bacterial NTRs ......... 118

5.2.3. Screening the core library for CB1954-reductase activity ................. 122 


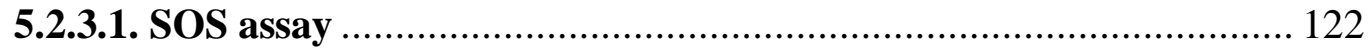

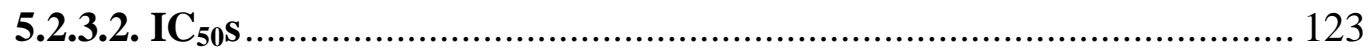

5.2.3.3. In vitro kinetics of purified enzymes............................................. 126

5.2.3.4. Identification of $\mathrm{CB} 1954$ reduction products............................... 128

5.2.4. Screening the core library for PR-104A-reductase activity ............... 131

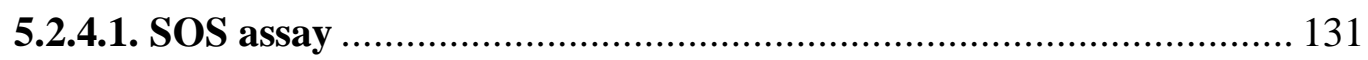

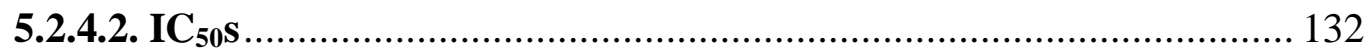

5.2.4.3. Identification of PR-104A reduction products............................ 136

5.2.4.4. In vitro kinetics of purified enzymes.............................................. 139

5.2.5. Activity screening of human AKR1C3 and select core library NTRs with mononitrobenzamide mustard prodrugs.............................................. 143

5.2.6. SOS screening of the core library with alternative nitroaromatic

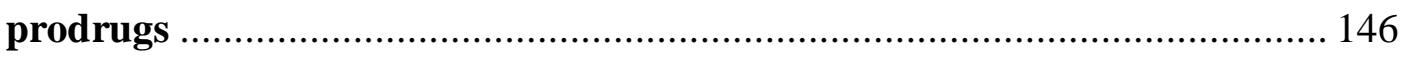

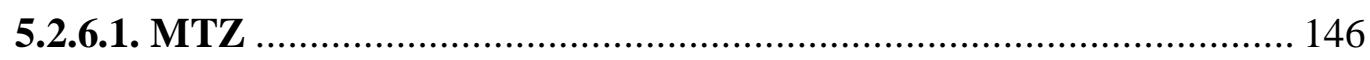

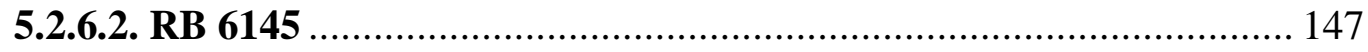

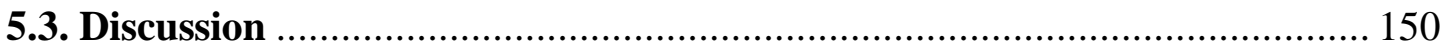

Chapter 6: Improving the PR-104A-reductase activity of Bacillus subtilis YcnD:

Saturation mutagenesis of the active site. .................................................... 155

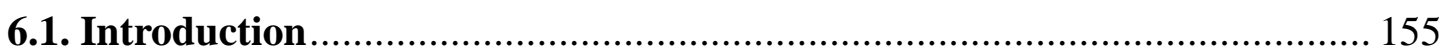

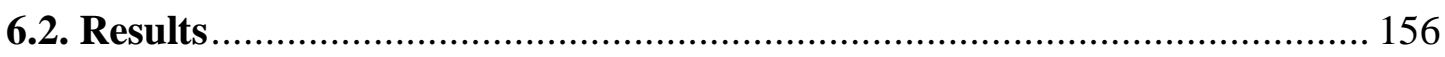

6.2.1. Generation of a positive selection vector ........................................ 156

6.2.2. Selection of YcnD (B.s) targeted residues ...................................... 161

6.2.3. Creation of mutant libraries and initial SOS screening .................... 163

6.2.4. Pairwise combinations of beneficial single mutations ....................... 171

6.2.5. $\mathrm{IC}_{50}$ determination and in vitro enzyme kinetics of lead candidate YcnD

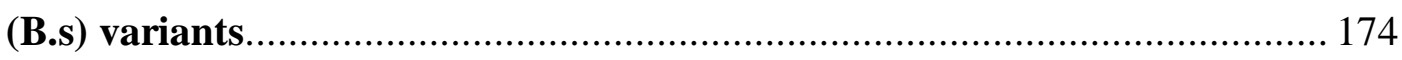

6.2.6. SOS activity with alternative nitroaromatic prodrugs .................... 178

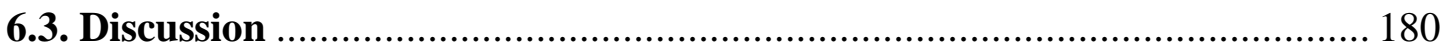

Chapter 7: Summary, conclusions and future directions. ................................ 185

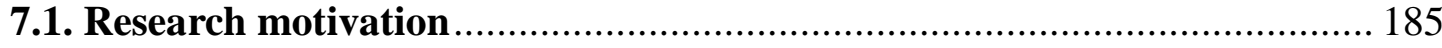

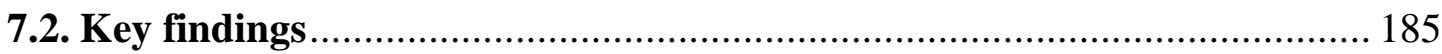

7.2.1. Development of a screen for nitroaromatic prodrug reduction ........... 185

7.2.2. Roles of various DNA repair pathways in processing and mutagenicity of CB1954-induced DNA adducts. . 
7.2.3. Testing an expanded library of bacterial NTRs for CB1954 and PR-

$104 \mathrm{~A}$ reductase activity

7.2.4. Improving the PR-104A reductase activity of $Y c n D$ (B.s) through active

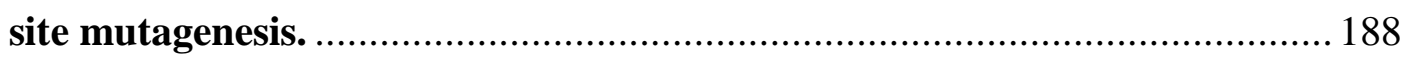

7.3. Critical evaluation and possible experimental improvements ................... 189

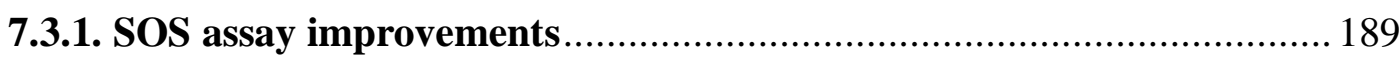

7.3.2. Validation of $E$. coli as model organism........................................ 190

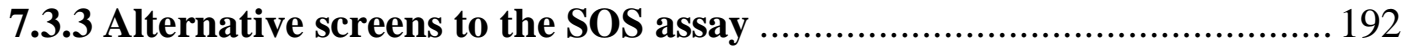

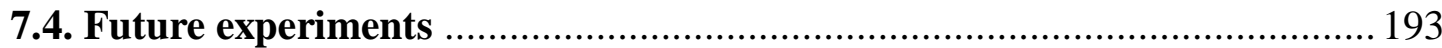

7.4.1 Structure-activity relationship studies: NTRs and prodrugs............... 193

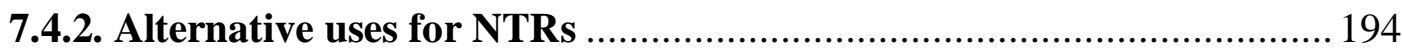

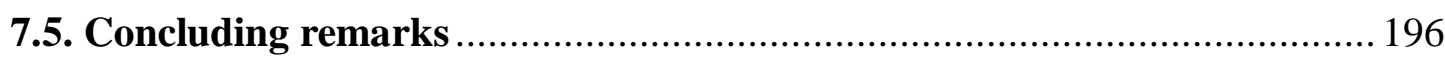

Appendix 1: NTR accession numbers and sequences ....................................... 197

Appendix 2: HPLC chromatograms of CB1954 and PR-104A reduction products 


\section{List of Figures}

Figures 1.1A \& B. Mechanism of action of hypoxia-activated prodrugs. ...................... 3

Figure 1.2. Hypoxia-activated prodrugs.............................................................. 5

Figure 1.3. Schematic overview of nitroaromatic compound reduction........................ 7

Figures 1.4A-C. Nitrogen mustards as anti-cancer chemotherapeutics........................ 8

Figures 1.5A \& B. Schematic overview of the bystander effect. ................................. 9

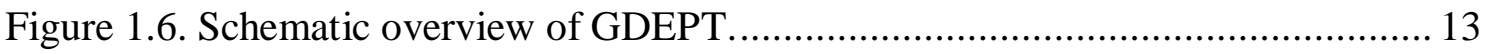

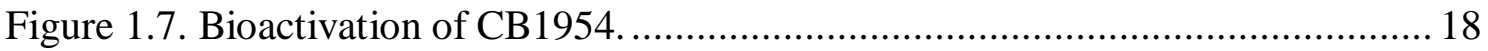

Figure 1.8. Structures of several nitroaromatic prodrugs that act as substrates for E. coli

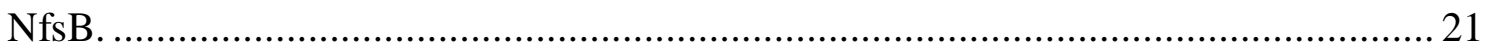

Figures 1.9A \& B. Schematic overview of the E. coli SOS response. .......................... 33

Figure 2.1. Overview of E. coli gene knockout procedure. .................................... 50

Figure 2.2. Map and MCS sequence of pUCX:empty............................................ 58

Figures 2.3A-C. pUCXKT and pUCXKG: vector maps, sequences and cloning procedure.

Figure 2.4. Schematic outline of overlap PCR protocol used for single codon mutagenesis.

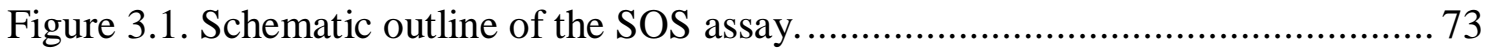

Figures 3.2A-E. Optimisation of SOS assay parameters. ........................................ 76

Figure 3.3. Relative expression levels of candidate NTRs in SOS-R1_.......................79

Figure 3.4. CB1954-induced SOS response induction of NTR over-expressing SOS-R1 strains

Figure 3.5. CB1954-mediated growth inhibition of NTR over-expressing E. coli SOS-

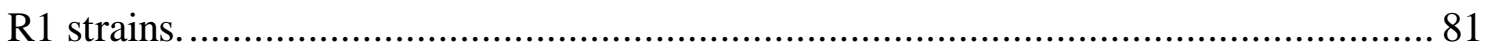

Figure 3.6. SDS-PAGE of purified, His6-tagged candidate NTRs ............................. 82

Figure 3.7. Expression of E. coli NTRs in the human colon carcinoma cell line HCT116. 86

Figures 3.8A-F. HPLC analysis of products of NTR-catalysed reduction of CB1954 . 88

Figure 4.1. Correlation between $\mathrm{OD}_{550}$ and $\mathrm{OD}_{600} \times v$ of samples used in SOS assays. 99 Figures 4.2A \& B. Variation in SOS response fold inductions from replicate cultures using different Miller equations. 
Figure 4.3. HPLC analysis of products of YfkO (B.s) and NfsA (K.p)-catalysed reduction of $\mathrm{CB} 1954$. 100

Figure 4.4. MNU-induced SOS responses from DNA repair-deficient and wildtype SOS-R1 strains.

Figure 4.5. CB1954-induced SOS responses in NTR over-expressing DNA repair proficient and deficient strains of SOS-R1

Figure 4.6. Expression of candidate NTRs from wildtype and $\Delta u v r B$ strains of SOS-R1.

Figure 4.7. CB1954 reduction metabolite-induced SOS responses from wildtype and NER-deficient strains of SOS-R1.

Figures 4.8A-C. SOS responses induced by alternative nitroaromatic prodrugs in NERproficient and -deficient NTR over-expressing strains of SOS-R1 106

Figure 4.9. CB1954-induced rifampicin resistance in wildtype and NER-deficient NTR over-expressing strains of SOS-R1. 108

Figure 5.1. Differences in nitroaromatic prodrug-induced SOS responses between tolC $^{+}$ and $t o l C^{-}$strains of SOS-R1B. 117

Figure 5.2. Phylogram of the 47 NTRs chosen for the core library. 119

Figure 5.3. Expression of core library NTRs in SOS-R2 121

Figure 5.4. CB1954-induced SOS responses from NTR over-expressing strains of SOS$\mathrm{R} 2$.

Figure 5.5. Logarithmic correlations between SOS assay results and $\mathrm{IC}_{50}$ s for CB1954challenged NTR over-expressing SOS-R2 strains. 126

Figure 5.6. SDS-PAGE of purified, His6-tagged NTRs used in this chapter. 127

Figures 5.7A \& B. Linear correlations between SOS assay results and purified NTR kinetic efficiency, CB954 as substrate.

Figure 5.8. PR-104A-induced SOS responses from NTR over-expressing strains of SOS-R2

Figures 5.9A \& B. Logarithmic correlations between SOS assay results and $\mathrm{IC}_{50} \mathrm{~s}$ for PR-104A-challenged NTR over-expressing strains of SOS-R2.

Figure 5.10. Different routes of reduction of PR-104A. 136

Figures 5.11A-F. HPLC and MS analysis of products of NTR-catalysed reduction of PR-104A.

Figures 5.12A-C. Calculation of extinction coefficient for spectrophotometric monitoring of PR-104A reduction. 
Figures 5.13A \& B. Linear correlation between SOS assay results and purified NTR catalytic efficiency, PR-104A as substrate.

Figure 5.14. Structures of mono- and dinitrobenzamide mustard prodrugs tested in this section.

Figure 5.16. SN 30444-induced SOS responses from NTR over-expressing strains of SOS-R2.

Figure 5.17. MTZ-induced SOS responses from NTR over-expressing strains of SOS-

R2. 148

Figure 5.18. RB 6145-induced SOS responses from NTR over-expressing strains of SOS-R2.

Figure 5.19. Structures of RB 6145 and selected 2-NI imaging agents. 153

Figure 6.1. Overview of cloning mechanism of pUCXKT and PUCXKG................. 158

Figure 6.2. Relative NTR expression levels from pUCX and pUCXKG. 160

Figure 6.3. Relative SOS response induction using either pUCX or pUCXKG for NTR over-expression. 160

Figures 6.4A \& B. Comparison of active site architecture between Frp (V.h) and YcnD (B.s).

Figures 6.5A-J. SOS responses of SOS-R2 strains over-expressing YcnD (B.s) variants following $3 \mathrm{~h}$ challenge with $10 \mu \mathrm{M}$ PR-104A. 168

Figure 6.6. PR-104A induced SOS responses from strains of SOS-R2 over-expressing the most active $\mathrm{YcnD}$ (B.s) single-site variants. 169

Figures 6.7A-C. PR-104A-induced SOS responses of SOS-R2 strains over-expressing double site variants of $\mathrm{YcnD}$ (B.s). 172

Figure 6.8. PR-104A-induced SOS responses from SOS-R2 strains over-expressing the most active single and double-site variants of $\mathrm{YcnD}$ (B.s).....

Figure 6.9. Logarithmic correlations between SOS assay results and $\mathrm{IC}_{50}$ values for PR104A-challenged strains of SOS-R2 over-expressing YcnD (B.s) single and double-site variants. 175

Figure 6.10. Kinetic characterisation of $\mathrm{YcnD}$ (B.s) and three active site mutants with PR-104A

Figure 6.11. Reduction of PR-104A by purified His6-tagged wildtype and mutant YcnD (B.s), using NADH or NADPH as co-substrate. 177

Figures 6.12A \& B. Reduction of alternative nitroaromatic prodrugs by purified His6tagged wildtype and mutant $\mathrm{YcnD}$ (B.s). 
xii

Figures 6.13A-C. SOS induction of wildtype and mutant YcnD (B.s) over-expressing strains of SOS-R2 following challenge with alternative nitroaromatic prodrugs......... 179 Figures 6.14A \& B. Mutagenesis-induced alterations in YcnD (B.s) active site topography. 182 


\section{List of Tables}

Table 1.1. Several GDEPT enzyme-prodrug combinations currently in clinical development.

Table 1.2. Examples of biochemically characterised bacterial NTRs.

Table 1.3. Kinetic parameters for all naturally-occurring CB1954-reducing enzymes characterised to date

Table 2.1. Oligonucleotide primers used in this study. 36

Table 2.2. Bacterial strains used in this study. 39

Table 2.3. Bacterial strains used as sources of NTRs in this study 40

Table 2.4. Plasmids used in this study. 40

Table 2.5. Properties of media supplements used in this work 43

Table 2.6. Composition of PCR reactions 44

Table 2.7. Thermal cycling parameters of PCR reactions, in chronological order. 44

Table 2.8. Composition of restriction digests. 45

Table 2.9. Composition of ligation reactions. 46

Table 2.10. Recipes for transformation buffers. 47

Table 2.11. Recipes of buffers used in miniprep protocol described in section 2.6.8 ... 48

Table 2.12. Recipes of gels and buffers used in SDS-PAGE. 53

Table 2.13. Recipes of buffers required for measurement of $\beta$-galactosidase activity in SOS assays. 60

Table 3.1: Steady-state kinetic parameters of purified His6-tagged candidate NTRs with CB1954.

Table 3.2. Relative sensitivity of the HCT-116 cell lines to CB1954 per unit NTR protein expressed.

Table 4.1. Kinetic parameters and end-products of CB1954 metabolism for each purified, His6-tagged candidate NTR.

Table 4.2. Frequency and specificity of base pair substitutions within the E. coli rpoB gene induced by each CB1954 reduction product

Table 4.3. Frequency and sequence specificity of mutations within the E. coli rpoB gene induced by the two principal reduction products of CB1954. 
xiv

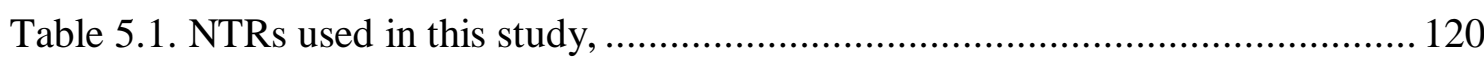

Table 5.2. $\mathrm{IC}_{50} \mathrm{~S}$ of CB1954 in NTR over-expressing strains of SOS-R2 .................. 125

Table 5.3. Kinetic parameters for the reduction of CB1954 by purified, His6-tagged

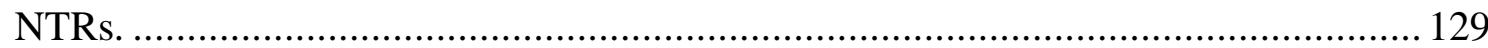

Table 5.4. $\mathrm{IC}_{50} \mathrm{~S}$ of PR-104A in NTR over-expressing strains of SOS-R2 ................. 134

Table 5.5. Kinetic parameters for the reduction of PR-104A by purified His6-tagged

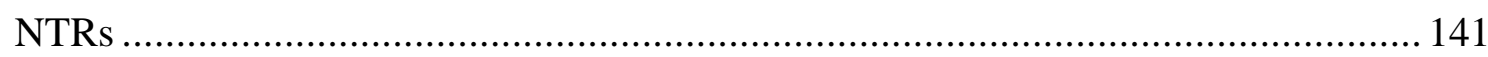

Table 5.6. Kinetic parameters for the reduction of selected DNBM and MNBM

prodrugs with purified his6-tagged AKR1C3.

Table 6.1. Amino acid substitutions of the 20 most active YcnD (B.s) single-site variants with PR-104A. 170

Table 6.2. $\mathrm{IC}_{50}$ values of PR-104A in SOS-R2 over-expressing wildtype and active site mutants of $\mathrm{YcnD}$ (B.s). 174

Table 6.3. Kinetic parameters for the reduction of PR-104A by purified His6-tagged wildtype and evolved YcnD (B.s) 176

Table A1.1. NCBI database accession numbers of NTRs used throughout this work. 197 


\section{List of abbreviations and definitions}

$\begin{array}{ll}\text { 5-FC } & \text { 5-fluorocytosine } \\ \text { 5-FU } & \text { 5-fluorouracil }\end{array}$

Acetyl CoA Acetyl Co-enzyme A

ACSRC Auckland Cancer Society Research Centre

ACV Aciclovir

AKR1C3

Aldo/keto reductase $1 \mathrm{C} 3$

Amp

Ampicillin

$\mathbf{A U}$

Absorbance units (arbitrary)

BER

Base excision repair

BLAST Basic Local Alignment Search Tool

BSA

Bovine Serum Albumin

CD

Cytosine deaminase

CPG2

Carboxypeptidase G2

CYP450

Cytochrome P450

CYP450R

Cytochrome $\mathrm{P} 450$ reductase

DMSO

Dimethylsulfoxide

DNBM

Dinitrobenzamide mustard

$\mathrm{e}^{-}$

Electron

FACS

Fluorescence-activated cell sorting

FMN

Flavin mononucleotide

GCV

Ganciclovir

GDEPT

Gene-directed enzyme-prodrug therapy

Gfp

Green fluorescent protein

HIF

Hypoxia-inducible factor

HPLC High-performance liquid chromatography

HSVtk Herpes simplex virus thymidine kinase

$\mathrm{IC}_{50}$

Concentration of test compound required to inhibit growth of test organism to $50 \%$ of control (unchallenged) levels

IFO

Ifosfamide

Kan

Kanamycin

$\boldsymbol{k}_{\text {cat }}$

Kinetic constant describing theoretical maximum rate of catalysis for a specific enzyme with a specific substrate, under the conditions tested.

Kinetic constant describing the concentration of substrate at which rate of

$K_{\mathbf{m}}$

LB catalysis of a specific enzyme is exactly half of the Kcat, under the conditions tested.

MCS

Lysogeny broth

MMC

Multi-cloning site

MMR

Mitomycin C

(DNA) Mismatch repair 


\begin{tabular}{|c|c|}
\hline MNBM & Mononitrobenzamide mustard \\
\hline MS & Mass spectrometry \\
\hline MTD & Maximum tolerated dose \\
\hline MTZ & Metronidazole \\
\hline NADH & Nicotinamide adenine dinucleotide \\
\hline NADPH & Nicotinamide adenine dinucleotide phosphate \\
\hline NCBI & National Centre for Biotechnology Information \\
\hline NER & Nucleotide excision repair \\
\hline NFZ & Nitrofurazone \\
\hline $\mathbf{N H}_{2}$ & Amino derivative \\
\hline NHOH & Hydroxylamino derivative \\
\hline NI & Nitroimidazole (2-NI, 5-NI: 2- or 5-nitroimidazole) \\
\hline $\mathrm{NO}_{2}$ & Nitro group \\
\hline NQO1/2 & NAD(P)H:Quinone oxidoreductase 1/2 \\
\hline NTR & Nitroreductase \\
\hline OD & Optical density (at a certain wavelength) \\
\hline OYE & Old yellow enzyme \\
\hline PCR & Polymerase chain reaction \\
\hline PET & Positron emission tomography \\
\hline RBS & Ribosome binding site \\
\hline Rif & Rifampicin \\
\hline rpm & Revolutions per minute \\
\hline SD & Standard deviation \\
\hline SEM & Standard error of the mean \\
\hline TLS & Translesion synthesis \\
\hline TNT & Trinitrotoluene \\
\hline TPZ & Tirapazamine \\
\hline Tris & Tris(hydroxymethyl)aminomethane \\
\hline$v /[\mathrm{E}]$ & Catalytic rate (reaction velocity/enzyme concentration) \\
\hline $\mathbf{v} / \mathbf{v}$ & Volume per volume \\
\hline $\mathbf{w} / \mathbf{v}$ & Weight per volume \\
\hline
\end{tabular}




\section{Chapter 1: Introduction}

\subsection{Chemotherapy for cancer}

Cancer is currently one of the leading causes of death worldwide, with the number of new cases and mortality rates only expected to rise over the next 50 years due to an expanding and ageing global population (1). Chemotherapy, the use of small molecular weight compounds to kill tumour cells, is currently a leading treatment option for many cancer types. "Traditional" chemotherapeutic drugs primarily exert their toxicity through the suppression of processes involved in cell division, thereby minimizing cancerous cell proliferation. Many classes of anti-cancer drugs have been developed and are in clinical use today, including DNA alkylating agents that directly damage DNA, nucleotide analogues (anti-metabolites) that interfere with DNA replication, microtubule stabilising and destabilising agents that prevent chromosomal segregation, and topoisomerase inhibitors, among others (2). Although they constitute the mainstay of current cancer chemotherapeutics, their effectiveness is severely hampered by high systemic toxicity (including myelosuppression, alopecia, gastrointestinal disorders and infertility) due to low cancer cell-type specificity, and high prevalence of drug resistance development (3-5). Chemotherapies that specifically target diseased cells, by exploiting unique molecular properties associated with cancer progression have therefore emerged in recent years to address many of these limitations (6). These chemotherapies have typically been small molecule inhibitors of, or monoclonal antibodies raised against certain receptor tyrosine kinases or their associated pathways, many of which are upregulated in cancer and found to be essential for tumour growth and survival (7). However, cross-reactivity of these therapies with molecular targets in healthy cells, and intratumoural molecular heterogeneity has been shown to be a major contributor to diminished therapeutic efficacy of many of these therapies $(8 ; 9)$; nevertheless, targeted chemotherapies are becoming more clinically prevalent, with over $70 \%$ of anti-cancer drugs approved by the US Food and Drug Administration in the last decade fitting this description (10). 


\subsection{Targeting tumour hypoxia}

To be effective, cancer chemotherapy need not solely target the cancer cells themselves. Growth and progression of tumours is dependent not only on internal genetic abnormalities and inter-cellular signalling, but also the microenvironment in which they reside, thus providing a novel target for chemotherapy. Factors such as tumour vasculature, stromal composition and tumour oxygenation have all been exploited in the design of chemotherapeutic drugs, with many beneficial results $(11 ; 12)$.

A significant feature of many solid tumours is the presence of regions of hypoxia, characterised by greatly reduced oxygen tensions $(<10 \mathrm{~mm} \mathrm{Hg}$ partial pressure) relative to those found under normal physiological conditions (40-60 $\mathrm{mm} \mathrm{Hg}$; (13)). Hypoxia is a product of the inadequate and disorganized nature of the tumour vasculature network, which prevents sufficient oxygenation of all tumour cells. As such, chronic hypoxia is largely a characteristic unique to solid tumours and not found in healthy tissue. Hypoxia is detrimental to the efficacy of traditional anticancer therapies for several reasons. First, the correlation between hypoxic regions and increasing distance from vasculature creates difficulties in tumour penetration for many circulating drugs (14). Second, the decreased cellular growth rate associated with regions of hypoxia renders them resistant to many antiproliferative chemotherapeutics (15). Third, a link between hypoxia and up-regulation of certain proteins involved in multi-drug resistance, including p-glycoproteins has been observed $(16 ; 17)$. Fourth, hypoxic cells are much less sensitive to the effects of radiation therapy, which requires molecular oxygen to aid in DNA damage induction (11). Finally, hypoxia is generally indicative of a poor prognosis, correlating with enhanced malignant phenotypes. This correlation is largely due to over-expression of hypoxia-inducible transcription factors (HIFs) in hypoxic cells, which mediate the expression of other genes involved in nutrient transport, cell survival, proliferation and metastasis (18). On the other hand, due to its unique association with solid tumours, hypoxia presents an attractive target for anticancer drug design. To this end, some success has been observed in the specific targeting and inhibition of the HIF family of proteins themselves, although many of the small molecule inhibitors identified to date are not specific for these enzymes and are therefore associated with much off-target toxicity (19). 


\subsubsection{Hypoxia-activated prodrugs}

More effective hypoxia-selectivity and toxicity has been demonstrated for prodrugs designed to specifically be activated under hypoxia. The best-characterised hypoxiaactivated prodrugs developed so far are the bioreductive prodrugs, which require reductive activation by endogenous cellular reductases to elicit cytotoxicity $(20 ; 21)$. Multiple cellular oxidoreductase enzymes such as cytochrome P450s (CYP450) and cytochrome $\mathrm{P} 450$ reductase (CYP450R) are capable of one-electron reduction of these compounds. The first released product of enzyme-catalysed reduction is an anion radical that is efficiently and non-enzymatically back-oxidised to the parental compound by molecular oxygen, if present. Such 'futile-cycling' results in the net production of rapidly degraded superoxide ions and therefore limited toxicity (see Figure 1.1). In the absence of molecular oxygen, this radical is stabilized and can either inflict DNA damage itself, or be further reduced or metabolized to intermediates capable of reacting readily with DNA and other macromolecules (11). Therefore, prodrug activation is limited to regions of extreme hypoxia, as typically found in solid tumours. Examples of hypoxia-activated prodrugs currently under various stages of clinical and pre-clinical development are illustrated in Figure 1.2.

\section{A Oxic cell}

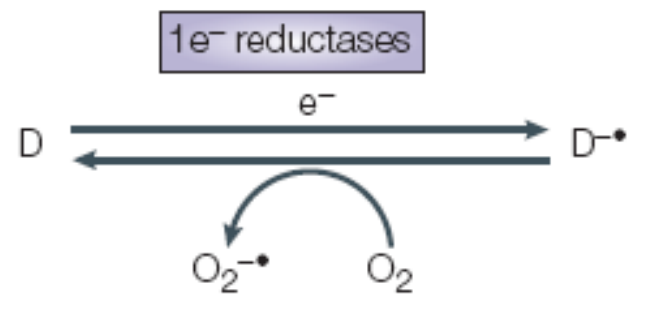

B Hypoxic cell

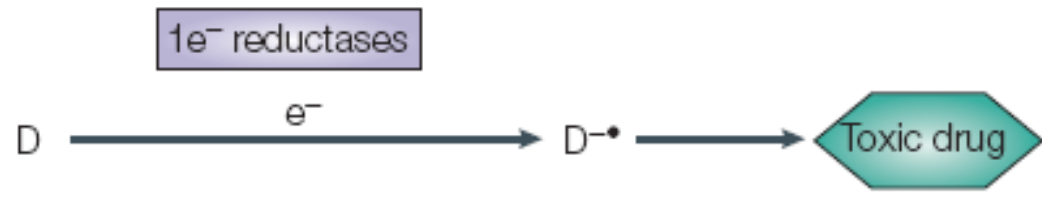

Figures 1.1A \& B. Mechanism of action of hypoxia-activated prodrugs.

A prodrug (D) is a substrate for one-electron reductases (such as CYP450R). In the presence of oxygen (A), the one-electron reduction product is efficiently and rapidly back-oxidised to the parent compound, with the net production of one super-oxide radical anion. In the absence of oxygen (B), further metabolism beyond the one-electron reduction product can take place, permitting accumulation of the desired toxic end product. Figure reproduced from Brown and Wilson (11). 


\subsubsection{1. $N$-oxide and quinone hypoxia-activated prodrugs}

One of the first hypoxia-activated prodrugs to be developed was the benzotriene di- $N$ oxide tirapazamine (TPZ; see Figure 1.2), which under hypoxia experiences bioreduction to an oxidizing nitroxide radical capable of DNA strand cleavage, topoisomerase II poisoning and inhibition of DNA replication (22-24). Despite a wealth of promising pre-clinical data, clinical efficacy of this prodrug has largely been limited by off-target toxicity (25) and minimal tumour penetration of the activated prodrug (due to the very short half-lives of the cytotoxic metabolites; (26)). Analogues of TPZ that can overcome these limitations are currently being investigated $(27 ; 28)$. Other well characterised hypoxia-activated prodrugs include the naturally-occurring quinone compound mitomycin C (MMC) and its synthetic analogues such as porfiromycin, EO9 and AZQ. Similar to TPZ, the reduced (semiquinone) derivatives of quinone hypoxiaactivated prodrugs are able to kill cells by directly inducing cytotoxic DNA lesions (29). However, the hypoxia selectivity ratio (drug dose required for equitoxicity in normoxic versus hypoxic cells) of the quinone hypoxia-activated prodrugs are low (3-7 for MMC, compared to up to 200 for TPZ; (30)) limiting their utility as a targeted therapy $(21 ; 31$; 32). This is largely a consequence of the efficient reduction of these compounds by twoelectron (aerobic, or oxygen-insensitive)-reductases, especially NAD(P)H:Quinone Oxidoreductase (NQO1, or DT-Diaphorase; (33)). These enzymes convert the parental prodrug directly to the final cytotoxin in a reduction step involving the simultaneous transfer of two electrons, therefore bypassing the initial unstable one electron radical reduction product. As such, they function in normoxic cells, including healthy tissue, and their presence is highly implicated in systemic toxicity imparted by the use of these compounds. 


\section{N-oxides}<smiles>Nc1n[n+]([O-])c2ccccc2[n+]1[O-]</smiles>

Tirapazamine<smiles>C[N+](C)([O-])CCNc1ccc(NCC[N+](C)(C)[O-])c2c1C(=O)c1c(O)ccc(O)c1C2=O</smiles><smiles>Cn1c(/C=C/CO)c(CO)c2c1C(=O)C(N1CC1)=CC2=O</smiles>

EO9<smiles>NC(=O)OCC1=C(N2CC2)C(=O)C(COC(N)=O)=C(N2CC2)C1=O</smiles>

AZQ

Porfiromycin $\quad \mathrm{R}=\mathrm{CH}_{3}$

\section{Nitroaromatics}<smiles>O=[N+]([O-])c1nccn1CC(O)CO</smiles>

Misonidazole<smiles>O=[N+]([O-])c1nccn1CC(O)CN1CC1</smiles>

RSU 1069<smiles>O=[N+]([O-])c1nccn1CC(O)CNCCBr</smiles>

RB 6145<smiles>Cn1c(COP(=O)(NCCBr)NCCBr)cnc1[N+](=O)[O-]</smiles>

TH-302

Figure 1.2. Hypoxia-activated prodrugs.

Structures of several compounds currently under investigation as hypoxia-activated prodrugs for anticancer therapy.

\subsubsection{Nitroaromatic hypoxia-activated prodrugs}

One of the most promising families of hypoxia-activated prodrugs is the nitroaromatic bioreductives. Hypoxia-selectivity has long been associated with compounds containing bioreducible nitroaromatic groups, ever since the initial elucidation of the mode of action of radiosensitizing compounds such as misonidazole and other 2-nitroimidazoles 
(2-NIs) (34). Nitroaromatic compounds are reduced sequentially at the nitro group to form nitroso ( 2 electron), hydroxylamino (4 electron) and amino (6 electron) derivatives (Figure 1.3); the hydroxylamino product, however, is the most common end metabolite observed for the reduction of the vast majority of nitroaromatics due to the very low redox potential required for further reduction to the amine (35). Nitro groups are inherently electron-withdrawing and provide stability to other functional groups. Conversely, nitroreduction causes a rearrangement of electron densities within the aromatic ring that converts the compound to a reactive and unstable electrophile. The change in electronic effect resulting from reduction of an aromatic nitro group to the hydroxylamine is among the largest known for a single catalytic step (36), making such a reaction highly attractive as an electronic 'switch' for the conversion of an inert prodrug to a potent cytotoxin. Further cytotoxicity can be applied to nitroaromatic prodrugs upon addition of other functional groups, the activity of which is suppressed until nitroreduction occurs. This characteristic was initially explored in development of the 2-NI prodrug RSU 1069, which contains a dormant aziridinyl functional group on the N1 side chain. Upon nitroreduction, the activation of this pre-positioned aziridine group converts this compound into a bifunctional DNA-crosslinking agent (37). Interstrand crosslinks produced by bifunctional DNA alkylating agents are much more cytotoxic than monoadducts, due to an inherent inability of host cell DNA-repair machinery to process them (38). Bifunctional alkylating agents are therefore much more attractive as anti-cancer agents than monofunctional drugs. RSU 1069 was shown to be 20-200-fold more toxic under hypoxia than normoxia when tested in a variety of mammalian cell lines $(39 ; 40)$ in vitro. However, a high incidence of gastrointestinal distress among trialled patients, considered to be caused by the incompletely repressed activity of the aziridinyl functional group of the parental compound, has impaired its entry into clinical use $(41 ; 42)$. An analogue with less toxic side effects but similar DNA-alkylating reactivity and hypoxia selectivity profiles, RB 6145, has also been unsuccessful due to induction of irreversible retinal damage in preclinical mouse models (43). Another 2-nitroimidazole hypoxia-activated prodrug, the ifophosphoramidereleasing prodrug TH-302, has shown enhanced pre-clinical potential, including greater hypoxic cell selectivity and a higher therapeutic index relative to other 2-NIs, and is currently in phase I clinical trials (44). 


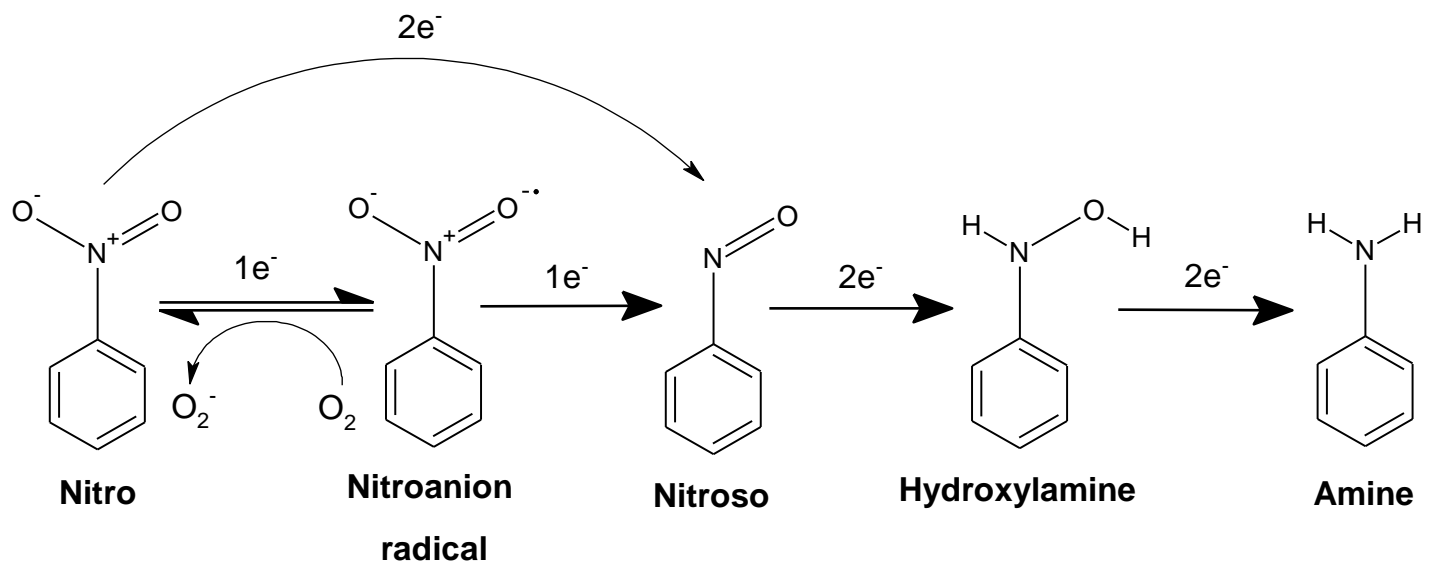

Figure 1.3. Schematic overview of nitroaromatic compound reduction.

The initial one-electron reduction step of the parental nitroaromatic, catalysed by various intracellular reductases, results in an unstable nitro-anion radical that can react with molecular oxygen to reform the parental nitro compound, with the concomitant production of superoxide. In the absence of oxygen, or via a two-electron reduction step (as catalysed by oxygen-insensitive nitroreductases) the nitro group can be irreversibly reduced to the nitroso metabolite, and further to the hydroxylamino (4-electron) and amino (6-electron) reduction products.

\subsection{Dinitrobenzamide mustards (DNBMs)}

The proven capacity of nitroaromatics to function as hypoxia-activated prodrugs has initiated the development of a host of next-generation lead compounds, chief among these being the dinitrobenzamide mustards (DNBMs; examples shown in Figure 1.4A). This class of compound was originally developed following investigations into the enhancement of toxicity and hypoxia selectivity of nitrophenyl prodrugs, through the addition of a nitrogen mustard functional group (45). The alkylating properties of the dormant mustard are restored following nitroreduction, in a mechanism akin to that of RSU 1069 and its aziridinyl functional group. The anti-cancer properties of nitrogen mustards have been acknowledged for over half a century, with some of the very first anti-cancer drugs belonging to this family of compounds (46). Nitrogen mustards act as bifunctional alkylating agents and are capable of forming long-lasting DNA interstrand crosslinks in cells, at high frequency (47) (see Figure 1.4C). Their effectiveness and cytotoxicity has ensured their inclusion as functional groups in a variety of clinically approved anti-cancer drugs, including chlorambucil, bendamustine and cyclophosphamide (Figure 1.4B). The nature of the lesions induced by these compounds is particularly attractive for hypoxia-activated prodrug therapy, as the non-cycling/slow growing nature of many hypoxic cells requires that toxic and stable DNA adducts be 
formed in order to ensure cell death occurs either in the absence of cell division or once cycling restarts $(48 ; 49)$.

A<smiles>NC(=O)c1cc(N(CCCl)CCCl)c([N+](=O)[O-])cc1[N+](=O)[O-]</smiles>

SN 23862<smiles>Cn1c(CCCC(=O)O)nc2cc(N(CCCl)CCCl)ccc21</smiles>

Bendamustine<smiles></smiles>

PR-104<smiles>O=C(O)CCCc1ccc(N(CCCl)CCCl)cc1</smiles>

Chlorambucil<smiles>O=P1(N(CCCl)CCCl)NCCCO1</smiles>

Cyclophosphamide

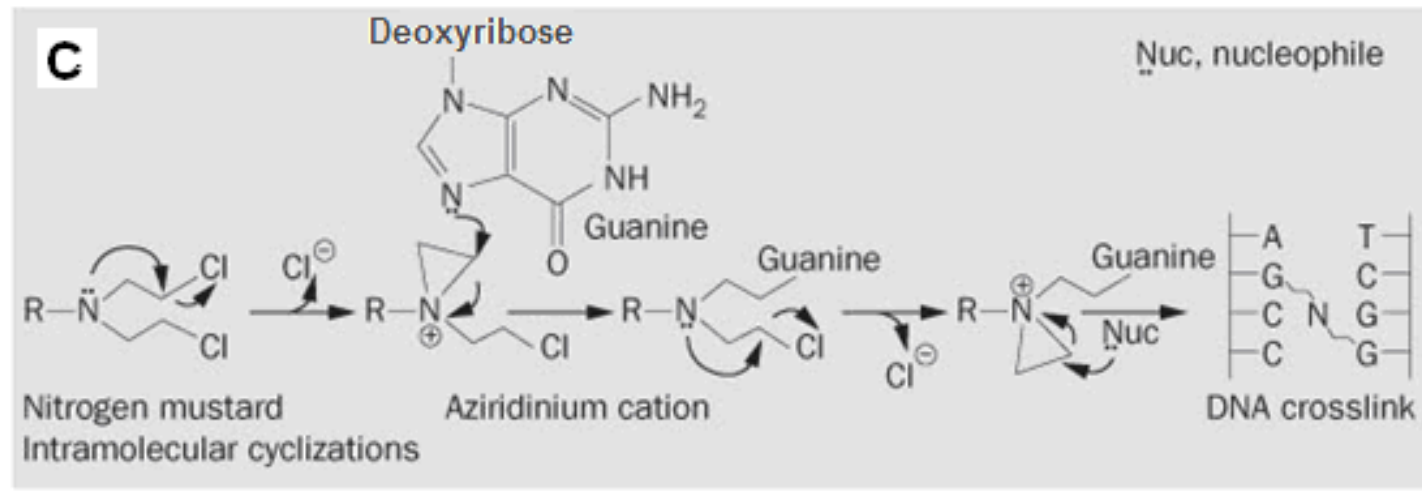

Figures 1.4A-C. Nitrogen mustards as anti-cancer chemotherapeutics.

(A) Structures of two DNBM prodrugs. (B) Structures of other nitrogen mustard containing anti-cancer drugs. (C) Mechanism of toxicity of nitrogen mustards. Figure 1.4C adapted from Emadi et al. (50). 
Another attractive characteristic of the DNBMs is their ability to impart highly efficient bystander-mediated killing in tumour models (51). The bystander effect, in the context of prodrug activation, involves the transfer of activated cytotoxins from the cell of origin to neighbouring and other nearby cells, either by passive diffusion through cell membranes or active transport mechanisms (52) (see Figure 1.5). This has become an essential component of hypoxia-activated prodrug design, as it enables drugs that are activated solely under strict conditions of anoxia to diffuse into nearby cells postreduction, including those with higher oxygen tensions. Thus, a greater volume of the tumour can be targeted via this method. As previously mentioned, TPZ has a poor bystander effect, due to the short half lives of the reduced metabolites, and therefore limited anti-tumour properties $(26 ; 53)$. Similarly, nitroimidazole-based prodrugs are devoid of a measurable bystander effect $(51 ; 54)$, also likely due to the short half-lives of the reactive metabolites (55). In contrast, the prototype DNBM prodrug SN 23862 shows efficient bystander killing in vitro in multi-layered three-dimensional cell cultures, because of the stability and longer half-lives of the toxic hydroxylamino derivatives (51). Similar results have been observed with other DNBM prodrugs (56; $57)$.

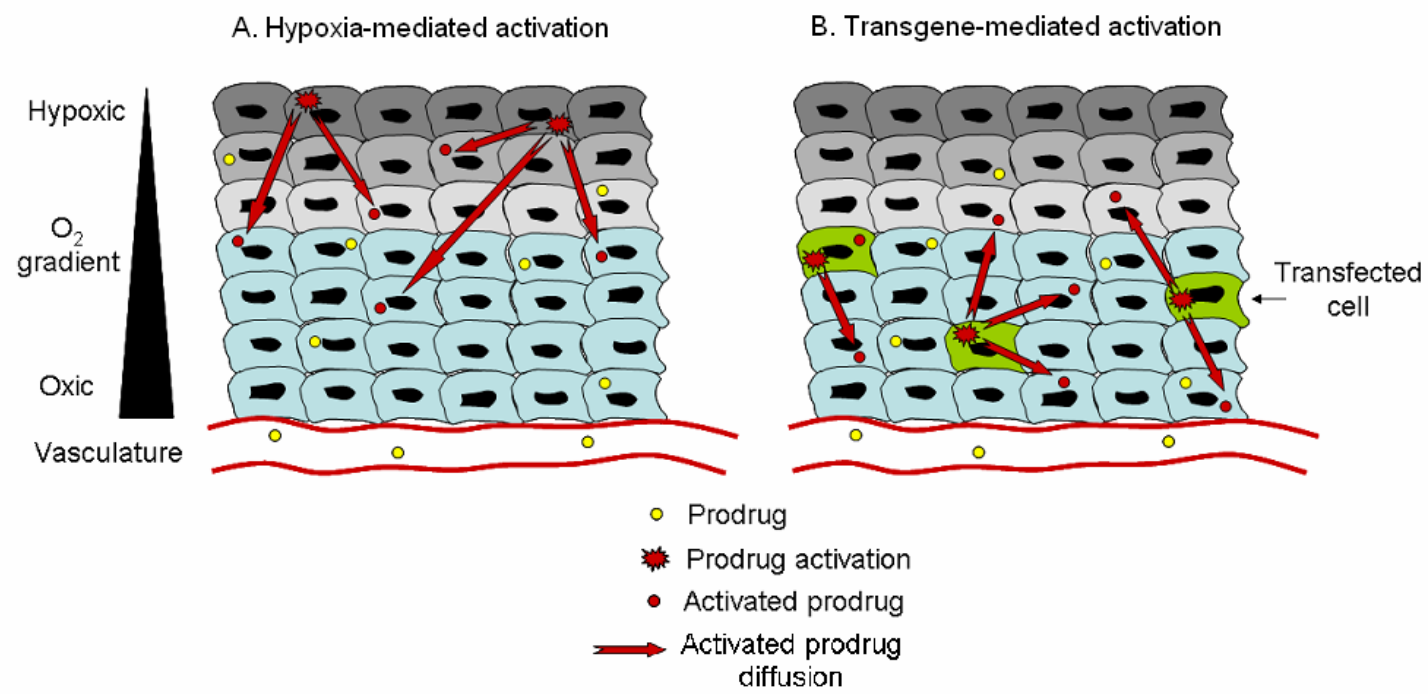

Figures 1.5A \& B. Schematic overview of the bystander effect.

Prodrugs activated either by (A) hypoxia or (B) delivered therapeutic transgenes are able to ablate surrounding oxygenated and/or non-transfected cells by passive diffusion through cell membranes or active cell transport out of the cell of origin. 
The 3, 5-dinitrobenzamide 2-mustard prodrug PR-104 (57) (see Figure 1.4A) is currently the DNBM in the most advanced stage of development, having already completed Phase I clinical trials as a hypoxia-activated cancer therapy (57). PR-104 shares the characteristic traits of its family members, including high bystander-mediated toxicity, hypoxia selectivity and a high frequency and stability of DNA interstrand crosslink formation $(48 ; 58 ; 59)$. Further, the relative positioning of the nitro groups and asymmetrical mustard leaving groups contribute to an overall enhanced toxicity profile relative to SN 23862 and other DNBMs $(56 ; 57 ; 60)$. Like other DNBMs, PR-104 is not a substrate for the oxygen-insensitive reductase NQO1 (61); however aerobic metabolism of the drug does occur and this is mediated principally through the aldoketo reductase AKR1C3 (62). Although undesirable in its capacity as a uniquely hypoxia-activated prodrug, levels of AKR1C3 have been shown to be elevated in many tumour types, suggesting a selective mechanism for PR-104 in dual hypoxia and aerobic targeting of certain cancer types (62). Furthermore, despite the aerobic reduction, a phase I clinical trial has demonstrated the safety of this prodrug in patients, with few reported dose-limiting toxicities and a maximum tolerated dose (MTD) exceeding the concentration required to achieve significant anti-tumour activity in cell culture and xenograft models (63).

\subsubsection{Combining hypoxia-activated prodrugs with other cancer therapies}

One disadvantage of hypoxia-activated prodrugs is their dependence on functional, intracellular reductases to enact prodrug reduction and cytotoxin release. This characteristic is further confounded by the heterogeneity in reductase expression profiles across different cancer types $(64 ; 65)$ as well as the differences in reductase specificity for different hypoxia-activated prodrugs (22). As previously mentioned, aerobic reduction by a variety of oxygen-insensitive reductases, including NQO1 and AKR1C3, can also affect the therapeutic outcomes of use of these drugs. Additionally, the hypoxia-selectivity of these drugs renders the oxygen-perfused portion of the tumour resistant to their cytotoxic effects, although the bystander effect of, for example, the DNBMs is theoretically able to compensate for this limitation. Overall, clinical results suggest that hypoxia-activated prodrugs are not likely to be completely curative as monoagent therapies. Instead, combinatorial therapy involving hypoxia-activated prodrugs and other treatment regimes, such as gene therapy, is potentially a much more effective means of successfully combating cancer. 


\subsection{Gene therapy for cancer}

An emerging technique for targeting treatment to cancer cells is gene therapy, which in its most general form involves the targeted delivery of therapeutic transgenes to specific target cells. In this way, host cells themselves bring about reversal of disease phenotype, through expression of delivered transgenes. Cancer is a particularly attractive candidate disorder for gene therapy due to its genetic basis and exploitable cellular characteristics for gene delivery. In fact, of the roughly 1,600 gene therapy clinical trials performed to date, more than $60 \%$ of these trials have been aimed at the treatment of cancer (66). Most, if not all cancers are characterized by loss-of-function mutations in tumour suppressor genes (e.g. p53) or gain-of-function mutations in oncogenes (e.g. bcl-2) (67). A major area of gene therapy research is therefore aimed at reversion of these aberrations to the native cellular state, either by delivering tumour suppressor genes themselves, or inhibition of the expression of oncogenes via anti-sense or siRNA delivery (68). Cancer however is generally typified by not one, but multiple genetic abnormalities, suggesting single gene-function restoration via gene therapy will not produce significant anti-tumour effects (69). Furthermore, as the action of the expressed transgene is limited to the host cell, the clinical success of gene therapy is largely dictated by the efficiency of gene delivery; even at the highest currently achievable cell transfection rates, the majority of tumour cells would remain unaffected with singlegene restoration therapy. In some instances, tumour cell death can trigger T-cell and NK-cell-mediated immune responses that can target surrounding, non-transfected tumour cells, providing a certain level of bystander effect $(70 ; 71)$. However, more substantial and prolonged bystander effects are required if significant tumour ablation is to be achieved. To attain this, other cancer gene therapies have been developed that aim to either stimulate the immune system to attack tumour cells (e.g. genes encoding cytokines, co-stimulatory molecules and/or tumour-specific antigens (72)), be antiangiogenic (e.g. by the inhibition of vascular endothelial growth factor by siRNA (73)), or cause cancer cell death by either direct oncolysis by replicating viral vectors (74) or through the activation of cytotoxic prodrugs; this latter approach is discussed in more detail below. 


\subsection{Gene-Directed Enzyme-Prodrug Therapy (GDEPT)}

Gene-directed enzyme-prodrug therapy (GDEPT), or suicide gene therapy, builds on the therapeutic potential of gene therapy by employing transgenes whose products are able to convert separately administered biologically inert prodrugs into potent cytotoxins (for an overview see Figure 1.6). Prodrug activation and the associated cytotoxicity are therefore confined to transgene expressing cells, even though the physiological distribution of the prodrug is widespread. Low systemic toxicity therefore allows much greater concentrations of chemotherapy to be administered, improving the therapeutic index of the treatment. The ability of activated prodrugs to transport out of the cell of origin, either passively or actively, and into neighbouring, non-transfected cells (the bystander effect, as previously described in the context of hypoxia-activated prodrugs) is an important, if not the most important, characteristic of GDEPT, for it provides an elegant solution to the unavoidable issue of low cell transfection rates (52). Studies using prodrugs with high bystander effects have demonstrated that significant tumour reduction can occur when less than $0.1 \%$ of the tumour population expresses the activating enzyme (75).

Although there are some exceptions, genes in the service of GDEPT are typically non-human in origin, as activation by endogenous enzymes in healthy tissue is obviously undesirable. One potential issue therefore is the elicitation of immune responses against the therapeutic protein (76). However, heightened immune responses can also result from cancer cell death and/or reactions to viral or bacterial delivery vectors and are therefore not specific to GDEPT systems (77). Furthermore, elicitation of an immune response towards cancer cells can have significant therapeutic benefits in the aiding of tumour ablation, suggesting that such a response is not entirely detrimental. A plethora of enzyme-drug combinations have been developed for cancer GDEPT over the last few decades, spanning a wide range of enzymatic activities (78). Several of the more well-known combinations tested in GDEPT are described briefly below (see also Table 1.1). 


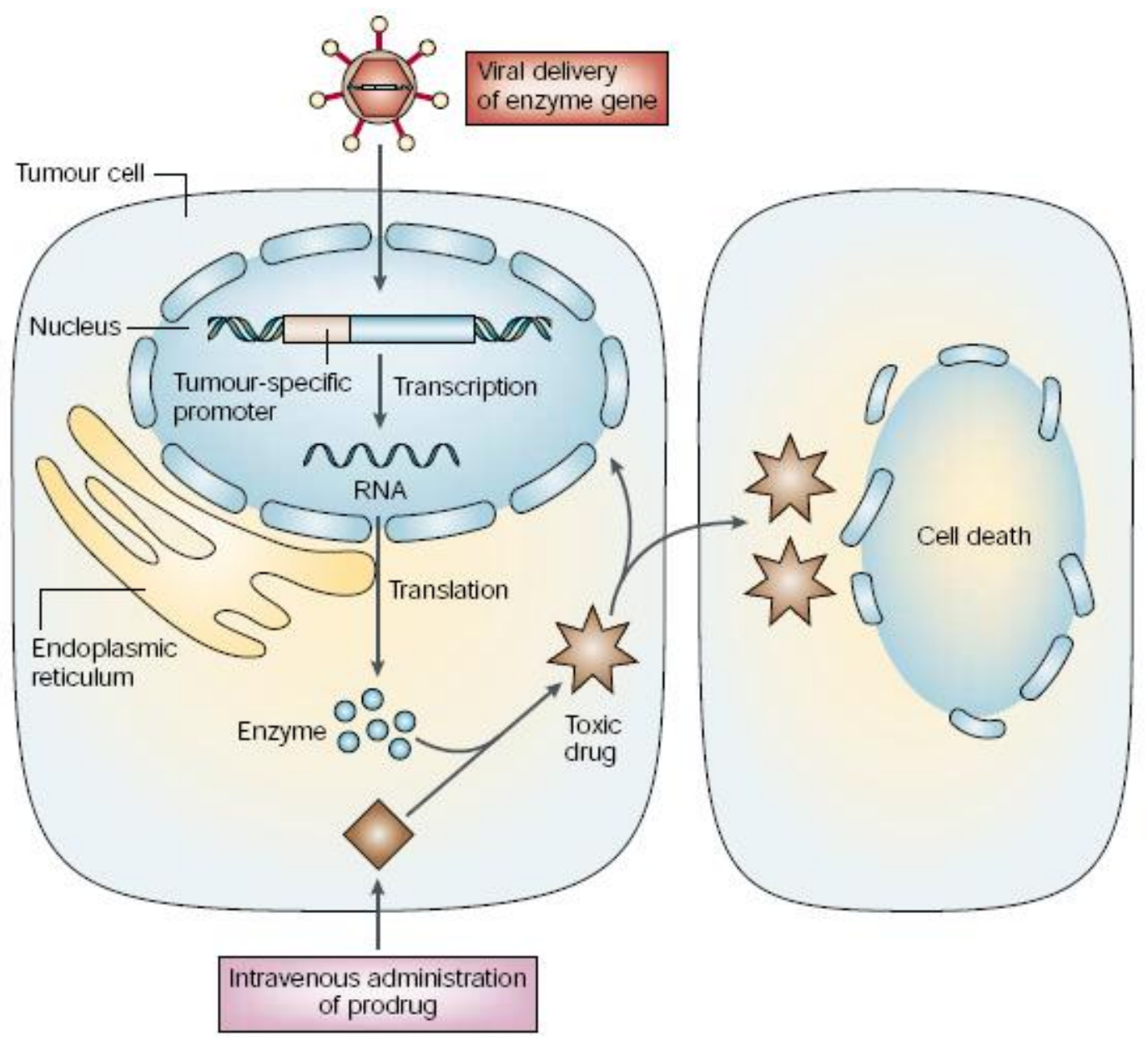

Figure 1.6. Schematic overview of GDEPT.

A therapeutic transgene is packaged into a targeted delivery vector (viral, bacterial, liposome) and delivered either locally to tumours, or systemically. Tumour cells that take up the vector then express the transgene, which is able to convert a systemically administered prodrug into its toxic form. In addition to killing the transgene expressing cell, the toxic metabolites can spread to and exert their toxic effects on neighbouring and nearby cells that have not been transfected (the bystander effect). Figure reproduced from Kerr (79).

\subsubsection{Herpes simplex virus thymidine kinase - ganciclovir}

The first GDEPT system to be designed specifically for cancer involves activation of the purine nucleoside analogue prodrug ganciclovir (GCV) by the herpes simplex virus thymidine kinase (HSVtk) enzyme (80). Phosphorylation of GCV to its triphosphate derivative converts the prodrug into a potent DNA polymerase inhibitor and lethal guanine nucleotide analogue (81). Although humans also carry thymidine kinase genes, these versions are roughly 1,000-fold less efficient at activating the prodrug compared to HSVtk (82), minimizing activation in healthy tissue. Clinical success has, however, been varied to date, with one phase III clinical trial reporting no clear benefit to patients with glioblastoma multiforme (83), while another targeting malignant glioma has 
demonstrated a significantly increased patient survival time (84) with this therapy. Another phase III clinical trial has recently been completed with results due in the course of 2011, potentially heralding approval of the first-ever cancer GDEPT product for clinical use (85).

A serious limitation to the success of the HSVtk-GCV system is the inability of highly-polar phosphorylated-GCV and analogues to freely diffuse through cell membranes. It has been shown that the observed bystander effect mediated by activated GCV is dependent on cell-cell contacts, primarily gap junctions, and possibly also through apoptotic vesicles (86). However, down-regulation or complete loss of cell-cell contacts often happens throughout progression of many cancer types, providing a barrier to efficient diffusion of this class of drug (87). Nevertheless, enhancement of this type of bystander effect has been demonstrated through the use of gap-junction protein upregulating drugs, (88), by up-regulating gap junction proteins themselves with gene therapy (89), or by fusing the HSVtk gene to genes involved in intercellular trafficking (90).

\subsubsection{Cytosine deaminase - 5-fluorocytosine}

Another prodrug used in GDEPT is 5-fluorocytosine (5-FC), which is converted into the toxic nucleotide analogue 5-fluorouracil (5-FU) by yeast and bacterial cytosine deaminases (CDs), enzymes not found in mammalian cells (91). Further metabolism of 5-FU by native cellular enzymes creates a variety of cellular toxins, including potent inhibitors of thymidylate synthase (involved in thymidine catabolism) and inhibitors of RNA and DNA processing (92). A major advantage of the CD/5-FC system over $\mathrm{HSVtk} / \mathrm{GCV}$ is that the bystander effect is much more pronounced and does not require cell-cell contacts (93). Significant tumour regression has been observed with as little as $1 \%$ of total tumour cells expressing CD $(94 ; 95)$. Conversely, it appears that one bottleneck to 5-FC metabolism is limited penetration of the parental prodrug into cells (96). Furthermore, hepatic metabolism of 5-FC is associated with moderate systemic toxicity (97). 
Table 1.1. Several GDEPT enzyme-prodrug combinations currently in clinical development.

\begin{tabular}{|c|c|c|c|c|c|c|}
\hline Enzyme & Origin & Prodrug & Released drug & Mechanism & Bystander effect & References \\
\hline TK & $\begin{array}{l}\text { Herpes } \\
\text { simplex virus }\end{array}$ & $\mathrm{GCV}, \mathrm{ACV}$ & $\begin{array}{l}\text { Monophosphate } \\
\text { derivatives }\end{array}$ & $\begin{array}{l}\text { DNA polymerase } \\
\text { inhibition, guanine } \\
\text { nucleotide analogue }\end{array}$ & $\begin{array}{l}\text { Gap-junction- } \\
\text { dependent }\end{array}$ & $(80 ; 84)$ \\
\hline $\mathrm{CD}$ & $\begin{array}{l}\text { E. coli, } \\
\text { S. Cerevisiae }\end{array}$ & $5-\mathrm{FC}$ & $5-\mathrm{FU}$ & $\begin{array}{l}\text { RNA, DNA polymerase } \\
\text { and thymidylate synthase } \\
\text { inhibition }\end{array}$ & Passive diffusion & $(91 ; 98)$ \\
\hline CYP450 & Human, rat & $\begin{array}{l}\text { Oxazaphosphorines } \\
\text { (e.g. CPA, IFO) }\end{array}$ & Phosphoramide mustards & $\begin{array}{l}\text { DNA alkylation (intra, } \\
\text { interstrand crosslinks) }\end{array}$ & $\begin{array}{l}\text { Passive diffusion, } \\
\text { 4-hydroxy } \\
\text { derivatives only }\end{array}$ & (99) \\
\hline NTR & E. coli & $\begin{array}{l}\text { Nitroaromatic prodrugs } \\
\text { (e.g. CB1954, DNBMs, } \\
\text { 2-NIs) }\end{array}$ & $\begin{array}{l}\text { Hydroxylamine/amine } \\
\text { derivatives }\end{array}$ & $\begin{array}{l}\text { DNA alkylation (intra, } \\
\text { interstrand crosslinks) }\end{array}$ & Passive diffusion & $(36 ; 100)$ \\
\hline
\end{tabular}




\subsubsection{Cytochrome P450 - cyclophosphamide.}

The oxazaphosphorine prodrugs cyclophophamide (CPA) and ifosfamide (IFO) have been used in general anti-cancer chemotherapeutic practices as sole agents for many years (103). They are metabolized by certain members of the CYP450 superfamily of enzymes to their 4-hydroxy forms, whereupon they rapidly degrade into phosphoramide mustards - potent DNA alkylating, interstrand crosslinking agents - and another toxin, acrolein (99). Although these compounds are effective anti-cancer compounds as sole agents, high levels of activating CYP450 enzymes in the liver limit their clinical usefulness. Therefore, GDEPT approaches, mainly using the catalytically superior rat CYP2B1 isoform as activating enzyme, have been in development since the mid 1990s (104). The activated 4-hydroxy forms of CPA and IFO freely diffuse through cell membranes, mediating a strong bystander effect in vitro and in vivo (104). However, the very short plasma half-lives of these metabolites ( $<6 \mathrm{~min}$; (105)) and the lipidimpenetrability of the mustard and acrolein degradation products are potential obstacles (99). A significant advantage of the CYP450-CPA/IFO system is the cell-cycle independent nature of the primary cytotoxin, the phosphoramide mustard. Directly DNA-damaging agents are able to kill cells whether replicating or not; as it is widely acknowledged that only a very small percentage of total tumour cells are dividing at any one time, this characteristic is a great advantage over the cell-cycle dependency of GCV and 5-FC.

\subsection{Nitroreductase GDEPT}

The aforementioned GDEPT systems have all proved efficacious in pre-clinical studies, and several have advanced into clinical trials for the treatment of a variety of cancer types. However, none has demonstrated all the ideal characteristics of the perfect GDEPT enzyme-drug combination. For example, activated GCV is devoid of a substantial, cell-cell contact-independent bystander effect, while both GCV and 5-FC, and their respective derivatives, require active cell division to exert their toxicity. Meanwhile, the prevalence and activity of native hepatic CYP450 enzymes limits the dose of the oxazaphosphorine prodrugs that can be administered in CYP-CPA/IFO gene therapy, with limited half lives of the activated derivatives also preventing efficient tumour penetration. Nitroreductase (NTR) enzymes coupled with nitroaromatic prodrugs are attractive combinations for GDEPT that address many of these problems, 
and as such represent some of the most promising GDEPT systems studied to date. Many of the advantages of the use of nitroaromatic prodrugs in cancer therapy have already been described, in the context of hypoxia activation (e.g. 2-NIs, DNBMs). The use of GDEPT to complement the therapeutic benefits of these drugs is therefore a promising avenue of research.

\subsubsection{CB1954 and the Walker 256 Carcinoma}

The prototypical nitroaromatic prodrug, 5-(aziridin-1-yl)-2,4-dinitrobenzamide, or CB1954, was originally discovered during a series of tests on $N$-substituted-aziridine compounds for their anti-tumour effects against the rat Walker-256 carcinoma, an established preclinical cancer model system (106). In an unprecedented display of antitumour activity, CB1954 was found to cause complete regression and cure of the Walker 256-carcinoma $(107 ; 108)$. Such marked activity was unusual for a compound that, based on its structure, was predicted to act as a monofunctional DNA-alkylating agent by virtue of the single aziridine functional group (107). The selective ablation of tumour cells also pointed towards a targeted mechanism of action. Further studies identified its mode of action to be that of a prodrug: reduction of the 4-carbon-linked nitro group (4- $\left.\mathrm{NO}_{2}\right)$ of the compound to its hydroxylamino derivative (5-(aziridin-1-yl)4-hydroxylamino-2-nitrobenzamide: 4- $\mathrm{NHOH}$ ), catalysed principally by the native oxygen-insensitive reductase NQO1, is required before cytotoxicity can be exerted $(109 ; 110)$. This reductive step allows the compound to undergo two important electronic and structural changes that contribute significantly to its overall DNAreactivity. Firstly, the aforementioned redistribution of electrons within the aromatic ring provides the aziridine group with enhanced electrophilic properties, effectively 'awakening' its repressed DNA-binding abilities. However, the difference in DNA alkylating reactivity of the aziridine group between the parental and 4-NHOH derivatives of CB1954 is modest, and does not account for the vast difference in DNAreactivity observed in vivo between the parental and reduced forms $(110 ; 111)$. Instead, it has been proposed that a non-enzymatic reaction occurs between the hydroxlyamino group of the 4-NHOH derivative and intracellular thioesters, in particular acetylcoenzyme A (acetyl-CoA), to form a highly reactive $N$-acetoxy derivative (110) (see Figure 1.7). This reaction provides the compound with a secondary DNA-reactive centre, and explains the ability of the activated compound to form DNA interstrand crosslinks at high frequency (up to $70 \%$ of total DNA lesions; (112)) in cells, but not 
naked DNA $(109 ; 110)$. Furthermore, the intrinsic toxicity of CB1954-induced DNA lesions is enhanced even further by the inability of host cell repair mechanisms to process them $(112 ; 113)$. In addition, CB1954 displays a marked bystander effect in vitro and in vivo (114-118), is not cell cycle-dependent, and kills cells via p53independent apoptosis (119); factors that further enhance the overall efficacy of this compound as an anti-cancer agent.

Although curative against well-established rat models of cancer, CB1954 as a sole agent was found to be 500-5,000 times less toxic to human cancer cells, including cell lines expressing high levels of the native NQO1 enzyme (120). Later biochemical studies with purified enzymes revealed dramatic differences in catalytic abilities between the rat and human forms of the enzyme $\left(k_{\mathrm{cat}}\right.$ of $4.1 \mathrm{~min}^{-1}$ for the rat form, compared to $0.64 \mathrm{~min}^{-1}$ for the human), confirming the inability of human NQO1 to efficiently metabolize CB1954 (120).

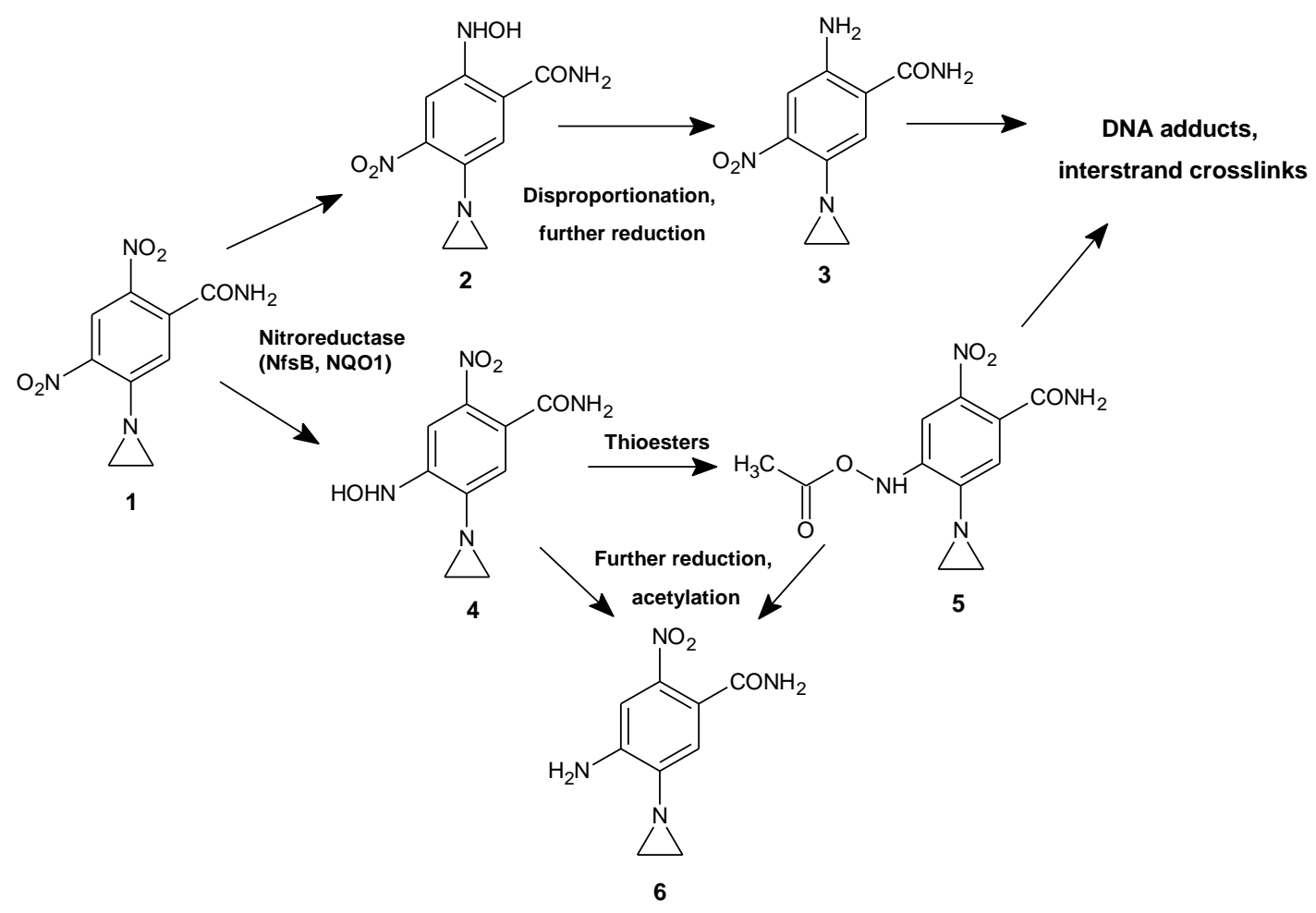

Figure 1.7. Bioactivation of CB1954.

The first step is enzymatic nitroreduction of the parental prodrug (1) to either the 2- (2) or 4hydroxylamine (4) (2-NHOH/4-NHOH respectively). 2 is unstable and rapidly disproportionate, in aqueous solution, to the nitroso (not shown) and amine (3) derivatives (111). 4 can undergo further reduction to the comparatively non-toxic amine (6), or react with intracellular thioesters (such as acetyl$\mathrm{CoA}$ ) to form the $N$-acetoxy derivative (5) (110). Over-acetylation of $\mathbf{5}$ can also form $\mathbf{6}$. Only $\mathbf{3}$ and $\mathbf{5}$ readily react with DNA to form monoadducts, intra- and interstrand crosslinks $(111 ; 121)$. 


\subsubsection{CB1954 and Escherichia coli NfsB}

A major step forward in CB1954 therapy was achieved upon the identification of an enzyme from the bacterium Escherichia coli that was not only able to efficiently reduce CB1954, but could do so 90-fold faster than even the rat form of NQO1 (122), opening up opportunities for CB1954-mediated GDEPT. The enzyme was purified and studied, and found to be a $24 \mathrm{kDa}$ flavoenzyme, with no discernible amino acid sequence or structural homology to NQO1. Instead, it has high sequence similarity to previously reported bacterial nitroreductases from Salmonella typhimurium and Enterobacter cloacae, enzymes involved in the bioreduction of nitroaromatics and quinones (123125) and was also identified as the same enzyme implicated in previous studies on the development of resistance to nitrofuran antibiotics $(126 ; 127)$. Thus it was named NfsB (for "nitrofuran sensitivity" enzyme B).

Unlike NQO1, reduction of CB1954 by NfsB yields equimolar amounts of both the 2- and 4- $\mathrm{NO}_{2}$ reduction products (however both nitro groups are unable to be reduced simultaneously on a single molecule). Original data suggested that the 2-NHOH metabolite was much less toxic than the 4-NHOH due to its inability to form DNA interstrand crosslinks (i.e. is a monofunctional agent) and was therefore undesirable as an end product (109). Recent data has however challenged this theory and suggests that the 2-amino $\left(2-\mathrm{NH}_{2}\right)$ derivative, which is produced non-enzymatically by hydrolysis and disproportionation of the unstable 2-NHOH (111), is the key metabolite responsible for the bystander-mediated toxicity of CB1954. Furthermore, this same metabolite displays potency towards nucleotide-excision repair mutant cancer cell lines at a level comparable to, or greater than, the 4-NHOH product (121).

E. coli $\mathrm{NfsB}$ has been tested preclinically in vitro and in vivo for its ability to confer CB1954 sensitivity to transgene expressing cancer cell lines and tumour xenografts. 10-2,000 fold improvements in sensitivity to the prodrug have been reported in vitro upon NTR over-expression, depending on the cell line, expression vector and CB1954 dose employed (116-118; 128-132). In NfsB over-expressing murine tumour xenograft models, several studies have reported an almost doubling of median survival times upon CB1954 administration relative to no treatment (131; 133). In addition, significant reduction in tumour size has been reported upon treatment with CB1954 when only a small percentage (5-10\%) of cells are expressing $\mathrm{NfsB}$, which demonstrates the in vivo utility of bystander-mediated cell killing $(115 ; 116 ; 130 ; 134)$. It has also been demonstrated that CB1954 is equally effective against NfsB over- 
expressing cell lines that are resistant to cisplatin and other common alkylating agents $(131 ; 135)$.

Clinical trials have also demonstrated the safety of systemically administered CB1954 (136) or $n f s B$ expressing adenovirus (137) alone in patients with gastrointestinal or resectable liver cancers, respectively. The results of two phase I/II clinical trials of adenovirus-delivered NfsB/CB1954 therapy in patients with prostate cancer were released in $2009(100 ; 138)$. Although primary aims were to investigate toxicity of the combined treatments, some observations were made on anti-tumour effects. Results were however ambiguous, with no clear advantage for the therapy in aiding tumour regression. Furthermore, dose escalation to improve anti-tumour effects has been hampered by hepatic toxicity of CB1954, with the maximum serum concentration achievable in patients being $\sim 6 \mu \mathrm{M}(136 ; 139)$. The basis of off-target toxicity of CB1954 has been examined in mouse and human liver preparations, and demonstrated to be the result of endogenous aerobic and anaerobic reductase activity independent of NQO1 $(139 ; 140)$. Experimental evidence has suggested a possible involvement of nitric oxide synthases in this activity (141).

\subsubsection{CB1954 and human NQO2}

An alternative native human enzyme, NQO2, has more recently been identified as having CB1954-reducing activity (142). NQO2 has high sequence homology to NQO1 (but not to NfsB) and is also known to be up-regulated in many cancer types. In addition, the catalytic efficiency of this enzyme in reducing CB1954 in vitro is 3,000 times greater than that of NQO1, while a 100-3,000-fold difference in CB1954 dosedependent toxicity has been observed in human cancer cell lines upon over-expression of NQO2; however a stern dependence on non-biogenic co-substrates (such as dihydronicotinamide riboside) has ensured that activity of the endogenous enzyme remains dormant in vivo, with the previously reported study employing separatelyadministered dihydronicotinamide riboside (NRH) as the electron donor. Investigations into less expensive synthetic co-factors which could act as substrates for NQO2 have identified several lead compounds, renewing interest in the possible use of CB1954 as a sole agent to control cancers in which high NQO2 levels have been reported (143). A phase I clinical trial involving CB1954 and the synthetic nicotinamide NQO2 co-factor EP0152R has recently been completed with favourable outcomes, demonstrating the feasibility of such an approach (144). 


\subsection{4. $E$. coli $\mathrm{NfsB}$ and alternative nitroaromatic prodrugs}

In order to improve potency, analogues of CB1954 and other nitroaromatic-based compounds have been studied as alternative prodrugs for NTR-mediated GDEPT (see Figure 1.8). In addition to direct variants and regioisomers of the base CB1954 structure (145), alternative strategies have been investigated including the attachment of nitrobenzylcarbamate moieties to a variety of cytotoxic agents, including mitomycin $\mathrm{C}$, anthracyclines and aniline mustards, in order to mask their activity prior to nitroreduction (146). Similarly, fusions of nitrobenzene and cyclophosphamide have been studied as possible NTR-mediated prodrugs (147). However, despite some promising in vitro data, many of the above species have failed to replace CB1954 as a lead compound due to poor solubility, extravascular diffusion and/or NTR-selectivity (148-150).<smiles>NC(=O)c1cc(N2CC2)c([N+](=O)[O-])cc1[N+](=O)[O-]</smiles>

CB1954<smiles>Cc1ncc([N+](=O)[O-])n1CCO</smiles>

Metronidazole<smiles>O=[N+]([O-])c1ccc2c(c1)OP(=O)(NCCCl)NC2</smiles>

Nitrobenzocyclophosphamide<smiles>CCPC(=O)OCc1ccc([N+](=O)[O-])cc1</smiles>

Nitrobenzylcarbamate<smiles>[R6]c1c([N+](=O)[O-])cc2c(c1[2H])C(CCl)CN2C(=O)c1cc2cc(OC)c(OC)c(OC)c2[nH]1</smiles>

Nitroindoline<smiles>NC(=O)N/N=C/c1ccc([N+](=O)[O-])o1</smiles>

Nitrofurazone

Figure 1.8. Structures of several nitroaromatic prodrugs that act as substrates for $E$. coli $\mathrm{NfsB}$. 


\subsubsection{1. $E$. coli NfsB and DNBMs}

Studies have also demonstrated that DNBM hypoxia-activated prodrugs act as substrates for NfsB, securing a potential role for them in GDEPT to complement their hypoxia-activated cytotoxicity $(151 ; 152)$. In fact, the catalytic efficiency of NfsB towards SN 23862 is superior to that of CB1954 (4-fold higher $k_{\text {cat }}$ ), while in NfsB over-expressing UV4 cell lines the improvement in toxicity upon administration of SN 23862 (140-fold) is over ten times that of CB1954 (12-fold) (151). Furthermore, the bystander effect and hypoxia selectivity of DNBMs are far in excess of those of CB1954 $(121 ; 153 ; 154)$. It has been shown that the differences in cytotoxicity between CB1954 and certain DNBMs, in particular SN 23862, is related to the nature and alkylating reactivity of the reduction metabolites of the two types of compound. NfsB catalyzes the formation of both the 2- and 4-NHOH metabolites of CB1954, while only the 2-NHOH is formed from reduction of SN 23862. The 2-NHOH of SN 23862 is however unstable in aqueous conditions, undergoing rapid disproportionation and hydrolysis to form the amine $\left(2-\mathrm{NH}_{2}\right)$. This amine, which can be considered the primary end product of SN 23862 metabolism, is roughly 2,200-fold more reactive than the parental compound, compared with only 17-fold for both the 4-NHOH N-acetoxy and 2- $\mathrm{NH}_{2}$ derivatives of CB1954 (111). In contrast to CB1954, alkylating reactivity of SN 23862 is not dependent on conjugation with intracellular thioesters, a potentially ratelimiting step, providing a simpler and more direct mechanism of bioactivation for this prodrug (111). Overall, results clearly demonstrate that the 2- $\mathrm{NH}_{2}$ derivative of $\mathrm{SN}$ 23862 is superior to both the 4-NHOH $N$-acetoxy and 2- $\mathrm{NH}_{2}$ of CB1954 in terms of alkylating reactivity and cytoxicity in vitro $(111 ; 121)$.

Results with regioisomers of SN 23862, including the more potent 3, 5-dinitro class of benzamide mustards, are consistent with those previously mentioned, particularly in regards to their substrate specificity towards NfsB $(152 ; 155)$. For example, a dibromo-analogue of PR-104, SN 27686, has been evaluated in vivo in mouse tumour xenograft models in conjunction with a conditionally replicating, NfsB over-expressing adenoviral vector. The mean survival time of animals treated with this regime was enhanced considerably over that of a CB1954-administered control group, specifically when only a fraction (10\%) of cells were expressing the transgene (56). The emerging use of DNBMs in GDEPT alongside nitroreductase enzymes therefore presents a promising avenue in cancer gene therapy. 


\subsection{Alternative enzymes for cancer gene therapy}

Most, if not all GDEPT strategies currently being investigated for cancer treatment are still in preclinical development and as such require extensive optimisation before they can be clinically useful. Because much of the success of GDEPT is currently limited by modest gene-delivery and expression methods, a lot of the emphasis on improving GDEPT systems will rely on enhancing alternative aspects of the therapy regime. Prodrug modification to improve potency and tumour selectivity is an obvious approach, and this has already been discussed in detail for NTR GDEPT. However, a major limiting factor in many GDEPT strategies is often the catalytic efficiency of the activating enzyme for the prodrug of interest. GDEPT enzyme choices are usually made on a first-come first-served basis, with $E$. coli being a major source of prodrugactivating enzymes $(75 ; 122 ; 156 ; 157)$. However, more active enzymes can often be found in other organisms. For example, the cytosine deaminase from Saccharomyces cerevisiae was found to have a $K_{\mathrm{m}} 22$-fold lower than that of the previously tested $E$. coli enzyme (158) towards 5-FC. More importantly, this improvement in activity was also seen in vitro and in vivo in transgene-expressing cancer models (159).

This issue is particularly relevant to NTR/CB1954 GDEPT, as plasma concentrations of CB1954 at the maximum tolerated dose (MTD) are more than 80 times lower than the lowest reported $K_{\mathrm{m}}$ of $E$. coli NfsB for this substrate (122). Furthermore, the $K_{\mathrm{m}}$ of NfsB for SN 23862, which displays very similar pharmacokinetics to CB1954 in mice (160), is 2-3 fold higher than that reported for CB1954 (151). Although in vitro kinetic data for members of the more potent 3, 5dinitro series of DNBMs are not available, our own work suggests that they are not very efficient substrates for NfsB and would suffer from similar low catalytic turnover in vivo, despite having higher MTDs in mice $(56 ; 161)$ and humans $(63 ; 136)$. Although compensated in part, relative to CB1954, through improved tissue diffusion and cytotoxicity, the low $K_{\mathrm{m}}$ of NfsB for these substrates suggests that enzyme improvement would have a significant impact on in vivo efficacy. 


\subsubsection{The bacterial nitroreductases: phylogenetic and biochemical properties}

E. coli NfsB belongs to a superfamily of enzymes, the bacterial nitroreductases (NTRs), which are widespread amongst prokaryotes, including both bacteria and archaea (162). NTRs are broadly defined as any enzyme with the capability of reducing nitro groups of nitroaromatic compounds, either through sequential one electron steps (oxygensensitive, or class II) or simultaneous two electron steps (oxygen-insensitive, or class I) (162; 163). In this regard, NQO1, which can reduce CB1954 aerobically, is a class I NTR, while CYP450R, which reduces CB1954 and many other nitroaromatic compounds via one electron steps, belongs to the class II NTRs. Most work with bacterial NTRs to date has focused on those belonging to class I, due to their ability to reduce nitro groups under physiological conditions (i.e. in the presence of oxygen). Nitroaromatic groups are found in a variety of chemical species, almost all of which have been produced over the last century through anthropogenic processes such as the manufacture of explosives, dyes, textiles and insecticides, and can therefore be regarded as recent additions to the chemical diversity available in the environment (162). As such, it is assumed that many, if not all, bacterial NTRs possess nitroreductase activity as a secondary, or 'promiscuous' function to their primary activity, as there has likely been insufficient time to evolve specific metabolic pathways directed at such compounds. One interesting case has been reported, however, of an NTR from Haemophilus influenzae able to reduce and thereby detoxify the natural nitroaromatic antibiotic chloramphenicol, suggesting a possible physiological role in antimicrobial detoxification for this specific enzyme (164). Otherwise, physiological roles associated with these enzymes are largely assumed to be a function independent of nitroreductase activity, such as quinone reduction or $\mathrm{NAD}(\mathrm{P}) \mathrm{H}$ homeostasis in oxidative stress protection (165), flavin reduction for bioluminescence $(166 ; 167)$, dihydropteridine reduction for aromatic amino acid hydroxylation (168), the production of vitamin K12 (169), or the production of $\mathrm{H}_{2} \mathrm{O}_{2}$ through $\mathrm{NAD}(\mathrm{P}) \mathrm{H}$ oxidation as a defence or $\mathrm{O}_{2}$ homeostasis mechanism (170; 171). Bacterial NTRs have been well-studied for a variety of reasons, including their role in development of resistance to nitroaromatic antimicrobials such as nitrofurazone (NFZ) and metronidazole (MTZ) $(172 ; 173)$ and their ability to biotransform recalcitrant nitroaromatic environmental pollutants such as di- and trinitrotoluenes (D/TNTs), thus affording them attractive properties for bioremediation processes (162). Many have been purified and biochemically 
characterised, including the elucidation of crystal structures in many cases, allowing detailed understanding of their molecular biology (see Table 1.2 for examples) (170; 174-183). Most bacterial NTRs are relatively small (20-40 kDa), homodimeric flavincontaining (typically FMN) enzymes that require $\mathrm{NAD}(\mathrm{P}) \mathrm{H}$ as co-substrates/electron donors to reduce other substrates. Substrate specificity is generally broad and can include quinones, flavins, azo-dyes, ferricyanide and chromate (VI), in addition to nitroaromatics $(176 ; 184)$. Nevertheless, the mechanism of catalysis is usually conserved between all substrates and all enzymes, and proceeds via a bi-bi ping-pong mechanism, whereby $\mathrm{NAD}(\mathrm{P}) \mathrm{H}$ is the first substrate to enter (leading to the twoelectron reduction of the enzymatic FMN group), followed by exit of $\mathrm{NAD}(\mathrm{P})^{+}$and entry of the second substrate. Electrons are then passed from reduced FMN to the second substrate, followed by dissociation of the reduced end metabolite. In the case of nitroaromatic compounds, this entire cycle is repeated once to form the hydroxylamine, or twice to produce the amine (see Figure 1.3). However, as previously mentioned, the hydroxylamine is the most common end product of the reaction, due to the lower reduction potential of the $\mathrm{NHOH}$ group relative to the parental nitro group, creating electrochemical difficulties in further reduction (35).

Phylogenetically, many of the bacterial NTRs characterised to date belong to one of two groups, based on their amino-acid homology to either E. coli NfsA (the major NTR) or NfsB (the minor NTR) $(176 ; 177)$. Little sequence homology is shared between these two groups (7\% amino acid identity between $E$. coli $\mathrm{NfsA}$ and NfsB, across entire sequence), although biochemically (as already described) and structurally they are very similar and contain elements similar to many flavoenzymes (core structure typically consisting of a multi-stranded parallel $\beta$-sheet core flanked on each side by multiple $\alpha$-helices; (162)). No mammalian enzyme with considerable homology to either of these classes has been identified to date, although several examples exist in protists, most likely arising through horizontal gene transfer from bacterial species (185; 186). Outside of these two principal families, nitroreductase activity has been associated with several other bacterial enzyme classes, in particular the Old Yellow Enzymes (OYEs) (187). 
Table 1.2. Examples of biochemically characterised bacterial NTRs.

\begin{tabular}{|c|c|c|c|}
\hline NTR & Organism & Substrates & Refs \\
\hline NfsA & E. coli & Quinones, chromate, ferricyanide, flavins, CB1954, TNT, NFZ, other nitroaromatics & $(188-190)$ \\
\hline Frp & Vibrio harveyi & Quinones, chromate, ferricyanide, flavins, NFZ, other nitroaromatics & $(188 ; 191)$ \\
\hline PnrA & Pseudomonas putida JLR11 & TNT, other nitroaromatics & $(192)$ \\
\hline SnrA & Salmonella typhimurium & NFZ & $(193)$ \\
\hline NfrA1 & Bacillus subtilis & Quinones, ferricyanide, flavins, NFZ, other nitroaromatics & $(167 ; 170)$ \\
\hline YcnD & Bacillus subtilis & Chromate, NFZ, other nitroaromatics & (194) \\
\hline NitA & Clostridium acetobutylicum & TNT, other nitroaromatics & $(181)$ \\
\hline NfrA & Staphylococcus aureus & Ferricyanide, FMN, NFZ & $(195)$ \\
\hline $\mathrm{NfsB}$ & E coli & Quinones, ferricyanide, flavins, CB1954, NFZ, TNT other nitroaromatics & $(122 ; 177 ; 190)$ \\
\hline FRaseI & Vibrio fischeri & Quinones, ferricyanide, flavins, NFZ, other nitroaromatics & $(166)$ \\
\hline $\mathrm{NR} / \mathrm{NfsI}$ & Enterobacter cloacae & Quinones, flavins, NFZ, TNT, other nitroaromatics & $(174 ; 196)$ \\
\hline $\mathrm{CnR}$ & Salmonella typhimurium & Quinones, flavins, NFZ, other nitroaromatics & $(197 ; 198)$ \\
\hline $\operatorname{RdxA}$ & Helicobacter pylori & Quinones, ferricyanide, NFZ, MTZ, CB1954, other nitroaromatics & $(171)$ \\
\hline YfkO & Bacillus licheniformis & NFZ, CB1954, other nitroaromatics & $(199 ; 200)$ \\
\hline NprA & Rhodobacter capsulatus & NFZ, other nitroaromatics & $(168)$ \\
\hline PETNr & Enterobacter cloacae & TNT, other nitroaromatics & $(201)$ \\
\hline XenB & Pseudomonas fluorescens & TNT, other nitroaromatics & $(202)$ \\
\hline AzoR & Rhodobacter sphaeroides & Quinones, chromate, ferricyanide, flavins, TNT, other nitroaromatics & $(203)$ \\
\hline DrgA & Synechocystis spp. & Quinones, ferricyanide, flavins, NFZ, other nitroaromatics & $(204)$ \\
\hline NitB & Clostridium acetobutylicum & TNT, other nitroaromatics & $(181)$ \\
\hline Frb & Bacillus subtilis & Quinones, ferricyanide, flavins, NFZ, other nitroaromatics & $(205)$ \\
\hline
\end{tabular}




\subsubsection{CB1954 and alternative bacterial NTRs}

The widespread abundance and phylogenetic diversity of NTRs in the environment suggests that there are likely to be enzymes that reduce nitroaromatic prodrugs more efficiently than $E$. coli $\mathrm{NfsB}$. Indeed, several research groups have focused their efforts on the characterisation of alternative NTRs in order to identify enzymes with enhanced prodrug reducing activities (see table 1.3. for examples). Many of these studies resulted from characterisation of NTRs in other contexts, including reduction of environmental contaminants and antibiotics. For example, the NTR NprA from Rhodobacter capsulatus was initially identified on the basis of its 2,4-dinitrophenol reducing abilities (182). It was later found that over-expression of this NTR in E. coli dramatically increased the sensitivity of the host towards CB1954 (168). Similarly, nitrobenzene reductase (NbzA) from Pseudomonas pseudoalcaligenes JS45, an organism able to survive on nitrobenzene as a sole carbon and nitrogen source, has demonstrated CB1954-reducing activity, with a $K_{\mathrm{m}}$ for this substrate almost 80 -fold lower than NfsB (206). The NTR RdxA from the gut pathogen Helicobacter pylori, an enzyme implicated in development of host resistance to MTZ, has also been purified and characterized and identified as having a 10-fold improved CB1954-reducing activity compared to NfsB (171). However, none of these reports have described in vivo or in vitro mammalian cell culture data comparing prodrug reducing activity directly alongside NfsB, rendering it difficult to gauge their utility in GDEPT. Indeed, only a few publications exist of NTR characterisation specifically focusing on prodrug reducing abilities, and therefore offer insight into comparative activities of these enzymes with NfsB in a more robust clinically relevant setting. Anlezark et al. (2002) were the first to characterise an alternative bacterial CB1954-reducing enzyme, that of YwrO from Bacillus amyloliquefaciens (207). Not only does YwrO have a slightly higher affinity than NfsB for CB1954 ( $K_{\mathrm{m}}$ of 617 compared with $862 \mu \mathrm{M}$ ), but it also exclusively generates the more cytotoxic 4-NHOH metabolite. Interestingly though, V79 mammalian cells incubated in the presence of this enzyme and the relevant cosubstrate were ten-fold less sensitive to CB1954 compared to those treated with E. coli NfsB. YwrO is also unable to catalyse reduction of the DNBM analogue SN 23862, in contrast to NfsB. Likewise, Theys et al. (2006) tested a panel of NfsB and YwrO homologues from diverse bacterial species for CB1954-induced cytotoxicity in a human cancer cell line and found that YwrO-like (shared significant amino acid homology with YwrO) enzymes were significantly outperformed by NfsB-like enzymes (208). As part 
of this study however, they did isolate an NfsB-homologue from $H$. influenzae which had a lower $\mathrm{ED}_{50}$ value (concentration of prodrug required to inhibit cell growth to 50 $\%$ of unchallenged control) for CB1954 than E. coli NfsB, although the difference was modest (4.7 compared to $6.3 \mu \mathrm{M}$ ). A separate study has characterised the NTR YfkO, isolated from the thermophilic bacterium Bacillus licheniformis, and found it to have activity with a range of nitroaromatic prodrugs, including a 5,000-fold increase in catalytic efficiency towards CB1954 and 5-fold increase in specific activity towards SN 23862 compared with NfsB (199). Unfortunately, no comparative cytotoxicity tests were carried out in human or mammalian cell lines. Although scarce, these reports have unequivocally demonstrated that alternative CB1954 and nitroaromatic prodrug reducing enzymes exist. Furthermore, sharing significant amino acid homology to NfsB is not a prerequisite for CB1954-reducing activity. YwrO, for example, shares no sequence homology to NfsB, but does share some identity with mammalian NQO1 and NQO2 (207). NbzA from $P$. pseudoalcaligenes JS45 shares only $27 \%$ identity with $\mathrm{NfsB}$ across a 48 amino acid region, while YfkO, RdxA and NprA only share 24\%, 25\% and $30 \%$ amino acid identity, respectively, with NfsB across the entire gene sequence.

Table 1.3. Kinetic parameters for all naturally-occurring CB1954-reducing enzymes characterised to date.

\begin{tabular}{lllll}
\hline Enzyme & $\boldsymbol{K}_{\mathbf{m}}(\boldsymbol{\mu M})$ & $\boldsymbol{k}_{\mathbf{c a t}}\left(\mathbf{s}^{-\mathbf{1}}\right)$ & End products & Reference \\
\hline NQO1 (human) & 1,370 & 0.01 & $4-\mathrm{NHOH}^{\mathrm{a}}$ & $(120)$ \\
NQO1 (rat) & 840 & 0.07 & $4-\mathrm{NHOH}$ & $(120)$ \\
NQO1 (mouse) & 1,280 & 0.01 & $4-\mathrm{NHOH}$ & $(209)$ \\
NfsB (E. coli) & 860 & 6.0 & $2 \& 4-\mathrm{NHOH}$ & $(122)$ \\
NQO2 (human) & 260 & 6.01 & $4-\mathrm{NHOH}$ & $(142)$ \\
YfkO (B. licheniformis) & 30 & 1,070 & $4-\mathrm{NHOH}$ & $(199)$ \\
YwrO (B. amyloliquefaciens) & 620 & 8.2 & $4-\mathrm{NHOH}$ & $(207)$ \\
YdgI (B. amyloliquefaciens) & 3,860 & 30.3 & $2 \& 4-\mathrm{NHOH}$ & $(208)$ \\
YodC (B. amyloliquefaciens) & 550 & 58 & $2 \& 4-\mathrm{NHOH}$ & $(208)$ \\
NfnB (H. influenzae) & 690 & 56.2 & $4-\mathrm{NHOH}$ & $(208)$ \\
NfnB (C. jejeuni) & 217 & 6.1 & $4-\mathrm{NHOH}$ & $(208)$ \\
YwrO (P. gingivalis) & 1,200 & 3.2 & $4-\mathrm{NHOH}$ & $(208)$ \\
RdxA (H. pylori) & 35 & 10 & $\mathrm{NR}$ & $(171)$ \\
NbzA (P. pseudoalcaligenes) & 2.3 & $\mathrm{NR}$ & $\mathrm{NR}$ & $(206)$ \\
NfsA (E. coli) & 140 & 21 & $2-\mathrm{NHOH}$ & $(189)$ \\
\hline
\end{tabular}

a2-/4-NHOH: 2- or 4-hydroxylamine respectively.

${ }^{b} \mathrm{NR}$ : Not reported 


\subsubsection{Enzyme engineering to improve activation of anti-cancer prodrugs}

Enzymes are flexible molecules, and as such are amenable to finite levels of mutational alterations without loss of considerable catalytic activity. Moreover, mutations can even, in some circumstances, enhance many properties of the enzyme including substrate specificity, catalytic efficiency and thermal and solvent stability (210). The purposeful targeted or random mutagenesis of enzyme sequences using standard laboratory genetic procedures, otherwise known as enzyme engineering or directed evolution, has therefore emerged as a powerful approach to hone enzyme activity towards desired end goals (211).

The activity of enzymes for use in GDEPT has been improved by several groups. For example, Black and co-workers performed extensive studies on variants of HSVtk, mutated randomly at several residues in the putative active site of the enzyme, and identified several that confer markedly improved sensitivities towards GCV and the analogue aciclovir (ACV) to E. coli and mammalian cells over-expressing these enzymes (212-214). Furthermore, certain of these mutants were able to improve sensitivity of tumour cells expressing these enzymes to GCV and ACV two to five-fold over the wild-type enzyme, demonstrating a clear in vivo benefit (215). Similar successes have also been reported with bacterial and yeast $\mathrm{CD}$, with mutant enzymes identified that can increase sensitivity of E. coli, mammalian cells and human tumour xenografts to the toxic effects of 5-FC compared to the wild-type enzyme (156; 216; 217).

Not surprisingly, NfsB has also been the subject of a series of ongoing mutagenesis studies, primarily carried out by Peter Searle and co-workers, in order to improve its affinity and catalytic efficiency towards CB1954. An early study used sitesaturation mutagenesis (testing of all alternative amino acid possibilities at a certain residue) of active site residues, as determined by previously-solved crystal structures of the enzyme, to identify amino acids involved in CB1954 reduction (218). Out of the nine amino acids targeted, improved mutants were found with changes at any one of six sites. Most significantly, a single amino-acid mutant (F124K) was isolated that conferred a5-foldincrease in sensitivity of human ovarian cancer cells (over-expressing the relevant NTR) towards CB1954, compared with wild-type NfsB. More recently, the effects of pair-wise combination of some of these beneficial single mutations has been evaluated, with vast improvements in enzyme activity and prodrug substrate affinity observed with certain double mutants $(219 ; 220)$. An alternative approach has been 
explored by Jackson and colleagues (2006), using the incorporation of non-natural amino acids into site 124 of NfsB to improve CB1954 reducing activity (221). Of the 30 synthetic amino acids tested, one was found that not only conferred 30-fold improved CB1954-reducing activity in vitro over wild-type NfsB, but also 2.3-fold improved activity over the best possible natural amino acid mutant of site 124. Together, these studies clearly demonstrate the potential of enhancing NTR activity towards CB1954 using enzyme engineering approaches.

Nevertheless, the main focus of these enzyme engineering studies is still $E$. coli NfsB. The breadth of NTR-diversity available in nature suggests that alternative enzymes may provide better starting scaffolds for evolution experiments. A pivotal study in this regard was carried out by Barak et al. (222), whereby a chromate reductase (YieF) from E. coli was randomly mutated in order to further improve chromate reducing activity. One enhanced mutant, Y6, was not only found to be superior in its chromate reducing activity, but was also able to confer up to 10 -fold improvement in sensitivity of HeLa cells towards CB1954, relative to wild-type YieF. Furthermore, this activity was also far in excess of that of $E$. coli NfsA, another NTR recently found to be superior to NfsB in CB1954-mediated cell killing (189). However, this being the sole report to date of evolution of an NTR other than NfsB towards improved nitroaromatic prodrug-reducing activity suggests that there is substantial work required in this field before clinically-significant results can be generated.

\subsection{Enzyme activity screening technologies}

Enzyme discovery and/or engineering studies rely on the ability to identify individual enzymes that are improved for a function of interest over a pre-existing scaffold from a pool of enzyme variants. Depending on the technique used to generate enzyme libraries, the number of variants that comprise this pool can exceed $10^{4}$ by many orders of magnitude meaning that precise quantitative assays such as kinetic evaluation of purified enzymes are far too costly and time-consuming to be employed as screening techniques. As such, the development of reliable and high-throughput enzyme activity screening methodologies has received considerable attention in these fields.

Strategies based on the ability of a target enzyme to complement null mutations in metabolic pathways and/or allow host organisms (such as bacteria or yeast) to grow on selective media are among the most efficient techniques applied so far. Examples of these selective techniques have been successfully employed in GDEPT studies for the 
identification of enzyme variants with enhanced prodrug activating abilities, such as HSVtk variants improved for GCV phosphorylation (212) and E. coli CD variants for improved 5-FC deamination (156). Alternatively, in the absence of an appropriate selective function, the use of fluorescent enzyme substrates can allow very highthroughput screening of enzyme variants through fluorescence-activated cell sorting (FACS). This has successfully been applied, for example, in the identification of 2'deoxynucleoside kinase variants improved in the activation of nucleoside analogue prodrugs (223).

\subsubsection{Screening for nitroaromatic prodrug reduction}

Unfortunately, no essential metabolic pathway or generally applicable fluorescence based method has yet been developed for detection of NTR-mediated nitroaromatic prodrug reduction, severely hindering the potential for evolution of these enzymes. One individual case of a nitroaromatic prodrug (6-chloro-9-nitro-5-oxo-5 $\mathrm{H}$ benzo( $(a)$ phenoxazine; $\mathrm{CNOB})$ that produces a fluorescent signal, in addition to cytotoxicity, upon reduction by NTRs has been reported (224). Although valuable for allowing quantitative assessment of in vitro and in vivo activation of this prodrug, fluorescence emission is a unique property of CNOB and not of nitroaromatic prodrugs in general, precluding the application of this approach for use with other NTR-prodrug combinations. On the other hand, the Auckland Cancer Society Research Centre (ACSRC) has developed a range of NTR-activated fluorophore compounds, which could act as prodrug surrogates for evaluation of NTR activity (Jeff Smail, personal communication). Assessment of the applicability of these compounds in enzyme screening is currently being carried out in our lab. However, in many cases fluorophore structure does not accurately represent that of the final application prodrug, potentially biasing identification of lead NTRs towards those superior in fluorophore activation, and not necessarily the desired prodrug activity.

Of the few directed-evolution/protein engineering studies of NTR enzymes that have been carried out, the main quantitative enzyme activity screen employed has been based on either host organism growth inhibition (in the presence of increasing concentrations of prodrug) or direct spectrophotometric kinetic analysis of purified enzymes $(218 ; 219 ; 221)$. Although these methods do test for the precise enzymatic properties desired, they are both costly, in terms of concentrations of prodrug used, and 
laborious. Not surprisingly then, the total number of colonies screened using these methods have not exceeded several thousand in a single round.

\subsubsection{Use of the $E$. coli SOS response as a screening tool}

One notable case of a direct positive selection strategy for nitroaromatic prodrugreduction has been recorded, based on induction of the $E$. coli SOS response (225). The SOS response, present in many bacterial species, involves an up-regulation of a number of genes primarily involved in DNA repair and cell cycle control, in response to DNA damage (see Figure 1.9A) (226). It has also been shown that induction of the SOS response can reactivate genome-integrated lambda phages to their lytic cycle (227). Therefore, by using the DNA-damaging abilities of activated CB1954 to induce the SOS response in E. coli, Guise et al (2007) were able to identify active NTRs through the harvesting of released phages, and sequencing of the inserted $n f s B$ genes, from CB1954-challenged lysogen cultures (225). Improved NTRs could thus be detected by using lower concentrations of CB1954 than was necessary to elicit a response from wild-type NfsB. Using this method, a library of $6.8 \times 10^{5}$ variants was screened and a number of improved mutants identified, including a triple amino-acid mutant able to confer equitoxicity to human carcinoma cells at CB1954 concentrations 40-80-fold lower than the wild-type enzyme. Although clearly a powerful technique for enrichment of improved CB1954-activating mutants, the necessity of integration of the NTR gene into the lambda-phage DNA backbone and the subsequent multiple packaging, infection and harvesting steps precludes its use as a tool for rapidly and quantitatively assessing multiple NTRs simultaneously. Nevertheless, the utility of the E. coli SOS response as a quantifiable measure of DNA-damaging prodrug activation has been firmly illustrated by this example, especially in light of the absence of any exploitable nitroreductaseassociated metabolic pathway able to be used as the basis of a selection strategy.

The E. coli SOS response has been exploited elsewhere in biotechnological research, in particular for the assessment of cytotoxicity of environmental pollutants. The most widely used technique in this field employs $E$. coli strains containing a reporter gene (lacZ, gfp, lux) under transcriptional control of an SOS response linked promoter (228-232). In this case, reporter gene activity is directly proportional to the extent of SOS response induction (see Figure 1.9B). Reporter gene products provide signals, typically colorimetric, fluorescent or luminescent, that are readily detectable from intact cells or whole cell lysates, permitting rapid evaluation of their presence and 
quantity using simple substrates and common laboratory equipment and procedures. Therefore both the ability and the magnitude of SOS induction by a test drug can be measured using this system, allowing relative assessment of genotoxic potencies of multiple test compounds. In addition to environmental toxins, studies have shown that activated nitroaromatic prodrugs including CB1954, RSU-1069 and nitrofurazone (233235) also induce strong, dose-dependent SOS responses using the SOS response reporter gene assay. The ability to relatively quantify SOS response induction between multiple strains/drugs and the ease with which the assay is performed suggests advantages for this system over the lambda phage induction method in assessment of prodrug reducing abilities of multiple NTRs. Furthermore, directly assessing transcription from SOS-linked promoters is considered to be a more sensitive measure of SOS response induction compared to lambda phage activation, due to the 10-fold higher rate of RecA-mediated LexA (SOS response inhibitor) cleavage relative to $\lambda \mathrm{cI}$ (lambda phage lytic cycle repressor) cleavage (228).
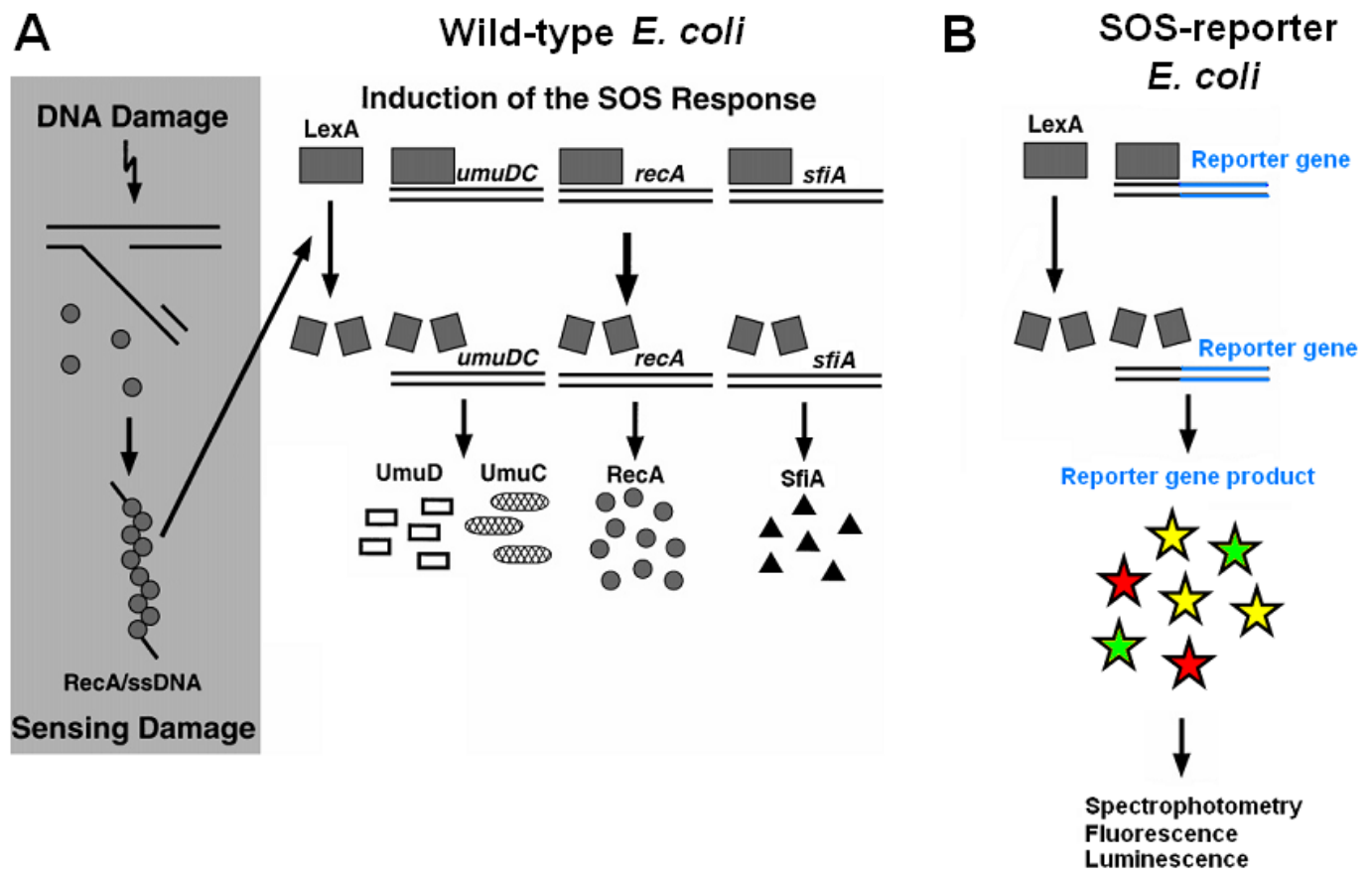

Figures 1.9A \& B. Schematic overview of the $E$. coli SOS response.

(A) In wildtype E. coli, DNA damage induces the formation of sections of single-stranded DNA, which is recognized and bound by RecA protein. RecA "activated" in this fashion then causes a conformational change to occur in the LexA protein, an SOS-gene repressor, permitting de-repression of SOS-linked genes. (B) In SOS reporter strains of E. coli, reporter genes such as lacZ or $g f p$ are transcriptionally or translationally fused downstream of an SOS-gene promoter. DNA damage occurring in this strain can therefore be relatively quantified by way of measurement of reporter-gene activity. Figure adapted from Sutton et al. (236). 


\subsection{Research objectives}

The overriding goals of this project were three-fold:

1) To develop a screening system, based on the E. coli SOS response reporter gene assay, able to rapidly and accurately measure relative levels of nitroaromatic prodrug (principally CB1954) reduction by candidate NTRs. These aims were investigated in Chapters 3 and 4.

2) To apply this screen to a range of phylogenetically diverse bacterial NTRs in order to identify lead candidate enzymes with the ability to reduce a variety of nitroaromatic prodrugs. These aims were investigated in Chapters 3, 4 and 5.

3) To further optimise a lead NTR (selected from results gained in goal 2 above) towards an enhanced catalytic rate of reduction of a specific nitroaromatic prodrug (in this case the DNBM PR-104A) via enzyme engineering techniques. This aim was investigated in Chapter 6. 


\section{Chapter 2: Materials and Methods}

\subsection{Chemicals, enzymes, reagents, media}

Chemicals, reagents and growth media were analytical grade and obtained from SigmaAldrich (St. Louis. MO, USA) or Thermo Fisher Scientific (Waltham, MA, USA) unless otherwise stated. PR-104A, RB 6145, SN 27686, SN 29176 and SN 30444 parental prodrugs and synthetic CB1954 reduction metabolites were generous gifts from the Auckland Cancer Society Research Centre (ACSRC; Auckland, NZ). IPTG (isopropyl $\beta$-D-thiogalactoside), BioMix ${ }^{\mathrm{TM}}$ Red and Accuzyme ${ }^{\mathrm{TM}}$ Mix were supplied by Bioline (London, UK). Restriction enzymes were supplied by New England Biolabs (NEB; Ipswich, MA, USA). T4 DNA ligase was supplied by Fermentas (Thermo Fisher Scientific) or Bioline. Phusion ${ }^{\mathrm{TM}}$ high-fidelity DNA polymerase was supplied by Finnzymes (Espoo, Finland).

\subsection{Oligonucleotide primers}

All primers used in this study were synthesized by Invitrogen (Carlsbad, CA, USA) or Integrated DNA Technologies (IDT; Coralville, IA, USA) and supplied lyophilized. For long-term storage, primers were resuspended in $1 \times \mathrm{TE} \mathrm{pH} 8.0(10 \mathrm{mM}$ Tris-Cl pH 8.0, $0.1 \mathrm{mM}$ EDTA) to $100 \mu \mathrm{M}$ and stored at $-20{ }^{\circ} \mathrm{C}$. For working stocks, aliquots were diluted in autoclaved, $0.22 \mu \mathrm{m}$ filter-sterilized distilled and deionised $\left(\mathrm{ddH}_{2} \mathrm{O}\right)$ water to $10 \mu \mathrm{M}$ final concentration. Primers are listed in Table 2.1. 
Table 2.1. Oligonucleotide primers used in this study.

Underlined sequences indicate restriction sites. Bold type indicates homology regions used in recombination.

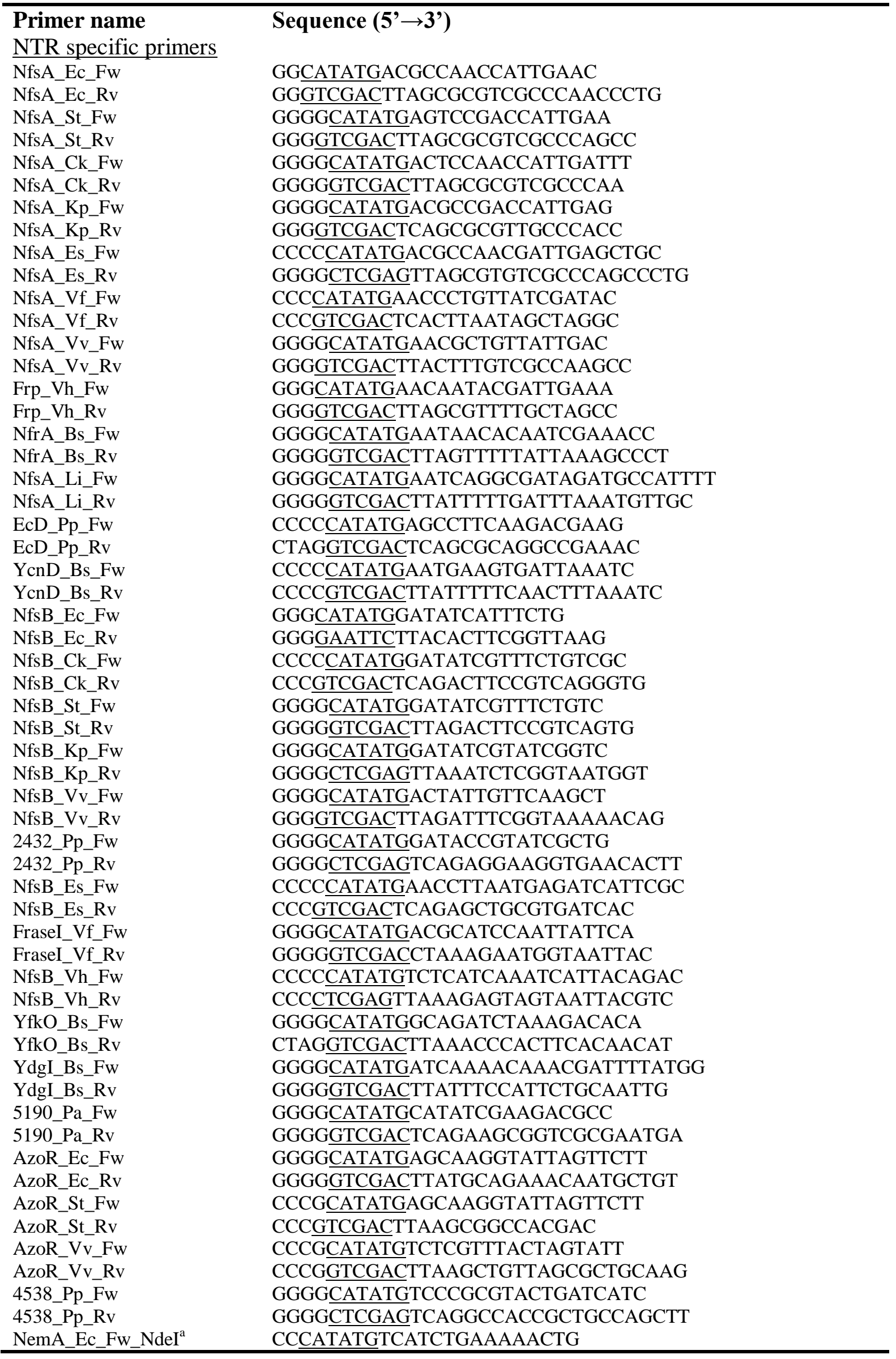




\begin{tabular}{|c|c|}
\hline NemA_Ec_Fw_BamHI ${ }^{\mathrm{a}}$ & CTAGGGATCCATGTCATCTGAAAAACTG \\
\hline NemA_Ec_Rv & CTAGGTCGACTTACAACGTCGGGTAATC \\
\hline NemA_St_Fw & CCCGGATCCATGTCATCAGCAAAACTG \\
\hline NemA_St_Rv & CCCGGTCGACTTACAGAGTAGGGTAGTC \\
\hline NemA_Kp_Fw & CCCCATATGTCGGAAGCAA \\
\hline NemA_Kp_Rv & CCCCTCGAGTTACAGAGTCGGGTAA \\
\hline NemA_Vv_Fw & CCC $\overline{\text { CATATGAGCAAACTGTTTGAA }}$ \\
\hline NemA_Vv_Rv & CCC $\overline{\text { GTCGACTCACACATGCAAAGCGGT }}$ \\
\hline YwrO_Bs_Fw & AAATTTGGATCCATGAAAATATTGGTTTTGGC \\
\hline YwrO_Bs_Rv & 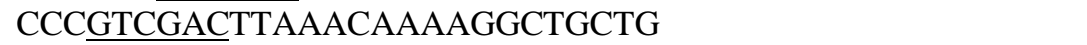 \\
\hline YwrO_Li_Fw & ССССCATATGAAAACATTAGTTATTAT \\
\hline YwrO_Li_Rv & CCCGTCGACCTAATTTAACGTTTTAATA \\
\hline YwrO_Vf_-Fw & AAATTTGGATCCATGACAAGCCCGGCT \\
\hline YwrO_Vf_Rv & CCCGTCEACTTATTCCTTAATAAATTTATC \\
\hline YieF_Ec_Ew & GGGGCATATGTCTGAAAAATTGCAGGTG \\
\hline YieF_Ec_Rv & 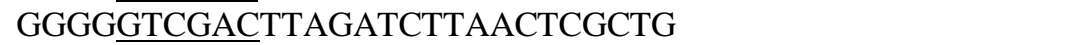 \\
\hline 1204_Pa_Fw & GGGG $\overline{\text { CATATG }}$ AGCGACGACATCAAG \\
\hline 1204_Pa_Rv & 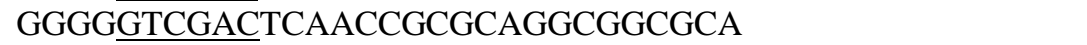 \\
\hline MdaB_Ec_Fw & GGGCATATGAGCAACATCCTG \\
\hline MdaB_Ec_Rv & 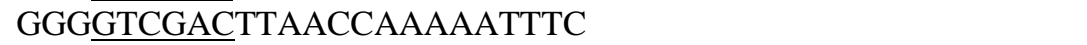 \\
\hline MdaB_Ps_Fw & GGGGCATATGAAAAAAGTATTGTTCCTCAAC \\
\hline MdaB_Ps_Rv & CTAGCTCGAGTTACTGCGCACCAAACACTT \\
\hline WrbA_Ec_Fw & GGGCATATGGCTAAAGTTCTGGTG \\
\hline WrbA_Ec_Rv & GGG $\overline{\text { GTCGACTTAGCCGTTAAGTTT }}$ \\
\hline WrbA_Ps_Fw & GGGGCATATGAGCAAACCTTACATTCTGG \\
\hline WrbA_Ps_Rv & 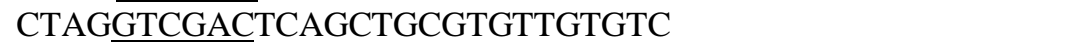 \\
\hline YdjA_Ec_Fw & CTAG $\overline{\text { CATATGGATGCACTCGAACTATTG }}$ \\
\hline YdjA_Ec_Rv & CTAGGTCGACTCAGAAATAAGTTACAAACGG \\
\hline YdjA_Kp_Fw & 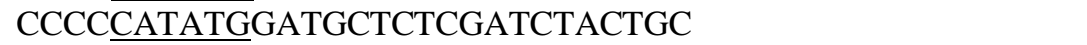 \\
\hline YdjA_Kp_Rv & CCCC $\overline{\text { GTCGACTCAGAATCGCGTCACAAACG }}$ \\
\hline 3720_Pp_Fw & GGGGCATATGCTTGTGAATGTACTG \\
\hline 3720_Pp_Rv & GGGGGTCGACTCACGTCAGCGGGTACAACG \\
\hline 4975_Pa_Fw & GGGG育ATATGAACGTACTGATCGTC \\
\hline 4975_Pa_Rv & GGGGGTCGACTCAGCGCGCCAGCGGCTGGA \\
\hline YcdI_Ec_Fw & 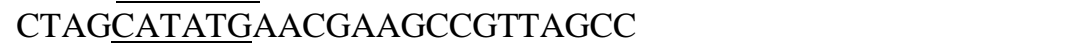 \\
\hline YcdI_Ec_Rv & 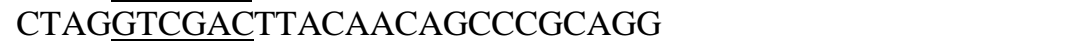 \\
\hline YcdI_Kp_Fw & GGGGCATATGAACGACGCGATAAACCA \\
\hline YcdI_Kp_Rv & CTAGCTCGAGTTATGCCAGGCAGGCT \\
\hline \multicolumn{2}{|l|}{ NTR KO primers } \\
\hline NfsA_KO_Fw & $\begin{array}{l}\text { GGGCAGAAAGAGAAAAAGATAATGACGCCAACCATTGAATGT } \\
\text { GTAGGCTGGAGCTGCTTC }\end{array}$ \\
\hline NfsA_KO_Rv & $\begin{array}{l}\text { GGGTACATCGACGTGGCGGTTTTAGCGCGTCGCCCAACCCAT } \\
\text { ATGAATATCCTCCTTAG }\end{array}$ \\
\hline NfsB_KO_Fw & $\begin{array}{l}\text { GGGCCCGGCAAGAGAGAATTACACTTCGGTTAAGGTGATTGT } \\
\text { GTAGGCTGGAGCTGCTTC }\end{array}$ \\
\hline NfsB_KO_Rv & $\begin{array}{l}\text { GGGTCACATGGAGTCTTTATGGATATCATTTCTGTCGCCCATA } \\
\text { TGAATATCCTCCTTAG }\end{array}$ \\
\hline AzoR_KO_Fw & $\begin{array}{l}\text { TTATGCAGAAACAATGCTGTCGATGGCTGCTTTTGCTGTGTAG } \\
\text { GCTGGAGCTGCTTG }\end{array}$ \\
\hline AzoR_KO_ext_Fw & САAACATCTATAAGGAAACACCATGAGCAAGGTATTAGTTC \\
\hline AzoR_KO_Rv & $\begin{array}{l}\text { ATGAGCAAGGTATTAGTTCTTAAATCCAGCATCCTGCATATGA } \\
\text { ATATCCTCCTTAG }\end{array}$ \\
\hline AzoR_KO_ext_Rv & CCACATCAAGACCGTGTCCGGTTATGCAGAAACAATGCTG \\
\hline NemĀ_KO_Fw' & $\begin{array}{l}\text { ATGTCATCTGAAAAACTGTATTCCCCACTGAAAGTGTGTGTAG } \\
\text { GCTGGAGCTGCTTC }\end{array}$ \\
\hline NemA_KO_ext_Fw & ATCACCAGACGACCGGGAGCCTTTATGTCATCTGAAAAACGT \\
\hline NemA_KO_Rv & $\begin{array}{l}\text { TTACAACGTCGGGTAATCGGTATAGCCTTCCGCGCCCATATGA } \\
\text { ATATCCTCCTTAG }\end{array}$ \\
\hline NemA_KOext_Rv & CTTTACGCCGCTCGCAATGTTGGATTACAACGTCGGGTAAT \\
\hline Other $\mathrm{KO}$ primers & \\
\hline
\end{tabular}




\begin{tabular}{|c|c|}
\hline TolC_KO_Fw & $\begin{array}{l}\text { ATCGGCCTGAGCCTTTCTGGGTTCAGTTCGTTGAGCTGTGTAG } \\
\text { GCTGGAGCTGCTTC }\end{array}$ \\
\hline TolC_KO_ext_Fw & ATGAAGAAATTGCTCCCCATTCTTATCGGCCTGAGCCTT \\
\hline TolC_KO_Rv & $\begin{array}{l}\text { GTTACTGGTGGTAGTGCGTGCGGATGTTTGCTGAACCATATG } \\
\text { ААТАТССТССТТАG }\end{array}$ \\
\hline TolC_KO_ext_Rv & TCAGTTACGGAAAGGGTTATGACCGTTACTGGTGGTAGT \\
\hline Ada_KO_Fw & $\begin{array}{l}\text { GCGAAAAAAATTAAAGCGCAAGATTGTTGGTTTTTGCGTGTGT } \\
\text { GTAGGCTGGAGCTGCTTC }\end{array}$ \\
\hline Ada_KO_ext_Fw & TCCTTAACCAGGGAGCTGATTATGAAAAAAGCC \\
\hline Ada_Fw2 & CCTGGATGTCACCACAG \\
\hline Ada_KO_Rv & $\begin{array}{l}\text { GGCTCTTGCCACGGTTCAGCATCGGCAAACAGATCCAACACA } \\
\text { TATGAАТАТССТССТТАG }\end{array}$ \\
\hline Ada_KO_ext_Rv & CTCTTGCCACGGTTCAGCATCGGCAAACAGATCCA \\
\hline Ada_Rv2 $2--$ & CTTGCCACGGTTCAGCATC \\
\hline Ogt_KO_Fw & $\begin{array}{l}\text { GGTCCGGGTATTTACCCACGTTTGTCTTAAGAGAGAACGGTG } \\
\text { TGTAGGCTGGAGCTGCTTC }\end{array}$ \\
\hline Ogt_KO_ext_Fw & GTTTGTCTTAAGAGAGAACGGATGCTGAGATTAC \\
\hline Ogt_Fw2 & CTTGTCGGTCTGCCGATAGGTC \\
\hline Ogt_KO_Rv & $\begin{array}{l}\text { CATAAAAGTGTGAACAAGCTGGCACAAATTGTTTAATGTCATA } \\
\text { TGAАТАТССТССТТАG }\end{array}$ \\
\hline Ogt_KO_ext_Rv & TGGCACAAATTGTTTAATGTTTACAGCAAAAG \\
\hline Ogt_Rv2 & CATAAAAGTGTGAACAAGCTGG \\
\hline MutS_KO_Fw & $\begin{array}{l}\text { ATCACACCCCATTTAATATCAGGGAACCGGACATAACCCCTGT } \\
\text { GTAGGCTGGAGCTGCTTC }\end{array}$ \\
\hline MutS_KO_ext_Fw & GATTACAACGAAAATAAAAACCATCACACCCCA \\
\hline MutS_Fw2 & GGTATCATGTGCGCCTTATG \\
\hline MutS_KO_Rv & $\begin{array}{l}\text { GTTAATATTCCCGATAGCAAAAGACTATCGGGAATTGTTACAT } \\
\text { ATGAАТАТССТССТТАG }\end{array}$ \\
\hline MutS_KO_ext_Rv & TATTTTATTCGTCAGTTGTCGTTAATATTCCCG \\
\hline MutS_Rv2 & CGTCAGTTGTCGTTAATATTCCCG \\
\hline UvrB_KO_Fw & $\begin{array}{l}\text { TCCTTCAGGTAGCGACTCATGAGTAAACCGTTCAAATGTGTAG } \\
\text { GCTGGAGCTGCTTC }\end{array}$ \\
\hline UvrB_Fw2 & GCTTTGGGGATAACCCGGTAAGGC \\
\hline UvrB_KO_Rv & $\begin{array}{l}\text { GTCTTCTTCGCTATCCTGTTACGATGCCGCGATAAACATATGA } \\
\text { АТАТССТССТТАG }\end{array}$ \\
\hline UvrB_Rv2 & CTGGAAAAGGCGTTACAGGCTTTA \\
\hline \multicolumn{2}{|c|}{ Primers for construction of pUCX and pUCXKT } \\
\hline pUCF1Fw & CTAGACCGGTGCTCTTCCGCTTCCT \\
\hline pUCF1Rv & GGGGACTAGTGTGCACTCTCAGTACAAT \\
\hline NewpUCF2Rv & CTAGACCGGTTCACTGCCCGCTTTCCA \\
\hline BlaPromFw & GGGGACTAGTCGGATACATATTTGAATG \\
\hline pETRBSRv & $\begin{array}{l}\text { GGGGCATATGATATCTCCTTCTTAAAGTTAAACAAAATTATTTCT } \\
\text { AGAGGGGAATTGTTATCCGCTC }\end{array}$ \\
\hline pUCXWTFw & CCCCCATATGGGATCCTCTAGAGTCGAC \\
\hline KantruncSalIFw3 & CCCCGTCGACTAATAGAGCTCGATGGATTGCACGCAGGTTC \\
\hline KantruncHindIIIRv & CCCCAAGCTTTCAGAAGAACTCGTCAAGAAGG \\
\hline pUCXSacIRv & 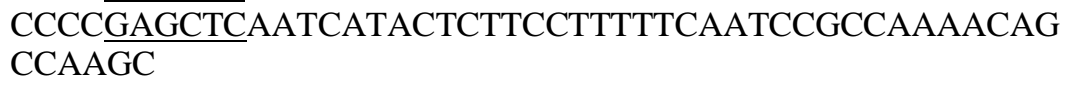 \\
\hline \multicolumn{2}{|c|}{ Primers for NTR site-directed mutagenesis } \\
\hline YcnD_NdeIdisruptFw & AGCTGACGTCTTACATTCAGGCTTACGATGAGAAAATGTCTG \\
\hline YcnD_NdeIdisruptRv & CTGAATGTAAGACGTCAGCTCATC \\
\hline YcnD_SDM15Fw & TAACAGACCACCGCTCGATTNNKAGCTATACAGATGAACC \\
\hline YcnD_SDM15Rv & AATCGAGCGGTGGTCTGTTAATG \\
\hline YcnD_SDM41Fw & TACAATCTGCCCCAACTTCTNNKAACGGGCAGCAAGTGAC \\
\hline YcnD_SDM41Rv & AGAAGTTGGGGCAGATTGTACCGC \\
\hline YcnD_SDM65Fw & AGAAAATCTCCGAGCTGTCANNKGGACAGCCTTGGATTG \\
\hline YcnD_SDM67Fw & $\begin{array}{l}\text { AGAAAATCTCCGAGCTGTCAGGCGGANNKCCTTGGATTGACCAG } \\
\text { GC }\end{array}$ \\
\hline YcnD_SDM69Fw & $\begin{array}{l}\text { AGAAAATCTCCGAGCTGTCAGGCGGACAGCCTNNKATTGACCAG } \\
\text { GCTCCTG }\end{array}$ \\
\hline
\end{tabular}




\begin{tabular}{|c|c|}
\hline YcnD_SDM65_67_69Rv & TGACAGCTCGGAGATTTTCTTTTTGCG \\
\hline YcnD_SDM105̄ Fw & TGGAAATCACAAATGGCTTANNKTCTGTTCTTGTCGGCG \\
\hline YcnD_SDM105Rv & TAAGCCATTTGTGATTTCCATTTTG \\
\hline YcnD_SDM139Fw & CGGTTCCGATCGGTGCGGTTNNKGGAAACCCTCAAGAGC \\
\hline YcnD_SDM140Fw & CGGTTCCGATCGGTGCGGTTCGCNNKAACCCTCAAGAGCTGATC \\
\hline YcnD_SDM139_140Rv & AACCGCACCGATCGGAACCG \\
\hline YcnD_SDM227Fw & GGTCACAGGGCATCGCATCCNNKTATGAGCGCCTGTAC \\
\hline YcnD_SDM227Rv & GGATGCGATGCCCTGTGACC \\
\hline YcnD_SDM233Fw & CCTATTATGAGCGCCTGTACNNKCCACACATCCGTGAAATG \\
\hline YcnD_SDM233Rv & GTACAGGCGCTCATAATAGGATGC \\
\hline YcnD_65upstreamFw & AGTCCAGGATAAAGAGCGC \\
\hline YcnD_139upstreamFw & TGAATCACTCGGACTTGGC \\
\hline YcnD_227upstreamFw & AGAAACTGGTCACAGGGC \\
\hline YcnD_233upstreamFw & TATGAGCGCCTGTAC \\
\hline YcnD_233upstreamRv & GTACAGGCGCTCATA \\
\hline \multicolumn{2}{|l|}{ Miscellaneous primers } \\
\hline pMMBFw & GGCTCGTATAATGTGTGG \\
\hline pMMBRv & GACCGCTTCTGCGTTCTGAT \\
\hline T7prom & TAATACGACTCACTATAGGG \\
\hline T7term & GCTAGTTATTGCTCAGCGG \\
\hline M13Fw & GTAAAACGACGGCCAG \\
\hline M13Rv & CAGGAAACAGCTATGAC \\
\hline T7FwpMMBFw & TAATACGACTCACTATAGGGGGCTCGTATAATGTGTGG \\
\hline T7FwpUCXpromBamHIFw & $\begin{array}{l}\text { TAATACGACTCACTATAGGGGGATCCTCACTGCATAATTCGTGTC } \\
\text { G }\end{array}$ \\
\hline V5Rv & ACCGAGGAGAGGGTTAGGGAT \\
\hline AKR1C3 Fw & ССССАTATGGATTCCAAACACCAGTG \\
\hline AKR1C3 Rv & 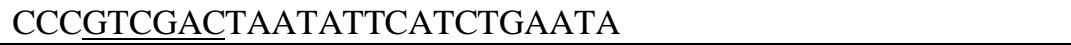 \\
\hline
\end{tabular}

\subsection{Bacterial strains and plasmids}

All bacterial strains used in this study are listed in Tables 2.2 and 2.3. Plasmids are listed in Table 2.4.

Table 2.2. Bacterial strains used in this study.

\begin{tabular}{|c|c|c|}
\hline Strain & Relevant characteristics & Source \\
\hline $\mathrm{DH} 5 \alpha$ & 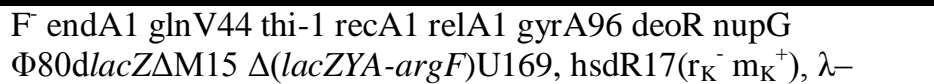 & Invitrogen \\
\hline DH5 $\alpha \lambda$ (pir) & 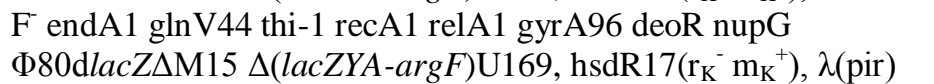 & Invitrogen \\
\hline BL21(DE3) & $\begin{array}{l}\mathrm{F}^{-} \text {ompT gal dem lon hsdS } \mathrm{B}_{\mathrm{B}}\left(\mathrm{r}_{\mathrm{B}}{ }^{-} \mathrm{m}_{\mathrm{B}}{ }^{-}\right) \lambda(\mathrm{DE} 3 \text { [lacI lacUV5-T7 gene } \\
1 \text { ind1 sam7 nin5]) }\end{array}$ & Novagen \\
\hline ADA510 & F lac-6(del) $\lambda \Phi($ sulA::lacZ $)$ & $(231)$ \\
\hline SOS-R1 & ADA510 $\Delta n f_{s A} \Delta n f_{s B}$ & This study \\
\hline SOS-R1 $\Delta$ adaogt & SOS-R1 $\Delta a d a \Delta o g t$ & This study \\
\hline SOS-R1 $\Delta m u t S$ & SOS-R1 $\Delta m u t S$ & This study \\
\hline SOS-R1 $\Delta u v r B$ & SOS-R1 $\Delta u v r B$ & This study \\
\hline SOS-R1B & SOS-R1 $\triangle a z o R \Delta n e m A$ & This study \\
\hline SOS-R2 & SOS-R1B $\Delta$ tolC & This study \\
\hline
\end{tabular}


Table 2.3. Bacterial strains used as sources of NTRs in this study

\begin{tabular}{|c|c|}
\hline Bacterial genus, species and strain ${ }^{a}$ & Source \\
\hline Escherichia coli $\mathrm{W} 3110$ & Lab stock \\
\hline Salmonella enterica (typhimurium) Ty2 & ESR culture collection ${ }^{\mathrm{b}}$ \\
\hline Klebsiella pneumoniae ATCC 13883 & ESR culture collection \\
\hline Vibrio vulnificus ATCC 27562 & ESR culture collection \\
\hline Vibrio harveyi ATCC 33843 & ESR culture collection \\
\hline Vibrio fischeri ATCC $7744^{\mathrm{b}}$ & ESR culture collection \\
\hline Pseudomonas aeruginosa $\mathrm{PAO} 1$ & Lab stock \\
\hline Pseudomonas putida KT2440 & Lab stock \\
\hline Pseudomonas syringae $1448 \mathrm{a}$ & Lab stock \\
\hline Bacillus subtilis subsp. Subtilis NZ isolate & ESR culture collection \\
\hline Enterobacter (Chronobacter) sakazaki ATCC 29544 & ESR culture collection \\
\hline Citrobacter koseri ATCC 27156 & ESR culture collection \\
\hline Listeria innocua Clip11262 & ESR culture collection \\
\hline
\end{tabular}

\footnotetext{
${ }^{a}$ All strains were propagated in LB media, with the exception of $V$. vulnificus and $V$. harveyi (marine broth).

${ }^{\mathrm{b}}$ Institute of Environmental Science and Research, Porirua, NZ.

${ }^{c}$ Only genomic DNA was available for $V$. fischeri.
}

Table 2.4. Plasmids used in this study.

\begin{tabular}{|c|c|c|}
\hline Plasmid & Relevant characteristics & Source \\
\hline$\overline{\text { pUC19 }}$ & $\begin{array}{l}\text { Amp }{ }^{\mathrm{R}} \text {. Cloning plasmid, pMB1 origin of replication. } \\
\text { Source of DNA for pUCX }\end{array}$ & Invitrogen \\
\hline pMMB67EH(nfsA) & $\begin{array}{l}\mathrm{Amp}^{\mathrm{R}} \text { E. coli-Pseudomonas expression vector. tac } \\
\text { promoter, lac operator, E. coli nfsA gene insert. } \\
\text { Source of DNA for pUCX }\end{array}$ & $(184)$ \\
\hline pUCX & $\begin{array}{l}\mathrm{Amp}^{\mathrm{R}} \text {. E. coli expression vector. tac promoter, lac } \\
\text { operator, pET } 28 \mathrm{a}(+) \mathrm{RBS} \text {. }\end{array}$ & This study \\
\hline pUCXKT & $\begin{array}{l}\mathrm{Amp}^{\mathrm{R}} \cdot \mathrm{pUCX} \text { with truncated } k a n^{\mathrm{R}} \text { gene downstream } \\
\text { of MCS. }\end{array}$ & This study \\
\hline pUCXKG:ntr & $\begin{array}{l}\mathrm{Amp}^{\mathrm{R}}, \mathrm{Kan}^{\mathrm{R}} \cdot \mathrm{pUCXKT}^{\mathrm{U} U \mathrm{~T}} \text { wh } n t r \text { gene insert and } \\
\text { functional } \text { kan }^{\mathrm{R}} \text { gene. }\end{array}$ & This study \\
\hline pET28a $(+)$ & $\begin{array}{l}\text { Kan }^{\mathrm{R}} \text {. Expression vector for } 6 \text { His-tagged enzyme } \\
\text { purification. T7 promoter. }\end{array}$ & Novagen \\
\hline pDONR221 & $\begin{array}{l}\text { Kan }{ }^{\mathrm{R}} \text {. Entry vector for Gateway recombination } \\
\text { cloning. }\end{array}$ & Invitrogen \\
\hline pF527-V5 & $\begin{array}{l}A m p^{\mathrm{R}} \text {. Destination vector for Gateway } \\
\text { recombination cloning. Mammalian expression } \\
\text { vector. Elongation-factor-1 } \alpha \text { promoter (constitutive) }\end{array}$ & ACSRC \\
\hline pKD4 & $\begin{array}{l}\text { Amp }^{\mathrm{R}}, \mathrm{Kan}^{\mathrm{R}} \text {. Pir-origin of replication. } \text { kan }^{\mathrm{R}} \text { gene- } \\
\text { template for KO-PCR. }\end{array}$ & $(237)$ \\
\hline pKD46 & $\begin{array}{l}\text { Amp }{ }^{R} \text {. Heat sensitive origin of replication. } \\
\text { Recombinase expression vector. ara operon } \\
\text { promoter. }\end{array}$ & $(237)$ \\
\hline pCP20 & $\mathrm{Amp}^{\mathrm{R}}$. Heat sensitive origin of replication. & $(237)$ \\
\hline
\end{tabular}

\subsection{Bioinformatics}

Protein homology searches were carried out in the National Centre for Biotechnology

Information (NCBI) protein database using the alignment search algorithm protein-

protein BLAST (blastp; http://blast.ncbi.nlm.nih.gov/; (238)). 


\subsection{Bacterial growth and maintenance}

\subsubsection{Growth media}

Unless otherwise stated, all media listed below were made up to the required volume with $\mathrm{ddH}_{2} \mathrm{O}$ and sterilized by autoclaving for $30 \mathrm{~min}$ at $121^{\circ} \mathrm{C}$ prior to use. Media were stored at either room temperature (no supplements), or $4{ }^{\circ} \mathrm{C}$ (supplemented with antibiotics or other heat-sensitive chemicals).

\subsubsection{Lysogeny Broth (LB) ${ }^{\mathrm{a}}$}

\begin{tabular}{ll}
\hline Component & Final concentration \\
\hline Tryptone & $10 \mathrm{~g} \mathrm{~L}^{-1}$ \\
Yeast extract & $5 \mathrm{~g} \mathrm{~L}^{-1}$ \\
$\mathrm{NaCl}$ & $10 \mathrm{~g} \mathrm{~L}^{-1}$ \\
\hline${ }^{\mathrm{a}} \mathrm{LB}$ was supplied from the manufacturer as a premixed powder.
\end{tabular}

\subsubsection{SOB, $\mathrm{SOC}^{\mathrm{a}}$}

\begin{tabular}{ll}
\hline Tryptone & $20 \mathrm{~g} \mathrm{~L}^{-1}$ \\
Yeast extract & $5 \mathrm{~g} \mathrm{~L}^{-1}$ \\
$\mathrm{NaCl}$ & $10 \mathrm{mM}$ \\
$\mathrm{KCl}$ & $2.5 \mathrm{mM}$ \\
$\mathrm{MgCl}_{2}{ }^{\mathrm{b}}$ & $10 \mathrm{mM}$ \\
$\mathrm{MgSO}_{4}{ }^{\mathrm{b}}$ & $10 \mathrm{mM}$ \\
\hline
\end{tabular}

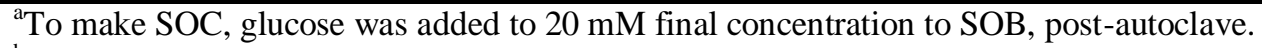

${ }^{\mathrm{b}} \mathrm{MgCl}_{2}$ and $\mathrm{MgSO}_{4}$ were added post-autoclave.

\subsubsection{ZYP5052 (autoinduction medium)}

\begin{tabular}{ll}
\hline ZY Base $^{\mathrm{a}}$ & $919 \mathrm{~mL} \mathrm{~L}^{-1}$ \\
$20 \times \mathrm{NPS}^{\mathrm{a}}$ & $50 \mathrm{~mL} \mathrm{~L}^{-1}$ \\
$50 \times 5052^{\mathrm{a}}$ & $20 \mathrm{~mL} \mathrm{~L}^{-1}$ \\
$100 \times$ metal salts $^{\mathrm{a}}$ & $10 \mathrm{~mL} \mathrm{~L}^{-1}$ \\
$\mathrm{MgSO}_{4}$ & $1 \mathrm{mM}$ \\
\hline${ }^{\mathrm{a}}$ All solutions were made up separately and autoclaved prior to mixing.
\end{tabular}

${ }^{a}$ All solutions were made up separately and autoclaved prior to mixing.

\subsection{ZY Base}

\begin{tabular}{ll}
\hline Tryptone & $10.9 \mathrm{~g} \mathrm{~L}^{-1}$ \\
Yeast extract & $5.4 \mathrm{~g} \mathrm{~L}^{-1}$ \\
\hline
\end{tabular}

2.5.1.3.2. $20 \times$ NPS

\begin{tabular}{ll}
\hline $\mathrm{NH}_{4} \mathrm{SO}_{2}$ & $0.5 \mathrm{M}$ \\
$\mathrm{KH}_{2} \mathrm{PO}_{4}$ & $1 \mathrm{M}$ \\
$\mathrm{Na}_{2} \mathrm{HPO}_{4}$ & $1 \mathrm{M}$ \\
\hline
\end{tabular}




\begin{tabular}{ll}
\hline Glycerol & $250 \mathrm{~g} \mathrm{~L}^{-1}$ \\
Glucose & $25 \mathrm{~g} \mathrm{~L}^{-1}$ \\
Lactose & $100 \mathrm{~g} \mathrm{~L}^{-1}$ \\
\hline
\end{tabular}

\subsubsection{4. $100 \times$ metal salts}

\begin{tabular}{|c|c|}
\hline $\mathrm{CaCl}_{2} \cdot 2 \mathrm{H}_{2} \mathrm{O}$ & $0.1 \mathrm{~g} \mathrm{~L}^{-1}$ \\
\hline $\mathrm{CoCl}_{2} \cdot 2 \mathrm{H}_{2} \mathrm{O}$ & $0.04 \mathrm{~g} \mathrm{~L}^{-1}$ \\
\hline $\mathrm{CuSO}_{4} .5 \mathrm{H}_{2} \mathrm{O}$ & $0.02 \mathrm{~g} \mathrm{~L}^{-1}$ \\
\hline EDTA & $1 \mathrm{~g} \mathrm{~L}^{-1}$ \\
\hline $\mathrm{FeSO}_{4} \cdot 7 \mathrm{H}_{2} \mathrm{O}$ & $0.5 \mathrm{~g} \mathrm{~L}^{-1}$ \\
\hline $\mathrm{MgCl}_{2} \cdot 6 \mathrm{H}_{2} \mathrm{O}$ & $10 \mathrm{~g} \mathrm{~L}^{-1}$ \\
\hline $\mathrm{MnCl}_{2} \cdot 2 \mathrm{H}_{2} \mathrm{O}$ & $0.1 \mathrm{~g} \mathrm{~L}^{-1}$ \\
\hline $\mathrm{NaMoO}_{4} \cdot 2 \mathrm{H}_{2} \mathrm{O}$ & $0.02 \mathrm{~g} \mathrm{~L}^{-1}$ \\
\hline $\mathrm{ZnSO}_{4} .7 \mathrm{H}_{2} \mathrm{O}$ & $0.2 \mathrm{~g} \mathrm{~L}^{-1}$ \\
\hline
\end{tabular}

\subsubsection{M63 minimal medium}

\begin{tabular}{ll}
\hline $5 \times \mathrm{M} 63^{\mathrm{a}}$ & $200 \mathrm{~mL} \mathrm{~L}^{-1}$ \\
$\mathrm{MgSO}_{4}{ }^{\mathrm{b}}$ & $2 \mathrm{mM}$ \\
Carbon source (glucose, lactose) $^{\mathrm{b}}$ & $4 \mathrm{~g} \mathrm{~L}^{-1}$ \\
Cas-amino acids $^{\mathrm{a}}$ & $2 \mathrm{~g} \mathrm{~L}^{-1}$ \\
Thiamine-HCl $^{\mathrm{b}}$ & $10 \mathrm{mg} \mathrm{L}^{-1}$ \\
\hline
\end{tabular}

${ }^{a}$ Solutions were made up and autoclaved separately.

${ }^{\mathrm{b}}$ Solutions were made up separately and sterilized by passage through a $0.22 \mu \mathrm{m}$ filter.

\subsubsection{1. $5 \times{\mathrm{M} 63^{\mathrm{a}}}^{\mathrm{a}}$}

\begin{tabular}{ll}
\hline $\mathrm{KH}_{2} \mathrm{PO}_{4}$ & $68 \mathrm{~g} \mathrm{~L}^{-1}$ \\
$\mathrm{FeSO}_{4} .7 \mathrm{H}_{2} \mathrm{O}$ & $2.5 \mathrm{mg} \mathrm{L}^{-1}$ \\
$\left(\mathrm{NH}_{4}\right)_{2} \mathrm{SO}_{4}$ & $10 \mathrm{~g} \mathrm{~L}^{-1}$ \\
\hline
\end{tabular}

${ }^{\text {a }}$ Solution was adjusted to $\mathrm{pH} 7.0$ with $\mathrm{KOH}$ prior to autoclaving.

\subsubsection{Marine Broth}

Marine broth was supplied by the manufacture (Sigma-Aldrich) as a ready mixed powder. Liquid Marine broth was prepared according to the manufacturer's instructions.

\subsubsection{Solid media}

To make solid media, molecular biology-grade agar was added to liquid media to $1.5 \%$ (w/v) final concentration, prior to autoclaving. If supplements were to be added, the agar was allowed to cool to $<50{ }^{\circ} \mathrm{C}$, post-autoclave, before addition. Subsequently, 
medium was aliquoted $(15-20 \mathrm{~mL}$ each) into sterile $90 \mathrm{~mm} \times 15 \mathrm{~mm}$ Petri dishes in a laminar fume hood and left to solidify. Plates were stored at $4{ }^{\circ} \mathrm{C}$.

\subsubsection{Antibiotics and other media supplements}

All antibiotics and media supplements used in this study were made up to the indicated stock concentration in the recommended solvent (see Table 2.5), filter-sterilized using a $0.22 \mu \mathrm{m}$ filter and stored indefinitely at $-20{ }^{\circ} \mathrm{C}$.

Table 2.5. Properties of media supplements used in this work

\begin{tabular}{lll}
\hline Supplement & $\begin{array}{l}\text { Stock } \\
\text { concentration }\end{array}$ & Solvent \\
\hline Ampicillin & $100 \mathrm{mg} \mathrm{mL}^{-1}$ & $\mathrm{ddH}_{2} \mathrm{O}$ \\
Kanamycin & $50 \mathrm{mg} \mathrm{mL}^{-1}$ & $\mathrm{ddH}_{2} \mathrm{O}$ \\
Rifampicin & $50 \mathrm{mg} \mathrm{mL}^{-1}$ & $\mathrm{DMSO}^{\mathrm{a}}$ \\
IPTG & $100 \mathrm{mg} \mathrm{mL}^{-1}$ & $\mathrm{ddH}_{2} \mathrm{O}$ \\
\hline
\end{tabular}

${ }^{\mathrm{a}}$ Rifampicin was stored wrapped in aluminium foil due to inactivation by light.

\subsubsection{Bacterial growth and storage}

For routine growth in liquid media, E. coli strains were propagated in LB medium, with relevant supplements, at $37{ }^{\circ} \mathrm{C}$ and shaking at 200 revolutions per minute (rpm), unless otherwise stated. Bacteria grown on solid media were stored at $4{ }^{\circ} \mathrm{C}$ for no longer than one month. For longer term storage, bacteria in liquid media were supplemented with glycerol to $40 \%(\mathrm{w} / \mathrm{v})$ final concentration, and kept at $-80{ }^{\circ} \mathrm{C}$.

\subsection{Cloning methods, routine molecular biology}

\subsubsection{Preparation of genomic DNA (gDNA)}

Bacterial gDNA was extracted from $3 \mathrm{~mL}$ overnight culture using the DNeasy ${ }^{\mathrm{TM}}$ Blood and Tissue kit (Qiagen, GmbH, Germany), following the manufacturer's instructions. Genomic DNA preparations were stored at $-20^{\circ} \mathrm{C}$. 


\subsubsection{PCR protocols}

For construction of vectors and amplification of NTR genes for cloning, either

Phusion $^{\mathrm{TM}}$ high-fidelity polymerase or Accuzyme ${ }^{\mathrm{TM}}$ Mix were used. For all other PCRs, including colony screening, diagnostic and gene-knockout PCR creation, BioMix ${ }^{\mathrm{TM}}$ Red was used. PCR reactions were set up as outlined in Table 2.6 and thermal cycling parameters were employed as outlined in Table 2.7.

Table 2.6. Composition of $\mathrm{PCR}$ reactions

\begin{tabular}{|c|c|}
\hline Biomix $^{\mathrm{TM}}$ Red, Accuzyme ${ }^{\mathrm{TM}}$ Mix & Volume per $20 \mu \mathrm{L}$ reaction \\
\hline Biomix $^{\text {TM }}$ Red/Accuzyme ${ }^{\text {TM }}$ Mix & $10 \mu \mathrm{L}$ \\
\hline Primer 1 & $1 \mu \mathrm{L}$ \\
\hline Primer 2 & $1 \mu \mathrm{L}$ \\
\hline Template DNA $^{\mathrm{a}}$ & $0.2-1 \mu \mathrm{L}$ \\
\hline $\mathrm{ddH}_{2} \mathrm{O}$ & To $20 \mu \mathrm{L}$ final volume \\
\hline
\end{tabular}

\begin{tabular}{ll}
\hline Phusion $^{\mathrm{TM}}$ & Volume per $20 \boldsymbol{\mu L}$ reaction \\
\hline $5 \times$ Phusion $^{\mathrm{TM}} \mathrm{HF}$ buffer & $4 \mu \mathrm{L}$ \\
$10 \mathrm{mM}^{\mathrm{m}} \mathrm{dP}$ mix & $0.4 \mu \mathrm{L}$ \\
Phusion $^{\mathrm{TM}}$ high-fidelity polymerase & $0.2 \mu \mathrm{L}$ \\
Primer 1 & $1 \mu \mathrm{L}$ \\
Primer 2 & $1 \mu \mathrm{L}$ \\
Template DNA & $0.2-1 \mu \mathrm{L}$ \\
$\mathrm{ddH}_{2} \mathrm{O}$ & To $20 \mu \mathrm{L}$ final volume \\
\hline${ }^{\mathrm{a}}$ For colony PCR screens, a small amount of E. coli colony, picked directly from an agar plate with a \\
sterile toothpick or pipette tip, was used as template DNA
\end{tabular}

Table 2.7. Thermal cycling parameters of PCR reactions, in chronological order.

\begin{tabular}{cll}
\hline${\text { Temperature }\left({ }^{\circ} \mathbf{C}\right)}^{\text {Phusion }}$ & Time & Cycles $^{\mathrm{T}}$ \\
98 & $1 \mathrm{~min}$ & \\
98 & $15 \mathrm{sec}$ & $30-35$ \\
72 & $30 \mathrm{sec}$ per kb & \\
72 & $5 \mathrm{~min}$ & \\
16 & Hold & \\
$\underline{\text { Biomix }}^{\mathrm{TM}}$ & Red, Accuzyme & \\
94 & $5 \mathrm{~min}$ & \\
94 & $20 \mathrm{sec}$ & \\
56 & $30 \mathrm{sec}$ & \\
72 & BioMix & \\
& Accuzyme Red: 1 min per kb & \\
94 & $20 \mathrm{sec}$ & \\
52 & $30 \mathrm{sec}$ & \\
72 & BioMix: 2 min per kb & \\
& Accuzyme Red: 1 min per kb & \\
72 & 5 min & \\
16 & Hold & \\
\hline
\end{tabular}

${ }^{\mathrm{a}}$ Where no number is indicated, cycle was only performed once. 


\subsubsection{PCR product purification}

Purification of PCR products was carried out using the commercially available DNA Clean and Concentrator ${ }^{\mathrm{TM}}$ kit (Zymo Research; Irvine, CA, USA), according to the manufacturer's instructions. Products were eluted using sterile-filtered $(0.22 \mu \mathrm{m})$ $\mathrm{ddH}_{2} \mathrm{O}$ and stored at $-20{ }^{\circ} \mathrm{C}$.

\subsubsection{Agarose gel electrophoresis}

To analyse the size and purity of PCR products, plasmid digests and other DNA samples, small aliquots were run on ethidium bromide-containing $1 \%$ agarose gels $(1 \%$ w/v agarose in $1 \times$ TAE buffer, $1 \mu \mathrm{g} \mathrm{mL}^{-1}$ ethidium bromide), submerged in $1 \times \mathrm{TAE}$ buffer in a suitable electrophoresis tank at 120-140 V for 20-40 min. DNA markers of known size were run alongside samples for band comparison. DNA bands were viewed under ultra-violet (UV) light.

\subsubsection{Restriction digests}

Restriction digests were set up as outlined in Table 2.8. Restriction digests were incubated at $37^{\circ} \mathrm{C}$ for $1-6 \mathrm{~h}$, prior to heat inactivation at the recommended (by the manufacturer) temperature and time for the enzyme(s) being used. Digested DNA was then either purified (using the PCR purification protocol outlined in section 2.6.3) and stored at $4{ }^{\circ} \mathrm{C}$, or analysed directly via agarose gel electrophoresis (see section 2.6.4). Purified digested DNA was used for downstream applications either immediately, or within 1-2 days.

Table 2.8. Composition of restriction digests.

\begin{tabular}{ll}
\hline Component & Volume per $20 \mu \mathrm{L}$ reaction \\
\hline $10 \times$ Restriction buffer ${ }^{\mathrm{a}}$ & $2 \mu \mathrm{L}$ \\
$20 \times$ Bovine Serum Albumin (BSA) & $1 \mu \mathrm{L}$ \\
Restriction enzyme 1 & $0.5-1 \mu \mathrm{L}$ \\
Restriction enzyme $2^{\mathrm{b}}$ & $0.5-1 \mu \mathrm{L}$ \\
DNA & As desired \\
dd ${ }_{2} \mathrm{O}$ & To $20 \mu \mathrm{L}$ final volume \\
\hline${ }^{\mathrm{a}}$ For single enzyme digests, the optimal restriction buffer for the enzyme was used. For double digests, the \\
buffer in which both enzymes had the highest activity was used. Restriction enzyme - buffer \\
compatibility data was obtained from the NEB website (http://www.neb.com/nebecomm/default.asp). \\
${ }^{b}$ Second enzyme only added if required.
\end{tabular}




\subsubsection{Ligation}

DNA ligation reactions were set up as outlined in Table 2.9. Reactions were left at room temperature for at least $4 \mathrm{~h}$ (typically overnight), prior to heat inactivation at $65{ }^{\circ} \mathrm{C}$ for 10 min. Products were then used directly to transform E. coli (see below), or stored indefinitely at $-20^{\circ} \mathrm{C}$.

Table 2.9. Composition of ligation reactions.

\begin{tabular}{ll}
\hline Component & Volume/quantity per $10 \mu \mathrm{L}$ reaction \\
\hline $10 \times$ DNA ligase buffer & $1 \mu \mathrm{L}$ \\
T4 DNA ligase & $0.2 \mu \mathrm{L}$ \\
Digested vector DNA & $40-60 \mathrm{ng}^{\mathrm{a}}$ \\
Digested insert DNA & $10-50 \mathrm{ng}^{\mathrm{a}}$ \\
$\mathrm{ddH}_{2} \mathrm{O}$ & To $10 \mu \mathrm{L}$ final volume \\
\hline
\end{tabular}

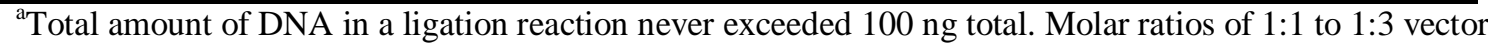
DNA:insert DNA were used.

\subsubsection{Transformation/Electroporation}

\subsubsection{Generation of chemically competent cells}

$3 \mathrm{~mL} \mathrm{LB}+10 \mathrm{mM} \mathrm{MgCl} 2$ cultures of $E$. coli strains to be made competent were inoculated from a single colony (on an agar plate) or a glycerol stock and grown overnight at $37^{\circ} \mathrm{C}, 200 \mathrm{rpm}$. The following day, the overnight culture was diluted 50fold into a fresh $10-50 \mathrm{~mL} \mathrm{LB}+10 \mathrm{mM} \mathrm{MgCl}{ }_{2}$ culture and grown at $37^{\circ} \mathrm{C}, 200 \mathrm{rpm}$ for several hours, until the $\mathrm{OD}_{600}$ reached $\sim 0.4-0.6$. At this point the cells were transferred to ice for $10-15 \mathrm{~min}$. Cells were then centrifuged at $4,000 \mathrm{rpm}$ for $10 \mathrm{~min}$ at $4{ }^{\circ} \mathrm{C}$, the supernatant discarded and the cell pellet resuspended in 1 volume ice-cold TFB I (see Table 2.10 for recipe). Resuspended cells remained on ice for $2 \mathrm{~h}$, prior to another round of centrifugation at $4,000 \mathrm{rpm}, 10 \mathrm{~min}$ at $4{ }^{\circ} \mathrm{C}$. The supernatant was discarded and the cell pellet resuspended in 0.1 volumes of ice-cold TFB II (see Table 2.10 for recipe). Cells were aliquoted $(100-200 \mu \mathrm{L})$ into sterile $1.5 \mathrm{~mL}$ microfuge tubes and snap frozen using a metal tube rack pre-chilled to $-80{ }^{\circ} \mathrm{C}$. Competent cell aliquots were stored at -80 ${ }^{\circ} \mathrm{C}$ indefinitely. 
Table 2.10. Recipes for transformation buffers.

\begin{tabular}{|c|c|}
\hline Component & Final concentration \\
\hline \multicolumn{2}{|l|}{ TFB I $^{\mathrm{a}}$} \\
\hline$\overline{\text { Potassium acetate }}$ & $30 \mathrm{mM}$ \\
\hline $\mathrm{MnCl}_{2}$ & $50 \mathrm{mM}$ \\
\hline $\mathrm{CaCl}_{2}$ & $10 \mathrm{mM}$ \\
\hline Glycerol & $15 \%(\mathrm{w} / \mathrm{v})$ \\
\hline \multicolumn{2}{|l|}{ TFB II $^{\mathrm{a}}$} \\
\hline MOPS, pH 7.0. & $10 \mathrm{mM}$ \\
\hline $\mathrm{CaCl}_{2}$ & $75 \mathrm{mM}$ \\
\hline $\mathrm{KCl}$ & $10 \mathrm{mM}$ \\
\hline Glycerol & $15 \%(\mathrm{w} / \mathrm{v})$ \\
\hline
\end{tabular}

\subsubsection{Transformation}

Chemically competent cell aliquots were removed from the $-80{ }^{\circ} \mathrm{C}$ storage and thawed on ice for 5-10 min. 50-100 $\mu \mathrm{L}$ competent cells were used per transformation. Plasmid DNA to be transformed was added (never more than 0.1 the volume of competent cells being used) to the cells and the mixture kept on ice for 15-20 min. Cells were then heatshocked at $42{ }^{\circ} \mathrm{C}$ for $90 \mathrm{sec}$, typically in a water bath or heating block, and then returned to ice for 1-2 min. 9 volumes of LB or SOC was then added to each tube, followed by incubation at $37{ }^{\circ} \mathrm{C}$ for $45 \mathrm{~min}$ to $1 \mathrm{~h}$. Cells were then spread directly onto LB agar plates containing the necessary antibiotics and supplements and incubated at $37{ }^{\circ} \mathrm{C}$ for at least $16 \mathrm{~h}$, or until colonies became visible.

\subsubsection{Miniprep}

Preparation of plasmid DNA was carried out using either the commercially available Zyppy $^{\mathrm{TM}}$ Plasmid Miniprep kit (Zymo Research; Irvine, CA, USA), according to the manufacturer's instructions, or by using the following protocol:

$3 \mathrm{~mL}$ of an overnight culture (LB) of $E$. coli carrying the plasmid of interest was transferred into $21.5 \mathrm{~mL}$ microfuge tubes and centrifuged at 13,000 rpm for $1 \mathrm{~min}$ at room temperature. The supernatants from both tubes were discarded and the remaining cell pellets resuspended in $250 \mu \mathrm{L}$ buffer P1 (per $3 \mathrm{~mL}$ of original culture; see Table 2.11 for recipes of all buffers used in this protocol) and transferred into a single microfuge tube. Cells were lysed by addition of $250 \mu \mathrm{L}$ buffer P2 and the tube gently 
inverted 10-15 times or until turbidity had dissipated (no longer than $3 \mathrm{~min}$ ). The lysis reaction was terminated by addition of $350 \mu \mathrm{L}$ buffer $\mathrm{N} 3$ and the tube gently inverted 10-15 times or until precipitation was uniform. The tube was then centrifuged at 13,000 rpm for $5 \mathrm{~min}$ at room temperature, following which the supernatant was carefully transferred to a clean miniprep column (either ZYMO or QIAGEN) inserted into a $2 \mathrm{~mL}$ collection tube. The column was then centrifuged for $30 \mathrm{sec}$ at 13,000 rpm and the flow through discarded. $200 \mu \mathrm{L}$ buffer PB was then added to the column, and centrifuged for $15 \mathrm{sec}$ at 13,000 rpm. $400 \mu \mathrm{L}$ buffer PE was next added to the column and centrifuged for $1 \mathrm{~min}$ at 13,000 rpm. The column was then carefully removed from the collection tube (being sure not to let the column come into contact with any ethanol or liquid remaining in the collection tube) and inserted into sterile a $1.5 \mathrm{~mL}$ microfuge tube. 30 $50 \mu \mathrm{L}$ of either sterile $\mathrm{ddH}_{2} \mathrm{O}$ or $1 \times \mathrm{TE}$ buffer was applied directly to the column matrix and left to stand at room temperature for at least one minute. The tube was then centrifuged for $1 \mathrm{~min}$ at 13,000 rpm to elute the plasmid.

All eluted plasmid samples were stored indefinitely at $-20^{\circ} \mathrm{C}$.

Table 2.11. Recipes of buffers used in miniprep protocol described in section 2.6.8.

\begin{tabular}{ll}
\hline Component & Concentration \\
\hline Buffer P1 & $50 \mathrm{mM}$ \\
Tric-HCl pH 8.0 & $10 \mathrm{mM}$ \\
EDTA pH 8.0 & $50 \mu \mathrm{gL}^{-1}$ \\
RNase A & \\
Buffer P2 & $200 \mathrm{mM}$ \\
NaOH & $1 \%$ \\
SDS & \\
Buffer N3 & $4.4 \mathrm{M}$ \\
Guanidine hydrochloride & $0.65 \mathrm{M}$ \\
Potassium acetate & $3.1 \mathrm{M}$ \\
Acetic acid & \\
Buffer PB & $5 \mathrm{M}$ \\
Guanidine hydrochloride & $10 \mathrm{mM}$ \\
Tris-HCl pH 6.6 & $30 \%$ \\
Ethanol & \\
Buffer PE & $10 \mathrm{mM}$ \\
Tris-HCl pH 7.5 & $80 \%$ \\
Ethanol &
\end{tabular}




\subsubsection{DNA quantification}

Measurement of DNA concentration and purity of purified PCR and plasmid samples was performed using a NanoDrop ND-1000 spectrophotometer (Thermo Scientific), according to the manufacturer's instructions.

\subsubsection{DNA Sequencing}

All vectors constructed throughout this study were sequence confirmed using commercial DNA sequencing services. All DNA sequencing was carried out either at the Allan Wilson Centre (Palmerston North, NZ) or by Macrogen Inc. (Seoul, South Korea).

\subsection{Gene knockout in E. coli}

In frame gene-deletion in $E$. coli was carried out using a modified version of the protocol of Datsenko and Wanner (237), described below.

\subsubsection{Gene knockout PCR amplification}

Firstly, plasmid pKD4 was used as a template to PCR-amplify a kanamycin resistance $\left(\mathrm{kan}^{\mathrm{R}}\right)$ gene flanked either side by FLP-recombinase recognition sites. Primers for amplification contained 15-20 bp of sequence at the 3 ' end for priming and amplification from pKD4, while the remaining 30-40 bp at the 5' end of the primers were homologous to either end of the genomic region targeted for deletion (see Table 2.1 for primer sequences). The complete process is illustrated in figure 2.1. In order to improve knock-out efficiency in certain cases, the genomic homologous regions at each end of the PCR-amplified $\mathrm{kan}^{\mathrm{R}}$ cassette were lengthened via a second PCR, using the first PCR product as template and knock-out extension primers (see Table 2.1) for amplification. The final PCR product was purified and concentrated to at least $100 \mathrm{ng}$ $\mu \mathrm{L}^{-1}$ in sterile-filtered $\mathrm{ddH}_{2} \mathrm{O}$. 
1. PCR amplification of FRT-flanked kanamycin resistance gene from pKD4 plasmid

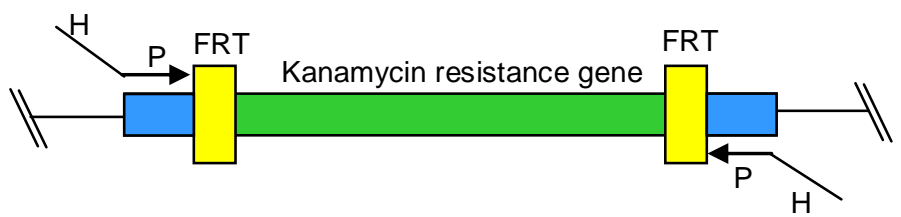

2. Electroporation into red-recombinase expressing host strain

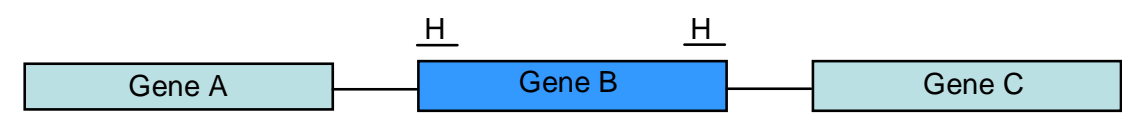

3. Selection of positive recombinants on kanamycin-containing media

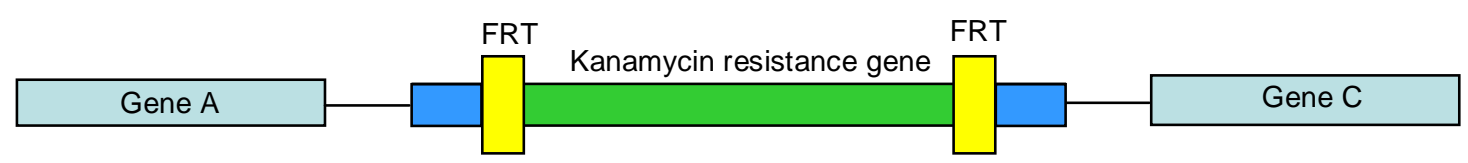

4. Deletion of kanamycin resistance gene with plasmid (pCP20) encoded

FLP recombinase

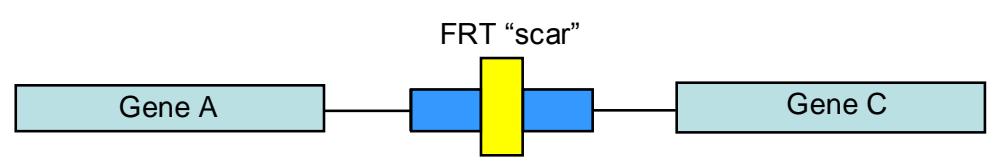

Figure 2.1. Overview of $\boldsymbol{E}$. coli gene knockout procedure.

Figure adapted from Datsenko and Wanner (237). See text (section 2.7) for details.

\subsubsection{Preparation of electrocompetent cells}

The E. coli strain to be genetically altered was first transformed with the heat-sensitive plasmid pKD46. Transformation plates were grown at $30{ }^{\circ} \mathrm{C}$, instead of $37{ }^{\circ} \mathrm{C} .3 \mathrm{~mL} \mathrm{LB}$ $+\operatorname{amp}\left(100 \mu \mathrm{g} \mathrm{mL}^{-1}\right)$ was inoculated with a single colony and grown overnight at $30{ }^{\circ} \mathrm{C}$, $200 \mathrm{rpm}$. The following day, $50 \mathrm{~mL} \mathrm{LB}+\operatorname{amp}\left(100 \mu \mathrm{g} \mathrm{mL}^{-1}\right)$ was inoculated with $1 \mathrm{~mL}$ overnight culture and incubated at $30{ }^{\circ} \mathrm{C}, 200 \mathrm{rpm}$ for several hours, until the $\mathrm{OD}_{600}$ reached 0.1. At this point, expression of recombinase genes from pKD46 was induced by the addition of L-arabinose to $0.1 \mathrm{M}$ final concentration. The culture was then left to incubate at $30{ }^{\circ} \mathrm{C}, 200 \mathrm{rpm}$ until the $\mathrm{OD}_{600}$ reached 0.4-0.6. Cells were then transferred to ice to chill for $10-15 \mathrm{~min}$, followed by centrifugation at 4,000 rpm, $10 \mathrm{~min}, 4{ }^{\circ} \mathrm{C}$. The supernatant was discarded and the cell pellet gently resuspended in $50 \mathrm{~mL}$ ice-cold 10 $\%(\mathrm{w} / \mathrm{v})$ glycerol. The cells were then centrifuged, as previously, the supernatant discarded and the cells resuspended in $25 \mathrm{~mL}$ fresh ice-cold $10 \%$ glycerol. Centrifugation and resuspension was repeated once more, then after a final centrifugation step, the cell pellet was resuspended in $250 \mu \mathrm{L} 10 \%$ glycerol. At this point, the concentration of $E$. coli was determined by measuring the $\mathrm{OD}_{600}$ of a 1:1,000 
dilution of the cell prep. Assuming that an $\mathrm{OD}_{600}$ value of 1.0 is equivalent to an E. coli density of $2.5 \times 10^{8}$ cells $\mathrm{mL}^{-1}(239)$, the cell prep was adjusted to $2-3 \times 10^{10}$ cells per $\mathrm{mL}$, either by the addition of extra $10 \%$ glycerol, or by re-centrifuging and resuspending in a lower volume. The electrocompetent cells were then split into $40 \mu \mathrm{L}$ aliquots and either used immediately, or stored at $-80{ }^{\circ} \mathrm{C}$ for future use.

\subsubsection{Electroporation}

Up to $4 \mu \mathrm{L}$ ( 0.1 volumes) of knock-out PCR product from step 2.7.1. was added to 40 $\mu \mathrm{L}$ electrocompetent cells and incubated on ice for 1-2 min. Cells were then added to a sterile, pre-chilled $2 \mathrm{~mm}$ gap electroporation cuvette and electroporated $(2.5 \mathrm{kV}, 25 \mu \mathrm{F}$, $100 \Omega$ ) using a Gene Pulser Xcell ${ }^{\mathrm{TM}}$ electroporation system (Bio-Rad) Time constants typically ranged from 5.0-5.2 msec. Immediately after electroporation, $1 \mathrm{~mL} \mathrm{SOC}+10$ $\mathrm{mM}$ L-arabinose was added to the cuvette, mixed thoroughly but gently, and transferred to a $1.5 \mathrm{~mL}$ microfuge tube. Electroporated cells were left to recover for $3 \mathrm{~h}$ at $37^{\circ} \mathrm{C}$, $200 \mathrm{rpm}$, before being spread onto $\mathrm{LB}+\mathrm{kan}\left(40 \mu \mathrm{g} \mathrm{mL}^{-1}\right)$ agar plates. These were left to incubate at $37{ }^{\circ} \mathrm{C}$ overnight.

\subsubsection{Curing of kanamycin resistance cassette}

Colonies were PCR screened for successful recombination of the $\mathrm{kan}^{\mathrm{R}}$ gene into the region of interest using knock-out diagnostic primers (see Table 2.1), which primed from regions upstream of the PCR integration sites. Bands of $\sim 1,600 \mathrm{bp}$ indicated a successful recombination. These colonies were then grown in $\mathrm{LB}+\mathrm{kan}\left(50 \mu \mathrm{g} \mathrm{mL}^{-1}\right)$ at $42{ }^{\circ} \mathrm{C}$, followed by dilution onto $\mathrm{LB}+\operatorname{kan}\left(50 \mu \mathrm{g} \mathrm{mL}^{-1}\right)$ agar plates to obtain single colonies. These colonies were then tested for loss of the pKD46 plasmid by replica streaking onto $\mathrm{LB}+\operatorname{amp}\left(100 \mu \mathrm{g} \mathrm{mL}^{-1}\right)$ and $\mathrm{LB}+\mathrm{kan}\left(50 \mu \mathrm{g} \mathrm{mL}^{-1}\right)$ agar plates. Colonies that were sensitive to ampicillin but still resistant to kanamycin were selected for further processing. These colonies were grown overnight in liquid media, made chemically competent (supplementing all growth media with kanamycin to $50 \mu \mathrm{g} \mathrm{mL}^{-1}$ ) and transformed with the heat-sensitive plasmid pCP20. Transformation plates were incubated at $30{ }^{\circ} \mathrm{C}, 200 \mathrm{rpm}$ until colonies appeared. Successful transformants were grown in $\mathrm{LB}+\operatorname{amp}\left(100 \mu \mathrm{g} \mathrm{mL}^{-1}\right)$ overnight at $30{ }^{\circ} \mathrm{C}, 200 \mathrm{rpm}$, re-innoculated into fresh $\mathrm{LB}+\operatorname{amp}\left(100 \mu \mathrm{g} \mathrm{mL^{-1 }}\right)$ the following day and incubated for another full day and night at $30{ }^{\circ} \mathrm{C}, 200 \mathrm{rpm}$. Afterwards, a fresh culture of LB (without antibiotics) was 
inoculated with the overnight culture and grown to an $\mathrm{OD}_{600}$ of $\sim 0.1$ at $30{ }^{\circ} \mathrm{C}, 200 \mathrm{rpm}$. The culture was then transferred to $42{ }^{\circ} \mathrm{C}, 200 \mathrm{rpm}$ and left to grow until stationary phase was reached $\left(\mathrm{OD}_{600}>0.8\right)$. Aliquots were then diluted 1:50,000 and spread onto LB (without antibiotics) agar plates and incubated overnight at $42{ }^{\circ} \mathrm{C}$. The following day, individual colonies from this plate were replica-streaked onto LB (without antibiotics $), \mathrm{LB}+\mathrm{kan}\left(50 \mu \mathrm{g} \mathrm{mL}^{-1}\right)$ and $\mathrm{LB}+\operatorname{amp}\left(100 \mu \mathrm{g} \mathrm{mL}^{-1}\right)$ agar plates and incubated at $37{ }^{\circ} \mathrm{C}$ until bacterial growth was evident. Colonies that failed to grow on both LB + kan and LB + amp agar plates were PCR tested for $k^{2} n^{\mathrm{R}}$ cassette curing using gene specific or knock-out primers. Colonies that produced PCR banding patterns indicative of loss of the $\operatorname{kan}^{\mathrm{R}}$ gene (i.e. retained the scar region only; Figure 2.1) were grown in LB medium (without antibiotic) overnight at $37^{\circ} \mathrm{C}, 200 \mathrm{rpm}$ and stored as a glycerol stock at $\left.-80{ }^{\circ} \mathrm{C}\right)$.

\subsection{SDS-PAGE}

Preparation and running of SDS-PAGE was carried out essentially as described by Laemmli et al. (240). 12-15\% polyacrylamide gels (1 mm thickness) were cast and run on a Bio-Rad Protean II ${ }^{\mathrm{TM}}$ apparatus, according to the manufacturer's instructions. Briefly, $\sim 5 \mathrm{~mL}$ of pre-set $12-15 \%$ separating gel (see Table 2.12 for all SDS-PAGE recipes) was applied to the gel cast and covered with $\sim 1 \mathrm{~mL} 100 \%$ isopropanol (drum grade). The gel was left to set for roughly $30 \mathrm{~min}$, followed by discarding of the isopropanol. Roughly $1.5 \mathrm{~mL}$ of pre-set $4 \%$ stacking gel was then poured over the top of the separating gel, a multi-well comb inserted and left to solidify for roughly $1 \mathrm{~h}$. At this point the gel could be stored (wrapped in glad-wrap) for 1-2 days.

Samples to be run on SDS-PAGE were added in a 2:1 ratio to $3 \times$ SDS-load buffer. Samples (typically $30 \mu \mathrm{L}$ total) were heated at $95{ }^{\circ} \mathrm{C}$ for $5 \mathrm{~min}$ prior to electrophoresis. Gels were run in $1 \times$ SDS-Run buffer, at constant voltage (200 V) for $45 \mathrm{~min}$ to $1 \mathrm{~h}$. Protein bands in gels were stained by gentle shaking in Coomassie blue stain solution for $30 \mathrm{~min}$ to $1 \mathrm{~h}$. Destaining was carried out by washing the gel of all Coomassie blue stain in running tap water, followed gentle shaking in destain solution for as long as necessary to achieve the desired contrast. 
Table 2.12. Recipes of gels and buffers used in SDS-PAGE.

\begin{tabular}{|c|c|}
\hline Component & Concentration \\
\hline $15 \%$ separating gel & Per $50 \mathrm{~mL}$ \\
\hline $40 \%$ acrylamide solution & $18.23 \mathrm{~mL}$ \\
\hline $2 \%$ Bis-acrylamide solution & $10.05 \mathrm{~mL}$ \\
\hline $1.5 \mathrm{M}$ Tris- $\mathrm{Cl} \mathrm{pH} 8.8$ & $12.5 \mathrm{~mL}$ \\
\hline $10 \%$ SDS & $0.5 \mathrm{~mL}$ \\
\hline $\mathrm{dd}_{2} \mathrm{O}$ & $8.72 \mathrm{~mL}$ \\
\hline $4 \%$ stacking gel & $\underline{\text { Per } 50 \mathrm{~mL}}$ \\
\hline $40 \%$ acrylamide solution & $4.8 \mathrm{~mL}$ \\
\hline $2 \%$ Bis-acrylamide solution & $2.6 \mathrm{~mL}$ \\
\hline $0.5 \mathrm{M}$ Tris- $\mathrm{Cl} \mathrm{pH} 6.8$ & $12.5 \mathrm{~mL}$ \\
\hline $10 \% \mathrm{SDS}$ & $0.5 \mathrm{~mL}$ \\
\hline $\mathrm{ddH}_{2} \mathrm{O}$ & $29.5 \mathrm{~mL}$ \\
\hline$\underline{3 \times \text { SDS-load buffer }}$ & $\underline{\text { Final concentration }}$ \\
\hline Tris-Cl, $\mathrm{pH} 6.8$ & $150 \mathrm{mM}$ \\
\hline SDS & $6 \%(\mathrm{w} / \mathrm{v})$ \\
\hline Bromophenol blue & $0.3 \%(\mathrm{w} / \mathrm{v})$ \\
\hline Glycerol & $30 \%(w / v)$ \\
\hline$\beta$-mercaptoethanol & $300 \mathrm{mM}$ \\
\hline$\underline{1 \times \text { SDS-run buffer }}{ }^{\mathrm{a}}$ & $\underline{\text { Per } 1 \mathrm{~L}}$ \\
\hline Glycine & $14.4 \mathrm{~g}$ \\
\hline Tris & $3.03 \mathrm{~g}$ \\
\hline SDS & $1 \mathrm{~g}$ \\
\hline Coomassie blue stain & $\underline{\text { Per } 1 \mathrm{~L}}$ \\
\hline Coomassie Brilliant Blue & $2.5 \mathrm{~g}$ \\
\hline $100 \%$ Ethanol & $450 \mathrm{~mL}$ \\
\hline $100 \%$ Acetic Acid & $100 \mathrm{~mL}$ \\
\hline $\mathrm{dd}_{2} \mathrm{O}$ & $450 \mathrm{~mL}$ \\
\hline Destain & $\underline{\text { Per } 1 \mathrm{~L}}$ \\
\hline $100 \%$ Methanol & $400 \mathrm{~mL}$ \\
\hline Acetic acid & $100 \mathrm{~mL}$ \\
\hline $\mathrm{ddH}_{2} \mathrm{O}$ & $500 \mathrm{~mL}$ \\
\hline
\end{tabular}

${ }^{a}$ SDS-run buffer was prepared as a $10 \times$ solution, with $\mathrm{ddH}_{2} \mathrm{O}$ used to dilute to $1 \times$ when necessary.

\subsection{Enzyme purification}

\subsubsection{Enzyme expression in LB media}

A single colony or small inoculum of $E$. coli BL21(DE3) harbouring pET28a(+):ntr (where $n t r$ denotes any NTR cloned into the MCS of pET28a(+)) was used to inoculate $3 \mathrm{~mL} \mathrm{LB}+\operatorname{kan}\left(50 \mu \mathrm{g} \mathrm{mL}^{-1}\right)$. This was incubated overnight at $37^{\circ} \mathrm{C}, 200 \mathrm{rpm}$. The following day, $50-200 \mathrm{~mL} \mathrm{LB}+\operatorname{kan}\left(50 \mu \mathrm{g} \mathrm{mL}^{-1}\right)$ was inoculated with a 1:100-fold 
dilution of the overnight culture. This was incubated at $37{ }^{\circ} \mathrm{C}, 200 \mathrm{rpm}$ until the $\mathrm{OD}_{600}$ of the culture reached 0.4-0.6. At this point, enzyme expression was induced by the addition of IPTG to $0.5 \mathrm{mM}$ final concentration. The culture was returned to the incubator $\left(37^{\circ} \mathrm{C}, 200 \mathrm{rpm}\right)$ for a further $3-5 \mathrm{~h}$, prior to chilling on ice for $15 \mathrm{~min}$. The cell pellet was then collected by centrifugation at $4,000 \mathrm{rpm}, 4{ }^{\circ} \mathrm{C}$ for $15 \mathrm{~min}$. After discarding the supernatant, the cell pellet was either processed immediately, or stored at $-80{ }^{\circ} \mathrm{C}$ until required.

\subsubsection{Enzyme expression in ZYP-5052 media}

A single colony or small inoculum of $E$. coli BL21(DE3) harbouring pET28a(+):ntr was used to inoculate $50 \mathrm{~mL}$ ZYP-5052 + kan $(100 \mu \mathrm{g} \mathrm{mL})$. This culture was left to incubate at $37^{\circ} \mathrm{C}, 200 \mathrm{rpm}$ for at least $16 \mathrm{~h}$, before chilling on ice for $15 \mathrm{~min}$. The cell pellet was then collected by centrifugation at $4,000 \mathrm{rpm}, 4{ }^{\circ} \mathrm{C}$ for $25 \mathrm{~min}$. After discarding the supernatant, the cell pellet was either processed immediately, or stored at $-80 \mathrm{C}$ until required.

\subsubsection{Cell lysis}

Lysis of cells to extract protein was achieved by one of three different methods, none of which appeared to have any detrimental downstream effect on enzyme activity (personal observations) and were therefore used interchangeably throughout this thesis, depending on availability of reagents/apparatus.

\subsubsection{BugBuster ${ }^{\mathrm{TM}}$}

The first method employed BugBuster ${ }^{\mathrm{TM}}$ protein-extraction reagent (Novagen; Merck, Darmstadt, Germany) and was carried out according to the manufacturer's protocol. Briefly, the cell pellet resulting from either of steps 2.9.1 or 2.9.2 above was resuspended in BugBuster ${ }^{\mathrm{TM}}$ reagent, to a final volume equating to either $2.5 \mathrm{~mL}$ reagent per $50 \mathrm{~mL}$ initial culture for $\mathrm{LB} / \mathrm{IPTG}$ expressed proteins, or $10 \mathrm{~mL}$ reagent per $50 \mathrm{~mL}$ initial culture for ZYP-5052-expressed proteins. The resuspended cell pellet was gently mixed on a rotating platform at room temperature for $20 \mathrm{~min}$, followed by chilling on ice for 10-15 min. 


\subsubsection{French press}

The second method involved lysis of cells by passage through a French press. Cell pellets were resuspended in 10-20 $\mathrm{mL} 1 \times$ His $\bullet$ Bind $^{\mathrm{TM}}$ Binding buffer (see below) and pressure at 40,000 psi applied three times to achieve uniform lysis. Lysed cells were then kept on ice until further processing

\subsubsection{Sonication}

The third method employed ultra-sonication. Cell pellets were resuspended in 10-20 mL $1 \times$ His $\bullet$ Bind $^{\mathrm{TM}}$ Binding buffer and sonicated, on ice, at $70 \%$ maximum output, with 50 $\%$ duty cycle for five $30 \mathrm{~s}$ bursts, alternated with 1 min cooling-down periods.

The soluble fraction of the cell lysate resulting from any of the lysis methods listed above was then separated from the insoluble fraction by centrifugation at 17,000 $\mathrm{g}, 4^{\circ} \mathrm{C}$ for $10 \mathrm{~min}$. All components were kept chilled (on ice) at all times.

\subsubsection{Enzyme purification and storage}

Recombinant his6-tagged NTRs were purified by nickel-affinity chromatography, employing the His•Bind Kit (Novagen) and following the manufacturer's instructions. The final settled-bed resin volume per column was between $500 \mu \mathrm{L}$ and $1 \mathrm{~mL}$. Upon elution, flow through was collected in a $1.5 \mathrm{~mL}$ microfuge tube, until the eluate started to become noticeably yellow. At this point, $1.5 \mathrm{~mL}$ of the flow-through was collected in a new sterile, pre-chilled $1.5 \mathrm{~mL}$ microfuge tube. $15 \mu \mathrm{L}$ of $100 \mathrm{mM}$ FMN (or FAD for $\mathrm{MdaB}$ (E.c)) was then added to the eluted protein and the tube kept on ice or at $4{ }^{\circ} \mathrm{C}$ for at least one hour, or overnight when necessary. Buffer-exchange into $40 \mathrm{mM}$ Tris-Cl pH 7.0 was then conducted using a $5 \mathrm{~mL}$ HiTrap ${ }^{\mathrm{TM}}$ Desalting column (GE Healthcare, Chalfont St. Giles, UK) according to the manufacturer's instructions. Only $1 \mathrm{~mL}$ of column flow-through was collected upon elution, to minimize contamination with excess free FMN. Samples were then mixed 1:1 with $80 \%$ glycerol and stored at $-20{ }^{\circ} \mathrm{C}$ indefinitely. The presence of glycerol was found to have no detrimental effect on enzyme activity (results not shown). 


\subsubsection{Removal of His6 tags from purified enzymes}

When required, the Novagen Thrombin Kit was used to cleave His6 tags from purified recombinant enzymes, according to the manufacturer's instructions.

\subsubsection{Determination of enzyme concentration}

Concentrations of purified protein samples were measured using the $\mathrm{Dc}^{\mathrm{TM}}$ protein assay kit (Bio-Rad), following the manufacturer's instructions. Bovine serum albumin (BSA), reconstituted in $20 \mathrm{mM}$ Tris- $\mathrm{Cl} \mathrm{pH} 7.0$ and $40 \%$ glycerol, was used to establish a standard curve with concentrations ranging from 0.25 to $1.5 \mathrm{mg} \mathrm{mL}^{-1}$.

\subsection{Determination of substrate extinction coefficients}

$100 \mu \mathrm{M}$ substrate (PR-104A, SN 27686, SN 29176 or SN 30444) in $10 \mathrm{mM}$ Tris-Cl pH 7.0, $4 \%$ DMSO was scanned for total absorbance between 325 and $500 \mathrm{~nm}$ wavelength, with a $1 \mathrm{~cm}$ path length, using a Helios $\gamma \mathrm{UV}$-Vis spectrophotometer (Thermo-Fisher). The same concentration of substrate was then incubated with $300 \mu \mathrm{M}$ NADPH and $\sim 1$ $\mu \mathrm{g}$ purified recombinant NfsA (E.c). This reaction was left to run for 15-20 min, or until NADPH oxidation had terminated (assessed by decrease in absorbance at $340 \mathrm{~nm}$; note that NfsA (E.c) has innate NADPH-oxidase activity, eliminating any excess reduced cosubstrate from the reaction mix). This reaction was then scanned for absorbance between 325 and $500 \mathrm{~nm}$ and any changes relative to the absorbance plot of parental substrate noted, especially at wavelengths $\geq 400 \mathrm{~nm}$, due to no interference by reduced or oxidised $\mathrm{NAD}(\mathrm{P}) \mathrm{H}$ co-substrate at these wavelengths. The wavelength at which this difference was maximal was chosen for determination of the extinction coefficient $(\varepsilon)$. This was calculated by measuring the difference in absorbance between the parental and reduced forms of the substrate and adjusting for concentration, so that the final constant was expressed in $\mathrm{M}^{-1} \mathrm{~cm}^{-1}$.

\subsection{In vitro kinetics of purified enzymes}

Steady-state enzyme kinetics of purified NTRs were assessed spectrophotometrically at either $420 \mathrm{~nm}$ for CB1954 (based on equal absorption of both 2- and 4-hydroxylamine reduction products of $\mathrm{CB} 1954$ at this wavelength; $\varepsilon=1,200 \mathrm{M}^{-1} \mathrm{~cm}^{-1} ;(241)$ ) or $400 \mathrm{~nm}$ 
for PR-104A ( $\varepsilon=6,000 \mathrm{M}^{-1} \mathrm{~cm}^{-1}$; see Figure 5.12), SN $27686\left(\varepsilon=7,000 \mathrm{M}^{-1} \mathrm{~cm}^{-1}\right), \mathrm{SN}$ $29176\left(\varepsilon=4,000 \mathrm{M}^{-1} \mathrm{~cm}^{-1}\right)$, SN $30444\left(\varepsilon=7,000 \mathrm{M}^{-1} \mathrm{~cm}^{-1}\right)$ and NFZ $\left(12,960 \mathrm{M}^{-1} \mathrm{~cm}^{-1}\right.$; (176)) using a Helios $\gamma \mathrm{UV}-\mathrm{V}$ is spectrophotometer. Reactions were performed in $60 \mu \mathrm{L}$ in UVettes ${ }^{\mathrm{TM}}$ (Eppendorf, Hamburg, Germany), containing $10 \mathrm{mM}$ Tris-Cl (pH 7.0), 4 $\%$ DMSO, $0.25 \mathrm{mM} \mathrm{NAD}(\mathrm{P}) \mathrm{H}$ and varying substrate concentrations (the range used was dependent on substrate and enzyme being tested, but was always within 10-2000 $\mu \mathrm{M})$. Reactions were commenced by addition of $6 \mu \mathrm{L}$ of an appropriate purified enzyme dilution and changes in absorbance were measured for the initial 15-30 s. For calculation of apparent $K_{\mathrm{m}}$ and $k_{\mathrm{cat}}$ values, substrate concentrations were varied from $\sim 0.2 \times$ to $5 \times K_{\mathrm{m}}$ (although CB1954 and PR-104A solubility and absorbance limits precluded analysis of the full range of concentrations for enzymes with high $K_{\mathrm{m}}$ values; maximum CB1954 concentration tested was $5 \mathrm{mM}$, maximum PR-104A concentration tested was $800 \mu \mathrm{M}$ ). Non-linear regression analyses and Michaelis-Menten curve fitting were performed using SigmaPlot ${ }^{\mathrm{TM}} 10.0$ (Systat Software, Richmond, CA, USA).

\subsection{2. $p U C X$ construction}

The inducible, high-copy number, E. coli expression vector pUCX was used for expression of native NTRs (without His6-tags or other sequence additions associated with pET28a(+) in E. coli SOS reporter strains (SOS-R1 and all derivatives thereof) for use in the SOS-chromotest and other in vivo E. coli assays of NTR activity. pUCX was originally constructed so that the completed vector would already contain a test-NTR (in this case azoR from E. coli) in the MCS. This was to facilitate expression testing of the final vector, prior to making an empty version for subsequent cloning of NTRs. Two templates were required for creation of pUCX:azoR. The first, pMM67EH:azoR, had previously been made in the lab by cloning the E. coli azoR gene (PCR-amplified using azoR_Ec_Fw and Rv primers) between the NdeI and Sall sites of digested pMMB(nfsA) (184). From this, a 2,669 bp fragment (encompassing the lacIq gene, promoter region, MCS and T0T1 transcriptional terminator sequence) was PCRamplified, using primers BlaPromFw and NewpUCF2Rv. The second template was pUC19 (Invitrogen), from which a 2,198 bp fragment (encompassing the $a m p^{\mathrm{R}}$ gene and origin of replication) was PCR-amplified using primers pUCF1Fw and pUCF1Rv. Both of these PCR products were purified, digested with AgeI and SpeI and ligated together to form pUC19X:azoR. To remove the His6-tag sequence from the expression region, a $4.7 \mathrm{~kb}$ segment was PCR-amplified from pUC19X:azoR using primers azoR_Ec_Fw 
and pETRBSRv. This PCR product was then digested with NdeI and self-ligated to form pUCX:azoR. The empty (no inserted NTR) vector was created by PCR-amplifying a $4.2 \mathrm{~kb}$ segment of pUCX:azoR using primers pUCXWTFw and pETRBSRv. This was then digested with NdeI and self ligated to form pUCX:empty.

All other NTRs were cloned directly into the MCS of pUCX after PCR amplification of the gene using the relevant NTR specific primers listed in Table 2.1.
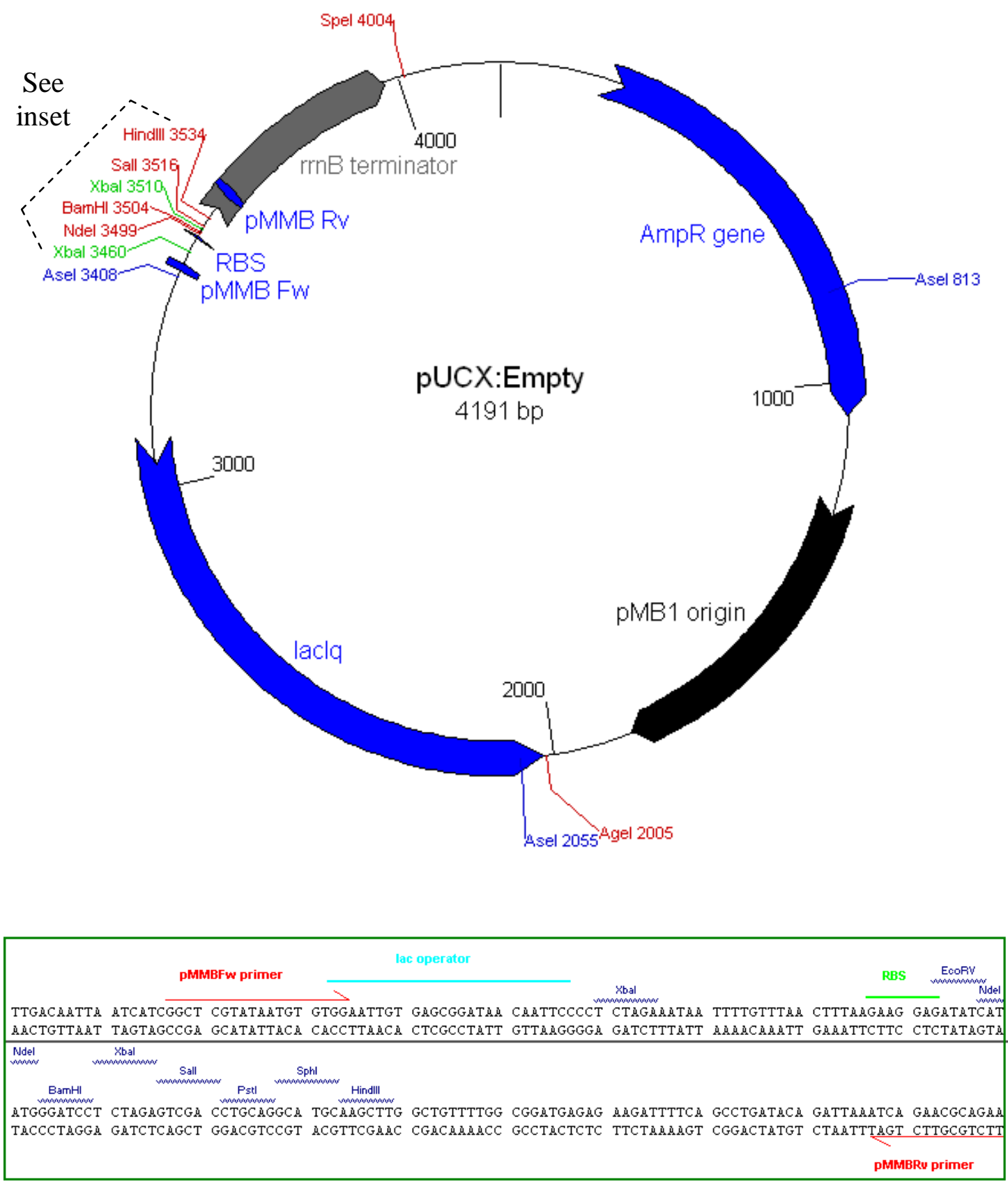

Figure 2.2. Map and MCS sequence of pUCX:empty. 


\subsection{SOS assay}

\subsubsection{Chapter 3 and 4 protocol}

Individual microtitre plate wells containing $200 \mu \mathrm{L} \mathrm{LB}+\operatorname{amp}\left(100 \mu \mathrm{g} \mathrm{mL} \mathrm{m}^{-1}\right)+0.4 \%$ (w/v) glucose were inoculated with SOS-R1 (and derivatives) pUCX:ntr strains and incubated overnight at $30{ }^{\circ} \mathrm{C}, 200 \mathrm{rpm} .10 \mu \mathrm{L}$ of the overnight cultures were used to inoculate $190 \mu \mathrm{L}$ of fresh assay media (LB + amp $100 \mu \mathrm{g} \mathrm{mL}^{-1}, 0.05 \mathrm{mM}$ IPTG, $0.2 \%$ glucose) in a sterile microtitre plate and incubated at $30{ }^{\circ} \mathrm{C}, 200 \mathrm{rpm}$ for $3 \mathrm{~h}$ (prechallenge growth). Cultures were then split into $100 \mu \mathrm{L}$ duplicates into a clean microtitre plate and supplemented with either $100 \mu \mathrm{L}$ challenge media (assay media + prodrug, $2 \%$ DMSO) or $100 \mu \mathrm{L}$ control media (assay media $+2 \%$ DMSO). OD 600 readings were recorded using either a VERSAmax ${ }^{\mathrm{TM}}$ microplate reader (Molecular Devices, Sunnyvale CA, USA) or an EnSpire ${ }^{\mathrm{TM}} 2300$ Multilabel Reader (PerkinElmer, Waltham, MA, USA) and the plate returned to the incubator (challenge; $30{ }^{\circ} \mathrm{C}, 200$ $\mathrm{rpm}$ ). Following the appropriate length of time (typically $3 \mathrm{~h}$ ), plates were removed, $\mathrm{OD}_{600}$ readings recorded and $10 \mu \mathrm{L}$ cells removed for $\beta$-galactosidase analysis (except for section 4.2.1, where cell volumes were varied from 2.5 to $20 \mu \mathrm{L}$ and compensated for by altering the amount of $40 \mathrm{mM}$ sodium phosphate buffer $\mathrm{pH} 7.0$ added to an accumulative total of $100 \mu \mathrm{L}$ ). If multiple time points were to be analysed, plates were returned to the incubator for further growth. The $10 \mu \mathrm{L}$ aliquots were added to $90 \mu \mathrm{L}$ of $40 \mathrm{mM}$ sodium phosphate buffer $\mathrm{pH} 7.0$ and $50 \mu \mathrm{L}$ ZOB buffer ( 4 parts $\mathrm{Z}$ buffer, 1 part T-Base, see Table 2.13 for recipes; (242)) in a sterile microtitre plate, which was incubated at $37^{\circ} \mathrm{C}$ without shaking for 20-40 min until sufficient colour development from the $o$-nitrophenyl- $\beta$-D-galactopyranoside (ONPG) substrate was observed. Reactions were terminated by addition of $50 \mu \mathrm{L}$ of $1 \mathrm{M} \mathrm{Na}_{2} \mathrm{CO}_{3}$ to each well. Absorbance readings at $420 \mathrm{~nm}$ and $550 \mathrm{~nm}$ were recorded and Miller units were calculated by the Miller equation (243). Miller unit calculation was altered from the original equation for all $\beta$-galactosidase activity measurements from Chapter 4 onwards, as described in section 4.2.1.

\subsubsection{Chapter 5 and 6 modifications}

Through general observations and increasing experience at running SOS assays, alterations to the SOS methodology that led to improved signal output and reliability 
were noted and incorporated into the protocol. These were as follows: $15 \mu \mathrm{L}$ overnight cultures were inoculated into $195 \mu \mathrm{L}$ fresh assay media at commencement of assay. Prechallenge incubation time was increased to $3.5 \mathrm{~h} .95 \mu \mathrm{L}$ of pre-challenge culture was added to $95 \mu \mathrm{L}$ challenge media at the commencement of challenge (both challenged and unchallenged cultures being transferred to new plates). The time allowed for colour development upon addition of ONPG and ZOB was kept constant at $25 \mathrm{~min}$. When working with SOS-R2 carrying pUCKG:ntr, IPTG concentration was increased to 0.25 $\mathrm{mM}$.

Table 2.13. Recipes of buffers required for measurement of $\beta$-galactosidase activity in SOS assays.

\begin{tabular}{|c|c|}
\hline Component & Concentration \\
\hline \multicolumn{2}{|c|}{1 M Sodium phosphate buffer $\mathrm{pH} 7.0$} \\
\hline $\mathrm{Na}_{2} \mathrm{HPO}_{4}$ & $0.577 \mathrm{M}$ \\
\hline $\mathrm{NaH}_{2} \mathrm{PO}_{4}$ & $0.423 \mathrm{M}$ \\
\hline \multicolumn{2}{|l|}{ Z-Buffer } \\
\hline$\overline{\mathrm{NaH}}_{2} \mathrm{PO}_{4}$ & $0.074 \mathrm{M}$ \\
\hline $\mathrm{Na}_{2} \mathrm{HPO}_{4}$ & $0.126 \mathrm{M}$ \\
\hline $\mathrm{MgSO}_{4}$ & $2 \mathrm{mM}$ \\
\hline $\mathrm{MnSO}_{4}$ & $0.4 \mathrm{mM}$ \\
\hline CTAB & $399 \mathrm{mg} \mathrm{L}^{-1}$ \\
\hline Sodium deoxycholate & $199.5 \mathrm{mg} \mathrm{L}^{-1}$ \\
\hline$\beta$-mercaptoethanol ${ }^{\mathrm{a}}$ & $0.174 \mathrm{M}$ \\
\hline \multicolumn{2}{|l|}{ T-Base } \\
\hline$\overline{\mathrm{K}}_{2} \mathrm{HPO}_{4}$ & $0.08 \mathrm{M}$ \\
\hline $\mathrm{KH}_{2} \mathrm{PO}_{4}$ & $0.044 \mathrm{M}$ \\
\hline$(\mathrm{NH} 4)_{2} \mathrm{SO}_{4}$ & $15.1 \mathrm{mM}$ \\
\hline Tri-sodium citrate & $1 \mathrm{~g} \mathrm{~L}^{-1}$ \\
\hline $\mathrm{ONPG}^{\mathrm{b}}$ & $8 \mathrm{mg} \mathrm{mL}^{-1}$ \\
\hline
\end{tabular}

${ }^{a} \beta$-mercaptoethanol was added to Z-buffer directly prior to use. Z-buffer without $\beta$-mercaptoethanol was stored long term at $4{ }^{\circ} \mathrm{C}$.

${ }^{\mathrm{b}}$ After ONPG was added, T-Base was stored in aliquots at $-80{ }^{\circ} \mathrm{C}$. T-Base without ONPG was made up and stored long term at $4{ }^{\circ} \mathrm{C}$.

\subsection{4. $\mathrm{IC}_{50}$ measurements}

\subsubsection{LB liquid media version (Chapter 3)}

Cultures of SOS-R1 pUCX:ntr were set up and challenged as described for the SOS assay (see section 2.13.1). CB1954 concentrations used ranged from 0-2,000 $\mu \mathrm{M}$. $\mathrm{IC}_{50}$ values were calculated by finding the concentration of CB1954 that inhibited growth of 
NTR over-expressing strains to $50 \%$ of unchallenged controls, after $3 \mathrm{~h}$ challenge with the prodrug. Cell growth was monitored by spectrophotometry (absorbance of cell culture at $600 \mathrm{~nm}$ wavelength). $\mathrm{IC}_{50}$ values were calculated using non-linear regression analysis in SigmaPlot, fitting the data to a 4-parameter logistics curve.

\subsubsection{M63 minimal media version (Chapters 5 and 6)}

Cultures of SOS-R2:pUCX or pUCXKG:ntr were grown overnight as described for the SOS assay (see section 2.13.2). The following day $100 \mu \mathrm{L}$ of overnight culture was used to inoculate $2 \mathrm{~mL}$ M63IA $(1 \times \mathrm{M} 63+\mathrm{amp}+50 \mu \mathrm{M}$ IPTG $)$ and incubated at $30{ }^{\circ} \mathrm{C}, 200$ rpm for $3.5 \mathrm{~h}$. This culture was then split into $100 \mu \mathrm{L}$ aliquots and added to $100 \mu \mathrm{L}$ aliquots of MD63AI + CB1954/PR-104A at varying concentrations (0-1,600 $\mu \mathrm{M}$ for CB1954, 0-800 $\mu \mathrm{M}$ for PR-104A, DMSO final concentration of $1 \%$ ) in a sterile 96 well plate. Alternatively, cultures were aliquoted ( $40 \mu \mathrm{L}$ each) into wells of a 384 well plate, each containing $40 \mu \mathrm{L}$ M63AI plus prodrug. Each concentration of prodrug was assayed in duplicate for each NTR over-expression strain. Culture turbidity was monitored at $600 \mathrm{~nm}$ every hour for at least three and up to six hours post-challenge. $\mathrm{IC}_{50}$ values were calculated as previously described (see section 2.14.1).

\subsection{Mutagenicity testing}

Overnight and day cultures of SOS-R1 and SOS-R1 $\Delta u v r B$ pUCX:ntr were set up as described in section 2.13.1 (using at least 6 wells for each strain to be tested). Following $3.5 \mathrm{~h}$ pre-challenge growth of the assay cultures, $\mathrm{OD}_{600}$ readings were taken and $800 \mu \mathrm{L}$ of each strain transferred to sterile $1.5 \mathrm{~mL}$ microfuge tubes on ice. Cells were chilled for $10 \mathrm{~min}$, centrifuged at 13,000 rpm for $1 \mathrm{~min}$ and the supernatant discarded. Cell pellets were then resuspended in $1 \times \mathrm{M} 63$ (without glucose or casamino acids) to an $\mathrm{OD}_{600}$ of 0.3 , and $200 \mu \mathrm{L}$ aliquots, in duplicate for each strain, transferred to sterile $1.5 \mathrm{~mL}$ microfuge tubes. CB1954 was added to one tube ("challenged") of each duplicate to the desired concentration with a final DMSO concentration of $1 \% \mathrm{v} / \mathrm{v}$. DMSO was added to $1 \% \mathrm{v} / \mathrm{v}$ concentration to the other tube ("unchallenged"). Cultures were then incubated at $30{ }^{\circ} \mathrm{C}, 200 \mathrm{rpm}$ for an additional $3 \mathrm{~h}$, prior to centrifugation at 13,000 rpm for $1 \mathrm{~min}$ and discarding of supernatant. Cell pellets were resuspended in 1 volume of $0.22 \mu \mathrm{m}$-filtered $1 \times \mathrm{PBS}\left(8 \mathrm{~g} \mathrm{~L}^{-1} \mathrm{NaCl}, 0.2 \mathrm{~g} \mathrm{~L}^{-1} \mathrm{KCl}, 1.44 \mathrm{~g} \mathrm{~L}^{-1} \mathrm{Na}_{2} \mathrm{HPO}_{4}, 0.24 \mathrm{~g} \mathrm{~L}^{-1}\right.$ $\left.\mathrm{KH}_{2} \mathrm{PO}_{4}\right)$ and $100 \mu \mathrm{L}$ used to inoculate $3 \mathrm{~mL}$ of $\mathrm{LB}+\operatorname{amp}\left(100 \mu \mathrm{g} \mathrm{mL}{ }^{-1}\right)$ media, which 
was left to incubate overnight at $37^{\circ} \mathrm{C}, 200 \mathrm{rpm}$. The following day, $100 \mu \mathrm{L}$ of overnight recovered cultures were spread onto LB agar plates containing amp and rif (both $100 \mu \mathrm{g} \mathrm{mL}^{-1}$ ). Aliquots from the remaining cultures were then diluted 1 in $10^{6}$ and 1 in $10^{7}$ in sterile $1 \times$ PBS and $100 \mu \mathrm{L}$ spread onto $\mathrm{LB}+\operatorname{amp}\left(100 \mu \mathrm{g} \mathrm{mL}^{-1}\right)$ agar plates. All agar plates were incubated at $37{ }^{\circ} \mathrm{C}$ overnight or until colonies were visible. Frequency of rifampicin resistance (expressed as $\mathrm{Rif}^{\mathrm{R}}$ mutants per $10^{8}$ cells) for each strain was then calculated by normalising the total number of Rif $^{\mathrm{R}}$ colony forming units (CFUs) to the total number of viable cells per $\mathrm{mL}$ of initial culture (as calculated from CFUs on ampicillin only-containing agar plates).

\subsection{HPLC}

\subsubsection{Identification of $\mathrm{CB} 1954$ nitroreduction products}

Reactions of $100 \mu \mathrm{L}$ containing $10 \mathrm{mM}$ Tris-Cl pH 7.0, $200 \mu \mathrm{M}$ CB1954, $1 \mathrm{mM}$ $\mathrm{NAD}(\mathrm{P}) \mathrm{H}$ and initiated by addition of $0.5-2 \mu \mathrm{M}$ purified enzyme were incubated for 10 $30 \mathrm{~min}$ at room temperature before being stopped by addition of 1 volume ice-cold 100 $\%$ methanol. Samples were immediately transferred to $-80^{\circ} \mathrm{C}$ for at least $1 \mathrm{~h}$ to precipitate proteins, followed by centrifugation at $12,000 \mathrm{~g}$ for $10 \mathrm{~min}$ at $4{ }^{\circ} \mathrm{C}$. The supernatant was then diluted 1:20 in $45 \mathrm{mM}$ formate buffer $\mathrm{pH} 6.5$ containing $2.5 \%$ methanol and $100 \mu \mathrm{L}$ of each sample was analysed by reverse phase-HPLC employing an Agilent 1100 system with an Altima ${ }^{\mathrm{TM}}$ C8 $5 \mu 150$ x $2.1 \mathrm{~mm}$ column. The mobile phase used $45 \mathrm{mM}$ formate buffer ( $\mathrm{pH}$ 6.5) as aqueous and $80 \%$ acetonitrile as organic. Assay conditions consisted of $4 \mathrm{~min}$ at $5 \%$ organic, a linear increase to $50 \%$ organic from 4-19mins, and a further gradient increase to $70 \%$ organic for $2 \mathrm{~min}$. Flow rate was constant at $0.3 \mathrm{~mL} \mathrm{~min}^{-1}$. The eluate was monitored at $262 \mathrm{~nm}$. Elution profiles from NTR-CB1954 reactions were compared against pure standards of each potential metabolite: CB1954, its 2- and 4-hydroxylamines (2-/4-NHOH), and 2- and 4-amines $\left(2-/ 4-\mathrm{NH}_{2}\right)$.

\subsubsection{Identification of PR-104A nitroreduction products}

Reactions with PR-104A as substrate were set up and carried out identically to those outlined for CB1954, in section 2.16.1. Following centrifugation, samples were diluted 1:20 in $45 \mathrm{mM}$ formate buffer $\mathrm{pH} 4.5,20 \%$ methanol. Column type used and volume of 
sample employed were the same as for CB1954. The mobile phase used $45 \mathrm{mM}$ formate buffer ( $\mathrm{pH} 4.5)$ as aqueous and $80 \% \mathrm{MeCN}$ as organic. Assay conditions consisted of 4 min at $20 \%$ organic, a linear increase to $100 \%$ organic over the following $21 \mathrm{~min}$, followed by an extra $1 \mathrm{~min}$ at $100 \%$ organic. Flow rate was constant at $0.3 \mathrm{~mL} \mathrm{~min}^{-1}$. Eluates were monitored continuously at $254 \mathrm{~nm}$. Reaction products were identified through comparison of chromatograms to those obtained from pure, chemically synthesized metabolites: PR-104A and the para nitro group reduction products PR104H (hydroxylamino derivative) and PR-104M (amino derivative).

Further identification and confirmation of reaction products was achieved through mass spectrometry, carried out as described elsewhere $(57 ; 244)$. Electrospray ionisation and quadrupole detection methods were employed, set at positive ionisation mode.

\subsection{HCT-116 general methods}

All methods outlined in this section were carried out by Dr. Sophie Syddall at the ACSRC.

Generation of mammalian expression vectors and testing of candidate NTRs in the human colon carcinoma cell line HCT-116 was performed by Dr. Sophie Syddall at the ACSRC. Each NTR gene was PCR-amplified using oligonucleotide primers that incorporated a mammalian kozak consensus sequence and a Shine-Dalgarno prokaryote consensus sequence up stream of the 5 ' end of the gene, a TAG stop codon in place of the native gene stop codon, and Invitrogen Gateway ${ }^{\mathrm{TM}}$ BP recombination sites both upstream of the 5' end and downstream of the 3' end of the gene sequence (consensus sequence for forward primer: GGGGACAAGTTTGTACAAAAAAGCAGGCTTCGA AGGAGATAGAACCATGGGC-3', followed by 15-20 bp homology to the 5' end of the desired gene, without including the initial ATG codon; consensus sequence for reverse primer: 5'-GGGGACCACTTTGTACAAGAAAGCTGGGTCCTA-3', followed by $15-20$ bp homology to the reverse complement of the 3' end of the desired gene, without including the native stop codon). PCR products were recombined into the pDONR221 $1^{\mathrm{TM}}$ vector (Invitrogen) and sequence verified using M13 sequencing

primers. NTR genes were then recombined into the F527-V5 vector (62). Plasmids were transfected into HCT-116 colon cancer cells using Fugene-6 (Roche, Basel, Switzerland), according to the manufacturer's instructions. Transiently transfected cells 
were assayed $1 \mathrm{~h}$ later, or to select stably transfected pools cells were left untreated for 3 days after which fresh $\alpha$ MEM media containing 5\% FCS and $1 \mu \mathrm{M}$ puromycin was added. Puromycin concentrations were increased daily until a maximum of $3 \mu \mathrm{M}$ was achieved. Media was changed daily until cell death was no longer evident and growth of transfected cells was achieved.

For $\mathrm{IC}_{50}$ cytotoxicity analysis of stably transfected pools, cells (500 per well) were seeded into 96-well plates and left to attach for $4 \mathrm{~h}$. After the attachment period cells were exposed to a range of CB1954 concentrations for $18 \mathrm{~h}$, washed 3 times, then left to grow for 5 days. Plates were stained with sulforhodamine B as previously described (245) and absorbance measured on an ELx 808 Absorbance Microplate Reader (Bio-tek Instruments, Winooski, VT, USA). $\mathrm{IC}_{50}$ values were defined as the drug concentration required to reduce absorbance to $50 \%$ of that of unchallenged controls on the same plate. Each cell line was independently tested 2-10 times.

NTR expression was assessed using expression of a V5 tag by infection of $5 \times 10^{5}$ cells (stably transfected cell lines, or simultaneously during transient transfections) with 50 MOI Tag-On-Demand ${ }^{\text {TM }}$ Suppressor Supernatant (Invitrogen). Cells were incubated for $27 \mathrm{~h}$ post-infection then lysed in situ in $50 \mathrm{mM}$ Tris-Cl pH 8.0 containing $150 \mathrm{mM}$ $\mathrm{NaCl}, 1 \mathrm{mM}$ EDTA, $1 \%$ (v/v) Nonidet P40 (Roche), 0.5\% sodium deoxycholate and 1\% protease inhibitor cocktail (Sigma-Aldrich). Samples were incubated on ice for $20 \mathrm{~min}$ with periodic agitation, collected by centrifugation and protein was quantified using the BCA assay (Pierce, Thermo-Fisher) with BSA as reference. Soluble proteins were fractionated by denaturing PAGE, transferred onto nitrocellulose (Bio-Rad) and blocked in PBS containing 5\% non-fat dry milk powder and 0.05\% Tween-20. Blots were incubated for $2 \mathrm{~h}$ with HRP-conjugated primary anti-V5 antibody (Invitrogen) diluted to 1:5,000 in PBS-Tween-20, or $1 \mathrm{~h}$ with mouse anti- $\beta$-actin diluted to 1:10,000 (Chemicon) followed by incubation for $1 \mathrm{~h}$ with a HRP-conjugated secondary antibody diluted 1:10,000 (Zymed). HRP signal was detected by enhanced chemiluminescence (Pierce). Images were acquired on an ImageReader LAS-3000 (Fujifilm).

\subsection{Site-directed mutagenesis}

\subsection{1. pUCXKT construction}

Plasmid pUCXKT is a derivative of pUCX that confers kanamycin resistance to host cells only when a gene amplified using the pUCXSacIRv primer has been correctly 
inserted into the MCS. As such, the frequency of false positive transformants that arise either through incomplete digestion of parental plasmid, re-circularized plasmid through ligation of degraded, blunt ended DNA ends, or insertion of rogue DNA sequences, is significantly reduced when using kanamycin in the selective media.

pUCXKT was constructed as follows: A $794 \mathrm{bp}$ fragment, containing (in order, 5' to 3') 2 stop codons (TAA, TAG), a SacI site and a truncated version of a kanamycin-resistance gene (missing the first 12 nucleotides), was PCR amplified from plasmid pKD4 using primers KantruncSalIFw3 and KantruncHindIII Rv. This was purified, digested with SalI and HindIII and ligated into similarly digested pUCX:empty, resulting in plasmid pUCXKT $\left(\mathrm{Amp}^{\mathrm{R}}\right.$, $\mathrm{Kan}^{\mathrm{S}}$; see Figure 2.3A)

\subsection{2. pUCXKG construction}

Genes to be inserted into pUCXKT were PCR amplified from previously constructed pUCX:ntr vectors using gene-specific Fw and pUCXSacIRv primers. pUCXSacIRv primer contains (in order, 5'-3') a SacI site, the first two codons of the kanamycin resistance gene and a 17-base pair linker region identical to the sequence separating the ampicillin and kanamycin resistance genes of plasmid pCR2.1 (Invitrogen). The primer also contains 18 base pairs of homology with pUCX, downstream of the MCS (terminating within the HindIII site). This latter property allows pUCXSacIRv to amplify any gene already inserted into pUCX. This scheme is illustrated in Figure 2.3B. Genes amplified in this manner were purified, digested with NdeI and SacI and ligated into similarly digested pUCXKT. Ligations were heat-inactivated and transformed into E. coli SOS-R2 as previously described. During the transformation process, $50 \mu \mathrm{M}$ IPTG was added to the recovery media to permit expression of the re-constituted $\mathrm{kan}^{\mathrm{R}}$ gene. Positive clones were selected for on LB agar containing amp $\left(100 \mu \mathrm{g} \mathrm{mL}^{-1}\right)$, kan $\left(50 \mu \mathrm{g} \mathrm{mL}^{-1}\right)$ and IPTG $(50 \mu \mathrm{M})$. Successful constructs were termed pUCXKG:ntr, where $n t r$ denotes the relevant inserted NTR gene. 


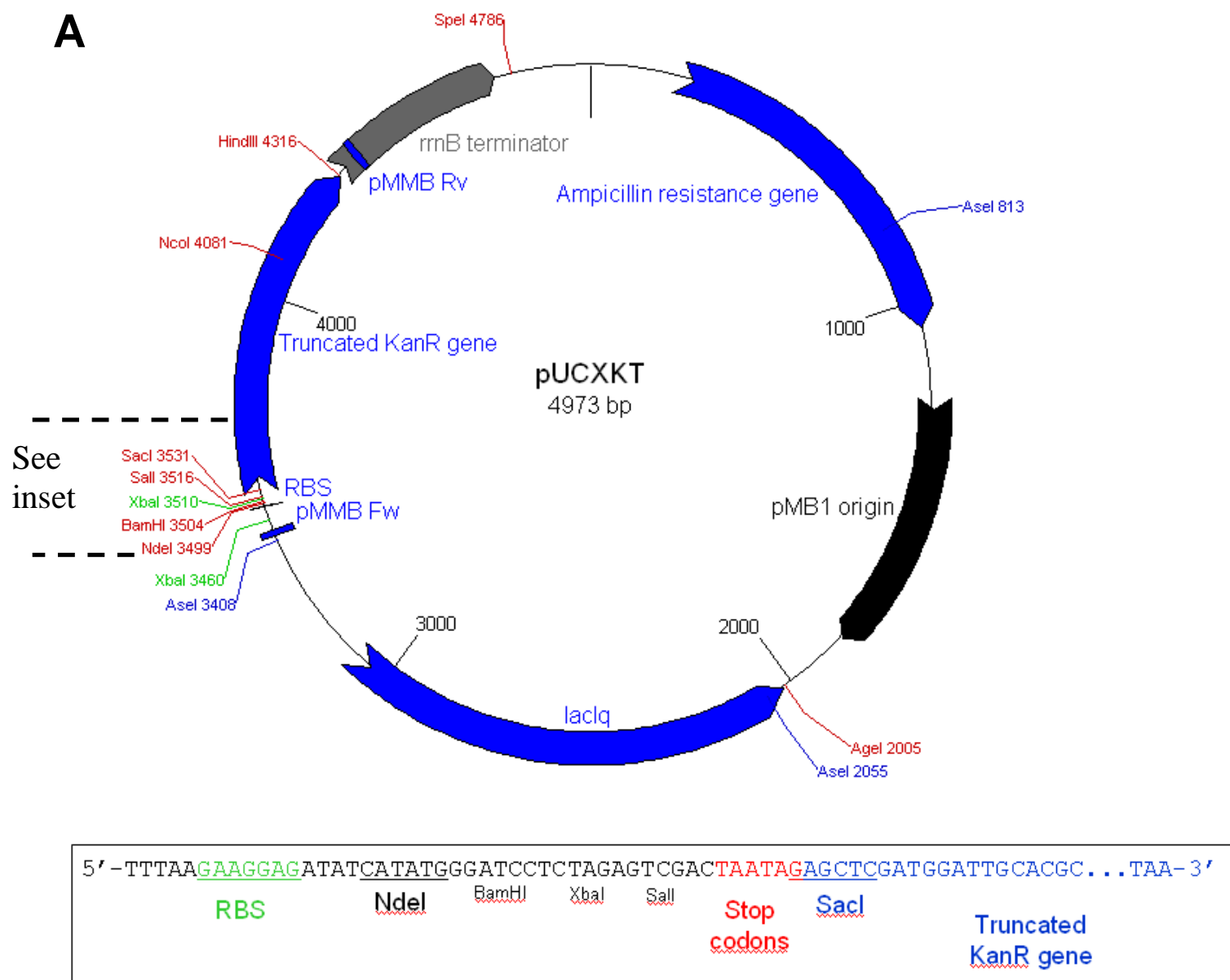

B

pUCX:ntr template

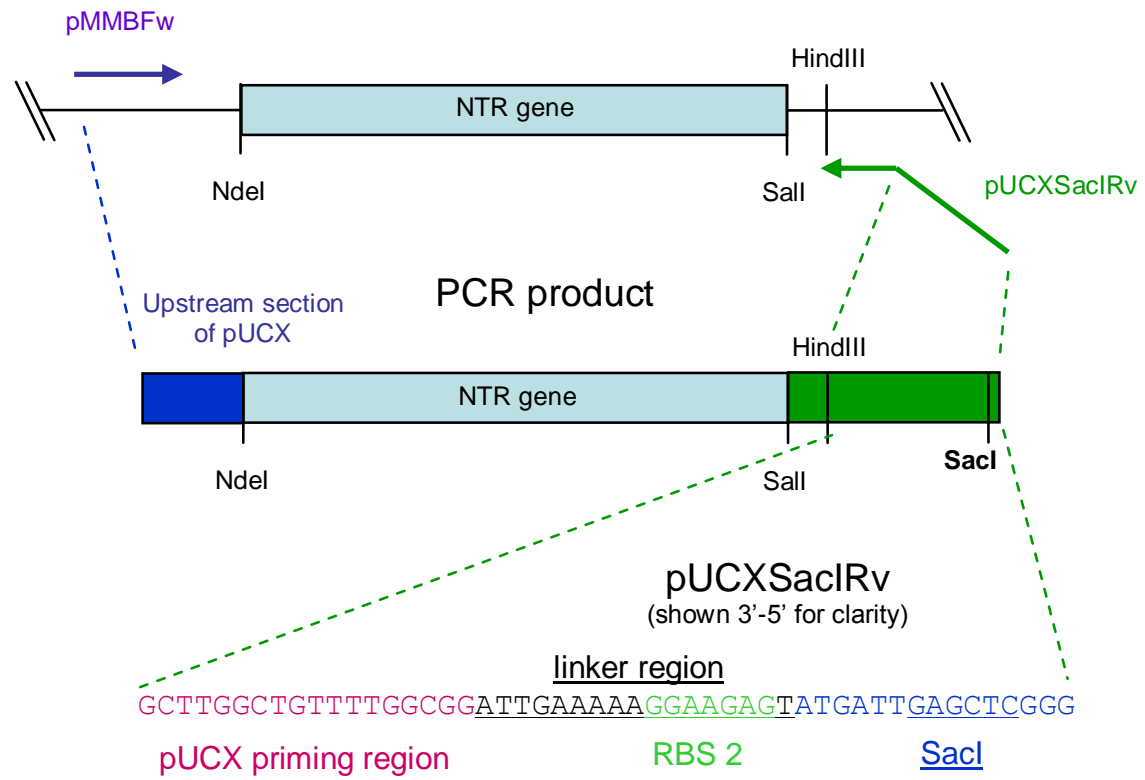

First 4 amino acids of kanR 


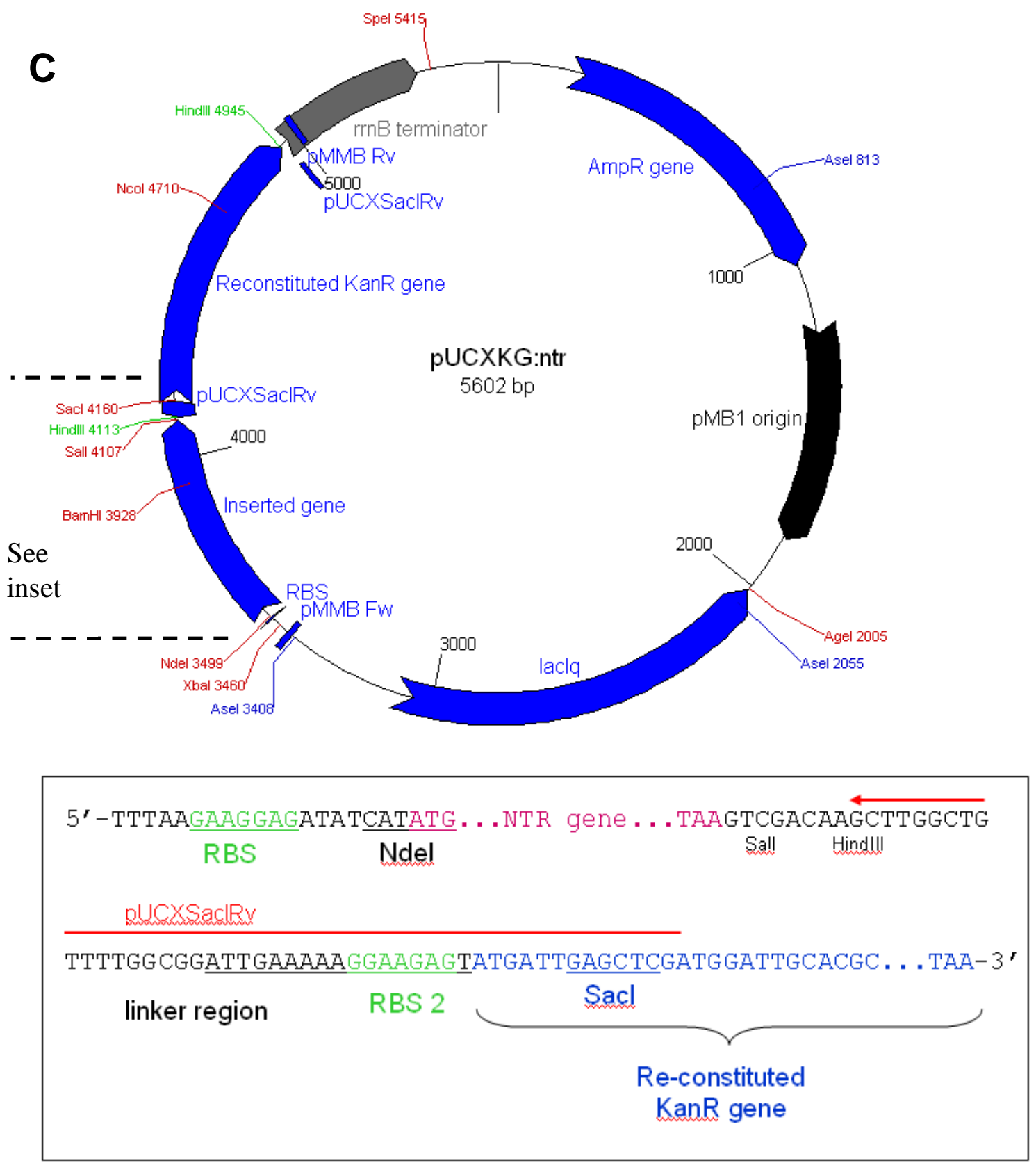

Figures 2.3A-C. pUCXKT and pUCXKG: vector maps, sequences and cloning procedure.

(A) Map and sequence of MCS of pUCXKT. (B) Diagram of PCR protocol used to create product for cloning into pUCXKT, to form pUCXKG:ntr. The sequence provided by the primer pUCXSacIRv is shown. (C) Map and sequence of MCS of pUCXKG:ntr (the product of ligation between NdeI-SacI digested pUCXKT and PCR product from (B). 


\subsection{Site directed mutagenesis}

\subsubsection{Overlap PCR}

To perform saturation mutagenesis at a specific codon of YcnD (B.s), an overlap PCR protocol was used (see Figure 2.4). A site-directed mutagenesis (SDM) forward primer containing (5' to 3') 20 nucleotides homologous to the 20 base pairs directly upstream of the codon to be mutated, the nucleotide sequence NNK in place of the codon to be mutated (where $\mathrm{N}$ denotes any of the four possible nucleotides and $\mathrm{K}$ denotes either guanine or thymine) and finally 15-20 nucleotides homologous to the region directly downstream of the codon to be mutated was used in conjunction with primer pMMBRv to amplify the relevant segment from the appropriate pUCX:ntr construct.

Simultaneously, a separate PCR was set up using a SDM Rv primer, consisting of 20 nucleotides directly complementary to the initial 5' 20 nucleotides of the SDM Fw primer, in conjunction with primer T7FwpMMBFw, to amplify the relevant segment from the appropriate pUCX:ntr construct. High-fidelity polymerase (Accuzyme ${ }^{\mathrm{TM}}$ or Phusion $^{\mathrm{TM}}$ ) was employed for both of these PCR steps. The total amount of template plasmid used did not exceed $0.01 \mathrm{ng} \mu \mathrm{L}^{-1}$ final concentration to minimise carry over of parental plasmid into the final PCR reaction. PCR products were purified, digested for 3 $\mathrm{h}$ with DpnI, heat-inactivated at $80{ }^{\circ} \mathrm{C}$ for $20 \mathrm{~min}$ and re-purified. Both PCR products were then added ( $\sim 10 \mathrm{ng}$ each) to triplicate primer-less PCR reactions (10 $\mu \mathrm{L}$ total, BioMix $^{\text {TM }}$ Red). Control reactions containing each of the PCR fragments alone were also set up. These PCRs were applied to thermal cycling as previously described, except the annealing temperature was reduced to $50{ }^{\circ} \mathrm{C}$. After 15 cycles, the reactions were held at $72{ }^{\circ} \mathrm{C}$ while primers (T7Fw and pUCXSacIRv) were added (to $0.5 \mu \mathrm{M}$ final concentration, each primer) along with extra BioMix ${ }^{\mathrm{TM}}$ Red (to a final volume of 12.5 $\mu \mathrm{L}$ for each reaction) to each tube. Amplification was then continued for an additional 15 cycles, with an annealing temperature of $56^{\circ} \mathrm{C}$. PCR products were analysed for correct length by agarose gel electrophoresis, purified and finally cloned into pUCXKT using the procedure described previously (see section 2.18.2). 
1. PCR-amplify individual fragments of gene to be mutated

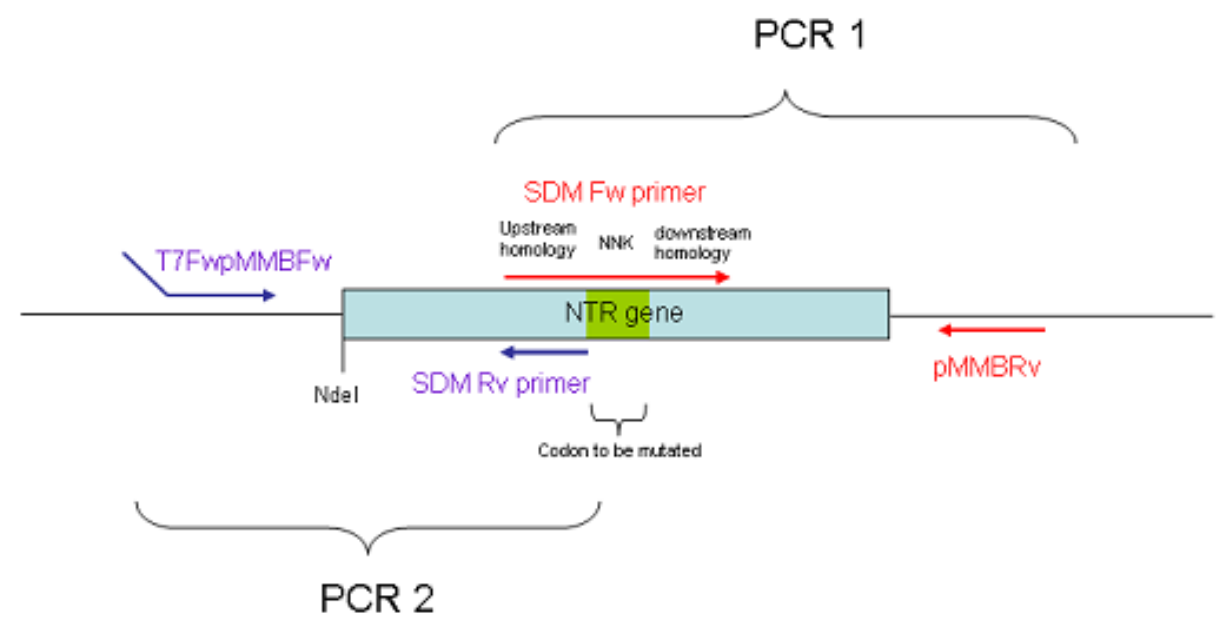

2. Primer-less PCR to allow fragments to self-anneal and extend, followed by primer addition to amplify entire gene

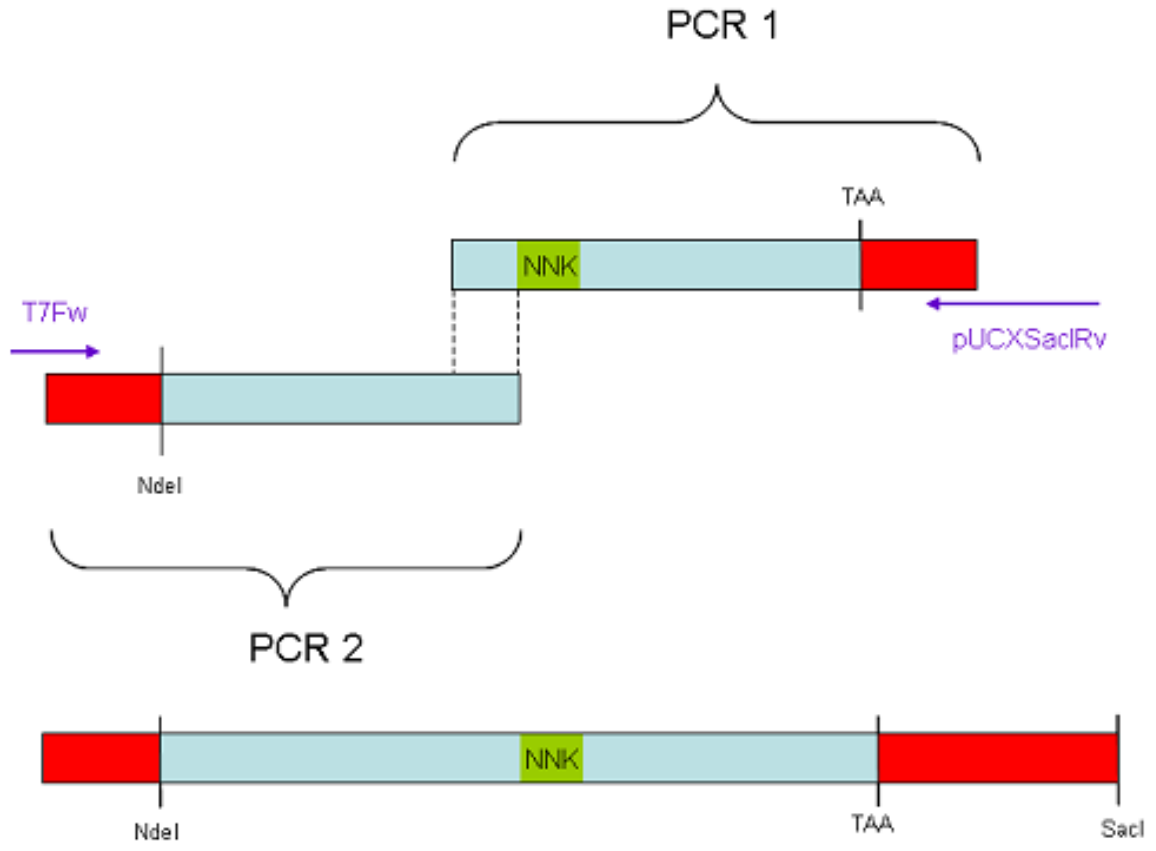

3. Digest (Ndel, Sacl) and ligate into pUCXKT

Figure 2.4. Schematic outline of overlap PCR protocol used for single codon mutagenesis. See text in section 2.19.1 for details. 


\subsubsection{Mutant enzyme library creation}

88-92 colonies of SOS-R2: pUCXKG:ycnD (B.s), mutated at a specific site as described in section 2.19.1, were picked directly from transformation agar plates and inoculated into separate wells of a 96-well plate containing $120 \mu \mathrm{L} \mathrm{LB}+\operatorname{amp}\left(100 \mu \mathrm{g} \mathrm{mL}^{-1}\right)$ and glucose $(0.2 \% \mathrm{w} v)$. At least 2 wells were reserved for SOS-R2 pUCXKG expressing the wildtype NTR, and one well for SOS-R2 pUCXKG:empty. One well was also reserved as a blank (no inoculation). Plates were then grown overnight at $30^{\circ} \mathrm{C}, 200$ rpm, before being diluted 1:1 with $80 \%$ (w v) glycerol, mixed thoroughly and stored indefinitely at $-80{ }^{\circ} \mathrm{C}$.

\subsection{Statistical analysis}

All pairwise comparisons of means were performed using the unpaired Student's t-test (online at http://www.graphpad.com/quickcalcs/ttest1.cfm). Regression analysis was performed using in-built statistical software in SigmaPlot.

\subsection{Enzyme active site modelling}

The protein 3D structure viewing program DeepView (SwissPDB) was used for all comparisons of NTR active sites and active site modelling (located at http://spdbv.vitalit.ch/; (246)) 


\section{Chapter 3: Screening putative nitroreductases of $\boldsymbol{E}$. coli for CB1954-reducing activity: validation of the SOS assay as a prodrug reduction screen}

\subsection{Introduction}

This chapter describes the preliminary testing and optimisation of the SOS assay as a potential screen for the assessment of CB1954 reduction by bacterial NTRs. In the first part of this chapter, the ability of the SOS assay to function in microplate format and to detect CB1954 reduction in a dose-dependent manner, was experimentally confirmed using over-expression of the prototypical NTR NfsB from E. coli, in the E. coli SOS reporter strain ADA510 (sfiA:lacZ). Secondly, the SOS assay was employed to assess the breadth of CB1954-reductase activity in E. coli by applying it to a small panel of phylogenetically diverse native $E$. coli enzymes with putative NTR activity, based on amino acid/structural homology to previously identified CB1954 or other nitroaromatic compound-reductases. Finally, the results obtained from the SOS assay were validated by comparison with those gained through a series of other in vivo and in vitro tests, including cytotoxicity in a human cancer cell line. Overall, the results from this study demonstrate the utility of the SOS response as a sensitive and efficient screen for detection and relative quantification of CB1954 activation by NTRs. Furthermore, CB1954-reductase activity was identified with two enzymes previously un-characterised in this regard, proving the existence of multiple enzyme families capable of nitroaromatic prodrug metabolism.

The majority of results presented in this chapter have been published independently in the peer-reviewed Journal Biochemical Pharmacology, as Prosser et al. (247).

\subsection{Initial testing and optimisation of SOS assay}

\subsubsection{Choice and optimisation of reporter strain}

A multitude of $E$. coli SOS reporter strains have been constructed over the last few decades, encompassing a variety of reporter genes (e.g. lacZ, gfp, the lux operon) and SOS promoters (e.g. recA, umuDC, sfiA, $c d a, u v r A)(229 ; 248-250)$. Some have been 
plasmid based, while others contain the SOS promoter-reporter gene fusion as a single genome-integrated copy $(228 ; 229 ; 251 ; 252)$. The strain chosen for this study (ADA510; (231)) belongs to the latter group, and consists of the reporter gene lacZ, encoding $\beta$-galactosidase, transcriptionally coupled to the sfiA gene promoter. ADA510 was chosen for several reasons. Firstly, $\beta$-galactosidase is one of the best-characterised reporter gene products available, having been used in a variety of studies over several decades (243). As such, its genetic and biochemical properties are well-understood and numerous colorimetric substrates are available for enzyme detection. The substrate employed throughout this body of work was $o$-nitrophenyl- $\beta$-D-galactopyranoside (ONPG), a colourless, water-soluble substrate that is converted into a yellow nitrophenol derivative upon cleavage by $\beta$-galactosidase. ONPG is cheap, easy to use and a highly sensitive substrate for $\beta$-galactosidase (243). The sfiA promoter employed is widely considered to have one of the most tightly regulated expression profiles of all SOS-linked genes (253). The SfiA protein itself is involved in inhibition of cell division, and as such, basal levels (in the absence of SOS induction) are minimal (253). Additionally, cytosolic SfiA levels can increase up to 125-fold upon SOS induction, the highest of all SOS genes studied to date, providing a high dynamic range for reporter gene expression from this promoter (254). Lastly, a genome-integrated reporter gene fusion strain was chosen over a plasmid-based system to prevent high background reporter activity or reporter gene stability problems arising $(230 ; 255)$, as well as to facilitate transformation and maintenance of NTR over-expression vectors, precluding plasmid-incompatibility issues.

ADA510 is a lac null mutant, preventing interference from high background rates of native $\beta$-galactosidase activity. In order to further optimise this strain for prodrug reduction screening, the native major and minor NTR genes ( $n f s A$ and $n f s B$ ) were deleted from the ADA510 chromosome, thereby minimizing background reduction of nitroaromatic prodrugs in this strain. Our justification of targeting NfsA was through preliminary data (not shown) that implied a substantial role of this enzyme in CB1954 metabolism. The work of Vass et al. (189) was not available at the time of this study. The resultant strain was termed SOS-R1 (SOS Reporter strain 1). 


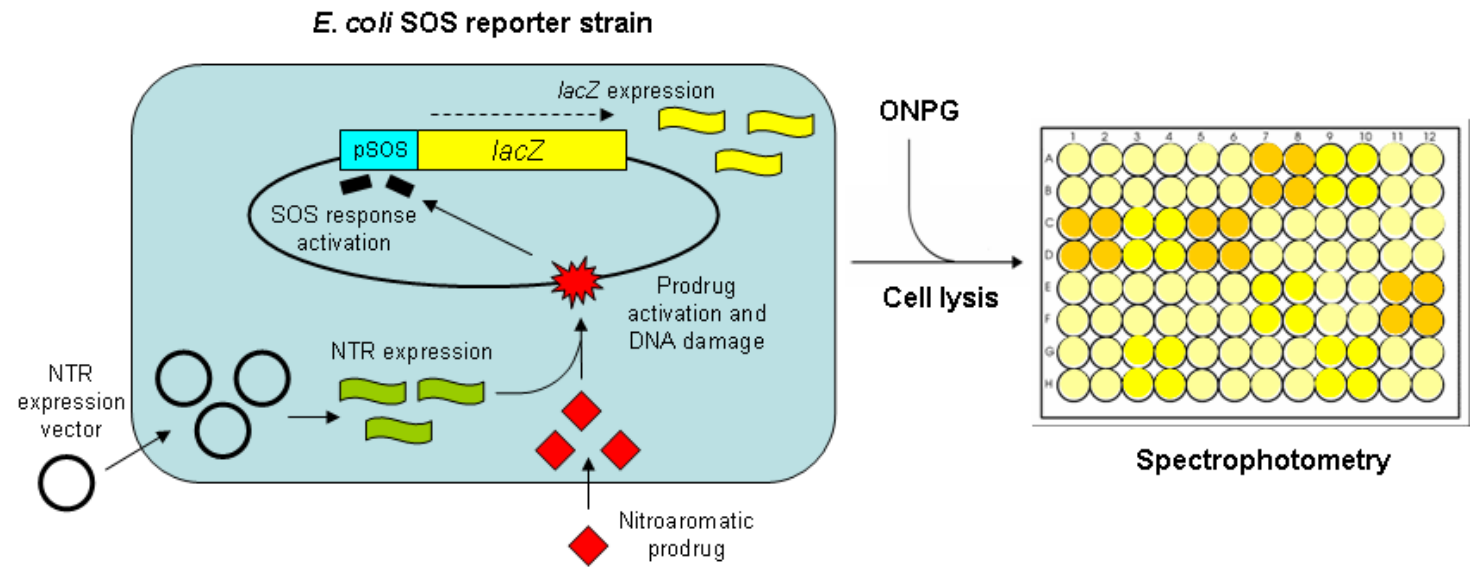

Figure 3.1. Schematic outline of the SOS assay.

An SOS reporter strain containing a genome-integrated reporter gene (in this case lac $Z$ encoding $\beta$ galactosidase) fused downstream of an SOS-responsive promoter (pSOS) is transformed with an NTR expression vector. Transformants are grown, induced for NTR expression and challenged with a nitroaromatic prodrug (e.g. CB1954). The prodrug is reduced by active NTRs leading to formation of DNA adducts. DNA damage activates the SOS response leading to expression of SOS-linked genes, including lacZ. Following prodrug challenge, cells are lysed and incubated with a reporter substrate (e.g. ONPG) to allow measurement of reporter gene activity (e.g. via spectrophotometry).

\subsubsection{NTR over-expression and SOS assay optimisation}

In order to over-express candidate NTRs in SOS-R1, an inducible expression plasmid (pUCX) was constructed by cloning the IPTG-inducible tac promoter region and ribosome binding site from pMMB67EH into the high-copy number cloning plasmid pUC19 (See section 2.12 for details on plasmid construction and Figure 2.2 for vector map). The native $E$. coli $n f s B$ gene was cloned into the MCS of pUCX, transformed into SOS-R1 and the resultant strain tested under a variety of conditions, alongside an empty plasmid (pUC19)-containing control, for its ability to elicit $\beta$-galactosidase activity upon incubation with CB1954. A schematic diagram of the SOS assay is illustrated in Figure 3.1. Variable parameters that were tested included IPTG concentration, CB1954 concentration and total CB1954 challenge time (length of incubation of prodrug with SOS-R1 prior to $\beta$-galactosidase detection). Figure 3.2 depicts the positive correlation between $\beta$-galactosidase (and therefore SOS) activity and the various parameters tested for NfsB-over-expressing SOS-R1. Minimal SOS activity was recorded with the empty plasmid control over the entire range of conditions tested (Figure 3.2C), except for a modest ( 2-fold) increase in activity at the highest CB1954 concentrations ( 0.5 and $1 \mathrm{mM})$ and the longest challenge time ( $5 \mathrm{~h}$ ) employed. Thus, NTR-mediated reduction of CB1954 is readily detectable over background levels using the SOS assay. Based on the 
results gained from NfsB over-expressing SOS-R1, optimal conditions were chosen for subsequent SOS assays. For IPTG concentration, no significant increase in $\beta$ galactosidase activity was observed between 50 and $200 \mu \mathrm{M}$ (Figure 3.2A) and therefore the former was selected for future experiments. Also, because IPTG is a known inhibitor of $\beta$-galactosidase activity (256), it was deemed beneficial for the SOS assay to work with this lower concentration. SOS activity correlated positively with CB1954 concentrations up to $200 \mu \mathrm{M}$, after which a steep decrease in SOS activity was observed (Figure 3.2B). This was most likely due to decreased cell viability (as measured by culture turbidity) at these high concentrations, as illustrated in Figure 3.2D. Optimal CB1954 concentrations were therefore judged as being $\geq 10 \mu \mathrm{M}$ and $\leq 100 \mu \mathrm{M}$, a range over which SOS activity was significantly higher than background and correlated positively with CB1954 concentration. For challenge time, a large increase in SOS activity was seen at each time point from 0 to $2 \mathrm{~h}$, after which increases in activity were more modest (Figure 3.2B). A challenge time of $3 \mathrm{~h}$ was selected for subsequent work, as it allowed sufficient time for SOS response pathways to be activated and was short enough to allow ease of assay processing over a single working day. 

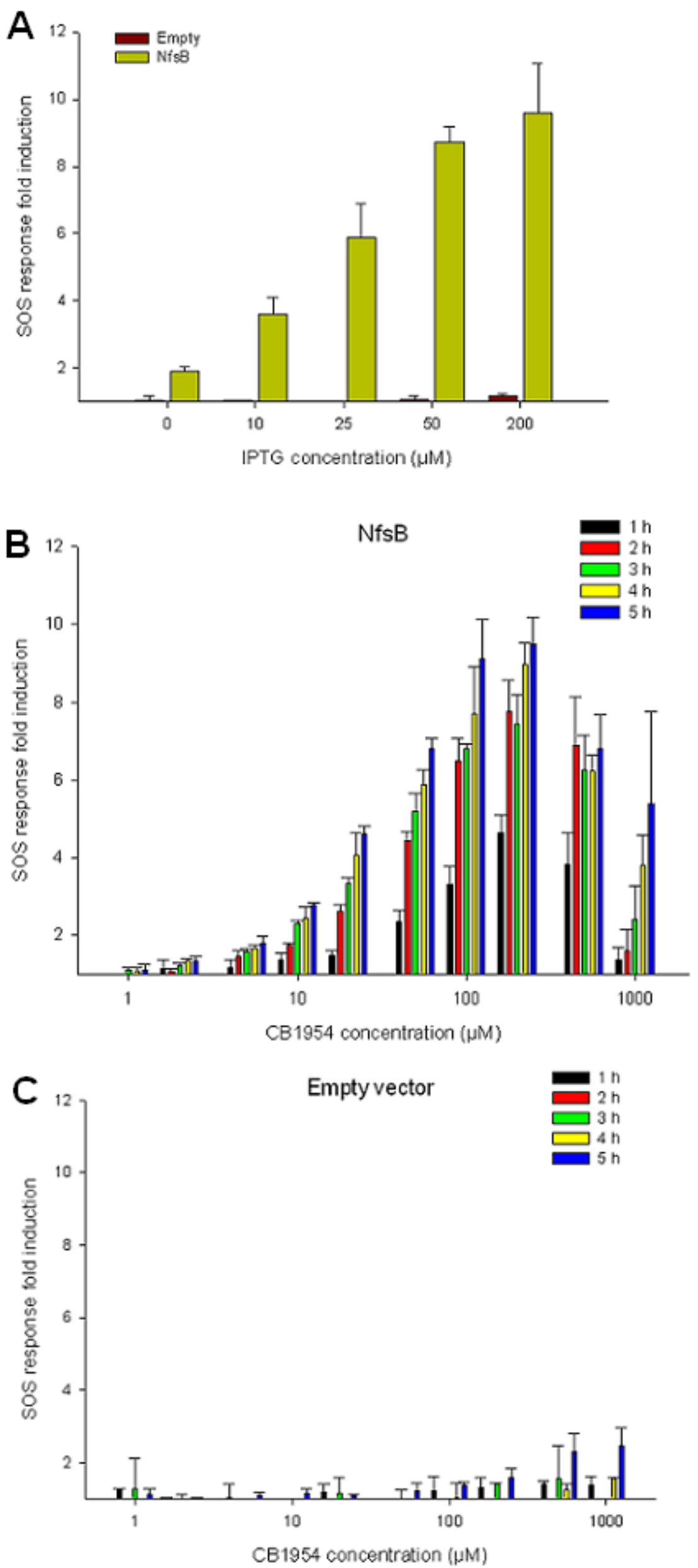

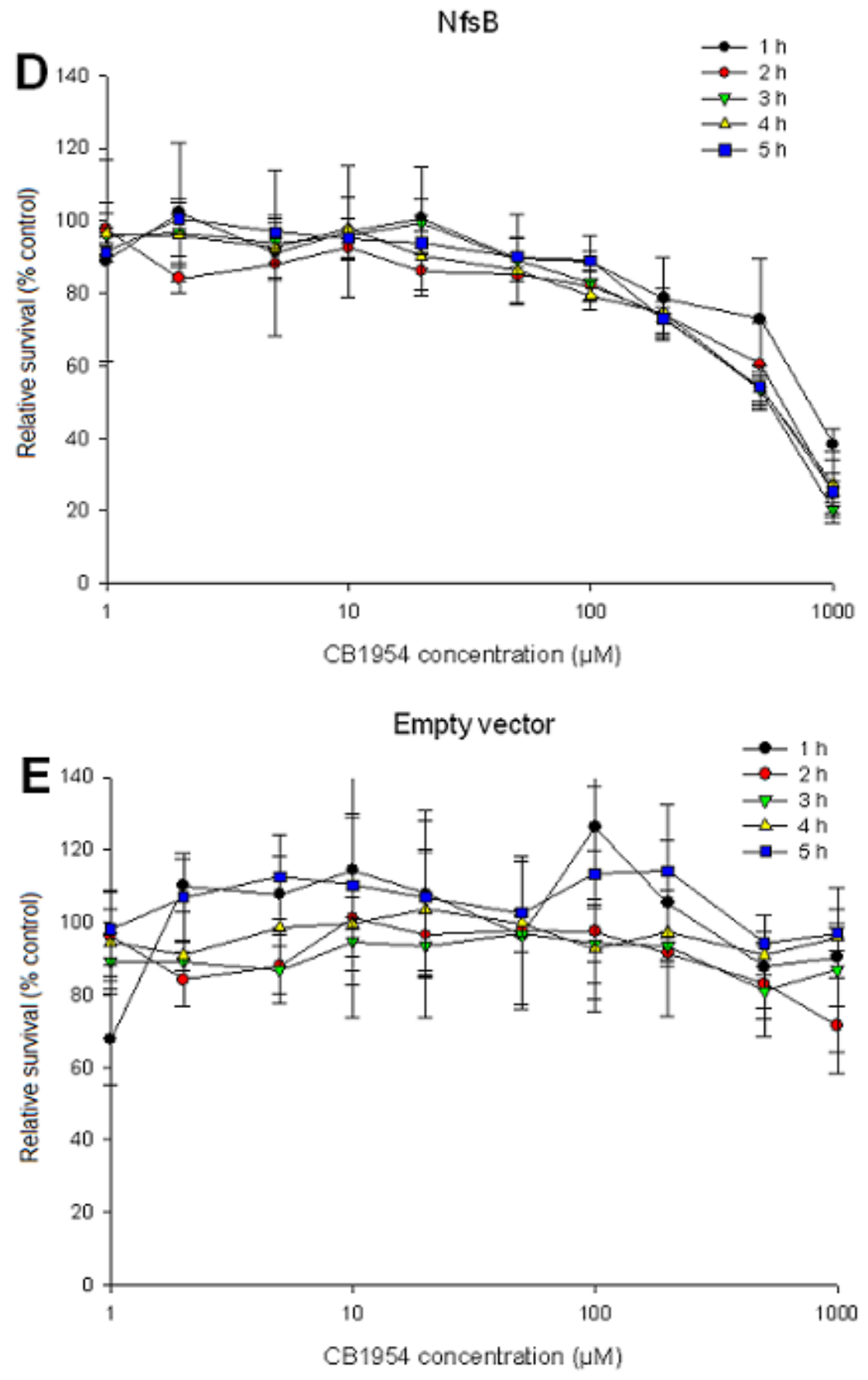

Figures 3.2A-E. Optimisation of SOS assay parameters.

(A) SOS response of SOS-R1 over-expressing E. coli $n f_{s} B$ (pUCX:nfs $B$ ) or no enzyme (empty vector) after $3 \mathrm{~h}$ challenge with $100 \mu \mathrm{M}$ CB1954 at varying IPTG concentrations. SOS response fold induction refers to the ratio of raw $\beta$-galactosidase activity (in Miller units, (243)) of challenged cultures to unchallenged isogenic strains. (B \& C) SOS response of (B) SOS-R1:pUCX:nfsB and (C) SOS-R1:empty vector after challenge with multiple concentrations of CB1954 at multiple time points. (D \& E) Relative cell survival (as measured by changes in culture turbidity at $600 \mathrm{~nm}$ and expressed as a percentage of unchallenged isogenic control cultures) of (D) SOS-R1 pUCX:nfsB or (E) SOS-R1 empty vector over multiple concentrations of CB1954 and at multiple time points. $50 \mu \mathrm{M}$ IPTG was employed in all assays used to generate figures B-E. Each data set is the average of 1-3 independent assays, \pm 1 standard deviation. 


\subsection{Candidate NTR selection}

In order to further assess the applicability and accuracy of the SOS assay as a measure of CB1954-reductase activity, additional candidate enzymes were tested. This also afforded the opportunity of exploring potential NTR activity in previously uncharacterised enzymes. The enzymes selected for this section were all native to $E$. coli, so as to minimize problems arising from heterologous gene expression. They also all adhered to at least one of the following criteria, chosen so as to maximise the possibility of locating novel nitroaromatic prodrug-reducing enzymes:

1. Proven (published) CB1954-reductase activity. Literature searches revealed that only three native $E$. coli enzymes had been directly associated with the ability to reduce CB1954. These included the major and minor NTRs (NfsA and $\mathrm{NfsB})$ and the chromate reductase YieF $(122 ; 222)$. We noted that although NfsA had previously been recorded as an enzyme with the capacity to reduce CB1954, this activity had never formally been characterised (222).

2. Amino acid sequence homology ( $\geq 25 \%)$ to proven CB1954-reductase enzymes. BLAST searches within the NCBI database using the protein sequences of $E$. coli $\mathrm{NfsA}, \mathrm{NfsB}, \mathrm{YieF}$, mammalian NQO1, $B$. amyloliquefaciens YwrO or P. pseudoalcaligenes NbzA (the report on YfkO from $B$. licheniformis had not been published at the time of these experiments) as query sequences were conducted to find possible native $E$. coli homologues. Three genes were located. KefF, annotated as a flavoprotein subunit of the KefC potassium efflux system (257) has 40\% amino acid identity to B. amyloliquefaciens YwrO across 162 residues. Similarly, the hypothetical protein YcaK is $44 \%$ homologous to Human NQO1 across 127 residues. Lastly, the "minimal nitroreductase" YdjA, of which a crystal structure has been determined despite a lack of experimental evidence for nitroreductase activity (258), was chosen due to both its annotation as an NTR and 53\% amino acid similarity across a 65 amino acid region with $P$. pseudoalcaligenes NbzA.

3. Proven nitroreductase activity. This encompassed any enzyme (or a close homologue thereof) with the ability to reduce nitroaromatic compounds other than CB1954. Two enzymes fitted this description: NemA (a member of the OYE family with proven TNT reductase activity (190)) and the azoreductase AzoR (proven quinone and azo dye reductase activity (259; 
260)), which is a close homologue of an enzyme possessing nitroreductase activity from Rhodobacter shpaeroides (203). Additionally, the enzyme YcdI, believed to play a role in pyrimidine metabolism, is annotated in the NCBI database as a nitroreductase, although no experimental data exists to confirm this (261). It shares $22 \%$ identity and $37 \%$ similarity with $\mathrm{NfsB}$, across the entire protein sequence.

4. Enzymes published as having structural similarity to known CB1954reductases. Two previously characterised NAD $(\mathrm{P}) \mathrm{H}$ :quinone oxidoreductases, MdaB and WrbA, belonged to this group. Both have had their crystal structure solved and similarities observed with that of human NQO1 $(262 ; 263)$. Furthermore, MdaB is considered orthologous with an NTR from H. pylori (264).

\subsection{Screening candidate NTRs for CB1954-reductase activity}

\subsubsection{SOS assay}

The genes of the 10 additional candidate NTRs listed above were PCR amplified and cloned into the MCS of pUCX (restriction sites as indicated in primer sequences listed in Table 2.1) for activity screening. Based on SDS-PAGE analysis, expression of 9 of the 11 genes from pUCX in SOS-R1 was equivalent under the conditions used in the SOS assay (see Figure 3.3). The remaining two, YcdI and KefF, were visibly reduced in expression relative to the other NTR candidates (30-60\% of other proteins based on densitometric calculations).

The panel of NTRs was assessed for CB1954 reductase activity via the SOS assay (see Figure 3.4). No substantial SOS response was induced in the absence of prodrug for any of the 11 over-expressed enzymes or empty plasmid control. However, clear and dose-dependant increases in $\beta$-galactosidase activity were observed with NfsA, NfsB, AzoR and NemA over-expression strains upon challenge with CB1954, over the range of prodrug concentrations tested. In case low level CB1954-reductase activity from other enzymes was missed due to prodrug concentrations being too low, SOS response induction was also tested at higher CB1954 concentrations. No increase in $\beta$-galactosidase activity relative to the empty plasmid control was observed for any of the remaining enzymes at concentrations of CB1954 up to and including the highest (1 $\mathrm{mM}$ ) tested (results not shown). 


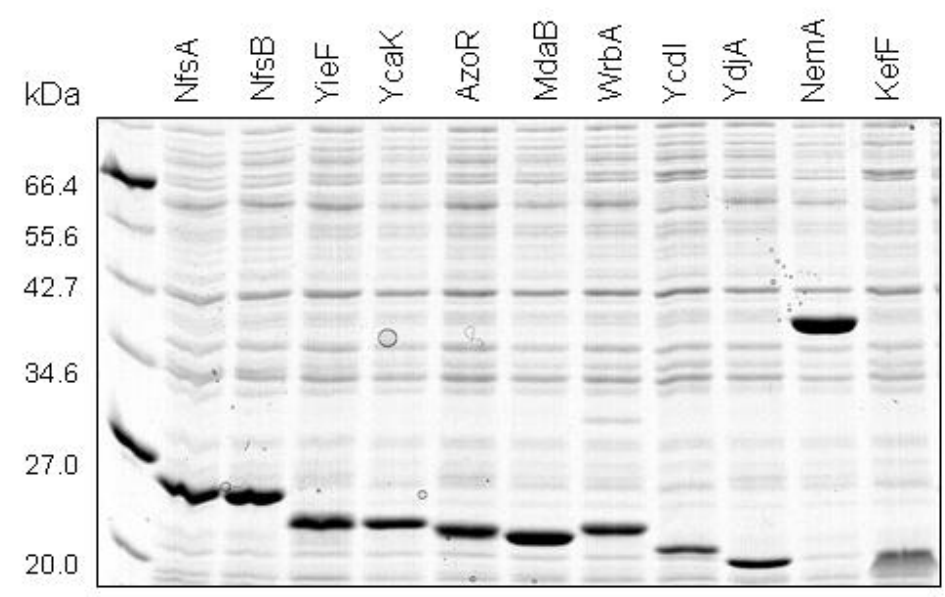

Figure 3.3. Relative expression levels of candidate NTRs in SOS-R1.

SDS-PAGE indicating relative levels of expression of each candidate NTR from pUCX in SOS-R1.

Samples for SDS-PAGE analysis were whole cell lysates taken directly from a completed SOS assay (50 $\mu \mathrm{M}$ IPTG) and normalised for cell density $\left(\mathrm{OD}_{600}\right)$ before electrophoresis.

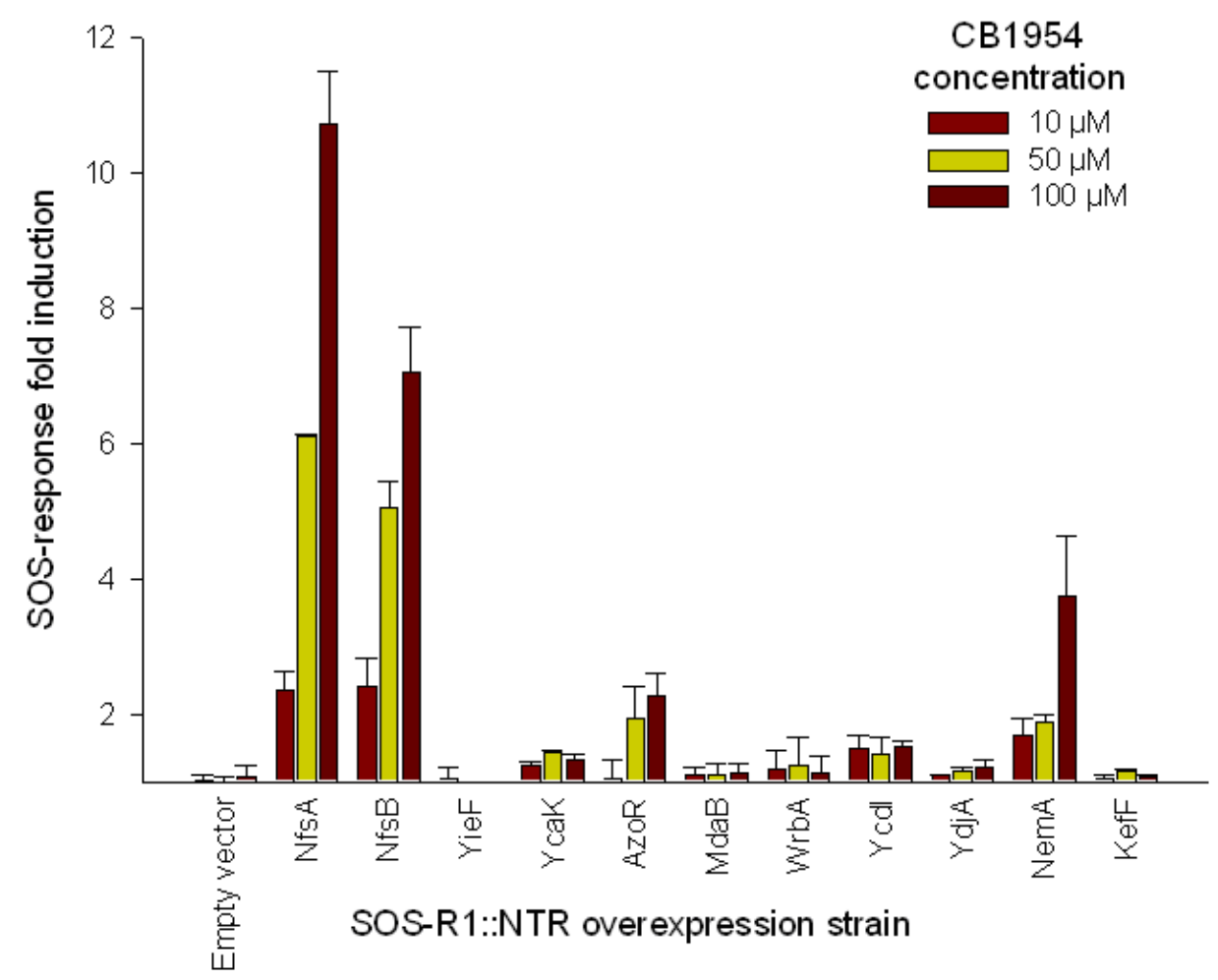

Figure 3.4. CB1954-induced SOS response induction of NTR over-expressing SOS-R1 strains

SOS-R1 strains over-expressing candidate NTRs were grown in liquid media and challenged with the indicated concentrations of CB1954 for $3 \mathrm{~h}$ prior to assessment of SOS response induction. Data are the average \pm 1 SEM of 4 independent assays. 
The development of colour during the $\beta$-galactosidase activity assessment stage of the assay is due to the enzymatic hydrolysis of ONPG into galactose and the chromogenic compound o-nitrophenolate (2-nitrophenol). Because this latter species is itself a nitroaromatic compound, we reasoned that the ability of candidate NTRs to use 2 -nitrophenol as a substrate might interfere with $\beta$-galactosidase activity measurements. Using purified His6-tagged enzymes (see section 3.4.3) and spectrophotometry, each candidate NTR was individually tested for its ability to reduce 2-nitrophenol in the presence of $\mathrm{NAD}(\mathrm{P}) \mathrm{H}$ co-substrates (measured as decrease in absorbance at $340 \mathrm{~nm}$ due to oxidation of $\mathrm{NAD}(\mathrm{P}) \mathrm{H} ;(265))$. No increase in $\mathrm{NAD}(\mathrm{P}) \mathrm{H}$ oxidation above background levels was observed for any enzyme using this method (results not shown), confirming that 2-nitrophenol is not a substrate for any of the candidate NTRs used in this study.

\subsubsection{Bacterial $\mathrm{IC}_{50} \mathrm{~S}$}

To validate the results obtained in the SOS assay, the candidate NTRs were subsequently assessed for CB1954-reductase activity using a variety of other in vivo and in vitro tests. The first was based on the ability of reduced CB1954 to arrest bacterial growth through its cytotoxicity, as already demonstrated in this study (Figure 3.2D) and elsewhere in the literature $(218 ; 219)$. This assay was carried out by subjecting NTR over-expressing SOS-R1 strains to increasing concentrations of CB1954 and monitoring growth rate (via optical density at $600 \mathrm{~nm}$ ) relative to unchallenged isogenic cultures over a $3 \mathrm{~h}$ challenge period. These results were then used to calculate $\mathrm{IC}_{50}$ values (defined as the concentration of prodrug required to inhibit bacterial growth to $50 \%$ of that of an unchallenged isogenic strain) of CB1954 for each candidate NTR overexpression strain. Survival curves and $\mathrm{IC}_{50}$ values for each over-expressed NTR are displayed in Figure 3.5. In agreement with SOS assay results, NfsA, NfsB and NemA over-expression strains showed greatly reduced $\mathrm{IC}_{50}$ values (higher sensitivity) relative to the empty plasmid control. Furthermore, the relative ranking of the NTRs was also the same (NfsA $>\mathrm{NfsB}>\mathrm{NemA})$. However, this assay was not able to distinguish any clear difference between AzoR (low level activity based on the SOS assay results) and the rest of the seemingly inactive NTRs, although there was a modest difference relative to the empty vector control. 


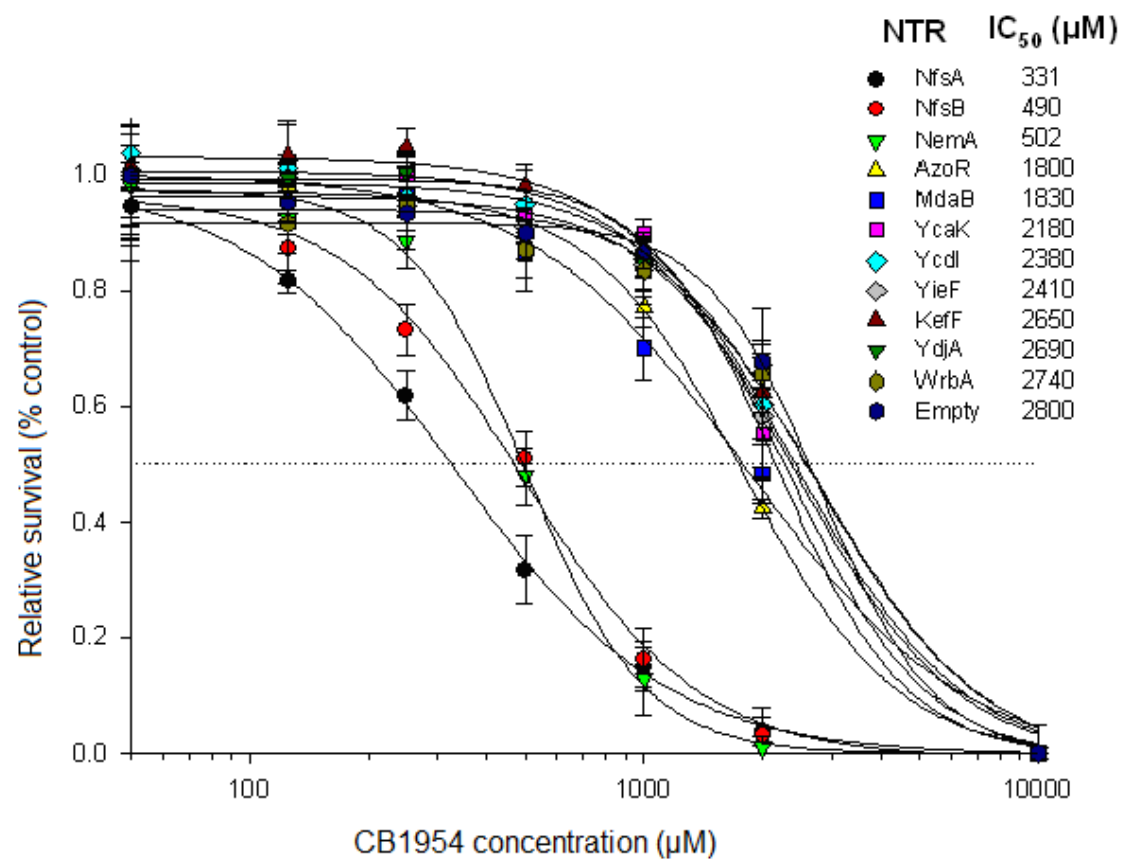

Figure 3.5. CB1954-mediated growth inhibition of NTR over-expressing $E$. coli SOS-R1 strains. Turbidity $\left(\mathrm{OD}_{600}\right)$ of NTR over-expressing cell cultures was recorded directly before and after $3 \mathrm{~h}$ incubation with varying concentrations of CB1954. Cell survival is expressed as the percentage of total growth (change in $\mathrm{OD}_{600}$ ) of challenged cells relative to the unchallenged control for each strain, over the $3 \mathrm{~h}$ challenge. $\mathrm{IC}_{50}$ values indicate the concentration of $\mathrm{CB} 1954$ at which bacterial growth is inhibited to $50 \%$ of that of unchallenged isogenic strains. Data are the average \pm 1 SEM of 4 independent assays.

\subsubsection{In vitro kinetics of purified NTR candidates with CB1954}

Reductive metabolism of CB1954 by candidate NTRs was further investigated through in vitro purified enzyme kinetics. Each candidate NTR gene was cloned into the expression vector pET28a(+), over-expressed in E. coli BL21 and recombinant enzymes purified to homogeneity as hexahistidine (His6) tagged derivatives. All NTRs except YcdI and KefF were able to be purified using the methods described in section 2.9. Variations in the parameters used for protein expression, including reduced growth temperature and addition of betaine and sorbitol (266) were also tested; however no soluble protein under any condition was able to be extracted for either of these two enzymes. Based on inactivity of these two enzymes from the previous studies (SOS and $\mathrm{IC}_{50}$ analysis), their purification was not pursued any further. SDS-PAGE was used to determine purity ( $>90 \%$ by densitometric analysis; results not shown) and correct size for each enzyme (Figure 3.6). Although most enzyme preparations displayed banding patterns indicative of a second protein species at higher molecular weight, size comparisons suggested that these were dimeric forms of the respective enzyme and therefore were not considered to be contaminants. 


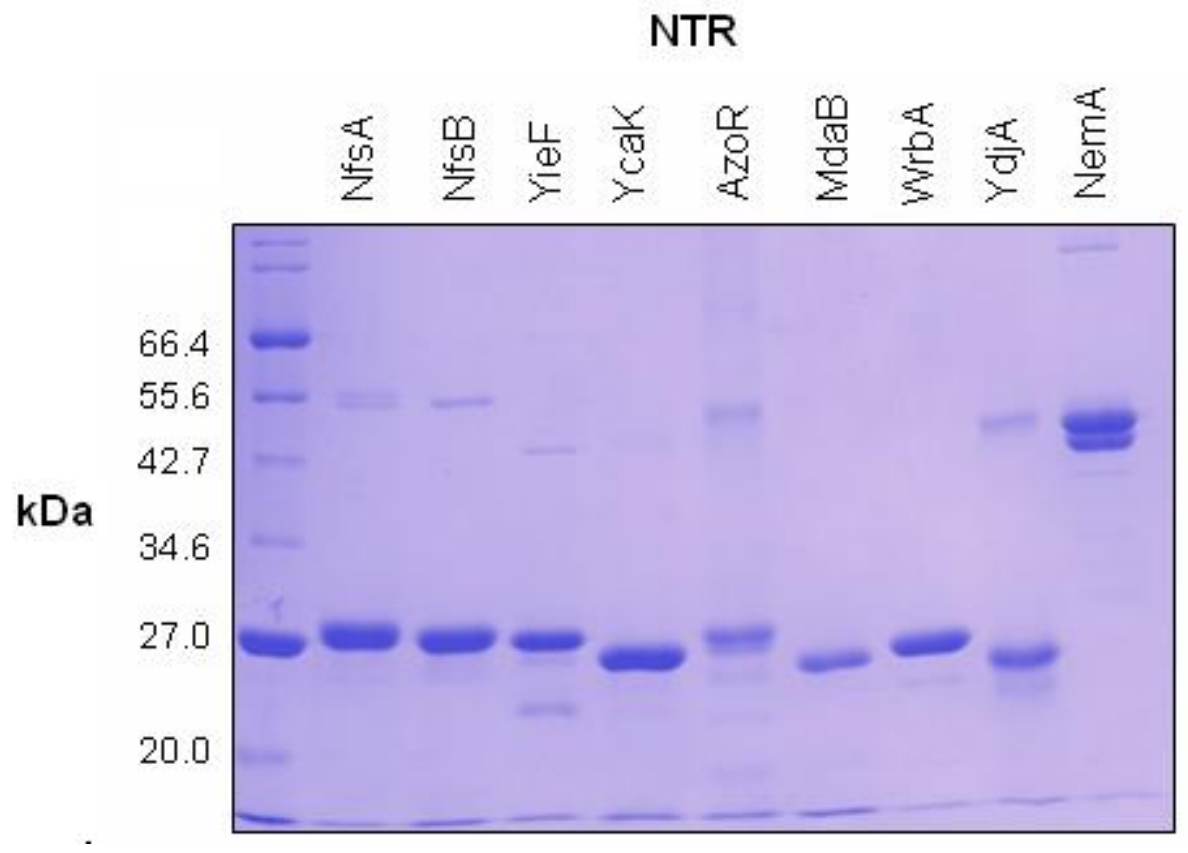

Figure 3.6. SDS-PAGE of purified, His6-tagged candidate NTRs

NTRs were cloned into pET28a(+) vectors and over-expressed in E. coli BL21, followed by purification of recombinant enzymes via nickel-affinity chromatography. Each lane was loaded with $10 \mu \mathrm{L}$ of enzyme sample at $0.1 \mathrm{mg} \mathrm{mL}^{-1}$.

Measurement of the rate of CB1954-reduction by individual purified enzymes was carried out using a spectrophotometric method devised by Race et al. (241). As previously demonstrated, CB1954 can be reduced at either the 2- or 4-NO ${ }_{2}$, but not both, resulting in either the 2- or 4-NHOH as an end metabolite, which each display distinct absorption spectra (241). Nitro group preference can differ between enzymes resulting in reduction of only the $4-\mathrm{NO}_{2}(109 ; 199 ; 207)$, only the $2-\mathrm{NO}_{2}(189)$ or mixtures of both in various ratios $(122 ; 208)$. Race and colleagues showed that both the 4-NHOH and 2-NHOH absorb equally at $420 \mathrm{~nm}$ wavelength, with an extinction coefficient of $1,200 \mathrm{M}^{-1} \mathrm{~cm}^{-1}$, allowing comparative assessment of enzymatic reduction of CB1954 without prior knowledge of the reduction pathway taken.

Buffered reactions containing CB1954, co-substrate (NAD(P)H) and purified enzyme were set up and monitored for CB1954 reduction by measuring the rate of change in absorbance of the reaction mixture at $420 \mathrm{~nm}$ wavelength. Detectable activity was only obtained from NfsA, NfsB, AzoR and NemA. Both NADH and NADPH were tested as possible co-substrates in the reaction. Consistent with previous results, NfsA and NemA both had a strong preference for NADPH (176; 267), while NfsB showed roughly equal activity with both (177). In contrast to previous reports, AzoR displayed higher activity with NADPH as co-substrate $(259 ; 260)$. Affinity $\left(K_{\mathrm{m}}\right)$ and catalytic $\left(k_{\text {cat }}\right)$ constants for each of the four active enzymes with CB1954 were then extrapolated from 
reaction rate data obtained over a range of CB1954 concentrations (see Table 3.1). All four of these enzymes have previously been determined to catalyze substrate reduction via a bi-bi ping pong mechanism of action (described in section 1.6.1), an implication of which is that kinetic parameters for the substrate being tested (in this case CB1954) can vary for a specific enzyme depending on the concentration of the second substrate $(\mathrm{NAD}(\mathrm{P}) \mathrm{H})$ used in the reaction (268). Co-substrate concentrations were kept constant throughout all these experiments $(250 \mu \mathrm{M})$ and as such $k_{\text {cat }}$ and $K_{\mathrm{m}}$ values are apparent (not global) indicators of substrate affinity and catalysis. However, the ratio of $k_{\text {cat }}: K_{\mathrm{m}}$ for such reactions remains constant across different co-substrate concentrations (268) and therefore it is this value, the specificity constant $\left(k_{\mathrm{cat}} / K_{\mathrm{m}}\right)$, that provides a true measure of relative enzyme activity towards CB1954 in the context of this study. Based on these principles, NfsA was $\sim 10$-fold more active with CB1954 than NfsB, which was in turn approximately twice as active as NemA and 60-fold more active than AzoR, when using their preferred co-substrates (Table 3.1).

To further investigate the presence or absence of any discernible nitroreductase activity from the remaining six enzymes, reductive metabolism of the archetypal nitroaromatic compound nitrofurazone (NFZ) was assessed (by monitoring the change in absorbance at $400 \mathrm{~nm}, \varepsilon=12,960 \mathrm{M}^{-1} \mathrm{~cm}^{-1}$; (176)) Surprisingly, all candidate NTRs, except YdjA, were capable of in vitro reduction of NFZ at the concentration tested (50 $\mu \mathrm{M}$ ), albeit with widely varying rates (Table 3.1). This activity therefore confirmed these enzymes as bona fide NTRs. These observations also confirmed that the enzyme preparations employed were active and that the lack of CB1954-reducing activity associated with these enzymes was unlikely due to poor quality of the purified sample.

The effect of the His6 tag on enzyme performance was also assessed. His6 tags were cleaved from enzyme preparations by thrombin treatment and the resultant native enzymes assessed for CB1954-reductase activity as previously described. No discernible difference in activity was detected between His6 tag-containing and His6 tag-deficient enzymes (data not shown). 
Table 3.1: Steady-state kinetic parameters of purified His6-tagged candidate NTRs with CB1954.

\begin{tabular}{|c|c|c|c|c|c|}
\hline Enzyme & Co-subs. $^{a}$ & $k_{\text {cat }}\left(\mathrm{s}^{-1}\right)$ & $K_{\mathrm{m}}(\mu \mathrm{M})$ & $\begin{array}{l}k_{\text {cat }} / K_{\mathrm{m}} \\
\left(\mathbf{M}^{-1} \mathbf{s}^{-1}\right)\end{array}$ & $\begin{array}{c}\text { NFZ activity } \\
\left(\mu \mathrm{mol} \min ^{-1} \mathrm{mg}^{-1}\right)^{b}\end{array}$ \\
\hline \multirow{2}{*}{$\mathrm{NfsA}$} & NADPH & $16.1 \pm 0.48$ & $219 \pm 18$ & $73,500 \pm 6,400$ & 10.5 \\
\hline & $\mathrm{NADH}$ & $2.88 \pm 0.15$ & $45.8 \pm 7.0$ & $62,800 \pm 10,000$ & 3.5 \\
\hline \multirow[t]{2}{*}{$\mathrm{NfsB}$} & NADPH & $25.9 \pm 2.6$ & $3,570 \pm 660$ & $7,250 \pm 1,500$ & 5.7 \\
\hline & $\mathrm{NADH}$ & $61.5 \pm 11$ & $11,000 \pm 2,600$ & $5,600 \pm 1,700$ & 6.8 \\
\hline \multirow[t]{2}{*}{ AzoR } & NADPH & $0.153 \pm 0.008$ & $1,420 \pm 160$ & $108 \pm 13$ & $1.1 \times 10^{-1}$ \\
\hline & $\mathrm{NADH}$ & $0.148 \pm 0.02$ & $6,600 \pm 1,000$ & $22.4 \pm 5$ & $8.7 \times 10^{-2}$ \\
\hline \multirow[t]{2}{*}{ NemA } & NADPH & $0.216 \pm 0.003$ & $55.7 \pm 2.2$ & $3,880 \pm 160$ & $3.2 \times 10^{-2}$ \\
\hline & $\mathrm{NADH}$ & $0.048 \pm 0.002$ & $54.7 \pm 5.1$ & $878 \pm 90$ & $5.8 \times 10^{-2}$ \\
\hline \multirow[t]{2}{*}{ MdaB } & $\mathrm{NADH}$ & $\mathrm{ND}^{\mathrm{c}}$ & ND & - & $6.0 \times 10^{-3}$ \\
\hline & NADPH & ND & ND & - & $2.3 \times 10^{-2}$ \\
\hline \multirow[t]{2}{*}{ WrbA } & $\mathrm{NADH}$ & ND & ND & - & $2.7 \times 10^{-3}$ \\
\hline & NADPH & ND & ND & - & $1.8 \times 10^{-2}$ \\
\hline \multirow[t]{2}{*}{ YcaK } & $\mathrm{NADH}$ & ND & ND & - & $1.6 \times 10^{-2}$ \\
\hline & NADPH & ND & ND & - & $1.4 \times 10^{-2}$ \\
\hline \multirow[t]{2}{*}{ YieF } & $\mathrm{NADH}$ & ND & ND & - & $2.9 \times 10^{-2}$ \\
\hline & NADPH & ND & ND & - & $6.3 \times 10^{-2}$ \\
\hline \multirow[t]{2}{*}{ YdjA } & $\mathrm{NADH}$ & ND & ND & - & ND \\
\hline & NADPH & ND & ND & - & ND \\
\hline
\end{tabular}

${ }^{\mathrm{a}}$ Initial co-substrate concentration in all protein assays was $0.25 \mathrm{mM}$

${ }^{\mathrm{b}}$ Catalytic rates were measured at $50 \mu \mathrm{M}$ initial concentration of NFZ

${ }^{\mathrm{c}} \mathrm{ND}$ : not detected

\subsubsection{In vivo cytotoxicity in HCT-116 human colon carcinoma cell line}

All results presented in this section were generated by Dr. Sophie Syddall, ACSRC.

To evaluate the ability of the candidate NTRs to confer CB1954-sensitivity to a human cell line model, genes encoding the eleven enzymes were individually recombined into the Gateway ${ }^{\mathrm{TM}}$ compatible expression plasmid F527-V5 and transfected into the cultured human colon carcinoma cell line HCT-116. Following transient transfection, protein translation was detected by concurrent cell infection with TAG stop suppressor adenovirus (Tag-On-Demand ${ }^{\mathrm{TM}}$, Invitrogen), permitting read through of all eleven 
TAG-terminated NTRs to give rise to an immunoreactive V5-epitope at the C-terminus. All transfected cell lines gave a detectable V5 band of the expected size in western blots, confirming that it was possible to express each enzyme in HCT-116 cells (Figure 3.7A). However, the amplitude of expression varied between the different candidates with the most highly expressed enzyme KefF being measured by densitometry at an 8.1fold greater level than $\mathrm{NfsB}$; and the lowest, $\mathrm{NfsA}$, at only 0.24 -fold the level of $\mathrm{NfsB}$ (Table 3.2).

The range of expression levels was even greater with stable expression in pooled cell line populations generated under puromycin selection (Figure 3.7B, Table 3.2). YcdI and YcaK could no longer be detected in V5 western blots, and while NfsA was now detected at 0.3 -fold the level of NfsB, KefF was found at 15.7-fold higher levels than NfsB. Nonetheless, transient and stable expression levels correlated $\left(\mathrm{r}^{2}=0.65 ; P=\right.$ 0.02; paired t-test). The relative protein expression levels did not appear related to the presence of codons with poor human bias, as artificially synthesized versions of $n f s A$ and $n f s B$ that were codon optimized for maximal human cell expression did not yield a detectably stronger signal on V5 western blots (results not shown). Thus, the variation in expression levels of the 11 NTR candidates appeared to be due to intrinsic variations in mRNA or protein stability in HCT-116 cells.

In contrast to results in bacterial assays and at a purified protein level, cytotoxicity testing identified $n f s B$ expressing cells as having the greatest sensitivity to CB1954, about twice as sensitive as those expressing $n f_{s} A$ and approximately 20 -fold more sensitive than those expressing nemA (in terms of $\mathrm{IC}_{50}$ normalized against the relative expression levels of each NTR; Table 3.2). Although each of the remaining E. coli NTR candidates appeared to slightly enhance sensitivity to CB1954 relative to untransfected HCT-116 cells ( $\mathrm{p}<0.05$; One-way ANOVA), none of these (including AzoR) were within three orders of magnitude of NfsB. 


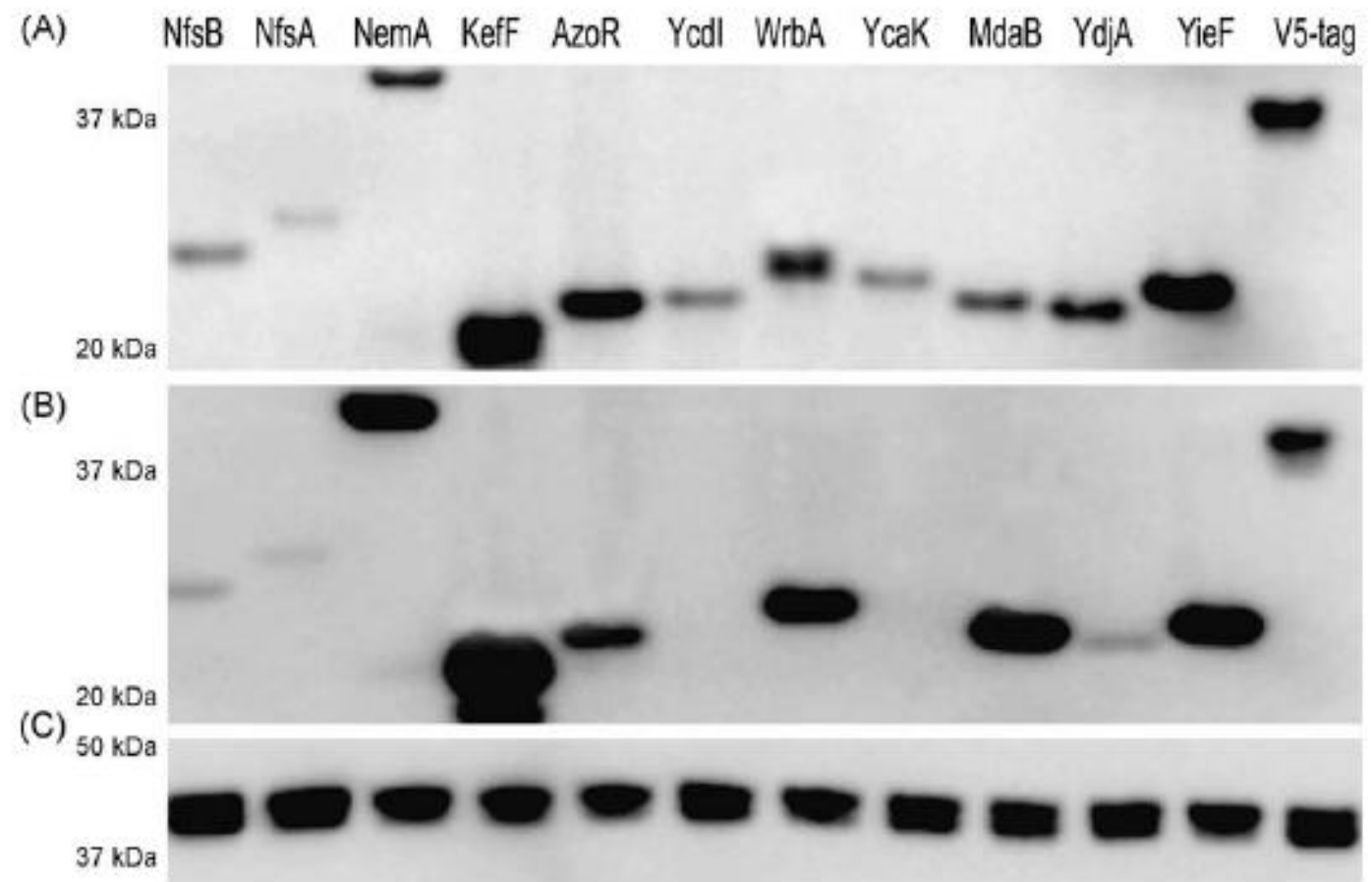

Figure 3.7. Expression of $E$. coli NTRs in the human colon carcinoma cell line HCT-116.

(A) HCT-116 cells were transiently transfected with F527-V5 vectors containing each of the E. coli NTRs. Cells were concurrently infected with Tag-On-Demand ${ }^{\text {TM }}$ Suppressor Supernatant, lysed, and immunoblotted for induced C-terminal V5 tag. Relative densitometry measurements for each NTR band (and a $40 \mathrm{kDa}$ V5 tag control) are presented in Table 3.2. (B). HCT-116 cells engineered for stable expression of each of the E. coli NTRs were infected, lysed, immunoblotted and quantified as in (A). (C) Representative $\beta$-actin loading control (in this instance derived from the transiently transfected immunoblot in (A)). 
Table 3.2. Relative sensitivity of the HCT-116 cell lines to CB1954 per unit NTR protein expressed.

\begin{tabular}{|c|c|c|c|c|}
\hline $\begin{array}{l}\text { NTR } \\
\text { candidate }\end{array}$ & $\begin{array}{l}\text { Expression } \\
\text { Transient }^{\mathrm{a}}\end{array}$ & $\begin{array}{l}\text { Expression } \\
\text { Stable }^{\mathrm{b}}\end{array}$ & $\mathrm{IC}_{50}(\mu \mathrm{M})^{\mathrm{c}}$ & $\begin{array}{l}\text { Relative } \\
\text { Sensitivity }^{\mathrm{d}}\end{array}$ \\
\hline NfsB & 1.00 & 1.00 & $0.18 \pm 0.03$ & 100 \\
\hline NfsA & 0.24 & 0.30 & $1.4 \pm 0.2$ & 43 \\
\hline $\operatorname{NemA}$ & 1.98 & 9.40 & $0.397 \pm 0.003$ & 4.8 \\
\hline KefF & 8.11 & 15.70 & $19 \pm 4$ & 0.060 \\
\hline YdjA & 2.76 & 2.40 & $192 \pm 48$ & 0.039 \\
\hline AzoR & 4.20 & 7.20 & $107 \pm 55$ & 0.023 \\
\hline MdaB & 2.16 & 10.90 & $83 \pm 43$ & 0.020 \\
\hline YieF & 5.12 & 11.40 & $178 \pm 64$ & 0.009 \\
\hline WrbA & 3.26 & 9.50 & $237 \pm 75$ & 0.008 \\
\hline YcdI & 1.45 & $\mathrm{ND}^{\mathrm{e}}$ & $356 \pm 93$ & $\mathbf{N A}{ }^{\mathbf{f}}$ \\
\hline YcaK & 1.34 & ND & $526 \pm 74$ & NA \\
\hline
\end{tabular}

${ }^{\mathrm{a}}$ Relative expression level of each NTR protein in the transiently transfected HCT-116 cell line (derived from densitometry of the immunoblot presented in Figure 3.7, with each NTR normalized against the corresponding actin control and presented relative to $\mathrm{NfsB}$ )

${ }^{b}$ Relative expression level of each NTR protein in the stably transfected HCT-116 cell line (normalized against actin and presented relative to $\mathrm{NfsB}$ )

${ }^{\mathrm{c}}$ Mean \pm 1 SEM $\left(\mathrm{IC}_{50}\right.$ for untransfected HCT-116 line was $\left.469 \pm 54 \mu \mathrm{M}\right)$

${ }^{\mathrm{d}} \mathrm{IC}_{50}$ values multiplied by the relative expression level of each NTR in the stable cell line and presented as a percentage relative to $\mathrm{NfsB}$

${ }^{\mathrm{e}} \mathrm{ND}$ : not detected

${ }^{\mathrm{f}} \mathrm{NA}$ : not applicable

\subsubsection{Identification of reduced CB1954 metabolites generated by the different $E$. coli NTRs}

The 2- and 4- $\mathrm{NHOH}$ derivatives of CB1954 have been shown to elicit cytotoxicity by distinct mechanisms $(110 ; 111)$. To investigate whether differences in the CB1954 metabolite ratios generated by each candidate NTR may have contributed to disparities between measurements of purified enzyme kinetics, SOS assays, and bacterial and mammalian cell $\mathrm{IC}_{50} \mathrm{~s}$, the relative levels of $2-\mathrm{NHOH}$ and $4-\mathrm{NHOH}$ produced by each of NfsA, NfsB, NemA and AzoR were measured by reverse-phase HPLC (Figure 3.8). Consistent with previous studies, NfsB was found to generate equimolar levels of 2$\mathrm{NHOH}$ and 4-NHOH. In contrast, NfsA was shown to reduce primarily the 2-nitro group to give 2-NHOH with a minimal HPLC peak corresponding to 4-NHOH, whereas AzoR and NemA both produced almost exclusively 4-NHOH, with only a very small 
peak corresponding to 2-NHOH for AzoR. As previously observed for $\mathrm{NfsB}$, reduction to the terminal (6e $)$ amino-product at either the 2- or the 4- position was not observed with any of the enzymes, irrespective of the duration of incubation or concentration of NAD(P)H employed.
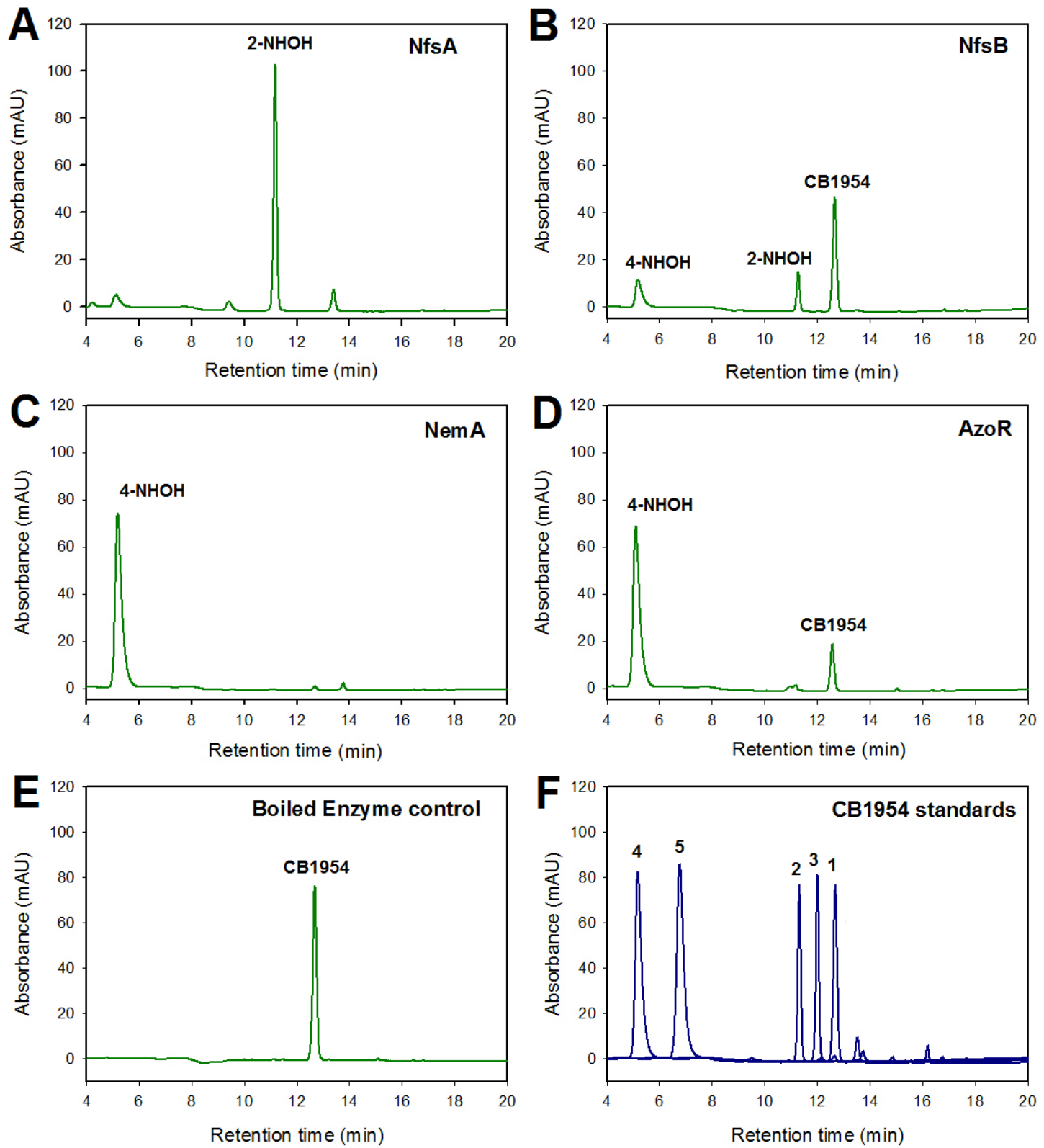

Figures 3.8A-F. HPLC analysis of products of NTR-catalysed reduction of CB1954.

Buffered reactions consisting of CB1954 $(200 \mu \mathrm{M})$, co-substrate (NAD(P)H; $1 \mathrm{mM})$ and purified candidate NTR $(0.5-2 \mu \mathrm{M})$ were incubated at room temperature for 10-30 minutes prior to chromatographic separation of reaction products (as outlined in section 2.16). Eluates were continuously monitored at $262 \mathrm{~nm}$. Authentic CB1954 metabolite standards were generous gifts from Prof. William Denny and Graham Atwell (ACSRC). CB1954 standards (Figure 3.8F) are (1) CB1954, (2) 2-NHOH, (3) 2- $\mathrm{NH}_{2},(4) 4-\mathrm{NHOH}$ and (5) 4- $\mathrm{NH}_{2}$. 


\subsection{Discussion}

This study investigated the ability of eleven native $E$. coli enzymes to reduce the nitroaromatic anti-cancer prodrug CB1954, via a panel of in vivo and in vitro assays, including a novel colorimetric screening method based on the E. coli SOS response. Of the candidate NTRs tested, four were found to produce detectable levels of CB1954 reduction products, two of which (AzoR and NemA) had not previously been studied in the context of nitroaromatic prodrug activation. Also, at the time of performing this study NfsA had only been characterised in minor detail regarding its CB1954 reductase activity (222), with the publication of Vass et al. (189) appearing subsequently. A major facet of this study was to assess the ability of a novel screening method, the SOS assay, to accurately determine relative CB1954-reductase activity among candidate enzymes. The SOS assay is an attractive technique for NTR-mediated prodrug activation screening, as it affords the possibility of moderate to high-throughput analysis of candidate enzyme activity and does not require high prodrug concentrations; both of which are key elements of any enzyme discovery study and have not been thoroughly developed for the NTR-CB1954 system. This study demonstrated that not only was the SOS assay able to detect NTR-mediated CB1954 reduction at very low substrate concentrations ( $<10 \mu \mathrm{M}$ depending on the enzyme, see Figure 3.4), but could also provide a semi-quantitative assessment of the relative CB1954-reducing abilities of multiple NTRs simultaneously. This latter point sets the SOS assay apart from the SOSmediated lambda-phage based assay previously developed for evaluation of CB1954 reduction (225): although the latter system provides a highly efficient means of selecting NTRs improved for CB1954 reductase activity from a pool of enzyme variants, it appears to lack the capability of rapidly analysing relative levels of individual candidate enzymes simultaneously. Using the SOS assay, it was shown that Nfs A was the most active CB1954-reducing enzyme tested, followed closely by NfsB and more distantly by NemA and AzoR. Results from E. coli $\mathrm{IC}_{50} \mathrm{~s}$ and purified enzyme kinetics were all in good agreement (in particular regarding the relative rankings of the four active NTRs) with those obtained in the SOS assay, proving the validity and accuracy of this technique as a suitable CB1954-reduction screening tool.

The final application of NTR-CB1954 GDEPT is for use in human tumours and therefore it is imperative that results gained through E. coli and in vitro testing are reliably indicative of the clinical scenario. Testing of the eleven candidate NTRs in the human colon carcinoma cell line HCT-116 yielded a CB1954-sensitivity pattern in 
general agreement with the $E$. coli results, with only NfsA, NfsB and NemA providing CB1954-sensitivities more than 3 orders of magnitude lower than the non-transfected control; however one significant discrepancy was the superiority of NfsB overexpression relative to NfsA over-expression in conferring CB1954 sensitivity, with NfsB generating an expression level normalized IC $_{50}$ in HCT-116 2-fold lower ( $p$ $<0.05$; t-test) than NfsA (see Table 3.2). It is noteworthy that in another study focusing on NfsA, a clear advantage of this enzyme was observed over NfsB in imparting CB1954 sensitivity towards the ovarian cancer cell lines SKOV-3. The basis of the variation among these different organisms and cell lines therefore requires significant discussion.

Firstly, western-blot analysis of NTR over-expression in HCT-116 indicated that expression levels varied widely between NTRs (Figure 3.7). In particular, overexpressed NfsA levels were the lowest out of all NTRs tested, being less than one third that of NfsB. Normalised CB1954 sensitivities were calculated by dividing raw $\mathrm{IC}_{50}$ values by relative protein expression levels (established by scanning densitometry of blots); however there is no data to definitively confirm that a direct linear relationship exists between NTR expression levels and sensitivity to CB1954, in particular at either very high or very low enzyme levels. Indeed, Chen et al. (1995) demonstrated a lack of correlation between HSV-TK expression levels in 9L glioma cells and sensitivity to GCV (269). Other studies on enhanced enzymes for prodrug activation either do not report or do not at all test enzyme expression levels in mammalian cell lines $(216 ; 270)$, including that of NfsA in SKOV-3 (189), impeding further investigation into this phenomenon. Therefore, NfsA levels in HCT-116 may have not been high enough to elicit a significantly toxic response, with the low levels of reduced metabolites possibly out-competed by cellular detoxification machinery.

Another possible source of variation is the difference in susceptibility between cell types to the various end products of CB1954 metabolism. Although much of the emphasis on CB1954-reduction studies has been towards the 4-NHOH metabolite, widely considered to be the primary toxin of CB1954 metabolism $(111 ; 121)$, studies have shown that products of reduction of the 2- $\mathrm{NO}_{2}$, in particular the $2-\mathrm{NH}_{2}$, can be equally, if not more toxic than the 4-NHOH in certain cell lines (121). Results described here, in agreement with other studies, demonstrate that $\mathrm{Nfs}$ A only reduces the 2- $\mathrm{NO}_{2}$ (189), while NfsB produces equimolar ratios of both the 2- and 4-NHOH metabolites (122). HCT-116 could therefore be inherently more resistant to the DNA adducts formed by $2-\mathrm{NO}_{2}$ reduction products, compared to both E. coli and SKOV-3, 
diminishing any cytotoxic effect conferred by NfsA-catalysed reduction of CB1954 in this cell line. It has previously been shown that cell sensitivity to the 4-NHOH of CB1954 is more pronounced in nucleotide excision repair (NER) deficient genetic backgrounds $(111 ; 121)$. Although E. coli, HCT-116 and SKOV-3 are NER-proficient, both HCT-116 and SKOV-3 (but not E. coli) are deficient in mismatch-repair (MMR), a pathway that is known to affect resistance of cells to certain DNA-alkylating compounds (271). More importantly, in contrast to HCT-116, SKOV-3 lacks a functional p53 gene $(272 ; 273)$, the product of which is involved in DNA repair and cell cycle control (274). Together, these factors suggest that heterogeneity in DNA repair pathway proficiencies could affect the relative sensitivities of cell types to the different CB1954 reduction products, and in particular provide an explanation to the differences observed in sensitivities towards CB1954 between various NfsA over-expressing cell lines.

It should also be noted that the intercellular diffusion properties of the two principal hydroxylamine metabolites are disparate (121) and consequently their cytotoxic effects are likely to be non-uniform in low cell density cultures. The 2- $\mathrm{NH}_{2}$ product is the major contributor to the bystander effect of CB1954 (121), and the efficiency of bystander killing is dependent upon cell density (153). In this study, 500 cells (HCT-116) were plated per well, whereas Vass et al. employed a density of $2 \times 10^{4}$ cells per well for cytotoxicity assays of transfected cell lines (189). Thus, the contribution of the bystander effect (and hence the contribution of NfsA) to cell killing may have been greater in the experiments of Vass et al.

Alternatively, differences in co-substrate availability between the cell types could have an impact on the relative abilities of each NTR to impart CB1954 sensitivity. This study, among others, has demonstrated a strong preference of NfsA for NADPH, while NfsB is equally capable of utilizing both NADH and NADPH as co-substrates (177). A few studies have shown that NADPH is typically at lower levels intracellularly, in both bacteria and human cells, compared to NADH $(275 ; 276)$, while relative levels of each co-factor can vary significantly between disease and non-disease states $(275 ; 277)$. NADPH levels could therefore be a limiting factor in HCT-116, relative to E. coli and SKOV-3, possibly explaining the lower NfsA-mediated CB1954sensitivity observed in the former. Consistent with this explanation, NemA and AzoR (which also exhibit a NADPH co-substrate preference) were also less active in HCT116 relative to $\mathrm{NfsB}$ than might have been predicted from the E. coli $\mathrm{IC}_{50}$ and $\mathrm{SOS}$ assay or in vitro purified protein kinetics. Lower levels of NADPH in E. coli could also 
explain the modest difference in SOS response induction relative to kinetic activity, compared with NfsB (1.2 and 1.5-fold increase in SOS activity for NfsA, over NfsB, at $50 \mu \mathrm{M}$ and $100 \mu \mathrm{M}$ CB1954 respectively, compared to a $\sim 10$-fold increase in $k_{\text {cat }} / K_{\mathrm{m}}$ for NfsA over NfsB, with CB1954).

Another salient message from the points discussed above is that there is an inherent heterogeneity in cellular metabolism and redox status of different human cell lines, especially those with malignant phenotypes; and hence results obtained in one cell line may only be generally predictive of those that would be obtained in a different cell line, or for a tumour in vivo. Consequently, the use of E. coli as a model organism to study enzyme-catalyzed prodrug activation may prove an equally reliable - and far more efficient - alternative to creating and testing panels of transfected tumour cell lines. In particular, the SOS assay provides a simple and elegant means of overcoming undesirable characteristics of other traditional negative-selection strategies, such as growth inhibition and cell survival assays. CB1954 concentrations required to elicit a response in the SOS assay were significantly lower than in the corresponding $\mathrm{IC}_{50}$ experiments and, unlike purified protein kinetics, the SOS assay is also able to distinguish between toxic and non-toxic end products of metabolism. While reduction of either the ortho or para nitro groups of CB1954 yields toxic species, this is not the case for the DNBM prodrugs; for example, the DNBM PR-104 undergoes internal cyclisation to a non-toxic derivative upon reduction of the ortho nitro group (57). Although application of the SOS assay is inherently limited to agents that either directly damage DNA or indirectly interfere with DNA replication and maintenance, there are a number of prodrugs under various stages of development that meet this criterion (for example 5-FU, which has been shown to induce the E. coli SOS response (278).

As well as the most active NTRs NfsA and NfsB, this study also identified two enzymes previously uncharacterized in their ability to reduce CB1954: AzoR and NemA. NemA is of particular interest, as at a purified level it has kinetic properties towards CB1954 of a similar magnitude to $\mathrm{NfsB}\left(k_{\mathrm{cat}} / K_{\mathrm{m}}\right.$ of $3,870 \mathrm{M}^{-1} \mathrm{~s}^{-1}$ for NemA, $7,250 \mathrm{M}^{-1} \mathrm{~s}^{-1}$, for NfsB, NADPH as co-factor), while unlike NfsB it only forms the 4$\mathrm{NHOH}$ reduction product, suggesting possible utility against cancer types with increased sensitivity towards this metabolite. Furthermore, NemA presents a completely novel enzyme structural scaffold for CB1954 reduction, having no sequence or structural homology to any previously characterised CB1954-reductase. In contrast, AzoR shares roughly 45\% amino acid sequence similarity to NQO1 across 152 residues and demonstrates close structural homology to this enzyme (279), especially within the 
enzyme active site, perhaps explaining the similar CB1954 reduction kinetics between these two enzymes $\left(k_{\text {cat }} / K_{\mathrm{m}}\right.$ of 22 and $7.8 \mathrm{M}^{-1} \mathrm{~s}^{-1}$ respectively with NADH as cosubstrate; Table 3.1, (120)). However, both MdaB and WrbA are also known to share structural (though little sequence) homology to NQO1 $(262 ; 263)$, yet in this study no CB1954-reductase activity was obtained from these two enzymes. It is possible that minor structural variations in the active sites of the enzymes could interfere with CB1954 binding. Alternatively, this lack of activity may be related to the low oneelectron reduction potential $\left(\mathrm{E}_{0}\right)$ of $\mathrm{CB} 1954\left(\mathrm{E}_{0}=-385 \mathrm{mV} ;(280)\right)$, relative to $\mathrm{NFZ}\left(\mathrm{E}_{0}\right.$ $=-257 \mathrm{mV} ;(281)$ ), the latter of which acted as a substrate for all three enzymes (see Table 3.1). Therefore, the 2 electron mid-point potentials of MdaB and WrbA (as well as the other NFZ-active/CB1954-inactive enzymes characterised in this study), but not AzoR and other CB1954-reductases, may not be low enough to reduce CB1954, yet sufficiently low to retain NFZ reductase activity. Such a mechanism has previously been described as the driving force behind the inability of most oxygen-insensitive NTRs to reduce nitroaromatic compounds beyond hydroxylamino derivatives (35). This hypothesis is also supported by the ability of all three enzymes to reduce menadione $\left(\mathrm{E}_{0}\right.$ $=-203 \mathrm{mV},(282))(259 ; 283 ; 284)$, while only AzoR has been documented to catalyse the reduction of the much more electronegative azo-dye methyl red $\left(\mathrm{E}_{0}=-395 \mathrm{mV}\right.$, (285)) (259). In contrast, both NfsA and NfsB are unable to aerobically reduce azodyes, despite high activity with CB1954 and other compounds with similar reduction potentials $(176 ; 177 ; 286)$. Together, these considerations demonstrate the importance of both active site conformation and enzyme reduction potential in substrate specificity and catalytic activity, providing strong support for the impetus behind screening of multiple, divergent enzyme families for prodrug reduction. 


\section{Chapter 4: Contribution of various DNA repair pathways to CB1954-adduct processing, and their effects on the SOS assay}

\subsection{Introduction}

The previous chapter introduced the E. coli-based SOS assay as a means of detecting and comparing rates of CB1954 reduction by over-expressed NTRs. For the SOS assay to be accurate and reliable in this context, SOS response induction must be proportional to both the total amount (as determined by rate of enzymatic reduction) and type (toxicity) of DNA adducts induced by the activated prodrug. However, the SOS response is not the only DNA repair pathway in E. coli that responds to DNA damage. For example, base-excision repair (BER), mismatch repair (MMR) and the adaptive (Ada) response are all SOS-independent DNA repair mechanisms that act on a variety of DNA lesions and base pair mismatches to maintain genome stability and integrity (287-289). Although adduct type and structure largely determine the DNA repair pathway that is activated for processing of a certain lesion, considerable overlap in substrate specificities can occur, often leading to complex interactions between DNA repair machineries for a specific adduct type (290-292). As such, levels of SOS response induction may not always correlate with the extent and magnitude of DNA damage within a cell, especially when multiple adduct types are involved. This may be particularly relevant to CB1954 due to the different DNA lesions induced by the two principal reduction products $(110 ; 121)$. In this chapter the mechanisms of repair in $E$. coli of the different CB1954-induced DNA adducts were investigated, to determine whether alternative DNA repair pathways affect SOS assay accuracy.

Additionally, as mentioned in Chapter 3, differences in DNA repair pathways between $E$. coli and mammalian cells may affect how each organism responds to the different reduction products of CB1954. As the utility of the SOS assay relies on an accurate representation of conditions within human cells, potential differences in DNA repair mechanisms should be investigated.

The primary aim of this chapter was to test the possible involvement of alternative, SOS-independent DNA repair pathways on processing of different CB1954induced DNA adducts in E. coli and how mutations in these repair mechanisms might affect SOS induction for each reduction product. This was achieved by comparison of relative SOS induction levels, in response to the different CB1954 metabolites, in SOS- 
$\mathrm{R} 1$ reporter strains deficient in the relevant repair genes (achieved through targetd gene knock out). The rationale was that the SOS response might increase in the absence of a repair pathway involved in processing of a specific lesion. Such a compensatory mechanism has previously been demonstrated, for example with oxidative DNA damage in strains deficient in oxidative stress response genes $(293 ; 294)$, or for strains deficient in either $\mathrm{O}^{6}$-methylguanine methyl transferase activity or mismatch repair in response to the anti-cancer alkylating agent chlorambucil (295).

Initially, the main pathway targeted to this end was the 'adaptive' response to DNA alkylation damage, a response primarily orchestrated by the $\mathrm{O}^{6}$-methylguanine methyl transferase enzyme Ada. The primary lesion recognized and repaired by the Ada protein and adaptive response is $\mathrm{O}^{6}$-methylguanine, a toxic DNA adduct that occurs at relatively high frequency and is induced by both endogenous and exogenous alkylating agents (296). However, Ada substrate specificity is also known to include much bulkier butyl, benzyl and pyridyloxobutyl adducts $(297 ; 298)$. Furthermore, ada-deficient mutant strains are much more sensitive to the toxic effects of aromatic amines (297), while the nitroaromatic antibiotic nitrofurazone and the nitrogen mustard-containing alkylating agent chlorambucil have also both been demonstrated to induce the Ada response $(295 ; 299)$. It is also noteworthy that the $\mathrm{O}^{6}$ position of guanine has been postulated as a primary target of the $\mathrm{N}$-acetoxy 4-NHOH derivative of CB1954 (110; 300). More importantly however, induction of the Ada response has been demonstrated to negatively affect subsequent induction of the SOS response by UV-irradiation (301). Together these observations indicate a potential involvement of the Ada response in both repair of CB1954-induced adducts and regulation of the SOS response.

The role of the MMR pathway was also investigated, as previous studies have found that the analogous system in mammalian cells is involved in the recognition and repair of a number of DNA adducts, including those induced by alkylating agents such as cisplatin, $N$-methyl- $N$ '-nitro- $N$-nitrosoguanidine and temozolomide $(271 ; 291 ; 302)$. Furthermore, HCT-116 cells are deficient in this pathway (271) while the E. coli reporter strain used in this study is proficient, suggesting a possible source of discrepancy between the two organisms in response to and tolerance of CB1954 induced adducts.

Finally, two SOS-dependent E. coli repair mechanisms were investigated, to further understand repair of CB1954 induced adducts in this organism. The first, nucleotide excision repair (NER), was targeted due to the fundamental role that the equivalent mammalian pathway plays in repair of 4-NHOH CB1954 adducts, but not 2- 
$\mathrm{NO}_{2}$ reduction product-induced adducts $(111 ; 121)$. Secondly, through assessment of mutagenic potential of the various prodrug metabolites, a possible role of damageinducible error-prone polymerases was investigated. Error-prone polymerases (such as PolB and UmuDC in E. coli) are employed in both mammalian and bacterial cells to bypass a variety of DNA lesions in a process known as translesion synthesis (TLS) (303). As this process is often mutagenic due to the low replication fidelity of these enzymes, their involvement in repair of certain DNA adducts can be monitored through mutagenesis screens. Investigating the involvement of error prone polymerases in $E$. coli in the repair of CB1954 induced adducts could provide valuable insight into the respective roles of the analogous enzymes in mammalian cells.

Select results from sections 4.2.2, 4.2.4 and 4.2.5 have been published independently in the peer-reviewed Journal of Biotechnology, as Prosser et al. (304).

\subsection{Results}

\subsubsection{Development of an improved Miller Unit calculation}

Prior to commencement of DNA repair mutant testing, a method for improving data calculation in the SOS assay was investigated, in order to improve assay reliability and accuracy.

The original calculation for determining $\beta$-galactosidase activity in a sample when ONPG is used as the reporter substrate (i.e. the Miller equation) is as follows:

Miller units $=\left(\mathrm{OD}_{420}-\left[1.75 \times \mathrm{OD}_{550}\right]\right) /\left(\mathrm{OD}_{600} \times t \times v\right)$

Where $t$ is the time allowed for ONPG metabolism (from addition of assay buffer to reaction termination with sodium carbonate) and $v$ is the volume of cells (in $\mathrm{mL}$ ) used for the final ONPG incubation step (243). In order to modify the original Miller assay protocol for use in microplate format, it was necessary to reduce all reagent volumes, including the volume of cells added. The culture volume used throughout all SOS assays in this work was $10 \mu \mathrm{L}$, a volume that was transferred from the original culture plate into the assay plate using a multi-channel pipette. I hypothesised that pipetting error at this crucial step would be a major source of variation in the final Miller unit calculation and therefore affect the quality of the overall results. 
With no available substitute to manual pipetting at this step, an alternative method of calculating Miller units was investigated in order to compensate for possible pipetting error. The $\mathrm{OD}_{550}$ measurement provides an estimation of the amount of cell debris present in the well and, in the original Miller equation, is used to compensate for the effect that this cellular matter has on absorbance at $420 \mathrm{~nm}$; (243). However, if the assumption is made that the degree of cell lysis is equivalent in each well of the assay, $\mathrm{OD}_{550}$ could instead be used to provide an indication of the total amount of cells originally transferred into the assay well, or in other words, be directly proportional to the product of $\mathrm{OD}_{600}$ (cell concentration) and cell volume transferred $(v) . \mathrm{OD}_{550}$ could then replace $\mathrm{OD}_{600} \times v$ in the Miller equation, providing ultimately more accuracy to SOS measurements.

The first step in testing this hypothesis was to demonstrate the existence of a direct and linear relationship between $\mathrm{OD}_{550}$ and $\mathrm{OD}_{600} \times v$. SOS assays were carried out as previously described except that the volume of cells transferred to the final assay plate was varied from 2.5 to $20 \mu \mathrm{L}$. Results, shown in Figure 4.1, demonstrated that there is a strong linear correlation between $\mathrm{OD}_{550}$ and $\mathrm{OD}_{600} \times v$, over the range of volumes tested. Next, the effect of substituting $\mathrm{OD}_{550}$ for $\mathrm{OD}_{600} \times v$ in the Miller equation on overall SOS response fold induction measurements was tested by comparing the variation in SOS response fold inductions generated from multiple replicates of identically treated (100 $\mu \mathrm{M}$ CB1954), isogenic NfsB over-expressing SOS-R1 reporter strains, as calculated by either the original or the adjusted Miller equations. As illustrated in Figure 4.2, altering the Miller equation had no significant effect on overall SOS response fold induction calculations (mean SOS response fold inductions of 5.62 and 5.29 for calculations based on the original and adjusted Miller equation, respectively; $\mathrm{p}>0.05$; t-test). Furthermore, the amount of variation (as indicated by standard deviation) observed across the data when using the altered Miller equation was almost 4-fold lower than when using the original equation (standard deviation:mean ratio of 0.08 compared to 0.28 for adjusted and original Miller equation calculations, respectively). 


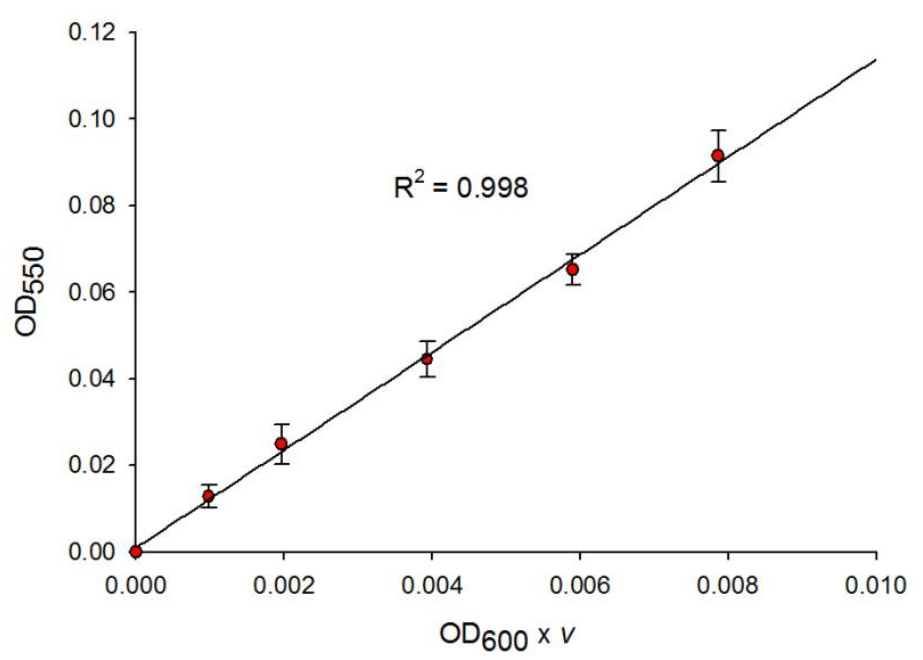

Figure 4.1. Correlation between $\mathrm{OD}_{550}$ and $\mathrm{OD}_{600} \times v$ of samples used in SOS assays.

Different volumes (2.5-20 $\mu \mathrm{L}$ ) of NfsA over-expressing SOS-R1 cultures challenged with CB1954 were transferred to fresh 96 well plates for SOS activity assessment. $\mathrm{OD}_{600}$ readings of the assay cultures multiplied by the volume of cells transferred ( $\mathrm{x}$ axis) were plotted against the respective $\mathrm{OD}_{550}$ readings of the SOS plate (y-axis). Results are the average \pm 1 SEM of duplicate assays, each performed with 12 replicates for each cell volume used.
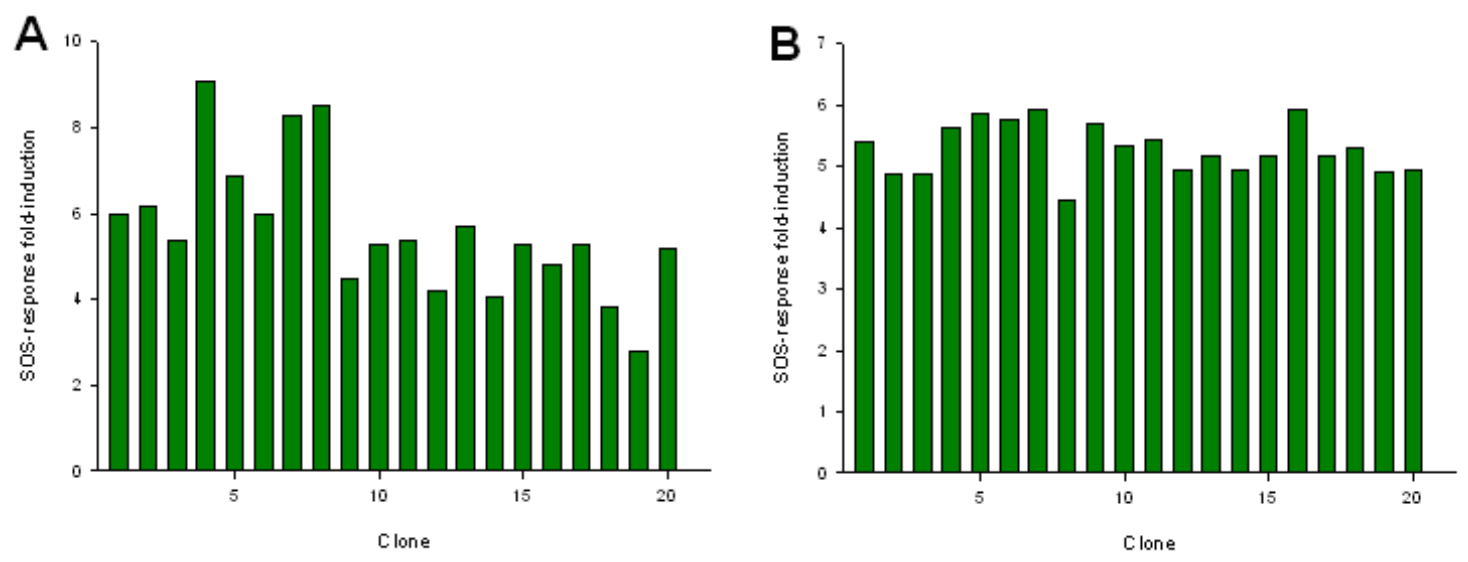

Figures 4.2A \& B. Variation in SOS response fold inductions from replicate cultures using different Miller equations.

20 individual replicates of NfsB over-expressing SOS-R1 were challenged for $3 \mathrm{~h}$ with $100 \mu \mathrm{M}$ CB1954 and then assessed for SOS activity as previously described. Miller units were calculated by either (A) the original Miller equation, or (B) the adjusted equation developed in this study.

In conclusion, due to the higher degree of accuracy and reliability associated with the adjusted Miller equation compared to the original, this method was used throughout this work for calculation of $\beta$-galactosidase activity in SOS response analysis. The adjusted equation is as follows:

Miller units $=\left(\mathrm{OD}_{420}-\left[1.75 \times \mathrm{OD}_{550}\right]\right) /\left(t \times \mathrm{OD}_{550}\right)$ 


\subsubsection{Identification of novel NTRs for reduction of either the 2- or 4- $\mathrm{NO}_{2}$ of CB1954}

In this chapter, generation of the different CB1954 reduction products was achieved through over-expression of the relevant NTRs in each test strain. Five NTRs were chosen, three of which had been characterised in Chapter 3: E. coli NfsA (2-NHOH producer), NfsB (2- and 4-NHOH, equimolar) and NemA (4-NHOH). Seeking to add an additional 2- $\mathrm{NO}_{2}$ and a 4- $\mathrm{NO}_{2}$ reducing enzyme to the panel, an Nfs A homologue from $K$. pneumoniae ( $83 \%$ amino acid identity) and a $B$. subtilis homologue of $B$. licheniformis YfkO (67\% identity) were selected for further investigation. These enzymes were chosen based on strain availability in the lab and with the assumption that metabolite production by each enzyme would be similar to their previously characterised homologues, i.e. 2-NHOH for K. pneumoniae NfsA and 4-NHOH for $B$. subtilis YfkO (as determined by Emptage et al. (2008); (199)). Kinetic characterisation of His6-tagged purified enzymes established that they each efficiently reduced CB1954 (see Table 4.1) and HPLC analysis of reaction products confirmed that NfsA (K.p) reduced solely the $2-\mathrm{NO}_{2}$ while $\mathrm{YfkO}$ (B.s) reduced solely the $4-\mathrm{NO}_{2}$ of CB1954 (Figure 4.3). Due to the employment of an increasing number of NTRs from different bacterial species, gene names are from here on (and in all remaining chapters) followed by the initials of the genus and species of origin, in parentheses (i.e. NfsA (E.c), NfsA (K.p) etc).
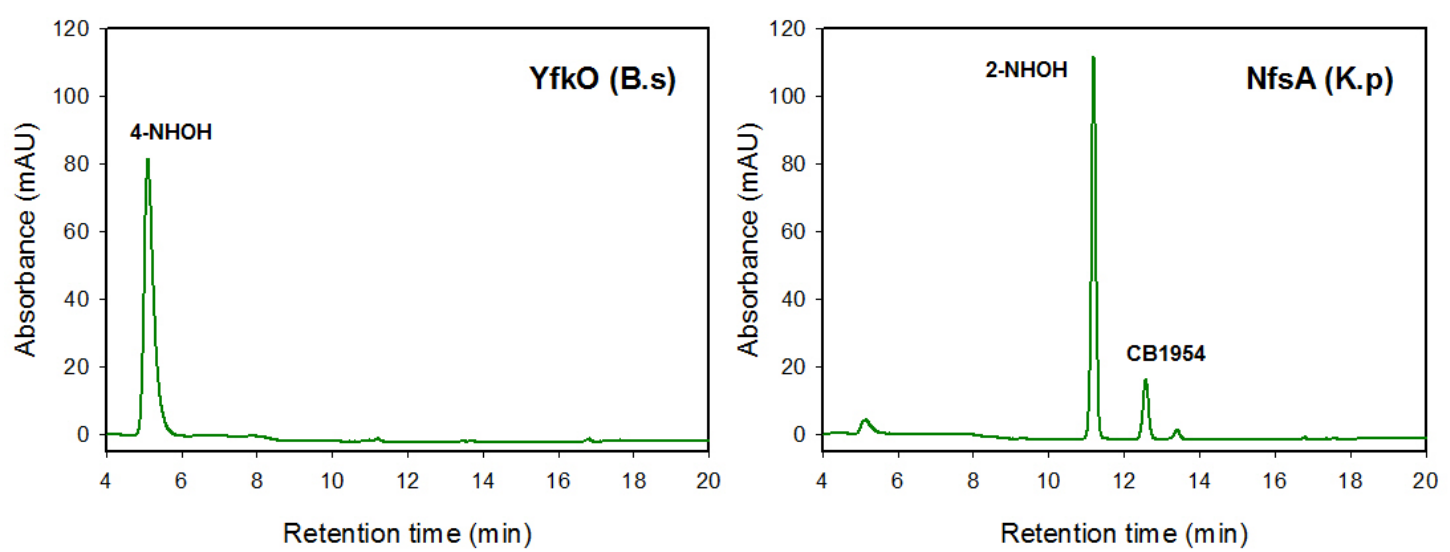

Figure 4.3. HPLC analysis of products of YfkO (B.s) and NfsA (K.p)-catalysed reduction of CB1954.

Reactions and HPLC conditions were set up and carried out as previously described (see Figure 3.8, section 2.16). Absorbance was measured at $262 \mathrm{~nm}$. 
Table 4.1. Kinetic parameters and end-products of CB1954 metabolism for each purified, His6-tagged candidate NTR.

\begin{tabular}{|c|c|c|c|c|}
\hline NTR & Metabolite & $k_{\text {cat }}\left(\mathrm{s}^{-1}\right)$ & $\overline{K_{m}}(\mu \mathrm{M})$ & $k_{\text {cat }} / K_{\mathrm{m}}\left(\mathrm{M}^{-1} \mathrm{~s}^{-1}\right)$ \\
\hline $\mathrm{NfsA}(\mathrm{E} . \mathrm{c})^{\mathrm{a}}$ & $2-\mathrm{NHOH}$ & $16 \pm 0.48$ & $220 \pm 18$ & $73,000 \pm 6,400$ \\
\hline $\mathrm{NfsA}(\mathrm{K} \cdot \mathrm{p})^{\mathrm{b}}$ & 2-NHOH & $37 \pm 1.3$ & $1,700 \pm 150$ & $22,000 \pm 2,200$ \\
\hline $\mathrm{NfsB}(\text { E.c })^{\mathrm{a}}$ & 2-/4-NHOH & $26 \pm 2.6$ & $3,600 \pm 660$ & $7,300 \pm 1,500$ \\
\hline $\operatorname{NemA}(\text { E.c })^{\mathrm{a}}$ & 4-NHOH & $0.22 \pm 0.003$ & $56 \pm 2.2$ & $3,900 \pm 160$ \\
\hline YfkO (B.s) ${ }^{b}$ & 4-NHOH & $60 \pm 2.9$ & $2,400 \pm 250$ & $25,000 \pm 2,800$ \\
\hline
\end{tabular}

${ }^{\mathrm{a}}$ Data taken from Table 3.1.

${ }^{\mathrm{b}} \mathrm{NADPH}$ was used as co-substrate, at an initial concentration of $0.25 \mathrm{mM}$ for all reactions.

\subsubsection{Generation and phenotypic characterisation of $\Delta a d a \Delta o g t, \Delta m u t S$ and $\Delta u v r B$ gene knockout strains of $E$. coli SOS-R1}

The genes encoding principal components of each DNA repair pathway under investigation were deleted, in-frame, from the SOS-R1 genome, resulting in a panel of reporter strains each with a unique DNA repair deficiency. These strains consisted of 1) a $\Delta a d a$ and $\Delta o g t$ null mutant, deficient in both inducible and constitutive $\mathrm{O}^{6}$ methylguanine methyl transferase activities, as well as deficient in the adaptive response; 2) a $\Delta m u t S$ null mutant, deficient in mismatch repair; and 3) a $\Delta u v r B$ null mutant, deficient in nucleotide excision repair. Genetic verification of loss of the relevant genes was achieved through size analysis of PCR-amplified regions of genomic DNA flanking the affected gene (results not shown). To biochemically verify that the $\Delta a d a \Delta o g t$ dual gene knockout had the expected phenotypic effect, all three repairdeficient and wildtype SOS reporter strains were assessed for SOS response induction following incubation with the known Ada response inducing agent $\mathrm{N}$-methyl $\mathrm{N}$ nitrosourea (MNU) (305). As expected, SOS activity in the $\Delta a d a \Delta o g t$ strain was almost 2-fold higher than in any other strain following $3 \mathrm{~h}$ challenge with this compound (see Figure 4.4). Appropriate drugs were not available to biochemically verify the other two mutant strains. 


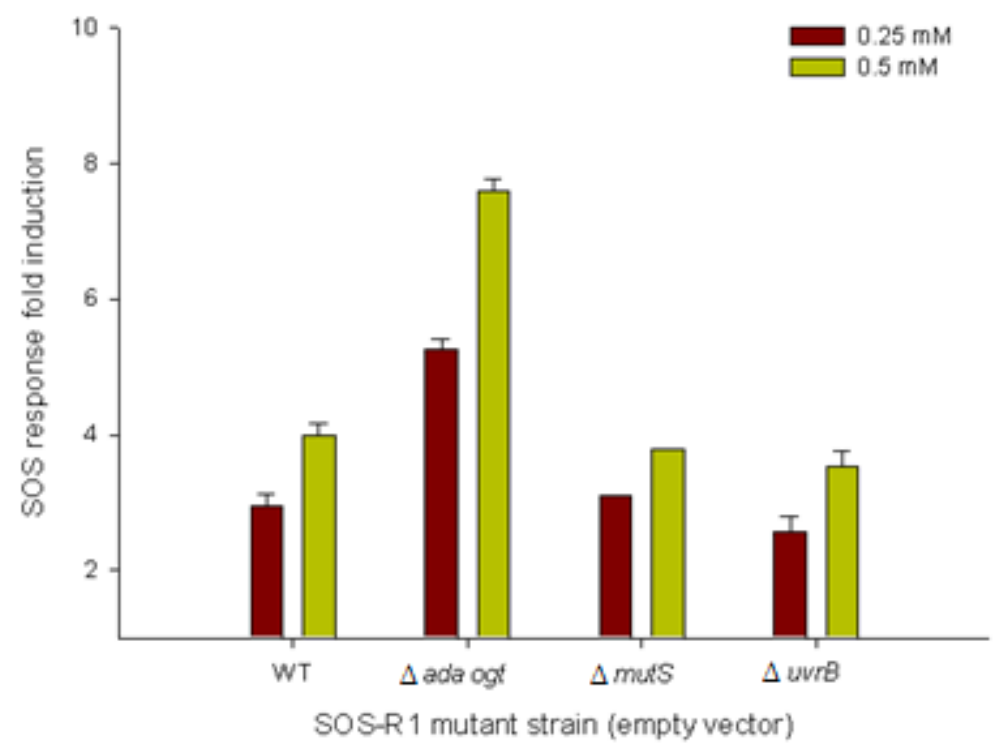

Figure 4.4. MNU-induced SOS responses from DNA repair-deficient and wildtype SOS-R1 strains. Wildtype SOS-R1 and strains deficient in certain DNA repair pathways carrying empty pUCX vector (xaxis) were grown and challenged with the indicated concentrations of MNU for $3 \mathrm{~h}$ prior to SOS activity assessment. Data is the average \pm 1 SEM of 2 independent assays.

\subsubsection{CB1954 SOS induction in DNA repair deficient and proficient strains of $E$. coli SOS-R1}

The genes encoding NfsA (K.p) and Yfko (B.s) were cloned into the MCS of pUCX, and individually transformed (together with pUCX constructs expressing the remaining three NTRs) into wildtype and each SOS-R1 repair-deficient reporter strain.

Measurement of SOS response induction upon challenge with $50 \mu \mathrm{M}$ CB1954 was then carried out as previously described. Results are displayed in Figure 4.5. No significant ( $p>0.05$; t-test) increase or decrease in SOS response was observed between wildtype and $\Delta a d a \Delta o g t$ or $\Delta m u t S$ strains, for any of the over-expressed NTRs. However, SOS induction was significantly enhanced in the $\Delta u v r B$ strain over wildtype when either NemA (E.c) or YfkO (B.s) (p=0.0182 and 0.0275 respectively; t-test), but not the remaining three enzymes, was over-expressed. To ensure that this difference was not due to alterations in enzyme expression levels between wildtype and $\Delta u v r B$ SOS-R1, SDS-PAGE was carried out on total cellular fractions remaining after a completed SOS assay. No substantial variation in over-expressed NTR levels was evident between the two strains (Figure 4.6). 


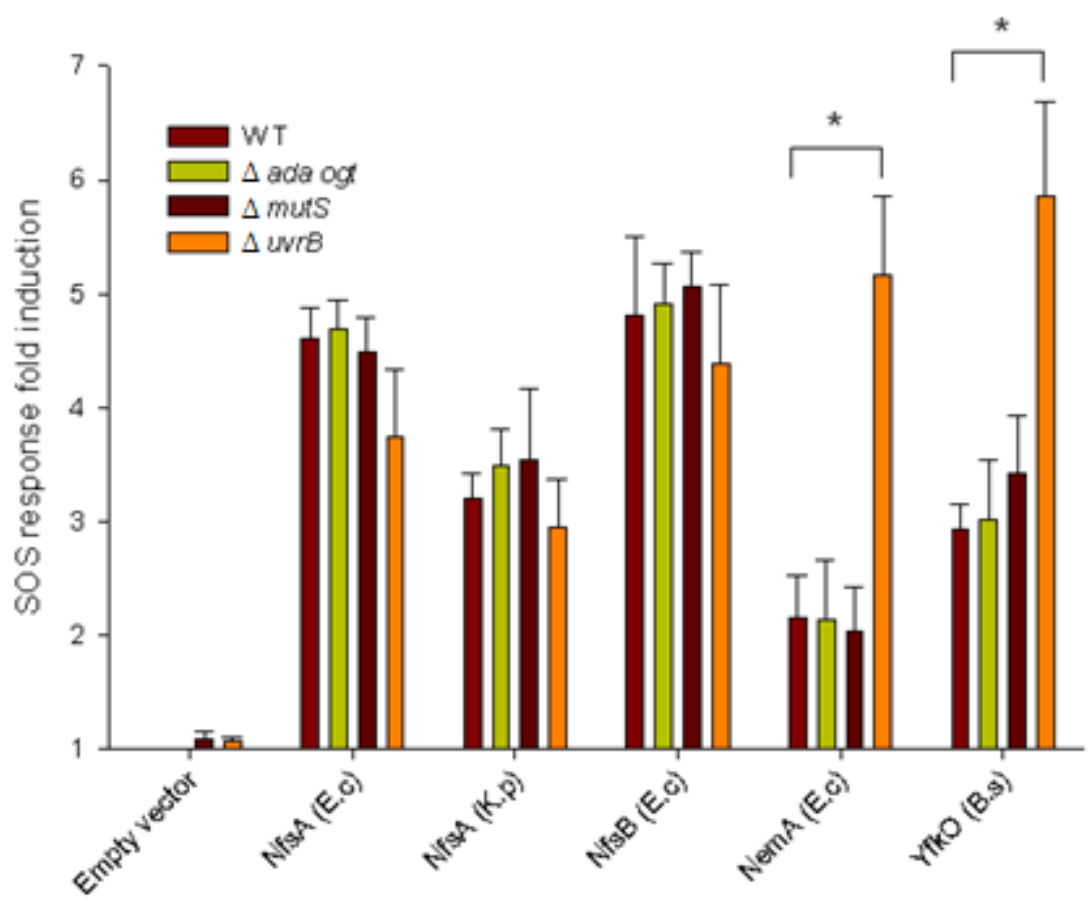

Overexpressed NTR

Figure 4.5. CB1954-induced SOS responses in NTR over-expressing DNA repair proficient and deficient strains of SOS-R1.

The relevant NTR over-expressing strains of wildtype (WT) and DNA repair-deficient SOS-R1 (x-axis) were grown and challenged with $50 \mu \mathrm{M} \mathrm{CB} 1954$ for $3 \mathrm{~h}$ prior to assessment of SOS activity. *p $<0.05$; ttest. Data is the mean \pm 1 SEM of 3 independent assays.

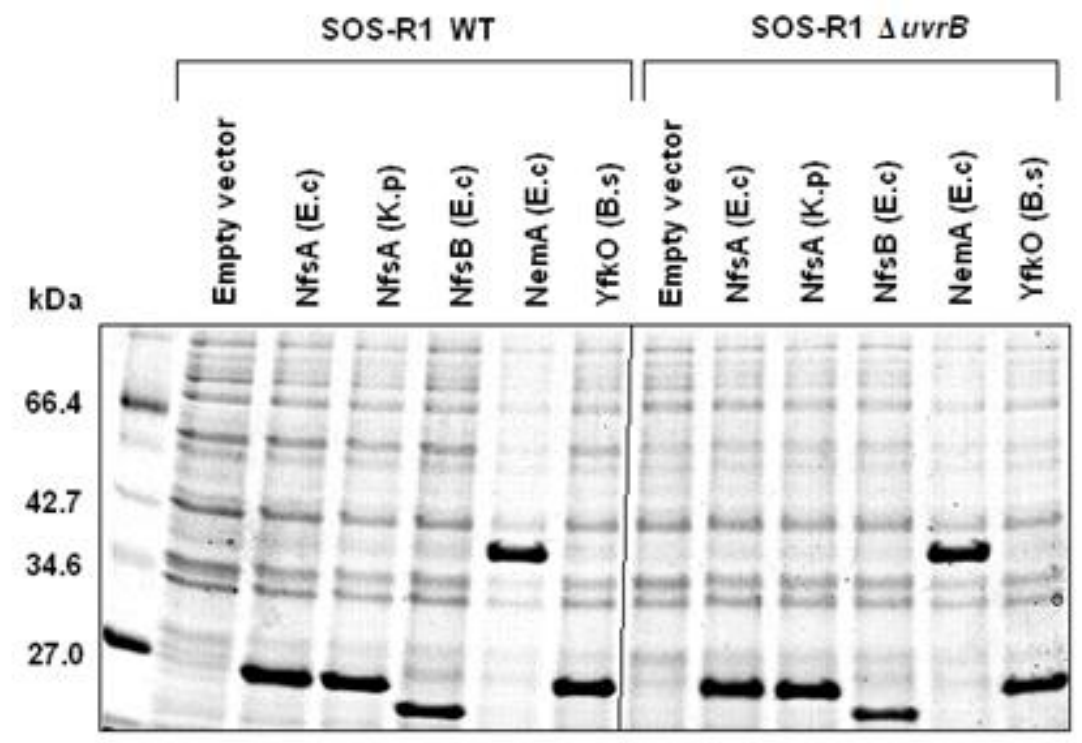

Figure 4.6. Expression of candidate NTRs from wildtype and $\triangle u v r B$ strains of SOS-R1.

SDS-PAGE gel scan indicating relative levels of expression of each candidate NTR from pUCX in wildtype (left) and $\triangle u v r B$ (right) strains of SOS-R1. Samples for SDS-PAGE analysis were whole cell lysates taken directly from a completed SOS assay (50 $\mu$ M IPTG, unchallenged cultures) and normalised for cell density $\left(\mathrm{OD}_{600}\right)$ prior to electrophoresis. 
These results were further tested and validated by comparing SOS response induction upon application of pure synthetic reduction metabolites of CB1954 (the 2$\mathrm{NH}_{2}, 4-\mathrm{NHOH}, 4-\mathrm{NH}_{2}$ and parental compound control (the 2-NHOH being too unstable as a purified compound to test in this manner (121)) to both SOS-R1 wildtype and $\Delta u v r B$, in the absence of NTR over-expression (empty vector only; Figure 4.7). Although the 2- $\mathrm{NH}_{2}$ metabolite induced the highest overall SOS response in both strains, there was a significant increase in activity $(\mathrm{p}<0.05$; $\mathrm{t}$-test $)$ with the $4-\mathrm{NHOH}$ metabolite in the $\Delta u v r B$ strain over wildtype that was not observed with any of the other compounds. In contrast, SOS activity was slightly, but significantly $(\mathrm{p}<0.05 ; \mathrm{t}$-test) decreased in the $\Delta u v r B$ strain relative to wildtype upon challenge with $200 \mu \mathrm{M} 2-\mathrm{NH}_{2}$, although this difference was not observed at $100 \mu \mathrm{M} 2-\mathrm{NH}_{2}$.

Therefore, in a NER-deficient genetic background, unique reduction of the 4$\mathrm{NO}_{2}$ of CB1954, but not the $2-\mathrm{NO}_{2}$ or mixtures of both, appears to elevate SOS induction levels over wildtype.

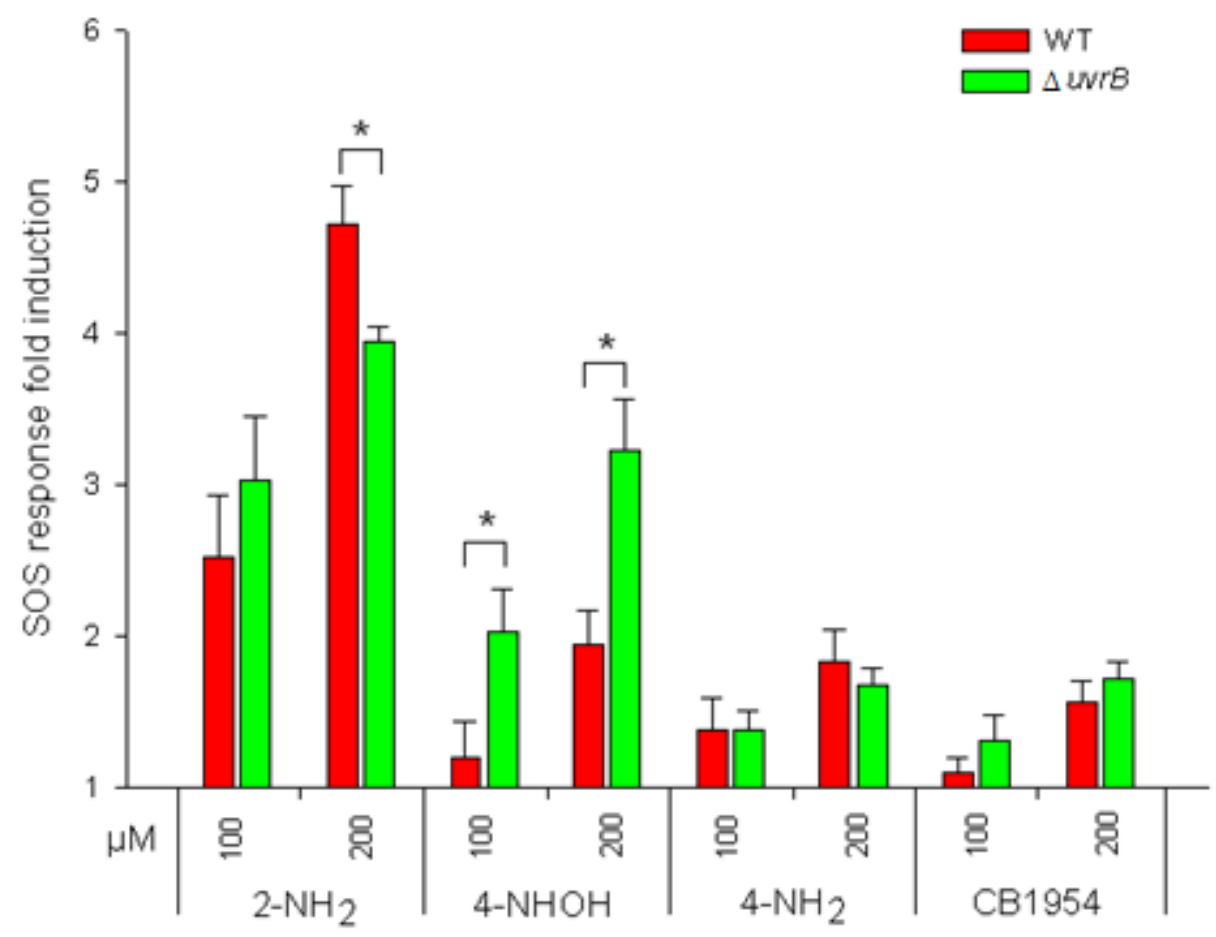

CB1954 metabolite

Figure 4.7. CB1954 reduction metabolite-induced SOS responses from wildtype and NER-deficient strains of SOS-R1.

Wildtype (WT) and $\Delta u v r B$ strains of SOS-R1 (carrying empty vector) were grown and challenged for $3 \mathrm{~h}$ with the indicated pure CB1954 reduction metabolites at the indicated concentrations prior to SOS activity assessment. 2/4- $\mathrm{NH}_{2}$ : 2/4-amino derivative. 4-NHOH: 4-hydroxylamino derivative. CB1954: parental prodrug. ${ }^{*} \mathrm{p}<0.05$; t-test. Data are the mean \pm 1 SEM of 2-5 independent assays. 


\subsubsection{Role of NER in processing adducts induced by alternative nitroaromatic prodrugs}

The ability to discriminate between NTR-catalysed reduction of either the 2- or 4- $\mathrm{NO}_{2}$ of CB1954, through the use of multiple SOS reporter strains that differ in DNA repair proficiencies, suggested that the same system might be useful to determine genotoxic mechanisms of alternative compounds. This could be particularly useful, for example, in the analysis of environmental pollutants or for isolation of novel DNA-damaging antibiotic or anticancer agents from large-scale chemical libraries. Alternatively, it might be possible to increase the sensitivity of the SOS assay for specific metabolites by using a mutant reporter strain deficient in an adduct-specific DNA repair pathway.

These hypotheses were tested by comparing the SOS induction of SOS-R1 wildtype and $\Delta u v r B$ strains, over-expressing the five candidate NTRs, following $3 \mathrm{~h}$ challenge with the 5-nitrofuran NFZ, the 5-nitroimidazole metronidazole (MTZ) or the DNBM prodrug PR-104A (the de-phosphorylated alcohol derivative of PR-104). Both of the former two compounds are established substrates for multiple bacterial NTRs $(171 ; 176 ; 177 ; 306)$, while PR-104A has yet to be investigated in the context of bacterial NTR-catalysed reduction. Furthermore, all substrates require nitroreduction prior to onset of genotoxicity $(57 ; 307 ; 308)$, and the reduced forms of both NFZ and MTZ have previously been shown to induce the SOS response $(235 ; 309)$. Previous work has also established that nucleotide excision repair is the primary repair pathway involved in processing of NFZ-induced lesions (310), while the repair mechanism of MTZ-induced adducts is less well understood (311; 312). Repair of PR-104A-induced adducts has been shown to require multiple repair machineries, including NER and homologous recombination, in human cells (48). Accordingly, results from this study demonstrated that SOS induction was enhanced, on average, in NTR over-expressing $\Delta u v r B$ strains of SOS-R1, over wildtype, upon challenge with nitrofurazone or PR104A, while little to no difference was observed with metronidazole (Figure 4.8). 


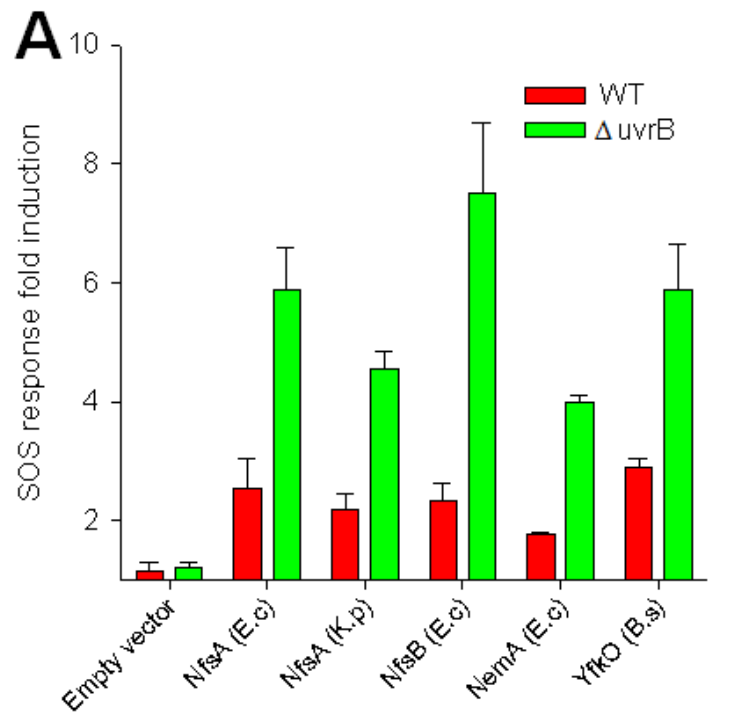

Overexpressed NTR

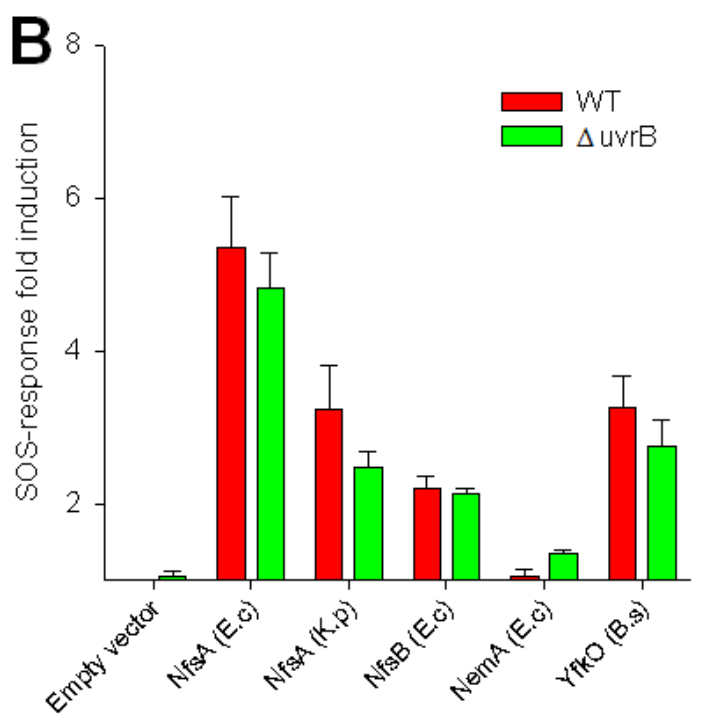

Overexpressed NTR

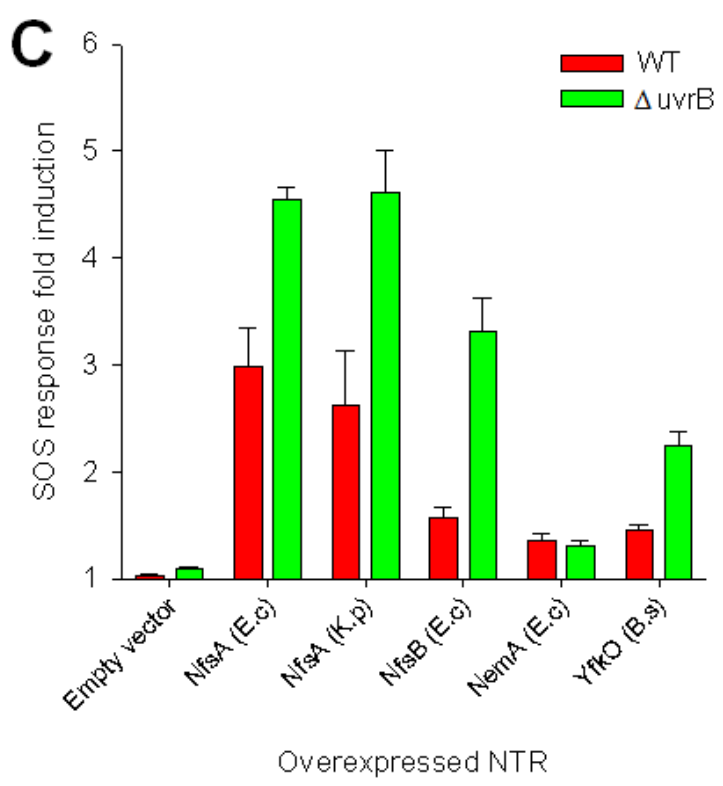

Figures 4.8A-C. SOS responses induced by alternative nitroaromatic prodrugs in NER-proficient and -deficient NTR over-expressing strains of SOS-R1.

NTR over-expressing strains of wildtype (WT) and $\triangle u v r B$ SOS-R1 were grown and challenged with either (A) $5 \mu \mathrm{M} \mathrm{NFZ,} \mathrm{(B)} 5 \mu \mathrm{M}$ MTZ or (C) $200 \mu \mathrm{M}$ PR-104A prior to analysis of SOS activity induction. Data are the average $\pm 1 \mathrm{SEM}$ of 3 independent assays. 


\subsubsection{Mutagenicity of $\mathrm{CB} 1954$ reduction products}

The role of error-prone polymerases and TLS in repair of CB1954-induced adducts was assessed through a mutagenicity screen involving selection for rifampicin-resistant $\left(\right.$ Rif $^{\mathrm{R}}$ ) phenotypes following CB1954 challenge of NTR over-expressing SOS-R1 reporter strains. Rifampicin (an inhibitor of bacterial RNA polymerase) resistance in many bacteria is obtained through specific mutations within the gene encoding RNA polymerase $($ rрoв) $(313 ; 314)$. A large number of base pair substitutions $(>60)$ across more than 30 nucleotide positions within the $r p o B$ gene can induce a Rif ${ }^{\mathrm{R}}$ phenotype, allowing detection of a wide variety of mutagens with this assay (315). Furthermore, as most of the mutational sites lie within a small $(<1,000$ base pair) region of the gene, characterisation of the mutational spectra (preferred substitution type and sequence specificity) of a test compound can easily be determined through PCR amplification and DNA sequencing of the relevant gene region.

Using this assay, mutagenicity of both the 2- $\mathrm{NO}_{2}$ and $4-\mathrm{NO}_{2}$ reduction products of CB1954 (via NfsA (E.c) and YfkO (B.s) over-expression, respectively) was assessed, in both NER-proficient (wildtype) and -deficient $(\Delta u v r B)$ genetic backgrounds. Briefly, exponential phase cultures of the relevant strains were chilled, resuspended in PBS, and then challenged with $\mathrm{CB} 1954$ for $3 \mathrm{~h}$ at $30^{\circ} \mathrm{C}$, followed by dilution and recovery in fresh (LB) media overnight. The following day aliquots were spread onto LB agar plates containing either just ampicillin (for assessment of total viable cells) or both ampicillin and rifampicin $\left(100 \mu \mathrm{g} \mathrm{mL}^{-1}\right)$ and incubated at $37^{\circ} \mathrm{C}$ for an additional $24 \mathrm{~h}$. Colonies were then counted and the frequency of iif $^{\mathrm{R}}$ isolates per $10^{8}$ cells calculated (Figure 4.9). Both the 2- and 4- $\mathrm{NO}_{2}$ reduction products of $\mathrm{CB} 1954$ demonstrated mutagenic potential in this assay, illustrated by substantially higher (up to 4,000-fold) mutation frequencies compared with unchallenged isogenic cultures. Also, these compounds were significantly more mutagenic than the parental compound (comparison to empty vector; $\mathrm{p}<0.01$; t-test). Furthermore, mutagenicity of both reduction products, as well as the parental compound, was significantly higher in the $\Delta u v r B$ strain compared to wildtype ( $<<0.05$ for all three compounds; t-test). This difference was highest for the 2- $\mathrm{NO}_{2}$ reduction product (22.5-fold difference compared to 3.3- and 5.5-fold for the 4- $\mathrm{NO}_{2}$ reduction product and parental compound respectively). Due to catalytic differences between the two enzymes (see table 4.1) used for CB1954 reduction, the relative mutagenic potential of the two principal reduction products could not be confidently assessed. 


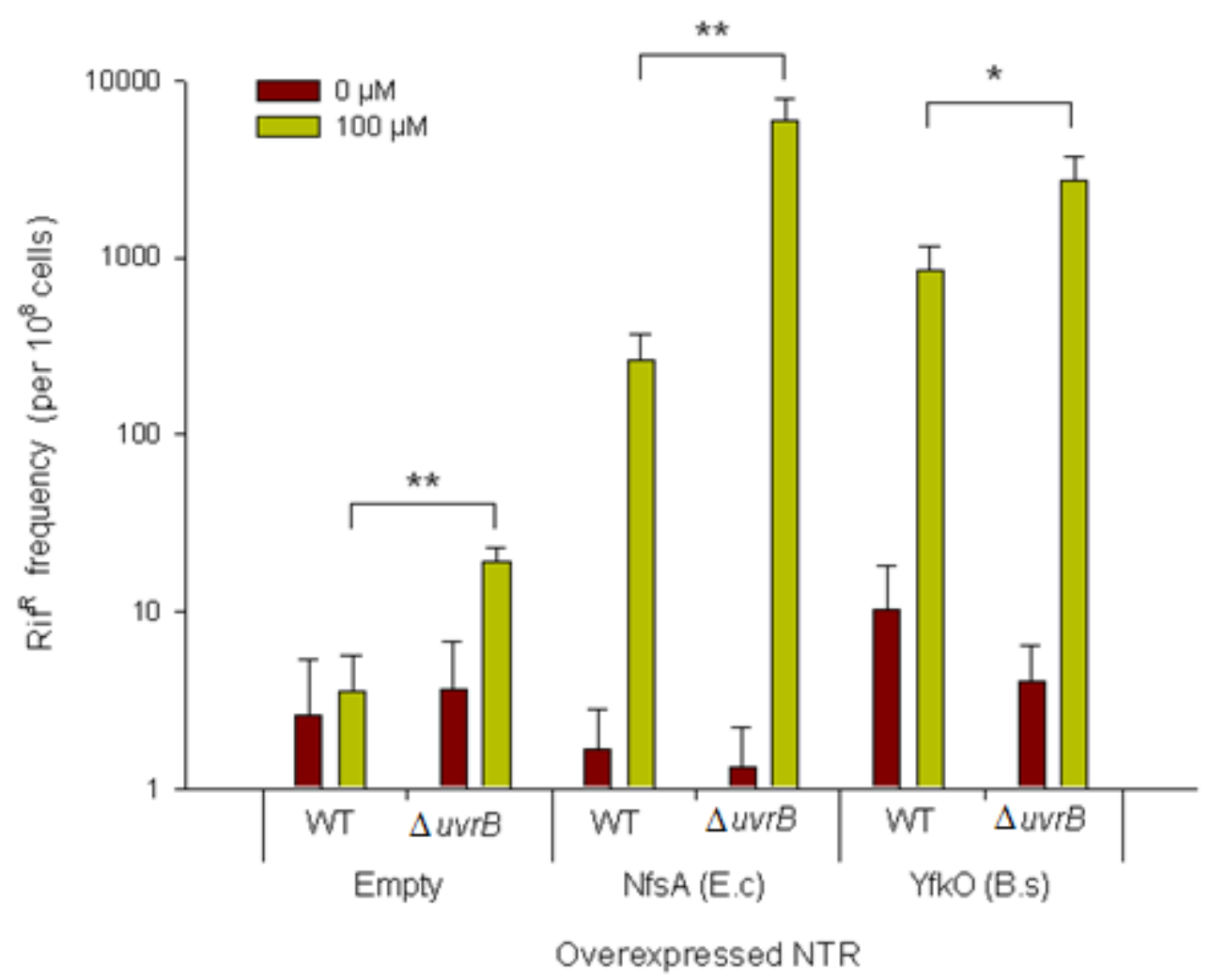

Figure 4.9. CB1954-induced rifampicin resistance in wildtype and NER-deficient NTR overexpressing strains of SOS-R1.

Strains of wildtype (WT) and $\Delta u v r B$ strains of SOS-R1, over-expressing NfsA (E.c) (reduces 2- $\mathrm{NO}_{2}$ of CB1954), YfkO (B.s) (reduces 4- $\mathrm{NO}_{2}$ of CB1954) or no enzyme (Empty) were grown and challenged with CB1954 (0 or $100 \mu \mathrm{M}$ ) for $3 \mathrm{~h}$, prior to dilution in fresh media and overnight recovery. Samples were then plated on solid media containing either just ampicillin (for calculation of total viable cells) or ampicillin and rifampicin (for detection of $\mathrm{Rif}^{\mathrm{R}}$ mutants). Data is expressed as number of $\mathrm{Rif}^{\mathrm{R}}$ colonies per $10^{8}$ viable cells. ${ }^{*} \mathrm{p}<0.05 .{ }^{*} \mathrm{p}<0.01$; t-test. Data is the average \pm 1 SEM of 3 independent assays.

\subsubsection{Mutational spectra (nucleotide substitutions, sequence specificity) of CB1954 reduction products}

The region comprising nucleotides 1351 to 2224 of the $r p o B$ genes (within which the majority of mutations that confer rifampicin resistance lie; (315)) from a selection of $\mathrm{Rif}^{\mathrm{R}}$ colonies obtained through CB1954-induced mutagenesis were PCR amplified, sequenced and assessed for mutation specificity (results displayed in Table 4.2). In the wildtype strain, the predominant mutations induced by both 2 - and $4-\mathrm{NO}_{2}$ reduction products were $\mathrm{GC} \rightarrow$ AT transitions, with the $2-\mathrm{NO}_{2}$ reduction product also inducing a single instance of a $\mathrm{GC} \rightarrow \mathrm{TA}$ transversion. In the $\Delta u v r B$ strain, $2-\mathrm{NO}_{2}$ reduction products only induced GC $\rightarrow$ AT transitions, while both GC $\rightarrow$ TA transversions (3 total) and $\mathrm{GC} \rightarrow \mathrm{AT}$ transitions (2 total) were induced by $4-\mathrm{NO}_{2}$ reduction products. 
Of the specific sites where mutagenesis occurred, 5 of the 10 sequenced mutations induced by the $2-\mathrm{NO}_{2}$ reduction products and 4 of 10 induced by the $4-\mathrm{NO}_{2}$ reduction products were all at the same site: guanine 1546 (Table 4.3). No other mutational hotspot was observed for either of the two CB1954 reduction products, with mutations distributed equally (1-2 each) over a range of nucleotide sites.

Table 4.2. Frequency and specificity of base pair substitutions within the $E$. coli $r p o B$ gene induced by each $\mathrm{CB} 1954$ reduction product

\begin{tabular}{llll}
\hline SOS-R1 strain & Base pair change & $\begin{array}{c}2-\mathrm{NO}_{2} \text { reduction } \\
\text { products }^{\mathrm{a}}\end{array}$ & $\begin{array}{l}4-\mathrm{NO}_{2} \text { reduction } \\
\text { products }^{\mathrm{b}}\end{array}$ \\
\hline Wildtype & $\mathrm{GC} \rightarrow \mathrm{AT}$ & 4 & 4 \\
& $\mathrm{GC} \rightarrow \mathrm{TA}$ & 1 & 0 \\
\multirow{2}{*}{$\Delta u v r B$} & $\mathrm{GC} \rightarrow \mathrm{AT}$ & 5 & 2 \\
& $\mathrm{GC} \rightarrow \mathrm{TA}$ & 0 & 3 \\
\hline
\end{tabular}

${ }^{\mathrm{a}}$ Generated by challenging NfsA (E.c) over-expressing cells with CB1954

${ }^{\mathrm{b}}$ Generated by challenging YfkO (B.s) over-expressing cells with CB1954

Table 4.3. Frequency and sequence specificity of mutations within the $E$. coli rpoB gene induced by the two principal reduction products of CB1954.

\begin{tabular}{llll}
\hline Nucleotide site $^{\mathrm{a}}$ & $\begin{array}{l}\text { Surrounding sequence } \\
\left(5^{\prime} \rightarrow 3^{\prime}\right)^{\mathrm{b}}\end{array}$ & $\begin{array}{l}2-\mathrm{NO}_{2} \text { reduction } \\
\text { products }\end{array}$ & $\begin{array}{l}4-\mathrm{NO}_{2} \text { reduction } \\
\text { products }\end{array}$ \\
\hline 1535 & GCTGTCTCAGT & 1 & 1 \\
1546 & TTATGGACCAG & 5 & 4 \\
1576 & TTACGCACAAA & 1 & 1 \\
1592 & TATCTCCGCAC & 1 & 0 \\
1595 & CTCCGCACTCG & 0 & 1 \\
1691 & AACCCCTGAAG & 0 & 1 \\
1721 & CAACTCTCTGT & 2 & 1 \\
\hline
\end{tabular}

\footnotetext{
${ }^{a}$ Nucleotide numbering is relative to the adenine of the ATG start codon

${ }^{\mathrm{b}}$ Nucleotide in bold indicates position of mutagenesis
} 


\subsection{Discussion}

The primary aim of this chapter was to investigate the roles of various $E$. coli DNA repair pathways, both SOS-dependent and -independent, on processing of the different DNA adducts induced by the principal reduction metabolites of CB1954. Results showed that SOS reporter strains deficient in either $\mathrm{O}^{6}$-methylguanine methyl transferase activity/adaptive response ( $\Delta a d a \Delta o g t$ null mutants) or mismatch repair ( $\Delta$ mutS null mutant) elicited levels of SOS response comparable to the wildtype strain, following challenge with any of the CB1954 reduction products (as generated by overexpression of nitro group-specific CB1954-reductases). In contrast, NER ( $\Delta u v r B)$ nullmutants displayed enhanced SOS activity (relative to wildtype) in the presence of the 4$\mathrm{NHOH}$ metabolite, but not the 2- $\mathrm{NO}_{2}$ reduction products (as shown by both NTRcatalysed generation of reduction products and with synthetic pure metabolites).

These observations have several implications. Firstly, it is unlikely that the SOSindependent adaptive response or mismatch repair pathways are involved in repair of any CB1954-induced adduct; or, if so, play only a very minor role, such that other repair pathways are able to compensate for their absence without necessitating increased SOS induction. Therefore the presence of these pathways in the E. coli reporter strains used throughout this work should not interfere with SOS assay results, in particular regarding the relative levels of SOS response induced by the different CB1954 reduction products. This is particularly relevant to the adaptive response, the activation of which has previously been shown to inhibit subsequent SOS induction in response to UV irradiation (301). However, other SOS-independent repair pathways, in particular BER, which were not investigated in this chapter may still be involved in CB1954 adduct repair and therefore warrant further study. For example, previous studies have shown that expression of the E. coli BER-linked DNA glycosylase Fpg in human cells reduces sensitivity to the toxic effects of both aziridine and the aziridinyl alkylating agent thiotepa $(316 ; 317)$. This suggests a role for this enzyme in repair of aziridinyl-induced adducts, which could include certain lesions produced by CB1954

Secondly, the enhanced SOS response relative to wildtype observed in SOS-R1 $\Delta u v r B$ strains over-expressing NemA (E.c) or YfkO (B.s) following challenge with CB1954 suggests a substantial role of NER in processing of 4-, but not 2- $\mathrm{NO}_{2}$ reduction metabolite-induced adducts in E. coli. This is analogous to results obtained in mammalian cells (121) and therefore implies that the role of NER in repairing CB1954induced DNA damage is similar in the two species. This lends support to the use of $E$. 
coli as a model organism for studying CB1954 reduction. Furthermore, the improved sensitivity of the SOS-R1 $\Delta u v r B$ strain towards unique production of the 4-NHOH metabolite could be exploited as a screening tool for preferentially detecting overexpressed NTRs that only reduce the $4-\mathrm{NO}_{2}$ of CB1954. This may be beneficial in gene therapy targeting of tumours that are known to be deficient in NER and therefore more sensitive to the toxic effects of the 4-NHOH metabolite. Similarly, this strain could be used to improve overall sensitivity of the SOS assay towards alternative prodrugs whose principal adducts are processed by the NER machinery. This was demonstrated with the nitroaromatic prodrugs NFZ and PR-104A, which induced substantially higher SOS responses in NTR over-expressing $\Delta u v r B$ strains than in wildtype at equimolar doses. In contrast, metronidazole did not demonstrate a similar pattern of activity, no doubt due to differences in repair of the induced adducts compared with the former compounds. Together these data demonstrate that the SOS assay is able to detect different genotoxic mechanisms of test compounds, potentially aiding in identification of novel therapeutic drugs with specific modes of action (e.g. DNA interstrand crosslinking agents versus specific inhibitors of DNA replication machinery).

This study also reports the first documented evidence of reduction of the DNBM prodrug PR-104A by bacterial NTRs, as demonstrated by enhanced SOS responses in SOS-R1 strains over-expressing NTRs relative to an empty vector control, following challenge with this prodrug. Previous work has established the ability of NfsB (E.c) to reduce the dibromo-derivative SN 27686 (56), in agreement with the results presented here. These preliminary findings therefore provide rationale for further testing of this clinically attractive nitroaromatic prodrug with bacterial NTRs in order to investigate its potential utility in GDEPT.

Both the 2- and 4- $\mathrm{NO}_{2}$ reduction products of $\mathrm{CB} 1954$ were able to induce mutations in the E. coli genome, as illustrated by the significantly higher frequencies of rifampicin resistance in CB1954 challenged NTR over-expressing strains relative to unchallenged isogenic cultures. Mutagenic activity of alkylating agents and other DNA damaging species arises, in both bacteria and humans, primarily through the activity of damage-inducible error-prone polymerases, whose functions are to replicate DNA over the induced lesion (translesion synthesis), thereby rescuing replicative polymerases that have stalled at these positions (303). Error-prone polymerases and TLS have been associated with the repair of a number of different DNA lesions, in both mammalian and bacterial cells (303), and furthermore their activity has been directly linked to generation of drug resistance in cancer cells, most likely via mutational activation of 
multi-drug resistance genes $(318 ; 319)$. Studies have also demonstrated that downregulation of many translesion polymerases with antisense RNA technology can sensitize drug-resistant cancer cell lines to the toxic effects of alkylating agents (319; 320). Therefore, as results presented in this chapter demonstrate that CB1954 induced lesions are substrates for translesion polymerases, clinical therapy with this prodrug may benefit from targeted inhibition of tumour cell translesion polymerases.

The mutagenicity of both 2 - and $4-\mathrm{NO}_{2}$ reduction products significantly increased in the absence of functional NER machinery, suggesting that TLS is compensatory to NER in repair of CB1954 induced adducts. This has previously been observed for a number of nitroaromatic mutagens (321). However, the increase in mutagenicity in the $\Delta u v r B$ strain was much greater in response to the $2-\mathrm{NO}_{2}$ reduction products (22.5-fold difference over wildtype) than to the 4- $\mathrm{NO}_{2}$ reduction products (3.3fold difference). These observations indicate that $2-\mathrm{NO}_{2}$ reduction product induced adducts may be preferentially repaired by NER, but are just as efficiently processed by TLS pathways in the absence of NER. In contrast, $4-\mathrm{NO}_{2}$ reduction product-induced adducts may not be as efficient subsrates for TLS, therefore leading to enhanced SOS induction in the absence of a functional NER mechanism.

The majority of mutations induced by both 2- and 4- $\mathrm{NO}_{2} \mathrm{CB} 1954$ reduction products were $\mathrm{GC} \rightarrow \mathrm{AT}$ transitions, which is in agreement with previous research that has shown that $\mathrm{GC} \rightarrow \mathrm{AT}$ transitions are the predominant mutation induced by a number of structurally diverse alkylating agents $(322 ; 323)$. However, while the spectrum of mutations induced by the $2-\mathrm{NO}_{2}$ reduction products was similar in both wildtype and NER-deficient strains, the $4-\mathrm{NO}_{2}$ reduction products induced more $\mathrm{GC} \rightarrow$ TA transversions in the $\Delta u v r B$ strain. Together, these results suggest that initial DNA binding of both 2- and 4- $\mathrm{NO}_{2}$ reduction metabolites is similar, the product of which (most likely a monoadduct) is able to be processed by NER (error-free) or certain TLS enzymes (introducing mainly GC $\rightarrow$ AT mutations ). If left unrepaired, the primary adduct formed by the 4-, but not the 2-, $\mathrm{NO}_{2}$ reduction metabolites is able to undergo a second reaction (due to bifunctional properties) to form intra- or interstrand crosslinks, which are primarily repaired by NER. However, in the absence of NER, persistent intraor interstrand 4-NHOH lesions induce a higher SOS response, leading to up-regulation of alternative translesion polymerases that can then repair these adducts. The difference in base pair substitutions between NER-proficient and deficient cell lines may be due to the type of polymerases employed to repair either the initial monoadduct or the crosslink, as supported by both the differences in substrate specificity and mutational 
bias that have previously been reported for differed TLS polymerases $(303 ; 324)$.

Further analysis of base pair substitutions and other mutations (insertions, deletions)

induced by CB1954 will aid in understanding of the mechanisms of action of each reduction metabolite. 


\section{Chapter 5: Screening a phylogenetically diverse library of bacterial nitroreductases for nitroaromatic prodrug reduction}

\subsection{Introduction}

The results gained in the previous two chapters have provided strong evidence for two principal hypotheses of this research: firstly, that a variety of phylogenetically diverse enzymes are able to bioreductively activate a range of structurally distinct DNA alkylating nitroaromatic prodrugs; and secondly, that our novel E. coli-based SOS assay is a generally applicable, accurate and moderately high-throughput screen for detection and quantification of NTR-mediated metabolism of these prodrugs. However, these studies have been performed using a very small $(<15)$ panel of candidate enzymes, the majority of which are of E. coli origin. As previous GDEPT research has illustrated that prodrug-reductase activity can vary dramatically among homologues, whether close or distant, of prodrug-reducing enzymes $(158 ; 206 ; 208)$, the next logical step in this research is to expand the breadth of candidate enzymes tested to include homologues of the most active NTRs, from a variety of genetic backgrounds. Not only will this potentially allow identification of more active nitroaromatic prodrug reducing NTRs, it will also enable further assessment of the ability of the SOS assay to function in a more high-throughput capacity, and allow insight into possible structure-activity relationships between prodrug and enzyme active site architecture. Lead NTR(s) could then be further enhanced for prodrug reducing activity via directed evolution/enzyme engineering approaches.

The initial aim of the research described in this chapter was to identify, through database homology searches, a variety of bacterial NTRs with significant sequence homology to the previously tested E. coli enzymes. These enzymes formed the basis of a 'core' library of NTRs that were subsequently examined, principally via the SOS assay, for their ability to reduce a number of structurally diverse nitroaromatic prodrugs with potential anti-cancer clinical applications. Finally, a selection of nitrobenzamide mustard prodrug analogues of PR-104A that induced strong SOS activity in the E. colibased screen were assessed for in vitro metabolism by recombinant human AKR1C3, in order to identify potential hypoxia and nitroreductase activated prodrugs with reduced aerobic off-target toxicity. 


\subsection{Results}

\subsubsection{Further optimisation of the SOS assay}

Prior to generation and screening of the expanded library of NTRs for nitroaromatic prodrug reduction, several modifications were made to the SOS assay to improve sensitivity and reduce potential background activity by native enzymes. Firstly, as results from Chapters 3 and 4 indicated small but significant roles of both AzoR (E.c) and NemA (E.c) in reduction of a variety of test drugs, the native genes of these two enzymes were deleted, in-frame (as previously described for NfsA (E.c) and NfsB (E.c)) from the SOS-R1 genome. The resulting strain was designated SOS-R1B. No observable growth disadvantages were detected between this strain and SOS-R1 (results not shown), indicating that these genes are not essential or important under standard laboratory conditions used for organism growth and maintenance. Secondly, enhancement of prodrug sensitivity was sought by investigating the role of a multi-drug efflux pump accessory protein (TolC) in promotion of resistance to the nitroaromatic prodrugs employed in this study. Higher sensitivity would guarantee lower prodrug concentrations able to be employed in the screens, preventing wastage of prodrugs that are either commercially expensive, or not commercially available and therefore supplied in limited amounts by generous donors. This is of particular relevance to PR-104A, of which very high concentrations $(200 \mu \mathrm{M})$ were required to elicit moderate levels of SOS response induction in NTR over-expressing strains of SOS-R1 in the previous chapter (see Figure 4.8C).

The outer membrane pore protein TolC is able to act in combination with at least four structurally distinct inner membrane pump systems (acrAB, acrEF, emrAB and $m a c A B$ ) to expel a wide array of low molecular weight compounds and proteins out of the cell (325). Inactivation of the tolC gene has been shown to be accompanied by greatly enhanced sensitivity to a variety of natural toxins and xenobiotics, including antibacterial drugs, in agreement with the important role this protein plays in expulsion of potentially harmful compounds (326-328). Furthermore, tolC mutants have been used widely in SOS reporter systems to improve assay sensitivity and decrease detection limits of genotoxic compounds (229-231). The role of TolC in efflux of the two principal nitroaromatic prodrugs studied in this work, CB1954 and PR-104A, was investigated through in-frame deletion of the tolC gene from the SOS-R1B genome. The resultant strain, termed SOS-R2, displayed a vastly enhanced sensitivity towards 
reduction of PR-104A (from 2.3 to 7.2-fold induction, for otherwise isogenic tolC ${ }^{+}$and tolC ${ }^{-}$strains respectively, with NfsA (E.c)-over-expression and $50 \mu \mathrm{M}$ PR-104A challenge; Figure 5.1). However, an increase in SOS response with the empty vector control was also noted in SOS-R2, suggesting a heightened sensitivity of this strain towards toxicity of the PR-104A parental prodrug. In contrast, no considerable difference in sensitivity towards CB1954 was observed between the two strains. Lack of a functional tolC gene did not affect cell growth rate (as compared to wildtype in the absence of prodrug; results not shown).

In light of these data, SOS-R2 was chosen as the host strain of choice for all SOS and $\mathrm{IC}_{50}$ assays for the remainder of this work.

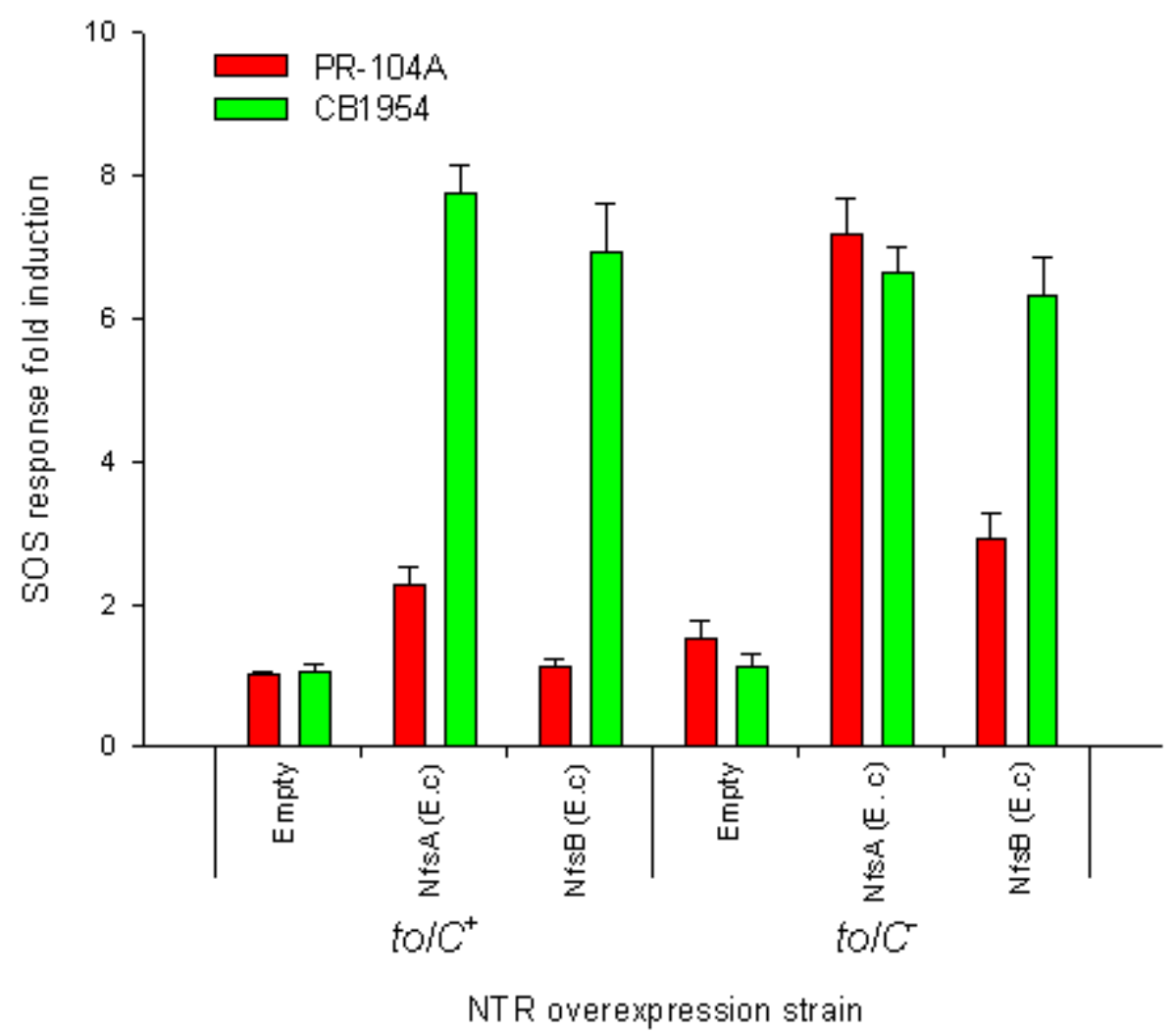

Figure 5.1. Differences in nitroaromatic prodrug-induced SOS responses between $\mathrm{tolC}^{+}$and $\mathrm{tolC}^{-}$ strains of SOS-R1B.

NTR over-expressing strains of SOS-R1B that either contained $\left(\mathrm{tolC}^{+}\right)$or lacked $\left(\mathrm{tolC} \mathrm{C}^{-}\right)$the outermembrane pore protein TolC were grown and challenged for $3 \mathrm{~h}$ with the indicated nitroaromatic prodrug at $50 \mu \mathrm{M}$ final concentration, prior to analysis of SOS activity. Data is the average \pm 1 SEM of 4 independent assays. 


\subsubsection{Generation and expression of a core library of bacterial NTRs}

NTRs that would form the basis of a core library to be screened for nitroaromatic prodrug-reductase activity were chosen from the NCBI protein database, through BLAST homology searches using each of the E. coli enzymes tested in Chapter 3 (as well as human NQO1 and B. amyloliquefaciens YwrO, see below) as query sequences. As such, the library was partitioned into separate NTR families, based around these key members. A total of 47 NTRs were chosen (plus the empty vector control), to facilitate assay screening of the entire library in duplicate in a single 96-well plate, at each prodrug concentration. NTRs were chosen from a range of bacterial species based on availability to our laboratory; this included both Gram positive and Gram negative strains, with the only other criteria for selection being a $\geq 25 \%$ amino acid identity to the key (query sequence) family member. Table 5.1 contains the complete list of enzymes chosen for the core library and their phylogenetic relationships are displayed in Figure 5.2. As can be seen, emphasis was given to both the NfsA and NfsB families (12 members each), as they were the most active enzymes with CB1954, as previously determined. Likewise, the AzoR and NemA families contain 4 members apiece, with the other families containing at least one homologue in addition to the E. coli enzyme. 2 bacterial homologues of mammalian NQO1 were also included. Finally, based on the results of Anlezark et al. (207), a new family containing homologues of $B$. amyloliquefaciens YwrO, of which no E. coli enzyme with significant homology exists, was also introduced.

NTR genes were PCR amplified from genomic DNA (strains used are listed in Table 2.3), cloned into pUCX and sequence-confirmed constructs were transformed into SOS-R2. SDS-PAGE was used to assess protein expression levels. High levels of expression were observed for most of the 47 NTRs tested, with only YwrO (B.s) and YwrO (V.f) failing to produce a visible band. Expression levels were consistent across the majority of NTRs, with the exception of a few for which expression was visibly lower (NfsA (V.f), EcD (P.p), YcdI (E.c), YcdI (K.p) and YdjA (E.c)). However, as the over-expressed NTR was the most prominent band in even these lanes, for simplicity of SOS response and $\mathrm{IC}_{50}$ data calculations, relative NTR expression levels were not analysed any further. 


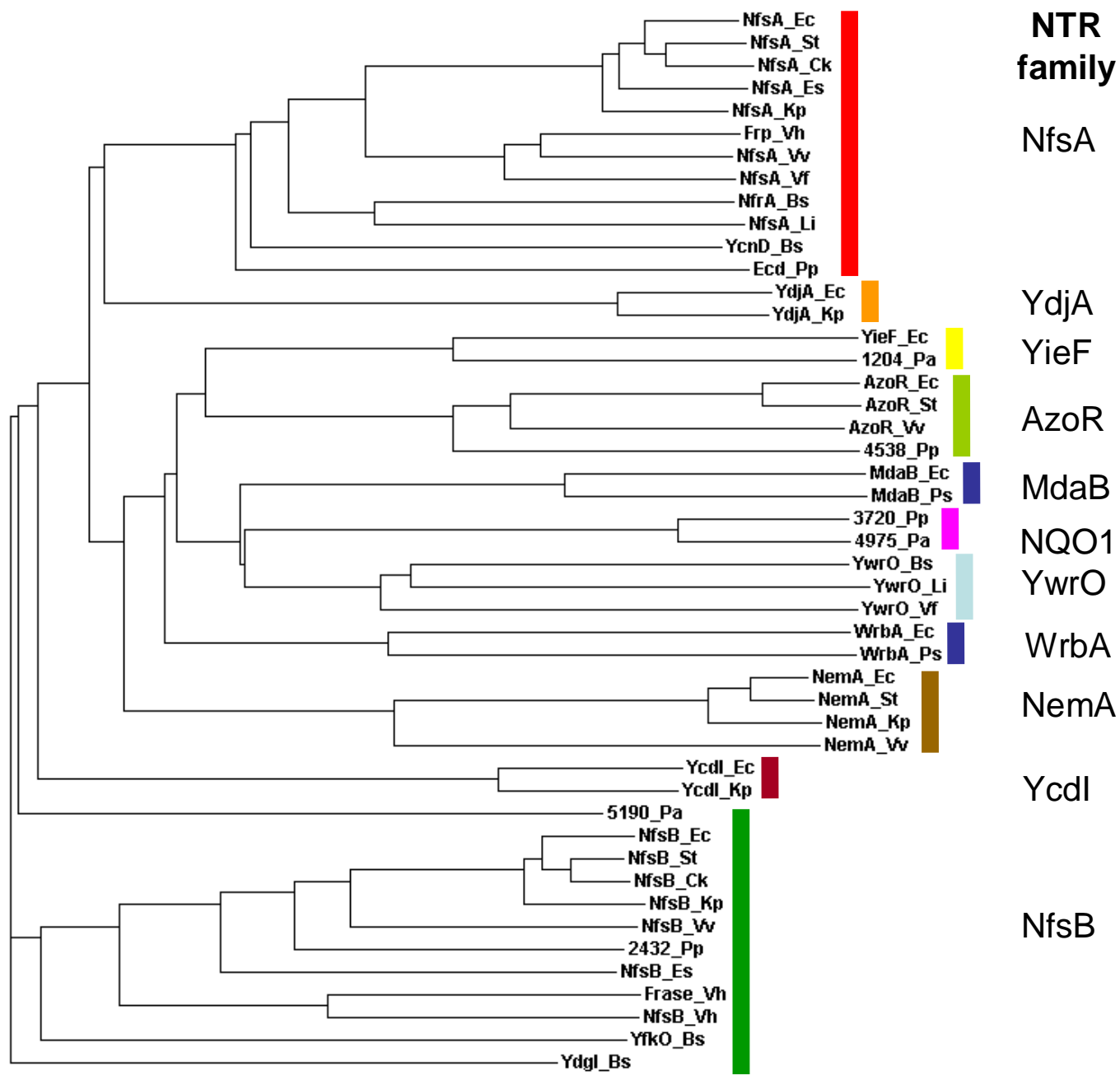

Figure 5.2. Phylogram of the 47 NTRs chosen for the core library.

Gene names used throughout this work are those before the underscore (_). Letters after the underscore refer to the bacterial origin of each gene. Ec: E. coli. St: S. typhimurium. Ck: C. koseri. Es: E. sakazakii. Kp: K. pneumoniae. Vh: V. harveyi. Vv: V. vulnificus. Vf: V. fischeri. Bs: B. subtilis. Li: L. innocua. Pp: P. putida. Pa: $P$. aeruginosa. Ps: $P$. syringae. Table 2.3 contains more details on strains used, including ATCC number and/or substrain designation. Coloured lines indicate the different families that NTRs are divided into, with designated family names for each indicated to the right. Multiple sequence alignment and phylogram generation was performed using ClustalW2 (http://www.ebi.ac.uk/Tools/msa/clustalw2/), on default settings. 
Table 5.1. NTRs used in this study,

\begin{tabular}{|c|c|c|c|c|}
\hline NTR & Family $^{\mathrm{a}}$ & $\% \mathrm{ID}^{\mathrm{b}}$ & $\% \mathrm{Sim}^{\mathrm{b}}$ & $\operatorname{Refs}^{c}$ \\
\hline NfsA (E.c) & NfsA & 100 & 100 & $(176 ; 189)$ \\
\hline NfsA (S.t) & NfsA & 87 & 95 & (193) \\
\hline NfsA (C.k) & NfsA & 86 & 92 & \\
\hline NfsA (K.p) & NfsA & 83 & 92 & \\
\hline NfsA (E.s) & NfsA & 82 & 94 & \\
\hline NfsA (V.f) & NfsA & 52 & 68 & \\
\hline NfsA (V.v) & NfsA & 51 & 65 & \\
\hline Frp (V.h) & NfsA & 51 & 65 & $(179 ; 191)$ \\
\hline NfrA (B.s) & NfsA & 39 & 62 & $(167 ; 170)$ \\
\hline NfsA (L.i) & NfsA & 39 & 60 & \\
\hline EcD (P.p) & NfsA & 35 & 57 & \\
\hline YcnD (B.s) & NfsA & 35 & 56 & $(194 ; 329)$ \\
\hline $\mathrm{NfsB}$ (E.c) & $\mathrm{NfsB}$ & 100 & 100 & $(122 ; 177)$ \\
\hline NfsB (C.k) & $\mathrm{NfsB}$ & 88 & 95 & \\
\hline NfsB (S.t) & $\mathrm{NfsB}$ & 88 & 91 & $(197 ; 198)$ \\
\hline NfsB (K.p) & $\mathrm{NfsB}$ & 84 & 93 & \\
\hline NfsB (V.v) & $\mathrm{NfsB}$ & 61 & 75 & \\
\hline 2432 (P.p) & NfsB & 52 & 71 & \\
\hline NfsB (E.s) & NfsB & 46 & 66 & \\
\hline FraseI (V.f) & NfsB & 34 & 53 & (166) \\
\hline NfsB (V.h) & NfsB & 30 & 48 & \\
\hline YfkO (B.s) & NfsB & 29 & 46 & \\
\hline YdgI (B.s) & NfsB & 25 & 47 & (208) \\
\hline 5190 (P.a) & NfsB & 25 & 42 & \\
\hline AzoR (E.c) & AzoR & 100 & 100 & $(259 ; 260)$ \\
\hline AzoR (S.t) & AzoR & 87 & 95 & \\
\hline AzoR (V.v) & AzoR & 56 & 73 & \\
\hline 4538 (P.p) & AzoR & 48 & 66 & \\
\hline NemA (E.c) & NemA & 100 & 100 & $(187 ; 190)$ \\
\hline NemA (S.t) & NemA & 92 & 96 & \\
\hline NemA (K.p) & NemA & 86 & 92 & \\
\hline NemA (V.v) & NemA & 47 & 65 & \\
\hline YwrO (B.s) & YwrO & $70^{\mathrm{d}}$ & $82^{\mathrm{d}}$ & \\
\hline YwrO (L.i) & YwrO & $42^{\mathrm{d}}$ & $57^{\mathrm{d}}$ & \\
\hline YwrO (V.f) & YwrO & $52^{\mathrm{d}}$ & $68^{\mathrm{d}}$ & \\
\hline
\end{tabular}


Table 5.1. continued.

\begin{tabular}{ccccc}
\hline NTR & Family $^{\mathrm{a}}$ & \% ID & \% Sim & Refs $^{\mathrm{c}}$ \\
\hline YieF (E.c) & YieF & 100 & 100 & $(184 ; 222)$ \\
1204 (P.a) & YieF & 45 & 60 & \\
MdaB (E.c) & MdaB & 100 & 100 & $(262 ; 330)$ \\
MdaB (P.s) & MdaB & 60 & 74 & \\
WrbA (E.c) & WrbA & 100 & 100 & $(263 ; 283)$ \\
WrbA (P.s) & WrbA & 39 & 55 & \\
YdjA (E.c) & YdjA & 100 & 100 & $(258)$ \\
YdjA (K.p) & YdjA & 79 & 89 & \\
3720 (P.p) & NQO1 & $38^{\mathrm{e}}$ & $57^{\mathrm{e}}$ & \\
4975 (P.a) & NQO1 & $42^{\mathrm{e}}$ & $55^{\mathrm{e}}$ & \\
YcdI (E.c) & YcdI & 100 & 100 & $(261 ; 315)$ \\
YcdI (K.p) & YcdI & 76 & 85 &
\end{tabular}

${ }^{a}$ NTR family names as designated in this study (based on homology groupings; see Figure 5.2)

${ }^{\mathrm{b}} \%$ ID and \% Sim. indicate amino acid identity and similarity, as calculated by the blastp program ("identities" and "positives", respectively) of each NTR to the designated family lead member (the lead member is indicated as having $100 \%$ ID and Sim)

${ }^{\mathrm{c}}$ Publications that report biochemical data for the respective NTR.

${ }^{\mathrm{d}} \mathrm{Sim}$. and ID values for all YwrO family enzymes are relative to B. amyloliquefaciens $\mathrm{YwrO}$ (207)

${ }^{\mathrm{e}} \mathrm{Sim}$. and ID values are relative to human NQO1

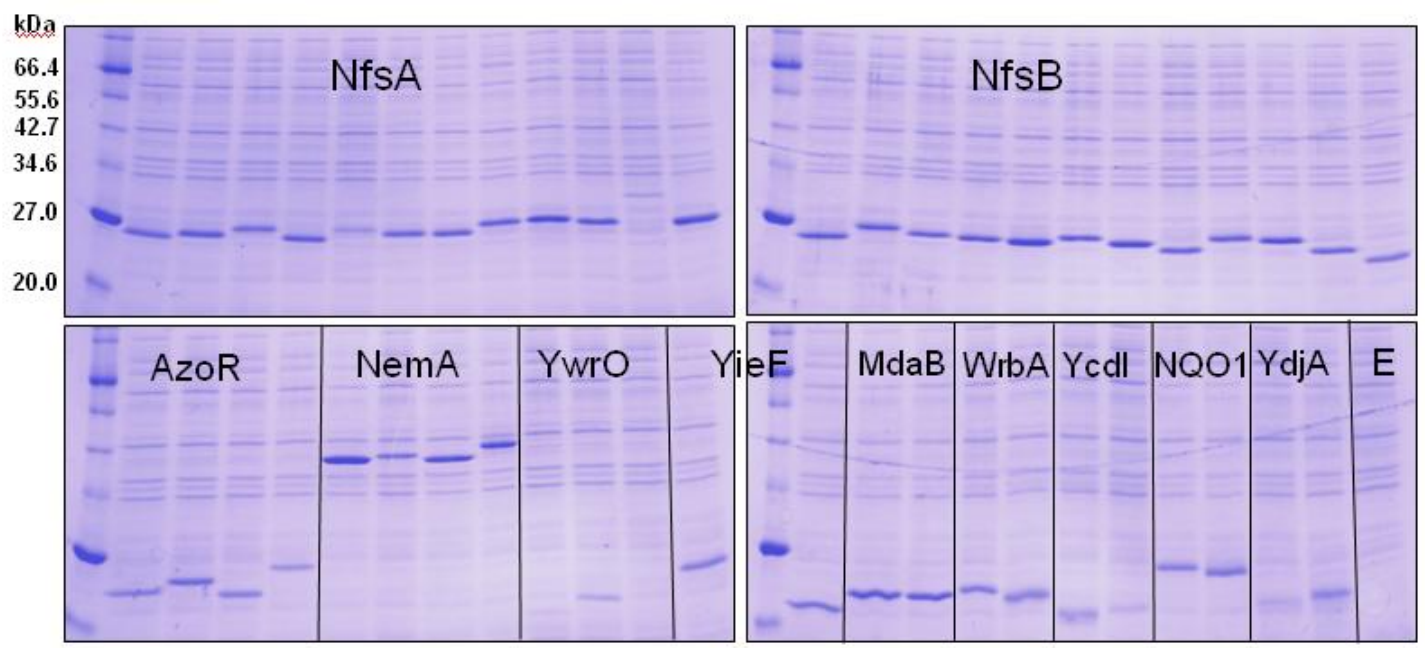

Figure 5.3. Expression of core library NTRs in SOS-R2.

SDS-PAGE analysis of expression levels of the 47 NTRs of the core library, from pUCX in SOS-R2. The order of enzymes is the same as that shown in Table 5.1 (the final sample, "E", is the empty vector control). Samples for electrophoresis were whole cell lysates taken from a single replicate at the completion of an SOS assay. Samples were normalized for cell density $\left(\mathrm{OD}_{600}\right)$ prior to gel loading. 


\subsubsection{Screening the core library for CB1954-reductase activity}

\subsubsection{SOS assay}

The core library of NTRs was screened for CB1954-reductase activity using the SOS assay, employing prodrug concentrations ranging from 10-50 $\mu \mathrm{M}$ and a challenge time of $3 \mathrm{~h}$ (Figure 5.4). Consistent with previous results for the E. coli enzymes (Chapter 3), the NfsA and NfsB families elicited the highest average SOS responses, with smaller contributions from AzoR and NemA families. No substantial increase in activity above empty vector levels was observed for any NTR outside of these families. A lot of variation in SOS response induction was also observed within the Nfs A and NfsB families. For example, within the NfsA family SOS fold inductions ranged from 2 to 10.7 at the midrange CB1954 concentration of $20 \mu \mathrm{M}$, while within the NfsB family the equivalent range was between 2.6 and 8.3. Although on average the NfsA family elicited a higher SOS response across all CB1954 concentrations compared to the NfsB family (3.13, 5.4 and 8.4-fold SOS response induction compared with 2.8, 4.4 and 7.1, respectively, for the increasing concentrations of prodrug), there was no statistical significance between these differences ( $p>0.05$; t-test). The highest overall response was obtained from the NfsA family enzyme YcnD (B.s), whose activity was roughly 2.5 times higher (at both 10 and $20 \mu \mathrm{M}$ CB1954) than that of NfsA (E.c) (6.7-and 10.7-fold compared to 2.4 and 4.3-fold, respectively). In fact, SOS response induction for $\mathrm{YcnD}$ (B.s) at the highest concentration tested $(50 \mu \mathrm{M})$ was lower than at $20 \mu \mathrm{M}$, most likely due to the onset of growth inhibition (58\% growth relative to unchallenged at $50 \mu \mathrm{M}$, whereas no other NTR induced growth inhibition below $70 \%$ of unchallenged levels; results not shown). Two other NfsA-family enzymes also showed significantly $(\mathrm{p}<0.05$; t-test) higher SOS activity across all concentrations tested relative to NfsA (E.c), these being NfsA (V.v) and Frp (V.h). 


\subsubsection{2. $\mathrm{IC}_{50} \mathrm{~S}$}

SOS assay results were next compared to $\mathrm{IC}_{50} \mathrm{~S}$ of $\mathrm{CB} 1954$ for each over-expressed NTR in SOS-R2. Due to the large number of NTRs required to be tested and therefore high volume of CB1954 needed for extensive $\mathrm{IC}_{50}$ testing, the assay protocol was adjusted from that used in Chapter 3 to minimize prodrug requirements and usage. This involved both a change in the media used (M63 minimal media, compared to LB) and adaptation of the assay for use in 384-well plates. The justification for use of minimal media was that a slower growth rate would allow more pronounced CB1954-mediated toxic effects to show through and therefore produce lower overall $\mathrm{IC}_{50}$ s. Growth was indeed slower overall in M63 relative to LB (results not shown) and subsequently $\mathrm{IC}_{50} \mathrm{~S}$ in this section were on average 2-3-fold lower than those previously measured (i.e. Chapter 3).

$\mathrm{IC}_{50}$ values for each NTR, calculated from these assays, are listed in Table 5.2. In agreement with SOS assay results, the most active NTRs (lowest $\mathrm{IC}_{50} \mathrm{~s}$ ) belonged to the NfsA and NfsB families, including 19 of the overall top 20 enzymes (NemA (E.c) taking $18^{\text {th }}$ place $)$. Overall, a strong and significant $\left(R^{2}>0.75, p<0.001\right)$ correlation between SOS (all concentrations tested) and $\mathrm{IC}_{50} \mathrm{~S}$ was observed (see Figure 5.5). Correlations between results from just the NfsA and NfsB family enzymes were also high $\left(\mathrm{R}^{2}>0.7\right.$; results not shown), demonstrating little correlation bias towards the many inactive enzymes. One consistent outlier was NfsA (S.t), which although eliciting only slightly higher SOS responses than NfsA (E.c) at all CB1954 concentrations tested yielded an $\mathrm{IC}_{50}$ value roughly 2 -fold higher. 


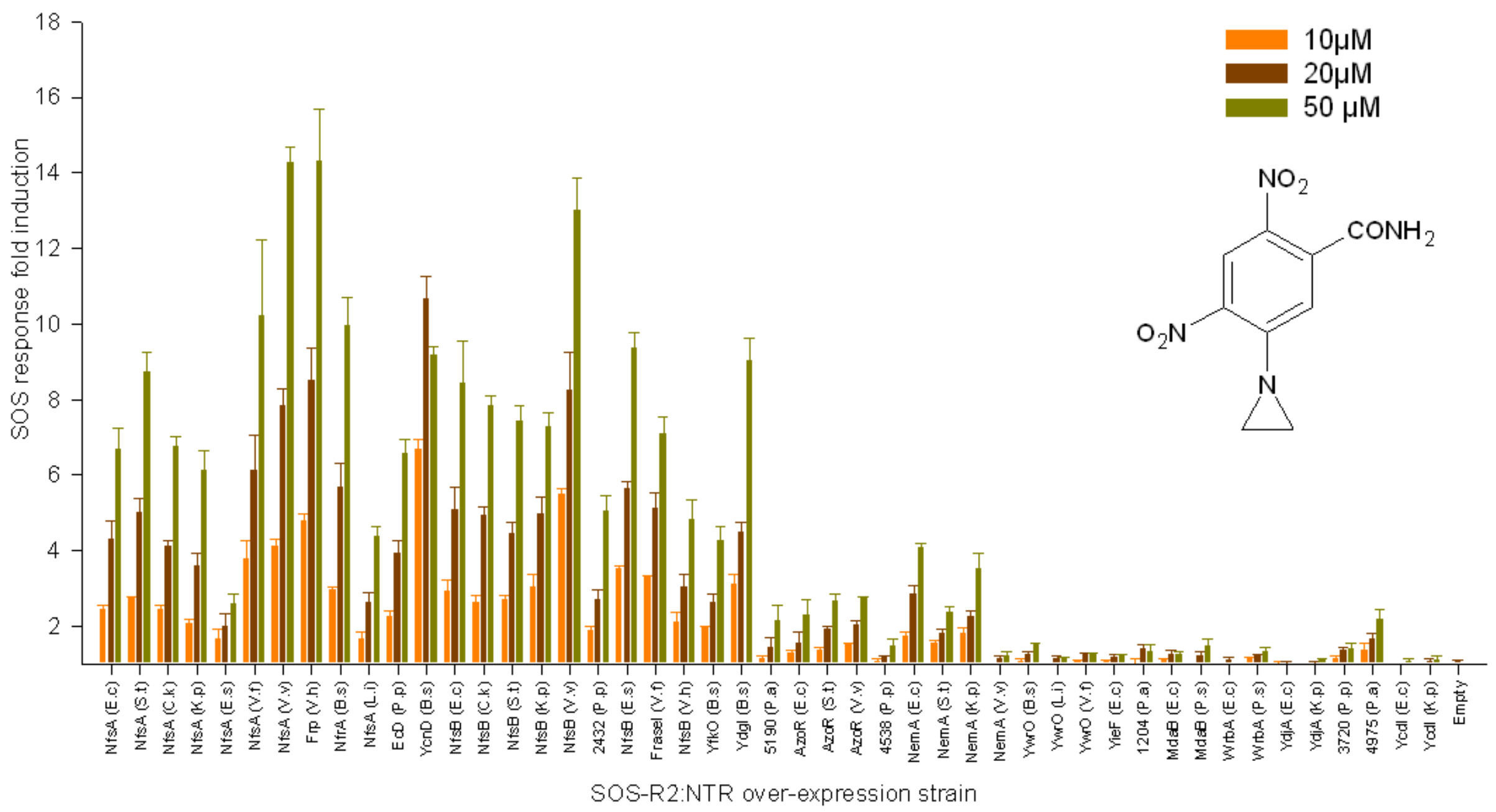

Figure 5.4. CB1954-induced SOS responses from NTR over-expressing strains of SOS-R2.

NTR over-expressing strains of SOS-R2 were grown and challenged with CB1954 (at the indicated concentrations) for $3 \mathrm{~h}$ prior to analysis of SOS activity. Data is the average \pm 1 SEM of 4 independent assays 
Table 5.2. IC $_{50}$ S of CB1954 in NTR over-expressing strains of SOS-R2.

$\mathrm{IC}_{50}$ values were calculated from growth measurements taken after $4 \mathrm{~h}$ challenge with increasing concentrations of CB1954.

\begin{tabular}{|c|c|c|c|c|c|c|c|c|c|c|c|}
\hline NTR & $\mathrm{IC}_{50}{ }^{\mathrm{a}}(\mu \mathrm{M})$ & Rank & SOS rank ${ }^{b}$ & NTR & $\mathrm{IC}_{50}(\mu \mathrm{M})$ & Rank & SOS rank & $\begin{array}{l}\text { NTR } \\
\end{array}$ & $\mathrm{IC}_{50}(\mu \mathrm{M})$ & Rank & SOS rank \\
\hline NfsA (E.c) & 150 & 11 & 15 & NfsB (V.v) & 82.8 & 5 & 3 & YwrO (B.s) & 1,380 & 42 & 35 \\
\hline NfsA (S.t) & 305 & 25 & 10 & 2432 (P.p) & 290 & 23 & 21 & YwrO (L.i) & 1,800 & 47 & 42 \\
\hline NfsA (C.k) & 154 & 13 & 16 & NfsB (E.s) & 183 & 15 & 7 & YwrO (V.f) & 1,600 & 44 & 36 \\
\hline NfsA (K.p) & 183 & 16 & 18 & FraseI (V.f) & 120 & 7 & 8 & YieF (E.c) & 1,750 & 46 & 40 \\
\hline NfsA (E.s) & 203 & 22 & 24 & NfsB (V.h) & 256 & 21 & 19 & 1204 (P.a) & 1,290 & 39 & 32 \\
\hline NfsA (V.f) & 79.2 & 4 & 5 & YfkO (B.s) & 201 & 20 & 23 & $\operatorname{MdaB}$ (E.c) & 400 & 26 & 34 \\
\hline NfsA (V.v) & 83.9 & 6 & 4 & YdgI (B.s) & 127 & 8 & 13 & MdaB (P.s) & 832 & 32 & 37 \\
\hline Frp (V.h) & 70 & 3 & 2 & 5190 (P.a) & 541 & 29 & 31 & WrbA (E.c) & 1,330 & 40 & 43 \\
\hline NfrA (B.s) & 46.1 & 2 & 6 & AzoR (E.c) & 866 & 33 & 30 & WrbA (P.s) & 944 & 35 & 38 \\
\hline NfsA (L.i) & 201 & 19 & 22 & AzoR (S.t) & 511 & 28 & 27 & YdjA (E.c) & 1,430 & 43 & 47 \\
\hline $\mathrm{EcD}(\mathrm{P} . \mathrm{p})$ & 148 & 10 & 17 & AzoR (V.v) & 466 & 27 & 26 & YdjA (K.p) & 1,980 & 48 & 46 \\
\hline YcnD (B.s) & 29.1 & 1 & 1 & 4538 (P.p) & 1,220 & 37 & 39 & 3720 (P.p) & 1,330 & 41 & 33 \\
\hline NfsB (E.c) & 154 & 12 & 9 & NemA (E.c) & 188 & 18 & 20 & 4975 (P.a) & 802 & 30 & 29 \\
\hline NfsB (C.k) & 157 & 14 & 12 & NemA (S.t) & 817 & 31 & 28 & YcdI (E.c) & 994 & 36 & 48 \\
\hline NfsB (S.t) & 188 & 17 & 14 & NemA (K.p) & 300 & 24 & 25 & YcdI (K.p) & 1,280 & 38 & 44 \\
\hline NfsB (K.p) & 136 & 9 & 11 & NemA (V.v) & 905 & 34 & 41 & Empty & 1,740 & 45 & 45 \\
\hline
\end{tabular}

${ }^{\mathrm{a}}$ Degrees of error associated with $\mathrm{IC}_{50}$ values are omitted for clarity. Standard deviations did not exceed $17 \%$ of the $\mathrm{IC}_{50}$ value, with the exception of YdjA (K.p) (23\%)

${ }^{\mathrm{b}} \mathrm{SOS}$ rankings are derived from the $20 \mu \mathrm{M}$ CB1954 challenge data 


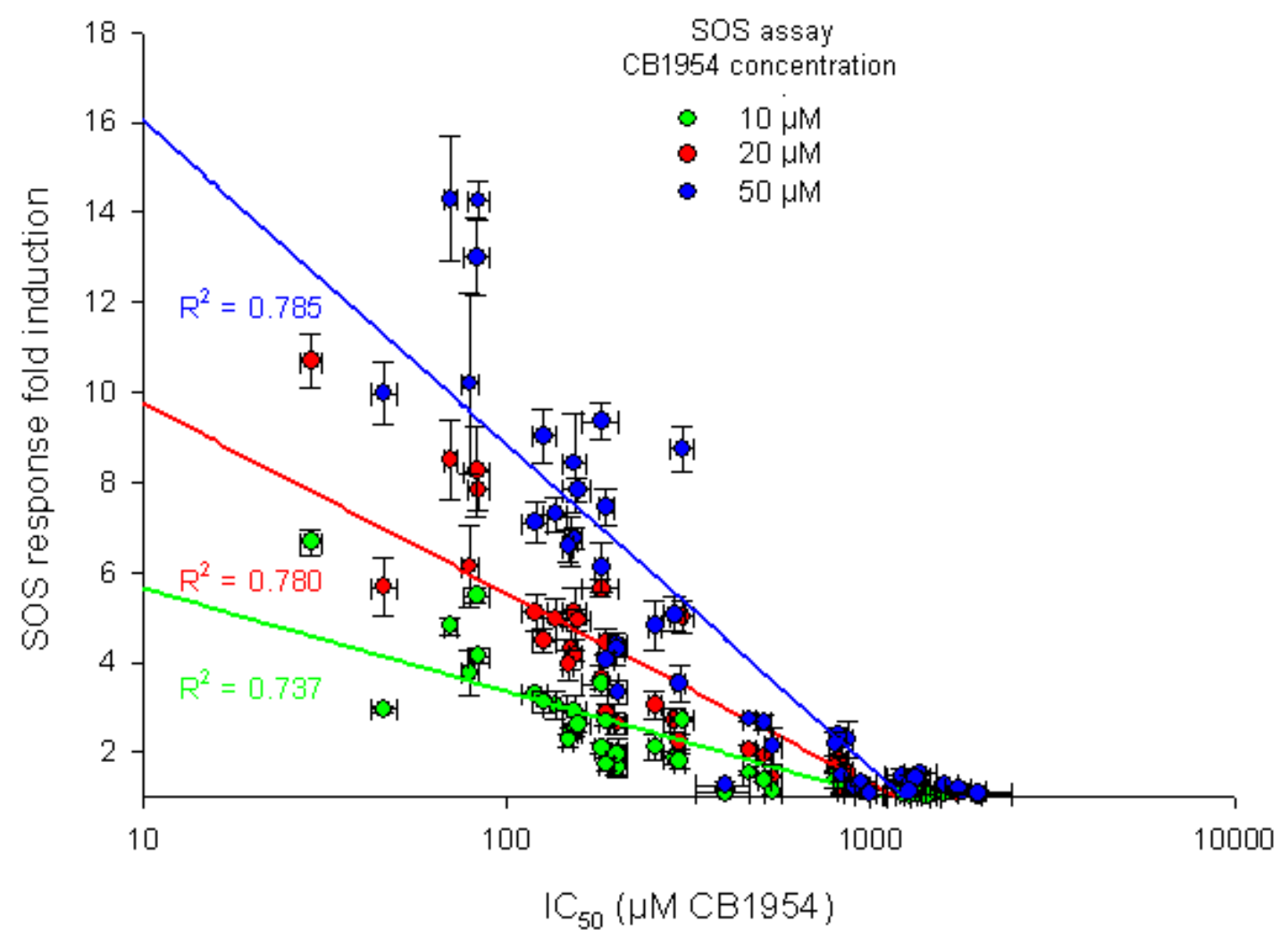

Figure 5.5. Logarithmic correlations between $\mathrm{SOS}$ assay results and $\mathrm{IC}_{50} \mathrm{~s}$ for $\mathrm{CB} 1954$-challenged NTR over-expressing SOS-R2 strains.

Data for SOS results were taken from Figure 5.4. Data for $\mathrm{IC}_{50} \mathrm{~s}$ were taken from Table 5.2. The SOS value for $50 \mu \mathrm{M}$ CB1954-challenged YcnD over-expressing SOS-R2 has been omitted, as growth inhibition impaired SOS output for this NTR at this concentration of prodrug.

\subsubsection{In vitro kinetics of purified enzymes}

A selection of NTRs that performed strongly in both SOS and $\mathrm{IC}_{50}$ assays were further characterised for CB1954-reductase activity by measuring their in vitro kinetic parameters using purified recombinant enzymes. In addition to the 4 native $E$. coli enzymes previously screened in this way (Chapter 3), 16 new enzymes were chosen, of which 9 were NfsA and 7 NfsB homologues. Genes were cloned into the expression vector pET28a(+), transformed into E. coli BL21 (DE3), over-expressed, and purified by nickel-affinity chromatography. Although all enzyme preparations retained yellow colour upon elution, to compensate for partial loss of loosely bound flavin co-factors during the purification steps, all eluted enzymes were incubated with pure FMN prior to buffer exchange. Absorbance spectrophotometry on FMN-reconstituted samples indicated that flavin:enzyme monomer ratios were roughly equimolar (flavin:monomer ratios of recombinant enzymes directly after elution from nickel-affinity columns were 
typically less than 0.5 ; results not shown), indicating roughly complete co-factor reconstitution by this method.

The purity of recombinant protein preparations was assessed by SDS-PAGE, the results of which are displayed in Figure 5.6. All proteins were deemed to be $>90 \%$ pure by scanning densitometry (results not shown).
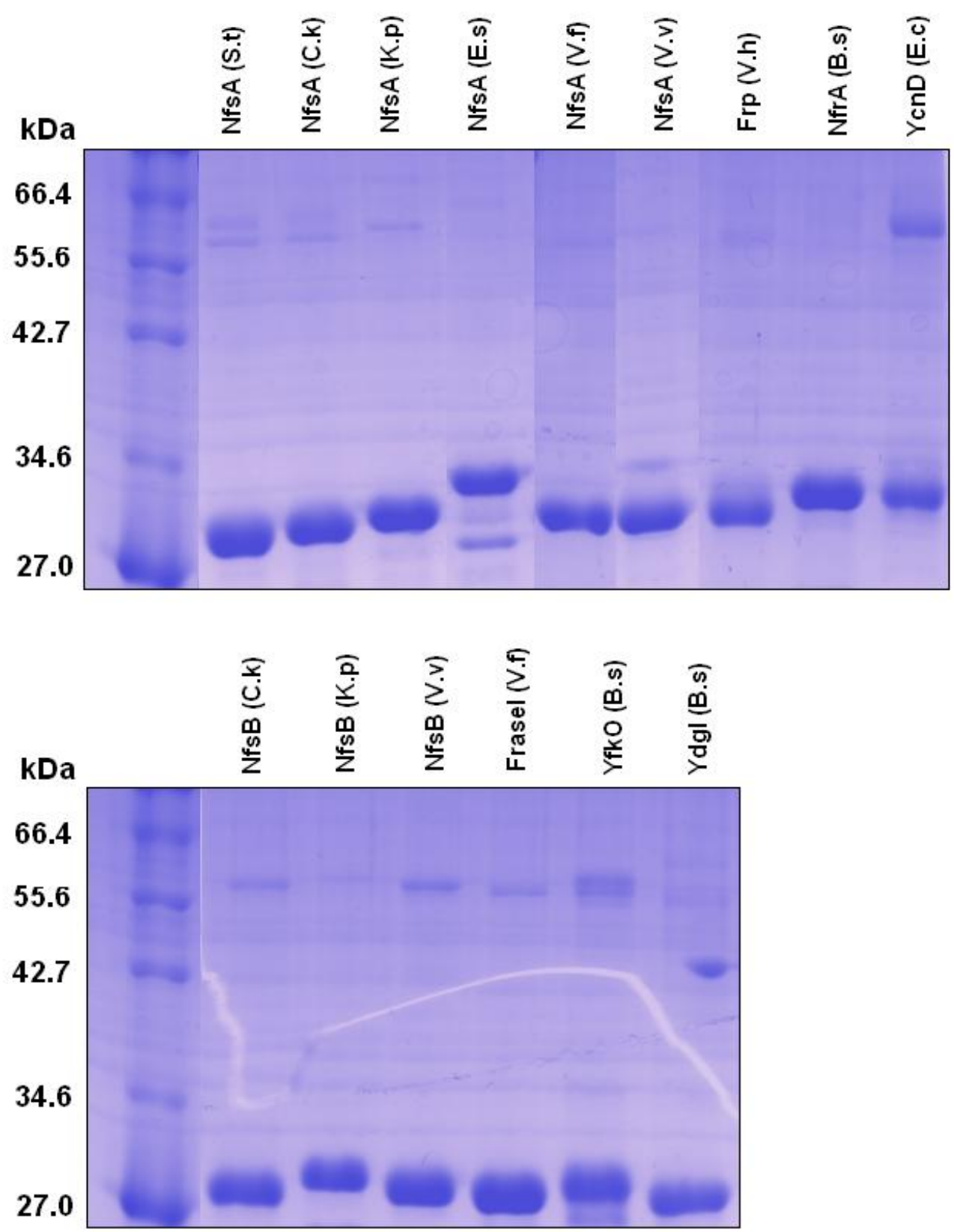

Figure 5.6. SDS-PAGE of purified, His6-tagged NTRs used in this chapter.

Genes of the NTRs to be assessed were cloned into pET28a(+) vector, over-expressed in E. coli BL21 and recombinant His6-tagged NTR purified to homogeneity using nickel-affinity chromotography. Each lane corresponds to $2 \mu \mathrm{g}$ of purified enzyme. 
CB1954-reductase activity of purified enzymes was assessed as previously described (Chapter 3). All enzymes tested demonstrated clear activity towards this substrate (with the exception of NfsA (S.t), which exhibited no activity in vitro with any substrate tested, despite high heterologous expression levels and purified enzyme yield). As predicted by SOS and $\mathrm{IC}_{50} \mathrm{~s}$, catalytic efficiencies varied dramatically (see Table 5.3). On average, NfsA-like enzymes were 10-fold more catalytically efficient than their $\mathrm{NfsB}$ counterparts (as measured by $k_{\mathrm{cat}} / K_{\mathrm{m}}$; averages of $174,000 \mathrm{M}^{-1} \mathrm{~s}^{-1}$ and $17,500 \mathrm{M}^{-1}$ $\mathrm{s}^{-1}$ for NfsA and NfsB-families, respectively), similar to the previous results obtained with the 2 native $E$. coli representatives of these families. The basis of this difference appeared to be primarily due to substrate affinity rather than catalytic turnover, as NfsBfamily $K_{\mathrm{m}}$ values were $>25$ times higher on average than those of NfsA-family enzymes. In contrast, NfsB $k_{\text {cat }}$ values were slightly higher, on average.

Correlations between SOS assay results and $k_{\text {cat }} / K_{\mathrm{m}}$ values for the tested NTRs (see Figure 5.7A; $\mathrm{R}^{2}=0.53$ and 0.55 ) were not as strong as correlations observed between SOS assay results and $\mathrm{IC}_{50}$ values, likely due to the large difference (10-fold on average) in $k_{\text {cat }} / K_{\mathrm{m}}$ values between $\mathrm{NfsA}$ and NfsB family enzymes, which was not mirrored in either SOS assay results or $\mathrm{IC}_{50} \mathrm{~S}$.

\subsubsection{Identification of CB1954 reduction products}

As the reduction pathway (nitro group preference) taken by each enzyme has already been shown to have a considerable effect on SOS response induction, largely independent of kinetic efficiency (see Chapter 4), metabolite ratios produced upon reduction of CB1954 were analysed, via HPLC, for a selection of the tested enzymes (see last column of Table 5.3; see Appendix 2 for HPLC chromatograms). As was observed previously with the $E$. coli NTRs, all NfsA-family enzymes tested were only able to reduce the $2-\mathrm{NO}_{2}$ to the hydroxylamino derivative, while $\mathrm{NfsB}$-family enzymes produced a mixture of both the 2 and 4-NHOH, albeit in varying ratios. The only exception was YfkO (B.s), which generates only the 4-NHOH, as described previously (Chapter 4). Consistent with these observations, the correlation between $k_{\mathrm{cat}} / K_{\mathrm{m}}$ and SOS induction increased sharply when only the NfsA-family enzymes (2-NHOH producers) were considered (see Figure 5.7B; $\mathrm{R}^{2}=0.85$ and 0.742 , SOS response induction with 10 and $20 \mu \mathrm{M}$ CB1954 respectively).

The most active NTR based on kinetics data was YcnD (B.s); this result was consistent with SOS data and $\mathrm{IC}_{50}$ s. NfsA (V.f), (V.v) and Frp (V.h) were also among 
the top six most catalytically active enzymes (based on $k_{\text {cat }} / K_{\mathrm{m}}$ values), as predicted by both SOS and $\mathrm{IC}_{50}$ s. Furthermore, NfsB (V.v), the lead NfsB-family member in both SOS assay results and $\mathrm{IC}_{50} \mathrm{~s}$, was also the most kinetically active $\mathrm{NfsB}$-family enzyme, with a $k_{\text {cat }} / K_{\mathrm{m}}$ more than 4 -fold higher than the majority of the NfsB-family enzymes.

Table 5.3. Kinetic parameters for the reduction of CB1954 by purified, His6tagged NTRs.

\begin{tabular}{|c|c|c|c|c|c|}
\hline NTR & Co-subs. $^{a}$ & $k_{\text {cat }}\left(\mathrm{s}^{-1}\right)$ & $K_{\mathrm{m}}(\mu \mathrm{M})$ & $\begin{array}{l}k_{\mathrm{cat}} / K_{\mathrm{m}} \\
\left(\mathrm{M}^{-1} \mathrm{~s}^{-1}\right)\end{array}$ & $\begin{array}{l}\mathrm{NO}_{2} \\
\text { pref. }^{b}\end{array}$ \\
\hline NfsA $(\text { E.c.c })^{\mathrm{c}}$ & NADPH & $16 \pm 0.48$ & $220 \pm 18$ & $73,000 \pm 6,400$ & 2 \\
\hline NfsA (S.t) & NADPH & $<1$ & $\mathrm{ND}^{\mathrm{d}}$ & $\mathrm{NA}^{\mathrm{e}}$ & 2 \\
\hline NfsA (C.k) & NADPH & $24.7 \pm 0.96$ & $163 \pm 18$ & $151,700 \pm 17,300$ & $\mathrm{NT}^{\mathrm{f}}$ \\
\hline NfsA (K.p) & NADPH & $37.1 \pm 1.4$ & $1,670 \pm 150$ & $22,270 \pm 2,180$ & 2 \\
\hline NfsA (E.s) & NADPH & $25.7 \pm 1.1$ & $260 \pm 28$ & $98,880 \pm 11,400$ & NT \\
\hline NfsA (V.f) & NADPH & $13.8 \pm 0.7$ & $52.5 \pm 6.8$ & $262,900 \pm 36,500$ & NT \\
\hline NfsA (V.v) & NADPH & $45.2 \pm 1.8$ & $210 \pm 21$ & $215,700 \pm 23,300$ & 2 \\
\hline Frp (V.h) & NADPH & $21.7 \pm 0.7$ & $99.6 \pm 10$ & $217,600 \pm 22,900$ & 2 \\
\hline NfrA (B.s) & NADPH & $14.2 \pm 0.8$ & $147 \pm 23$ & $96,520 \pm 15,900$ & 2 \\
\hline YcnD (B.s) & NADPH & $15.2 \pm 0.4$ & $35.4 \pm 4.1$ & $430,800 \pm 51,300$ & NT \\
\hline $\mathrm{NfsB}(\mathrm{E} . \mathrm{c})^{\mathrm{c}}$ & $\mathrm{NADH}$ & $62 \pm 11$ & $11,000 \pm 2,600$ & $5,600 \pm 1,660$ & $2,4(50: 50)$ \\
\hline NfsB (C.k) & NADH & $72.7 \pm 12$ & $12,200 \pm 2,400$ & $4,920 \pm 1,230$ & NT \\
\hline NfsB (K.p) & NADH & $204 \pm 76$ & $21,140 \pm 8,900$ & $9,630 \pm 5,440$ & $2,4(50: 50)$ \\
\hline NfsB (V.v) & NADH & $72.9 \pm 5.2$ & $1,260 \pm 160$ & $58,000 \pm 8,470$ & $2,4(25: 75)$ \\
\hline FraseI (V.f) & NADH & $12.4 \pm 3.3$ & $903 \pm 12$ & $13,660 \pm 3,640$ & NT \\
\hline YfkO (B.s) & NADPH & $60.2 \pm 2.9$ & $2,440 \pm 250$ & $24,740 \pm 2,840$ & 4 \\
\hline YdgI (B.s) & NADH & $7.68 \pm 0.5$ & $1,780 \pm 170$ & $4,330 \pm 493$ & $2,4(60: 40)$ \\
\hline AzoR (E.c) ${ }^{c}$ & NADPH & $0.15 \pm 0.008$ & $1,400 \pm 160$ & $110 \pm 14$ & 4 \\
\hline $\operatorname{NemA}(\text { E.c })^{c}$ & NADPH & $0.22 \pm 0.003$ & $56 \pm 2.2$ & $3,900 \pm 164$ & 4 \\
\hline
\end{tabular}

${ }^{\mathrm{a}}$ Preferred co-substrates were used for all enzymes at an initial concentration of $0.25 \mathrm{mM}$.

${ }^{b}$ Preferred nitro group (at 2 or 4 position) of CB1954 reduced by each enzyme, as determined by HPLC.

Numbers in parentheses indicate relative percentages of each nitro group reduced

${ }^{\mathrm{c}}$ Native $E$. coli NTR kinetic parameters and nitro group $\left(\mathrm{NO}_{2}\right)$ preference data taken from Chapter 3

${ }^{\mathrm{d}} \mathrm{ND}$ : not detected

eNA: not applicable

${ }^{\mathrm{f}} \mathrm{NT}$ : not tested 

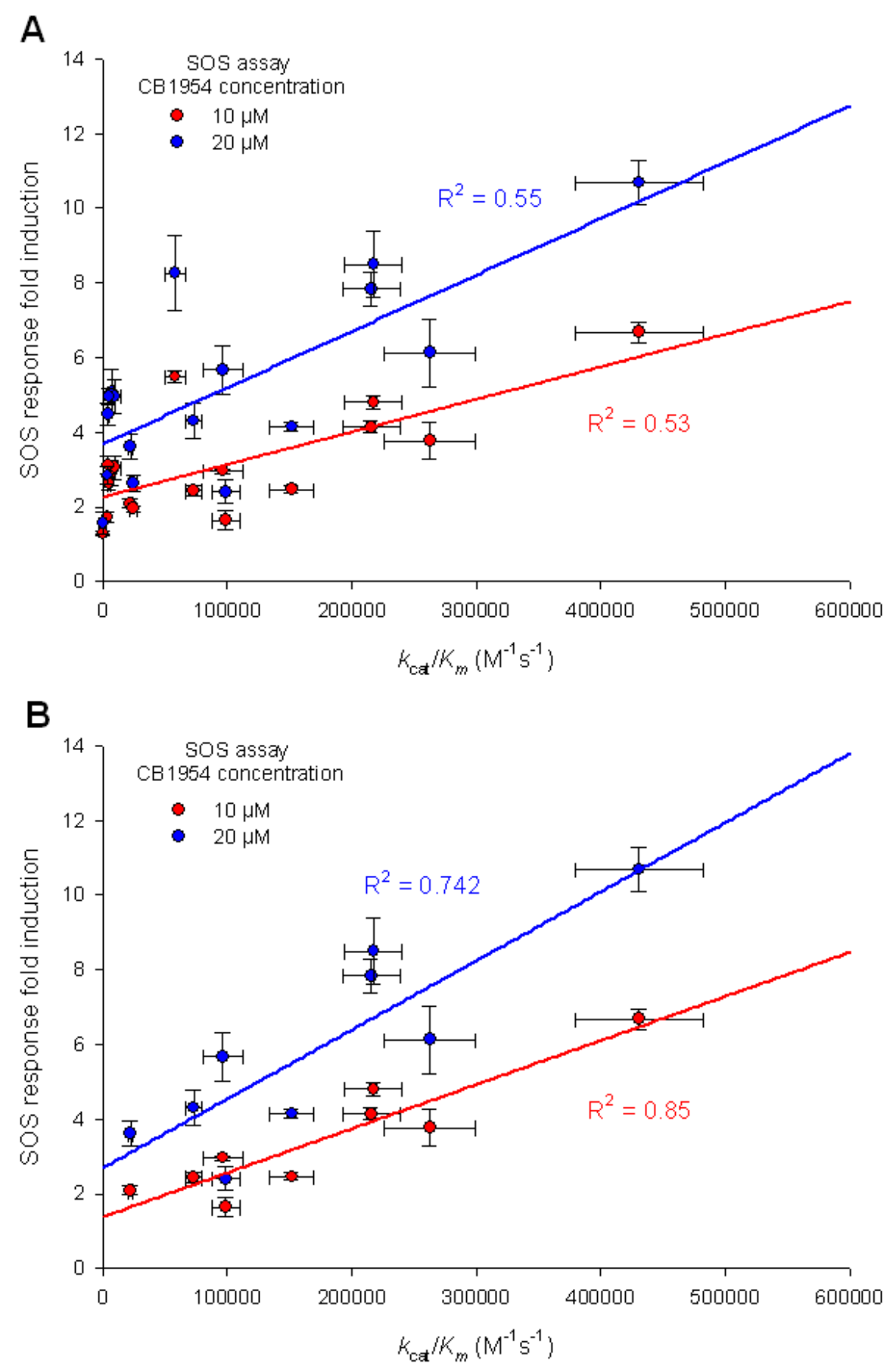

Figures 5.7A \& B. Linear correlations between SOS assay results and purified NTR kinetic efficiency, CB954 as substrate.

Data for SOS assay results were taken from Figure 5.4. Data for kinetic efficiencies (as measured by $k_{\text {cat }} / K_{\mathrm{m}}$ ) were taken from Table 5.3. (A) All NTRs assessed kinetically. (B) Only $2-\mathrm{NO}_{2}$ reducing enzymes. 


\subsubsection{Screening the core library for $P R-104 A$-reductase activity}

\subsubsection{SOS assay}

Following on from CB1954-activity screening, the core library was next subjected to assessment of PR-104A-reductase activity. As previously, the first screen employed was the SOS assay (Figure 5.8). In contrast to CB1954, a family-specific pattern was less evident from these results, with NfsA, NfsB and AzoR families all displaying considerably higher activity than the empty vector control. Furthermore, a selection of individual members from other families (MdaB (E.c), WrbA (P.s), 4975 (P.a) and YcdI (K.p) also elicited responses greater than 1.5-times that of the empty vector with at least one of the prodrug concentrations tested. It is noteworthy that at $50 \mu \mathrm{M}$ PR-104A, the highest concentration tested, considerable background SOS induction was observed, as is illustrated by the almost 3-fold induction of the empty vector control and at least 2 fold induction of the majority ( $>85 \%$ ) of the NTR-over-expressing strains at this concentration. Although background activity was still evident at the lower concentrations tested, the levels were overall much lower, with induction ratios only marginally above 1 for the empty vector control ( 1.1 and 1.36 for 10 and $20 \mu \mathrm{M}$ respectively). As such, only $10 \mu \mathrm{M}$ and $20 \mu \mathrm{M}$ SOS data are considered in subsequent comparison and correlation analyses.

The top 6 SOS-inducing NTRs with PR-104A all belonged to the NfsA family (in the order NfsA (S.t), NfsA (C.k), YcnD (B.s), NfrA (B.s), NfsA (E.c) and NfsA (K.p) at $20 \mu \mathrm{M}$ prodrug). Likewise, the average fold-induction in SOS response across the NfsA family at both concentrations (2.57 and 4.43 at $10 \mu \mathrm{M}$ and $20 \mu \mathrm{M}$ PR-104A respectively) was significantly higher ( $\mathrm{p}<0.05$; $\mathrm{t}$-test) than was the case for both the NfsB (1.78 and 2.70) and AzoR (1.42 and 2.26) families. In contrast, the average NfsB response was only significantly higher than the AzoR family at $10 \mu \mathrm{M}$ PR-104A $(\mathrm{p}<0.05$; t-test).

Within the NfsA family, substantial variation in SOS response was apparent, with fold-induction values ranging from 1.73 to 4.9 at $10 \mu \mathrm{M}$ prodrug and 2.72 to 8.57 at $20 \mu \mathrm{M}$. The most active NfsA family member, and the overall lead NTR from these results, NfsA (S.t), generated SOS responses almost twice that of the average NfsA response and 1.5-fold higher than the next best enzyme. Much less variation was observed within the NfsB family, with fold-induction values for the majority of 
enzymes lying between 1.49 and 1.97 at $10 \mu \mathrm{M}$ and between 2.21 and 3.16 at $20 \mu \mathrm{M}$. The only exception was YdgI (B.s), whose SOS fold induction values of 2.58 and 4.05 were considerably higher than the average NfsB family response, and on a par with the average $\mathrm{Nfs} A$-family response.

\subsubsection{2. $\mathrm{IC}_{50} \mathrm{~S}$}

The SOS data above were next compared to $\mathrm{IC}_{50} \mathrm{~S}$ of PR-104A, obtained for all NTRs in SOS-R2 after a $4 \mathrm{~h}$ PR-104A challenge time. IC $_{50}$ values are listed in Table 5.4. In contrast to SOS data, only 10 of the 20 lowest $\mathrm{IC}_{50}$ s were obtained from NfsA or NfsB family enzymes. In fact, the two lowest overall $\mathrm{IC}_{50} \mathrm{~S}$ came from alternative family enzymes: MdaB (E.c) and AzoR (S.t). On the other hand, NfsA (S.t), which elicited a considerably higher SOS response than any other enzyme tested, was much less active based on $\mathrm{IC}_{50}$ results, producing an $\mathrm{IC}_{50}$ on a par with many of the other NfsA-family enzymes ( IC $_{50}$ of $295 \mu \mathrm{M}$, compared to $292 \mu \mathrm{M}$ for NfsA (E.c) and $290 \mu \mathrm{M}$ for NfsA (C.k); see table 5.4). These discrepancies, among others, were evident in the overall, very weak correlations (for all enzymes) between SOS data and $\mathrm{IC}_{50} \mathrm{~S}\left(\mathrm{R}^{2}=0.263\right.$ and 0.337; see Figure 5.9A). However, much of this lack of correlation could possibly be attributed to the large degrees of error associated with many of the $\mathrm{IC}_{50}$ values, especially those at the higher end (less active), as is evident in the correlation graph. In fact, 21 of the 47 tested enzymes had standard deviations $>20 \%$ of the $\mathrm{IC}_{50}$ value, compared with just one $\mathrm{IC}_{50}$ value meeting this criteria among the CB1954 data. These large errors were likely a result of assay concentrations not reaching adequate levels to induce significant growth inhibition (due to both limited prodrug stocks and higher concentrations required, relative to CB1954), therefore preventing accurate calculation of $\mathrm{IC}_{50}$ s for the less active enzymes. Indeed, by omitting many of these data points, the strength of correlations between $\mathrm{IC}_{50}$ and SOS assay results was substantially increased

(in this case only focusing on NfsA and NfsB-family enzymes, $\mathrm{R}^{2}=0.626$ and 0.670 ; see Figure 5.9B). 


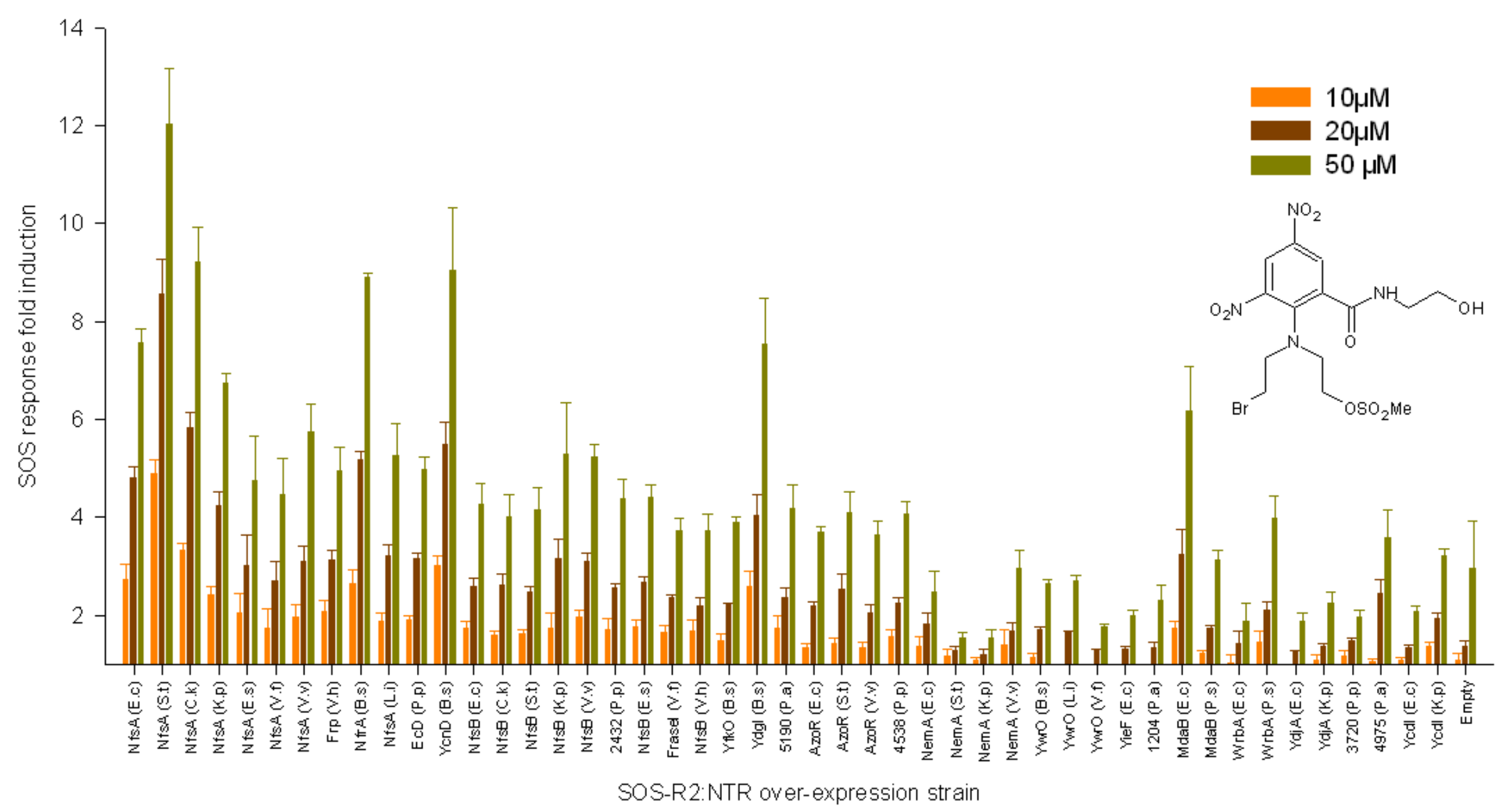

Figure 5.8. PR-104A-induced SOS responses from NTR over-expressing strains of SOS-R2.

NTR over-expressing strains of SOS-R2 were grown and challenged with PR-104A (at the indicated concentrations) for $3 \mathrm{~h}$ prior to analysis of SOS activity. Data is the average \pm SEM of 4 independent assays 
Table 5.4. $\mathrm{IC}_{50} \mathrm{~S}$ of PR-104A in NTR over-expressing strains of SOS-R2.

$\mathrm{IC}_{50}$ values were calculated from growth measurements taken after $4 \mathrm{~h}$ challenge with increasing concentrations of PR-104A.

\begin{tabular}{|c|c|c|c|c|c|c|c|c|c|c|c|}
\hline NTR & $\mathrm{IC}_{50}(\boldsymbol{\mu M})$ & Rank & SOS rank ${ }^{a}$ & NTR & $\mathrm{IC}_{50}(\boldsymbol{\mu M})$ & Rank & SOS rank & NTR & $\mathrm{IC}_{50}(\boldsymbol{\mu M})$ & Rank & SOS rank \\
\hline NfsA (E.c) & 292 & 5 & 5 & NfsB (V.v) & 496 & 27 & 13 & YwrO (B.s) & 492 & 26 & 35 \\
\hline NfsA (S.t) & 295 & 6 & 1 & 2432 (P.p) & 407 & 17 & 20 & YwrO (L.i) & 539 & 39 & 37 \\
\hline NfsA (C.k) & 290 & 3 & 2 & NfsB (E.s) & 520 & 37 & 17 & YwrO (V.f) & 501 & 30 & 45 \\
\hline NfsA (K.p) & 378 & 11 & 6 & FraseI (V.f) & 511 & 33 & 25 & YieF (E.c) & 496 & 28 & 44 \\
\hline NfsA (E.s) & 384 & 12 & 15 & NfsB (V.h) & 516 & 35 & 28 & 1204 (P.a) & 808 & 46 & 43 \\
\hline NfsA (V.f) & 529 & 38 & 16 & YfkO (B.s) & 480 & 25 & 27 & $\operatorname{MdaB}$ (E.c) & 205 & 1 & 8 \\
\hline NfsA (V.v) & 471 & 24 & 14 & YdgI (B.s) & 334 & 9 & 7 & MdaB (P.s) & 385 & 13 & 34 \\
\hline Frp (V.h) & 497 & 29 & 12 & 5190 (P.a) & 601 & 44 & 24 & WrbA (E.c) & 517 & 36 & 39 \\
\hline NfrA (B.s) & 315 & 7 & 4 & AzoR (E.c) & 419 & 19 & 29 & WrbA (P.s) & 346 & 10 & 30 \\
\hline NfsA (L.i) & 511 & 32 & 9 & AzoR (S.t) & 263 & 2 & 21 & YdjA (E.c) & 544 & 40 & 47 \\
\hline $\mathrm{EcD}(\mathrm{P} . \mathrm{p})$ & 433 & 20 & 10 & AzoR (V.v) & 388 & 14 & 31 & YdjA (K.p) & 588 & 43 & 40 \\
\hline YcnD (B.s) & 291 & 4 & 3 & 4538 (P.p) & 449 & 21 & 26 & 3720 (P.p) & 514 & 34 & 38 \\
\hline NfsB (E.c) & 418 & 18 & 19 & NemA (E.c) & 941 & 48 & 33 & 4975 (P.a) & 400 & 16 & 23 \\
\hline NfsB (C.k) & 502 & 31 & 18 & NemA (S.t) & 927 & 47 & 46 & YcdI (E.c) & 321 & 8 & 42 \\
\hline NfsB (S.t) & 465 & 23 & 22 & NemA (K.p) & 585 & 42 & 48 & YcdI (K.p) & 390 & 15 & 32 \\
\hline NfsB (K.p) & 566 & 41 & 11 & $\operatorname{NemA}($ V.v) & 456 & 22 & 36 & Empty & 666 & 45 & 41 \\
\hline
\end{tabular}

${ }^{\mathrm{a}} \mathrm{SOS}$ rankings are taken from $20 \mu \mathrm{M}$ challenge data 

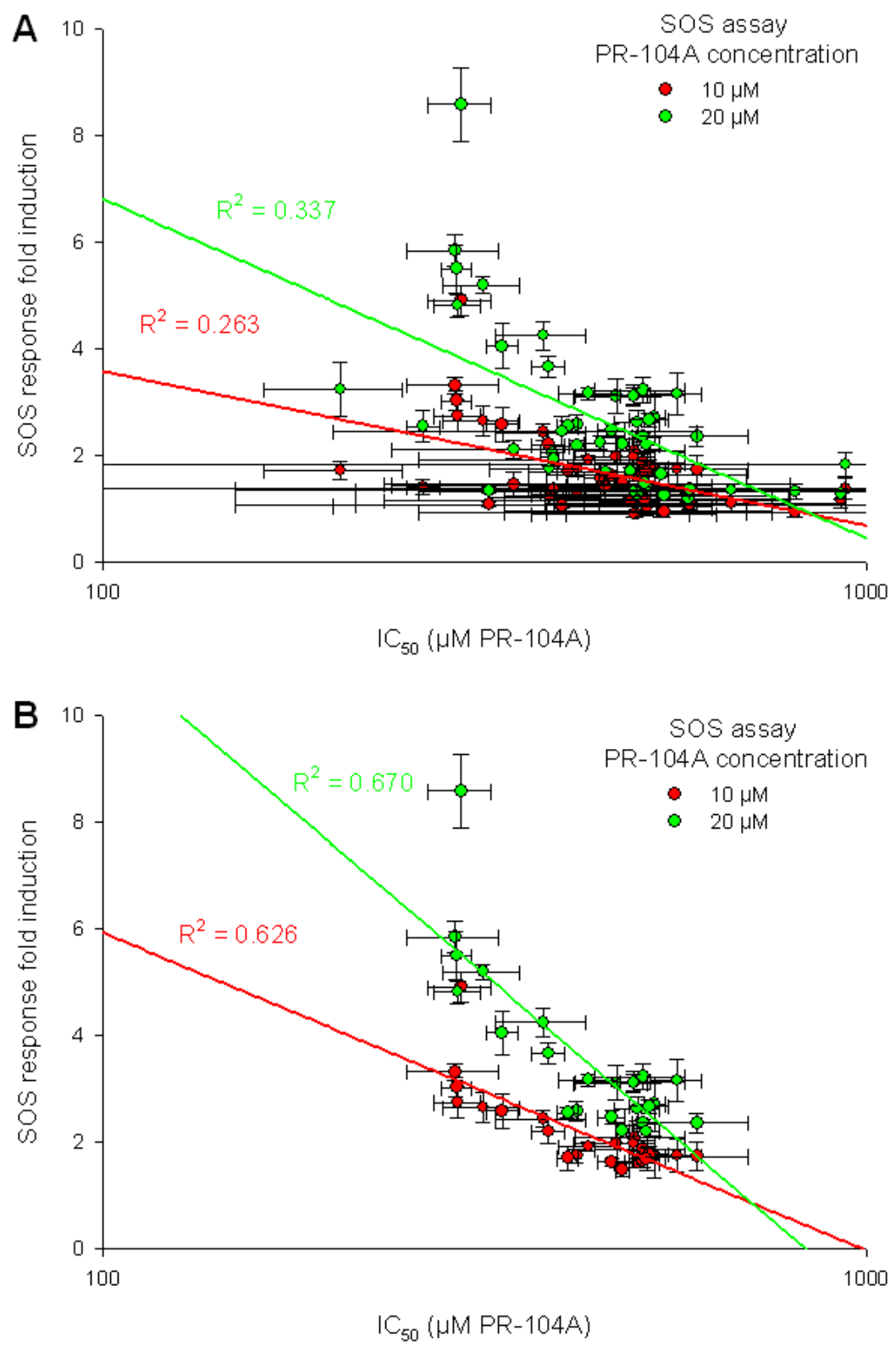

Figures 5.9A \& B. Logarithmic correlations between SOS assay results and $\mathrm{IC}_{50}$ S for PR-104Achallenged NTR over-expressing strains of SOS-R2.

Data for SOS assay results were taken from Figure 5.8. Data for $\mathrm{IC}_{50}$ s were taken from Table 5.4. (A) All NTRs tested. (B) Only NfsA and NfsB-family enzymes. 


\subsubsection{Identification of $P R-104 A$ reduction products}

PR-104A, like CB1954, contains two nitro groups available for reduction (the para and ortho nitro groups, relative to the mustard nitrogen). Unlike CB1954, however, only reduction of the para nitro group yields a toxic derivative. Reduction of the ortho nitro group is accompanied by non-enzymatic intramolecular alkylation and cyclisation to form a non-toxic tetrahydroquinoxaline product (Product Y; see Figure 5.10) (57; 331).<smiles>CO[R6](=O)OCCN(CCBr)c1c(C(=O)NCCO)cc([N+](=O)[O-])cc1C(=O)NCCO</smiles><smiles>[Y]CCN1CCN(O)c2cc([N+](=O)[O-])cc(C(=O)NCCO)c21</smiles>

Figure 5.10. Different routes of reduction of PR-104A.

Nitroreduction of PR-104A can occur via either the para nitro group, to form the DNA alkylating hydroxylamine PR-104H, or the ortho nitro group to form the unstable hydroxylamine $\mathbf{X}$, which subsequently undergoes spontaneous intramolecular alkylation and cyclisation to form the non-toxic tetrahydroquinoxaline product $\mathbf{Y}$. Figure adapted from Paterson et al. (57).

In order to confirm that NTR-mediated reduction of PR-104A was occurring via the desired para nitro group pathway, reaction products obtained from incubation of purified enzymes, co-substrate and prodrug were analysed by HPLC-MS.

All enzymes tested in this manner were able to readily reduce PR-104A to HPLC-detectable metabolites (listed in final column of Table 5.5). No reduction products were observed in the absence of enzyme (see Figure 5.11E). Furthermore, all enzymes, with the exception of NemA (E.c), solely reduced the para-nitro group, as was demonstrated by both identical retention times ( $13.8 \mathrm{~min})$ of the major reaction metabolite with that of the synthetic pure compound (as exemplified by NfsA (E.c); see Figures 5.11A and F), and by mass spectrometry (primary species $\mathrm{m} / \mathrm{z}=485$ and 487 , 
bromine isotope pattern; PR-104H molecular mass $=484$ see Figure 5.11B). In contrast, reaction products formed by NemA (E.c) created no chromotographic peak characteristic of para-nitroreduction (including the hydroxylamine, amine and all minor electrophilic displacement products; see Paterson et al. (57) for details). Instead, a single peak (with low absorbance at $254 \mathrm{~nm}$ ) was observed at around 9 min retention time (see Figure 5.11C). Mass spectrometry indicated a compound with a molecular mass of 388 and a bromine isotope pattern (see Figure 5.11D) - properties matched only by the ortho-nitro group reduction and ring-closed product $\mathbf{Y}$ (see Figure 5.10), among all known products of PR-104A metabolism (Dianne Ferry, personal communication). Therefore it is likely that NemA (E.c) reduces solely the ortho-nitro group of PR-104A, while all other NTRs tested reduce the para-nitro group. 

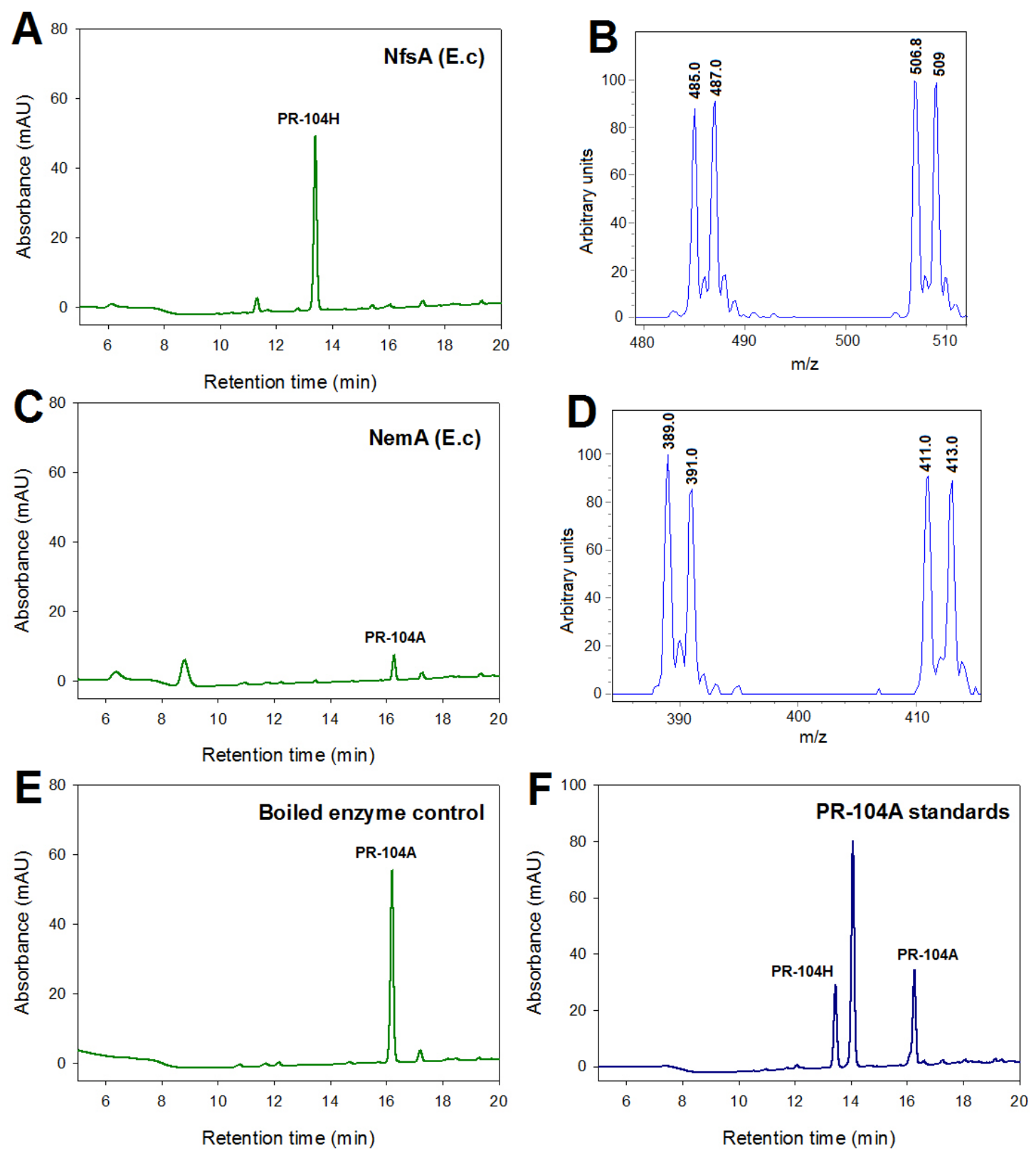

Figures 5.11A-F. HPLC and MS analysis of products of NTR-catalysed reduction of PR-104A.

Reactions containing PR-104A ( $200 \mu \mathrm{M})$, preferred co-substrate $(1 \mathrm{mM})$ and purified His6-tagged NTR (0.5-2 $\mu \mathrm{M})$ were set up and incubated at room temperature for 10-30 min prior to chromatographic separation. (A) Chromatogram of NfsA (E.c)-mediated PR-104A reduction. (B) Mass spectrum of major metabolite, both with and without sodium adducts, identified in (A) at retention time of $\sim 13.8$ min (PR104H). (C) Chromatogram of NemA (E.c)-mediated PR-104A reduction. (D) Mass spectrum of major metabolite identified in (C) at $\sim 9$ min retention time. (E) Chromatogram of reaction products following incubation of substrates with inactivated (boiled) enzyme. (F) Chromatogram of synthetic PR-104A (parental), PR-104H (hydroxylamine) and PR-104M (amine) chemical species (1.5 $\mu \mathrm{M})$. No authentic standards were available for ortho nitro group reduction products. Absorbance for all eluates was monitored at $254 \mathrm{~nm}$. The first 5 minutes are omitted from each chromatogram for clarity $(\mathrm{NAD}(\mathrm{P}) \mathrm{H}$ species eluted during this retention time). 


\subsubsection{In vitro kinetics of purified enzymes}

Assessment of reduction kinetics of PR-104A by purified His6-tagged enzymes was carried out in a similar manner to CB1954. Identification of a wavelength and extinction coefficient at which reduction could be monitored was achieved by comparing the absorption spectrum (from 330 to $550 \mathrm{~nm}$ ) of the parental compound to the reduced para hydroxyamino derivative; the latter being generated by incubation of PR-104A with purified NfsA (E.c) protein and NADPH co-substrate. Excess NADPH in this reaction was removed automatically using the intrinsic low-level NADPH-oxidase activity of NfsA (E.c), by letting the reaction run until no further decrease in absorbance at $340 \mathrm{~nm}$ was evident. PR-104A displayed an absorbance spectrum with a single peak around $350 \mathrm{~nm}$ (see Figure 5.12A), which was lost upon reduction of the para nitro group to the hydroxylamine (PR-104H; see Figure 5.12C). Although not at its absorbance maximum, PR-104A reduction in this study was monitored as loss in absorbance at $400 \mathrm{~nm}\left(\varepsilon_{400 \mathrm{~nm}}=6,000 \mathrm{M}^{-1} \mathrm{~cm}^{-1}\right)$, so as to minimize the impact and overlap of absorbance imparted by the nicotinamide co-substrates and their oxidation products (see Figure 5.12B).

The kinetic parameters of candidate NTRs with PR-104A are listed in Table 5.5. NTR-family specific patterns are evident, including $k_{\text {cat }} / K_{\mathrm{m}}$ values of NfsA-family enzymes being on average almost 5-times higher than those of NfsB-family enzymes. As with CB1954, this difference appeared to be related largely to substrate affinity, based on substantially larger $K_{\mathrm{m}}$ values for NfsB-family enzymes relative to NfsAfamily enzymes (mean $K_{\mathrm{m}}$ s of $1770 \mu \mathrm{M}$ compared to $88 \mu \mathrm{M}$, respectively). A major exception to this relationship was that of $\mathrm{YdgI}$ (B.s), whose $k_{\mathrm{cat}} / K_{\mathrm{m}}$ was on a par with many NfsA-family enzymes. As previously, purified NfsA (S.t) displayed very low reductase activity with PR-104A as substrate. As this was most likely due to issues with the purified enzyme sample and not an intrinsic inability to reduce the prodrug (based on E. coli in vivo results), kinetic results for this enzyme were disregarded in all subsequent correlations and comparative analyses.

No kinetic data was extracted from NemA (E.c) due to its preference in reduction of the ortho nitro group, the product of which displays different spectrophotometric properties to $\mathrm{PR}-104 \mathrm{H}$, and is unsuitable for anti-cancer therapy.

Overall, positive but very weak linear correlations were generated from comparisons of $k_{\text {cat }} / K_{\mathrm{m}}$ values and SOS data across all NTRs tested with PR-104A $\left(\mathrm{R}^{2}=\right.$ 0.247 and 0.242; see Figure 5.13A). Rationale for this poor correlation appeared to be 
largely related to NfsA-family enzymes, as a distinct lack of correlation was observed when data for only these enzymes were compared $\left(\mathrm{R}^{2}<0.01\right.$; data not shown). In contrast, a vast improvement in the degree of correlation between SOS and kinetic results was observed when only NfsB-family enzymes were considered $\left(R^{2}=0.873\right.$ and 0.859; see Figure 5.13B).
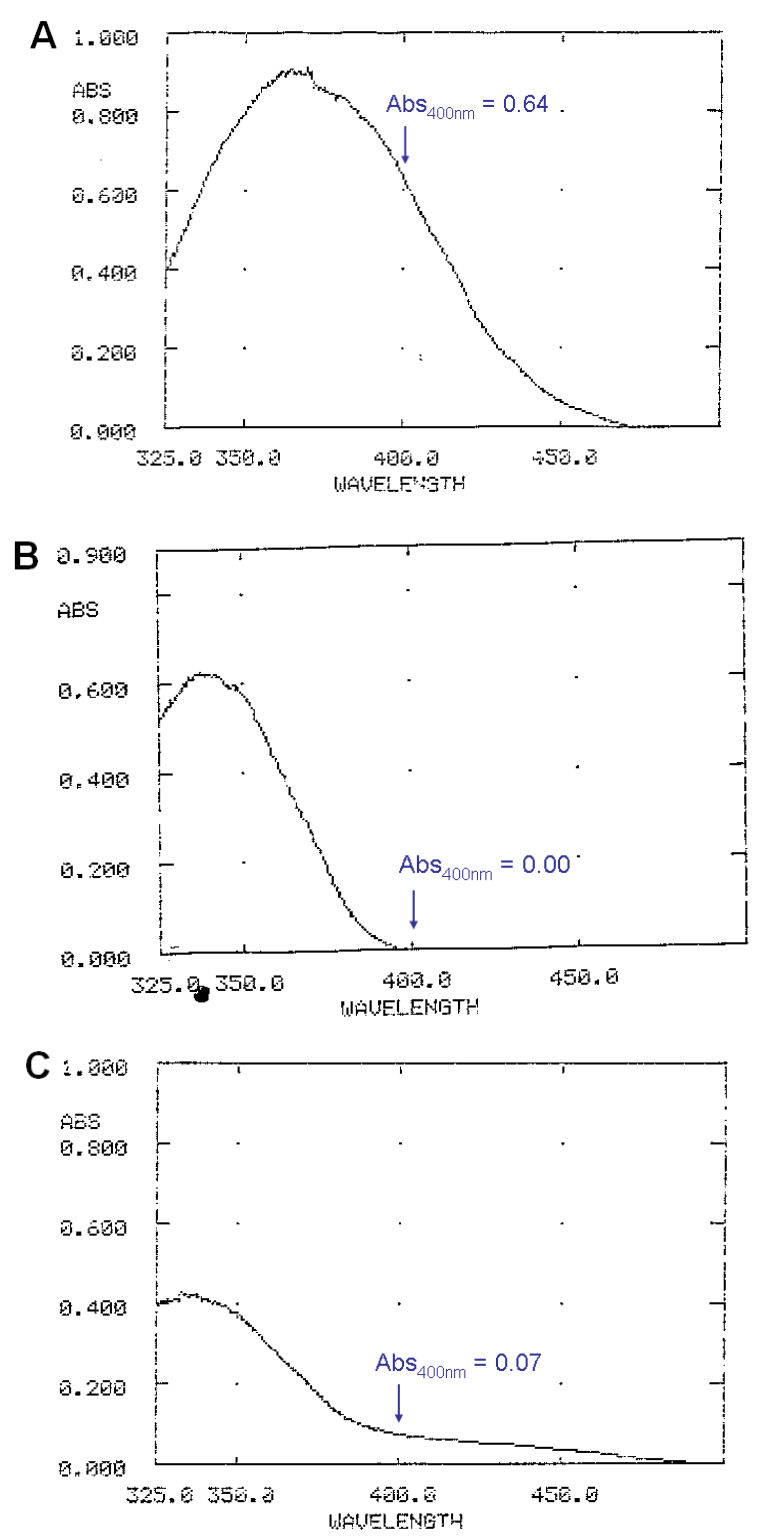

Figures 5.12A-C. Calculation of extinction coefficient for spectrophotometric monitoring of PR104A reduction.

Absorption spectra of (A) $100 \mu \mathrm{M}$ PR-104A, (B) $100 \mu \mathrm{M}$ NADPH and (C) $100 \mu \mathrm{M}$ reduced PR-104A between 325 and $500 \mathrm{~nm}$ wavelength. PR-104A reduction was achieved by incubation of $100 \mu \mathrm{M}$ PR104A, $400 \mu \mathrm{M}$ NADPH and $200 \mathrm{nM}$ NfsA (E.c) at room temperature for $\sim 10 \mathrm{~min}$. The peak at $340 \mathrm{~nm}$ in (C) corresponds to excess NADPH in the reaction. Kinetic analysis of PR-104A reduction was monitored at $400 \mathrm{~nm}$ due to no interference by $\mathrm{NAD}(\mathrm{P}) \mathrm{H}$ or its oxidation products at this wavelength. The extinction coefficient for PR-104A at $400 \mathrm{~nm}$ was calculated to be $6,000 \mathrm{M}^{-1} \mathrm{~cm}^{-1}\left(\mathrm{abs}_{400 \mathrm{~nm}} \mathrm{M}^{-1} \mathrm{~cm}^{-1}\right.$ of PR-104A - abs $400 \mathrm{~mm} \mathrm{M}^{-1} \mathrm{~cm}^{-1}$ of reduced PR-104A), rounded up to the nearest $1,000 \mathrm{M}^{-1} \mathrm{~cm}^{-1}$. 
Table 5.5. Kinetic parameters for the reduction of PR-104A by purified His6tagged NTRs

\begin{tabular}{llllll}
\hline NTR & Co-Subs. & $\boldsymbol{k}_{\text {cat }}\left(\mathbf{s}^{-1}\right)$ & $\boldsymbol{K}_{\mathbf{m}}(\boldsymbol{\mu M})$ & $\boldsymbol{k}_{\text {cat }} / \boldsymbol{K}_{\mathbf{m}}\left(\mathbf{M}^{-1} \mathbf{s}^{-1}\right)$ & NO $_{2}$ pref. $^{\mathbf{a}}$ \\
\hline NfsA (E.c) & NADPH & $12.1 \pm 0.51$ & $101 \pm 11$ & $119,900 \pm 14,000$ & para \\
NfsA (S.t) & NADPH & $<1$ & ND $^{\mathrm{b}}$ & NA $^{\mathrm{c}}$ & para \\
NfsA (C.k) & NADPH & $17.1 \pm 0.92$ & $155 \pm 19$ & $110,300 \pm 14,600$ & NT $^{\text {d }}$ \\
NfsA (K.p) & NADPH & $29.5 \pm 1.9$ & $245 \pm 30$ & $120,400 \pm 16,700$ & para \\
NfsA (E.s) & NADPH & $22.0 \pm 1.3$ & $99.5 \pm 19$ & $220,800 \pm 44,600$ & NT \\
NfsA (V.f) & NADPH & $6.2 \pm 0.18$ & $18.8 \pm 2.0$ & $329,800 \pm 36,400$ & NT \\
NfsA (V.v) & NADPH & $24.4 \pm 0.73$ & $77.7 \pm 7.8$ & $314,000 \pm 32,900$ & para \\
Frp (V.h) & NADPH & $17.6 \pm 0.35$ & $61.3 \pm 4.3$ & $287,800 \pm 21,000$ & para \\
NfrA (B.s) & NADPH & $10.1 \pm 0.39$ & $25.5 \pm 3.8$ & $397,600 \pm 61,200$ & para \\
YcnD (B.s) & NADPH & $5.15 \pm 0.13$ & $10.8 \pm 1.1$ & $479,100 \pm 50,500$ & NT \\
NfsB (E.c) & NADH & $60 \pm 23$ & $4,490 \pm 2,000$ & $13,280 \pm 7,760$ & para \\
NfsB (C.k) & NADH & $34.8 \pm 6.9$ & $2,060 \pm 530$ & $16,880 \pm 5,480$ & NT \\
NfsB (K.p) & NADH & $90 \pm 7.5$ & $1,930 \pm 210$ & $46,610 \pm 6,440$ & para \\
NfsB (V.v) & NADH & $54.5 \pm 3.8$ & $541 \pm 58$ & $100,700 \pm 12,900$ & para \\
YfkO (B.s) & NADPH & $15.1 \pm 6.8$ & $2,537 \pm 1,400$ & $5,970 \pm 4,280$ & para \\
YdgI (B.s) & NADH & $33.7 \pm 1.5$ & $244 \pm 26$ & $137,900 \pm 15,800$ & para \\
MdaB (E.c) & NADPH & $0.14 \pm 0.09$ & $4,290 \pm 3,000$ & $33 \pm 31$ & NT \\
AzoR (E.c) & NADPH & $0.076 \pm 0.017$ & $1,320 \pm 430$ & $57 \pm 23$ & NT \\
NemA (E.c) & NADPH & ND & ND & ND & ortho \\
\hline Nreffred & & & & & \\
\hline
\end{tabular}

${ }^{a}$ Preferred nitro group (para or ortho) of PR-104A reduced by each enzyme, as determined by HPLC (see section 5.4.2.3).

${ }^{b} \mathrm{ND}$ : not detected

cNA: not applicable

${ }^{\mathrm{d}} \mathrm{NT}$ : not tested 


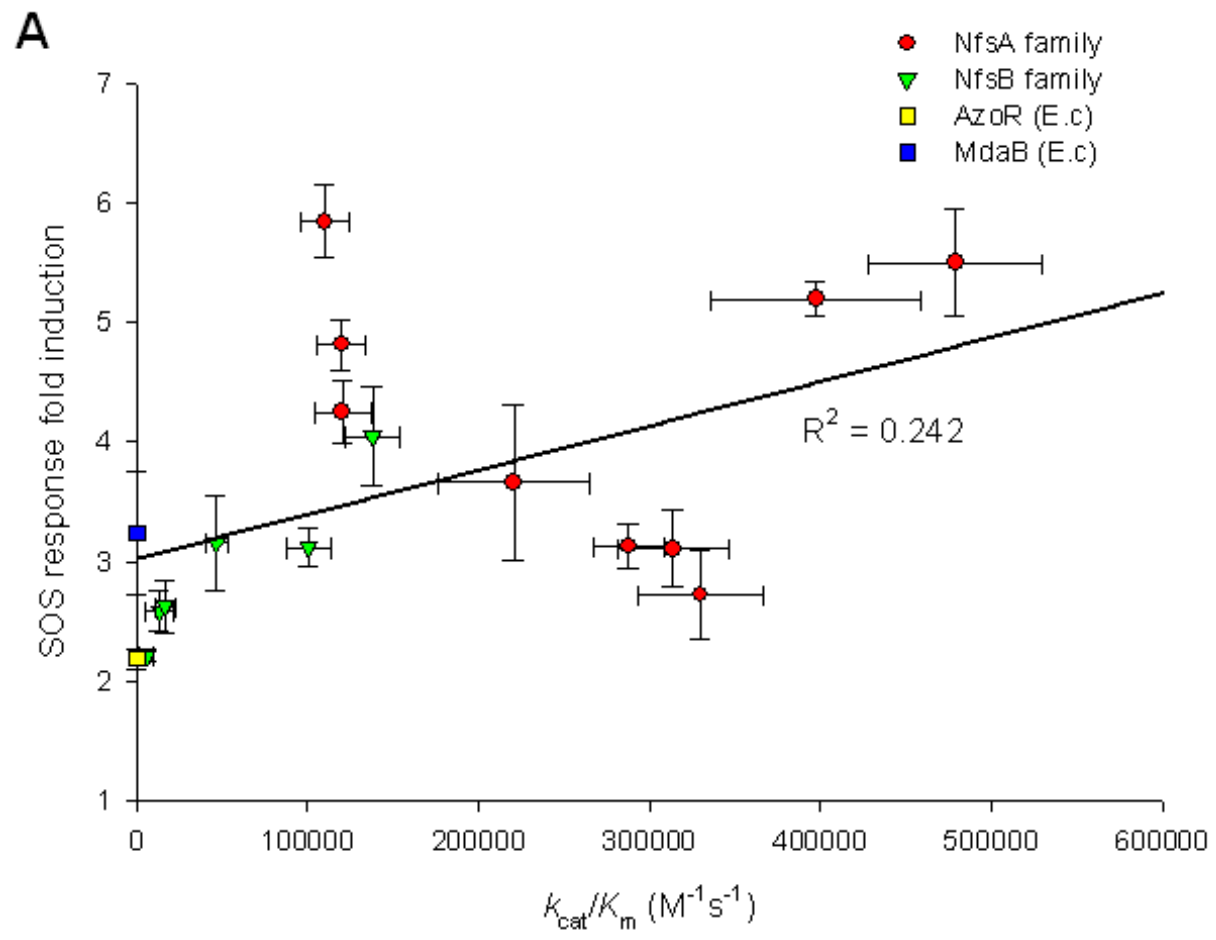

B

SOS assay

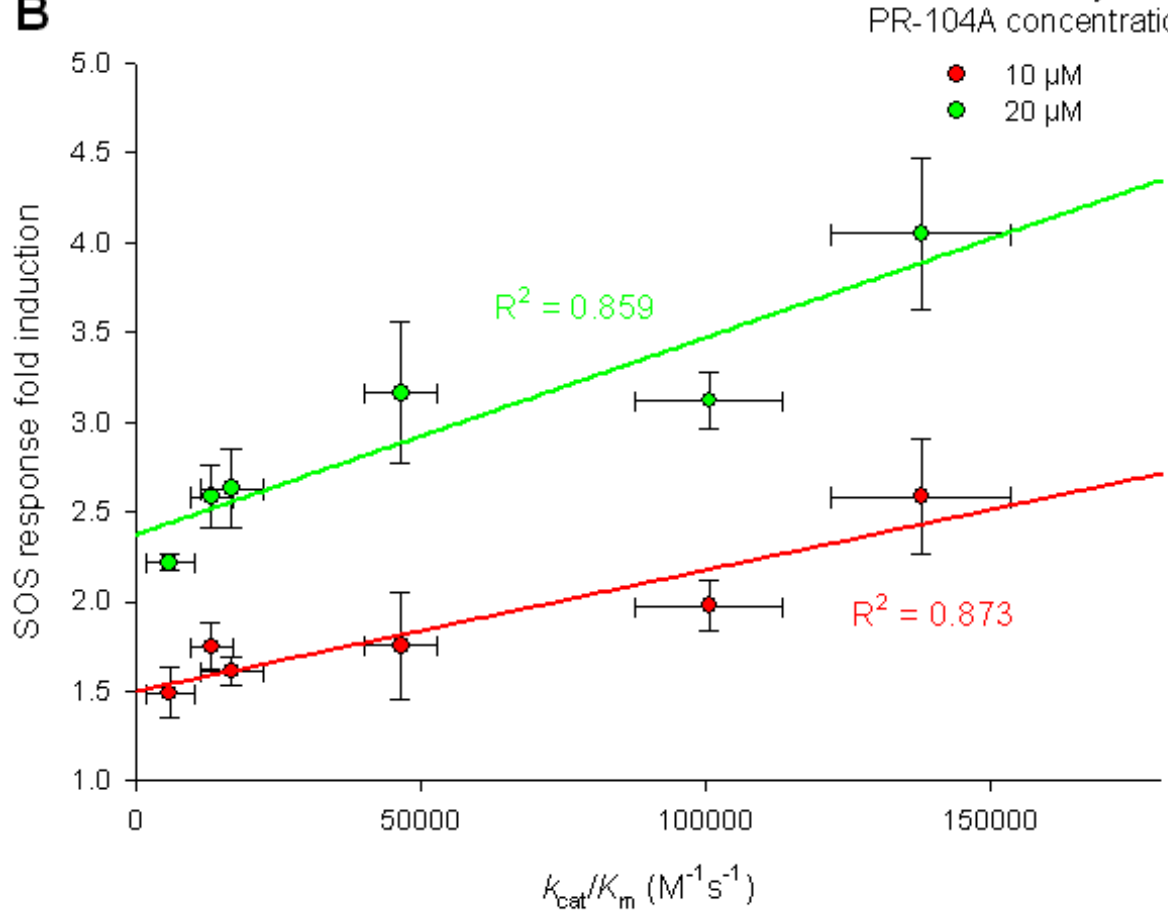

Figures 5.13A \& B. Linear correlation between SOS assay results and purified NTR catalytic efficiency, PR-104A as substrate.

Data for SOS assay results were taken from Figure 5.8. Data for kinetic efficiencies (as measured by $\left.k_{\mathrm{cat}} / K_{\mathrm{m}}\right)$ were taken from Table 5.5. (A) All enzymes tested. Only $20 \mu \mathrm{M}$ SOS data is used for clarity. Enzyme families are colour coded for reference. (B) Only NfsB-family enzymes, with both 10 and $20 \mu \mathrm{M}$ SOS data shown. All correlations are statistically significant $(\mathrm{p}<0.01)$. 


\subsubsection{Activity screening of human AKR1C3 and select core library NTRs with mononitrobenzamide mustard prodrugs}

A significant disadvantage of PR-104A (as well as several other DNBM prodrugs we have tested; results not shown) is its ability to act as a substrate for the aerobic (human) reductase AKR1C3 (62). As previously mentioned, such activity is undesirable for both hypoxia-selective and gene-therapy approaches for the treatment of human tumours, as it is implicated in off-target toxicity and therefore lower therapeutic efficiency.

Structural analogues of DNBMs are currently being investigated in order to identify compounds that demonstrate diminished AKR1C3 activity (Adam Paterson, personal communication). One promising class of compounds is the mononitrobenzamide mustards (MNBMs), which may be less efficient AKR1C3 substrates than their DNBM analogues by virtue of their lower reduction potentials (154) and/or diminished affinity for the AKR1C3 active site (Adam Patterson, personal communication).

In this section, the catalytic efficiency of AKR1C3 towards a panel of MNBM and DNBM prodrugs was experimentally investigated. The open reading frame encoding AKR1C3 was firstly PCR amplified from the vector pF527:AKR1C3 (62) using restriction site-containing AKR1C3-specific primers and cloned into pET28a(+). The enzyme was then over-expressed from pET28a(+) in E. coli BL21, purified to homogeneity and kinetically tested with a selection of DNBM and MNBM prodrugs (shown in Figure 5.14). Results are shown in Figure 5.15 and kinetic parameters listed in Table 5.6. In agreement with theoretical expectations, AKR1C3 was both less catalytically active and had lower substrate affinity for the MNBM prodrugs relative to the DNBMs, with overall catalytic efficiencies for the former being 5-7 fold lower than the latter. 
MNBMs<smiles>O=C(NCCO)c1cc([N+](=O)[O-])ccc1N(CCBr)CCBr</smiles><smiles>CS(=O)(=O)c1cc([N+](=O)[O-])c(C(=O)NCCO)cc1N(CCBr)CCBr</smiles>

SN30444

SN29176<smiles>CO[R5](C)(C)CCN(CCBr)c1c(C(=O)NCCO)cc([N+](=O)[O-])cc1[N+](=O)[O-]</smiles>

PR-104A<smiles>O=C(NCCO)c1cc([N+](=O)[O-])cc([N+](=O)[O-])c1N(CCBr)CCBr</smiles>

SN27686

Figure 5.14. Structures of mono- and dinitrobenzamide mustard prodrugs tested in this section.

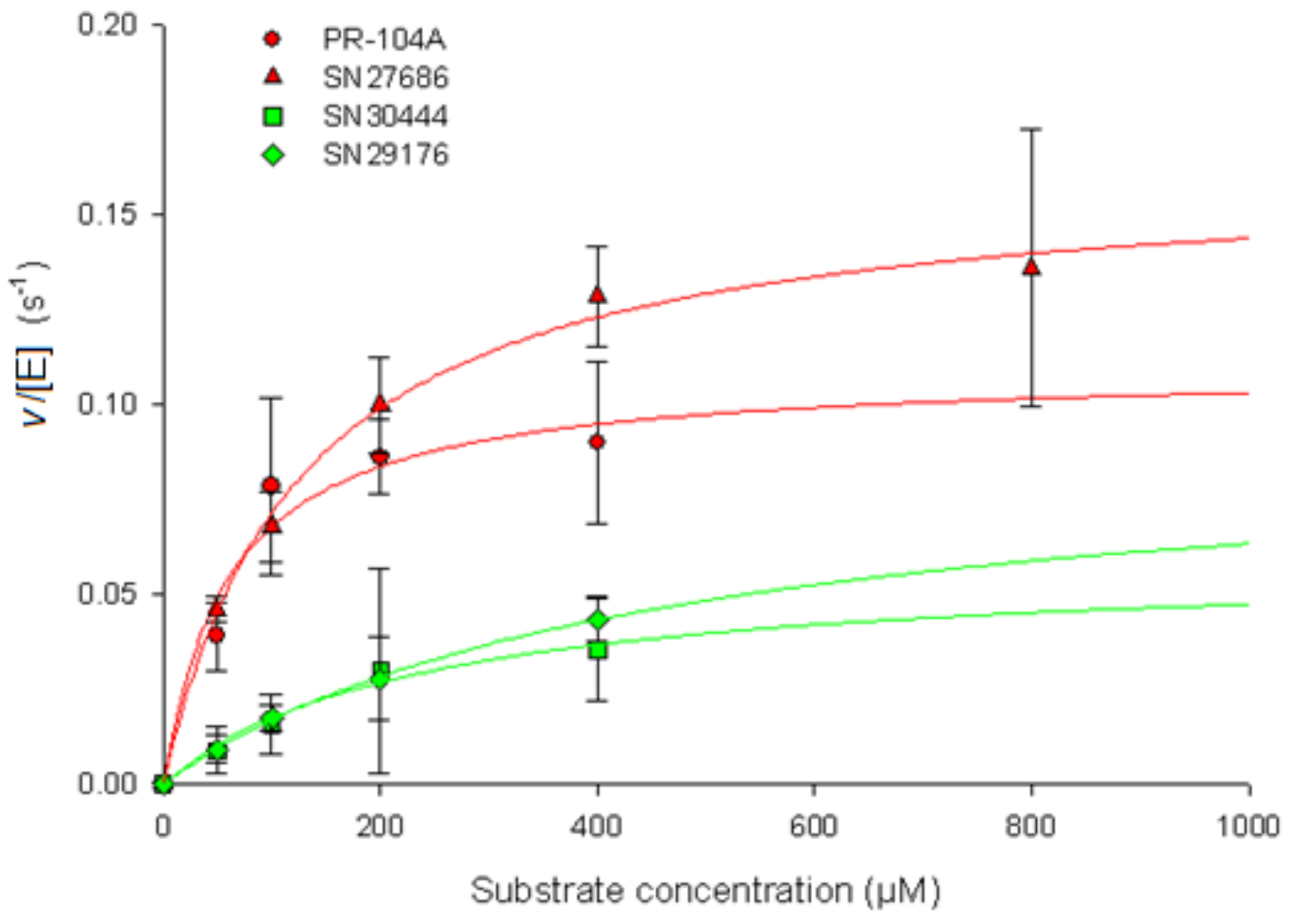

Figure 5.15. Kinetic activity of AKR1C3 with mono- and dinitrobenzamide prodrugs.

Plots of catalytic rate of reduction (initial rate, $v /$ enzyme concentration, [E]) versus substrate concentration for purified, His6-tagged recombinant human AKR1C3 with two examples each of DNBM (red) and MNBM (green) prodrugs. Each data point is the average \pm 1 SD of at least 4 individual replicate assays. Extinction coefficients used for monitoring reduction of prodrugs are as follows: SN 27686: $\varepsilon_{400 \mathrm{~nm}}$ $=7,000 \mathrm{M}^{-1} \mathrm{~cm}^{-1}$; SN 30444: $\varepsilon_{400 \mathrm{~nm}}=7,000 \mathrm{M}^{-1} \mathrm{~cm}^{-1}$; SN 29176: $\varepsilon_{400 \mathrm{~nm}}=4,000 \mathrm{M}^{-1} \mathrm{~cm}^{-1}$. 
Table 5.6. Kinetic parameters for the reduction of selected DNBM and MNBM prodrugs with purified his6-tagged AKR1C3.

\begin{tabular}{cccc}
\hline Substrate $^{\mathbf{a}}$ & $\boldsymbol{k}_{\text {cat }}\left(\mathbf{s}^{-1}\right)$ & $\boldsymbol{K}_{\mathbf{m}}(\boldsymbol{\mu M})$ & $\boldsymbol{k}_{\text {cat }} / \boldsymbol{K}_{\mathbf{m}}\left(\mathbf{M}^{-1} \mathbf{s}^{-1}\right)$ \\
\hline PR-104A & $0.114 \pm 0.007$ & $73.6 \pm 13$ & $1,540 \pm 288$ \\
SN 27686 & $0.162 \pm 0.007$ & $128 \pm 18$ & $1,270 \pm 187$ \\
SN 30444 & $0.059 \pm 0.009$ & $241 \pm 93$ & $244 \pm 101$ \\
SN 29716 & $0.092 \pm 0.02$ & $451 \pm 160$ & $204 \pm 84$ \\
\hline
\end{tabular}

${ }^{a} \mathrm{NADPH}(0.25 \mathrm{mM})$ was used as co-substrate.

Next, the ability of MNBMs to act as substrates for bacterial NTRs, and therefore as potential prodrugs for GDEPT strategies, was assessed by SOS assay using the prototypical MNBM SN 30444. Due to limited prodrug stocks, only a small selection of NTRs were able to be tested; however, results (shown in Figure 5.16) clearly demonstrate that this MNBM can be reductively activated by both NfsA and NfsB-family enzymes, although not by AzoR (E.c) or NemA (E.c).

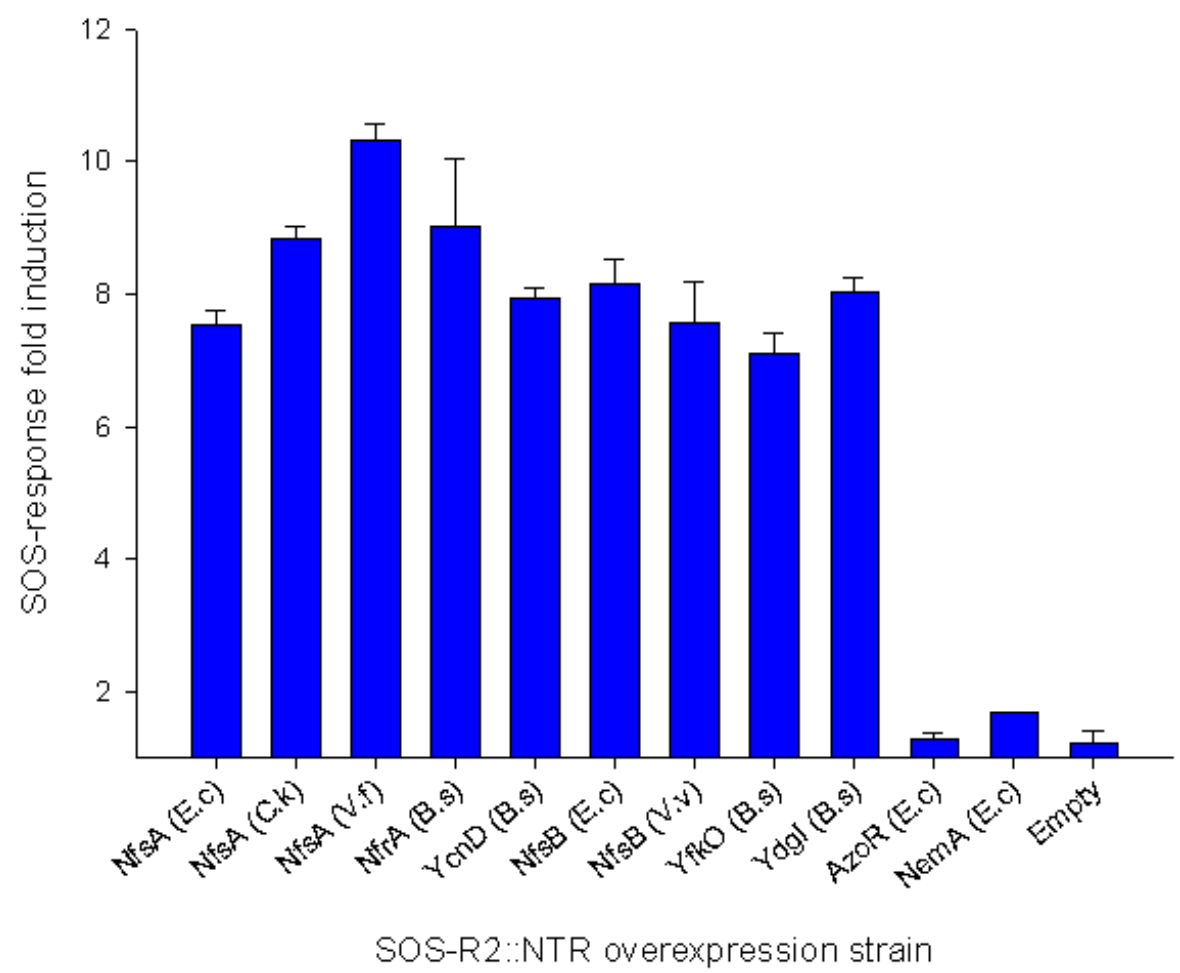

Figure 5.16. SN 30444-induced SOS responses from NTR over-expressing strains of SOS-R2. NTR over-expressing strains of SOS-R2 were grown and challenged with the MNBM prodrug SN 30444 $(10 \mu)$ for $3 \mathrm{~h}$ prior to analysis of SOS activity. Data is the average \pm 1 SEM of 3 independent assays. 


\subsubsection{SOS screening of the core library with alternative nitroaromatic prodrugs}

Many different classes of nitroaromatic prodrugs have been developed for clinical application to date, based on a wide variety of core chemical structures, including nitroimidazoles, nitrochloromethylbenzindolines and nitrobenzamides, among others $(37 ; 44 ; 57 ; 332)$. This section has so far only dealt with those belonging to the latter family, yet the diversity of compounds available suggests that NTR specificity may differ considerably between different chemical classes. Furthermore, the majority of nitroaromatic compounds developed to date have been for hypoxia activation therapy and therefore their application in GDEPT has been largely unexplored $(44 ; 51 ; 332)$. Many of these compounds display distinct properties, such as improved hypoxia selectivity (e.g. the nitrochloromethylbenzindolines; (332)) or radio-sensitising properties (nitroimidazoles; (40)) that could render them more attractive than the nitrobenzamides in certain clinical applications. Therefore it would be beneficial to test the compatibility of such nitroaromatic prodrugs for nitroreduction by diverse bacterial NTRs, in order to assess their utility in GDEPT strategies. To this end, the SOS assay was once again used to evaluate the NTR specificity of two alternative nitroaromatic prodrugs, the 5-NI MTZ and the 2-NI RB 6145.

\subsubsection{MTZ}

MTZ has already been introduced in this study (see Chapter 4), where it was shown to be selectively reduced by certain members of a small panel of NTRs, the products of which were detectable by SOS assay. Screening this compound against the full core library therefore allowed greater understanding of NTR specificity.

Results of SOS assay screening are presented in Figure 5.17. Both NfsA- and NfsB-family enzymes readily reduced MTZ; however no NTR from any of the other represented families elicited SOS responses above background levels. Even within the $\mathrm{NfsA}$ and NfsB families, a wide variety of SOS responses was elicited, ranging from less than 2-fold, to 7-fold above unchallenged levels at the lowest concentration of MTZ tested $(1.25 \mu \mathrm{M})$. Mean responses between NfsA and NfsB families, at both MTZ concentrations tested, did not significantly differ (e.g. mean SOS fold inductions of 5.47 for the NfsA family and 4.26 for the NfsB family, for $2.5 \mu \mathrm{M}$ MTZ challenge data; p>0.05; t-test), demonstrating broad acceptor specificity for this compound. The highest 
overall responses were obtained from NfsB (V.h), although these did not differ considerably, especially at the lower concentration tested, from those of $\mathrm{YcnD}$ (B.s) and NfsA (V.f).

\subsubsection{RB 6145}

RB 6145 is a hypoxia-activated nitroimidazole prodrug that has previously been investigated as a sole agent for use in clinical anti-cancer therapy (43). No reports exist however (to the best of our knowledge) on the ability of this prodrug to act as a substrate for bacterial NTRs.

Results from SOS assays employing RB 6145 and the core library of NTRs demonstrated that NfsA-family enzymes were (on average) the most active RB 6145reductases (e.g. mean SOS fold inductions of 5.5 for the NfsA family and 3.0 for the NfsB family, for $5 \mu \mathrm{M}$ RB 6145 challenge data; see Figure 5.18). In particular, NfsA (S.t) and Frp (V.h) induced SOS responses substantially higher than any other overexpressed NTR. With a couple of exceptions (WrbA (E.c) and YfkO (B.s), $5 \mu \mathrm{M}$ RB 6145), no other NTR induced an SOS response more than 1.5-fold that of the empty vector control. 


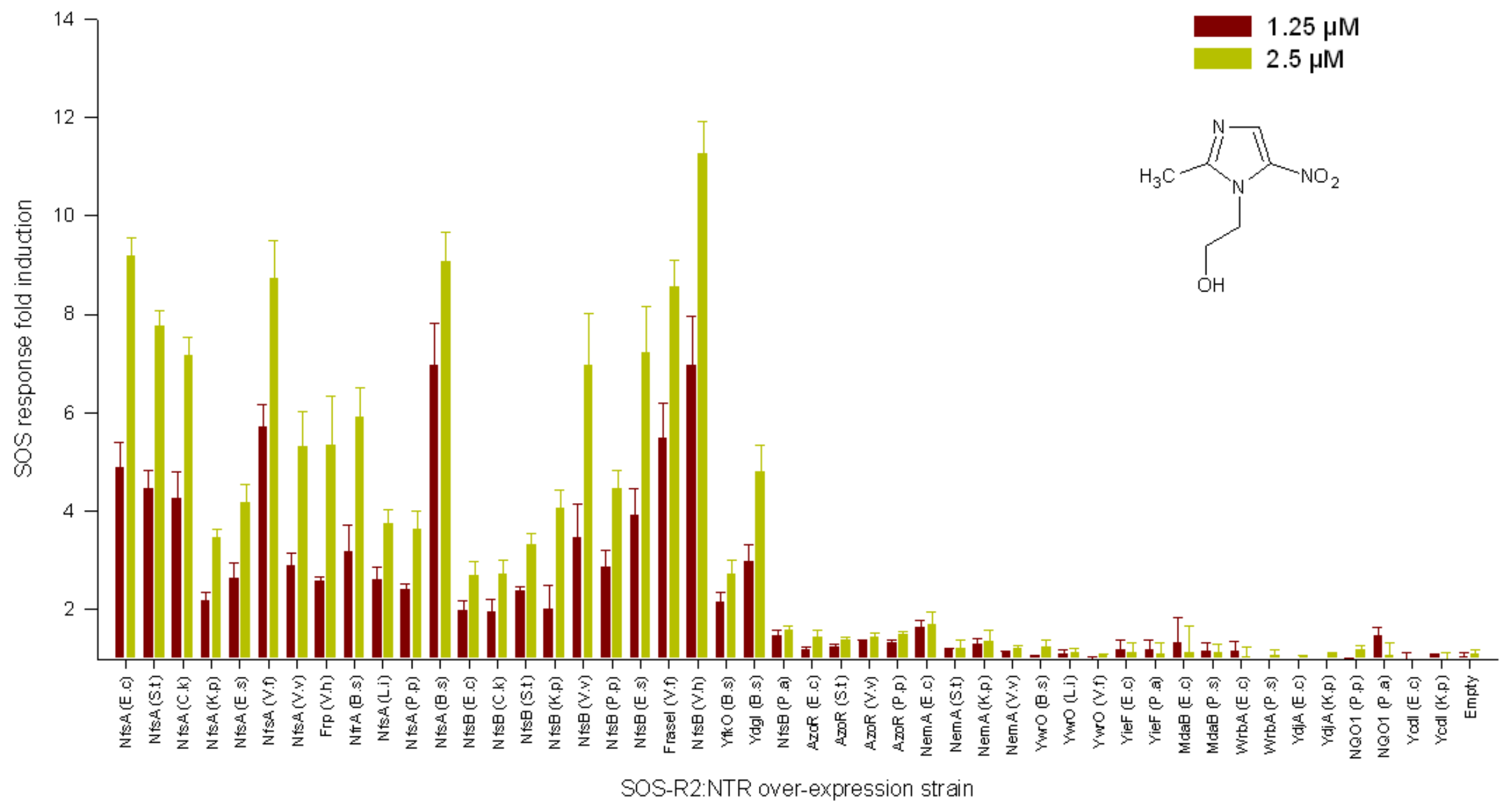

gure 5.17. MTZ-induced SOS responses from NTR over-expressing strains of SOS-R2.

NTR over-expressing strains of SOS-R2 were grown and challenged with MTZ (at the indicated concentrations) for $3 \mathrm{~h}$ prior to analysis of SOS activity. Data is the average \pm 1 SEM of 4 independent assays. 


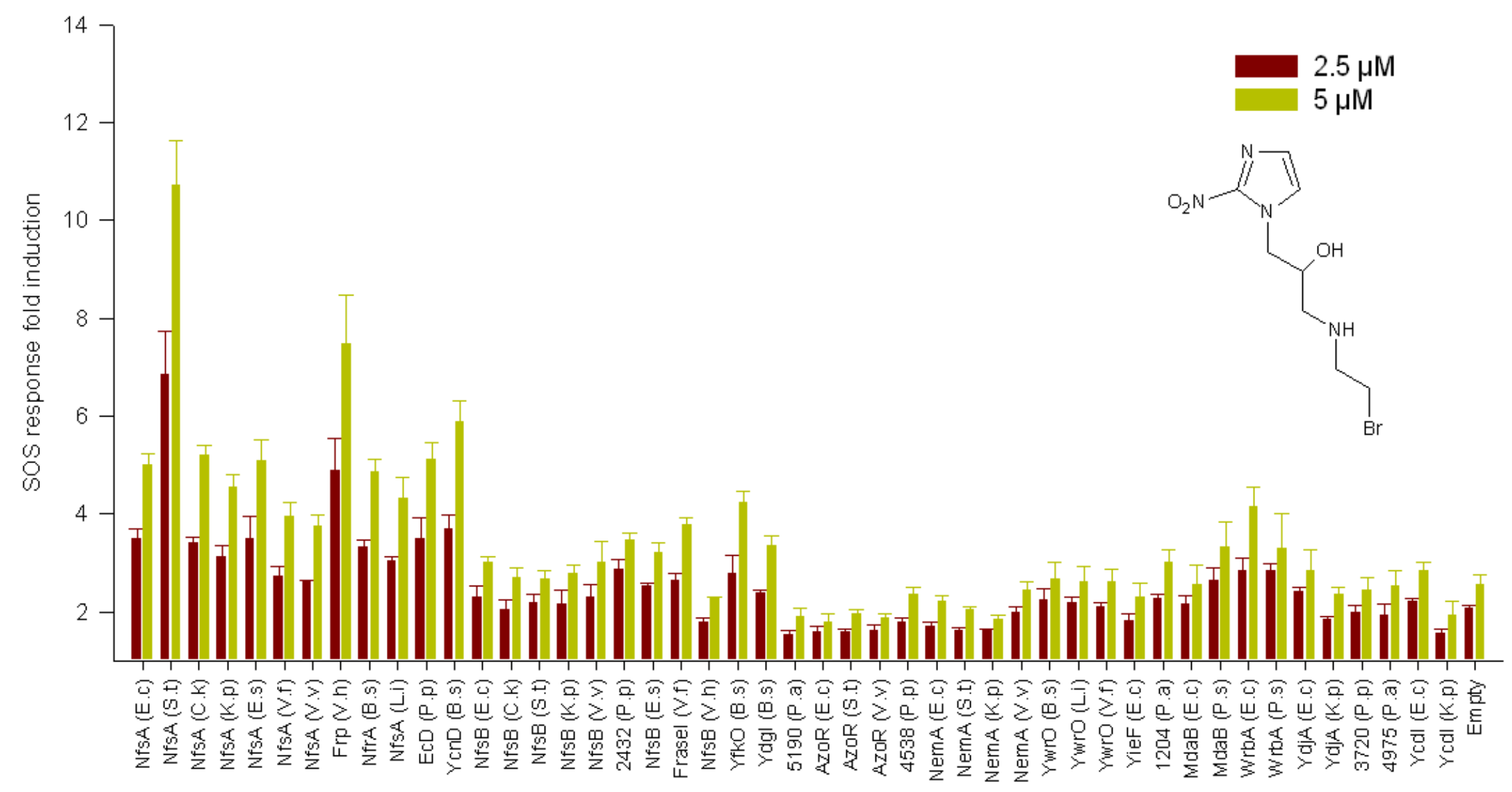

SOS-R2:NTR overexpression strain

Figure 5.18. RB 6145-induced SOS responses from NTR over-expressing strains of SOS-R2.

NTR over-expressing strains of SOS-R2 were grown and challenged with RB 6145 (at the indicated concentrations) for 3 h prior to analysis of SOS activity. Data is the average $\pm 1 \vec{f}$ SEM of 3 independent assays 


\subsection{Discussion}

The ultimate aim of this section was to screen multiple, phylogenetically diverse NTRs for their ability to reduce a variety of nitroaromatic prodrugs, in order to identify lead candidate enzymes that would form the basis of further improvement work via enzyme engineering and directed evolution.

With CB1954 as substrate, correlations between results from the different assays employed were generally strong $\left(R^{2}>0.7\right)$, with the overall most efficient enzyme across all screens being the NfsA-family member YcnD (B.s). Other NfsA-family members, including NfsA (V.f), NfsA (V.v) and Frp (V.h) also featured within the top six of each screen, demonstrating a clear NfsA family-superiority towards CB1954, consistent with results from Chapter 3 and elsewhere (189). It is noteworthy that these latter three enzymes are all from Vibrio species and consequently share close protein sequence homology with one another ( $>80 \%$ sequence similarity). This therefore suggests a structural basis for the improvement in CB1954 activity over, for example, NTRs with closer homology to NfsA (E.c) (e.g. NfsA (S.t), NfsA (C.k), NfsA (K.p); see Figure 5.2).

NfsA family enzymes also induced the highest SOS response and were the most catalytically active (highest $k_{\text {cat }} / K_{\mathrm{m}}$ ), on average, towards the DNBM PR-104A. However, in contrast to CB1954, no significant level of correlation existed between SOS assay results and $k_{\text {cat }} / K_{\mathrm{m}}$ values of the NfsA-family members with PR-104A as substrate $\left(\mathrm{R}^{2}<0.01\right)$, leading to greater ambiguity in overall enzyme rankings. Furthermore, SOS assay results did not correlate with purified enzyme $k_{\text {cat }}$ values $\left(\mathrm{R}^{2}<\right.$ 0.01 ); positive correlations have previously been observed between toxicity of several DNBMs in an NfsB (E.c) over-expressing cancer cell line and the $k_{\text {cat }}$ value of the purified enzyme with each substrate (151). In the absence of additional mammalian cell PR-104A toxicity data for these enzymes, it is difficult to ascertain which set of results (SOS or kinetics) to be the more globally meaningful. Although $\mathrm{IC}_{50}$ values for each over-expressed NfsA-family NTR correlated more closely with SOS results than kinetics (data not shown), this was likely due to the shared $E$. coli in vivo conditions employed for each assay. Nevertheless, the ability of each NfsA-family NTR to induce strong, dose-dependent SOS responses with PR-104A as substrate indicates that discrepancies between SOS results and kinetics between NfsA-family members should not impact on the ability to use the SOS assay for screening of improved mutants of a specific NfsA-family enzyme. 
Unlike the NfsA family, NfsB-family enzymes displayed a strong correlation between SOS and kinetics results with both CB1954 and PR-104A as substrates. Although ultimately less catalytically efficient in terms of $k_{\text {cat }} / K_{\mathrm{m}}$ than their NfsA counterparts, NfsB-like enzymes may be preferable as therapeutic proteins for gene therapy, depending on the administered prodrug and target cell type. For example, despite a lack of mammalian cell data in this chapter, results from Chapter 3 indicate that NfsB (E.c) performs better than NfsA (E.c) with CB1954 in the colon carcinoma cell line HCT-116. Furthermore, despite substantially lower in vitro purified enzyme activity measurements, NfsB-family enzymes elicited SOS responses with CB1954 and PR-104A only marginally lower than those of NfsA-family enzymes; these observations suggest that factors independent of catalytic ability may affect enzyme activity in vivo (for example co-substrate availability, or the reduction pathway taken), as has been discussed previously. Whatever the bases of these observations are, results here have demonstrated that NfsB-family enzymes that are superior to the well characterised $E$. coli representative exist, and could therefore provide improved alternatives for clinical use. Of particular note is NfsB (V.v), which produced the highest SOS response inductions out of all NfsB enzymes for CB1954 (at a level comparable to many NfsA enzymes) and also ranked among the highest for both PR-104A and MTZ activity by this same assay. Furthermore these results were validated at the purified enzyme level, illustrating the clear catalytic superiority of this enzyme among the NfsB-family members. The relatively high sequence homology of this enzyme to more well characterised enzymes (such as NfsB (E.c); 61\% identity, $75 \%$ similarity), for which multiple crystal structures and biochemically characterised mutants are available, could also facilitate investigations into specific residues involved in prodrug recognition and catalysis; this being fundamental knowledge required for any attempts at further improvement of enzyme activity through rational protein engineering techniques.

Another interesting observation from the results of this chapter was the alternative pathway taken by NemA (E.c) in the reduction of PR-104A. While all other enzymes clearly reduced the para nitro group of this compound to the hydroxylamine, HPLC-MS tentatively identified the major metabolite of NemA (E.c)-mediated reduction as being the tetrahydroquinoxaline product $\mathbf{Y}$ (see Figure $5.10 ; \mathrm{m} / \mathrm{z}=388$, positive scan mode), which is known to form spontaneously from the orthonitroreduction product of DNBM prodrugs via intramolecular alkylation and cyclisation (331). Furthermore, SOS assay and $\mathrm{IC}_{50}$ results with NemA (E.c) were roughly consistent with the formation of a product less toxic than both the para hydroxylamine 
and the parental compound itself (lower SOS response induction and higher $\mathrm{IC}_{50}$ relative to the empty vector control; see Figure 5.8 and Table 5.4). As it has been well established that the tetrahydroquinoxaline is of a very low cytotoxic potency by virtue of the loss of bifunctionality, these results support an ortho-nitroreduction mechanism of action for NemA (E.c). This is of interest as no specific enzyme able to uniquely reduce the ortho nitro group of a DNBM has previously been identified, and suggests a novel substrate-binding and catalytic mechanism for this enzyme. Furthermore, although ultimately ineffective as a PR-104A activating enzyme for tumour cell targeting, its ability to deactivate this prodrug, and potentially other DNBMs, could render it useful in gene therapy approaches where protection from prodrug activation is the desired outcome. In this way, NemA (E.c) could be over-expressed in certain healthy non-malignant tissue types that are particularly prone to off-target toxicity from alkylating agents (for example the bone marrow and liver), therefore enhancing resistance of these tissues to the prodrug. Such strategies have previously been trialled in vitro with moderate success, for example through the expression of the multi-drug resistance gene MDR-1 in hematopoietic stem cells (333), therefore demonstrating the feasibility of such an approach.

Other than the nitrobenzamide prodrugs already discussed, this study also investigated the ability of NTRs to activate two nitroimidazole prodrugs: MTZ and RB 6145. The first, MTZ, is most commonly employed as an antibiotic for the treatment of anaerobic pathogens; however MTZ has also been studied in the context of targeted cell ablation in developmental studies, in combination with NfsB (E.c) as an activating enzyme. MTZ is an ideal prodrug in this context due to its distinct lack of bystander effect $(54 ; 114)$, permitting highly specific targeted cell killing. Furthermore, lack of bystander-mediated cell killing has also been proposed as an attractive property for specific ablation of tumour vasculature in the treatment of cancer (334). Results shown here indicate that NfsB (E.c), although a proven effective MTZ reducing enzyme, is not the most efficient NTR available. In fact, NfsB (E.c), along with the very close relative NfsB (C.k), induced the lowest SOS activity out of all NfsA and NfsB-family enzymes tested. Therefore significant improvements in MTZ-based gene therapy could potentially be achieved through the use of an alternative NTR, such as NfsB (V.h) or YcnD (B.s).

The second prodrug, RB 6145, is a 2-NI, which along with the structurally and mechanistically similar RSU1069, has been extensively studied as a hypoxia-activated cytotoxin $(37 ; 39 ; 43)$. Although ultimately ineffective as anti-cancer agents due to their 
high systemic cytotoxicity, their structural relatedness to another class of compounds, the 2-NI positron emission tomography (PET) imaging agents, could render them useful as model drugs for the study of NTR-mediated activation of the latter. 2-NI PET imaging agents, such as F-MISO (335) and EF5 (336) are hypoxia-selective compounds that once reduced, are retained intracellularly due to reactivity with cellular macromolecules. In this way their mechanism of action is akin to the hypoxia-activated prodrugs described previously, except that they lack DNA alkylative activity, and therefore toxicity. They can however be radio-labelled via their incorporated fluorine groups, enabling PET imaging of their presence and location within a patient, once reduced, without the requirement of invasive procedures (337). The ability of these compounds to also undergo NTR-mediated reduction, as demonstrated here, could permit their use as imaging agents to assess the temporal and spatial aspects of transgene expression, in GDEPT procedures, prior to prodrug administration. However, their lack of DNA-reactivity renders them ineffective as substrates for the SOS assay, or any toxicity-based screen. As results described in this chapter show that RB 6145 activation can be effectively monitored by way of the SOS assay, this compound could be used as a replacement molecule, in order to employ the SOS assay as a screening procedure, to indirectly assess NTR-mediated reduction of 2-NI imaging agents. Validation of this approach is currently ongoing in the Ackerley laboratory.

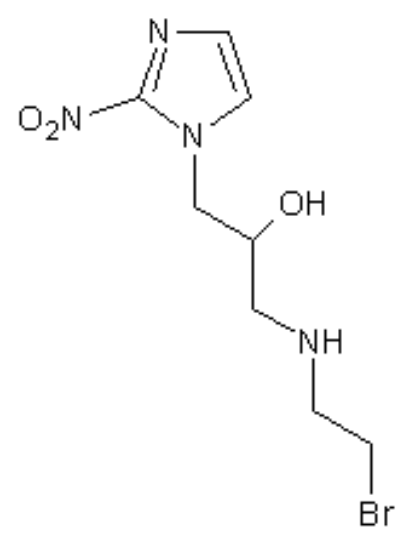

RB6145

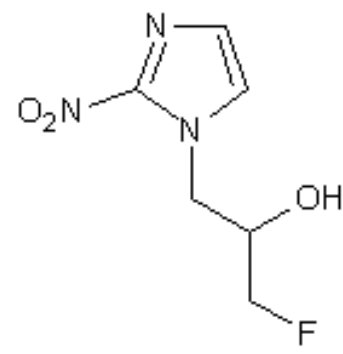

F-MISO

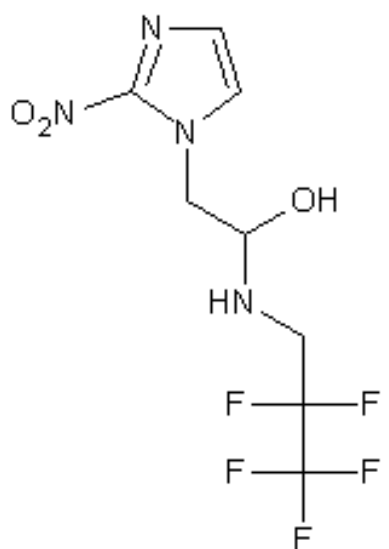

EF5

Figure 5.19. Structures of RB 6145 and selected 2-NI imaging agents.

In summary, NfsA-family enzymes were found to be the most active NTRs with all prodrugs tested in this section, across all screens employed. More specifically, YcnD (B.s) was the overall superior enzyme with CB1954 as a substrate, followed by NfsA (V.f), (V.v) and Frp (V.h), while it was less clear as to the identity of the lead enzyme 
with PR-104A as substrate. However, YcnD (B.s) had the highest $k_{\text {cat }} / K_{\mathrm{m}}$ and produced a substantial SOS response with this prodrug, suggesting this enzyme as a lead candidate for further mutagenesis and enzyme engineering studies. Furthermore, this enzyme displayed high SOS activity with the 5-NI nitroaromatic prodrug MTZ and moderate SOS activity with the 2-NI RB 6145. Alternatively, NfsA (S.t), which induced the highest SOS response for PR-104A and RB 6145 could equally be considered a lead NTR candidate, despite an inability to extract active purified enzyme of this NTR. 


\section{Chapter 6: Improving the PR-104A-reductase activity of Bacillus subtilis YcnD: Saturation mutagenesis of the active site.}

\subsection{Introduction}

The previous chapter characterised the nitroaromatic prodrug reductase activity of a variety of bacterial NTRs. In this chapter, one of the most active of these NTRs, YcnD (B.s), was selected for further enhancement of PR-104A reductase activity through sitedirected mutagenesis of the enzyme active site. Protein mutagenesis, when coupled with an effective screening or selection process, is a powerful tool for enhancing specific properties of an enzyme, such as catalytic efficiency. This has previously been shown to be an effective means of maximising rates of prodrug activation by GDEPT enzymes $(156 ; 213 ; 218 ; 223 ; 338)$. The only prior mutagenesis work carried out for NTR GDEPT has focused on NfsB (E.c) with CB1954 as substrate $(218 ; 221 ; 225)$; this chapter therefore presents the first reported case of mutagenesis of an NfsA-family enzyme towards improved activity with a DNBM prodrug.

YcnD (B.s) was chosen for mutagenesis in this chapter due to its high level of native reductase activity towards a variety of nitroaromatic prodrugs (including CB1954, PR-104A, MTZ and RB 6145, as determined by SOS assay and in vitro purified enzyme kinetics). PR-104A was selected as the substrate towards which enzyme activity was to be screened and enhanced due to its greater clinical appeal (increased potency, hypoxia activation, strong bystander effect) than CB1954 and many other nitroaromatic anticancer prodrugs. Lastly, site-directed mutagenesis of active site residues was used to create enzyme variants, as opposed to other commonly used methods of enzyme engineering such as full sequence random mutagenesis or DNA shuffling, for several reasons: a) previous work has demonstrated site-directed mutagenesis to be effective in generating mutant NfsB (E.c) variants with improved CB1954-reductase activity $(218 ; 225)$; b) a crystal structure in complex with the flavin co-factor was available for YcnD (B.s) (194) allowing insight into active site architecture; and c), the lower number of sites targeted with SDM techniques compared to full gene sequence mutagenesis is compatible with the lacZ SOS screen developed in this work, which is more amenable to "medium" than high throughput screening applications. 
In summary, this chapter investigated the effects of individually mutating 10 active site residues of YcnD (B.s), identified by structural analysis, on the PR-104A reductase activity of this enzyme. Double site mutants, created through pairwise combination of the most active single mutations, as identified by SOS assay, were subsequently tested for further PR-104A activity improvement by SOS assay. A selection of the overall most active $\mathrm{YcnD}$ (B.s) variants were finally subjected to $\mathrm{IC}_{50}$ and in vitro kinetic analysis with PR-104A and SOS response induction with alternative nitroaromatic prodrugs.

\subsection{Results}

\subsubsection{Generation of a positive selection vector}

PCR-based mutagenesis techniques, such as the one used in this chapter, typically generate a pool of variant gene sequences in a single PCR reaction, by substituting the codon of interest with a primer-encoded degenerate oligonucleotide sequence. This pool of gene variants is then cloned into the desired expression vector, transformed into the host organism, and a suitable number of colonies picked and screened to ensure coverage of all possible mutations. When using restriction enzyme and ligase-based cloning techniques it is often the case that a proportion of transformants carry vectors that either have no insert (due to, for example, degradation of restriction enzymegenerated 'sticky' DNA ends by contaminating exonucleases, followed by re-annealing of blunt ended DNA (339)) or that are the parental plasmid (due to insufficient restriction digestion prior to insert ligation). To account for the likely presence of these undesirable products, library sizes need to be increased, greatly enhancing workload and substrate usage during screening. One way to overcome this limitation is to use a positive-selection vector, whereby successful (in frame) cloning of a desired gene confers a selectable phenotype (for example antibiotic resistance) to the host organism. The feasibility of this type of cloning has been demonstrated in several publications (339-342), however many of the positive selection vectors described in these examples are limited in their scope of use due to either the requirement of unique restriction enzymes $(340 ; 341)$, dependence on a specific selectable marker (340), or generation of translational fusions between the cloned gene product and selectable marker (342). The first aim of this chapter was therefore to develop and test a positive selection vector that was compatible with the cloning methods used in the lab and that also permitted 
inducible expression of inserted genes, enabling its use in SOS assays and other activity screens.

The resultant plasmid, pUCXKT (short for pUCX Kan ${ }^{\mathrm{R}}$-Truncated; for full map and scheme outlining its use see Figures 2.3 and 6.1), was generated by the insertion of a truncated kanamycin resistance $\left(\mathrm{kan}^{\mathrm{R}}\right)$ gene, missing the initial (N-terminal) 4 codons, between the SalI and HindIII restriction sites of pUCX. The lack of a start (ATG) codon, in addition to two stop codons introduced (in-frame) between the ATG codon of the pUCX NdeI site and the start of the truncated kan $^{\mathrm{R}}$ gene prevented expression of this gene in host cells carrying this vector. A SacI site was also introduced directly upstream of and in-frame with the $\operatorname{kan}^{\mathrm{R}}$ gene, in effect constituting the $3^{\text {rd }}$ and $4^{\text {th }}$ codons of this gene. Plating tests indicated that this plasmid did not confer kanamycin resistance on host cells (SOS-R2), based on the inability of transformants to grow on LB agar plates containing $50 \mu \mathrm{g} \mathrm{mL}^{-1}$ kanamycin (results not shown).

Kanamycin resistance, and therefore positive selection, is provided by this plasmid only in the event of successful, in-frame cloning of a gene of insert, between a restriction site of the pUCX MCS (NdeI was used in all instances in this study) and the SacI site of the $\operatorname{kan}^{\mathrm{R}}$ gene. Initial PCR amplification of this gene requires the use of a unique reverse primer (pUCXSacIRv) that contains a SacI site followed directly by the first 2 codons of the $\mathrm{kan}^{\mathrm{R}}$ gene, as well as a 'linker' region containing a ribosome binding site. The resultant plasmid, termed pUCXKG $\left(\mathrm{pUCX} \mathbf{K a n}^{\mathrm{R}}\right.$ Gained) therefore contains the desired insert (NTR gene) directly downstream of the native pUCX promoter region, followed by a linker region containing a second ribosome binding site directly upstream of the reconstituted $\operatorname{kan}^{\mathrm{R}}$ gene. Both genes are therefore transcribed as a single operon; however translation of each gene is disengaged by virtue of the individual ribosome binding sites for each gene. The reconstituted $\mathrm{kan}^{\mathrm{R}}$ gene contains the SacI sequence (GAGCTC) in place of the native $3^{\text {rd }}$ and $4^{\text {th }}$ codons (sequence of GAACAA), therefore inducing a single amino acid change at position 4 from a glutamine to a leucine (the sequence change at codon three, GAG to GAA, is silent as both codons encode glutamic acid). Nevertheless, both agar plating methods (as described above) and growth in liquid media demonstrated that this plasmid conferred kanamycin (at $50 \mu \mathrm{g} \mathrm{mL}^{-1}$ ) resistance to transformed SOS-R2 host strains (results not shown), indicating that the $\mathrm{kan}^{\mathrm{R}}$ gene was positively expressed from this plasmid and that the Q4L amino acid substitution had little effect on $\operatorname{Kan}^{\mathrm{R}}$ enzyme activity. 


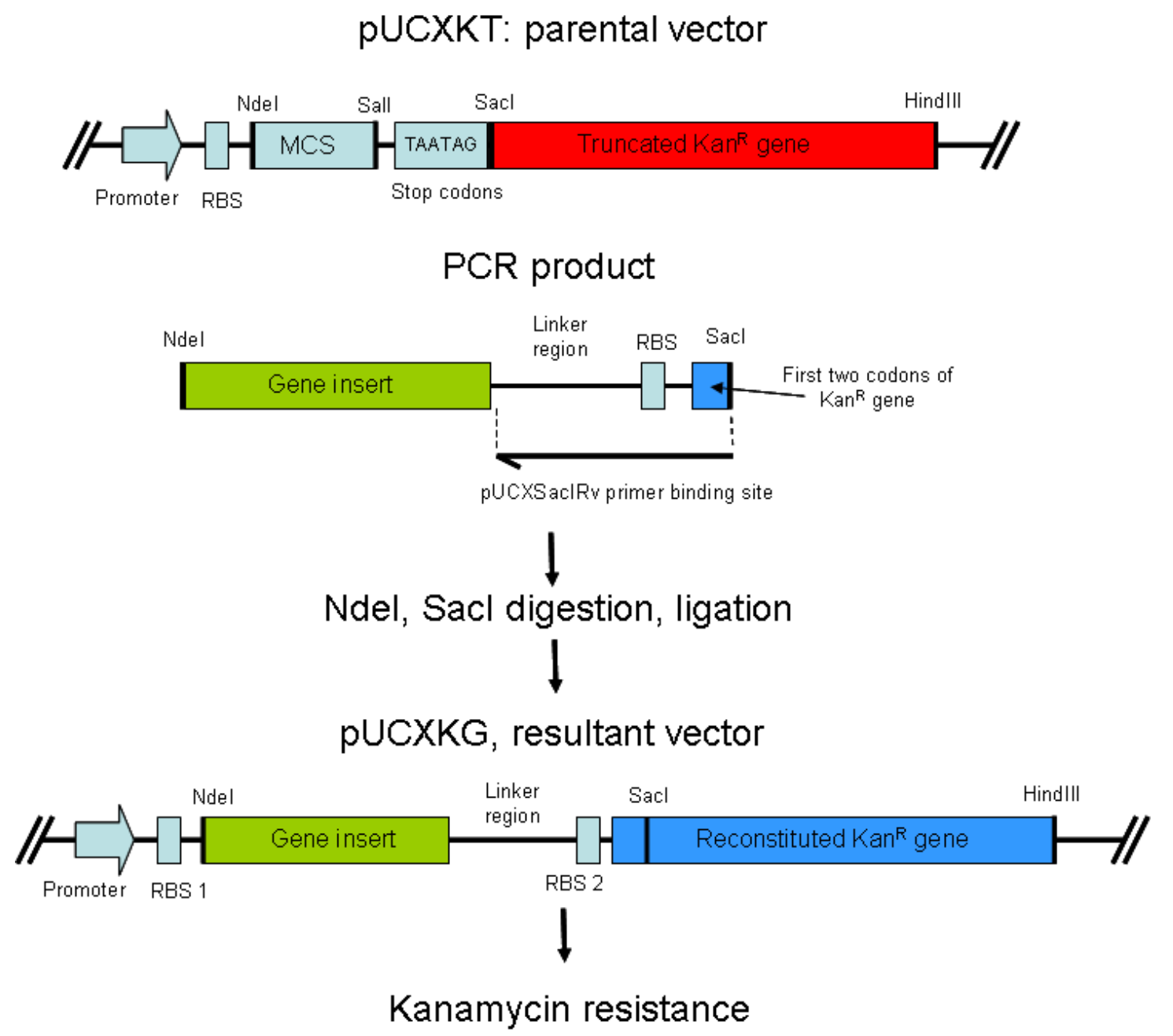

Figure 6.1. Overview of cloning mechanism of pUCXKT and PUCXKG.

See text (sections 2.18 and 6.2.1) for details.

The cloning efficiencies of pUCXKT and pUCXKG were tested by transforming E. coli SOS-R2 with either NdeI-SacI digested pUCXKT that had been treated just with ligase, or with ligase plus NdeI-SacI digested PCR product that had been generated using the pUCXSacIRv primer. Transformation mixes were plated onto solid media containing ampicillin and kanamycin. With one exception, in this control experiment and all subsequent cloning experiments with this system, transformation of NdeI-SacI digested pUCXKT that was re-ligated without insert yielded no colonies on kanamycincontaining solid media (results not shown). The single case recorded to date of $\operatorname{Kan}^{\mathrm{R}}$ colonies appearing after this treatment was found (upon sequence confirmation) to be due to ligation of a NdeI-SacI recognition site-flanked 105 bp region of the E. coli $\mathrm{F}$ plasmid into the respective site of pUCXKT (results not shown). This therefore did not constitute a case of kanamycin resistance afforded by transformation of parental plasmid (pUCXKT) or through degradation and re-ligation of digested plasmid sticky ends, the most common causes of appearance of false positives. In contrast, 
transformation of digested pUCXKT that was ligated with the relevant insert consistently yielded colonies, at high efficiency, on solid media containing ampicillin and kanamycin. PCR screening of a selection of these colonies yielded only bands corresponding to correctly sized and oriented inserts (results not shown).

To ensure that pUCXKG permitted expression of functional inserted genes, a selection of NTRs with a range of CB1954-reductase activities were cloned into this vector and transformed into SOS-R2. These strains were then compared to SOS-R2 strains expressing the NTRs from pUCX for both SOS induction and recombinant protein expression levels following challenge with or without CB1954. To maintain equivalence in assay conditions between the two vectors, kanamycin was not included in media used for strains harbouring pUCXKG (ampicillin being sufficient to maintain plasmid propagation in host cells). Overall, SDS PAGE analysis demonstrated that native sized NTRs were expressed from pUCXKG, although expression levels were much lower at equimolar IPTG concentrations (results not shown). Further assay optimisation established that raising the IPTG concentration to $250 \mu \mathrm{M}$ when expressing from pUCXKG achieved roughly equal NTR expression to that obtained from pUCX at $50 \mu \mathrm{M}$ IPTG (see Figure 6.2). No visible bands corresponding to the kanamycin resistance gene product $(29.1 \mathrm{kDa})$ encoded by pUCXKG were observed on SDS-PAGE gels, suggesting that translational regulation of the insert and $\mathrm{kan}^{\mathrm{R}}$ gene is separate.

SOS responses following CB1954 challenge were similar when NTRs were expressed from either pUCX or pUCXKG in SOS-R2, provided that IPTG was employed at the respective concentrations ( $50 \mu \mathrm{M}$ for pUCX, $250 \mu \mathrm{M}$ for pUCXKG; see Figure 6.3).

Based on these results, pUCXKT was used for cloning of all mutant YcnD (B.s) variants in this chapter and the resulting pUCXKG plasmids used for gene expression in SOS and $\mathrm{IC}_{50}$ assays ( $250 \mu \mathrm{M}$ IPTG in all instances). 


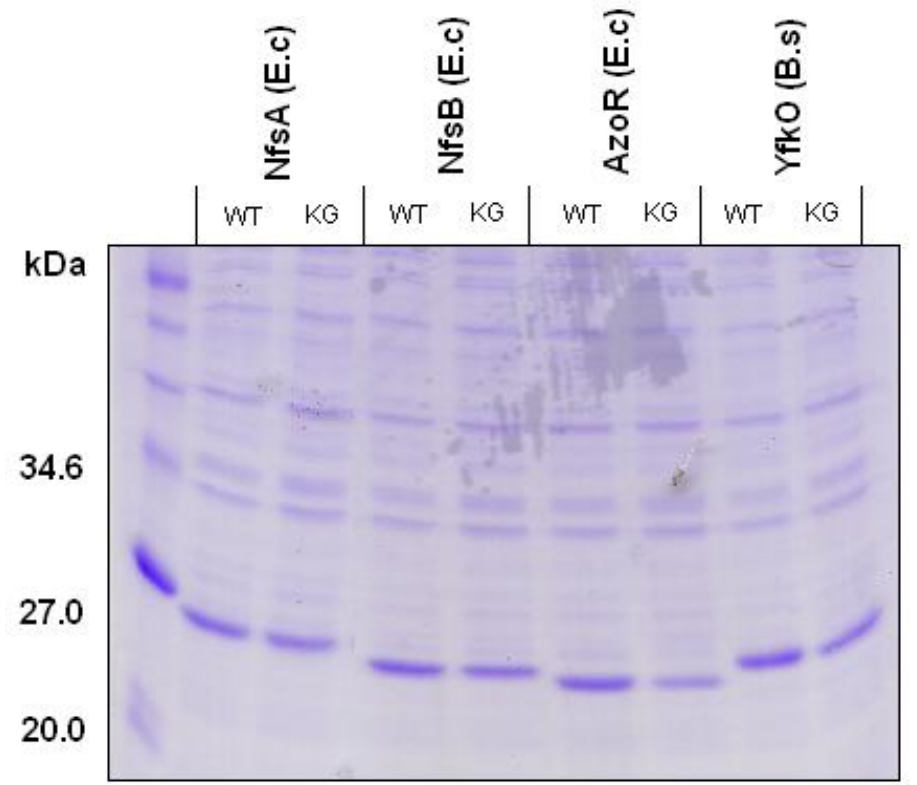

Figure 6.2. Relative NTR expression levels from pUCX and pUCXKG.

SDS PAGE gel scan demonstrating expression levels of various NTRs from either pUCX (indicated by 'WT') or pUCXKG (indicated by 'KG') in SOS-R2, following the completion of an SOS assay. Strains harbouring pUCX constructs were induced for protein expression at $50 \mu \mathrm{M}$ IPTG, those with pUCXKG at $250 \mu \mathrm{M}$ IPTG. Whole cell lysates were normalised for culture density $\left(\mathrm{OD}_{600}\right)$ prior to gel loading. The kanamycin resistance gene product encoded on PUCXKG is unable to be distinguished in any lane (molecular weight is $29.1 \mathrm{kDa}$ )

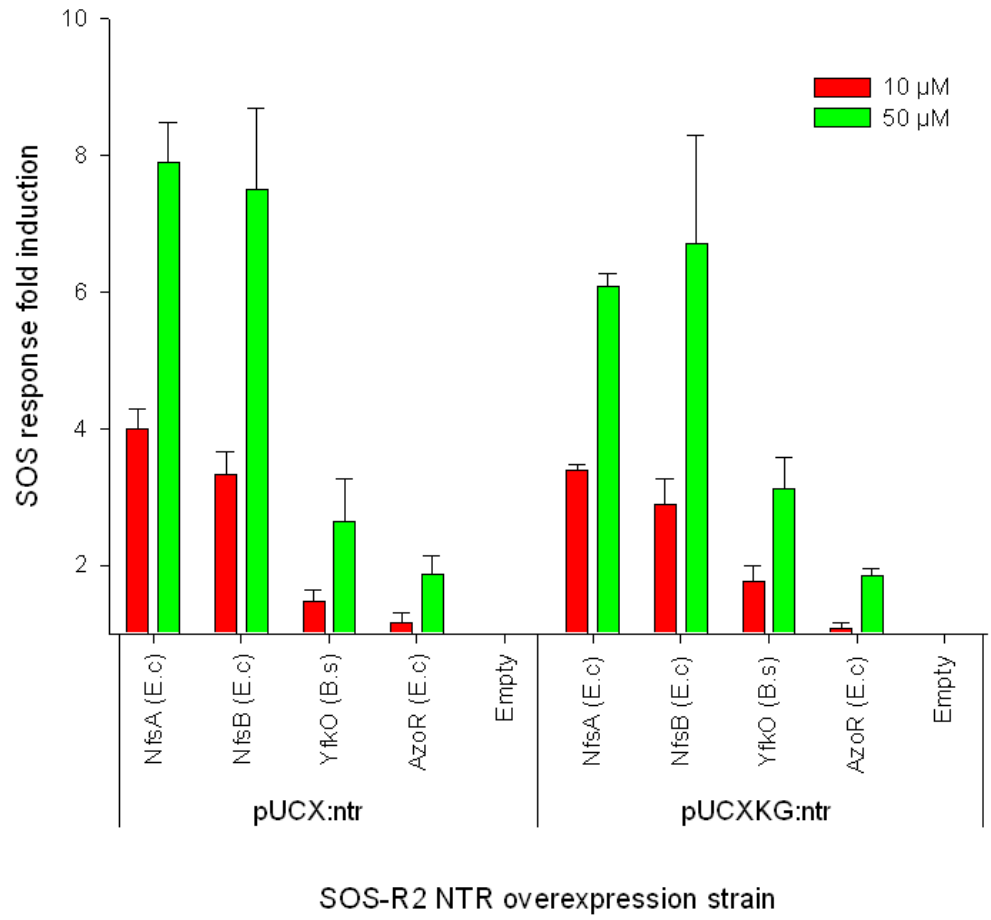

Figure 6.3. Relative SOS response induction using either PUCX or pUCXKG for NTR overexpression.

Strains of SOS-R2 over-expressing NTRs from either pUCX (left side of graph) or pUCXKG (right) were grown and challenged with the indicated concentrations of CB1954 for $3 \mathrm{~h}$ prior to analysis of SOS activity. Strain harbouring pUCX were induced for protein expression with $50 \mu \mathrm{M} \mathrm{IPTG}$; those with pUCXKG were induced with $250 \mu$ M IPTG. Data is the average \pm 1 SEM of 3 independent assays. 


\subsubsection{Selection of $Y \mathrm{cnD}$ (B.s) targeted residues}

The availability of a crystal structure of YcnD (B.s) facilitated selection of active site residues for targeted mutagenesis (194); however, the absence of any data for substrate binding within the active site of this enzyme complicated the process of assigning roles to specific residues. Therefore, the $\mathrm{YcnD}$ (B.s) active site structure was superimposed onto that of the homologous enzyme Frp (V.h), of which a crystal structure in complex with an nicotinamide adenine dinucleotide (NAD) inhibitor has previously been solved (179). Although amino acid sequence similarity between these two enzymes is only 54 $\%$, structural homology is strong, with the root mean square deviation (a measure of structural similarity) of $\alpha$ carbon atoms across the entire sequence being $1.0 \AA$ (194)). This therefore allows mapping of potential interactions of $\mathrm{YcnD}$ (B.s) active site residues with substrates by using Frp (V.h) as a model structure. Sites in YcnD (B.s) to be targeted were therefore chosen based on equivalent Frp (V.h) residues that formed hydrogen bonds with the NAD molecule, or that had potential roles in substrate and active site stabilisation, as previously reported for a selection of NfsA-family enzymes (see Figures 6.4A and B) $(179 ; 194 ; 343)$. The YcnD (B.s) active site differed significantly at one region relative to Frp (V.h), around amino acids 227-233 (corresponding to amino acids 219-225 in Frp (V.h)). In particular, four tyrosine residues (Y227, Y228, Y232 and Y233) extend into the active site of YcnD (B.s) that are not present in Frp (V.h) or any other NfsA-family enzyme structure solved to date. Two of these residues, Y227 and Y233, were chosen as targets based on their closer proximity to the assumed substrate binding site. It is noteworthy that Y233 is in the position corresponding to R225 of Frp (V.h), which is able to hydrogen bond with NAD in this enzyme. Furthermore, the equivalent residue in NfsA (E.c) has been shown to be important for substrate binding and catalysis with a number of nitroaromatic compounds, based on mutagenesis work performed in our laboratory (Dr. Janine Copp, personal communication).

In total, 10 sites in $\mathrm{YcnD}$ (B.s) were selected for mutagenesis. These were R15, I41, G65, Q67, W69, E105, R139, G140, Y227 and Y233 (displayed in Figure 6.4). 


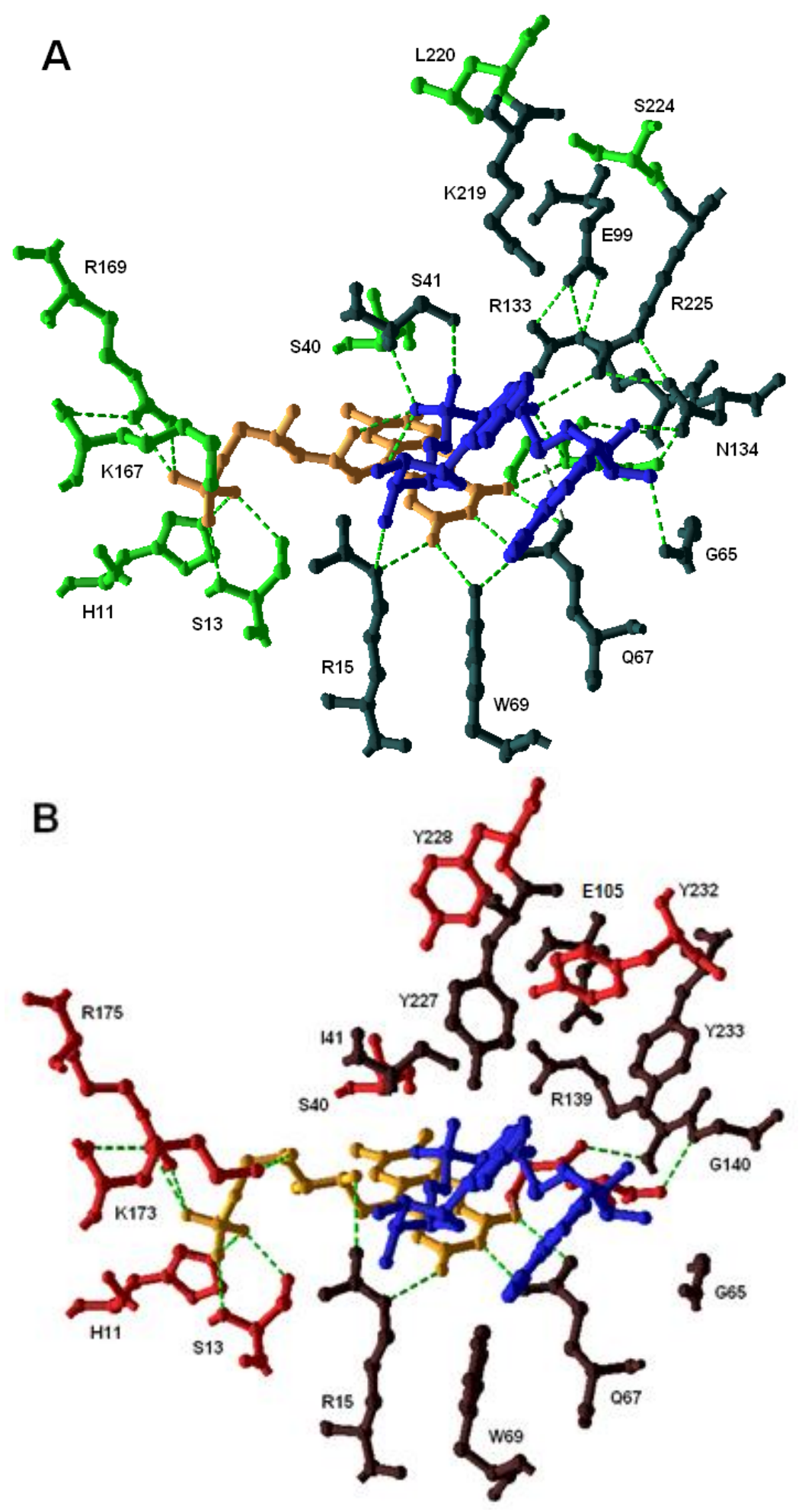

Figures 6.4A \& B. Comparison of active site architecture between Frp (V.h) and YcnD (B.s).

Residues surrounding the active site of (A) Frp (V.h) and (B) YcnD (B.s) are displayed. FMN co-factors are coloured in orange, while the NAD molecule is coloured in blue. Positioning of the NAD molecule in the YcnD active site was achieved by superimposing the active site of Frp (V.h) on that of YcnD (therefore no hydrogen bonds are displayed between YcnD residues and the NAD molecule, due to an inability of the employed software to compute them). Residues targeted in this chapter are shaded in dark red in the YcnD structure, with the equivalent residues of Frp (V.h) shaded in dark green. Protein structural alignments and images were generated in Swiss-Pdb Viewer (DeepView) (http://www.expasy.org/spdbv) 


\subsubsection{Creation of mutant libraries and initial SOS screening}

Targeted residues of YcnD (B.s) were individually mutated using an overlap PCR protocol with primers that led to the incorporation of the degenerate oligonucleotide sequence $\mathrm{NNK}$, where $\mathrm{N}$ designates any nucleotide and $\mathrm{K}$ is either thymine or guanine, in place of the targeted codon. The codon NNK is able to code for all 20 proteinogenic amino acids in just 32 codons, permitting coverage of all possible amino acid substitutions at a single site. Following transformation of pUCXKG:ycnD (B.s) mutant constructs into SOS-R2, gene variant libraries of 92 clones (per targeted site; analysis of 92 clones providing an estimated $94 \%$ chance of obtaining full library coverage (344)) were picked and grown in 96 well plates. Each plate also contained 2 wells of SOS-R2 over-expressing wildtype YcnD (B.s) and a single well with an empty vector control. The remaining well was left blank as a control for media contamination. Prior to activity screening, plasmids were extracted from several randomly selected clones for each library and sequenced, with results confirming that variation was being introduced into the correct codon for each library (results not shown). All 10 libraries were then screened for SOS response induction following $3 \mathrm{~h}$ challenge with $10 \mu \mathrm{M}$ PR-104A. Results are shown in Figures 6.5A-J. To facilitate screening of the large number of libraries, unchallenged cultures were not tested. Therefore all SOS responses are reported as net SOS response (as measured in Miller units) rather than fold inductions. Preliminary screening of unchallenged cultures for several libraries revealed very little difference in basal SOS responses (Miller units) between individual clones of a specific library (results not shown), indicating that net SOS response was a suitable substitute for fold induction in this instance.

The majority of libraries contained clones that induced SOS responses significantly higher than wildtype $\mathrm{YcnD}$ (B.s), in particular those encoding variants for sites I41, G65, R139, Y227 and Y233. At least one third of the clones within each of these libraries were superior to wildtype $\mathrm{YcnD}$ (B.s) in SOS response induction, with the greatest activities being 1.7-2.3-fold higher. In contrast, wildtype YcnD (B.s) ranked in the top five SOS inducers for R15, Q67 and E105 mutant libraries, while slightly more clones induced higher SOS responses than wildtype in W69 and G140 mutant libraries, although fold improvements were not as substantial as those previously mentioned (highest activities were 1.2 and 1.4-fold higher than wildtype for W69 and G140 libraries, respectively). 
To gain a better understanding of the comparative activities of different sitespecific mutants of $\mathrm{YcnD}$ (B.s), as well as to strengthen the data set already obtained, the top 9 hits of each of the I41, G65, R139, Y227 and Y233 mutant libraries and the top 4 hits of the W69 and G140 mutant libraries were combined in a single 96 well plate and screened (alongside wildtype YcnD (B.s) and empty vector controls) for PR-104A induced SOS activity. Only the middle 60 wells of the plate were used so as to prevent edge effects from influencing results (a phenomenon that was observed to a moderate degree with the initial mutant library testing; results not shown). In agreement with the previous data, results demonstrated that the majority of mutants tested induced higher SOS responses than wildtype, the only exceptions being two of the Q67 mutants (Figure 6.6). The most active clones overall originated from the I41, G65, R139 and Y227 mutant libraries, which altogether constituted the top 20 hits. Plasmids were subsequently extracted from these twenty clones and the $y c n D$ (B.s) gene inserts sequenced to identify the introduced mutations. These are displayed in Table 6.1. In total, 11 different mutations were represented by these 20 clones, with 5 specific mutations occurring more than once. These five mutations were I41G, I41M, G65I, G65V and Y227G. Of particular note were the I41G mutations, which appeared three times within the top 5 hits, and the Y227G mutations, which appeared five times in total. The remaining mutations were at sites R139 (Phe, His and Leu substitutions), Y227 (Phe and Cys substitutions) and G65 (Glu substitution). It is noteworthy that two of these sequenced mutants, encoding G65E and Y227C amino acid substitutions respectively, contained nucleotide mutations elsewhere in the gene leading to amino acid changes (see Table 6.1 for details). These double mutants were, however, not investigated and further, due to both their modest activity profiles relative to the most active single mutants identified, and an already substantial yield of genuine single mutants with improved function that was sufficient for further experimentation. 

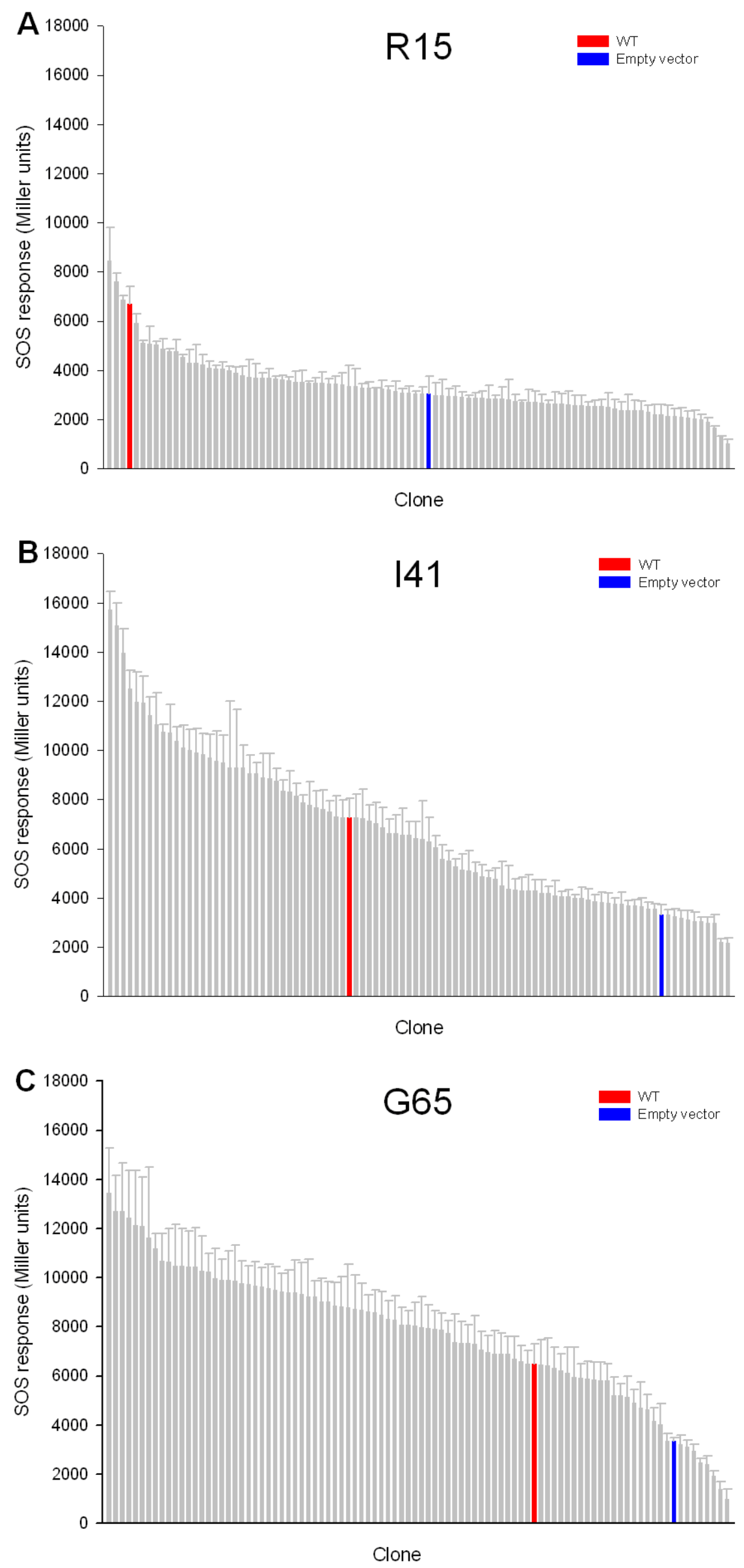

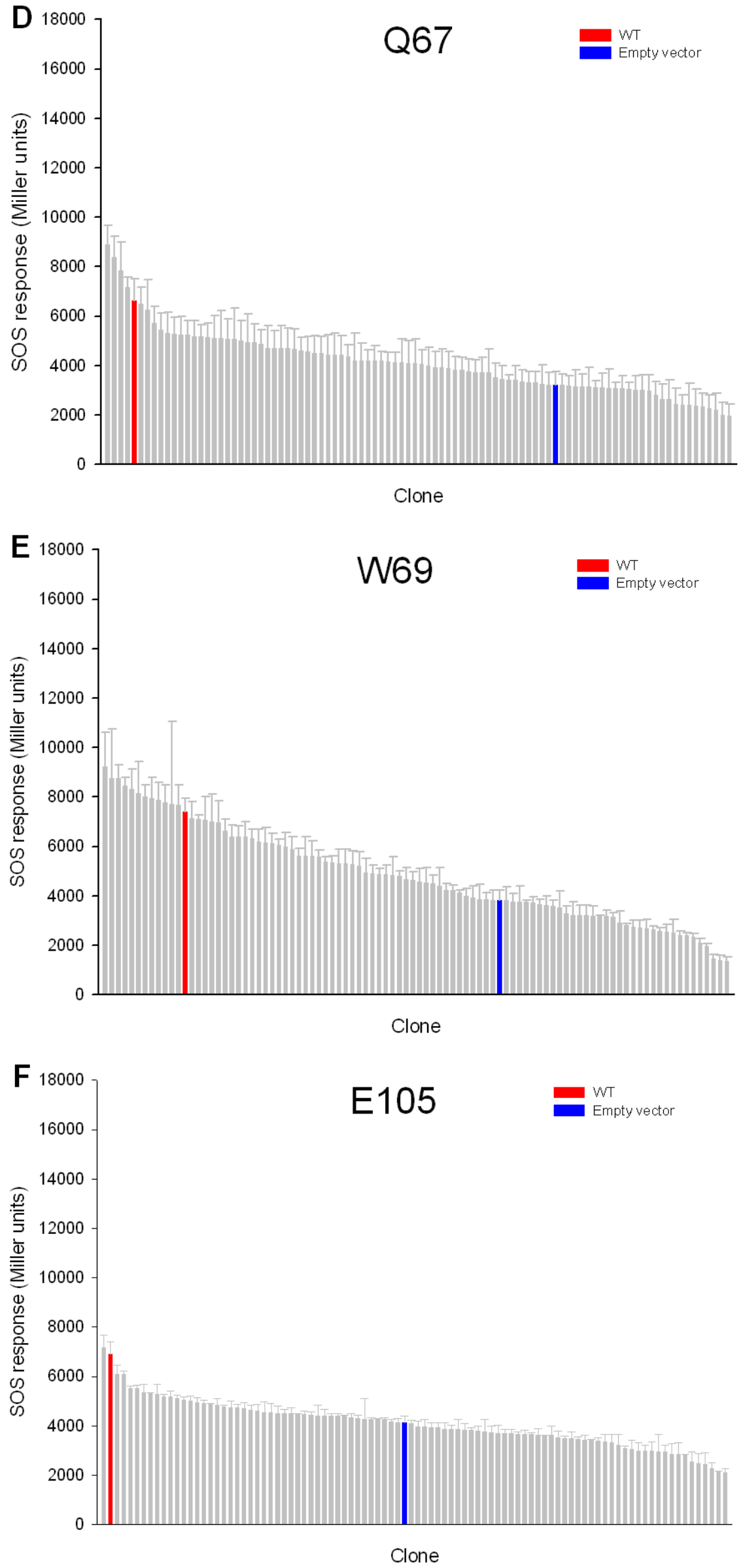

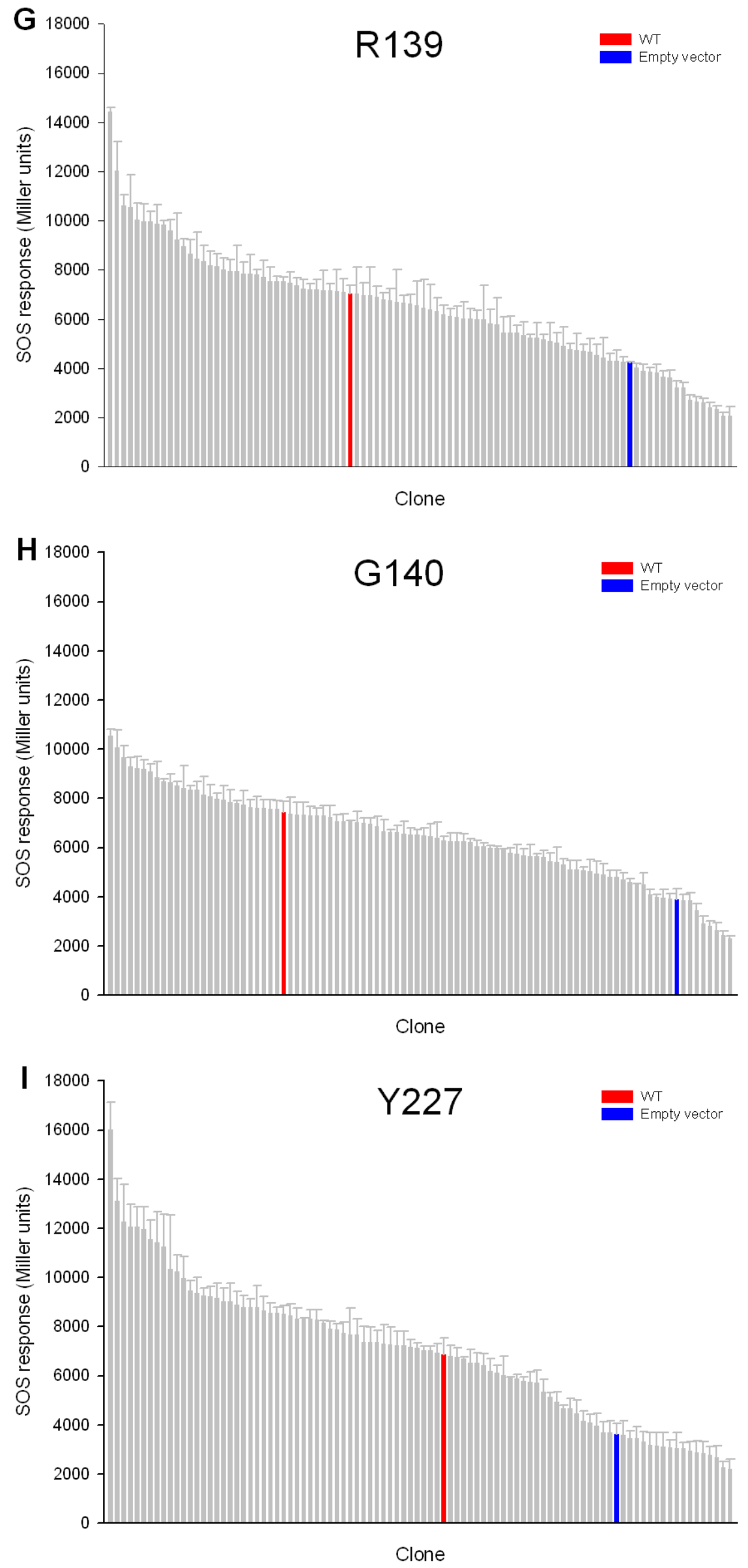
168

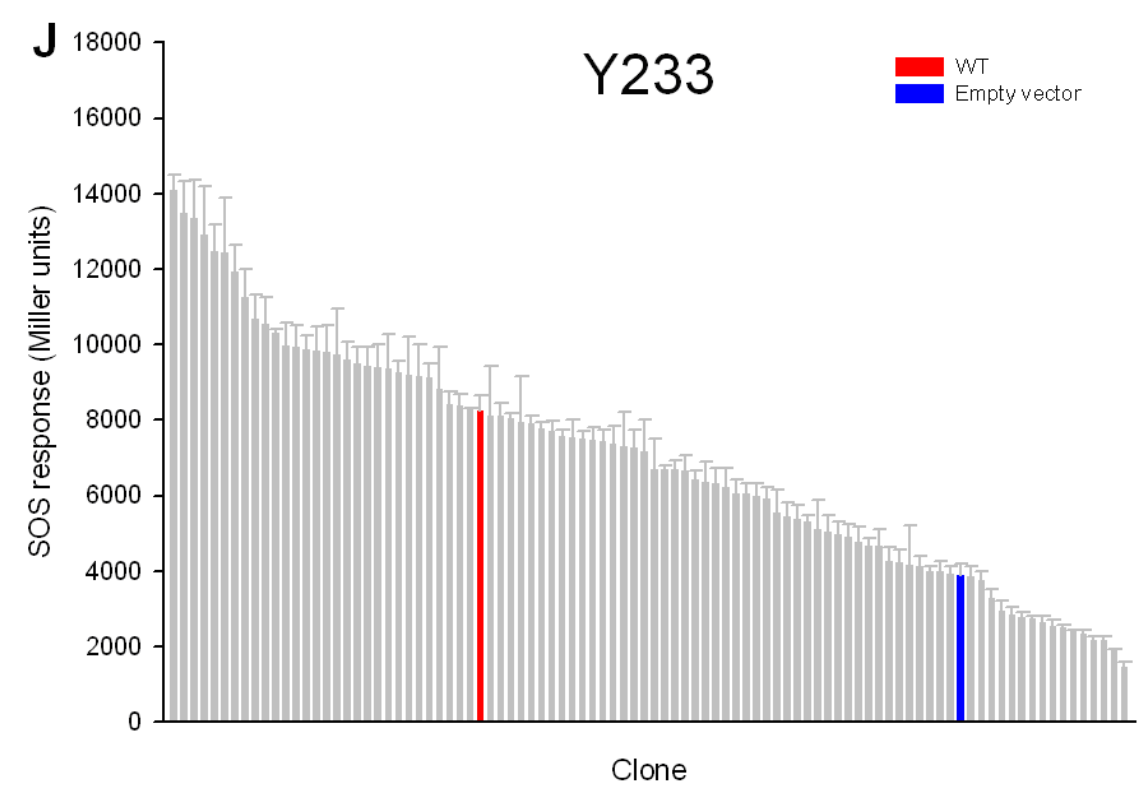

Figures 6.5A-J. SOS responses of SOS-R2 strains over-expressing YcnD (B.s) variants following $3 \mathrm{~h}$ challenge with $10 \mu \mathrm{M}$ PR-104A.

Single site mutants of YcnD (B.s) were created by PCR amplification of the $y c n D$ (B.s) gene, using primers that substituted the targeted wildtype codon for the degenerate sequence NNK. Libraries of 92 clones were created for each targeted site to ensure close to $95 \%$ probability of obtaining all possible codon variants (344). The site targeted is indicated at the top of each individual graph. SOS responses are displayed as total Miller units instead of fold induction, because unchallenged samples were not measured (to facilitate efficient library screening). Each graph is the mean \pm 1 SEM of at least 2 independent assays (each performed in duplicate). 


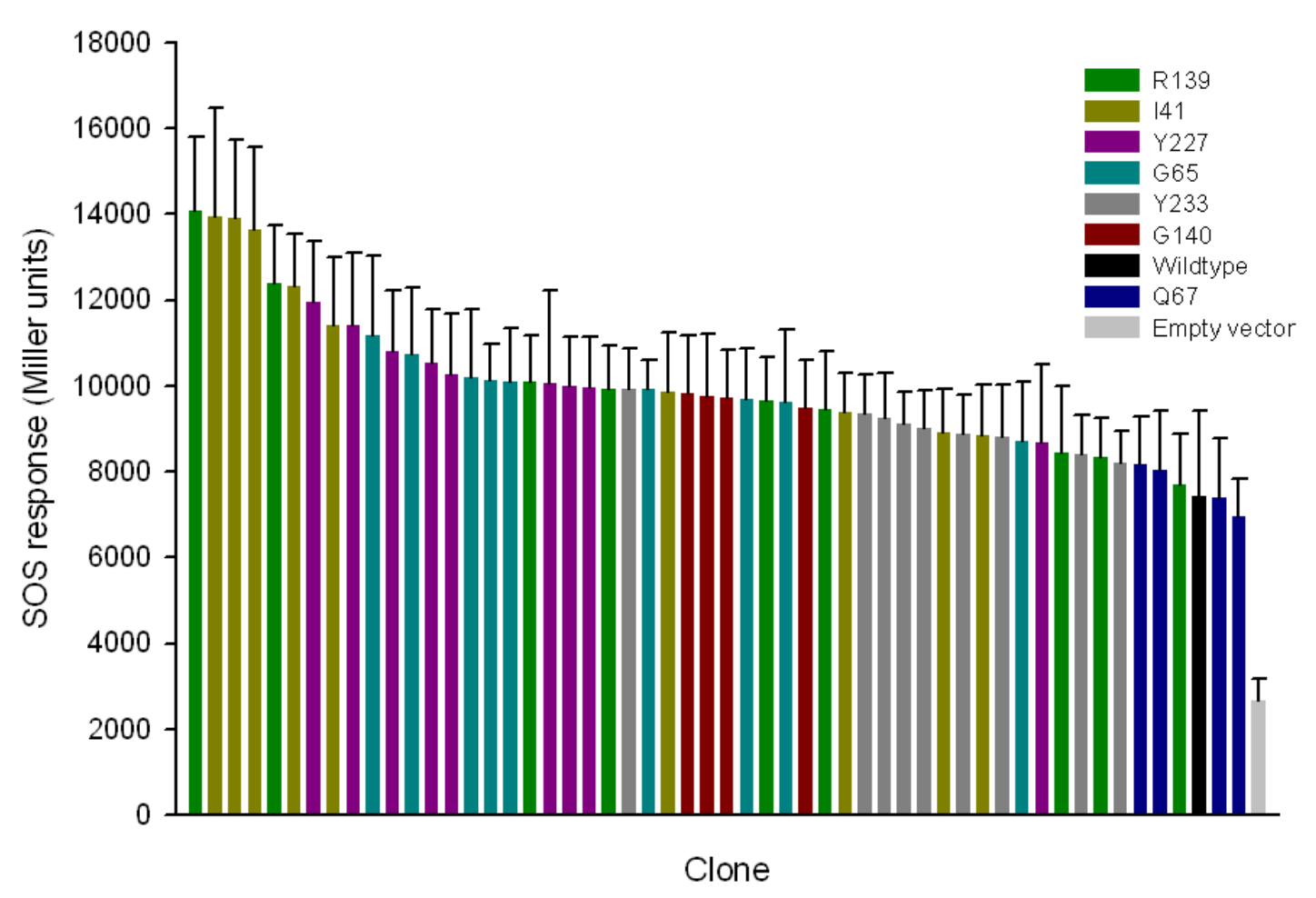

Figure 6.6. PR-104A induced SOS responses from strains of SOS-R2 over-expressing the most active YcnD (B.s) single-site variants.

The most active $\mathrm{YcnD}$ (B.s) over-expressing clones identified previously (see Figures 6.5A-J), along with wildtype $\mathrm{YcnD}$ (B.s) and empty vector controls, were combined in a single 96 well plate (middle 60 wells to avoid edge effects) and assessed for SOS activity following $3 \mathrm{~h}$ challenge with $10 \mu \mathrm{M}$ PR-104A. Bars are colour coded to indicate the mutation specificity (library of origin) of each tested clone. Data is the mean \pm 1 SEM of 3 independent assays (each performed in duplicate). 
Table 6.1. Amino acid substitutions of the 20 most active single-site variants of YcnD (B.s) with PR-104A.

Activity is based on SOS response induction, as displayed in Figure 6.6. Specific mutations that occurred multiple times are colour coded.

\begin{tabular}{|c|c|c|c|}
\hline Clone $^{\mathrm{a}}$ & $\begin{array}{l}\text { Original } \\
\text { amino acid }\end{array}$ & $\begin{array}{l}\text { Altered codon } \\
\text { sequence }\end{array}$ & $\begin{array}{l}\text { Amino acid } \\
\text { substitution }\end{array}$ \\
\hline 139_43 & Arg & TTT & Phe \\
\hline $41 \_80$ & Ile & GGG & Gly \\
\hline $41 \_57$ & Ile & GGG & Gly \\
\hline $41 \_15$ & Ile & GGG & Gly \\
\hline 139_15 & Arg & CAT & His \\
\hline $41 \_72$ & Ile & ATG & Met \\
\hline 227_90 & Tyr & GGT & Gly \\
\hline 41_9 & Ile & ATG & Met \\
\hline $227 \_5$ & Tyr & TTT & Phe \\
\hline 65_32 & Gly & ATT & Ile \\
\hline 227_39 & Tyr & GGG & Gly \\
\hline 65_90 & Gly & ATT & Ile \\
\hline $227 \_73$ & Tyr & GGT & Gly \\
\hline 227_47 & Tyr & GGG & Gly \\
\hline $65 \_88^{\mathrm{b}}$ & Gly & GAG & Glu \\
\hline $65 \_72$ & Gly & GTG & Val \\
\hline $65 \_24$ & Gly & GTT & Val \\
\hline 139_88 & Arg & TTG & Leu \\
\hline 227_87 & Tyr & GGG & Gly \\
\hline $2273^{c}$ & Tyr & TGT & Cys \\
\hline
\end{tabular}

${ }^{a}$ Clones are listed in descending order of activity, based on results displayed in Figure 6.6. Numbers before the underscore of each clone designation indicate the targeted residue; numbers after the underscore refer to the relative position on original screening plates.

${ }^{b} Y c n D$ (B.s) gene also carried an Asp to Gly substitution at codon 71

${ }^{\mathrm{c}} \mathrm{YcnD}$ (B.s) gene also carried a Trp to Arg substitution at codon 211. 


\subsubsection{Pairwise combinations of beneficial single mutations}

In order to further improve PR-104A reductase activity of $\mathrm{YcnD}$ (B.s), beneficial single mutations identified in the previous section were combined (pairwise) on the same gene to create various combinations of $\mathrm{YcnD}$ (B.s) double mutants. Firstly, due to the multiple amino acid substitutions at site R139 that led to heightened SOS activity with PR-104A, new NNK libraries (92 clones) of R139 were created using either I41G or Y227G gene variants as templates. Secondly, an NNK library of I41 was created using the Y227G gene variant as template, due to the different beneficial mutations available at site I41. These three libraries, along with internal controls consisting of wildtype and parental single mutant YcnD (B.s) expressing clones, were screened for SOS response induction, as previously described, following challenge with PR-104A. Few clones in the I41G R139 NNK and Y227 R139 NNK libraries demonstrated improved activity over the respective single mutant parents (see Figures 6.7A and B); however more than a third of the Y227G I41 NNK library elicited substantially heightened SOS responses relative to the Y227G single mutant (see Figure 6.7C). pUCXKG:ycnD plasmids were purified and the $y c n D$ (B.s) gene insert sequenced from the top 5 hits of the Y227G I41 NNK library and the top 3 hits of the I41G 139 NNK library. All three sequenced clones from the I41G 139 NNK library coded for glycine at site 139, while three of the five sequenced clones from the Y227G I41 NNK library coded for glycine at position 41. The remaining two sequenced Y227G I41 NNK clones coded for alanine at amino acid 41. 

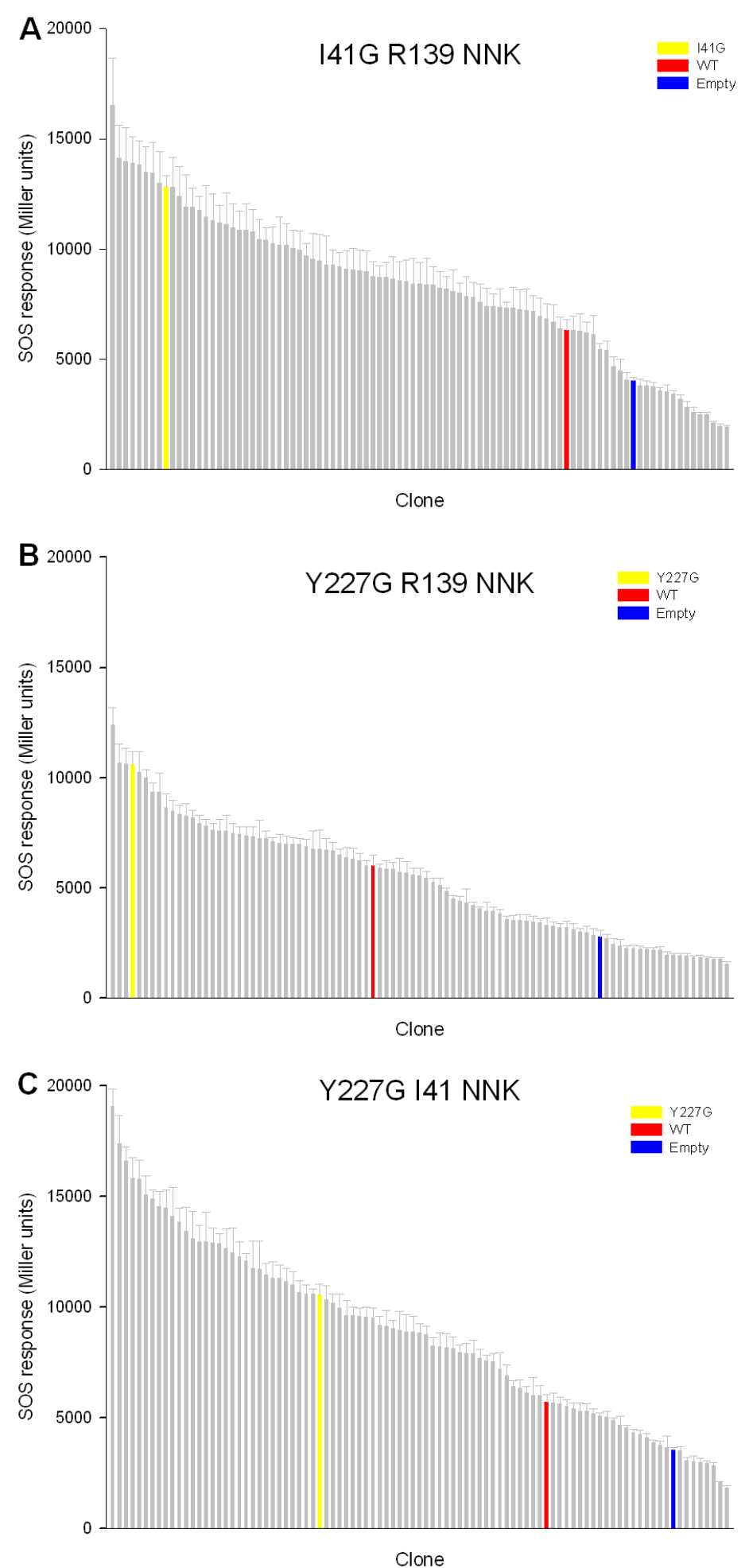

Figures 6.7A-C. PR-104A-induced SOS responses of SOS-R2 strains over-expressing double site variants of $Y \mathrm{cnD}$ (B.s).

Selected YcnD (B.s) single-site mutants (with improved PR-104A reductase activity over wildtype) were used as templates for further PCR-based (NNK codon substitution) site-saturation mutagenesis. Targeted residues included (A) R139 in an I41G genetic background, (B) R139 in an Y227G genetic background, and (C) I41 in an Y227G genetic background. Mutant libraries were created as previously described and each one individually assessed for SOS activity induction following $3 \mathrm{~h}$ challenge with $5 \mu \mathrm{M}$ PR-104A. Data displayed is the mean \pm 1 SEM of 3 independent assays. 
Four additional double mutants of $y c n D$ (B.s) were generated through pairwise combinations of $\mathrm{I} 41 \mathrm{G}$ or $\mathrm{Y} 227 \mathrm{G}$ mutations with either G65I or G65V mutations (resultant variants were I41G G65I, I41G G65V, Y227G G65I and Y227 G65V). These four double mutants, along with the I41G R139G, I41A Y227G and I41G Y227G double mutants identified previously, the individual single mutant parents of the above (where available), a selection of other single site mutants that demonstrated improved activity over wildtype, and wildtype YcnD (B.s) were combined on a single 96 well plate for comparative activity testing with PR-104A by SOS assay. Results demonstrated that the overall most active $\mathrm{YcnD}$ (B.s) variants were the double mutants I41A Y227G and I41G Y227G, followed very closely by the double mutants I41G G65I and I41G G65V (Figure 6.8). These enzymes elicited significantly higher ( $\mathrm{p}<0.05$; $\mathrm{t}$ test) SOS responses than wildtype YcnD (B.s); however the difference between the SOS response inductions of these enzymes and the most active parental single mutant $\mathrm{I} 41 \mathrm{G}$ (or I41A) was not significant ( $p>0.05$; $t$-test). Likewise, the Y227G G65I/V double mutants elicited SOS responses no higher than the parental Y227G single mutant.

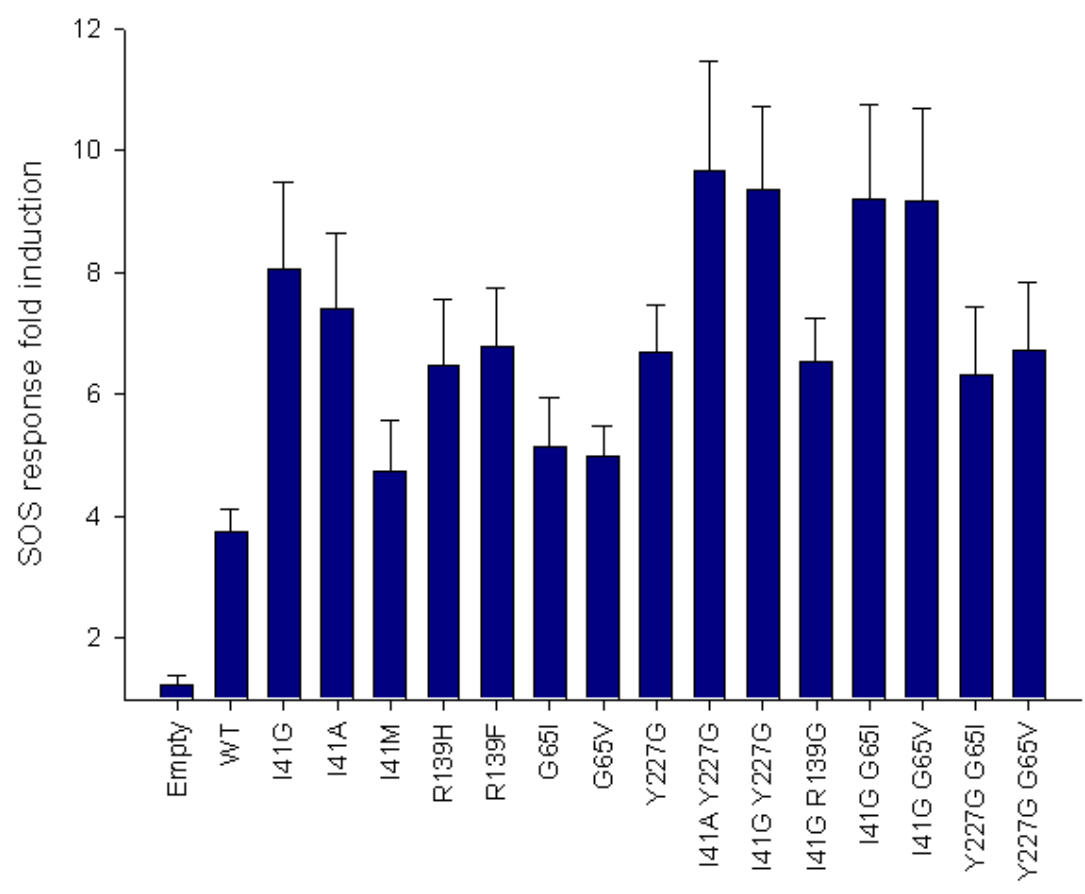

SOS-R2 YcnD (B.s) variant overexpression strain

Figure 6.8. PR-104A-induced SOS responses from SOS-R2 strains over-expressing the most active single and double-site variants of $Y \mathrm{cnD}$ (B.s).

Relevant strains were grown and challenged with $5 \mu \mathrm{M}$ PR-104A for $3 \mathrm{~h}$ prior to analysis of SOS activity. Data is the mean \pm 1 SEM of 4 independent assays. 


\subsection{5. $\mathrm{IC}_{50}$ determination and in vitro enzyme kinetics of lead candidate YcnD (B.s) variants}

To verify the results observed in the SOS assays, $\mathrm{IC}_{50}$ values of PR-104A were calculated for a selection of $\mathrm{YcnD}$ (B.s) variants following $3 \mathrm{~h}$ challenge with increasing concentrations of prodrug. Results are displayed in Table 6.2. The enzymes that conferred the highest sensitivity to PR-104A were the double mutants I41A Y227G and I41G Y227G; $\mathrm{IC}_{50}$ values from these variants were roughly 2.5 -fold lower than that obtained for wildtype $\mathrm{YcnD}$ (B.s). Furthermore, these $\mathrm{IC}_{50}$ values were significantly lower than those obtained from the $\mathrm{I} 41 \mathrm{G}$ and $\mathrm{Y} 227 \mathrm{G}$ single mutants $(\mathrm{p}<0.05$; $\mathrm{t}$-test). Expression levels in SOS-R2 of all the $\mathrm{YcnD}$ (B.s) variants tested were equivalent, as measured by SDS-PAGE (results not shown). Overall, the correlations between SOS assay results and $\mathrm{IC}_{50}$ s were very strong $\left(\mathrm{R}^{2}>0.95\right.$; Figure 6.9).

Table 6.2. $\mathrm{IC}_{50}$ values of PR-104A in SOS-R2 over-expressing wildtype and active site mutants of YcnD (B.s).

\begin{tabular}{ll}
\hline YcnD (B.s) variant & IC $_{\mathbf{5 0}}(\boldsymbol{\mu M})^{\mathbf{a}}$ \\
\hline Wildtype & $226 \pm 9.9$ \\
I41G & $112 \pm 7.9$ \\
G65I & $177 \pm 4.4$ \\
G65V & $176 \pm 9.1$ \\
Y227G & $141 \pm 6.4$ \\
I41G G65I & $107 \pm 12$ \\
I41G G65V & $101 \pm 8.6$ \\
I41A Y227G & $88.5 \pm 11$ \\
I41G Y227G & $88.9 \pm 9.9$
\end{tabular}

${ }^{\mathrm{a}} \mathrm{IC}_{50}$ values were calculated from growth measurements taken after $4 \mathrm{~h}$ challenge with increasing concentrations of PR-104A. 


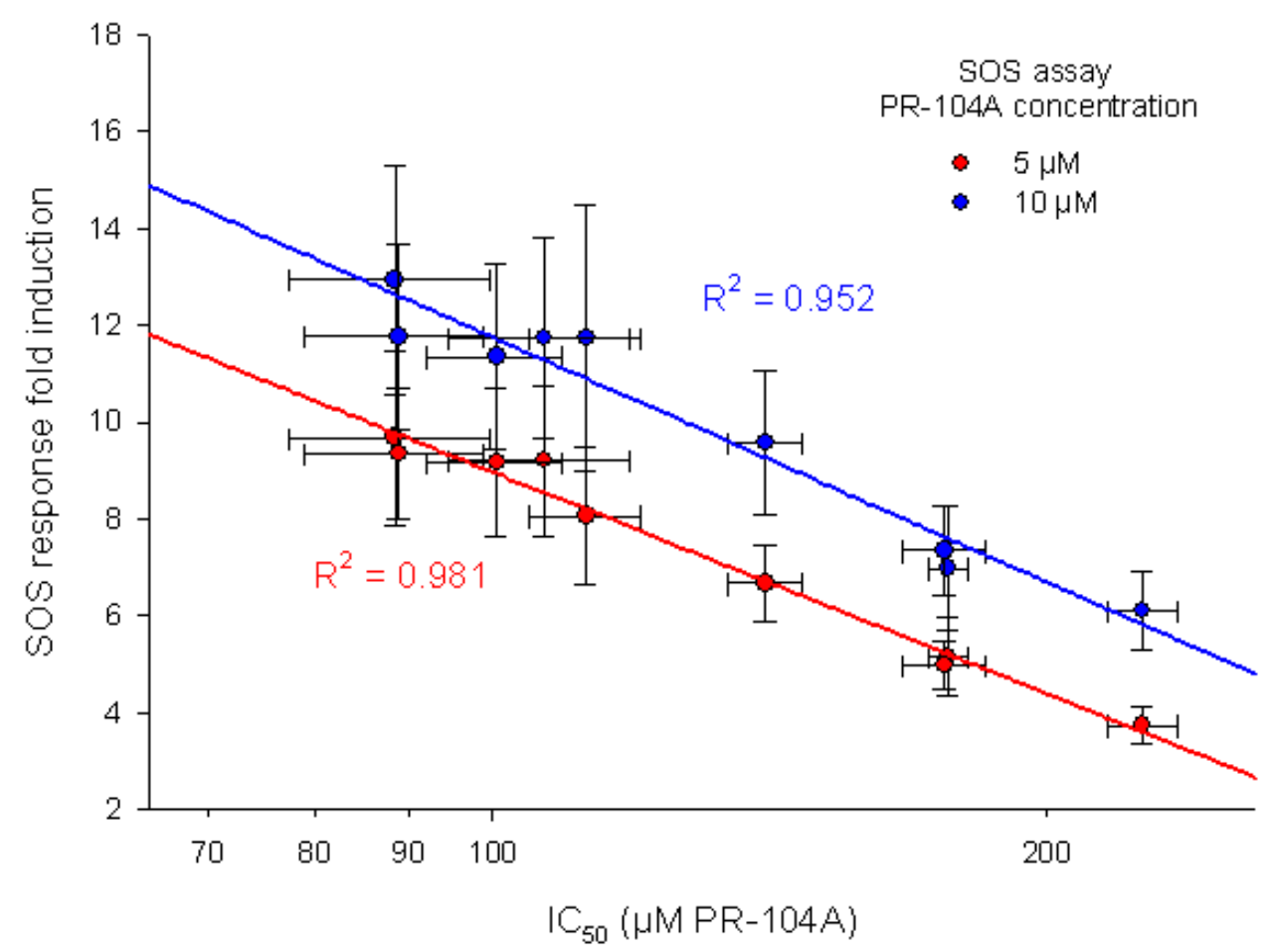

Figure 6.9. Logarithmic correlations between $S O S$ assay results and $I_{50}$ values for PR-104Achallenged strains of SOS-R2 over-expressing Y $\mathrm{cnD}$ (B.s) single and double-site variants. Data for SOS results was taken from Figure 6.8. IC $_{50}$ values were taken from Table 6.2.

The highly active (based on SOS and $\mathrm{IC}_{50}$ results) $\mathrm{YcnD}$ (B.s) double mutant I41G Y227G, along with its parental single site mutants I41G and Y227G, were further investigated for PR-104A reductase activity through in vitro purified enzyme kinetics. Genes were cloned into the expression vector pET28a(+), transformed into E. coli BL21 and recombinant His6-tagged enzymes over-expressed and purified as described previously. Wildtype YcnD (B.s) was also re-purified and tested alongside the single and double-site mutants for consistency. All enzymes were deemed to be $>95 \%$ pure based on densitometric analysis of bands after SDS PAGE of purified enzyme preparations (results not shown).

Spectrophotometric analysis of PR-104A reduction kinetics was assessed for each purified enzyme as previously described, using NADPH as co-substrate in all instances. In contrast to SOS assay and $\mathrm{IC}_{50}$ results, the catalytic activity of the mutant YcnD (B.s) enzymes (based on $k_{\text {cat }} / K_{\mathrm{m}}$ values) was on average considerably lower than that of the wildtype enzyme (see Table 6.3 and Figure 6.10). The I41G and I41G Y227G mutants displayed the lowest activity (3-4-fold lower $k_{\text {cat }} / K_{\mathrm{m}} \mathrm{s}$ than wildtype), while the $\mathrm{Y} 227 \mathrm{G}$ mutant yielded a $k_{\text {cat }} / K_{\mathrm{m}}$ roughly equal to that of wildtype $\left(248,000 \mathrm{M}^{-}\right.$ ${ }^{1} \mathrm{~cm}^{-1}$ compared to $255,000 \mathrm{M}^{-1} \mathrm{~cm}^{-1}, k_{\mathrm{cat}} / K_{\mathrm{m}} \mathrm{S}$ for $\mathrm{Y} 227 \mathrm{G}$ mutant and wildtype YcnD (B.s), respectively). Further testing revealed that co-substrate preferences among the 
mutant YcnDs had not been substantially altered relative to wildtype, as rates of reduction of PR-104A with $\mathrm{NADH}$ as co-substrate were consistently less than $50 \%$ of rates achieved with NADPH (at equimolar PR-104A concentrations) for all four enzymes (see Figure 6.11). Lastly, the in vitro activity of these enzymes with two alternative nitroaromatic compounds, CB1954 and NFZ, was also tested. Results (displayed in Figures 6.12A and B) demonstrated that relative levels of reduction between the four enzymes were highly consistent among the different substrates tested (i.e. Wildtype $>$ Y227G $>$ I41G $>$ I41G Y227G), and furthermore that these relative levels of activity also correlated with those seen with PR-104A as substrate (as illustrated in Figure 6.11).

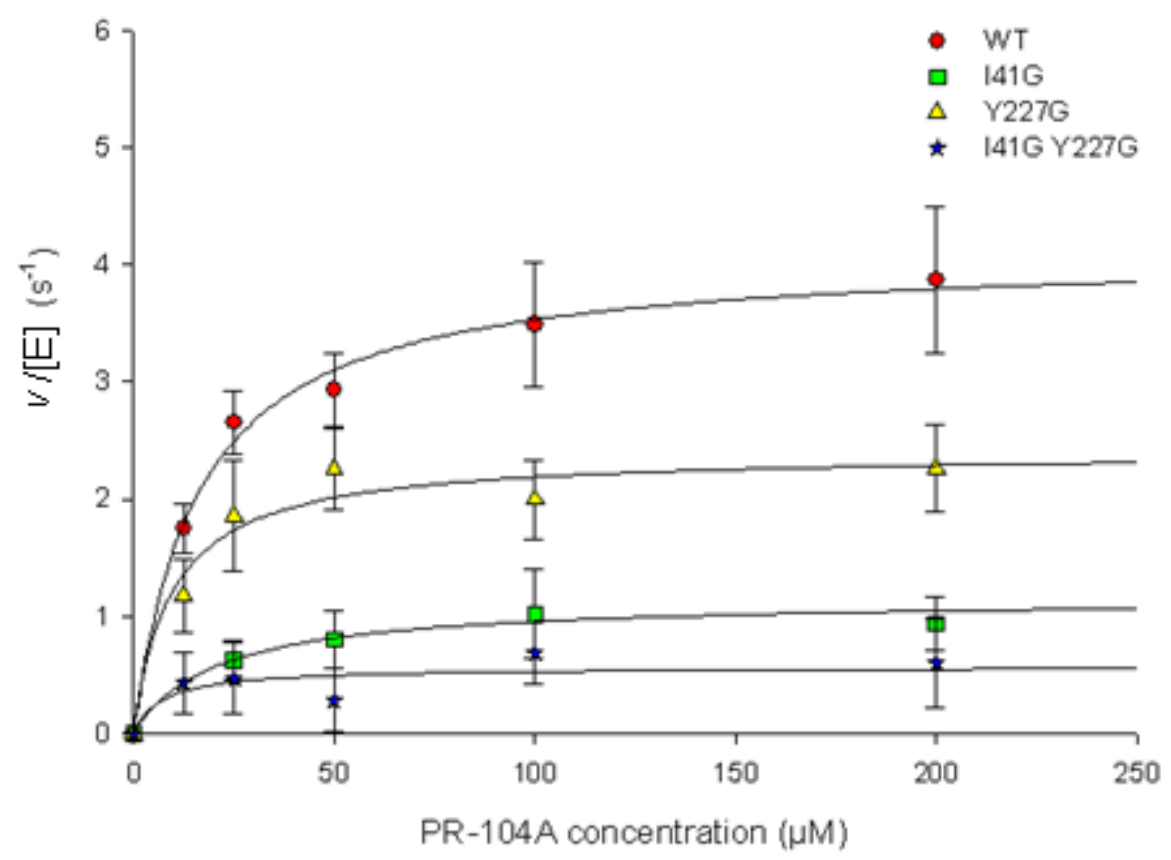

Figure 6.10. Kinetic characterisation of $Y \mathrm{cnD}$ (B.s) and three active site mutants with PR-104A. Catalytic rate of reduction (initial rate, $v /$ enzyme concentration, [E]) plotted against substrate concentration. NADPH was used as co-substrate, at $0.25 \mathrm{mM}$, for all reactions. Reduction of PR-104A was monitored as the decrease in absorbance at $400 \mathrm{~nm}\left(\varepsilon=6,000 \mathrm{M}^{-1} \mathrm{~cm}^{-1}\right)$. Results are the mean $\pm 1 \mathrm{SD}$ of at least 4 replicates for each PR-104A concentration tested.

Table 6.3. Kinetic parameters for the reduction of PR-104A by purified His6tagged wildtype and evolved YcnD (B.s). See legend of Figure 6.10 for more details.

\begin{tabular}{llll}
\hline YcnD (B.s) variant & $\boldsymbol{k}_{\text {cat }}\left(\mathbf{s}^{-1}\right)$ & $\boldsymbol{K}_{\mathbf{m}}(\boldsymbol{\mu M})$ & $\boldsymbol{k}_{\text {cat }} / \boldsymbol{K}_{\mathbf{m}}\left(\mathbf{M}^{-1} \mathbf{s}^{-1}\right)$ \\
\hline Wildtype & $4.10 \pm 0.11$ & $16.1 \pm 1.7$ & $255,000 \pm 28,000$ \\
I41G & $1.2 \pm 0.08$ & $20.9 \pm 5.5$ & $57,400 \pm 16,000$ \\
Y227G & $2.4 \pm 0.11$ & $9.66 \pm 2.2$ & $248,000 \pm 58,000$ \\
I41G Y227G & $0.57 \pm 0.08$ & $7.37 \pm 6.0$ & $77,300 \pm 64,000$ \\
\hline
\end{tabular}




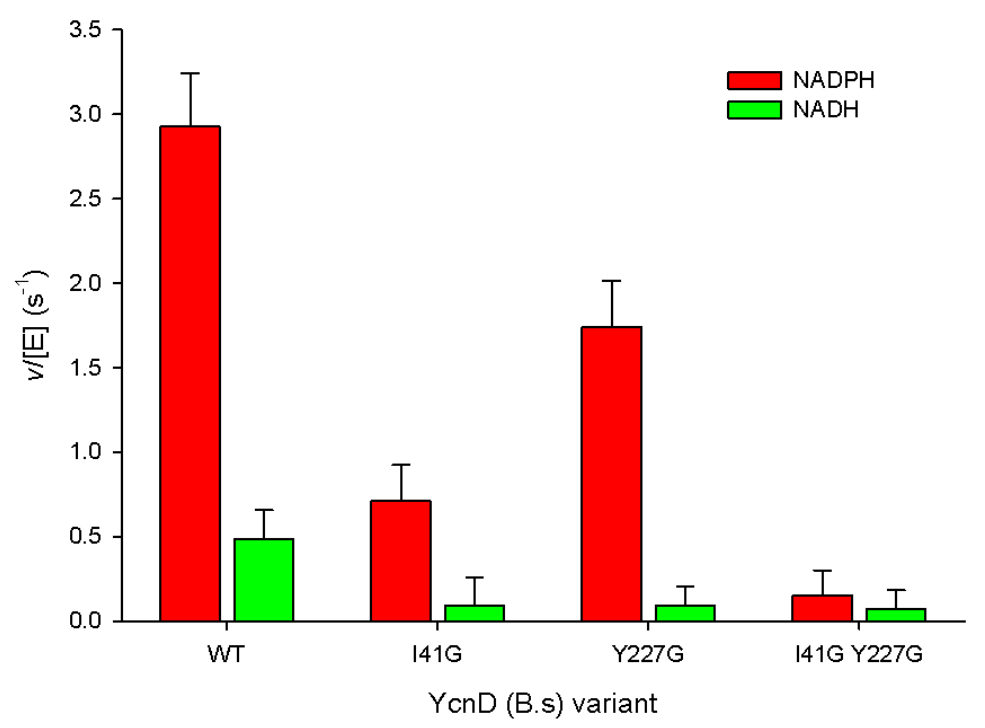

Figure 6.11. Reduction of PR-104A by purified His6-tagged wildtype and mutant YcnD (B.s), using NADH or NADPH as co-substrate.

Catalytic rates of PR-104A reduction by purified His6-tagged YcnD (B.s) and select mutants was measured as described previously, using PR-104A at $50 \mu \mathrm{M}$ and either NADH or NADPH as co-substrate (at $0.25 \mathrm{mM}$ fixed concentration). Data is the mean $\pm 1 \mathrm{SD}$ of at least 4 replicate assays for each enzymeco-substrate condition tested.

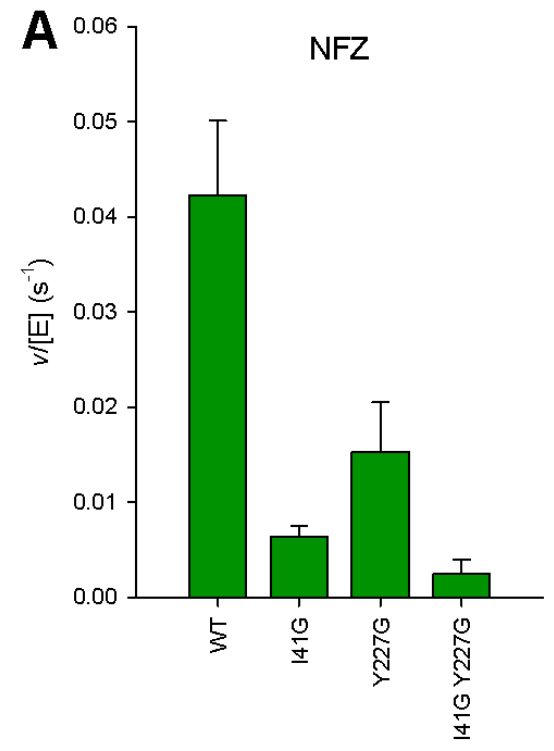

YcnD (B.s) variant

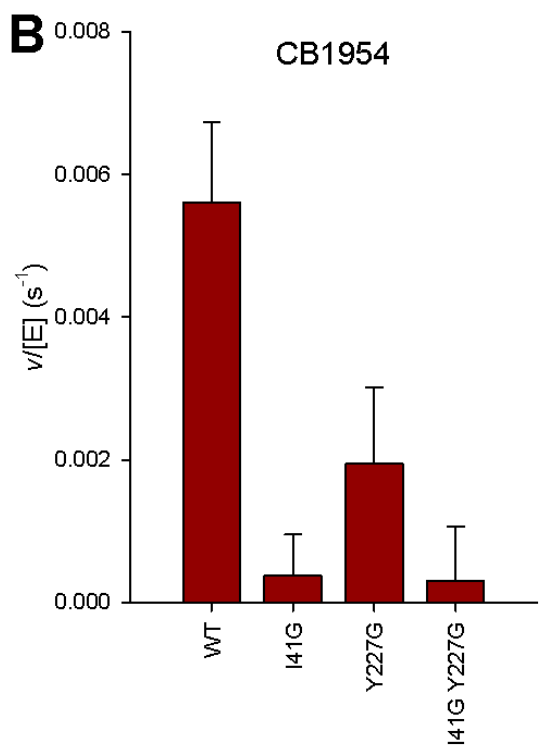

YcnD (B.s) variant

Figures 6.12A \& B. Reduction of alternative nitroaromatic prodrugs by purified His6-tagged wildtype and mutant $Y c n D$ (B.s).

Catalytic rates of reduction of either (A) NFZ $(50 \mu \mathrm{M})$ or (B) CB1954 (100 $\mu \mathrm{M})$ by purified his6-tagged YcnD (B.s) and select mutants were measured as previously reported for these substrates. NADPH was used as co-substrate $(0.25 \mathrm{mM})$. Data is the mean \pm 1 SD of at least 4 replicate assays for each enzymeprodrug combination tested. 


\subsubsection{SOS activity with alternative nitroaromatic prodrugs}

The effect of active site mutations on the ability of $\mathrm{YcnD}$ (B.s) to reduce nitroaromatic prodrugs other than PR-104A was tested next. The same panel of SOS-R2 strains overexpressing single and double-site $\mathrm{YcnD}$ (B.s) mutants that was used to generate data for Figure 6.8 was assessed for SOS response induction following challenge with either CB1954, MTZ or RB 6145. Overall, results shown in Figures 6.13A-C demonstrate that activity of mutant YcnD (B.s) enzymes differed substantially, relative to wildtype, across the different substrates tested. For example, the double mutants I41A/G Y227G, which demonstrated the highest SOS activity and lowest $\mathrm{IC}_{50}$ S with PR-104A, elicited lower SOS responses than wildtype across all three substrates tested in this section, with a particularly large difference (more than 2.2-fold lower SOS responses relative to wildtype) observed with MTZ as substrate. Furthermore, the R139H/F mutations caused a significantly higher SOS response than wildtype and the majority of mutants upon challenge with RB 6145, while these same R139 mutants produced decreased responses relative to wildtype upon challenge with CB1954 and MTZ. Overall, no YcnD (B.s) variant elicited an SOS response substantially higher than wildtype with either CB1954 or MTZ as substrate. In fact, with MTZ as substrate, the majority of mutations tested appeared to diminish activity relative to wildtype. 


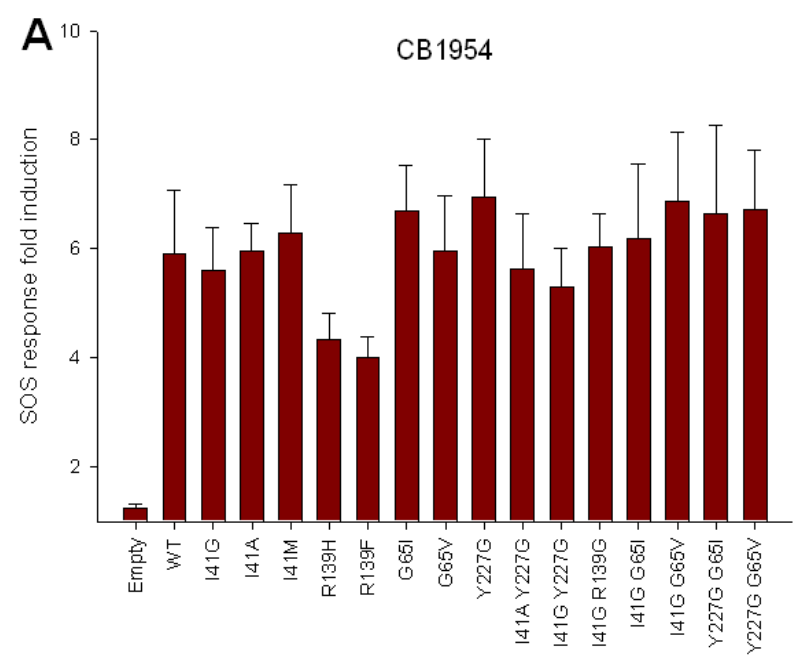

SOS-R2 YcnD (B.s) variant overexpression strain

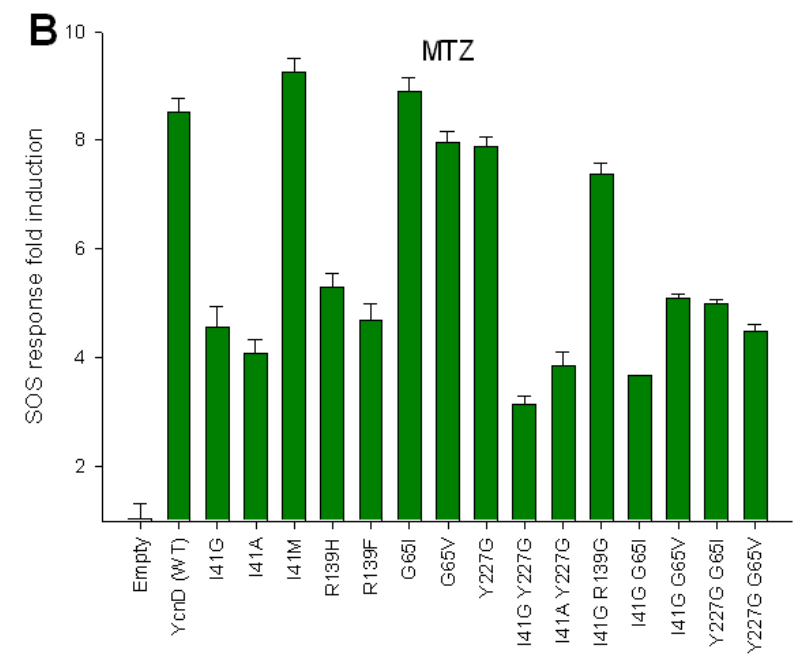

SOS-R2 YcnD (B.s) overexpression strain

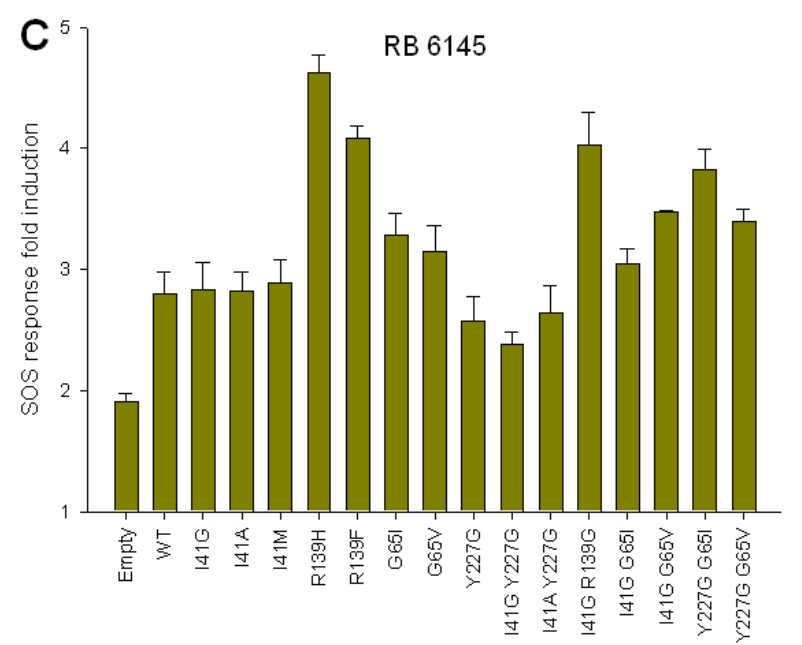

SOS-R2 YcnD (B.s) variant overexpression strain

Figures 6.13A-C. SOS induction of wildtype and mutant YcnD (B.s) over-expressing strains of SOS-

$\mathbf{R} 2$ following challenge with alternative nitroaromatic prodrugs.

Strains of SOS-R2 over-expressing wildtype or mutant (most active single- and double-site variants)

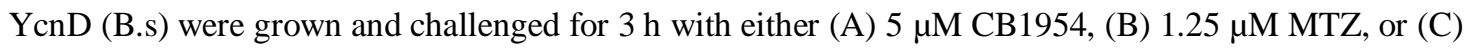
$2.5 \mu \mathrm{M}$ RB 6145 , prior to analysis of SOS activity. Each data set is the mean \pm 1 SEM of 4 independent assays. 


\subsection{Discussion}

The overall aim of the experiments described in this chapter was to improve the PR104A reductase activity of the Bacillus subtilis NTR YcnD through site directed mutagenesis of residues considered to be involved in substrate binding and catalysis. Using the SOS assay as a screening tool, 10 active site amino acids identified by analysis of crystal structure data were individually mutated and tested for PR-104A reductase activity. Double mutants containing paired combinations of beneficial single mutations were subsequently created and also tested for PR-104A reductase activity. The most active variants generated, the double mutants I41G/A Y227G, elicited SOS responses 2.5 -fold higher than the wildtype enzyme, following challenge of the relevant SOS-R2 over-expression strains with 5-10 $\mu \mathrm{M}$ of PR-104A. A similar difference in activity between these two strains was also observed in $\mathrm{IC}_{50}$ values, confirming the results gained with the SOS assay.

However, these results were not matched in vitro by purified enzyme kinetics. By this metric, the I41G Y227G mutant was judged to be approximately 3-fold less active (as measured by $k_{\mathrm{cat}} / K_{\mathrm{m}}$ ) as the wildtype enzyme, with diminished in vitro activity also observed for the two parental single mutants relative to their SOS activity profiles. It is unclear as to why the in vitro and E. coli in vivo results in this chapter were contradictory; however the purified mutant enzymes also displayed less activity with the alternative substrates CB1954 and NFZ relative to wildtype YcnD (B.s). This may be due to an unexplained issue with the mutant protein preparations, or may indicate that the mutant proteins are less stable or less active under the specific in vitro assay conditions employed in this chapter. Alternatively, assuming that the purified enzyme kinetic parameters measured in this chapter were an accurate measure of the enzyme activity, one possible explanation is that the mutant enzymes may nonetheless exhibit greater activity than wild type $\mathrm{YcnD}$ (B.s) in vivo due to significantly decreased catalytic activity with alternative substrates that are present naturally in E. coli. If YcnD has high affinity for such substrates, they could potentially compete with PR-104A in vivo; whereas the mutants may exhibit less activity with PR-104A in isolation, but higher effective activity in vivo due to reduced competitive inhibition. A similar theory was suggested as the rationale for the lower in vitro activity, but more than 1,000-fold higher sensitivity conferred towards rat glioma cells in vivo, of a mutant HSVtk enzyme with the prodrug substrate GCV, compared to the wildtype enzyme (345). In this case, the mutant enzyme demonstrated an even greater decrease in catalytic activity (as 
determined in vitro, relative to the decrease observed with GCV) with the native substrate thymidine, considered to be a competitive substrate for GCV (212). In the case of $Y \mathrm{cnD}$ (B.s), the competing substrates could be quinones, which are naturally found in abundance in E. coli and other bacteria and are known substrates for many bacterial NTRs $(167 ; 260 ; 286 ; 330 ; 346)$. Further investigation of this phenomenon could be achieved through kinetic analysis of quinone reduction by mutant and wildtype enzymes, as well as observing the effects on SOS induction and $\mathrm{IC}_{50}$ s upon addition of exogenous quinones to cell cultures during relevant assays. Unfortunately, attempts at over-expressing YcnD (B.s) and mutant variants in the human carcinoma cell line HCT116 were unsuccessful, preventing analysis of the relative activities of these enzymes with PR-104A in a more clinically relevant mammalian cell system.

A recurring motif in the mutagenesis results presented here was the substitution of relatively bulky amino acids (e.g. isoleucine, arginine, tyrosine) by glycine, resulting in improved SOS activity with PR-104A as substrate. Mutation of sites I41, Y227 and R139 to glycine all conferred beneficial effects on PR-104A reductase activity, with further improvement afforded by the combination of two of these changes (I41G Y227G). A possible reason for this pattern is that enlargement of the active site by decreasing resident amino acid side chain size (see Figures 6.14A \& B) results in greater affinity for bulkier substrates. PR-104A (molecular weight $498 \mathrm{~g} \mathrm{~mol}^{-1}$ ) is a substantially larger molecule than many quinones (molecular weights 100-200 $\mathrm{g} \mathrm{mol}^{-1}$ ), the latter widely considered to be natural substrates of NTRs. Furthermore, an I41A mutant was almost indistinguishable in SOS activity from the I41G mutant (see Figure 6.8), which is consistent with smaller side chains being beneficial in that residue position. A similar scenario was observed during mutagenesis of NfsA (E.c) towards improved reductase activity of flavins, where the greatest improvement in activity was conferred by an E99G mutation (188). It is noteworthy that other work in our lab has demonstrated that this same mutation in NfsA (E.c) substantially improves PR-104A reductase activity of this enzyme; however the analogous mutation in YcnD (B.s) (E105G) was not identified as being able to confer improved activity towards PR-104A in this study. In published site-directed mutagenesis work on NfsB (E.c), substitutions for glycine at either sites Y68 or S40 were demonstrated to be among the most beneficial mutations at these sites for improving CB1954 reductase activity of this enzyme (218; 219). 

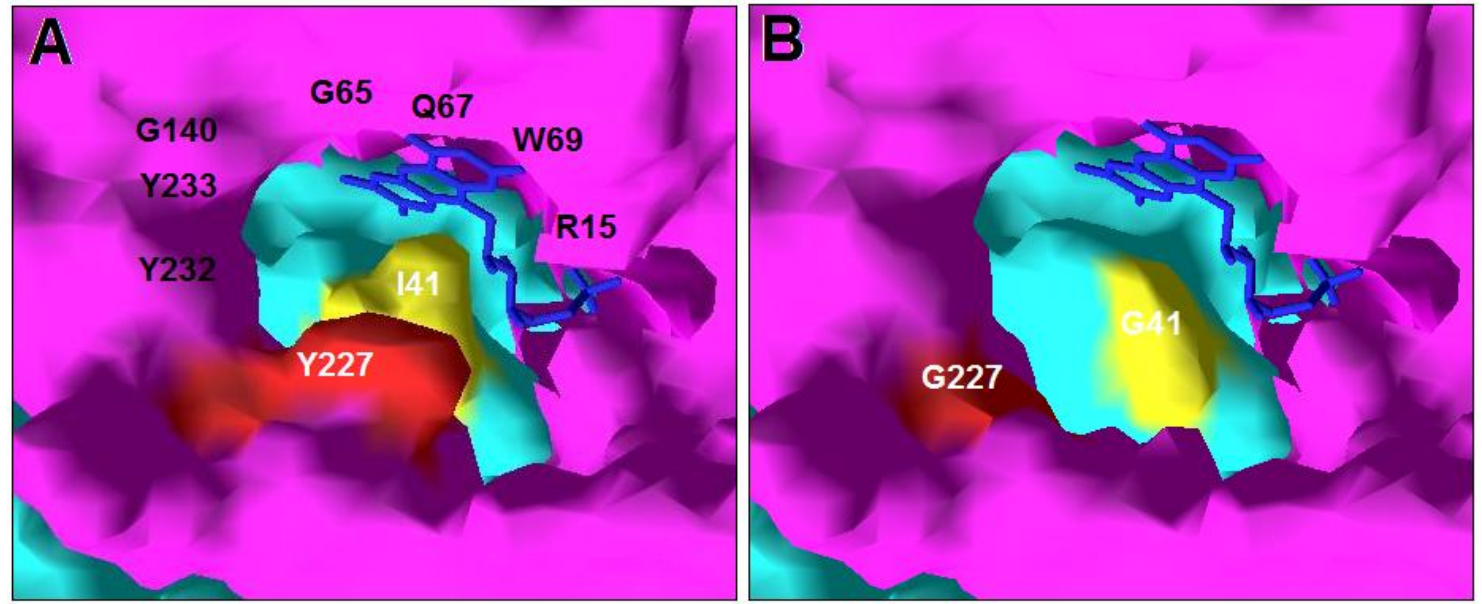

Figures 6.14A \& B. Mutagenesis-induced alterations in YcnD (B.s) active site topography.

Representation of the molecular surfaces of the substrate binding pockets of (A) wildtype and (B) I41G Y227G double mutant YcnD (B.s). Amino acids belonging to the separate monomeric YcnD (B.s) subunits are coloured in cyan and magenta, respectively, and the FMN co-factor is coloured blue. The approximate locations of other active site residues are shown in (A). Molecular surfaces were calculated and imaged with Swiss-PdB Viewer (Deepview), using lowest-energy conformations.

In contrast, for $Y c n D$ (B.s), substituting glycine by either isoleucine or valine at amino acid 65, or arginine by phenylalanine, leucine or histidine at amino acid 139 all appeared to enhance PR-104A reductase activity of this enzyme relative to wildtype. This suggests that enlargement of the active site is not the only alteration required for improved PR-104A catalysis, but that remodelling of active site architecture is also necessary.

It is noteworthy that many of the mutations that improved activity with PR-104A had either little effect, or were deleterious for activity of $Y \operatorname{cnD}$ (B.s) with alternative nitroaromatic prodrugs (CB1954, MTZ and RB 6145). In fact, the only mutation that substantially improved activity (more than 1.5-fold) of YcnD (B.s), relative to wildtype, towards any of these substrates was $\mathrm{R} 139 \mathrm{H}$, conferring a modest 1.65 -fold increase in SOS response activity over wildtype with RB 6145. These data suggest that mechanisms of binding and catalysis of YcnD (B.s) vary widely for different nitroaromatic substrates, and that development of a single lead mutant enzyme for optimal reduction of one prodrug may not be ideal for others. It might therefore be beneficial to develop a panel of mutant YcnD (B.s) enzymes for GDEPT clinical use, with the prodrug being administered ultimately determining which $\mathrm{YcnD}$ (B.s) variant is employed.

Overall, the best single and double mutant $\mathrm{YcnD}$ (B.s) enzymes described in this work (I41G and I41A/G Y227G) were 2 and 2.5-fold improved, respectively, over the 
wildtype enzyme (based on $\mathrm{SOS}$ and $\mathrm{IC}_{50}$ results), for PR-104A reduction. This is modest in comparison with enzyme engineering work performed elsewhere, in particular that of active site mutagenesis of NfsB (E.c). For this enzyme, the best single and double mutant variants identified were roughly 8 and 12-fold improved (respectively) for CB1954 reductase activity over wildtype (219). Although our results are not directly comparable to those of Searle and colleagues due to fundamental differences in the enzymes, substrates and experimental methodology, the differences in fold-improvement observed between the two enzymes following active site mutagenesis is worth discussing. In particular, the high PR-104A reductase activity exhibited by wildtype $Y c n D$ (B.s) suggests that native active site architecture may already be very good for reduction of this prodrug, and that small alterations (e.g. single or double mutations) are not going to substantially improve activity. Wildtype NfsB (E.c), on the other hand, has a $k_{\text {cat }} / K_{\mathrm{m}}$ almost 50-fold lower than that of YcnD (B.s) for both CB1954 and PR-104A (see Chapters 3 and 5), indicating a greater capacity for this enzyme to undergo catalytic improvement through active site mutagenesis. Alternatively, catalytically important residues of $\mathrm{YcnD}$ (B.s) may have been missed in this study, as the targeted sites were only chosen based on their proposed interactions with an NAD molecule following superimposition of the active site with that of the homologous NTR Frp (V.h). Binding of PR-104A and nitroaromatic substrates within the YcnD (B.s) active site could differ to that carried out by co-substrates, or additionally, binding of both substrates and co-substrates could differ between YcnD (B.s) and Frp (V.h). A more extensive site-directed mutagenesis study of $\mathrm{YcnD}$ (B.s) active site residues may therefore identify mutations that are more beneficial for PR-104A reduction than those identified in this study.

As previously mentioned, YcnD (B.s) contains four tyrosine residues (Y227, Y228, Y232 and Y233) within the active site that are absent in all other NfsA-family enzymes (based on sequence and structural alignments). Alteration of one of these residues, Y227, to a glycine significantly improved the PR-104A-reductase activity of YcnD (B.s) in this study, based on SOS and $\mathrm{IC}_{50}$ results. The rationale for this observation could be that steric hindrance of PR-104A binding and product release conferred by the phenyl ring of the tyrosine residue is alleviated upon conversion to a less bulky amino acid. This theory has previously been suggested in a mutagenesis study with NfsB (E.c), where it was demonstrated that mutation of a phenylalanine residue at site 124 to any of 14 alternative amino acids significantly improved the CB1954-reductase activity of this enzyme (218). Although no Y233 mutations 
conferred substantially higher PR-104A reductase activity over the wildtype enzyme, the remaining two tyrosines (Y228 and Y232) that were not investigated in this chapter present attractive candidates for future mutagenesis studies, based on the positive results obtained with alterations to residue Y227.

The site directed mutagenesis work performed in this chapter was greatly facilitated by the creation and employment of the positive selection vector pUCXKG. Using the pUCXKT-KG vector system has so far resulted in close to $100 \%$ efficiency in selection of transformants carrying correctly constructed vectors. The principal advantage of this system over other commonly used cloning techniques (such as blue/white selection or toxic allele replacement) is the complete reduction in frequency of false positive clones, the latter being principally generated through self-ligation of digested parental plasmid, following degradation and blunt ending of DNA ends by contaminating exonucleases (339). This situation is precluded in the pUCXKT/KG system due to the absolute requirement on incorporation of primer-encoded DNA sequences and regulatory regions into the parental plasmid, in order to confer the selective property (kanamycin resistance) on host cells.

Other positive selection vectors that work on similar principals to pUCXKG have previously been developed. For example, positive selection afforded by the vector RHP-AmpS designed by Haag and Ostermeier (2009) involves the reconstitution of a truncated antibiotic resistance gene, the $\beta$-lactamase encoding bla gene, by in frame, correctly oriented cloning of a PCR product that includes (and correctly restores) the final C-terminal codon (W290) of this gene (340). As described in the publication, this system is able to function due to the essentiality of residue W290 to the catalytic function of the $\beta$-lactamase enzyme. Therefore, although effective, this system is limited to the use of $\beta$-lactamase activity as the selective property. Additionally, both the inserted gene and bla are expressed in complementary directions (towards each other), suggesting (although not mentioned by the authors) the possibility of translational inhibition of both enzymes through generation of antisense RNA during transcription of each gene. pUCXKG overcomes both of these limitations, because it does not depend on essential residues for regulating activity of the selective enzyme, and the expression of both genes in the same orientation and from different RBSs allows separate translational control of each gene product. Selective functions other than kanamycin resistance are therefore able to be employed in the pUCXKG system, widening its potential utility. 


\section{Chapter 7: Summary, conclusions and future directions.}

\subsection{Research motivation}

To date, the E. coli NTR NfsB is the best studied nitroaromatic prodrug reducing enzyme for GDEPT, having already been employed, in combination with the prototypical nitroaromatic prodrug CB1954, in phase I/II clinical trials for the treatment of prostate cancer $(100 ; 136 ; 138)$. The observed clinical benefits of this system in aiding tumour regression have however been modest, with a likely contributing factor being insufficient catalytic turnover of CB1954 (at its MTD) by NfsB (122; 136). Therefore, the discovery and design of novel nitroaromatic drugs and activating enzymes with improved clinical efficacy are attractive areas of research $(36 ; 199 ; 219)$. Identification of alternative nitroaromatic prodrug reducing enzymes is facilitated by the vast availability and diversity of naturally occurring bacterial NTRs (162). Additionally, nitroaromatic prodrug reductase activity of NTRs can be enhanced through enzyme engineering methods, enabling unique tailoring of enzyme-prodrug combinations (218; 221). This avenue of research has been limited, however, by a lack of effective and flexible methodologies for high throughput screening of candidate NTRs that exhibit improved nitroaromatic prodrug activation. The research described in this thesis has addressed many of these issues, in particular through the development of a moderately high throughput screen for NTR-catalysed nitroaromatic prodrug reduction, and its application in identification and engineering of novel bacterial NTRs for enhanced activity towards several key nitroaromatic prodrugs.

\subsection{Key findings}

\subsubsection{Development of a screen for nitroaromatic prodrug reduction}

The first outcome of this research was the development and validation of a microtitre plate-based screen for the detection and relative quantification of nitroaromatic prodrug reduction catalysed by candidate NTRs. The screening system chosen was based on the previously reported SOS chromotest, an E. coli-based bioassay that employs a genetically engineered reporter strain containing a reporter gene (in this case the $\beta$ galactosidase-encoding gene lacZ) under the control of an SOS responsive promoter (in this case the $s f i$ promoter $)(231 ; 250)$. Expression of the reporter gene in this host is 
therefore directly linked to activation of the SOS response, which in turn is up-regulated by accumulation of DNA damage within the cell. Consequently, the relative rates of nitroaromatic prodrug reduction by candidate NTRs over-expressed in this host organism can be indirectly assessed by measuring the degree of SOS response induced by DNA adducts caused by the activated prodrugs.

Over-expression of NfsB (E.c) in the SOS reporter strain SOS-R1 (sfiA::lacZ) led to a dose-dependent increase in SOS response relative to an empty plasmid control, following challenge with CB1954 (Figure 3.2), proving the feasibility of this approach for monitoring nitroaromatic prodrug reduction. Further testing with a panel of ten additional native E. coli candidate NTRs identified three enzymes (NfsA, AzoR and NemA) also capable of inducing SOS responses substantially higher than empty vector levels (Figure 3.4). Furthermore, one of these NTRs, NfsA, displayed superior SOS activity to NfsB. SOS assay results were consistent with both $\mathrm{IC}_{50}$ values of $E$. coli grown in the presence of CB1954 and in vitro kinetics using His6-tagged purified enzymes (Figure 3.5, Table 3.1). In contrast, cytotoxicity studies in the human carcinoma cell line HCT-116 demonstrated an apparent (over 2-fold) superiority of NfsB over NfsA in conferring CB1954 sensitivity to host cells; otherwise, results were consistent with those obtained in E. coli regarding relative rankings of NTRs (Table 3.2). Although the basis for the discrepancy between NfsA and NfsB results observed in HCT-116 and E. coli is not clear, it is possible that it was related to either different cosubstrate availability between the cell types (NfsA is NADPH-dependent, NfsB has no preference), different sensitivities to the various CB1954 reduction products (NfsA reduces the 2- $\mathrm{NO}_{2}, \mathrm{NfsB}$ reduces both the 2 and 4- $\mathrm{NO}_{2}$ of $\mathrm{CB} 1954$; Figure 3.8) or other as yet unknown differences between the cell types employed. Overall however, the metabolic heterogeneity of different cancer types and cell lines suggests that results obtained in HCT-116 may not be representative of cancer as a whole. Therefore E. coli may provide a valid, as well as simple, rapid and more generally applicable, alternative to human cell lines for studying nitroaromatic prodrug reduction.

\subsubsection{Roles of various DNA repair pathways in processing and mutagenicity of CB1954-induced DNA adducts.}

CB1954 can be reduced by NTRs at either the 2- or 4-NO 2 (but not both) resulting in metabolites (hydroxylamino- and amino-derivatives) with disparate cytotoxic properties $(110 ; 121)$. Previous work in mammalian cells has demonstrated that the $4-\mathrm{NO}_{2}$ 
reduction product can induce DNA interstrand crosslinks, which are significantly more lethal in cells deficient in NER than in wildtype cells (121). The 2-NO $\mathrm{NO}_{2}$ reduction products on the other hand are generally presumed to form far less toxic mono-adducts (109), although toxicity of the $2-\mathrm{NH}_{2}$ and $4-\mathrm{NHOH}$ metabolites were very similar in an NER-proficient cell line (121). These differences led us to investigate the roles of both SOS-dependent and -independent DNA repair pathways in E. coli in CB1954-induced DNA lesion repair, and how the presence or absence of these pathways may affect SOS inductions in response to CB1954. SOS-R1 strains deficient in either $\mathrm{O}^{6}$-methylguanine transferase activity, the adaptive response to DNA alkylation damage, or DNA mismatch repair induced SOS responses of equal magnitude to wildtype strains upon challenge with either 2- or 4- $\mathrm{NO}_{2}$ reduction products of CB1954, suggesting little to no role for these pathways in CB1954 adduct repair. In contrast, strains lacking the $u v r B$ gene, essential for NER to function, induced SOS functions significantly higher than WT strains in response to the $4-\mathrm{NO}_{2}$, but not the $2-\mathrm{NO}_{2}$ reduction products of CB1954 (Figure 4.5). Therefore, NER is likely to be a critical pathway for repair of 4- $\mathrm{NO}_{2}$ reduction product induced adducts, similar to results observed in mammalian cells (121). Likewise, reduced forms of other nitroaromatic prodrugs (NFZ, PR-104A) that generate adducts also known to be repaired by NER (in bacteria and/or mammalian cells) induced SOS responses in SOS-R1 $\triangle u v r B$ strains substantially higher than in WT strains (Figure 4.8). Mutagenicity testing of each of the CB1954 reduction products in SOS-R1 wildtype and $\Delta u v r B$ strains demonstrated that error-prone polymerases, through TLS repair pathways, are likely to be involved in repair of DNA adducts formed by both metabolites. Furthermore, CB1954-induced mutagenicity was higher in the $\Delta u v r B$ strain relative to wildtype, particularly in response to the 2- $\mathrm{NO}_{2}$ reduction products (Figure 4.9). These results suggest that NER may be the primary repair pathway for adducts induced by both CB1954 reduction metabolites; however, in its absence, adducts induced by $2-\mathrm{NO}_{2}$, but not $4-\mathrm{NO}_{2}$ reduction products are equally capable of being repaired through the TLS pathway.

\subsubsection{Testing an expanded library of bacterial NTRs for CB1954 and PR- $104 \mathrm{~A}$ reductase activity}

To identify novel NTRs with improved nitroaromatic prodrug reductase activity, a core library of 47 bacterial NTRs of diverse origins and sequence/structure were selected for activity screening (Figure 5.2). Prior to screening, the SOS reporter strain was optimised 
for enhanced sensitivity to nitroaromatic prodrugs by in-frame deletion of the outer membrane channel-encoding gene tolC. This resulted in up to 3-fold higher SOS responses relative to $\mathrm{tolC}^{+}$strains upon challenge with equimolar concentrations of PR104A (Figure 5.1), greatly reducing levels of prodrug consumed in SOS assays. The library of NTRs was screened for CB1954 and PR-104A reductase activity, initially via the SOS assay and subsequently through $\mathrm{IC}_{50}$ assays and in vitro measurement of purified enzyme kinetics. Results correlated positively and strongly across all assays with CB1954 (Figures 5.4 to 5.7), with NfsA family enzymes being the most active class of NTRs on average, in agreement with results achieved in Chapter 3. Although correlations between results of the various assays with PR-104A as substrate were not as strong as those with CB1954 (Figures 5.8, 5.9 and 5.13), overall results indicated a superiority of NfsA-family enzymes in metabolism of this substrate. NfsA-family enzymes were also, on average, the most active enzymes (in terms of SOS response induction) with the nitroimidazole prodrugs MTZ and RB 6145 (Figures 5.17 and 5.18, respectively).

A potential clinical weakness of PR-104A and related DNBMs is their ability to undergo metabolism by the endogenous human oxygen insensitive reductase AKR1C3, resulting in off-target toxicity in healthy cells. Seeking to identify alternative nitrobenzamide mustard prodrugs that do not share this limitation, two mono nitrobenzamide mustards (MNBMs) were tested with AKR1C3 in vitro and found to be poor substrates for this enzyme (Figure 5.15). Furthermore, SOS testing with a selection of NTRs demonstrated that MNBMs are efficient substrates for NfsA and NfsB-family NTRs (Figure 5.16), suggesting a potential application in GDEPT for these compounds.

\subsubsection{Improving the PR-104A reductase activity of $Y c n D$ (B.s) through active site mutagenesis.}

YcnD (B.s) was identified in Chapter 5 as an ideal candidate NTR, based on high CB1954 and PR-104A reductase activity, for further enhancement of nitroreductase activity by site saturation mutagenesis of active site residues. Using the SOS assay as a screen, YcnD (B.s) variants randomly mutated at one of ten active site residues (chosen through analysis of the solved enzyme structure; (194)) were screened for improved PR104A reductase activity (Figure 6.5). Individual mutations that conferred the highest improved activity were combined, pairwise, within the same gene and the subsequent double site variants subsequently tested for PR-104A reductase activity. The most active 
single and double mutants (I41G and I41A/G Y227G) that were identified through these assays induced SOS responses that were on average 2- and 2.5-fold higher than wildtype YcnD (B.s), respectively, at equimolar concentrations of PR-104A (Figure 6.8). These results were in agreement with $\mathrm{IC}_{50}$ measurements of the same strains (Table 6.2). Among the amino acid substitutions that conferred enhanced PR-104A reductase activity to $\mathrm{YcnD}$ (B.s), many involved changes to glycine, suggesting that enlargement of the active site cavity was beneficial in enzyme catalytic activity with PR-104A. However, purified His6-tagged recombinant enzymes of three of the most active $\mathrm{YcnD}$ (B.s) variants demonstrated lower PR-104A reductase activity in vitro, on average, relative to the wildtype enzyme (Figure 6.10). This pattern was also observed with other nitroaromatic substrates (Figure 6.12), suggesting that the low mutant enzyme activity observed in vitro was a product of poor quality or unstable purified enzyme preparations. Alternatively, the introduced mutations may have lowered the activity of $\mathrm{YcnD}$ (B.s) with alternative substrates that naturally occur within the E. coli host (e.g. quinones). Therefore, mutant enzymes, although kinetically inferior to wildtype $\mathrm{YcnD}$ (B.s) in reduction of PR-104A, may have demonstrated superior activity in vivo due to substantially reduced competition from endogenous substrates. If this hypothesis is correct, the evolved enzyme variants may only prove superior to native YcnD in GDEPT models if these competing substrates are also present in human cells.

To facilitate mutant library creation and screening, a novel positive selection expression vector, pUCXKT, was developed and used for over-expression of variant YcnD (B.s) enzymes in this section. The cloning efficiency (frequency of correctly formed constructs among all transformants) of this vector was close to $100 \%$; substantially higher than success rates typically encountered with restriction digestion/ligation based cloning techniques. Therefore, these results indicate that this vector is a useful tool for efficient preparation of large expression libraries for protein evolution and engineering studies.

\subsection{Critical evaluation and possible experimental improvements}

\subsubsection{SOS assay improvements}

A significant component of this thesis was the development and employment of the SOS assay as a rapid and reliable screening technology for detection and relative quantification of nitroaromatic prodrug reduction by candidate NTRs. Despite its 
obvious advantages, the screening potential of this assay is largely limited by the necessity of working in 96-well plates and the extra handling steps (cell lysis, addition of reporter substrates) that are required prior to measurement of SOS activity. For a single researcher working without automated liquid-handling robots it would be difficult to use this assay to screen more than 1,000 variants per day, an inadequate number if more comprehensive enzyme evolution and engineering studies were to be performed. One approach for obtaining higher assay throughput could be the replacement of the colorimetric LacZ substrate ONPG for one of the many available cell-permeable fluorescent LacZ substrates (e.g. MUG, FDG) $(347$; 348). This would not only obviate the need for cell lysis prior to SOS response detection, thereby decreasing manual screening labour, but also permit real-time assessment of SOS induction, providing deeper insight into the kinetics of SOS induction in response to different drugs. A particularly attractive property of fluorescent reporter substrates is their potential application in flow cytometry and FACS, which would provide a substantially more efficient means of screening, and selecting, for improved enzymes. However, the high cost associated with many of these fluorescent substrates could render the screen economically unviable; instead, replacement of the SOS-controlled lacZ gene with that for green fluorescent protein $(g f p)$ is potentially a more cost-effective means of applying fluorescence-based screening to the detection of active enzymes. Although an inherently less sensitive reporter molecule than $\beta$-galactosidase (349; 350), Gfp signal production and detection can be enhanced by incorporating the SOS reporter gene fusion into a multi-copy plasmid (229). Attempts to improve SOS screening using this method are ongoing in our laboratory (Janine Copp, personal communication).

Alternatively, it might be possible to place selectable markers such as genes that confer antibiotic resistance or that complement null mutations in es sential metabolic pathways (e.g. amino acid biosynthesis) under SOS control, permitting ultrahighthroughput selection of active NTR over-expressing clones on prodrug-containing selective-solid media. However, SOS response induction caused by antibiotic-mediated cellular damage $(351)$ or nutrient starvation $(352 ; 353)$ could potentially impact the effectiveness of these techniques.

\subsubsection{Validation of $E$. coli as model organism}

Another credible limitation of the experimental procedures employed in this thesis was the use of E. coli as a model organism. Although prokaryotic models provide significant 
advantages in enzyme activity screens, such as rapid growth time, ease of genetic manipulation and robust enzyme expression, fundamental genetic and physical differences between $E$. coli and human cells raise issues concerning the reliability and applicability of results gained in the former in the context of actual therapeutic performance in human GDEPT. The contrasting results observed with CB1954 toxicity between NfsA (E.c) and NfsB (E.c) over-expressing E. coli and HCT-116 carcinoma cells (see section 3.4.5) suggest potential discrepancies between the different cell types. More comprehensive cytotoxicity testing of prodrugs in NTR over-expressing mammalian cell lines, however, is required before definite conclusions can be reached; such studies are currently underway at the ACSRC (Adam Patterson, personal communication). On the other hand, many other GDEPT studies that have used E. coli as a model organism, including those investigating NfsB (E.c) variants for improved CB1954-reductase activity, describe positive correlations between enzyme activity results gained in this organism and mammalian cells $(156 ; 213 ; 218)$. It should be noted, however, that divergent results may be less likely to be reported in the literature. Nevertheless, if $E$. coli is to remain a core component in screening of NTRs for prodrug reduction, more detailed knowledge of prodrug metabolism in this species is required before results can be regarded with confidence. In particular, properties such as intracellular co-substrate levels, the ultimate fate of drug reduction products, resistance mechanisms to drugs and their metabolites, and the nature and repair of induced DNA lesions should ideally all be investigated and compared to the same properties in mammalian cells. This way, the basis of any differences in results observed between the organism types can be understood.

A method of avoiding the potential pitfalls of $E$. coli could be to use fastgrowing eukaryotic microorganisms as host strains for testing prodrug reduction. Thus, screening would retain the benefits of short assay time, but also gain the significant advantage of using an organism more closely related to the final application. One attractive candidate is the yeast $S$. cerevisiae, which has been well-characterised at the biochemical and genetic levels and is often employed in high throughput enzyme screens $(354 ; 355)$ and as a model organism for anticancer drug testing (356). Furthermore, DNA damage reporter strains of this species have been developed (357360 ), which would allow transfer of the screening benefits of the E. coli SOS assay to this new organism. 


\subsubsection{Alternative screens to the SOS assay}

It is important to note that SOS response induction in the SOS assay is an indirect effect of prodrug reduction; as such, internal or external factors independent of prodrug reduction and associated DNA damage could potentially influence SOS activity. More importantly, NTR-specific properties that may affect the SOS response (stress arising from heterologous protein expression or NTR activity with competing substrates, for instance) could have confounding effects on relative activity rankings. For example, many, but not all biochemically characterised NTRs have demonstrated NAD $(\mathrm{P}) \mathrm{H}$ oxidase activity, whereby $\mathrm{NAD}(\mathrm{P}) \mathrm{H}$ is oxidised at the expense of molecular oxygen, leading to the formation of hydrogen peroxide $(170 ; 171 ; 329) . \mathrm{As}_{2} \mathrm{O}_{2}$ is itself a harmful oxidant with DNA damaging properties, generation of this species by overexpression of such NTRs in host cells could impact on their SOS and cytotoxicity measurements relative to NTRs that lack such oxidase activity. As such, NTR activity screens that directly test for the desired end product of prodrug reduction (i.e. the hydroxylamino/amino derivatives) may be advantageous and, overall, more accurate and reliable in the assessment of relative NTR prodrug reductase activity. As an example of such a screening mechanism, the replacement of prodrugs with non-toxic fluorescent analogues in a whole cell assay would permit direct measurement of NTR activity via fluorescence spectrophotometry or flow cytometry/FACS, a technique that has previously been employed in the evolution of other enzymes $(223 ; 361 ; 362)$. However, the cost and effort involved in design and synthesis of such analogues could be a limiting factor. Also, catalytic activity of enzymes towards such analogues may not precisely reflect activity towards the prodrugs of interest, due to subtle structural differences between the compounds. Alternatively, reagents that can react directly and selectively with reduced prodrug derivatives, producing a detectable signal in the process (e.g. colour change, fluorescence), would permit rapid and direct quantification of prodrug reduction by candidate enzymes. As already mentioned, a similar screen has previously been applied successfully to the detection of hexavalent chromium reduction $(222 ; 363)$. Such a technique may be particularly relevant to nitroaromatic prodrug reduction due to the reactive nature of the reduced (hydroxylamino) species. Examples of reagents that are able to detect the presence of aromatic amines in complex mixtures have already been reported $(164 ; 364-366)$, although interactions of these reagents with aromatic hydroxylamino compounds - the typical end product of NTR-catalysed reduction of nitroaromatic compounds - have not been examined. Furthermore, it is 
expected that cell lysis and other handling steps would still be required prior to reagent addition and product detection, which would hinder assay efficiency.

\subsection{Future experiments}

\subsubsection{Structure-activity relationship studies: NTRs and prodrugs}

An appropriate future direction for this research would be the continuation of $Y \mathrm{cnD}$ (B.s) active site mutagenesis to further enhance PR-104A reductase activity. Although this study included a comprehensive coverage of active site single-residue variants of YcnD (B.s), the highest activity improvement detected was modest (ca. 2-fold over wildtype). Previous research has demonstrated that multiple simultaneous mutations within the active site can have synergistic effects on enhancement of the desired activity; activity improvements beyond those that might be predicted by addition of the effects of the individual single mutants $(367 ; 368)$. Therefore more detailed investigations into double or triple site mutants of $\mathrm{YcnD}$ (B.s) may uncover unexpected improvements in enzyme activity. Additionally, random mutagenesis techniques that target the entire gene sequence could identify mutations outside of the active site that improve enzyme activity and/or stability that would be difficult or impossible to predict based on structural analyses alone (369). This would however require more efficient methods for high-throughput screening, as discussed above. Finally, determination of crystal structures of both native enzymes and improved mutant NTRs in complex with prodrug substrates could provide highly valuable information regarding key residues involved in prodrug binding and catalysis and therefore enable more rational design of improved biocatalysts for prodrug reduction.

Understanding the role of competing substrates (e.g. quinones) in vivo on the prodrug-reductase activity of NTRs is also an important direction for further research. This is underscored by the likely heterogeneity in intracellular quinone pool composition between different human tumour types (370-372), which could lead to varied success rates of NTR-GDEPT between different cancers depending on the degree of competition by quinones on NTR activity. A similar scenario has previously been observed for mutant HSVtk enzymes, whose success in conferring GCV sensitivity to different human cell types depended on intracellular thymidine (competitive substrate) concentrations $(345 ; 373)$. Furthermore, differences in the composition of quinone pools between $E$. coli and human cells could constitute a source of discrepancy between NTR 
activity results (e.g. SOS, $\mathrm{IC}_{50}$ ) gained from the two organisms. Firstly however, a role of quinone competition on NTR prodrug reductase activity needs to be firmly established, through purified enzyme and in vivo assays. If confirmed, NTRs would then ideally need to be engineered to lose quinone affinity but retain or gain prodrug affinity; in this case a high throughput screen with the ability to detect quinone reduction would need to be developed and employed alongside prodrug reduction assays.

\subsubsection{Alternative uses for NTRs}

The biotechnological use of NTRs has been reported in areas of research other than cancer therapy. In particular, NTRs have been well-studied for their ability to reduce environmental contaminants such as hexavalent chromium $(184 ; 374)$, azo dyes (286; 375 ) and nitroaromatic by-products of industrial processes (162), thereby aiding in the detoxification and eventual removal of these compounds from the environment. As such, the native and evolved NTR libraries generated in this thesis may be of significance for the identification of enzymes with improved catalytic activity towards any of these alternative substrates. However, as reduced species of many of these compounds do not display strong genotoxic properties, the SOS assay is not likely to be an effective means of screening for enzyme activity. Instead, an assay based on $\mathrm{NAD}(\mathrm{P}) \mathrm{H}$ consumption that uses tetrazolium salts as indicator dyes of nicotinamide cosubstrate concentration (such as NBT or INT; $(247 ; 376)$ ) could be used as a generic screening tool for evaluation of NTR reductase activity. Validation of this technique is ongoing in the Ackerley laboratory (Elsie Williams, personal communication). Alternatively, a moderate throughput screen has previously been developed for evaluation of chromate reduction (363), while assessment of azo-dye reduction is facilitated by the change in visible colouration that accompanies this process. An alternative and potentially very high throughput screen for assessing rates of nitroaromatic compound reduction could be to couple NTR activity with dioxygenase enzymes that are able to release nitrite from aromatic amines, many of which have previously been characterised $(377 ; 378)$; as such, reaction products of NTR directed catalysis of nitroaromatic compounds would become substrates for the dioxygenase enzymes. When performed in an $E$. coli host, nitrite released from the latter reaction could act as a source of nitrogen for cell growth, thereby providing a selective mechanism for detection of active NTR enzymes by growing cells on solid media with the nitroaromatic compound as the sole nitrogen source. However, a dioxygenase 
enzyme with wide substrate specificity (yet lacking parental nitroaromatic compound dioxygenase activity) would first need to be identified, or as is more likely, engineered for this technique to function efficiently. If feasible, such a selection strategy could also be employed in assessing nitroaromatic prodrug reduction, thereby greatly enhancing rates of detection of improved enzymes.

Apart from bioremedial processes, NTRs have also been employed in developmental research, most notably by exploiting the ability of NfsB (E.c) to reduce the non-bystander prodrug MTZ, thereby presenting a strategy for targeted ablation of NTR over-expressing transgenic cell lines (379). This thesis presents evidence indicating not only that alternative NTRs may be better suited for MTZ reduction, but also that other NTR-prodrug combinations that do not overlap with MTZ reduction could be identified or developed, thereby enabling the study of multiple transgenic cell lines simultaneously. Work to this end, along with identification of fluorescent nitroaromatic reporter compounds for imaging of separate NTR over-expressing cell lines, is ongoing in the Ackerley laboratory (Claire Horvat, personal communication). 


\subsection{Concluding remarks}

This thesis has described both the development of techniques for evaluation of enzymecatalysed genotoxic prodrug reduction, and the assessment of novel bacterial NTRs for their ability to reduce a range of clinically attractive nitroaromatic prodrugs. In particular, NTRs superior to NfsB, the 'gold-standard' of NTR GDEPT, with CB1954 as well as other nitroaromatic prodrugs of interest, were identified. These results are not only relevant to the field of cancer gene therapy, but also hold significance for other areas of research that require efficient NTR-mediated catalysis, such as bioremediation, targeted cell ablation and reporter compound development. Similarly, the novel SOS assay developed in this study has the potential to be applied to the discovery of enzymes that activate other genotoxic prodrugs, such as cyclophosphamide and 5-FC, or simply to the discovery of novel DNA-damaging antimicrobial or anti-cancer agents from large-scale chemical libraries. However, deeper investigation is required before this assay and the $E$. coli host organism can confidently be viewed as a model system for drug metabolism in cancer.

GDEPT presents a novel strategy for combating cancer, with the particularly attractive property of limiting toxicity to cancerous cells. While improving activity of prodrug activating enzymes is one method for enhancing clinical efficacy of the system, there is likely to be an upper limit to the extent to which the catalytic rate of the activating enzyme can be improved before other factors, such as prodrug diffusion and toxicity, and gene delivery/transfection rates become the principal limitations in therapeutic efficacy of the treatment regimen. It is therefore pertinent that research into these other avenues is continued, to maximise the chances of GDEPT becoming a major anti-cancer therapy system. 


\section{Appendix 1: NTR accession numbers and sequences}

NCBI accession numbers of NTRs employed throughout this thesis are listed,

alphabetically by NTR name, in Table A1.1. The sequences the accession numbers refer to were used for design of oligonucleotide primers for PCR amplification of the respective genes. However, due to the use of non-type strains, in many instances, for the amplification and extraction of NTR genes, many gene and protein sequences of NTRs used throughout this work were divergent from those given in the NCBI database. Where this is the case, accession numbers are coloured in red in Table A1.1, and the actual nucleotide and amino acid sequences are listed below. All others (coloured in blue), refer to gene and protein sequences that were identical to those used in this study.

Table A1.1. NCBI database accession numbers of NTRs used throughout this work.

See text for details on colour coding.

\begin{tabular}{|c|c|c|c|c|c|}
\hline NTR & $\begin{array}{l}\text { Accession } \\
\text { number }\end{array}$ & NTR & $\begin{array}{l}\text { Accession } \\
\text { number }\end{array}$ & NTR & $\begin{array}{l}\text { Accession } \\
\text { number }\end{array}$ \\
\hline 1204 (P.a) & NP 249895 & $\overline{N e m A ~(S . t)}$ & NP 805105 & NfsB (V.v) & NP 762888 \\
\hline 2432 (P.p) & NP 744580 & NemA (V.v) & NP 762994 & WrbA (E.c) & $\underline{\text { AP } 001635}$ \\
\hline 3720 (P.p) & NP 745853 & NfrA (B.s) & $\underline{\mathrm{CAB} 15837}$ & WrbA (P.s) & YP 275866 \\
\hline 4538 (P.p) & NP 746647 & NfsA (C.k) & $\underline{\text { YP } 001453815}$ & YcaK (E.c) & $\underline{\text { AP } 001531}$ \\
\hline 4975 (P.a) & NP 253662 & NfsA (E.c) & AP 001482 & YcdI (E.c) & $\underline{\text { AP } 001639}$ \\
\hline 5190 (P.a) & NP 253877 & NfsA (E.s) ${ }^{c}$ & YP 001438568 & YcdI (K.p) & YP 001334699 \\
\hline AzoR (E.c) & AP 002037 & NfsA (K.p) & GU726296.1 & YcnD (B.s) & $\underline{\mathrm{CAB} 12194}$ \\
\hline AzoR (S.t) & NP 805329 & NfsA (L.i $)^{d}$ & NP 470273 & YdgI (B.s) & $\underline{\mathrm{CAB} 12385}$ \\
\hline AzoR (V.v) & NP 761189 & NfsA (S.t) & NP 455401 & YdjA (E.c) & AP 002384 \\
\hline $\mathrm{EcD}$ (P.p) & AAL09699 & NfsA (V.f) & YP 002158635 & YdjA (K.p) & YP 001334868 \\
\hline FraseI (V.f) ${ }^{a}$ & $\underline{B A A 04595}$ & NfsA (V.v) & NP 763284 & YfkO (B.s) & GU726295.1 \\
\hline $\operatorname{Frp}(\mathrm{V} . \mathrm{h})^{\mathrm{b}}$ & AAA21331 & $\mathrm{NfsB}(\mathrm{C} . \mathrm{k})$ & YP 001454141 & YieF (E.c) & AP 004074 \\
\hline $\operatorname{KefF}($ E.c) & AP 000710 & $\mathrm{NfsB}$ (E.c) & AP 001223 & YwrO (B.s) & NP 391480 \\
\hline MdaB (E.c) & $\underline{\mathrm{AP} 003578}$ & $\mathrm{NfsB}$ (E.s) & YP 001437964 & YwrO (L.i) & NP 471232 \\
\hline MdaB (P.s) & $\underline{\text { YP } 273002}$ & NfsB (K.p) & $\underline{\text { YP } 001334233}$ & YwrO (V.f) & YP 002157853 \\
\hline NemA (E.c) & AP 002272 & $\mathrm{NfsB}$ (S.t) & NP 806031 & & \\
\hline NemA (K.p) & $\underline{\text { YP } 001335650}$ & NfsB (V.h) & ZP 01985918 & & \\
\hline
\end{tabular}

${ }^{\mathrm{a}}$ Our fraseI nucleotide sequence contained 1 base pair change from the database sequence: $\mathrm{C} \rightarrow \mathrm{T}$ at position 651. This was a silent mutation (codon change from ATC $\rightarrow$ ATT, both coding for Ile)

${ }^{b}$ Out Frp nucleotide sequence was synthesized to contain all optimal E. coli codons. Protein sequence was identical to that given by the indicated accession number.

${ }^{c}$ The initial 17 amino acids indicated in the database sequence were not incorporated into the NfsA (E.s) sequence used in this work.

${ }^{\mathrm{d}}$ Initial codon was changed from GTG to ATG for the sequence used in this work. 


\section{$\operatorname{AzoR}($ V.v)}

Nucleotide sequence:

ATGTCTCGTTTACTAGTATTAAAATCAAGCATCCTTGgTGATTACTCTCAATCAAACAAACTGGTTGACG AGTTCATCAATAAATTCGACCAACAAGACGTTGTCGTTCGTGATCTGGCCCAGCAACCATTGCCTGTTTT AGATTTCCAAGTGGCGACAGCATTGCGCGCAAGCGGTGATTTAAGTGAAGCGCAACAGGCTATTGTTGAT СTTTCTGACCAACTGATTGCCGAAATCAAACACGCTGACACCTTAATTATTGCTGCCCCTATGTACAACT TCACCGTCCCGACCCAGCTTAAAAACTGGATCGATTTGATTGCTCGTGCTGGCGTTACCTTCACTTATAC CGAGCAAGGTCCGAAAGGACTGATCGAAGGTAAGAAAGCGGTTGTTGTAACGACTCGTGGTGGTATTCAT AAAGACGCAGCGAGTGACATCATCACTCCATACCTAAAAACCGTATTAGGGTTTGTTGGTATCACAGAGG TTGAATTTGTTACGCTGAAGCACTCAACATGGGTGAAGACTTTGCCTCTAAAGGCTTGGCTTCAGCAAG TGAGCACCTTGCAGCGCTAACAGCTTAA

\section{Amino acid sequence:}

MSRLLVLKSS ILGDYSQSNKLVDEF INKFDQQDVVVRDLAQQPLPVLDFQVATALRASGDLSEAQQAIVD LSDQLIAEIKHADTLI IAAPMYNFTVPTQLKNWI DLIARAGVTFTYTEQGPKGLIEGKKAVVVTTRGGIH KDAASDI ITPYLKTVLGFVGITEVEFVYAEALNMGEDFASKGLASASEHLAALTA

\section{NemA (K.p)}

Nucleotide sequence:

ATGTCGGAAGCAAAATTATTCAGCCCACTGAAAGTCGGTGCGGTCACCGTCCCTAATCGCGTATTTATGG CGCCGTTGACGCGTCTGCGCAGCATTGAGCCGGGCGATATCCCTACTCCGCTGATGGGCGAATACTACCG CCAGCGCGCCAGTTCCGGGCTGATCATCACCGAAGCGACGCAGATCTCCGCGCAGGCCAAAGGCTACGCC GGCGCGCCTGGGCTGCACAGCCCGGAGCAGATTGCCGCCTGGCAGAAAATCACCGCCGGCGTTCATGCCG AAAACGGTCATATCGCCGTCCAGCTGTGGCACACCGGGCGTATTTCCCATAGCAGCCTGCAGCCTGGCGG CGCCGCCCCGGTAGCGCCTTCCGCGCTGAGCGCGGGCACCCGTACCTCACTGCGTTACGAAAACGGCCAC GCCATTCGCGTCGACACCTCAATGCCCCGCGCCCTGGAAACCGCAGAGATCCCAGGGATCGTCAACGATT TCCGTCAGGCGGTCGGCAATGCCCGTGACGCCGGCTTCGATCTGGTCGAGCTGCACTCAGCCCACGGCTA TCTGCTGCACCAGTTCCTCTCGCCATCGGCGAACCAGCGTACCGATCAGTACGGCGGCAGCGTGGAGAAC CGTGCCCGTCTGGTGCTGGAAGTGGTTGACGCCGTGAGTCAAGAGTGGAGCGCCGAGCGCATTGGCATCC GCGTGTCGCCGATTGGCAGCTTCCAGAATGTGGATAATGGCCCGAACGAAGAAGAAGACGCGCTGTATCT GATTAGCGAGCTGGCGAAACGCGGTATCGCCTATCTGCACATGTCCGAGCCGGACTGGGCGGGCGGCAAA CCTTACAGTGAAGCTTTCCGTCAGAAAGTCCGCGACCGCTTCCCGGGGGTGATCATTGGCGCTGGCGCTT ACACAGTAGAAAAAGCCAACGATCTTATCAATAAAGGGCTGATTGATGCCGTGGCATTTGGCCGCGATTA CATCGCCAACCCGGATCTGGTGGCTCGCCTGCAGAAAAAAGCGGCGCTTAACCCGCAACGCCCGGAATCC TTCTACGGCGGCGGCGCGGAAGGCTACACCGATTACCCGACTCTGTAA

\section{Amino acid sequence:}

MSEAKLFS PLKVGAVTVPNRVFMAPLTRLRS IEPGDI PTPLMGEYYRQRAS S GLI ITEATQISAQAKGYA GAPGLHSPEQIAAWQKITAGVHAENGH IAVQLWHTGRISHSSLQPGGAAPVAPSALSAGTRTSLRYENGH AIRVDTSMPRALETAEIPGIVNDFRQAVGNARDAGFDLVELHSAHGYLLHQFLSPSANQRTDQYGGSVEN RARLVLEVVDAVSQEWSAERIGIRVSPIGSFQNVDNGPNEEEDALYLISELAKRGIAYLHMSEP DWAGGK PYSEAFRQKVRDRFPGVI I GAGAYTVEKANDL INKGL I DAVAFGRDYIANP DLVARLQKKAALNPQRPES FYGGGAEGYTDYPTL

\section{$\operatorname{NemA}($ S.t)}

\section{Nucleotide sequence:}

ATGTCATCAGCAAAACTGTTTACCCCCCTCAAGGTGGGTGCCATCACGGCGACAAGTCGCGTATTTATGG CGCCTTTGACGCGTTTGCGGAGTATTGAACCGGGCGACATTCCTACGCCGTTAATGGCGGAATATTACCG TCAACGCGCCAGCGCCGGATTGATCATCAGCGAAGCCACACAAATCTCCGCGCAGGCGAAAGGCTATGCC GGCGCACCGGGATTACACAGCGATGAGCAGATCGCCGCCTGGAAAAAAATCACTCAGGGAGTCCATGCCC AGGGCGGCCATATGGCGGTGCAACTGTGGCATACGGGCCGTATTTCACACGCCAGCCTGCAACCAGGCGG ACAAGCGCCCGTCGCGCCATCCGCTATCAATGCGGGAACCCGCACCTCTTTGCGTGACGAAAACGGCCAG GCCATCCGCGTAGAAACTTCCACGCCGCGCGCGCTGGATACTCATGAGATCCCCGGGATCGTCAACGATT TCCGCCAGGCTATCGCCAATGCCCGAGAGGCAGGCTTTGATCTGGTGGAGCTGCACTCCGCGCACGGTTA TCTGCTGCACCAGTTCCTCTCACCGTCTTCTAATCACCGTACCGATCGGTACGGCGGCAGCGTAGAGAAT CGCGCCCGTCTGGTGCTGGAGGTGGTTGATGCCGGCATTAAGGAATGGGGCGCGGACCGTATCGGCATCC GCCTTTCTCCTGTCGGCACTTTCCAGAATGTCGATAACGGCCCGAATGAAGAAGCTGACGCCCTTTATCT GATTGAAGCGTTAGGCAAACGCGGCATCGCTTATCTGCATATGTCTGAACCAGACTGGGCGGGCGGCGAA CCGTACAGCGACGCGTTCCGTGAAAAAGTGCGCGCGCGTTTCCACGGTCCCATCATCGGCGCTGGCGCCT 
ATACGCCAGAAAAAGCGGAAGATCTGATCGAAAAAGGGCTGATTGACGCCGTGGCGTTTGGTCGCGATTA CATCGCTAACCCGGATCTGGTGGCGCGTCTGCAACGTAAAGCGGAACTTAATCCACAGCGTCCCGAAAGT TTTTATGGTGGCGGCGCGGAAGGCTACACAGACTACCCTACTCTGTAA

\section{Amino acid sequence:}

MS SAKLFTPLKVGAITATSRVFMAPLTRLRS IEPGDI PTPLMAEYYRQRASAGLI I SEATQISAQAKGYA GAPGLHSDEQIAAWKKITQGVHAQGGHMAVQLWHTGRISHASLQPGGQAPVAPSAINAGTRTSLRDENGQ AIRVETSTPRALDTHEIPGIVNDFRQAIANAREAGFDLVELHSAHGYLLHQFLSPSSNHRTDRYGGSVEN RARLVLEVVDAGIKEWGADRIGIRLS PVGTFQNVDNGPNEEADALYLIEALGKRGIAYLHMSEPDWAGGE PYSDAFREKVRARFHGP I I GAGAYTPEKAEDLIEKGLIDAVAFGRDY IANPDLVARLQRKAELNPQRPES FYGGGAEGYTDYPTL

\section{$\operatorname{NemA}(\mathbf{V} \cdot \mathbf{v})$}

\section{Nucleotide sequence:}

ATGAGCAAACTGTTTGAACCAACACAGCTAAAACAACTGGATCTACTAAACCGTGTGGTGATGGCGCCAA TGACCCGAGCTCGCACTAGTCAACCGGGAAACATTCCAAACCAAATGATGGCGACTTACTACAAGCAGCG CGCTACCGCAGGCTTGATCATCTCTGAAGCCACCCAGATTTCCGATGATTCACAAGGCTACTCCTTCACA CCCGGCGTTTATACCGATGAACAAGTGGCCGGTTGGAAGTTAGTCACTCAAGCGGTGAAATCACAAGGCG CCGCCATGTTCTGCCAGCTTTGGCATGTGGGCCGAGTATCGCACCCAGTTTTCCAAAAAGGTGAACAGCC CATCGCACCATCTGCGCTTGCTCCGGTAGAGACTAAAGTGTGGATCGCCGATGAGCAAGGCAACGGCAAT ATGGTCGATTGTGTAGAGCCACGAGCCATGACTCAAGCGGATATTGACCGTGTTGTGAGTGATTTTGCCT ATGCCGCGAAACGGGCGATTGAAGCAGGCTTCGATGGCATCGAAATCCACGGCGGCAATGGTTACCTGAT TGACCAGTTCCTACGTACCAATTCAAACCACAGAACCGACAACTACGGCGGTACTCGAGAAAATCGACTT CGCTTTTTGTTAGAAGTGGTCGATGCGGTGAGTAAAGCCATAGGTGCCAACAAAGTAGGAGTACGACTCG CGCCTTTCATCACTTTCAAGGACATGAACTGCCCAGACATTGTGCCAACCATCCTCGATGCATCGAAACA ACTTCAAGCTCGTGATATCGCGTACTTGCATCTGTCTGAAGCGGATTGGGACGACGCGCCCGTGATTCCA GAGAGTTTCCGCATCGAATTGCGTGAATACTTCACCAACACCATTATCGTCGCTGGCAGTTACACTCAAG CGAGAGCCGATGAGGTACTTGAGAAGGGGTATGCAGATTTAGTGGCGTTTGGCCGCCCATTTGTCTCTAA TCCAGATTTGGTGGCACGCTTAAAGCATCAACAACCTCTGGCAGAACTGGATGGAGCAACCCTATTTGGC GGCAATGAACGAGGTTATACAGATTACACCGCTTTGCATGTGTGA

\section{Amino acid sequence:}

MSKLFEPTQLKQLDLLNRVVMAPMTRARTSQPGNI PNQMMATYYKQRATAGLI ISEATQISDDSQGYSFT PGVYTDEQVAGWKLVTQAVKSQGAAMFCQLWHVGRVSHPVFQKGEQP IAPSALAPVETKVWIADEQGNGN MVDCVEPRAMTQADI DRVVS DFAYAAKRAIEAGFDGIEIHGGNGYLIDQFLRTNSNHRTDNYGGTRENRL RFLLEVVDAVSKAIGANKVGVRLAPF ITFKDMNCPDIVPTILDASKQLQARDIAYLHLSEADWDDAPVIP ESERIELREYFTNTI IVAGSYTQARADEVLEKGYADLVAFGRPFVSNPDLVARLKHQQPLAELDGATLFG GNERGYTDYTALHV

\section{NfrA (B.s)}

\section{Nucleotide sequence:}

ATGAATAACACAATCGAAACCATTTTAAATCATCGGTCGATCCGGTCTTTTACTGATCAGCTTCTGACAG CTGAAGAAATTGATATATTAGTGAAGAGTGCGCAGGCTGCGTCTACGTCCAGCTATGTACAGGCGTATTC GATTATCGGGGTTTCCGATCCTGAGAAAAAACGTGAGCTGTCTGTGCTTGCCGGAAATCAGCCTTACGTT GAGAACAACGGGCACTTTTTTGTATTTTGCGCGGACTTGCACCGCCACCAAAAACTGGCTGAGGAAAAAG GCGAAAATATTTCTGAGCTGCTCGAAAATACGGAAATGTTTATGGTTAGCTTAATTGATGCAGCGCTGGC AGCGCAAAATATGAGCGTTGCCGCTGAATCTATGGGGCTTGGCATTTGTTATATCGGCGGCATTCGGAAT GAACTGGACAAGGTGACAGAGGTGTTGCAAACGCCTGACCATGTGCTTCCGCTTTTCGGTCTGGCAGTAG GCCATCCTGCGAACCTATCCGGCAAAAAACCAAGATTGCCAAAGCAGGCTGTCTATCATGAGAATACGTA TAATGTAAACGCAGACGATTTCCGCGACACCATGAATGCGTATGATCAAACCATTTCTGATTATTATAGA GAAAGAACAAACGGACAGCGGGAAGAAACGTGGTCTGATCAGATTCTGAACTTTATGAAGCAGAAGCCGC GTACCTATTTAAATGACTATGTGAAGGAAAAGGGCTTTAATAAAAACTAA

\section{Amino acid sequence:}

MNNTIETILNHRS IRSFTDQLLTAEEIDILVKSAQAASTSSYVQAYS I IGVSDPEKKRELSVLAGNQPYV ENNGHFFVFCADLHRHQKLAEEKGENI SELLENTEMFMVSLIDAALAAQNMSVAAESMGLGICYIGGIRN ELDKVTEVLQTPDHVLPLFGLAVGHPANLSGKKPRLPKQAVYHENTYNVNADDFRDTMNAYDQTISDYYR ERTNGQREETWSDQI LNFMKQKPRTYLNDYVKEKGFNKN 


\section{NfsA (C.k)}

Nucleotide sequence:

ATGACTCCAACCATTGATTTAATCCGTGGTCACCGTTCTATTCGTCATTTTACCGATGAGCCGATTTCCG ATGCGCAGCGTGAGTCGATTATTGCCGCCGCGCGGGGAACCTCAAGCTCCAGTTTTTTACAGTGCAGTTC CATTATCCGCATTACCGATAAAGCCATGCGCGAAGCGCTGGTGCCCCTGACGGGCGGGCAAAAGCACGTT GCGCAGGCCGCGGAATTTTGGGTTTTCTGCGCTGATTTTAATCGTCACTTACAGATTTGCCCGGACGCGC AGCTCGGGCTGGCGGAACAGCTGCTGCTGGGCACGGTCGATACGGCGATGATGGGGCAAAATGCGCTGAC CGCTGCCGAATCCCTGGGGCTGGGCGGCGTCTACATCGGCGGTATCCGCAATCATATTGAAGCGGTTACG GAACGGCTGAAATTACCGAAATATGTCCTGCCGCTGTTTGGACTGTGCCTCGGCTGGCCTGCGGATAACC CTGGCGTTAAGCCGCGTTTGCCCGCAGAACTGGTGGTGCATGAAAATCACTATCAGCCTGTGGATGCCGC GCTGTTGGCGCAATATGATGAGCAGATCGCGGAGTATTACCTGACCCGCGACAGCAATACGCGCCGCGAT ACCTGGAGCGATCATATCCGTCGTACCATCATCAAAGAGAATCGCCCGTTTATTCTGGACTATTTGCATA AACAGGGTTGGGCGACGCGCTAA

\section{Amino acid sequence:}

MTPTIDLIRGHRS IRHFTDEP I SDAQRES I IAAARGTSSSSFLQCSSI IRITDKAMREALVPLTGGQKHV AQAAEFWVFCADFNRHLQICPDAQLGLAEQLLLGTVDTAMMGQNALTAAESLGLGGVYIGGIRNHIEAVT ERLKLPKYVLPLFGLCLGWPADNPGVKPRLPAELVVHENHYQPVDAALLAQYDEQIAEYYLTRDSNTRRD TWSDHIRRTIIKENRPFILDYLHKQGWATR

\section{NfsA (E.s)}

\section{Nucleotide sequence:}

ATGAGCCCATCAGCGACGCCCAGCGTGAAGCGATTATCCACGCGGCGCAGTCCGCCTCCAGCTCCAGTTT CCTGCAGTGCAGCTCCATTATTCGTGTAACCGACCGCGCGATGCGTGAGCAACTGGTGACGCTGACCGGC GGTCAGCCGCACGTCGCGAAAGCCGCCGAGTTCTGGGTTTTCTGCGCCGATTTCAACCGTCATCTGCAAA TCTGCCCGGATGCGCAACTGGGGCTGGCTGAACAGCTTTTGCTTGGCGTGGTAGATACCGCGATGCTCGG ACAAAACGCGCTGGTCGCGGCGGAATCGCTTGGCCTCGGCGGGGTTTACATCGGCGGTATTCGTAACAGC ATTGAAGCGGTCACAGAACTGCTGGGCCTGCCGAAACATGTGCTGCCGCTGTTTGGCCTGTGCCTCGGCT GGCCGGCGGACAATCCGCAGGTGAAACCGCGTATGCCTGCGGGCCTGATGGTGCACGAAAACCGCTACCA GCCGGTCGACCGTGAACTGCTTGCTGAATATGACGAAGAGATAGCCGACTACTACCTGCATCGCGACAGC AACGCCCGCCGCGACACCTGGAGCGATCAGATCCGCCGCACCATCATTAAAGAGAACCGTCCGTTTATTC TCGACTATCTGCACAAGCAGGGCTGGGCGACACGCTAA

\section{Amino acid sequence:}

MTPTIELLCSHRS I RHYTDEP I SDAQREAI I HAAQSAS S S SF LQCSS I IRVTDRAMREQLVTLTGGQPHV AKAAEFWVFCADFNRHLQICPDAQLGLAEQLLLGVVDTAMLGQNALVAAESLGLGGVYIGGIRNSIEAVT ELLGLPKHVLPLFGLCLGWPADNPQVKPRMPAGLMVHENRYQPVDRELLAEYDEEIADYYLHRDSNARRD TWSDQIRRTIIKENRPFILDYLHKQGWATR

\section{NfsA (V.f)}

\section{Nucleotide sequence:}

ATGAACCCTGTTATCGATACTATTTTAGAGCATCGCTCTATTCGCTCTTTTACTAATGAACCTATCTCTA AAGAGCAATTAGATACCATCATCAGTGCGGGTATTGCAGCCTCTTCTTCTAGCTTGCTCCAAGTCAATTC TATTATTCGAATTACCGATAAGGAAAAACGAAAAGCATTAGTTGAGCTATCAGGAGGCCAGCCTTATGTT GAAGGTGCGGCGGAGTTTCTTGTTTTCTGTATTGATTTTCAACGTCATTATGAAATGAACCCGGAGATCA AAGCCGAATTTACCGAACTTACTCTTATTGGTGCTGTTGATGCAGGTATTATGGCGCAAAATTGTCTTTT AGCTGCAGAATCTATGGGATTAGGAGGTGTGTATATCGGTGGGTTACGTACAAACGCACAAGGTGTCGAT GATTTATTAGAACTGCCAAAAAACACAGCTGTTTTGTTTGGTATGTGCCTAGGTTACCCAAATCAAGCGC CTCAAAAAAAGCCACGTTTAAGTCCTGATGTTATTGTTCATGAAAATAGTTATCAGCCACTGGATAAAAG TAAAATTGATGAATATGACGAAATAATGCAAAGCTACTATGCAACGCGCTCGACGAATCAAAAACAAAGT TCATGGTCAGAGCAAATTACAGGAAAACTATCTCAAGAATCTCGCCCTCACATTAAAGGCTACTTAAATA ATAAAGGCCTAGCTATTAAGTGA

\section{Amino acid sequence:}

MNPVIDTILEHRS IRSFTNEP ISKEQLDTI ISAGIAAS S S SLLQVNS I IRITDKEKRKALVELSGGQPYV EGAAEFLVFCIDFQRHYEMNPEIKAEFTELTLIGAVDAGIMAQNCLLAAESMGLGGVY IGGLRTNAQGVD DLLELPKNTAVLFGMCLGYPNQAPQKKPRLSPDVIVHENSYQPLDKSKI DEYDE IMQSYYATRSTNQKQS SWSEQITGKLSQESRPHIKGYLNNKGLAIK 
NfsA (V.v)

Nucleotide sequence:

ATGAACGCTGTTATTGACACTCTGCTTTCCCACCGTTCTATTCGCAAATTTACCGACCAAGCGATAACGC CAGAGCAACTCGACACCATCATTCGAGCAGGGCTAGCCGCTTCTTCTTCCAGTTTGCTACAAGTGGTCTC AATCATTCGCATTACCGATCCAGCCAAACGTCAGCAGCTTGCCGAGCTCGCGGGGCCACAACACTATGTC GAAACGGCTGCCGAATTCCTCGTGTTTTGCATTGATTATCAACGCCACGCCACCCTCAACTCAGAAGTAC AAGCGGGCTTTACCGAGCTGACATTGATCGGCGCGGTGGATGCCGGCATCATGGCGCAAAACTGCTTATT GGCCGCAGAGTCGATGGGGTTAGGTGGCGTTTACATCGGTGGGCTGAGAAACAAAGCCGCCGAGGTGGAT GCCCTTCTCGAACTGCCTCCATTCAGTGCCGTCTTGTTTGGTATGTGCTTAGGCCATCCAGACCAAGACC CTGATCTCAAACCACGGCTGCCCGCGGAGGTCATTCTCCACGAGAACCACTATCAGCCGCTCGATCTCAA TAAAGTAGAGCAATACGATCAAACCATGCTCGACTACTATGGCAAGCGTTCATCAAACCAAAAACAGGCC AGCTGGTCTGAACAAGTAACGGGCAAGCTGGCGGGGGAATCTCGCCCTCATATCTTGCCTTATTTACACA GCAAAGGCTTGGCGACAAAGTAA

\section{Amino acid sequence:}

MNAVIDTLLSHRS IRKFTDQA ITPEQLDTI IRAGLAAS S S SLLQVVS I IRITDPAKRQQLAELAGPQHYV ETAAEFLVFCIDYQRHATLNSEVQAGFTELTLIGAVDAGIMAQNCLLAAESMGLGGVYIGGLRNKAAEVD ALLELPPFSAVLFGMCLGHPDQDPDLKPRLPAEVILHENHYQPLDLNKVEQYDQTMLDYYGKRSSNQKQA SWSEQVTGKLAGESRPHILPYLHSKGLATK

\section{NfsB (C.k)}

\section{Nucleotide sequence:}

ATGGATATCGTTTCTGTCGCCTTAAAACGCTACTCCACTAAGGCGTTCGATCCCAGCAAACAACTGACCG CAGACGAAGCGGAAAAACTCAAAACATTGCTGCAATATAGCCCTTCCAGCACCAACTCTCAGCCGTGGCA TTTCATCGTCGCCAGTACCGAAGAAGGGAAAGCGCGCGTTGCGAAATCCGCCGCCGGTAATTTCGTGTTT AACGAGCGCAAAATGCTGGATGCTTCCCATGTCGTCGTGTTTTGCGCAAAAACCGCAATGGATGACGCCT GGCTGGATCGCGTTGTCGATCAGGAAGATGCCGATGGCCGTTTTGCCACGCCGGAAGCCAAAGCGGCAAA TAACAAAGGCCGTCGCTTTTTCGCGGATCTGCACCGCCGCGACCTGAAAGATGACGATCAGTGGATGGCG AAACAGGTATACCTGAACGTTGGCAACTTCCTGCTGGGCGTCGCCGCGATGGGTCTGGACGCCGTACCGA TTGAAGGCTTCGACGCTGCGGTTCTTGACGCTGAATTTGGCCTGAAAGAGAAAGGTTATACCAGCCTGGT CGTGGTGCCTGTCGGCCATCACAGCGTAGAAGATTTTAACGCTGCGCTGCCGAAATCCCGCCTGCCGCAG GAAACCACCCTGACGGAAGTCTGA

\section{Amino acid sequence:}

MDIVSVALKRYSTKAFDPSKQLTADEAEKLKTLLQYSPSSTNSQPWHF IVASTEEGKARVAKSAAGNFVF NERKMLDASHVVVFCAKTAMDDAWLDRVVDQEDADGRFATPEAKAANNKGRRFFADLHRRDLKDDDQWMA KQVYLNVGNFLLGVAAMGLDAVP IEGFDAAVLDAEFGLKEKGYTSLVVVPVGHHSVEDFNAALPKSRLPQ ETTLTEV

\section{NfsB (E.s)}

\section{Nucleotide sequence:}

ATGAACCTTAATGAGATCATTCGCACTCGCCATACCAGCAAAGCCTACGATAACAGCCGTAAACTGACGG CCGAACAGCAGCAGGAGCTGCTGGATCTGCTGCGTTTTAGCCCCTCTTCCGTCAACTCACAGCCGTGGCA CTTTTTTGCCGTGACTACTGAAGAAGGCAAAGCGCAGATCCTGCCAGCGCTGATGGACGCTAATCAGGTA AAAGCGAAAAACGCCGCCATGACCGTCGTGTTCACCATTAAAGAAGAGCTGAACGAAGCGCACCTGCTGC AACTGCTGGAAAAAGAGCAGCAGGATGGTCGCTACGACGGCGAGGAAGCGCGCGCCGCGAACGATAAAGG CCGCCGCTTTTTTGTGGGTCTGAACAGCGAAACGCCGGAGCAGCAGCGCGAATGGATGACCCGTCAGGCC TATCTGGCGCTCGGTTTCCTGCTGCTGGGCGCAGCCGCGATGGGCCTCGACGCGACGCCTATCGAAGGTT TCCATCCGGAGAAAATGGATGAAGTGCTGGGCCTGAAAGAAAAAGGTCTACGCAGCGTGGTGGTAGCGAC CATCGGTTATCGCAGCGATGCCGACTTCAACGCCACACTGCCGAAATCCCGTCTGGATCAGGATGTGGTG ATCACGCAGCTCTGA

\section{Amino acid sequence:}

MNLNEI IRTRHTSKAYDNSRKLTAEQQQELLDLLRFS PS SVNSQPWHFFAVTTEEGKAQILPALMDANQV KAKNAAMTVVFTIKEELNEAHLLQLLEKEQQDGRYDGEEARAANDKGRRFFVGLNSETPEQQREWMTRQA YLALGFLLLGAAAMGLDATP IEGFHPEKMDEVLGLKEKGLRSVVVATIGYRSDADFNATLPKSRLDQDVV ITQL 


\section{NfsB (K.p)}

Nucleotide sequence:

ATGGATATCGTATCGGTCGCTTTGAAGCGCTATTCCACCAAGGCCTTTGACGCCACGAAAAAGCTGACTG CCGGGGAAGCGGAACAGCTGAAAACGCTGCTGCAGTACAGCCCGTCTAGCACCAACTCCCAGCCGTGGCA CTTTATTGTCGCCAGCACCGATGAAGGCAAAGCTCGCGTGGCGAAAGCCGCCAGCGGCACCTACGTGTTC AACGAACGTAAAATTCTCGACGCCTCGCACGTGGTGGTGTTCTGCGCGAAAACCGCGATGGACGACGCCT GGCTGCAGCGCGTGGTGGATCAGGAAGAGGCCGATGGCCGTTTCGCCACCCCGGACGCAAAAGCCGCTAA CCACAAGGGTCGCACCTTCTTTGCCGATATGCACCGCAAAGAGCTGAAAGATGACGATCAGTGGATGGCG AAACAGGTGTATCTCAACGTCGGCAATTTCCTGCTGGGCGTAGCGGCGATGGGTCTCGACGCCGTGCCGA TTGAAGGGGTCGATTTTGCGATCCTCGATGAGGAATTTGACCTGAAAGCCCAGGGCTACACCAGCCTGGT GGTGGTCCCGGTCGGCCACCATAGCGTAGAAGATTTCAACGCTACCCTGCCGAAGTCGCGTCTGCCGCAG TCGACGACCATTACCGAGATTTAA

\section{Amino acid sequence:}

MDIVSVALKRYSTKAFDATKKLTAGEAEQLKTLLQYSPSSTNSQPWHF IVASTDEGKARVAKAASGTYVF NERKILDASHVVVFCAKTAMDDAWLQRVVDQEEADGRFATPDAKAANHKGRTFFADMHRKELKDDDQWMA KQVYLNVGNFLLGVAAMGLDAVPIEGVDFAILDEEFDLKAQGYTSLVVVPVGHHSVEDFNATLPKSRLPQ STTITEI

\section{NfsB (V.h)}

\section{Nucleotide sequence:}

ATGTCTCATCAAATCATTACAGACCTAAACAACCGTTACACAGCTAAAAAATACGACGAAGAGAAACGCA TCTCTCAAGAAGATATGGCAATCATCAAAGAAGCAATCCGCCTATCTGCGTCTTCTATCAACTCTCAGCC TTGGAAATTCATCGTAATTGAGAGCGACGAAGCGAAACAGCGTTTCCATGACACGTTTGCTAACATGCAC CAGTTCAACCAACCGCACGCGAAAGCAGCATCCCACACTATTCTGCTAGCGTACGATCCAAAATTCACTA AAGAGAAATTTGCGAAGCGTGTTGATGCTGAAGTAACTTCTGGTCACCTACCAGCAGACATGTACGACAT GTTCATGGGTGCTTACGCATTTGCTGAAGCAAACACAGATGAAAACGGCTACAACGGCGGCTGGACGAAA GCGCAAGTTTATATCGCGCTTGGTAACCTACTGCACACACTAGCGCGTCTAGGTATCGACTCTACTCCAA TGGAAGGTGTTGACCCAACACTGATCGGCGAAGAGTTCAAAGCAGAACTAGACGGTCACGTATGTGAAGT TGCACTAGCAATCGGCTACCACAAAGACGGCGAAGACTACAACCACGGTCTACCAAAAGCACGTCTAAAC ATGGATGACGTAATTACTACTCTTTAA

\section{Amino acid sequence:}

MSHQI ITDLNNRYTAKKY DEEKRISQEDMA I I KEAIRLSASS INSQPWKF IVIESDEAKQRFHDTFANMH QFNQPHAKAASHTILLAYDPKFTKEKFAKRVDAEVTSGHLPADMYDMFMGAYAFAEANTDENGYNGGWTK AQVYIALGNLLHTLARLGIDSTPMEGVDPTLIGEEFKAELDGHVCEVALAIGYHKDGEDYNHGLPKARLN MDDVITTL

\section{NfsB (V.v)}

\section{Nucleotide sequence:}

ATGACTATTGTTCAAGCTGCCCAATCCCGCTATTCCACGAAAGCCTTTGATGCTTCGCGCAAATTGCCTG AAGAAAAAGTCGCGGCAGTGAAAGAGTTAATCCGCATGAGTGCGTCCAGTGTCAACTCGCAACCTTGGCA TTTCATTGTCGCGAGCAGTGAAGAAGGAAAAGCGCGCATCGCGAAAGCAACACAAGGTGGTTTTGCTTTT AATGAGCGCAAGATTTTGGATGCCTCTCACGTAGTGGTGTTTTGCGCCAAAACATCGATTGATGAAGCGT ATTTACTCGACCTTTTGCAGAGCGAAGATAAAGATGGCCGCTTTGCCGATGTCGAAGCAAAAAATGGTAT GCATGCTGGCCGCTCATTTTTCGTCAACATGCATCGCTTTGACTTGAAAGACGCGCATCACTGGATGGAA AAGCAAGTTTACCTCAATGTCGGGACGCTGCTATTGGGGGCCTCTGCGATGGAGATCGACGCAGTGCCAA TTGAAGGCTTCGATGCCAAGGTACTTGATGAAGAGTTTGGTCTGCGTGAGAAGGGCTTTACCAGCGTGGT GATTGTGCCGCTGGGTTACCATAGCGAAGACGATTTTAATGCTAAGCTGCCAAAATCACGTTGGCCAGCA GAGACTGTTTTTACCGAAATCTAA

\section{Amino acid sequence:}

MTIVQAAQSRYSTKAFDASRKLPEEKVAAVKELIRMSASSVNSQPWHFIVASSEEGKARIAKATQGGFAF NERKILDASHVVVFCAKTS I DEAYLLDLLQSEDKDGRFADVEAKNGMHAGRSFFVNMHRFDLKDAHHWME KQVYLNVGTLLLGASAMEIDAVPIEGFDAKVLDEEFGLREKGFTSVVIVPLGYHSEDDFNAKLPKSRWPA ETVFTEI 


\section{YcnD (B.s)}

Nucleotide sequence:

ATGAATGAAGTGATTAAATCATTAACAGACCACCGCTCGATTCGCAGCTATACAGATGAACCTGTAGCTA AGGAACAATTGGACCAAATCATTCAAGCGGTACAATCTGCCCCAACTTCTATCAACGGGCAGCAAGTGAC TGTGATCACAGTCCAGGATAAAGAGCGCAAAAAGAAAATCTCCGAGCTGTCAGGCGGACAGCCTTGGATT GACCAGGCTCCTGTTTTCTTGCTGTTCTGCGCAGACTTCAACCGGGCCAAAATCGCGCTTGAGGATCTGA ATGATATCAAAATGGAAATCACAAATGGCTTAGAGTCTGTTCTTGTCGGCGCAGTAGACGCTGGTATTGC CCTCGGCACAGCGACAGCAGCAGCTGAATCACTCGGACTTGGCACGGTTCCGATCGGTGCGGTTCGCGGA AACCCTCAAGAGCTGATCGAACTGCTTGAGCTTCCGAAATACGTGTTCCCTGTATCCGGCCTTGTCATCG GCCATCCTGCTGACCGTTCAGCGAAAAAACCGCGCCTTCCGCAGGAAGCAGTCAATTATCAGGAAACATA TTTGAATCAAGATGAGCTGACGTCTTACATTCAGGCATATGATGAGAAAATGTCTGAATACATGAATAAG CGGACAAACGGAAAAGAAACAAGAAACTGGTCACAGGGCATCGCATCCTATTATGAGCGCCTGTACTATC CACACATCCGTGAAATGCTTGAGAAACAAGGATTTAAAGTTGAAAAATAA

\section{Amino acid sequence:}

MNEVIKSLTDHRS IRSYTDEPVAKEQLDQI IQAVQSAPTS INGQQVTVITVQDKERKKKISELSGGQPWI DQAPVFLLFCADFNRAKIALEDLNDIKMEITNGLESVLVGAVDAGIALGTATAAAESLGLGTVPIGAVRG NPQELIELLELPKYVFPVSGLVIGHPADRSAKKPRLPQEAVNYQETYLNQDELTSYIQAYDEKMSEYMNK RTNGKETRNWSQGIASYYERLYYPHIREMLEKQGEKVEK

\section{YdgI (B.s)}

\section{Nucleotide sequence:}

ATGATCAAAACAAACGATTTTATGGAGATTATGAAAGGACGCCGTTCCATTCGCAACTATGATCCGACAG TGAAAATCAGCAAAGAAGAAATGACAGAAATCTTAGAAGAAGCCACAACTGCTCCATCTTCTGTCAACGC GCAGCCATGGCGTTTTCTCGTCATTGACAGCCCGGAAGGAAAAGAAAAGCTCGCACCGCTTGCAAGCTTT AACCAAACTCAAGTCACAACATCATCTGCCGTCATCGCTGTATTTGCAGATATGAACAATGGAGACTACT TAGAAGAAATCTATTCAAAAGCCGTGGAACTCGGTTACATGCCGCAGGAAGTCAAAGACAGACAAATCGC CGCTCTGACCGCTCATTTTGAAAAGCTTCCGGCACAGGTGAACCGCGAAACGATCCTGATTGACGGAGGT CTTGTTTCAATGCAGCTGATGCTTACTGCACGTGCGCACGGCTATGATACAAACCCGATCGGCGGATACG ATAAAGAAAACATCGCGGAAACATTCGGTTTAGATAAAGAACGTTATGTACCGGTCATGCTTCTTTCTAT CGGAAAAGCAGCAGACGAAGGCTATGCTTCCTACCGTCTGCCGATTGATAAAATTGCAGAATGGAAATAA

\section{Amino acid sequence:}

MIKTNDEME IMKGRRS IRNYDPTVKISKEEMTEILEEATTAP SSVNAQPWRFLVIDS PEGKEKLAPLASE NQTQVTTSSAVIAVFADMNNGDYLEEIYSKAVELGYMPQEVKDRQIAALTAHFEKLPAQVNRETILIDGG LVSMQLMLTARAHGYDTNP IGGYDKENIAETFGLDKERYVPVMLLSIGKAADEGYASYRLP I DKIAEWK

\section{YwrO (B.s)}

\section{Nucleotide sequence:}

ATGAAAATATTGGTTTGGCAGTGCATCCTCATATGGAAACCTCAGTTGTGAATAAGGCATGGACAGAGG AATTGAGTCAACATGACAATATCACAGTACGGGAGCTTTATAAAGAATACCCGAATGAAGCGATAGATGT TGCGAAGGAGCAGCAGCTGTGCGAGCAATACGACCGGATTGTCTTCCAATTTCCGTTTTATTGGTACGGC TCTCCGCCGCTCTTAAAAAAATGGCAGGATCTTGTGCTGACTTACGGCTGGGCCTTTGGTTCAGAAGGAA ACGCCTTGCACGGCAAGGAGCTGATGCTGGCTGTATCAACAGGGAGCGAAGCGGAAAAATATCAGGCGGG CGGAGCAAATCATTACTCGATCAGTGAGCTTTTGAAACCATTTCAGGCCACGAGTAATCTGATCGGCATG AAATACCTGCCACCATATGTGTTCTATGGCGTGAATGATGCAACTGCAGAGGATATCTCTCTCAGTGCAA AACGGTTAGCCGAATACATCCAGCAGCCTTTTGTTTAA

\section{Amino acid sequence:}

MKILVLAVHPHMETSVVNKAWTEELSQHDNITVRELYKEYPNEA I DVAKEQQLCEQYDRIVFQFPFYWYG SPPLLKKWQDLVLTYGWAFGSEGNALHGKELMLAVSTGSEAEKYQAGGANHYS ISELLKPFQATSNLIGM KYLPPYVFYGVNDATAEDISLSAKRLAEYIQQPFV 


\section{4}

\section{YwrO (L.i)}

\section{Nucleotide sequence:}

ATGAAAACATTAGTTATTATCGCACATCCGAATATCGAAAATTCACGCGTAAATAGAGTTTGGAAAGAAG CACTTTTAAAAAGTACAGAAGAGGTGGCAATTCATGAGTTATATGAAGTTTATCCTAACTGGGATATTGA TGTAACCTTTGAACAACAACAATTGCAAAACTACGATAAAGTCATTATCCAATTTCCATTTTATTGGTAC AGCTACCCGCCGTTATTAAAAAAATGGTTCGATGATGTCTTTTCGTATGGCTGGGCTTACGGCTCAAAAG GGGACAAGATGGCTGGGAAAAAGCTTGCTCTTGCAGTTTCAATTGGCGATAAAAAATATAATTATAAAGA AGATGCACCAATTGGCTATTCACTAGACACATTACTTACACCATTTCGAGCAACAATAAACCACATTCGA GCTGACTATAGAGGGGCCCACACGATATATGGCAGCTCCTTTGAAGTCACAGATGAAGAAATAGTAGAAA ATGCGTATGTGTATGCAAACAAGTTTATTAAAACGTTAAATTAG

\section{Amino acid sequence:}

MKTLVI IAHPNIENSRVNRVWKEALLKSTEEVAI HELYEVYPNWDI DVTFEQQQLQNYDKVI IQFPFYWY SYPPLLKKWFDDVFSYGWAYGSKGDKMAGKKLALAVS I GDKKYNYKEDAPIGYSLDTLLTPFRATINHIR ADYRGAHTIYGSSFEVTDEEIVENAYVYANKFIKTLN 


\section{Appendix 2: HPLC chromatograms of CB1954 and PR-104A}

\section{reduction products}
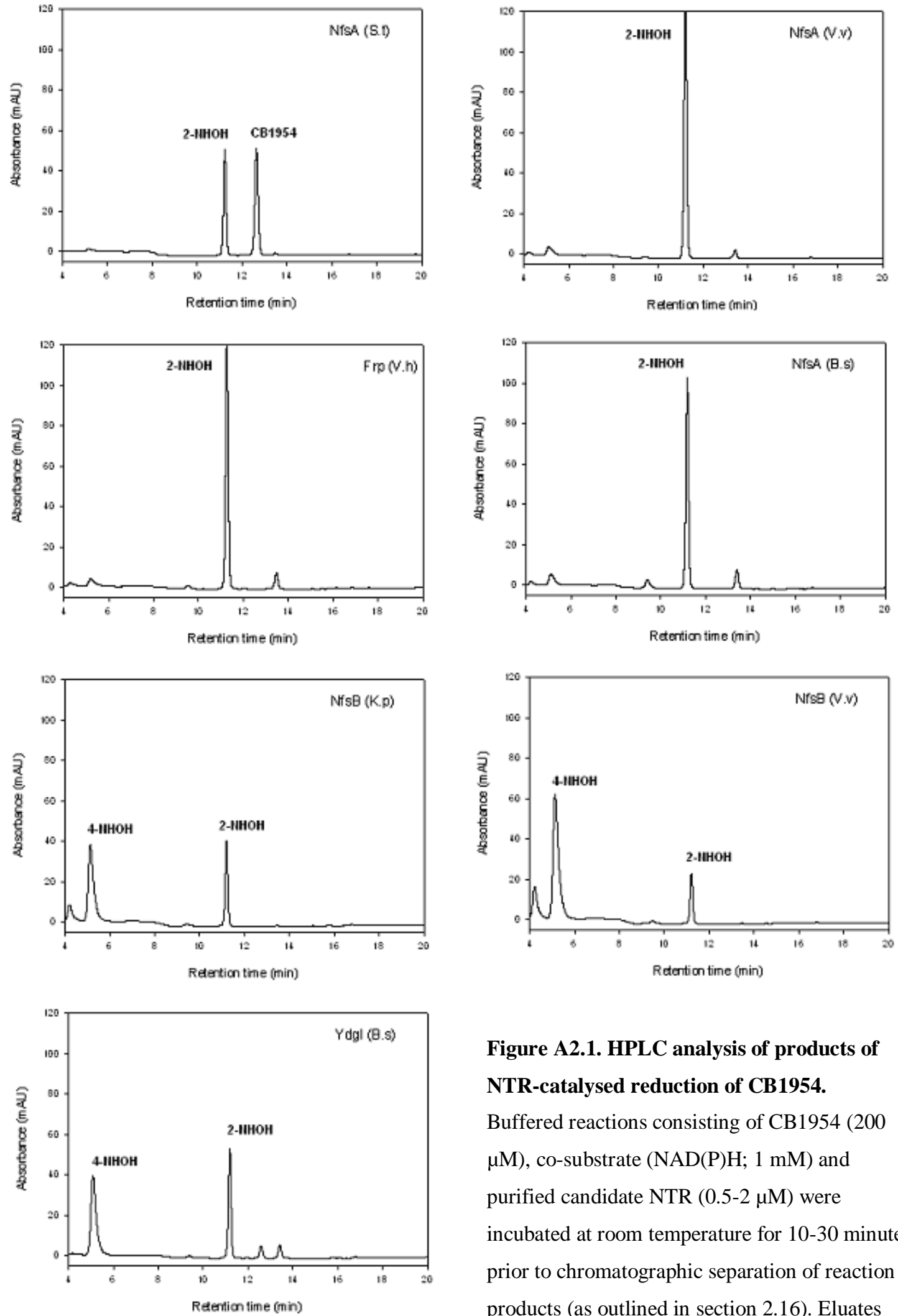

Figure A2.1. HPLC analysis of products of NTR-catalysed reduction of CB1954.

Buffered reactions consisting of CB1954 (200 $\mu \mathrm{M})$, co-substrate $(\mathrm{NAD}(\mathrm{P}) \mathrm{H} ; 1 \mathrm{mM})$ and purified candidate NTR $(0.5-2 \mu \mathrm{M})$ were incubated at room temperature for 10-30 minutes prior to chromatographic separation of reaction products (as outlined in section 2.16). Eluates were continuously monitored at $262 \mathrm{~nm}$. 

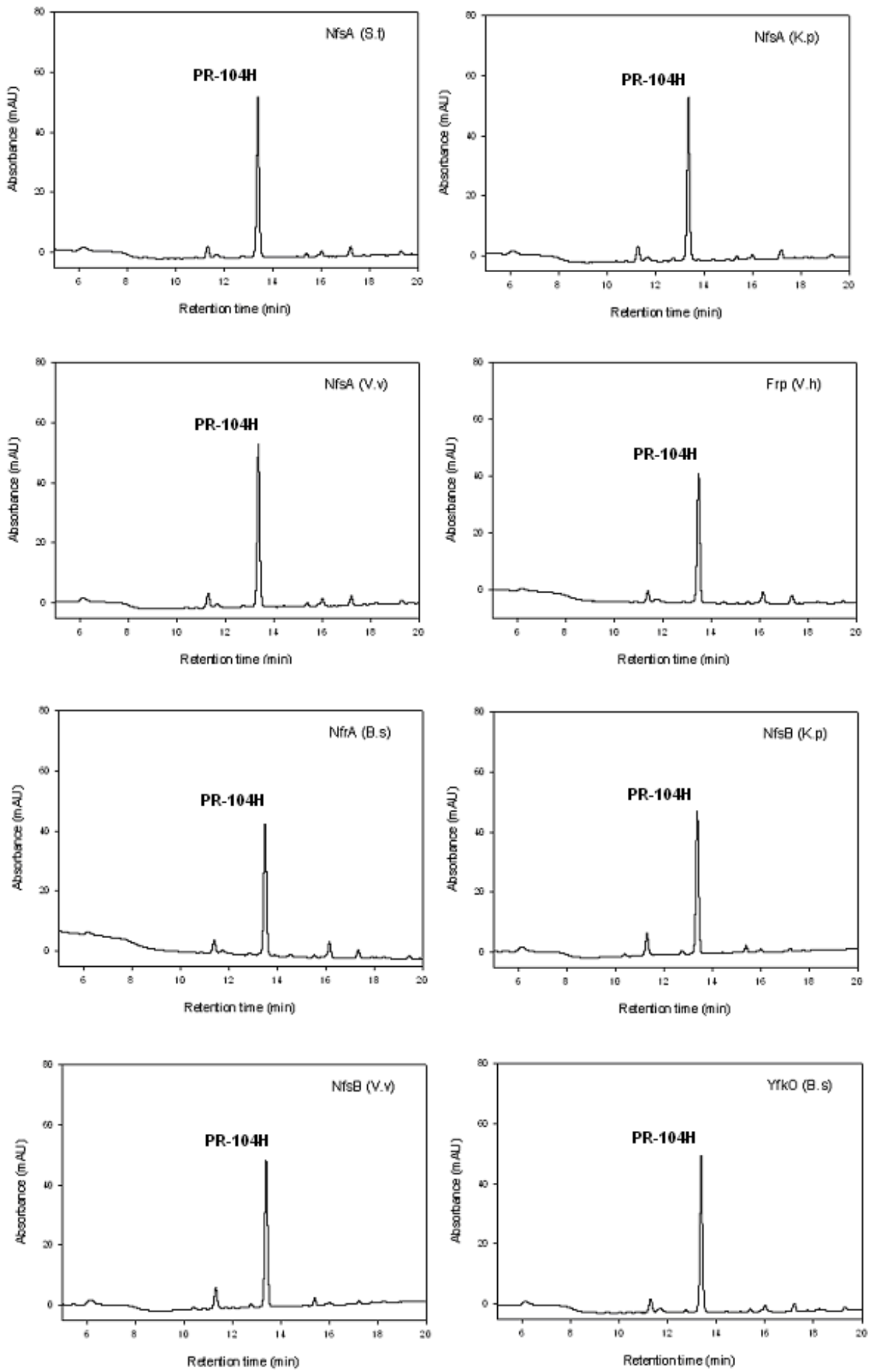

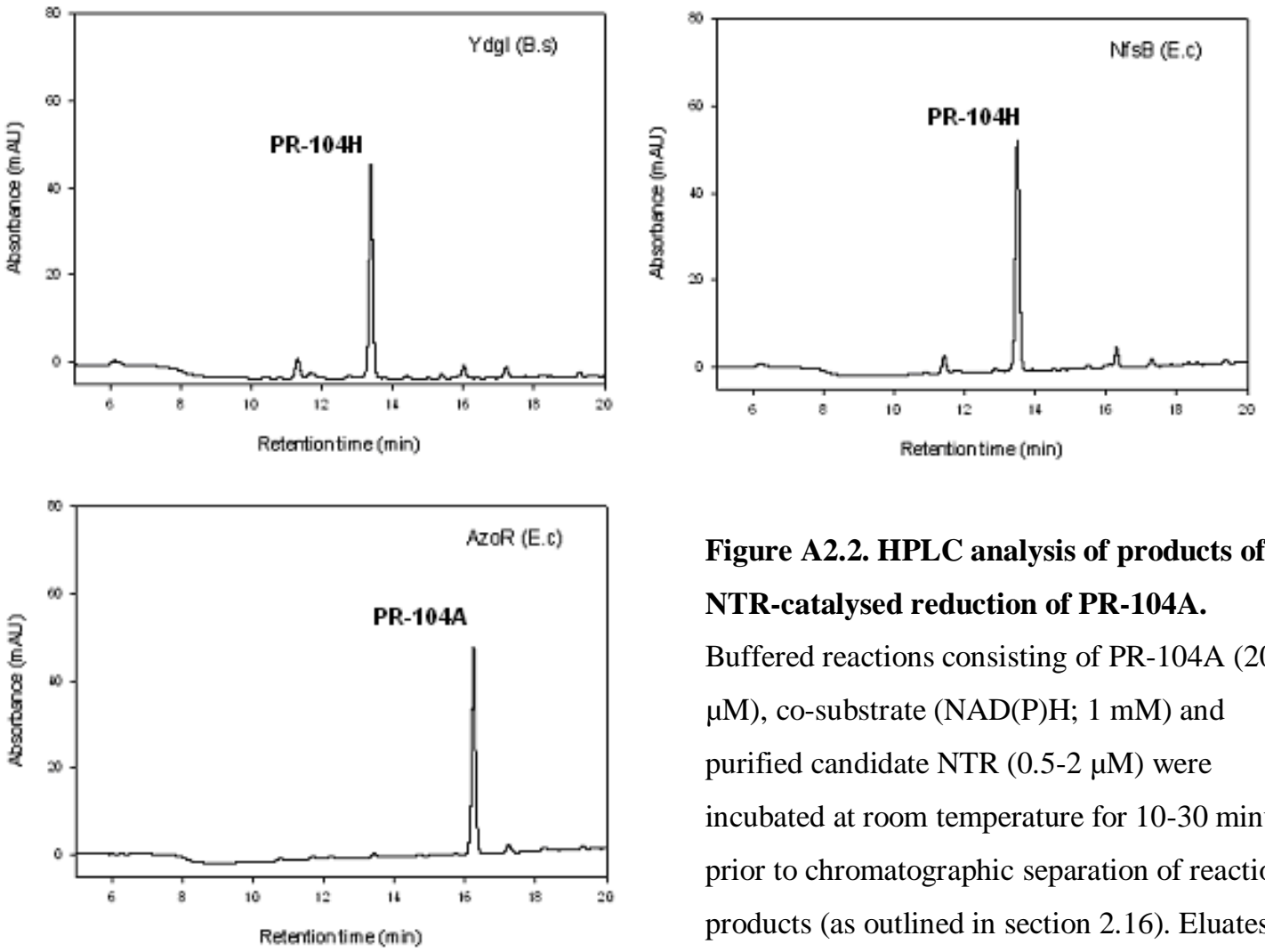

Figure A2.2. HPLC analysis of products of NTR-catalysed reduction of PR-104A.

Buffered reactions consisting of PR-104A (200 $\mu \mathrm{M})$, co-substrate $(\mathrm{NAD}(\mathrm{P}) \mathrm{H} ; 1 \mathrm{mM})$ and purified candidate NTR $(0.5-2 \mu \mathrm{M})$ were incubated at room temperature for 10-30 minutes prior to chromatographic separation of reaction products (as outlined in section 2.16). Eluates were continuously monitored at $254 \mathrm{~nm}$. 


\section{References}

1. American Cancer Society. Cancer Facts \& Figures 2010. Atlanta: American Cancer Society; 2010.

2. McKnight JA. Principles of chemotherapy. Clinical Techniques in Small Animal Practice 2003;18(2):67-72.

3. Peters BG. An overview of chemotherapy toxicities. Topics in hospital pharmacy management / Aspen Systems Corporation 1994;14(2):59-88.

4. Perez-Tomas R. Multidrug resistance: retrospect and prospects in anti-cancer drug treatment. Current Medicinal Chemistry 2006;13(16):1859-1876.

5. Mitchell EP. Gastrointestinal toxicity of chemotherapeutic agents. Seminars in Oncology 2006 Feb;33(1):106-120.

6. Gerber DE. Targeted therapies: a new generation of cancer treatments. American Family Physician 2008 Feb;77(3):311-319.

7. Bennasroune A, Gardin A, Aunis D, Cremel G, Hubert P. Tyrosine kinase receptors as attractive targets of cancer therapy. Critical Reviews in Oncology/Hematology 2004;50(1):23-38.

8. Cheng H, Force T. Why do kinase inhibitors cause cardiotoxicity and what can be done about it? Progress in Cardiovascular Diseases 2010;53(2):114-20.

9. Penas-Prado M, Gilbert MR. Molecularly targeted therapies for malignant gliomas: advances and challenges. Expert Review of Anticancer Therapy 2007;7(5):641-61.

10. http://www.centerwatch.com/drug-information/fda-approvals/ (accessed 28/10/10)

11. Brown JM, Wilson WR. Exploiting tumour hypoxia in cancer treatment. Nature Reviews Cancer 2004;4(6):437-47.

12. Sawyers C. Targeted cancer therapy. Nature 2004 Nov;432(7015):294-297.

13. Cowen RL, Garside EJ, Fitzpatrick B, Papadopoulou MV, Williams KJ. Gene therapy approaches to enhance bioreductive drug treatment. The British Journal of Radiology 2008;81 Spec No 1:S45-56.

14. Durand RE. The influence of microenvironmental factors during cancer therapy. In vivo (Athens, Greece) 1994;8(5):691-702.

15. Kennedy AS, Raleigh JA, Perez GM, Calkins DP, Thrall DE, Novotny DB, Varia MA. Proliferation and hypoxia in human squamous cell carcinoma of the cervix: first report of combined immunohistochemical assays. International Journal of Radiation Oncology, Biology, Physics 1997;37(4):897-905. 
16. Comerford KM, Wallace TJ, Karhausen J, Louis NA, Montalto MC, Colgan SP. Hypoxia-inducible factor-1-dependent regulation of the multidrug resistance (MDR1) gene. Cancer Research 2002;62(12):3387-94.

17. Wartenberg M, Ling FC, Muschen M, Klein F, Acker H, Gassmann M, Petrat K, Putz V, Hescheler J, Sauer H. Regulation of the multidrug resistance transporter P-glycoprotein in multicellular tumor spheroids by hypoxia-inducible factor (HIF-1) and reactive oxygen species. The FASEB Journal 2003;17(3):503-5.

18. Lu X, Kang Y. Hypoxia and hypoxia-inducible factors: master regulators of metastasis. Clinical Cancer Research 2010;16(24):5928-35.

19. Melillo G. Hypoxia-inducible factor 1 inhibitors. Methods in Enzymology 2007;435:385-402.

20. Ahn G-O, Brown M. Targeting tumors with hypoxia-activated cytotoxins. Frontiers in Bioscience 2007;12:3483-3501.

21. McKeown SR, Cowen RL, Williams KJ. Bioreductive drugs: from concept to clinic. Clinical Oncology (Royal College of Radiologists (Great Britain)) 2007;19(6):427-42.

22. Chen Y, Hu L. Design of anticancer prodrugs for reductive activation. Medicinal Research Reviews 2009;29(1):29-64.

23. Peters KB, Brown JM. Tirapazamine: a hypoxia-activated topoisomerase II poison. Cancer Research 2002;62(18):5248-53.

24. Peters KB, Wang H, Brown JM, Iliakis G. Inhibition of DNA replication by tirapazamine. Cancer Research 2001;61(14):5425-31.

25. Lee AE, Wilson WR. Hypoxia-dependent retinal toxicity of bioreductive anticancer prodrugs in mice. Toxicology and Applied Pharmacology 2000;163(1):50-9.

26. Kyle AH, Minchinton AI. Measurement of delivery and metabolism of tirapazamine to tumour tissue using the multilayered cell culture model. Cancer Chemotherapy and Pharmacology 1999;43(3):213-20.

27. Hay MP, Pchalek K, Pruijn FB, Hicks KO, Siim BG, Anderson RF, Shinde SS, Phillips V, Denny WA, Wilson WR. Hypoxia-selective 3-alkyl 1,2,4benzotriazine 1,4-dioxides: the influence of hydrogen bond donors on extravascular transport and antitumor activity. Journal of Medicinal Chemistry 2007;50(26):6654-64.

28. Hay MP, Gamage SA, Kovacs MS, Pruijn FB, Anderson RF, Patterson AV, Wilson WR, Brown JM, Denny WA. Structure-activity relationships of 1,2,4benzotriazine 1,4-dioxides as hypoxia-selective analogues of tirapazamine. Journal of Medicinal Chemistry 2003;46(1):169-82.

29. Tomasz M. Mitomycin C: small, fast and deadly (but very selective). Chemistry and Biology 1995;2(9):575-9. 
30. Zeman EM, Brown JM, Lemmon MJ, Hirst VK, Lee WW. SR-4233: a new bioreductive agent with high selective toxicity for hypoxic mammalian cells. International Journal of Radiation Oncology, Biology, Physics 1986;12(7):123942 .

31. Rockwell S, Hughes CS. Effects of mitomycin C and porfiromycin on exponentially growing and plateau phase cultures. Cell Proliferation 1994;27(3):153-63.

32. Cowen RL, Patterson AV, Telfer BA, Airley RE, Hobbs S, Phillips RM, Jaffar M, Stratford IJ, Williams KJ. Viral delivery of P450 reductase recapitulates the ability of constitutive overexpression of reductase enzymes to potentiate the activity of mitomycin $\mathrm{C}$ in human breast cancer xenografts. Molecular Cancer Therapeutics 2003;2(9):901-9.

33. Gutierrez PL. The role of NAD(P)H oxidoreductase (DT-Diaphorase) in the bioactivation of quinone-containing antitumor agents: a review. Free Radical Biology and Medicine 2000;29(3-4):263-75.

34. Adams GE, Stratford IJ. Hypoxia-mediated nitro-heterocyclic drugs in the radioand chemotherapy of cancer. An overview. Biochemical Pharmacology 1986;35(1):71-6.

35. Koder RL, Haynes CA, Rodgers ME, Rodgers DW, Miller AF. Flavin thermodynamics explain the oxygen insensitivity of enteric nitroreductases. Biochemistry 2002;41(48):14197-205.

36. Denny WA. Nitroreductase-based GDEPT. Current Pharmaceutical Design 2002;8(15):1349-61.

37. Adams GE, Ahmed I, Sheldon PW, Stratford IJ. RSU 1069, a 2-nitroimidazole containing an alkylating group: high efficiency as a radio- and chemosensitizer in vitro and in vivo. International Journal of Radiation Oncology, Biology, Physics 1984;10(9):1653-6.

38. Dronkert ML, Kanaar R. Repair of DNA interstrand cross-links. Mutation Research 2001;486(4):217-47.

39. Walling JM, Stratford IJ, Adams GE, Silver AR, Ahmed I, Jenkins TC, Fielden EM. Studies on the mechanisms of the radiosensitizing and cytotoxic properties of RSU-1069 and its analogues. International Journal of Radiation Oncology, Biology, Physics 1986;12(7):1083-6.

40. Hill RP, Gulyas S, Whitmore GF. Studies of the in vivo and in vitro cytotoxicity of the drug RSU-1069. British Journal of Cancer 1986;53(6):743-51.

41. Naylor MA, Threadgill MD, Webb P, Stratford IJ, Stephens MA, Fielden EM, Adams GE. 2-Nitroimidazole dual-function bioreductive drugs: studies on the effects of regioisomerism and side-chain structural modifications on differential cytotoxicity and radiosensitization by aziridinyl and oxiranyl derivatives. Journal of Medicinal Chemistry 1992;35(19):3573-8. 
42. Horwich A, Holliday SB, Deacon JM, Peckham MJ. A toxicity and pharmacokinetic study in man of the hypoxic-cell radiosensitiser RSU-1069. The British Journal of Radiology 1986;59(708):1238-40.

43. Breider MA, Pilcher GD, Graziano MJ, Gough AW. Retinal degeneration in rats induced by CI-1010, a 2-nitroimidazole radiosensitizer. Toxicologic Pathology 1998;26(2):234-9.

44. Duan JX, Jiao H, Kaizerman J, Stanton T, Evans JW, Lan L, Lorente G, Banica M, Jung D, Wang J, Ma H, Li X, Yang Z, Hoffman RM, Ammons WS, Hart CP, Matteucci M. Potent and highly selective hypoxia-activated achiral phosphoramidate mustards as anticancer drugs. Journal of Medicinal Chemistry 2008;51(8):2412-20.

45. Denny WA, Wilson WR. Considerations for the design of nitrophenyl mustards as agents with selective toxicity for hypoxic tumor cells. Journal of Medicinal Chemistry 1986;29(6):879-87.

46. DeVita VT, Chu E. A history of cancer chemotherapy. Cancer Research 2008;68(21):8643-53.

47. Povirk LF, Shuker DE. DNA damage and mutagenesis induced by nitrogen mustards. Mutation Research 1994;318(3):205-26.

48. Singleton RS, Guise CP, Ferry DM, Pullen SM, Dorie MJ, Brown JM, Patterson AV, Wilson WR. DNA cross-links in human tumor cells exposed to the prodrug PR-104A: relationships to hypoxia, bioreductive metabolism, and cytotoxicity. Cancer Research 2009;69(9):3884-91.

49. Green SL, Giaccia AJ. Tumor hypoxia and the cell cycle: implications for malignant progression and response to therapy. The Cancer Journal from Scientific American 1998;4(4):218-23.

50. Emadi A, Jones RJ, Brodsky RA. Cyclophosphamide and cancer: golden anniversary. Nature Reviews Clinical Oncology 2009 Nov;6(11):638-647.

51. Wilson WR, Hicks KO, Pullen SM, Ferry DM, Helsby NA, Patterson AV. Bystander effects of bioreductive drugs: potential for exploiting pathological tumor hypoxia with dinitrobenzamide mustards. Radiation Research 2007;167(6):625-36.

52. Freeman SM, Abboud CN, Whartenby KA, Packman CH, Koeplin DS, Moolten FL, Abraham GN. The "bystander effect": tumor regression when a fraction of the tumor mass is genetically modified. Cancer Research 1993;53(21):5274-83.

53. Miller VA, Ng KK, Grant SC, Kindler H, Pizzo B, Heelan RT, von Roemeling R, Kris MG. Phase II study of the combination of the novel bioreductive agent, tirapazamine, with cisplatin in patients with advanced non-small-cell lung cancer. Annals of Oncology 1997;8(12):1269-71.

54. Pisharath H, Rhee JM, Swanson MA, Leach SD, Parsons MJ. Targeted ablation of beta cells in the embryonic zebrafish pancreas using E. coli nitroreductase. Mechanisms of Development 2007;124(3):218-29. 
55. Bolton JL, McClelland RA. Kinetics and mechanism of the decomposition in aqueous solutions of 2-(hydroxyamino)imidazoles. Journal of the American Chemical Society 1989;111(21):8172-8181.

56. Singleton DC, Li D, Bai SY, Syddall SP, Smaill JB, Shen Y, Denny WA, Wilson WR, Patterson AV. The nitroreductase prodrug SN 28343 enhances the potency of systemically administered armed oncolytic adenovirus ONYX-411(NTR). Cancer Gene Therapy 2007;14(12):953-67.

57. Patterson AV, Ferry DM, Edmunds SJ, Gu Y, Singleton RS, Patel K, Pullen SM, Hicks KO, Syddall SP, Atwell GJ, Yang S, Denny WA, Wilson WR. Mechanism of action and preclinical antitumor activity of the novel hypoxia-activated DNA cross-linking agent PR-104. Clinical Cancer Research 2007;13(13):3922-32.

58. Hicks KO, Myint H, Patterson AV, Pruijn FB, Siim BG, Patel K, Wilson WR. Oxygen dependence and extravascular transport of hypoxia-activated prodrugs: comparison of the dinitrobenzamide mustard PR-104A and tirapazamine. International Journal of Radiation Oncology, Biology, Physics 2007;69(2):56071.

59. Gu Y, Patterson AV, Atwell GJ, Chernikova SB, Brown JM, Thompson LH, Wilson WR. Roles of DNA repair and reductase activity in the cytotoxicity of the hypoxia-activated dinitrobenzamide mustard PR-104A. Molecular Cancer Therapeutics 2009;8(6):1714-23.

60. Helsby NA, Goldthorpe MA, Tang MH, Atwell GJ, Smith EM, Wilson WR, Tingle MD. Influence of mustard group structure on pathways of in vitro metabolism of anticancer N-(2-hydroxyethyl)-3,5-dinitrobenzamide 2-mustard prodrugs. Drug Metabolism and Disposition: The Biological Fate of Chemicals 2008;36(2):353-60.

61. Guise CP, Wang AT, Theil A, Bridewell DJ, Wilson WR, Patterson AV. Identification of human reductases that activate the dinitrobenzamide mustard prodrug PR-104A: a role for NADPH:cytochrome P450 oxidoreductase under hypoxia. Biochemical Pharmacology 2007;74(6):810-20.

62. Guise CP, Abbattista MR, Singleton RS, Holford SD, Connolly J, Dachs GU, Fox SB, Pollock R, Harvey J, Guilford P, Donate F, Wilson WR, Patterson AV. The bioreductive prodrug PR-104A is activated under aerobic conditions by human aldo-keto reductase 1C3. Cancer Research 2010;70(4):1573-84.

63. Jameson MB, Rischin D, Pegram M, Gutheil J, Patterson AV, Denny WA, Wilson WR. A phase I trial of PR-104, a nitrogen mustard prodrug activated by both hypoxia and aldo-keto reductase $1 \mathrm{C} 3$, in patients with solid tumors. Cancer Chemotherapy and Pharmacology 2009;65(4):791-801.

64. Philip PA, Kaklamanis L, Ryley N, Stratford I, Wolf R, Harris A, Carmichael J. Expression of xenobiotic-metabolizing enzymes by primary and secondary hepatic tumors in man. International Journal of Radiation Oncology, Biology, Physics 1994;29(2):277-83.

65. Fitzsimmons SA, Workman P, Grever M, Paull K, Camalier R, Lewis AD. Reductase enzyme expression across the National Cancer Institute Tumor cell 
line panel: correlation with sensitivity to mitomycin C and EO9. Journal of the National Cancer Institute 1996;88(5):259-69.

66. http://www.wiley.com//legacy/wileychi/genmed/clinical/ (accessed 30/10/10)

67. Vogelstein B, Kinzler KW. The genetic basis of human cancer, 2nd Edition. McGraw-Hill, Medical Pub. Division; 2002.

68. Cao S, Cripps A, Wei MQ. New strategies for cancer gene therapy: progress and opportunities. Clinical and Experimental Pharmacology \& Physiology 2010;37(1):108-14.

69. McCormick F. Cancer gene therapy: fringe or cutting edge? Nature Reviews Cancer 2001;1(2):130-41.

70. Kawamura K, Tasaki K, Hamada H, Takenaga K, Sakiyama S, Tagawa M. Expression of Escherichia coli uracil phosphoribosyltransferase gene in murine colon carcinoma cells augments the antitumoral effect of 5-fluorouracil and induces protective immunity. Cancer Gene Therapy 2000;7(4):637-43.

71. Greco O, Dachs GU. Gene directed enzyme/prodrug therapy of cancer: historical appraisal and future prospectives. Journal of Cellular Physiology 2001;187(1):2236.

72. Rosenberg SA. Shedding light on immunotherapy for cancer. The New England Journal of Medicine 2004;350(14):1461-3.

73. Hadj-Slimane R, Lepelletier Y, Lopez N, Garbay C, Raynaud F. Short interfering RNA (siRNA), a novel therapeutic tool acting on angiogenesis. Biochimie 2007;89(10):1234-44.

74. Vaha-Koskela MJ, Heikkila JE, Hinkkanen AE. Oncolytic viruses in cancer therapy. Cancer Letters 2007;254(2):178-216.

75. Gadi VK, Alexander SD, Kudlow JE, Allan P, Parker WB, Sorscher EJ. In vivo sensitization of ovarian tumors to chemotherapy by expression of E. coli purine nucleoside phosphorylase in a small fraction of cells. Gene Therapy 2000;7(20):1738-43.

76. Nawa A, Tanino T, Luo C, Iwaki M, Kajiyama H, Shibata K, Yamamoto E, Ino K, Nishiyama Y, Kikkawa F. Gene directed enzyme prodrug therapy for ovarian cancer: could GDEPT become a promising treatment against ovarian cancer? Anti-Cancer Agents in Medicinal Chemistry 2008;8(2):232-9.

77. Liu TC, Galanis E, Kirn D. Clinical trial results with oncolytic virotherapy: a century of promise, a decade of progress. Nature Clinical Practice Oncology 2007;4(2):101-17.

78. Rooseboom M, Commandeur JN, Vermeulen NP. Enzyme-catalyzed activation of anticancer prodrugs. Pharmacological Reviews 2004;56(1):53-102.

79. Kerr D. Clinical development of gene therapy for colorectal cancer. Nature Reviews Cancer 2003;3(8):615-22. 
80. Moolten FL. Tumor chemosensitivity conferred by inserted herpes thymidine kinase genes: paradigm for a prospective cancer control strategy. Cancer Research 1986;46(10):5276-81.

81. Elion GB. The biochemistry and mechanism of action of acyclovir. The Journal of Antimicrobial Chemotherapy 1983;12 Suppl B:9-17.

82. Elion GB, Furman PA, Fyfe JA, de Miranda P, Beauchamp L, Schaeffer HJ. Selectivity of action of an antiherpetic agent, 9-(2-hydroxyethoxymethyl) guanine. Proceedings of the National Academy of Sciences of the United States of America 1977;74(12):5716-20.

83. Rainov NG. A phase III clinical evaluation of herpes simplex virus type 1 thymidine kinase and ganciclovir gene therapy as an adjuvant to surgical resection and radiation in adults with previously untreated glioblastoma multiforme. Human Gene Therapy 2000;11(17):2389-401.

84. Immonen A, Vapalahti M, Tyynela K, Hurskainen H, Sandmair A, Vanninen R, Langford G, Murray N, Yla-Herttuala S. AdvHSV-tk gene therapy with intravenous ganciclovir improves survival in human malignant glioma: a randomised, controlled study. Molecular Therapy 2004;10(5):967-72.

85. Both GW. Gene-directed enzyme prodrug therapy for cancer: a glimpse into the future? Discovery Medicine 2009;8(42):97-103.

86. Mesnil M, Yamasaki H. Bystander effect in herpes simplex virus-thymidine kinase/ganciclovir cancer gene therapy: role of gap-junctional intercellular communication. Cancer Research 2000;60(15):3989-99.

87. Yamasaki H, Krutovskikh V, Mesnil M, Tanaka T, Zaidan-Dagli ML, Omori Y. Role of connexin (gap junction) genes in cell growth control and carcinogenesis. Comptes Rendus De l'Académie Des Sciences. Série III, Sciences De La Vie 1999 Mar;322(2-3):151-159.

88. Touraine RL, Vahanian N, Ramsey WJ, Blaese RM. Enhancement of the herpes simplex virus thymidine kinase/ganciclovir bystander effect and its antitumor efficacy in vivo by pharmacologic manipulation of gap junctions. Human Gene Therapy 1998;9(16):2385-91.

89. Nicholas TW, Read SB, Burrows FJ, Kruse CA. Suicide gene therapy with Herpes simplex virus thymidine kinase and ganciclovir is enhanced with connexins to improve gap junctions and bystander effects. Histology and Histopathology 2003;18(2):495-507.

90. Tasciotti E, Giacca M. Fusion of the human immunodeficiency virus type 1 tat protein transduction domain to thymidine kinase increases bystander effect and induces enhanced tumor killing in vivo. Human Gene Therapy 2005;16(12):1389403.

91. Mullen CA, Kilstrup M, Blaese RM. Transfer of the bacterial gene for cytosine deaminase to mammalian cells confers lethal sensitivity to 5-fluorocytosine: a negative selection system. Proceedings of the National Academy of Sciences of the United States of America 1992;89(1):33-7. 
92. Longley DB, Harkin DP, Johnston PG. 5-fluorouracil: mechanisms of action and clinical strategies. Nature Reviews Cancer 2003;3(5):330-8.

93. Domin BA, Mahony WB, Zimmerman TP. Transport of 5-fluorouracil and uracil into human erythrocytes. Biochemical Pharmacology 1993;46(3):503-10.

94. Huber BE, Austin EA, Richards CA, Davis ST, Good SS. Metabolism of 5fluorocytosine to 5-fluorouracil in human colorectal tumor cells transduced with the cytosine deaminase gene: significant antitumor effects when only a small percentage of tumor cells express cytosine deaminase. Proceedings of the National Academy of Sciences of the United States of America 1994;91(17):8302-6.

95. Ichikawa T, Tamiya T, Adachi Y, Ono Y, Matsumoto K, Furuta T, Yoshida Y, Hamada $\mathrm{H}$, Ohmoto T. In vivo efficacy and toxicity of 5-fluorocytosine/cytosine deaminase gene therapy for malignant gliomas mediated by adenovirus. Cancer Gene Therapy 2000;7(1):74-82.

96. Haberkorn U, Oberdorfer F, Gebert J, Morr I, Haack K, Weber K, Lindauer M, van Kaick G, Schackert HK. Monitoring gene therapy with cytosine deaminase: in vitro studies using tritiated-5-fluorocytosine. Journal of Nuclear Medecine 1996;37(1):87-94.

97. van Kuilenburg AB. Dihydropyrimidine dehydrogenase and the efficacy and toxicity of 5-fluorouracil. European Journal of Cancer 2004;40(7):939-50.

98. Freytag SO, Khil M, Stricker H, Peabody J, Menon M, DePeralta-Venturina M, Nafziger D, Pegg J, Paielli D, Brown S, Barton K, Lu M, Aguilar-Cordova E, Kim JH. Phase I study of replication-competent adenovirus-mediated double suicide gene therapy for the treatment of locally recurrent prostate cancer. Cancer Research 2002;62(17):4968-76.

99. Roy P, Waxman DJ. Activation of oxazaphosphorines by cytochrome P450: application to gene-directed enzyme prodrug therapy for cancer. Toxicology In Vitro 2006;20(2):176-86.

100. Patel P, Young JG, Mautner V, Ashdown D, Bonney S, Pineda RG, Collins SI, Searle PF, Hull D, Peers E, Chester J, Wallace DM, Doherty A, Leung H, Young LS, James ND. A phase I/II clinical trial in localized prostate cancer of an adenovirus expressing nitroreductase with CB1954. Molecular Therapy 2009;17(7):1292-9.

101. Marais R, Spooner RA, Light Y, Martin J, Springer CJ. Gene-directed enzyme prodrug therapy with a mustard prodrug/carboxypeptidase G2 combination. Cancer Research 1996;56(20):4735-42.

102. Mayer A, Francis RJ, Sharma SK, Tolner B, Springer CJ, Martin J, Boxer GM, Bell J, Green AJ, Hartley JA, Cruickshank C, Wren J, Chester KA, Begent RH. A phase I study of single administration of antibody-directed enzyme prodrug therapy with the recombinant anti-carcinoembryonic antigen antibody-enzyme fusion protein MFECP1 and a bis-iodo phenol mustard prodrug. Clinical Cancer Research 2006;12(21):6509-16. 
103. Sladek NE. Metabolism of oxazaphosphorines. Pharmacology and Therapeutics 1988;37(3):301-55.

104. Chen L, Waxman DJ. Intratumoral activation and enhanced chemotherapeutic effect of oxazaphosphorines following cytochrome P-450 gene transfer: development of a combined chemotherapy/cancer gene therapy strategy. Cancer Research 1995;55(3):581-9.

105. Hong PS, Srigritsanapol A, Chan KK. Pharmacokinetics of 4hydroxycyclophosphamide and metabolites in the rat. Drug Metabolism and Disposition: The Biological Fate of Chemicals 1991;19(1):1-7.

106. Khan AH, Ross WC. Tumour-growth inhibitory nitrophenylaziridines and related compounds: structure-activity relationships. Chemico-Biological Interactions 1969 Oct;1(1):27-47.

107. Cobb LM, Connors TA, Elson LA, Khan AH, Mitchley BC, Ross WC, Whisson ME. 2,4-dinitro-5-ethyleneiminobenzamide (CB 1954): a potent and selective inhibitor of the growth of the Walker carcinoma 256. Biochemical Pharmacology 1969 Jun;18(6):1519-1527.

108. Connors TA, Melzack DH. Studies on the mechanism of action of 5-aziridinyl2,4-dinitrobenzamide (CB 1954), a selective inhibitor of the Walker tumour. International Journal of Cancer 1971 Jan;7(1):86-92.

109. Knox RJ, Friedlos F, Jarman M, Roberts JJ. A new cytotoxic, DNA interstrand crosslinking agent, 5-(aziridin-1-yl)-4-hydroxylamino-2-nitrobenzamide, is formed from 5-(aziridin-1-yl)-2,4-dinitrobenzamide (CB 1954) by a nitroreductase enzyme in Walker carcinoma cells. Biochemical Pharmacology 1988 Dec;37(24):4661-4669.

110. Knox RJ, Friedlos F, Marchbank T, Roberts JJ. Bioactivation of CB 1954: reaction of the active 4-hydroxylamino derivative with thioesters to form the ultimate DNA-DNA interstrand crosslinking species. Biochemical Pharmacology 1991 Oct;42(9):1691-1697.

111. Helsby NA, Wheeler SJ, Pruijn FB, Palmer BD, Yang S, Denny WA, Wilson WR. Effect of nitroreduction on the alkylating reactivity and cytotoxicity of the 2,4-dinitrobenzamide-5-aziridine CB 1954 and the corresponding nitrogen mustard SN 23862: distinct mechanisms of bioreductive activation. Chemical Research in Toxicology 2003 Apr;16(4):469-478.

112. Friedlos F, Quinn J, Knox RJ, Roberts JJ. The properties of total adducts and interstrand crosslinks in the DNA of cells treated with CB 1954. Exceptional frequency and stability of the crosslink. Biochemical Pharmacology 1992 Mar;43(6):1249-1254.

113. Knox RJ, Burke PJ, Chen S, Kerr DJ. CB 1954: from the Walker tumor to NQO2 and VDEPT. Current Pharmaceutical Design 2003;9(26):2091-2104.

114. Bridgewater JA, Knox RJ, Pitts JD, Collins MK, Springer CJ. The bystander effect of the nitroreductase/CB1954 enzyme/prodrug system is due to a cellpermeable metabolite. Human Gene Therapy 1997;8(6):709-17. 
115. Djeha AH, Hulme A, Dexter MT, Mountain A, Young LS, Searle PF, Kerr DJ, Wrighton CJ. Expression of Escherichia coli B nitroreductase in established human tumor xenografts in mice results in potent antitumoral and bystander effects upon systemic administration of the prodrug CB1954. Cancer Gene Therapy 2000 May;7(5):721-731.

116. Green NK, Youngs DJ, Neoptolemos JP, Friedlos F, Knox RJ, Springer CJ, Anlezark GM, Michael NP, Melton RG, Ford MJ, Young LS, Kerr DJ, Searle PF. Sensitization of colorectal and pancreatic cancer cell lines to the prodrug 5(aziridin-1-yl)-2,4-dinitrobenzamide (CB1954) by retroviral transduction and expression of the E. coli nitroreductase gene. Cancer Gene Therapy 1997 Aug;4(4):229-238.

117. McNeish IA, Green NK, Gilligan MG, Ford MJ, Mautner V, Young LS, Kerr DJ, Searle PF. Virus directed enzyme prodrug therapy for ovarian and pancreatic cancer using retrovirally delivered E. coli nitroreductase and CB1954. Gene Therapy 1998 Aug;5(8):1061-1069.

118. Friedlos F, Court S, Ford M, Denny WA, Springer C. Gene-directed enzyme prodrug therapy: quantitative bystander cytotoxicity and DNA damage induced by CB1954 in cells expressing bacterial nitroreductase. Gene Therapy 1998 Jan;5(1):105-112.

119. Cui W, Gusterson B, Clark AJ. Nitroreductase-mediated cell ablation is very rapid and mediated by a p53-independent apoptotic pathway. Gene Therapy 1999 May;6(5):764-770.

120. Boland MP, Knox RJ, Roberts JJ. The differences in kinetics of rat and human DT diaphorase result in a differential sensitivity of derived cell lines to CB 1954 (5-(aziridin-1-yl)-2,4-dinitrobenzamide). Biochemical Pharmacology 1991 Apr;41(6-7):867-875.

121. Helsby NA, Ferry DM, Patterson AV, Pullen SM, Wilson WR. 2-Amino metabolites are key mediators of CB 1954 and SN 23862 bystander effects in nitroreductase GDEPT. British Journal of Cancer 2004 Mar;90(5):1084-1092.

122. Anlezark GM, Melton RG, Sherwood RF, Coles B, Friedlos F, Knox RJ. The bioactivation of 5-(aziridin-1-yl)-2,4-dinitrobenzamide (CB1954)--I. Purification and properties of a nitroreductase enzyme from Escherichia coli--a potential enzyme for antibody-directed enzyme prodrug therapy (ADEPT). Biochemical Pharmacology 1992 Dec;44(12):2289-2295.

123. Bryant C, DeLuca M. Purification and characterization of an oxygen-insensitive $\mathrm{NAD}(\mathrm{P}) \mathrm{H}$ nitroreductase from Enterobacter cloacae. Journal of Biological Chemistry 1991 Mar;266(7):4119-4125.

124. Bryant C, Hubbard L, McElroy WD. Cloning, nucleotide sequence, and expression of the nitroreductase gene from Enterobacter cloacae. Journal of Biological Chemistry 1991 Mar;266(7):4126-4130.

125. Watanabe M, Ishidate M, Nohmi T. Nucleotide sequence of Salmonella typhimurium nitroreductase gene. Nucleic Acids Research 1990 Feb;18(4):1059. 
126. McCalla DR, Kaiser C, Green MH. Genetics of nitrofurazone resistance in Escherichia coli. Journal of Bacteriology 1978 Jan;133(1):10-16.

127. Sastry SS, Jayaraman R. Nitrofurantoin-resistant mutants of Escherichia coli: isolation and mapping. Molecular and General Genetics 1984;196(2):379-380.

128. Bridgewater JA, Springer CJ, Knox RJ, Minton NP, Michael NP, Collins MK. Expression of the bacterial nitroreductase enzyme in mammalian cells renders them selectively sensitive to killing by the prodrug CB1954. European Journal of Cancer 1995 Dec;31A(13-14):2362-2370.

129. Drabek D, Guy J, Craig R, Grosveld F. The expression of bacterial nitroreductase in transgenic mice results in specific cell killing by the prodrug CB1954. Gene Therapy 1997 Feb;4(2):93-100.

130. Westphal EM, Ge J, Catchpole JR, Ford M, Kenney SC. The nitroreductase/CB1954 combination in Epstein-Barr virus-positive B-cell lines: induction of bystander killing in vitro and in vivo. Cancer Gene Therapy 2000 Jan;7(1):97-106.

131. Weedon SJ, Green NK, McNeish IA, Gilligan MG, Mautner V, Wrighton CJ, Mountain A, Young LS, Kerr DJ, Searle PF. Sensitisation of human carcinoma cells to the prodrug CB1954 by adenovirus vector-mediated expression of E. coli nitroreductase. International Journal of Cancer 2000 Jun;86(6):848-854.

132. Blackwood L, O’Shaughnessy PJ, Reid SW, Argyle DJ. E. coli nitroreductase/CB1954: in vitro studies into a potential system for feline cancer gene therapy. The Veterinary Journal 2001 May;161(3):269-279.

133. Chen M-J, Green NK, Reynolds GM, Flavell JR, Mautner V, Kerr DJ, Young LS, Searle PF. Enhanced efficacy of Escherichia coli nitroreductase/CB1954 prodrug activation gene therapy using an E1B-55K-deleted oncolytic adenovirus vector. Gene Therapy 2004 Jul;11(14):1126-1136.

134. Benouchan M, Do Nascimento F, Perret GY, Colombo BM. Delivery of the bacterial nitroreductase gene into endothelial cells prolongs the survival of tumour-bearing mice by bystander mechanisms. International Journal of Oncology 2006 Feb;28(2):457-462.

135. Bilsland AE, Anderson CJ, Fletcher-Monaghan AJ, McGregor F, Evans TRJ, Ganly I, Knox RJ, Plumb JA, Keith WN. Selective ablation of human cancer cells by telomerase-specific adenoviral suicide gene therapy vectors expressing bacterial nitroreductase. Oncogene 2003 Jan;22(3):370-380.

136. Chung-Faye G, Palmer D, Anderson D, Clark J, Downes M, Baddeley J, Hussain S, Murray PI, Searle P, Seymour L, Harris PA, Ferry D, Kerr DJ. Virus-directed, enzyme prodrug therapy with nitroimidazole reductase: a phase I and pharmacokinetic study of its prodrug, CB1954. Clinical Cancer Research 2001 Sep;7(9):2662-2668.

137. Palmer DH, Mautner V, Mirza D, Oliff S, Gerritsen W, van der Sijp JRM, Hubscher S, Reynolds G, Bonney S, Rajaratnam R, Hull D, Horne M, Ellis J, Mountain A, Hill S, Harris PA, Searle PF, Young LS, James ND, Kerr DJ. Virusdirected enzyme prodrug therapy: intratumoral administration of a replication- 
deficient adenovirus encoding nitroreductase to patients with resectable liver cancer. Journal of Clinical Oncology 2004 May;22(9):1546-1552.

138. Onion D, Patel P, Pineda RG, James N, Mautner V. Antivector and tumor immune responses following adenovirus-directed enzyme prodrug therapy for the treatment of prostate cancer. Human Gene Therapy 2009 Nov;20(11):1249-1258.

139. Tang MHY, Helsby NA, Goldthorpe MA, Thompson KM, Al-Ali S, Tingle MD. Hepatic nitroreduction, toxicity and toxicokinetics of the anti-tumour prodrug CB 1954 in mouse and rat. Toxicology 2007 Oct;240(1-2):70-85.

140. Tang MHY, Helsby NA, Wilson WR, Tingle MD. Aerobic 2- and 4nitroreduction of CB 1954 by human liver. Toxicology 2005 Dec;216(2-3):129139.

141. Chandor A, Dijols S, Ramassamy B, Frapart Y, Mansuy D, Stuehr D, Helsby N, Boucher J-L. Metabolic activation of the antitumor drug 5-(Aziridin-1-yl)-2,4dinitrobenzamide (CB1954) by NO synthases. Chemical Research in Toxicology 2008 Apr;21(4):836-843.

142. Wu K, Knox R, Sun XZ, Joseph P, Jaiswal AK, Zhang D, Deng PS, Chen S. Catalytic properties of NAD(P)H:quinone oxidoreductase-2 (NQO2), a dihydronicotinamide riboside dependent oxidoreductase. Archives of Biochemistry and Biophysics 1997 Nov;347(2):221-228.

143. Knox RJ, Jenkins TC, Hobbs SM, Chen S, Melton RG, Burke PJ. Bioactivation of 5-(aziridin-1-yl)-2,4-dinitrobenzamide (CB 1954) by human NAD(P)H quinone oxidoreductase 2: a novel co-substrate-mediated antitumor prodrug therapy. Cancer Research 2000 Aug;60(15):4179-4186.

144. Middleton MR, Knox R, Cattell E, Oppermann U, Midgley R, Ali R, Auton T, Agarwal R, Anderson D, Sarker D, Judson I, Osawa T, Spanswick VJ, Davies S, Hartley JA, Kerr DJ. Quinone oxidoreductase-2-mediated prodrug cancer therapy. Science Translational Medicine 2010 Jul;2(40):40-50.

145. Helsby NA, Atwell GJ, Yang S, Palmer BD, Anderson RF, Pullen SM, Ferry DM, Hogg A, Wilson WR, Denny WA. Aziridinyldinitrobenzamides: synthesis and structure-activity relationships for activation by E. coli nitroreductase. Journal of Medicinal Chemistry 2004 Jun;47(12):3295-3307.

146. Mauger AB, Burke PJ, Somani HH, Friedlos F, Knox RJ. Self-immolative prodrugs: candidates for antibody-directed enzyme prodrug therapy in conjunction with a nitroreductase enzyme. Journal of Medicinal Chemistry 1994 Oct;37(21):3452-3458.

147. Hu L, Yu C, Jiang Y, Han J, Li Z, Browne P, Race PR, Knox RJ, Searle PF, Hyde EI. Nitroaryl phosphoramides as novel prodrugs for E. coli nitroreductase activation in enzyme prodrug therapy. Journal of Medicinal Chemistry 2003 Nov;46(23):4818-4821.

148. Sagnou MJ, Howard PW, Gregson SJ, Eno-Amooquaye E, Burke PJ, Thurston DE. Design and synthesis of novel pyrrolobenzodiazepine (PBD) prodrugs for ADEPT and GDEPT. Bioorganic and Medicinal Chemistry Letters 2000 Sep;10(18):2083-2086. 
149. Hay MP, Atwell GJ, Wilson WR, Pullen SM, Denny WA. Structure-activity relationships for 4-nitrobenzyl carbamates of 5-aminobenz[e]indoline minor groove alkylating agents as prodrugs for GDEPT in conjunction with E. coli nitroreductase. Journal of Medicinal Chemistry 2003 Jun;46(12):2456-2466.

150. Hay MP, Anderson RF, Ferry DM, Wilson WR, Denny WA. Synthesis and evaluation of nitroheterocyclic carbamate prodrugs for use with nitroreductasemediated gene-directed enzyme prodrug therapy. Journal of Medicinal Chemistry 2003 Dec;46(25):5533-5545.

151. Anlezark GM, Melton RG, Sherwood RF, Wilson WR, Denny WA, Palmer BD, Knox RJ, Friedlos F, Williams A. Bioactivation of dinitrobenzamide mustards by an E. coli B nitroreductase. Biochemical Pharmacology 1995 Aug;50(5):609-618.

152. Friedlos F, Denny WA, Palmer BD, Springer CJ. Mustard prodrugs for activation by Escherichia coli nitroreductase in gene-directed enzyme prodrug therapy. Journal of Medicinal Chemistry 1997 Apr;40(8):1270-1275.

153. Wilson WR, Pullen SM, Hogg A, Helsby NA, Hicks KO, Denny WA. Quantitation of bystander effects in nitroreductase suicide gene therapy using three-dimensional cell cultures. Cancer Research 2002 Mar;62(5):1425-1432.

154. Palmer BD, Wilson WR, Cliffe S, Denny WA. Hypoxia-selective antitumor agents. 5. Synthesis of water-soluble nitroaniline mustards with selective cytotoxicity for hypoxic mammalian cells. Journal of Medicinal Chemistry 1992 Aug;35(17):3214-3222.

155. Atwell GJ, Yang S, Pruijn FB, Pullen SM, Hogg A, Patterson AV, Wilson WR, Denny WA. Synthesis and structure-activity relationships for 2,4dinitrobenzamide-5-mustards as prodrugs for the Escherichia coli nfsB nitroreductase in gene therapy. Journal of Medicinal Chemistry 2007;50(6):1197212.

156. Mahan SD, Ireton GC, Knoeber C, Stoddard BL, Black ME. Random mutagenesis and selection of Escherichia coli cytosine deaminase for cancer gene therapy. Protein Engineering, Design and Selection 2004 Aug;17(8):625-633.

157. Seubert CM, Stritzker J, Hess M, Donat U, Sturm JB, Chen N, von Hof JM, Krewer B, Tietze LF, Gentschev I, Szalay AA. Enhanced tumor therapy using vaccinia virus strain GLV-1h68 in combination with a $\beta$-galactosidase-activatable prodrug seco-analog of duocarmycin SA. Cancer Gene Therapy 2011 Jan;18(1):42-52.

158. Kievit E, Bershad E, Ng E, Sethna P, Dev I, Lawrence TS, Rehemtulla A. Superiority of yeast over bacterial cytosine deaminase for enzyme/prodrug gene therapy in colon cancer xenografts. Cancer Research 1999 Apr;59(7):1417-1421.

159. Hamstra DA, Rice DJ, Fahmy S, Ross BD, Rehemtulla A. Enzyme/prodrug therapy for head and neck cancer using a catalytically superior cytosine deaminase. Human Gene Therapy 1999 Aug;10(12):1993-2003.

160. Kestell P, Pruijn FB, Siim BG, Palmer BD, Wilson WR. Pharmacokinetics and metabolism of the nitrogen mustard bioreductive drug 5. Cancer Chemotherapy and Pharmacology 2000;46(5):365-374. 
161. Patel K, Choy SSF, Hicks KO, Melink TJ, Holford NHG, Wilson WR. A combined pharmacokinetic model for the hypoxia-targeted prodrug PR-104A in humans, dogs, rats and mice predicts species differences in clearance and toxicity [Internet]. Cancer Chemotherapy and Pharmacology 2011 May;67(5):1145-1155

162. Roldán MD, Pérez-Reinado E, Castillo F, Moreno-Vivián C. Reduction of polynitroaromatic compounds: the bacterial nitroreductases. FEMS Microbiology Reviews 2008 May;32(3):474-500.

163. Peterson FJ, Mason RP, Hovsepian J, Holtzman JL. Oxygen-sensitive and insensitive nitroreduction by Escherichia coli and rat hepatic microsomes. Journal of Biological Chemistry 1979 May;254(10):4009-4014.

164. Smith AL, Erwin AL, Kline T, Unrath WCT, Nelson K, Weber A, Howald WN. Chloramphenicol is a substrate for a novel nitroreductase pathway in Haemophilus influenzae. Antimicrobial Agents and Chemotherapy 2007 Aug;51(8):2820-2829.

165. Liochev SI, Hausladen A, Fridovich I. Nitroreductase A is regulated as a member of the soxRS regulon of Escherichia coli. Proceedings of the National Academy of Sciences of the United States of America 1999 Mar;96(7):3537-3539.

166. Zenno S, Saigo K, Kanoh H, Inouye S. Identification of the gene encoding the major NAD(P)H-flavin oxidoreductase of the bioluminescent bacterium Vibrio fischeri ATCC 7744. Journal of Bacteriology 1994 Jun;176(12):3536-3543.

167. Zenno S, Kobori T, Tanokura M, Saigo K. Purification and characterization of NfrA1, a Bacillus subtilis nitro/flavin reductase capable of interacting with the bacterial luciferase. Bioscience, Biotechnology, and Biochemistry 1998 Oct;62(10):1978-1987.

168. Pérez-Reinado E, Roldán MD, Castillo F, Moreno-Vivián C. The NprA nitroreductase required for 2,4-dinitrophenol reduction in Rhodobacter capsulatus is a dihydropteridine reductase. Environmental Microbiology 2008 Nov;10(11):3174-3183.

169. Taga ME, Larsen NA, Howard-Jones AR, Walsh CT, Walker GC. BluB cannibalizes flavin to form the lower ligand of vitamin B12. Nature 2007 Mar;446(7134):449-453.

170. Cortial S, Chaignon P, Iorga BI, Aymerich S, Truan G, Gueguen-Chaignon V, Meyer P, Moréra S, Ouazzani J. NADH oxidase activity of Bacillus subtilis nitroreductase NfrA1: insight into its biological role. FEBS Letters 2010 Sep;584(18):3916-3922.

171. Olekhnovich IN, Goodwin A, Hoffman PS. Characterization of the NAD(P)H oxidase and metronidazole reductase activities of the RdxA nitroreductase of Helicobacter pylori. FEBS Journal 2009 Jun;276(12):3354-3364.

172. Jenks PJ, Edwards DI. Metronidazole resistance in Helicobacter pylori. International Journal of Antimicrobial Agents 2002 Jan;19(1):1-7.

173. Whiteway J, Koziarz P, Veall J, Sandhu N, Kumar P, Hoecher B, Lambert IB. Oxygen-insensitive nitroreductases: analysis of the roles of nfs A and nfsB in 
development of resistance to 5-nitrofuran derivatives in Escherichia coli. Journal of Bacteriology 1998 Nov;180(21):5529-5539.

174. Koder RL, Miller AF. Steady-state kinetic mechanism, stereospecificity, substrate and inhibitor specificity of Enterobacter cloacae nitroreductase. Biochimica et Biophysica Acta 1998 Sep;1387(1-2):395-405.

175. Haynes CA, Koder RL, Miller A-F, Rodgers DW. Structures of nitroreductase in three states: effects of inhibitor binding and reduction. Journal of Biological Chemistry 2002 Mar;277(13):11513-11520.

176. Zenno S, Koike H, Kumar AN, Jayaraman R, Tanokura M, Saigo K. Biochemical characterization of NfsA, the Escherichia coli major nitroreductase exhibiting a high amino acid sequence homology to Frp, a Vibrio harveyi flavin oxidoreductase. Journal of Bacteriology 1996 Aug;178(15):4508-4514.

177. Zenno S, Koike H, Tanokura M, Saigo K. Gene cloning, purification, and characterization of $\mathrm{NfsB}$, a minor oxygen-insensitive nitroreductase from Escherichia coli, similar in biochemical properties to FRase I, the major flavin reductase in Vibrio fischeri. Journal of Biochemistry 1996 Oct;120(4):736-744.

178. Kobori T, Lee WC, Akagi T, Sasaki H, Zenno S, Saigo K, Tanokura M. Crystallization and preliminary crystallographic analysis of major nitroreductase from Escherichia coli. Acta Crystallographica. Section D, Biological Crystallography 1999 Nov;55(Pt 11):1901-1902.

179. Tanner JJ, Lei B, Tu SC, Krause KL. Flavin reductase P: structure of a dimeric enzyme that reduces flavin. Biochemistry 1996 Oct;35(42):13531-13539.

180. Lovering AL, Hyde EI, Searle PF, White SA. The structure of Escherichia coli nitroreductase complexed with nicotinic acid: three crystal forms at $1.7 \mathrm{~A}, 1.8 \mathrm{~A}$ and 2.4 A resolution. Journal of Molecular Biology 2001 May;309(1):203-213.

181. Kutty R, Bennett GN. Biochemical characterization of trinitrotoluene transforming oxygen-insensitive nitroreductases from Clostridium acetobutylicum ATCC 824. Archives of Microbiology 2005 Nov;184(3):158-167.

182. Pérez-Reinado E, Blasco R, Castillo F, Moreno-Vivián C, Roldán MD. Regulation and characterization of two nitroreductase genes, nprA and nprB, of Rhodobacter capsulatus. Applied and Environmental Microbiology 2005 Dec;71(12):7643-7649.

183. Manina G, Bellinzoni M, Pasca MR, Neres J, Milano A, Ribeiro ALDJL, Buroni S, Skovierová H, Dianišková P, Mikušová K, Marák J, Makarov V, Giganti D, Haouz A, Lucarelli AP, Degiacomi G, Piazza A, Chiarelli LR, De Rossi E, Salina E, Cole ST, Alzari PM, Riccardi G. Biological and structural characterization of the Mycobacterium smegmatis nitroreductase $\mathrm{NfnB}$, and its role in benzothiazinone resistance. Molecular Microbiology 2010 Sep;77(5):1172-1185.

184. Ackerley DF, Gonzalez CF, Keyhan M, Blake R, Matin A. Mechanism of chromate reduction by the Escherichia coli protein, $\mathrm{NfsA}$, and the role of different chromate reductases in minimizing oxidative stress during chromate reduction. Environmental Microbiology 2004 Aug;6(8):851-860. 
185. Hall BS, Wu X, Hu L, Wilkinson SR. Exploiting the drug-activating properties of a novel trypanosomal nitroreductase. Antimicrobial Agents and Chemotherapy 2010 Mar;54(3):1193-1199.

186. Wilkinson SR, Taylor MC, Horn D, Kelly JM, Cheeseman I. A mechanism for cross-resistance to nifurtimox and benznidazole in trypanosomes. Proceedings of the National Academy of Sciences of the United States of America 2008 Apr;105(13):5022-5027.

187. Williams RE, Rathbone DA, Scrutton NS, Bruce NC. Biotransformation of explosives by the old yellow enzyme family of flavoproteins. Applied and Environmental Microbiology 2004 Jun;70(6):3566-3574.

188. Zenno S, Kobori T, Tanokura M, Saigo K. Conversion of NfsA, the major Escherichia coli nitroreductase, to a flavin reductase with an activity similar to that of Frp, a flavin reductase in Vibrio harveyi, by a single amino acid substitution. Journal of Bacteriology 1998 Jan;180(2):422-425.

189. Vass SO, Jarrom D, Wilson WR, Hyde EI, Searle PF. E. coli NfsA: an alternative nitroreductase for prodrug activation gene therapy in combination with CB1954. British Journal of Cancer 2009 Jun;100(12):1903-1911.

190. González-Pérez MM, van Dillewijn P, Wittich R-M, Ramos JL. Escherichia coli has multiple enzymes that attack TNT and release nitrogen for growth. Environmental Microbiology 2007 Jun;9(6):1535-1540.

191. Lei B, Liu M, Huang S, Tu SC. Vibrio harveyi NADPH-flavin oxidoreductase: cloning, sequencing and overexpression of the gene and purification and characterization of the cloned enzyme. Journal of Bacteriology 1994 Jun;176(12):3552-3558.

192. Caballero A, Lázaro JJ, Ramos JL, Esteve-Núñez A. PnrA, a new nitroreductasefamily enzyme in the TNT-degrading strain Pseudomonas putida JLR11. Environmental Microbiology 2005 Aug;7(8):1211-1219.

193. Nokhbeh MR, Boroumandi S, Pokorny N, Koziarz P, Paterson ES, Lambert IB. Identification and characterization of SnrA, an inducible oxygen-insensitive nitroreductase in Salmonella enterica serovar Typhimurium TA1535. Mutation Research 2002 Oct;508(1-2):59-70.

194. Morokutti A, Lyskowski A, Sollner S, Pointner E, Fitzpatrick TB, Kratky C, Gruber K, Macheroux P. Structure and function of YcnD from Bacillus subtilis, a flavin-containing oxidoreductase. Biochemistry 2005 Oct;44(42):13724-13733.

195. Streker K, Freiberg C, Labischinski H, Hacker J, Ohlsen K. Staphylococcus aureus NfrA (SA0367) is a flavin mononucleotide-dependent NADPH oxidase involved in oxidative stress response. Journal of Bacteriology 2005 Apr;187(7):2249-2256.

196. Nivinskas H, Staskeviciene S, Sarlauskas J, Koder RL, Miller AF, Cenas N. Twoelectron reduction of quinones by Enterobacter cloacae NAD $(\mathrm{P}) \mathrm{H}$ :nitroreductase: quantitative structure-activity relationships. Archives of Biochemistry and Biophysics 2002 Jul;403(2):249-258. 
197. Watanabe M, Nishino T, Takio K, Sofuni T, Nohmi T. Purification and characterization of wild-type and mutant "classical" nitroreductases of Salmonella typhimurium. L33R mutation greatly diminishes binding of FMN to the nitroreductase of S. typhimurium. Journal of Biological Chemistry 1998 Sep;273(37):23922-23928.

198. Yanto Y, Hall M, Bommarius AS. Nitroreductase from Salmonella typhimurium: characterization and catalytic activity. Organic and Biomolecular Chemistry 2010 Apr;8(8):1826-1832.

199. Emptage CD, Knox RJ, Danson MJ, Hough DW. Nitroreductase from Bacillus licheniformis: a stable enzyme for prodrug activation. Biochemical Pharmacology 2009 Jan;77(1):21-29.

200. Gwenin CD, Kalaji M, Williams PA, Kay CM. A kinetic analysis of three modified novel nitroreductases. Biodegradation 2011 Apr;22(2):463-474

201. French CE, Nicklin S, Bruce NC. Aerobic degradation of 2,4,6-trinitrotoluene by Enterobacter cloacae PB2 and by pentaerythritol tetranitrate reductase. Applied and Environmental Microbiology 1998 Aug;64(8):2864-2868.

202. Pak JW, Knoke KL, Noguera DR, Fox BG, Chambliss GH. Transformation of 2,4,6-trinitrotoluene by purified xenobiotic reductase B from Pseudomonas fluorescens I-C. Applied and Environmental Microbiology 2000 Nov;66(11):4742-4750.

203. Liu G, Zhou J, Lv H, Xiang X, Wang J, Zhou M, Qv Y. Azoreductase from Rhodobacter sphaeroides AS1.1737 is a flavodoxin that also functions as nitroreductase and flavin mononucleotide reductase. Applied Microbiology and Biotechnology 2007 Oct;76(6):1271-1279.

204. Takeda K, Iizuka M, Watanabe T, Nakagawa J, Kawasaki S, Niimura Y. Synechocystis DrgA protein functioning as nitroreductase and ferric reductase is capable of catalyzing the Fenton reaction. FEBS Journal 2007 Mar;274(5):13181327.

205. Takahashi S, Furuya T, Ishii Y, Kino K, Kirimura K. Characterization of a flavin reductase from a thermophilic dibenzothiophene-desulfurizing bacterium, Bacillus subtilis WU-S2B. Journal of Bioscience and Bioengineering 2009 Jan;107(1):38-41.

206. Berne C, Betancor L, Luckarift HR, Spain JC. Application of a microfluidic reactor for screening cancer prodrug activation using silica-immobilized nitrobenzene nitroreductase. Biomacromolecules 2006 Sep;7(9):2631-2636.

207. Anlezark GM, Vaughan T, Fashola-Stone E, Michael NP, Murdoch H, Sims MA, Stubbs S, Wigley S, Minton NP. Bacillus amyloliquefaciens orthologue of Bacillus subtilis ywrO encodes a nitroreductase enzyme which activates the prodrug CB 1954. Microbiology (Reading, England) 2002 Jan;148(Pt 1):297-306.

208. Theys J, Pennington O, Dubois L, Anlezark G, Vaughan T, Mengesha A, Landuyt W, Anné J, Burke PJ, Dûrre P, Wouters BG, Minton NP, Lambin P. Repeated cycles of Clostridium-directed enzyme prodrug therapy result in 
sustained antitumour effects in vivo. British Journal of Cancer 2006 Nov;95(9):1212-1219.

209. Chen S, Knox R, Lewis AD, Friedlos F, Workman P, Deng PS, Fung M, Ebenstein D, Wu K, Tsai TM. Catalytic properties of NAD $(\mathrm{P}) \mathrm{H}$ :quinone acceptor oxidoreductase: study involving mouse, rat, human, and mouse-rat chimeric enzymes. Molecular Pharmacology 1995 May;47(5):934-939.

210. Morley KL, Kazlauskas RJ. Improving enzyme properties: when are closer mutations better? Trends in Biotechnology 2005 May;23(5):231-237.

211. Bloom JD, Meyer MM, Meinhold P, Otey CR, MacMillan D, Arnold FH. Evolving strategies for enzyme engineering. Current Opinion in Structural Biology 2005 Aug;15(4):447-452.

212. Black ME, Newcomb TG, Wilson HM, Loeb LA. Creation of drug-specific herpes simplex virus type 1 thymidine kinase mutants for gene therapy. Proceedings of the National Academy of Sciences of the United States of America 1996 Apr;93(8):3525-3529.

213. Black ME, Kokoris MS, Sabo P. Herpes simplex virus-1 thymidine kinase mutants created by semi-random sequence mutagenesis improve prodrugmediated tumor cell killing. Cancer Research 2001 Apr;61(7):3022-3026.

214. Kokoris MS, Black ME. Characterization of herpes simplex virus type 1 thymidine kinase mutants engineered for improved ganciclovir or acyclovir activity. Protein Science 2002 Sep;11(9):2267-2272.

215. Wiewrodt R, Amin K, Kiefer M, Jovanovic VP, Kapoor V, Force S, Chang M, Lanuti M, Black ME, Kaiser LR, Albelda SM. Adenovirus-mediated gene transfer of enhanced Herpes simplex virus thymidine kinase mutants improves prodrug-mediated tumor cell killing. Cancer Gene Therapy 2003 May;10(5):353364.

216. Stolworthy TS, Korkegian AM, Willmon CL, Ardiani A, Cundiff J, Stoddard BL, Black ME. Yeast cytosine deaminase mutants with increased thermostability impart sensitivity to 5-fluorocytosine. Journal of Molecular Biology 2008 Mar;377(3):854-869.

217. Kaliberov SA, Market JM, Gillespie GY, Krendelchtchikova V, Della Manna D, Sellers JC, Kaliberova LN, Black ME, Buchsbaum DJ. Mutation of Escherichia coli cytosine deaminase significantly enhances molecular chemotherapy of human glioma. Gene Therapy 2007 Jul;14(14):1111-1119.

218. Grove JI, Lovering AL, Guise C, Race PR, Wrighton CJ, White SA, Hyde EI, Searle PF. Generation of Escherichia coli nitroreductase mutants conferring improved cell sensitization to the prodrug CB1954. Cancer Research 2003 Sep;63(17):5532-5537.

219. Jaberipour M, Vass SO, Guise CP, Grove JI, Knox RJ, Hu L, Hyde EI, Searle PF. Testing double mutants of the enzyme nitroreductase for enhanced cell sensitisation to prodrugs: effects of combining beneficial single mutations. Biochemical Pharmacology 2010;79(2):102-11. 
220. Jarrom D, Jaberipour M, Guise CP, Daff S, White SA, Searle PF, Hyde EI. Steady-state and stopped-flow kinetic studies of three Escherichia coli NfsB mutants with enhanced activity for the prodrug CB1954. Biochemistry 2009 Aug;48(32):7665-7672.

221. Jackson JC, Duffy SP, Hess KR, Mehl RA. Improving nature's enzyme active site with genetically encoded unnatural amino acids. Journal of the American Chemical Society 2006 Aug;128(34):11124-11127.

222. Barak Y, Thorne SH, Ackerley DF, Lynch SV, Contag CH, Matin A. New enzyme for reductive cancer chemotherapy, YieF, and its improvement by directed evolution. Molecular Cancer Therapeutics 2006 Jan;5(1):97-103.

223. Liu L, Li Y, Liotta D, Lutz S. Directed evolution of an orthogonal nucleoside analog kinase via fluorescence-activated cell sorting. Nucleic Acids Research 2009 Jul;37(13):4472-4481.

224. Thorne SH, Barak Y, Liang W, Bachmann MH, Rao J, Contag CH, Matin A. CNOB/ChrR6, a new prodrug enzyme cancer chemotherapy. Molecular Cancer Therapeutics 2009 Feb;8(2):333-341.

225. Guise CP, Grove JI, Hyde EI, Searle PF. Direct positive selection for improved nitroreductase variants using SOS triggering of bacteriophage lambda lytic cycle. Gene Therapy 2007;14(8):690-8.

226. Janion C. Inducible SOS response system of DNA repair and mutagenesis in Escherichia coli. International Journal of Biological Sciences 2008;4(6):338-344.

227. Roberts JW, Roberts CW. Proteolytic cleavage of bacteriophage lambda repressor in induction. Proceedings of the National Academy of Sciences of the United States of America 1975 Jan;72(1):147-151.

228. Quillardet P, Huisman O, D’Ari R, Hofnung M. SOS chromotest, a direct assay of induction of an SOS function in Escherichia coli K-12 to measure genotoxicity. Proceedings of the National Academy of Sciences of the United States of America 1982 Oct;79(19):5971-5975.

229. Norman A, Hestbjerg Hansen L, Sørensen SJ. Construction of a ColD cda promoter-based SOS-green fluorescent protein whole-cell biosensor with higher sensitivity toward genotoxic compounds than constructs based on recA, umuDC, or sulA promoters. Applied and Environmental Microbiology 2005 May;71(5):2338-2346.

230. Davidov Y, Rozen R, Smulski DR, Van Dyk TK, Vollmer AC, Elsemore DA, LaRossa RA, Belkin S. Improved bacterial SOS promoter::lux fusions for genotoxicity detection. Mutation Research 2000 Mar;466(1):97-107.

231. Shapiro E, Baneyx F. Stress-based identification and classification of antibacterial agents: second-generation Escherichia coli reporter strains and optimization of detection. Antimicrobial Agents and Chemotherapy 2002 Aug;46(8):2490-2497. 
232. Bartolome A, Mandap K, David KJ, Sevilla F, Villanueva J. SOS-red fluorescent protein (RFP) bioassay system for monitoring of antigenotoxic activity in plant extracts. Biosensors and Bioelectronics 2006 May;21(11):2114-2120.

233. Venitt S, Crofton-Sleigh C. The toxicity and mutagenicity of the anti-tumour drug 5-aziridino-2,4-dinitrobenzamide (CB1954) is greatly reduced in a nitroreductase-deficient strain of E. coli. Mutagenesis 1987 Sep;2(5):375-381.

234. Dale LD, Widdick DA, Edwards DI, Biol GI. Comparative DNA damage and repair induced by misonidazole, CB 1954 and RSU 1069. International Journal of Radiation Oncology, Biology, Physics 1989 Apr;16(4):995-999.

235. Gajewska J, Szczypka M, Tudek B, Szymczyk T. Studies on the effect of ascorbic acid and selenium on the genotoxicity of nitrofurans: nitrofurazone and furazolidone. Mutation Research 1990 Oct;232(2):191-197.

236. Sutton MD, Smith BT, Godoy VG, Walker GC. The SOS response: recent insights into umuDC-dependent mutagenesis and DNA damage tolerance. Annual Review of Genetics 2000;34:479-497.

237. Datsenko KA, Wanner BL. One-step inactivation of chromosomal genes in Escherichia coli K-12 using PCR products. Proceedings of the National Academy of Sciences of the United States of America 2000 Jun;97(12):6640-6645.

238. Altschul SF, Gish W, Miller W, Myers EW, Lipman DJ. Basic local alignment search tool. Journal of Molecular Biology 1990 Oct;215(3):403-410.

239. Sambrook J, Russel DW. Molecular cloning: a laboratory manual, 3rd Edition, Volume 1. Cold Spring Harbour Laboratory Press, New York; 2001.

240. Laemmli UK. Cleavage of structural proteins during the assembly of the head of bacteriophage T4. Nature 1970 Aug;227(5259):680-685.

241. Race PR, Lovering AL, White SA, Grove JI, Searle PF, Wrighton CW, Hyde EI. Kinetic and structural characterisation of Escherichia coli nitroreductase mutants showing improved efficacy for the prodrug substrate CB1954. Journal of Molecular Biology 2007 Apr;368(2):481-492.

242. Alksne LE, Burgio P, Hu W, Feld B, Singh MP, Tuckman M, Petersen PJ, Labthavikul P, McGlynn M, Barbieri L, McDonald L, Bradford P, Dushin RG, Rothstein D, Projan SJ. Identification and analysis of bacterial protein secretion inhibitors utilizing a SecA-LacZ reporter fusion system. Antimicrobial Agents and Chemotherapy 2000 Jun;44(6):1418-1427.

243. Miller JH. Experiments in molecular genetics. Cold Spring Harbor Laboratory Press, New York; 1972.

244. Patel K, Lewiston D, Gu Y, Hicks KO, Wilson WR. Analysis of the hypoxiaactivated dinitrobenzamide mustard phosphate pre-prodrug PR-104 and its alcohol metabolite PR-104A in plasma and tissues by liquid chromatographymass spectrometry. Journal of Chromatography B. Analytical Technologies in the Biomedical and Life Sciences 2007 Sep;856(1-2):302-311. 
245. Skehan P, Storeng R, Scudiero D, Monks A, McMahon J, Vistica D, Warren JT, Bokesch H, Kenney S, Boyd MR. New colorimetric cytotoxicity assay for anticancer-drug screening. Journal of the National Cancer Institute 1990 Jul;82(13):1107-1112.

246. Guex N, Peitsch MC. SWISS-MODEL and the Swiss-PdbViewer: an environment for comparative protein modeling. Electrophoresis 1997 Dec;18(15):2714-2723.

247. Prosser GA, Copp JN, Syddall SP, Williams EM, Smaill JB, Wilson WR, Patterson AV, Ackerley DF. Discovery and evaluation of Escherichia coli nitroreductases that activate the anti-cancer prodrug CB1954. Biochemical Pharmacology 2010;79(5):678-87.

248. Vollmer AC, Belkin S, Smulski DR, Van Dyk TK, LaRossa RA. Detection of DNA damage by use of Escherichia coli carrying recA'::lux, uvrA'::lux, or alkA'::lux reporter plasmids. Applied and Environmental Microbiology 1997 Jul;63(7):2566-2571.

249. Oda Y, Nakamura S, Oki I, Kato T, Shinagawa H. Evaluation of the new system (umu-test) for the detection of environmental mutagens and carcinogens. Mutation Research 1985 Oct;147(5):219-229.

250. Hofnung M, Quillardet P. The SOS Chromotest, a colorimetric assay based on the primary cellular responses to genotoxic agents. Annals of the New York Academy of Sciences 1988;534:817-825.

251. Gu MB, Min J, LaRossa RA. Bacterial bioluminescent emission from recombinant Escherichia coli harboring a recA::luxCDABE fusion. Journal of Biochemical and Biophysical Methods 2000 Aug;45(1):45-56.

252. McCool JD, Long E, Petrosino JF, Sandler HA, Rosenberg SM, Sandler SJ. Measurement of SOS expression in individual Escherichia coli K-12 cells using fluorescence microscopy. Molecular Microbiology 2004 Sep;53(5):1343-1357.

253. Kuzminov A. Recombinational repair of DNA damage in Escherichia coli and bacteriophage lambda. Microbiology and Molecular Biology Reviews 1999 Dec;63(4):751-813, table of contents.

254. Janion C. Some aspects of the SOS response system--a critical survey. Acta Biochimica Polonica 2001;48(3):599-610.

255. Rosen R, Davidov Y, LaRossa RA, Belkin S. Microbial sensors of ultraviolet radiation based on recA'::lux fusions. Applied Biochemistry and Biotechnology 2000 Dec;89(2-3):151-160.

256. Wentworth DF, Wolfenden R. Slow binding of D-galactal, a "reversible" inhibitor of bacterial beta-galactosidase. Biochemistry 1974 Nov;13(23):47154720 .

257. Miller S, Ness LS, Wood CM, Fox BC, Booth IR. Identification of an ancillary protein, YabF, required for activity of the KefC glutathione-gated potassium efflux system in Escherichia coli. Journal of Bacteriology 2000 Nov;182(22):6536-6540. 
258. Choi JW, Lee J, Kosuke N, Jung CH, Kim JS. Crystallization and preliminary Xray diffraction analysis of ydjA, a minimal nitroreductase from Escherichia coli K12. Acta Crystallographica. Section F, Structural Biology and Crystallization Communications 2007 Dec;63(Pt 12):1064-1066.

259. Nakanishi M, Yatome C, Ishida N, Kitade Y. Putative ACP phosphodiesterase gene (acpD) encodes an azoreductase. Journal of Biological Chemistry 2001 Dec;276(49):46394-46399.

260. Liu G, Zhou J, Fu QS, Wang J. The Escherichia coli azoreductase AzoR Is involved in resistance to thiol-specific stress caused by electrophilic quinones. Journal of Bacteriology 2009 Oct;191(20):6394-6400.

261. Loh KD, Gyaneshwar P, Markenscoff Papadimitriou E, Fong R, Kim K-S, Parales R, Zhou Z, Inwood W, Kustu S. A previously undescribed pathway for pyrimidine catabolism. Proceedings of the National Academy of Sciences of the United States of America 2006 Mar;103(13):5114-5119.

262. Adams MA, Jia Z. Modulator of drug activity B from Escherichia coli: crystal structure of a prokaryotic homologue of DT-diaphorase. Journal of Molecular Biology 2006 Jun;359(2):455-465.

263. Carey J, Brynda J, Wolfová J, Grandori R, Gustavsson T, Ettrich R, Smatanová IK. WrbA bridges bacterial flavodoxins and eukaryotic NAD $(\mathrm{P}) \mathrm{H}$ :quinone oxidoreductases. Protein Science 2007 Oct;16(10):2301-2305.

264. Jorgensen MA, Trend MA, Hazell SL, Mendz GL. Potential involvement of several nitroreductases in metronidazole resistance in Helicobacter pylori. Archives of Biochemistry and Biophysics 2001 Aug;392(2):180-191.

265. Dawson RMC, Elliott DC, Elliott WH. Data for Biochemical Research. Oxford University Press, Oxford; 1989.

266. Blackwell JR, Horgan R. A novel strategy for production of a highly expressed recombinant protein in an active form. FEBS Letters 1991 Dec;295(1-3):10-12.

267. van Dillewijn P, Wittich R-M, Caballero A, Ramos J-L. Type II hydride transferases from different microorganisms yield nitrite and diarylamines from polynitroaromatic compounds. Applied and Environmental Microbiology 2008 Nov;74(21):6820-6823.

268. Cleland WW. The kinetics of enzyme-catalyzed reactions with two or more substrates or products. I. Nomenclature and rate equations. 1963. Biochimica et Biophysica Acta 1989;1000:213-220.

269. Chen CY, Chang YN, Ryan P, Linscott M, McGarrity GJ, Chiang YL. Effect of herpes simplex virus thymidine kinase expression levels on ganciclovir-mediated cytotoxicity and the "bystander effect". Human Gene Therapy 1995 Nov;6(11):1467-1476.

270. Fuchita M, Ardiani A, Zhao L, Serve K, Stoddard BL, Black ME. Bacterial cytosine deaminase mutants created by molecular engineering show improved 5fluorocytosine-mediated cell killing in vitro and in vivo. Cancer Research 2009 Jun;69(11):4791-4799. 
271. Taverna P, Liu L, Hanson AJ, Monks A, Gerson SL. Characterization of MLH1 and MSH2 DNA mismatch repair proteins in cell lines of the NCI anticancer drug screen. Cancer Chemotherapy and Pharmacology 2000;46(6):507-516.

272. Yaginuma $\mathrm{Y}$, Westphal $\mathrm{H}$. Abnormal structure and expression of the p53 gene in human ovarian carcinoma cell lines. Cancer Research 1992 Aug;52(15):41964199.

273. Russo P, Arzani D, Trombino S, Falugi C. c-myc down-regulation induces apoptosis in human cancer cell lines exposed to RPR-115135 (C31H29NO4), a non-peptidomimetic farnesyltransferase inhibitor. Journal of Pharmacology and Experimental Therapeutics 2003 Jan;304(1):37-47.

274. Adimoolam S, Ford JM. p53 and regulation of DNA damage recognition during nucleotide excision repair. DNA Repair (Amst) 2003 Sep;2(9):947-954.

275. Klaidman LK, Leung AC, Adams JD. High-performance liquid chromatography analysis of oxidized and reduced pyridine dinucleotides in specific brain regions. Analytical Biochemistry 1995 Jul;228(2):312-317.

276. London J, Knight M. Concentrations of nicotinamide nucleotide coenzymes in micro-organisms. Journal of General Microbiology 1966 Aug;44(2):241-254.

277. Yu Q, Heikal AA. Two-photon autofluorescence dynamics imaging reveals sensitivity of intracellular NADH concentration and conformation to cell physiology at the single-cell level. Journal of Photochemistry and Photobiology B: Biology 2009 Apr;95(1):46-57.

278. Oda Y. Induction of SOS responses in Escherichia coli by 5-fluorouracil. Mutation Research 1987 Mar;183(2):103-108.

279. Ito K, Nakanishi M, Lee W-C, Sasaki H, Zenno S, Saigo K, Kitade Y, Tanokura M. Three-dimensional structure of AzoR from Escherichia coli. An oxidereductase conserved in microorganisms. Journal of Biological Chemistry 2006 Jul;281(29):20567-20576.

280. Workman P, White RA, Talbot K. CB 1954 revisited. I. Disposition kinetics and metabolism. Cancer Chemotherapy and Pharmacology 1986;16(1):1-8.

281. Smith MA, Edwards DI. Redox potential and oxygen concentration as factors in the susceptibility of Helicobacter pylori to nitroheterocyclic drugs. Journal of Antimicrobial Chemotherapy 1995 Jun;35(6):751-764.

282. Meisel D, Czapski G. One-electron transfer equilibriums and redox potentials of radicals studied by pulse radiolysis. The Journal of Physical Chemistry 1975 Jul;79(15):1503-1509.

283. Patridge EV, Ferry JG. WrbA from Escherichia coli and Archaeoglobus fulgidus is an $\mathrm{NAD}(\mathrm{P}) \mathrm{H}$ :quinone oxidoreductase. Journal of Bacteriology 2006 May;188(10):3498-3506.

284. Hayashi M, Ohzeki H, Shimada H, Unemoto T. NADPH-specific quinone reductase is induced by 2-methylene-4-butyrolactone in Escherichia coli. Biochimica et Biophysica Acta 1996 Feb;1273(2):165-170. 
285. Gale PJ, Bentz BL, Chait BT, Field FH, Cotter RJ. Reduction in liquid secondary ion mass spectrometry. Comparison of the fission fragment and liquid secondary ion mass spectra of organic dyestuffs. Analytical Chemistry 1986 May;58(6):1070-1076.

286. Rau J, Stolz A. Oxygen-insensitive nitroreductases NfsA and NfsB of Escherichia coli function under anaerobic conditions as lawsone-dependent Azo reductases. Applied and Environmental Microbiology 2003 Jun;69(6):3448-3455.

287. Krwawicz J, Arczewska KD, Speina E, Maciejewska A, Grzesiuk E. Bacterial DNA repair genes and their eukaryotic homologues: 1. Mutations in genes involved in base excision repair (BER) and DNA-end processors and their implication in mutagenesis and human disease. Acta Biochimica Polonica 2007;54(3):413-434.

288. Kleibl K. Molecular mechanisms of adaptive response to alkylating agents in Escherichia coli and some remarks on O(6)-methylguanine DNAmethyltransferase in other organisms. Mutation Research 2002 Sep;512(1):67-84.

289. Kunkel TA, Erie DA. DNA mismatch repair. Annual Review of Biochemistry 2005;74:681-710.

290. Daviet S, Couvé-Privat S, Gros L, Shinozuka K, Ide H, Saparbaev M, Ishchenko AA. Major oxidative products of cytosine are substrates for the nucleotide incision repair pathway. DNA Repair (Amst) 2007 Jan;6(1):8-18.

291. Rasmussen LJ, Samson L. The Escherichia coli MutS DNA mismatch binding protein specifically binds O(6)-methylguanine DNA lesions. Carcinogenesis 1996 Sep;17(9):2085-2088.

292. Pitsikas P, Polosina YY, Cupples CG. Interaction between the mismatch repair and nucleotide excision repair pathways in the prevention of 5-azacytidineinduced CG-to-GC mutations in Escherichia coli. DNA Repair (Amst) 2009 Mar;8(3):354-359.

293. Goerlich O, Quillardet P, Hofnung M. Induction of the SOS response by hydrogen peroxide in various Escherichia coli mutants with altered protection against oxidative DNA damage. Journal of Bacteriology 1989 Nov;171(11):61416147.

294. Müller J, Janz S. Assessment of oxidative DNA damage in the oxyR-deficient SOS chromotest strain Escherichia coli PQ300. Environmental and Molecular Mutagenesis 1992;20(4):297-306.

295. Salmelin C, Vilpo J. Induction of SOS response, cellular efflux and oxidative stress response genes by chlorambucil in DNA repair-deficient Escherichia coli cells (ada, ogt and mutS). Mutation Research 2003 Jan;522(1-2):33-44.

296. Wyatt MD, Pittman DL. Methylating agents and DNA repair responses: Methylated bases and sources of strand breaks. Chemical Research in Toxicology 2006 Dec;19(12):1580-1594.

297. Duarte MP, Palma BB, Laires A, Oliveira JS, Rueff J, Kranendonk M.

Escherichia coli BTC, a human cytochrome P450 competent tester strain with a 
high sensitivity towards alkylating agents: involvement of alkyltransferases in the repair of DNA damage induced by aromatic amines. Mutagenesis 2005 May;20(3):199-208.

298. Wang L, Spratt TE, Liu XK, Hecht SS, Pegg AE, Peterson LA. Pyridyloxobutyl adduct O6-[4-oxo-4-(3-pyridyl)butyl]guanine is present in 4(acetoxymethylnitrosamino)-1-(3-pyridyl)-1-butanone-treated DNA and is a substrate for O6-alkylguanine-DNA alkyltransferase. Chemical Research in Toxicology 1997 May;10(5):562-567.

299. Basak J, Mukherjee U, Chatterjee SN. Adaptive response of Vibrio cholerae and Escherichia coli to nitrofurantoin. Environmental and Molecular Mutagenesis 1992;20(1):53-60.

300. Knox RJ, Friedlos F, Biggs PJ, Flitter WD, Gaskell M, Goddard P, Davies L, Jarman M. Identification, synthesis and properties of 5-(aziridin-1-yl)-2-nitro-4nitrosobenzamide, a novel DNA crosslinking agent derived from CB1954. Biochemical Pharmacology 1993 Sep;46(5):797-803.

301. Vericat JA, Guerrero R, Barbé J. Inhibition of the SOS response of Escherichia coli by the Ada protein. Journal of Bacteriology 1988 Mar;170(3):1354-1359.

302. Fourrier L, Brooks P, Malinge J-M. Binding discrimination of MutS to a set of lesions and compound lesions (base damage and mismatch) reveals its potential role as a cisplatin-damaged DNA sensing protein. Journal of Biological Chemistry 2003 Jun;278(23):21267-21275.

303. Ho TV, Schärer OD. Translesion DNA synthesis polymerases in DNA interstrand crosslink repair. Environmental and Molecular Mutagenesis 2010 Jul;51(6):552566.

304. Prosser GA, Patterson AV, Ackerley DF. uvrB gene deletion enhances SOS chromotest sensitivity for nitroreductases that preferentially generate the 4hydroxylamine metabolite of the anti-cancer prodrug CB1954. Journal of Biotechnology 2010 Oct;150(1):190-194.

305. Sedgwick B, Lindahl T. Recent progress on the Ada response for inducible repair of DNA alkylation damage. Oncogene 2002 Dec;21(58):8886-8894.

306. Verdijk RM, Wilke M, Beslier V, Kloosterman A, Brand A, Goulmy E, Mutis T. Escherichia coli-nitroreductase suicide gene control of human telomerase reverse transcriptase-transduced minor histocompatibility antigen-specific cytotoxic $\mathrm{T}$ cells. Bone Marrow Transplant 2004 May;33(9):963-967.

307. Lindmark DG, Müller M. Antitrichomonad action, mutagenicity, and reduction of metronidazole and other nitroimidazoles. Antimicrobical Agents and Chemotherapy 1976 Sep;10(3):476-482.

308. McCalla DR, Olive P, Tu Y, Fan ML. Nitrofurazone-reducing enzymes in E. coli and their role in drug activation in vivo. Canadian Journal of Microbiology 1975 Oct;21(10):1484-1491.

309. De Méo M, Vanelle P, Bernadini E, Laget M, Maldonado J, Jentzer O, Crozet MP, Duménil G. Evaluation of the mutagenic and genotoxic activities of 48 
nitroimidazoles and related imidazole derivatives by the Ames test and the SOS chromotest. Environmental and Molecular Mutagenesis 1992;19(2):167-181.

310. Ona KR, Courcelle CT, Courcelle J. Nucleotide excision repair is a predominant mechanism for processing nitrofurazone-induced DNA damage in Escherichia coli. Journal of Bacteriology 2009 Aug;191(15):4959-4965.

311. Buschini A, Ferrarini L, Franzoni S, Galati S, Lazzaretti M, Mussi F, Northfleet de Albuquerque C, Maria Araújo Domingues Zucchi T, Poli P. Genotoxicity revaluation of three commercial nitroheterocyclic drugs: nifurtimox, benznidazole, and metronidazole. Journal of Parasitology Research 2009;2009:463575.

312. Elizondo G, Gonsebatt ME, Salazar AM, Lares I, Santiago P, Herrera J, Hong E, Ostrosky-Wegman P. Genotoxic effects of metronidazole. Mutation Research 1996 Sep;370(2):75-80.

313. Jin DJ, Gross CA. Mapping and sequencing of mutations in the Escherichia coli rpoB gene that lead to rifampicin resistance. Journal of Molecular Biology 1988 Jul;202(1):45-58.

314. Nicholson WL, Maughan $\mathrm{H}$. The spectrum of spontaneous rifampin resistance mutations in the rpoB gene of Bacillus subtilis 168 spores differs from that of vegetative cells and resembles that of Mycobacterium tuberculosis. Journal of Bacteriology 2002 Sep;184(17):4936-4940.

315. Garibyan L, Huang T, Kim M, Wolff E, Nguyen A, Nguyen T, Diep A, Hu K, Iverson A, Yang H, Miller JH. Use of the rpoB gene to determine the specificity of base substitution mutations on the Escherichia coli chromosome. DNA Repair (Amst) 2003 May;2(5):593-608.

316. Cussac C, Laval F. Reduction of the toxicity and mutagenicity of aziridine in mammalian cells harboring the Escherichia coli fpg gene. Nucleic Acids Research 1996 May;24(9):1742-1746.

317. Gill RD, Cussac C, Souhami RL, Laval F. Increased resistance to N,N',N"triethylenethiophosphoramide (thiotepa) in cells expressing the Escherichia coli formamidopyrimidine-DNA glycosylase. Cancer Research 1996 Aug;56(16):3721-3724.

318. Wang H, Zhang S-Y, Wang S, Lu J, Wu W, Weng L, Chen D, Zhang Y, Lu Z, Yang J, Chen Y, Zhang X, Chen X, Xi C, Lu D, Zhao S. REV3L confers chemoresistance to cisplatin in human gliomas: the potential of its RNAi for synergistic therapy. Neuro-oncology 2009 Dec;11(6):790-802.

319. Xie K, Doles J, Hemann MT, Walker GC. Error-prone translesion synthesis mediates acquired chemoresistance. Proceedings of the National Academy of Sciences of the United States of America 2010 Nov;107(48):20792-20797.

320. Okuda T, Lin X, Trang J, Howell SB. Suppression of hREV1 expression reduces the rate at which human ovarian carcinoma cells acquire resistance to cisplatin. Molecular Pharmacology 2005 Jun;67(6):1852-1860. 
321. Purohit V, Basu AK. Mutagenicity of nitroaromatic compounds. Chemical Research in Toxicology 2000 Aug;13(8):673-692.

322. Benigni R, Palombo F, Dogliotti E. Multivariate statistical analysis of mutational spectra of alkylating agents. Mutation Research 1992 May;267(1):77-88.

323. Ohta T, Ohmae S, Yamaya K, Kanemichi Y, Tokishita S, Yamagata H. Characterization of the mutational specificity of DNA cross-linking mutagens by the Lac+ reversion assay with Escherichia coli. Teratogenesis, Carcinogenesis, and Mutagenesis 2001;21(4):275-282.

324. Matsui K, Yamada M, Imai M, Yamamoto K, Nohmi T. Specificity of replicative and SOS-inducible DNA polymerases in frameshift mutagenesis: mutability of Salmonella typhimurium strains overexpressing SOS-inducible DNA polymerases to 30 chemical mutagens. DNA Repair (Amst) 2006 Apr;5(4):465478.

325. Koronakis V, Eswaran J, Hughes C. Structure and function of TolC: the bacterial exit duct for proteins and drugs. Annual Review of Biochemistry 2004;73:467489.

326. Sulavik MC, Houseweart C, Cramer C, Jiwani N, Murgolo N, Greene J, DiDomenico B, Shaw KJ, Miller GH, Hare R, Shimer G. Antibiotic susceptibility profiles of Escherichia coli strains lacking multidrug efflux pump genes. Antimicrobial Agents and Chemotherapy 2001 Apr;45(4):1126-1136.

327. Kuete V, Alibert-Franco S, Eyong KO, Ngameni B, Folefoc GN, Nguemeving JR, Tangmouo JG, Fotso GW, Komguem J, Ouahouo BMW, Bolla J-M, Chevalier J, Ngadjui BT, Nkengfack AE, Pagès J-M. Antibacterial activity of some natural products against bacteria expressing a multidrug-resistant phenotype. International Journal of Antimicrobial Agents 2011 Feb;37(2):156161.

328. Aono R, Tsukagoshi N, Yamamoto M. Involvement of outer membrane protein TolC, a possible member of the mar-sox regulon, in maintenance and improvement of organic solvent tolerance of Escherichia coli K-12. Journal of Bacteriology 1998 Feb;180(4):938-944.

329. Mueller NJ, Stueckler C, Hall M, Macheroux P, Faber K. Epoxidation of conjugated $\mathrm{C}=\mathrm{C}$-bonds and sulfur-oxidation of thioethers mediated by NADH:FMN-dependent oxidoreductases. Organic and Biomolecular Chemistry 2009 Mar;7(6):1115-1119.

330. Adams MA, Jia Z. Structural and biochemical evidence for an enzymatic quinone redox cycle in Escherichia coli: identification of a novel quinol monooxygenase. Journal of Biological Chemistry 2005 Mar;280(9):8358-8363.

331. Palmer BD, van Zijl P, Denny WA, Wilson WR. Reductive chemistry of the novel hypoxia-selective cytotoxin 5-[N,N-bis(2-chloroethyl)amino]-2,4dinitrobenzamide. Journal of Medicinal Chemistry 1995 Mar;38(7):1229-1241.

332. Wilson WR, Stribbling SM, Pruijn FB, Syddall SP, Patterson AV, Liyanage HDS, Smith E, Botting KJ, Tercel M. Nitro-chloromethylbenzindolines: hypoxia- 
activated prodrugs of potent adenine N3 DNA minor groove alkylators. Molecular Cancer Therapeutics 2009 Oct;8(10):2903-2913.

333. Sorrentino BP. Gene therapy to protect haematopoietic cells from cytotoxic cancer drugs. Nature Reviews Cancer 2002 Jun;2(6):431-441.

334. Dachs GU, Hunt MA, Syddall S, Singleton DC, Patterson AV. Bystander or no bystander for gene directed enzyme prodrug therapy. Molecules 2009;14(11):4517-4545.

335. Koh WJ, Rasey JS, Evans ML, Grierson JR, Lewellen TK, Graham MM, Krohn KA, Griffin TW. Imaging of hypoxia in human tumors with [F18]fluoromisonidazole. International Journal of Radiation Oncology, Biology, Physics 1992;22(1):199-212.

336. Komar G, Seppänen M, Eskola O, Lindholm P, Grönroos TJ, Forsback S, Sipilä H, Evans SM, Solin O, Minn H. 18F-EF5: a new PET tracer for imaging hypoxia in head and neck cancer. Journal of Nuclear Medicine 2008 Dec;49(12):19441951.

337. Padhani A. PET imaging of tumour hypoxia. Cancer Imaging 2006;6:S117-121.

338. Chen C-S, Lin JT, Goss KA, He Y-ai, Halpert JR, Waxman DJ. Activation of the anticancer prodrugs cyclophosphamide and ifosfamide: identification of cytochrome P450 2B enzymes and site-specific mutants with improved enzyme kinetics. Molecular Pharmacology 2004 May;65(5):1278-1285.

339. Malo MS, Husain Z. Positive selection vectors for high-fidelity PCR cloning. BioTechniques 2003 Jun;34(6):1250-1258.

340. Haag AF, Ostermeier C. Positive-selection vector for direct protein expression. BioTechniques 2009 May;46(6):453-457.

341. Ohashi-Kunihiro S, Yohda M, Masaki H, Machida M. A novel vector for positive selection of inserts harboring an open reading frame by translational coupling. BioTechniques 2007 Dec;43(6):751-752, 754.

342. Rombel IT, Sykes KF, Rayner S, Johnston SA. ORF-FINDER: a vector for highthroughput gene identification. Gene 2002 Jan;282(1-2):33-41.

343. Kobori T, Sasaki H, Lee WC, Zenno S, Saigo K, Murphy ME, Tanokura M. Structure and site-directed mutagenesis of a flavoprotein from Escherichia coli that reduces nitrocompounds: alteration of pyridine nucleotide binding by a single amino acid substitution. Journal of Biological Chemistry 2001 Jan;276(4):28162823.

344. Reetz MT, Kahakeaw D, Lohmer R. Addressing the numbers problem in directed evolution. Chembiochem 2008 Jul;9(11):1797-1804.

345. Kokoris MS, Sabo P, Adman ET, Black ME. Enhancement of tumor ablation by a selected HSV-1 thymidine kinase mutant. Gene Therapy 1999 Aug;6(8):14151426. 
346. Miseviciene L, Anusevicius Z, Sarlauskas J, Harris RJ, Scrutton NS, Cenas N. Two-electron reduction of quinones by Enterobacter cloacae PB2 pentaerythritol tetranitrate reductase: quantitative structure-activity relationships. Acta Biochimica Polonica 2007;54(2):379-385.

347. Vidal-Aroca F, Giannattasio M, Brunelli E, Vezzoli A, Plevani P, Muzi-Falconi $\mathrm{M}$, Bertoni G. One-step high-throughput assay for quantitative detection of betagalactosidase activity in intact gram-negative bacteria, yeast, and mammalian cells. BioTechniques 2006 Apr;40(4):433-434, 436, 438 passim.

348. Plovins A, Alvarez AM, Ibañez M, Molina M, Nombela C. Use of fluorescein-dibeta-D-galactopyranoside (FDG) and C12-FDG as substrates for betagalactosidase detection by flow cytometry in animal, bacterial, and yeast cells. Applied and Environmental Microbiology 1994 Dec;60(12):4638-4641.

349. Timmons L, Becker J, Barthmaier P, Fyrberg C, Shearn A, Fyrberg E. Green fluorescent protein/beta-galactosidase double reporters for visualizing Drosophila gene expression patterns. Developmental Genetics 1997;20(4):338-347.

350. Chalfie M, Kain S. Green fluorescent protein: properties, applications, and protocols. John Wiley and Sons; 2006.

351. Miller C, Thomsen LE, Gaggero C, Mosseri R, Ingmer H, Cohen SN. SOS response induction by beta-lactams and bacterial defense against antibiotic lethality. Science 2004 Sep;305(5690):1629-1631.

352. Janion C, Sikora A, Nowosielska A, Grzesiuk E. Induction of the SOS response in starved Escherichia coli. Environmental and Molecular Mutagenesis 2002;40(2):129-133.

353. Kunz BA, Glickman BW. Mechanism of mutation by thymine starvation in Escherichia coli: clues from mutagenic specificity. Journal of Bacteriology 1985 Jun;162(3):859-864.

354. García-Ruiz E, Maté D, Ballesteros A, Martinez AT, Alcalde M. Evolving thermostability in mutant libraries of ligninolytic oxidoreductases expressed in yeast. Microbial Cell Factories 2010;9:17.

355. Alcalde M. Mutagenesis protocols in Saccharomyces cerevisiae by in vivo overlap extension. Methods in Molecular Biology 2010;634:3-14.

356. Menacho-Márquez M, Murguía JR. Yeast on drugs: Saccharomyces cerevisiae as a tool for anticancer drug research. Clinical and Translational Oncology 2007 Apr;9(4):221-228.

357. Liu X, Kramer JA, Swaffield JC, Hu Y, Chai G, Wilson AGE. Development of a highthroughput yeast-based assay for detection of metabolically activated genotoxins. Mutation Research 2008 May;653(1-2):63-69.

358. Ichikawa K, Eki T. A novel yeast-based reporter assay system for the sensitive detection of genotoxic agents mediated by a DNA damage-inducible LexAGAL4 protein. Journal of Biochemistry 2006 Jan;139(1):105-112. 
359. Jia X, Zhu Y, Xiao W. A stable and sensitive genotoxic testing system based on DNA damage induced gene expression in Saccharomyces cerevisiae. Mutation Research 2002 Aug;519(1-2):83-92.

360. Jia X, Xiao W. Compromised DNA repair enhances sensitivity of the yeast RNR3-lacZ genotoxicity testing system. Toxicological Sciences 2003 Sep;75(1):82-88.

361. Aharoni A, Thieme K, Chiu CPC, Buchini S, Lairson LL, Chen H, Strynadka NCJ, Wakarchuk WW, Withers SG. High-throughput screening methodology for the directed evolution of glycosyltransferases. Nature Methods 2006 Aug;3(8):609-614.

362. Yang G, Withers SG. Ultrahigh-throughput FACS-based screening for directed enzyme evolution. Chembiochem 2009 Nov;10(17):2704-2715.

363. Park CH, Keyhan M, Wielinga B, Fendorf S, Matin A. Purification to homogeneity and characterization of a novel Pseudomonas putida chromate reductase. Applied and Environmental Microbiology 2000 May;66(5):1788-1795.

364. Meyer D, Witholt B, Schmid A. Suitability of recombinant Escherichia coli and Pseudomonas putida strains for selective biotransformation of m-nitrotoluene by xylene monooxygenase. Applied and Environmental Microbiology 2005 Nov;71(11):6624-6632.

365. Rinde E, Troll W. Colorimetric assay for aromatic amines. Analytical Chemistry 1976 Mar;48(3):542-544.

366. Ikawa M, Schaper TD, Dollard CA, Sasner JJ. Utilization of Folin-Ciocalteu phenol reagent for the detection of certain nitrogen compounds. Journal of Agricultural and Food Chemistry 2003 Mar;51(7):1811-1815.

367. De Wals P-Y, Doucet N, Pelletier JN. High tolerance to simultaneous active-site mutations in TEM-1 beta-lactamase: Distinct mutational paths provide more generalized beta-lactam recognition. Protein Science 2009 Jan;18(1):147-160.

368. Volpato JP, Fossati E, Pelletier JN. Increasing methotrexate resistance by combination of active-site mutations in human dihydrofolate reductase. Journal of Molecular Biology 2007 Oct;373(3):599-611.

369. Morley KL, Kazlauskas RJ. Improving enzyme properties: when are closer mutations better? Trends in Biotechnology 2005 May;23(5):231-237.

370. Chipperfield B. Ubiquinone concentrations in some tumour-bearing tissues. Ubiquinone concentrations in tumours and some normal tissues in man. Nature 1966 Mar;209(5029):1207-1208.

371. Picardo M, Grammatico P, Roccella F, Roccella M, Grandinetti M, Del Porto G, Passi S. Imbalance in the antioxidant pool in melanoma cells and normal melanocytes from patients with melanoma. Journal of Investigative Dermatology 1996 Sep;107(3):322-326. 
372. Portakal O, Ozkaya O, Erden Inal M, Bozan B, Koşan M, Sayek I. Coenzyme Q10 concentrations and antioxidant status in tissues of breast cancer patients. Clinical Biochemistry 2000 Jun;33(4):279-284.

373. Pantuck AJ, Matherly J, Zisman A, Nguyen D, Berger F, Gambhir SS, Black ME, Belldegrun A, Wu L. Optimizing prostate cancer suicide gene therapy using herpes simplex virus thymidine kinase active site variants. Human Gene Therapy 2002 May;13(7):777-789.

374. Ackerley DF, Gonzalez CF, Park CH, Blake R, Keyhan M, Matin A. Chromatereducing properties of soluble flavoproteins from Pseudomonas putida and Escherichia coli. Applied and Environmental Microbiology 2004 Feb;70(2):873882.

375. Chen H, Hopper SL, Cerniglia CE. Biochemical and molecular characterization of an azoreductase from Staphylococcus aureus, a tetrameric NADPH-dependent flavoprotein. Microbiology (Reading, England) 2005 May;151(Pt 5):1433-1441.

376. Luo C, Wang X, Long J, Liu J. An NADH-tetrazolium-coupled sensitive assay for malate dehydrogenase in mitochondria and crude tissue homogenates. Journal of Biochemical and Biophysical Methods 2006 Aug;68(2):101-111.

377. Johnson GR, Smets BF, Spain JC. Oxidative transformation of aminodinitrotoluene isomers by multicomponent dioxygenases. Applied and Environmental Microbiology 2001 Dec;67(12):5460-5466.

378. Ang EL, Obbard JP, Zhao H. Directed evolution of aniline dioxygenase for enhanced bioremediation of aromatic amines. Applied Microbiology and Biotechnology 2009 Jan;81(6):1063-1070.

379. Pisharath H, Parsons MJ. Nitroreductase-mediated cell ablation in transgenic zebrafish embryos. Methods in Molecular Biology 2009;546:133-143. 